\title{
Harnessing New Genomics Technologies to Assess Environmental Risk Factors That May Cause Heritable Genetic Disease.
}

\author{
by \\ Marc Andrew Beal
}

A thesis submitted to the Faculty of Graduate and Postdoctoral Affairs in partial fulfillment of the requirements for the degree of

Doctor of Philosophy

in

Biology

Carleton University

Ottawa, Ontario

(C) 2017, Marc Andrew Beal 


\section{Abstract}

It is becoming increasingly apparent that heritable mutations play a major role in developmental disorders, yet we still know little about the role of environmental agents in the etiology of heritable mutations. This is because germ cell mutations are rare and difficult to detect. In this thesis, novel methods were developed to address data gaps in our understanding of mutation induction in sperm and offspring. To test the hypothesis that mutagens induce germline mutations that are inherited in the offspring, MutaMouse males were exposed to $100 \mathrm{mg} / \mathrm{kg} /$ day of benzo[a]pyrene $(\mathrm{BaP})$, a known mutagen and common environmental pollutant, and mated to produce offspring. First, sperm from exposed males were analyzed for microsatellite mutations using a single-molecule amplification approach, and results confirmed that $\mathrm{BaP}$ induces microsatellite mutations. Second, the MutaMouse model, which uses a mutation reporting transgene, was adapted to facilitate mutation detection in sperm. Pairing this method with next-generation sequencing (NGS) revealed that BaP mutation spectrum in sperm differs from somatic tissues. Third, offspring of BaP-exposed males were screened for the induction of large copy number variations (CNVs). The quantity of $\mathrm{CNVs}$ in the $\mathrm{BaP}$ group was not significantly greater than controls; however, the types of CNVs were different, suggesting that $\mathrm{BaP}$ preferentially induces higher numbers of duplications. Fourth, genomes of the offspring were examined using NGS to detect all de novo mutations that were transmitted. Offspring of BaP-treated sires showed a 2-fold increase in genomewide mutations compared to controls. Induced mutations observed in BaP-treated mice were consistent with the expected BaP mutation spectrum. Lastly, using data from cigarette smoke exposure studies, a common route of BaP exposure, the global impact of 
smoking on genetic disease burden (1.4 million aneuploidies, 2-8 million mutations) was estimated conservatively at 86 billion dollars per generation for intellectual disease alone. Overall, this work furthers our understanding of heritable mutagenesis and allows for predictions to be made on the genetic disease burden of human exposures. Furthermore, these developments will be useful for future analyses of germ cell mutagens, and will be important for making recommendations on future assessments of mutagenic hazards to the germline. 


\section{Acknowledgements}

I am grateful for the 5 amazing years I have enjoyed as a $\mathrm{PhD}$ student at Health Canada and Carleton University. I would have never imagined how much my life would change when I headed to the Environmental Mutagenesis and Genomics Society meeting in Montreal in 2011. There, I spoke with Dr. Carole Yauk about a potential $\mathrm{PhD}$ and just over 5 years later here is my finished thesis! It has been a wonderful journey and I have many people I need to thank.

I'd like to start by thanking my supervisors Drs. Carole Yauk, Francesco Marchetti, and Iain Lambert. Your enthusiasm, patience, supportiveness, expertise, creativity, resourcefulness, brilliance (shall I go on?) made my experience more enjoyable, kept me motivated, and elevated the quality of my work. You may not even realize how many times I was anxious about something but after a quick meeting with you my mind was at ease. For example, early on I was eager to impress but was having issues with SM-PCR. After a rational discussion, I put my worries aside, and like many other instances, you gave me the drive to keep pushing on. I am also grateful for the countless other things you have done for me (support, advice, references, helpful edits, etc.). I hope this thesis serves as a testament to the great work we have accomplished together.

I have had the great fortune of working with some very talented and brilliant people. First, I have to give credit to my fellow members of the Sequencing Potential/Possible/Probable Environmentally Related Mutations (SPERM) group. It was a pleasure, to not only share with you any exciting updates, but to see the amazing things that you were working on. I am truly thankful for being able to share this experience with 
my SPERM brothers. Thank you Dr. Jason O'Brien for taking me under your wing early on, and Dr. Matt Meier for later filling that mentorship role (and of course analyzing Terabytes of genome data!). Thanks to my SPERM sister Andrea Rowan-Carroll for all her hard work helping me with SM-PCR.

Outside of the SPERM group there have been many other individuals at Health Canada that have helped make this thesis possible. First, I'd like to thank Remi Gagne for working closely with me on some very novel projects, and for your friendship as well. I look forward to more card games of "trop de queue" as I incorrectly say it. I owe many thanks to Andrew Williams for all his work with the CNV project and all his invaluable help with statistics. I would also like to thank John Gingerich, Lynda Soper, Beverly Allan, and Clotilde Maurice for all their support in the lab. Lastly, I need to say what a delight it was working alongside all my fellow students and postdoctoral fellows who have been great friends to me. We had some amazing times travelling to conferences (and some occasional extensions). I'll never forget our trip to Rio de Janeiro, especially the more dangerous memories! I'd also like to make a special mention of my fellow OttawaCarleton Institute of Biology Symposium co-chairs. Putting that symposium together with you was a truly wonderful experience and I am quite proud of our success.

Outside of the research group at Health Canada there are some important people to thank. I'd like to thank my external collaborators at the Wellcome Trust Sanger Institute, Dr. Matthew Hurles, and Dr. Sarah Lindsay, for their major contributions to the genome sequencing project. Thank you to my committee members Dr. Amanda MacFarlane and Dr. Alex Wong for meeting with me throughout my $\mathrm{PhD}$ and providing me with helpful suggestions. Furthermore, I would like to thank the members my 
examining committee, Dr. Thomas Glover and Dr. Kristin Connor, for their insightful comments and helpful suggestions.

I have been very fortunate to receive stipend support during my $\mathrm{PhD}$. I am specifically thankful for the support from Carleton University, the Natural Sciences and Engineering Research Council, and the Canadian Institutes of Health Research Training Program in Reproduction, Early Development, and the Impact on Health. I would also like to thank Dr. John Stavrinides and Dr. Chris Somers for providing me with the references that were crucial to obtain the support. Furthermore, I would also like to thank Dr. Somers for first sparking my interest in the field and encouraging me to pursue graduate studies.

I would like to thank my parents for all their support that has brought me to where I am today. This spans from reading to me at a young age and teaching me multiplication at the curling rink to letting me live at home during university. Also, I cannot thank you enough for having me at a young age and minimizing the number of de novo mutations you gave me!

Lastly, this section could not be completed without acknowledging the most important person of all: my wife Brittany. To you I owe the greatest thanks. You gave up your career and life in Regina to take a chance with me on a new life in Ottawa. What a journey it has been: several trips, an ultra-marathon (holding hands), a puppy, a house in the suburbs, and now our most cherished prize, a baby boy. Thank you for being by my side during graduate school. I'm excited for our next adventure together. 


\section{Table of Contents (brief)}

Chapter

Page

$\underline{1}$

Introduction

1

$\underline{2}$ Single-Molecule PCR Analys is of an Unstable Micros atellite for Detecting Mutations in Sperm of Mice Exposed

to Che mical Mutagens

3 Dose-Response Mutation and Spectrum Analyses Reveal Similar Responses at Two Micros atellite Loci in Benzo[a]pyre ne-Exposed Mouse Spermatogonia

$\underline{4}$ Characte rizing Benzo[a]pyrene-Induced lacZ Mutation Spectrum in Trans ge nic Mice Using Next-Generation Sequencing

$\underline{5}$ Benzo(a)pyrene is Mutagenic in Mouse Spermatogonial Stem

110 Cells and Dividing Spe rmatogonia

6 Next Generation Se quencing of B enzo(a)pyrene-Induced lac Z Mutants Identifies a Germ Cell-Specific Mutation Spectrum

7 Paternal Expos ure to Benzo(a)pyre ne Induces Genome-Wide Mutations in Offspring

8 From Sperm to Offspring: Ass essing the Heritable Genetic Consequences of Paternal Smoking and Potential Public Health Impacts

Discussion

Appendix

$\underline{\text { A }} \quad$ Trans genic Rodent Assay for Quantifying Male Germ Cell $\quad 308$ Mutant Frequency

B In Utero Exposure to Benzo[a]pyre ne Incre ases Mutation Burden in the Soma and Sperm of Adult Mice 


\section{Table of Contents (full)}

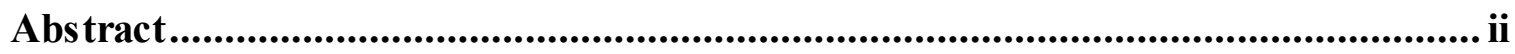

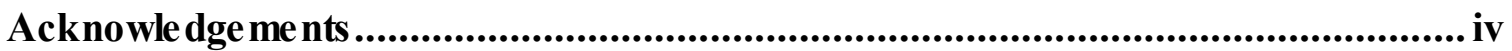

Table of Contents (brief) ............................................................................ vii

Table of Contents (full) ............................................................................ viii

List of Table s ................................................................................................. $\mathrm{xvi}$

List of Figures........................................................................................ xviii

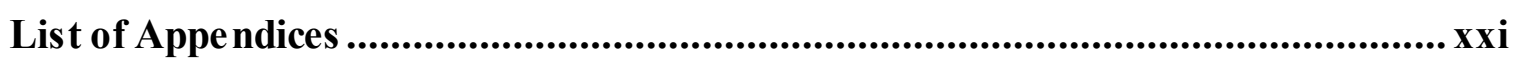

List of Abbre viations ....................................................................................... xxiii

State me nt of Contributions ............................................................................ xxv

Chapter 1: Introduction .............................................................................1

1.1 Importance of Studying de novo Mutations in Ge rm Cells ........................... 1

1.2 Spermatogenes is, Fe rtilization, and De velopme nt......................................3

1.3 His torical Overvie w of Methods Used to Study Germ Cell Mutage nicity ......... 7

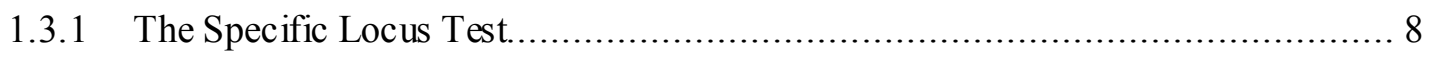

1.3.2 The Dominant Lethal Test...................................................... 9

1.3.3 The Heritable Translocation Test............................................................ 11

1.3.4 Tandem Repeat Mutation Analysis ................................................ 12

1.3.5 Transgenic Rodent Gene Mutation Assays........................................ 16

1.4 Whole Genome Analyses of Germline Mutation ....................................... 18

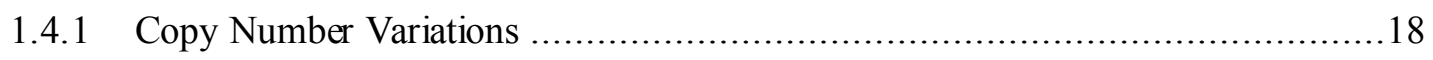

1.4.2 Next-Generation Sequenc ing of Whole Genomes..................................20

1.5 Test compound Benzo(a)pyre ne as a Model For Environmental Expos ures... 22

1.6 Thes is Hypothes is and Study Design ...................................................... 25 
$1.7 \quad$ Thes is Objectives............................................................................................... 27

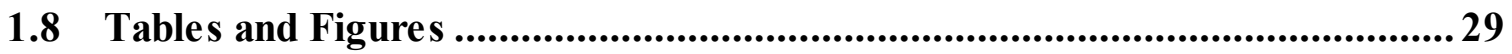

Chapter 2: Single-Molecule PCR Analysis of an Uns table Microsatellite for Detecting Mutations in Sperm of Mice Exposed to Chemical Mutagens ................... 30

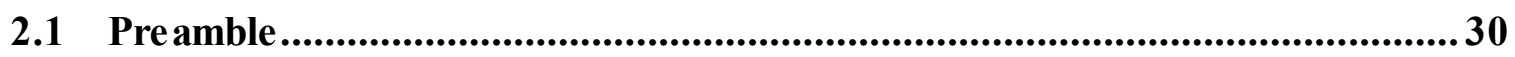

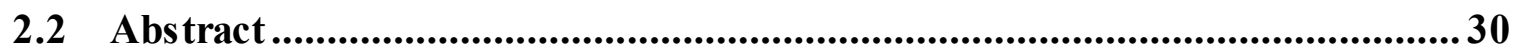

$2.3 \quad$ Introduction........................................................................................................... 31

2.4 Materials and Methods ......................................................................................35

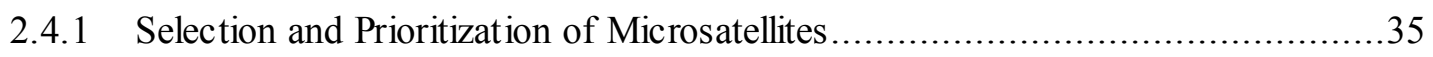

2.4.2 Spontaneous and Induc ed Germline Mutation Frequencies .......................... 36

2.4.3 DNA Isolation and Preparation for Mutation Analysis .............................. 37

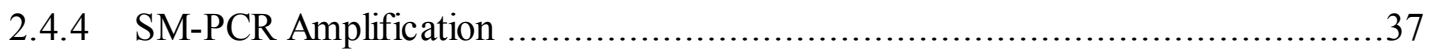

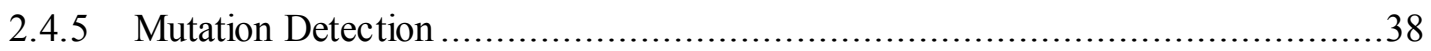

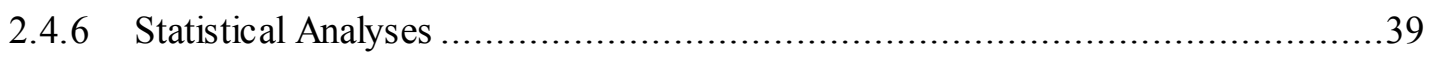

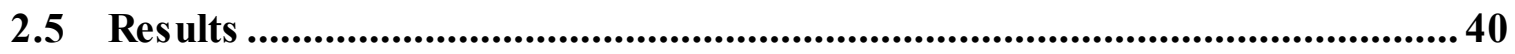

2.5.1 Selection and Prioritization of Microsatellites........................................40

2.5.2 Spontaneous and Induced Germline Mutation Frequencies ..........................40

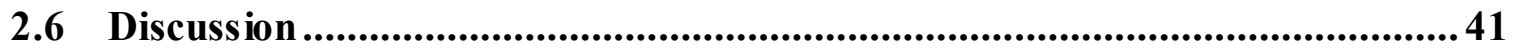

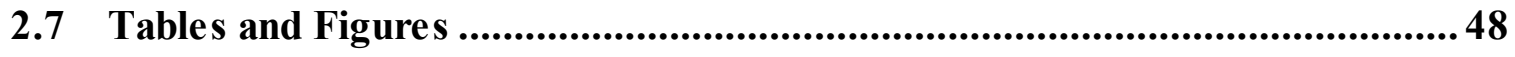

Chapter 3: Dose-Response Mutation and Spectrum Analyses Reveal Similar

Responses at Two Microsate llite Loci in Benzo[a]pyre ne-Exposed Mouse

Spe rmatogonia .............................................................................................................5

$3.1 \quad$ Pre amble .............................................................................................................5 53

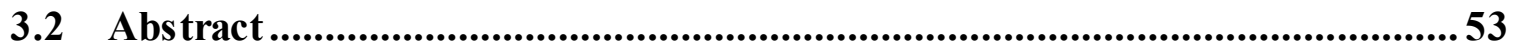

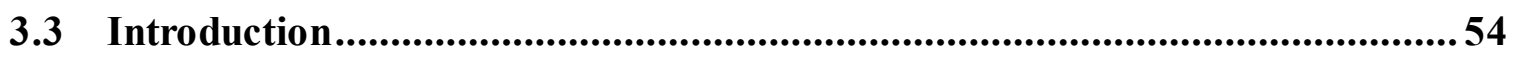


3.4 Mate rials and Methods ...................................................................5

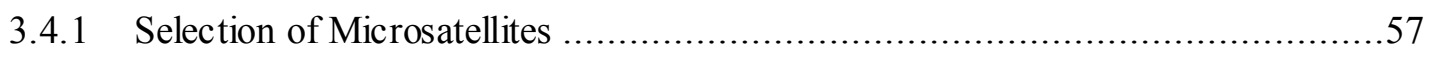

3.4.2 Animal Treatment and Sample Collection..............................................58

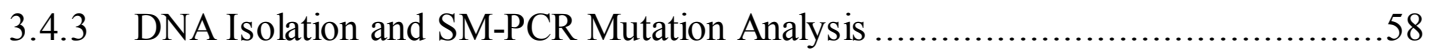

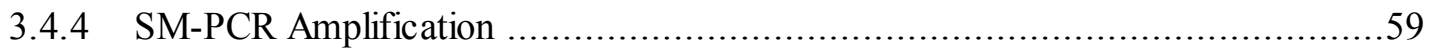

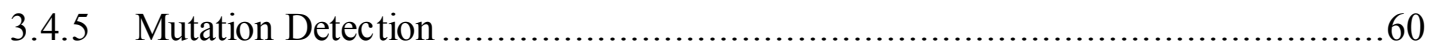

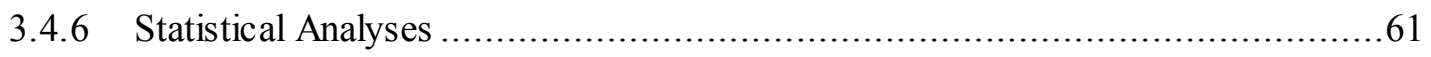

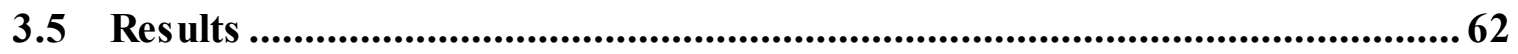

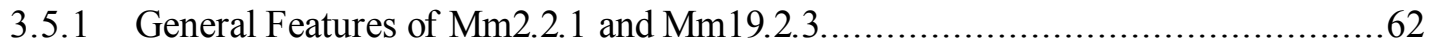

3.5.2 Induced Germline Mutation Frequencies ........................................62

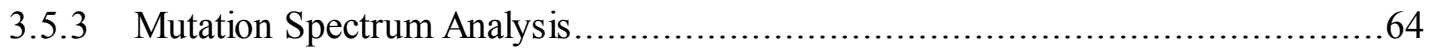

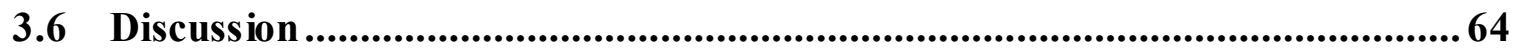

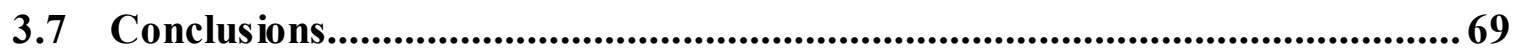

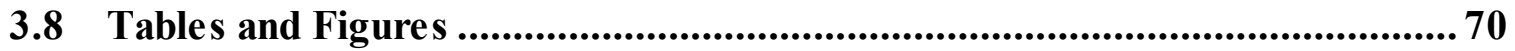

Chapter 4: Characterizing Benzo[a]pyre ne-Induced lacZ Mutation Spectrum in Transgenic Mice Using Next-Gene ration Sequencing....................................... 75

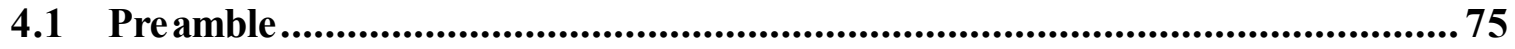

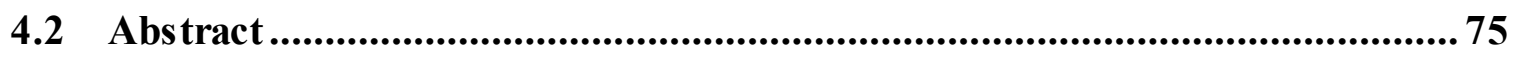

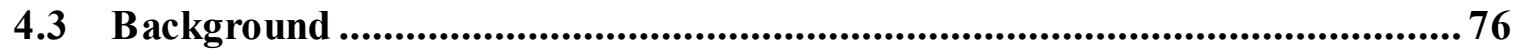

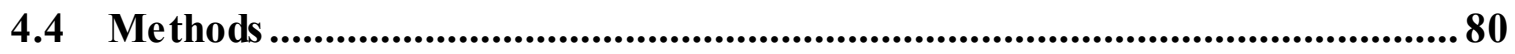

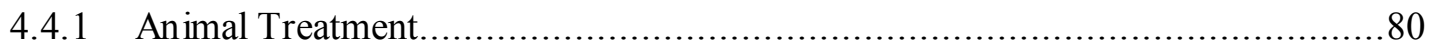

4.4.2 MutaMouse lacZ Mutation Analysis ............................................. 80

4.4.3 lacZ Mutant Plaque Collection and Preparation for Next-Generation Sequencing. 81

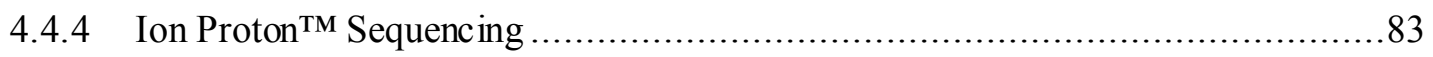

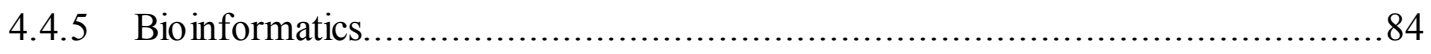




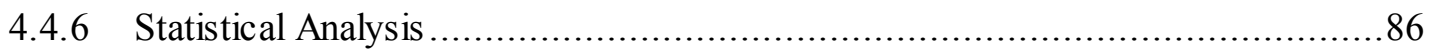

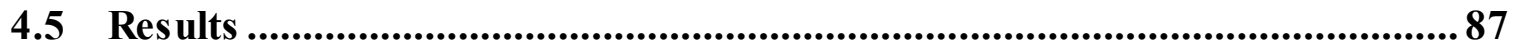

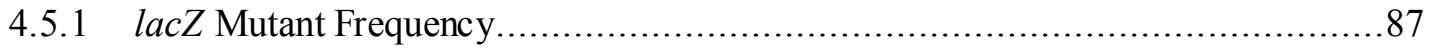

4.5.2 Sequencing Output and Read Coverage.............................................. 88

4.5.3 False Mutation Proportion Subtraction and Threshold Comparisons...................88

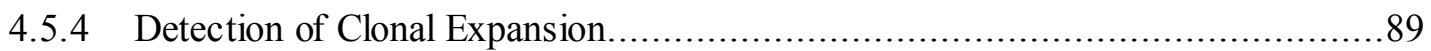

4.5.5 lacZ Mutation Spectra and Distribution .............................................91

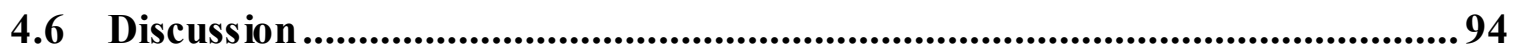

4.7 Conclusions............................................................................................. 100

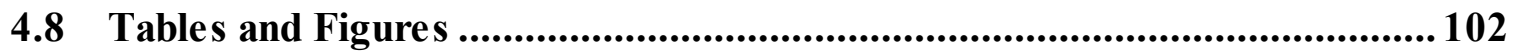

Chapter 5: Benzo(a)pyrene is Mutagenic in Mouse Spermatogonial Stem Cells and

Dividing Spermatogonia..................................................................................... 110

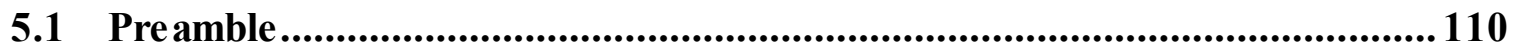

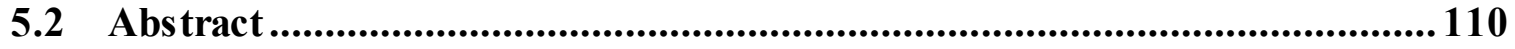

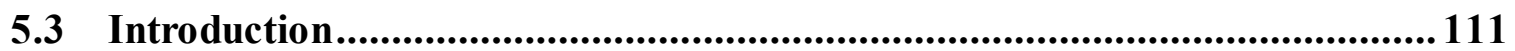

5.4 Materials and Methods .................................................................. 114

5.4.1 Exposures and Tissue Collection ............................................... 114

5.4.2 lacZ Mutation Assay ................................................................... 116

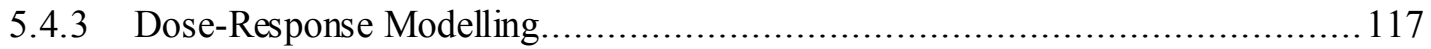

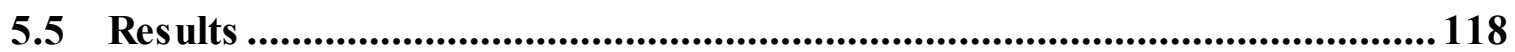

5.5.1 BaP Exposures and lacZ Mutant Frequencies in Germ Cells ....................... 118

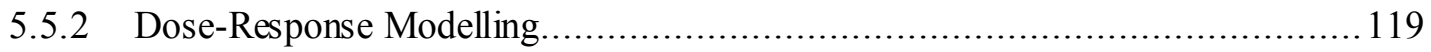

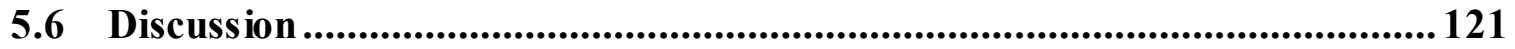

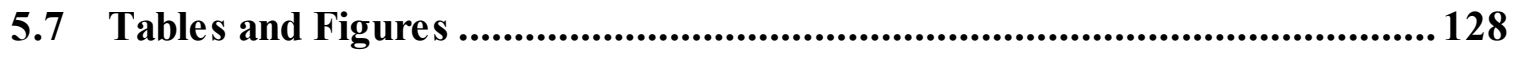


Chapter 6: Next Generation Seque ncing of Benzo(a)pyrene-Induced lacZ Mutants

Identifies a Germ Cell-Spe cific Mutation Spectrum ............................................ 132

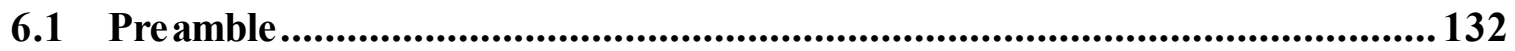

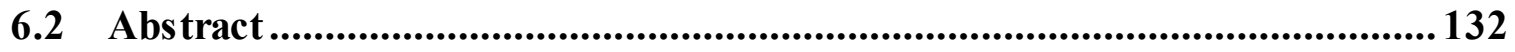

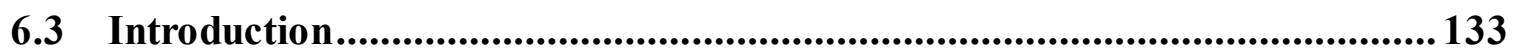

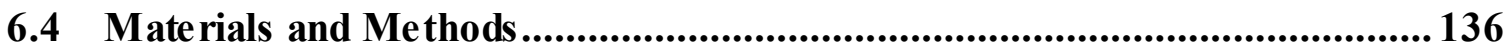

6.4.1 Animal Exposures and Tissue Collection.............................................. 136

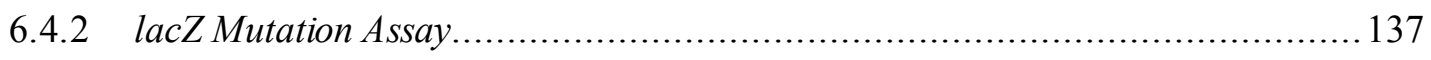

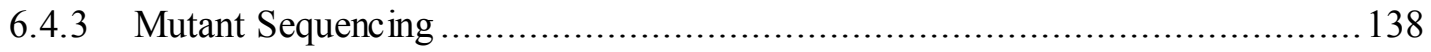

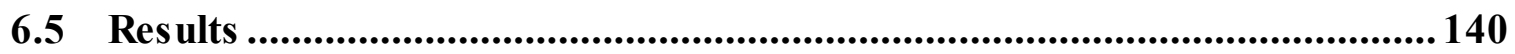

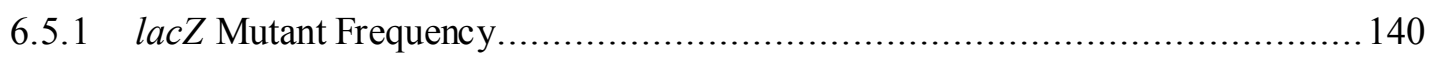

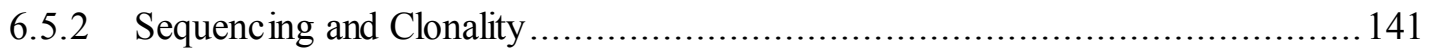

6.5.3 BaP-Induced Mutation Spectrum in Germ Cells.................................. 142

6.5.4 Comparison of Sperm and Bone Marrow Mutation Spectra .......................... 144

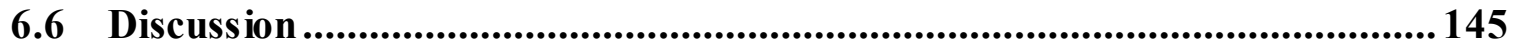

6.7 Tables and Figures .............................................................................. 152

Chapter 7: Paternal Exposure to Benzo(a)pyrene Induces Genome-Wide Mutations in Offs pring

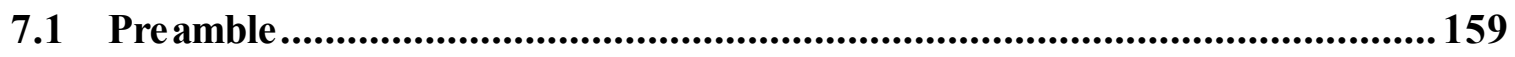

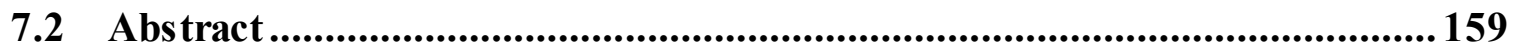

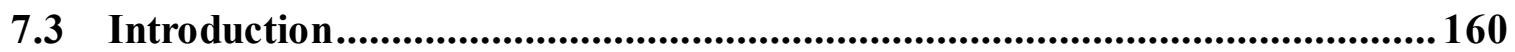

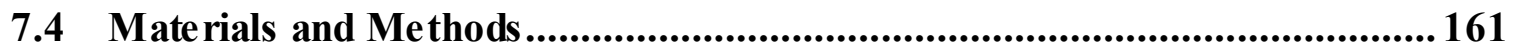

7.4.1 Benzo(a)pyrene Exposure and Mouse Breeding ..................................... 161

7.4.2 Comparative Genomic Hybridization and CNV Detection.......................... 162

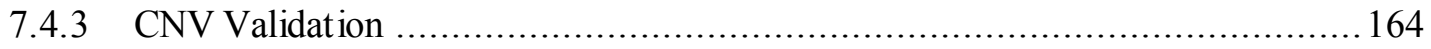


7.4.4 Identifying CNV Breakpoint and Parental Origin ................................ 164

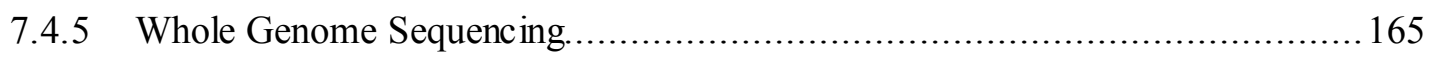

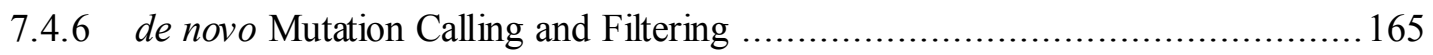

7.4.7 Cross-Platform Targeted Re-sequencing Validation .................................. 166

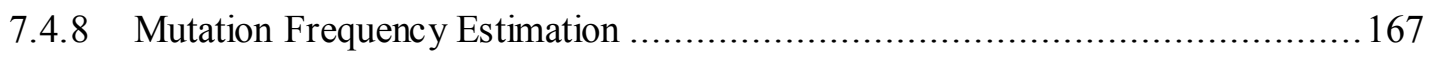

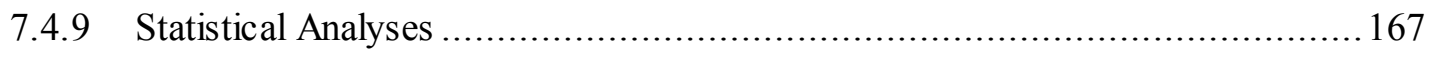

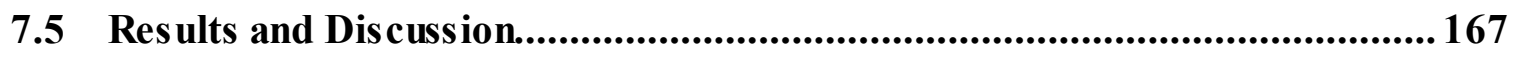

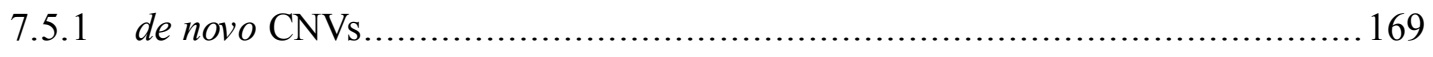

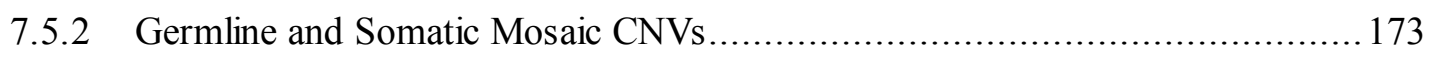

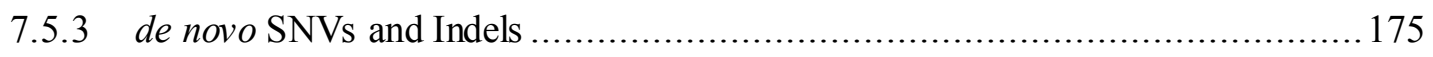

7.6 Conclusions........................................................................................... 180

7.7 Tables and Figures .................................................................................. 182

Chapter 8: From Sperm to Offs pring: Ass essing the Heritable Ge netic

Conseque nces of Pate rnal Smoking and Pote ntial Public Health Impacts ............ 191

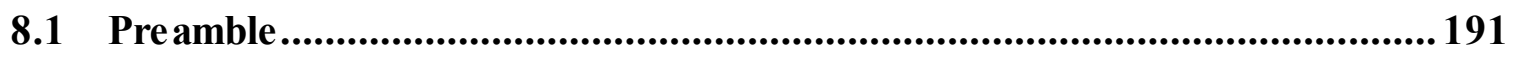

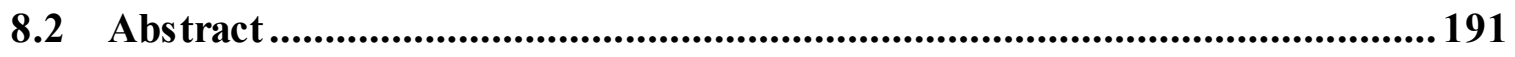

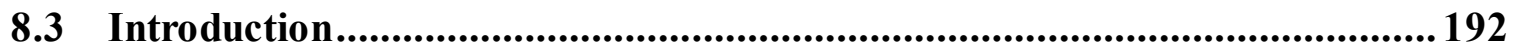

8.4 Human Spermatogenes is .............................................................. 195

8.5 Male Smoking and Fertility ....................................................................... 197

8.6 Evidence that tobacco smoke induces DNA damage in sperm ..................... 200

8.7 Evidence that tobacco smoke induces ane uploidy in spe rm ......................... 204

8.8 Evidence that tobacco s moke induces ge rmline mutations.......................... 206

8.9 Evidence that tobacco smoke induces ge nome instability ............................. 208

8.10 Preconception paternal smoking and potential phe notypic conse que nces .... 209

8.11 Evidence that tobacco s moke components induce germline mutations .........212 
8.12 Ge netic dise ase and socio-economic burden of smoke expos ure .................. 214

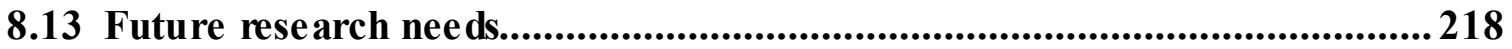

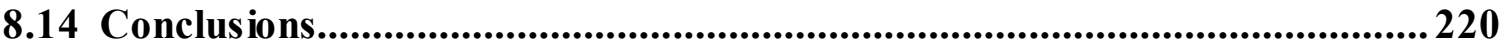

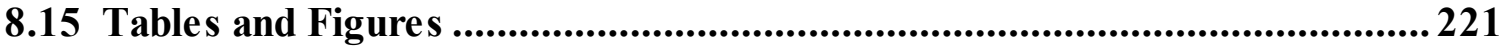

Chapter 9: Dis cuss ion............................................................................ 226

9.1 Thes is Outcomes............................................................................... 226

9.2 Advancements in Science and Contribution to Knowle dge.......................... 232

9.3 Future Directions................................................................................ 238

9.3.1 Tandem Repeat Mutations as a Biomarker for Mutagen Exposure ..................238

9.3.2 Understanding the Correlation Between Mutations in Reporter Transgenes and

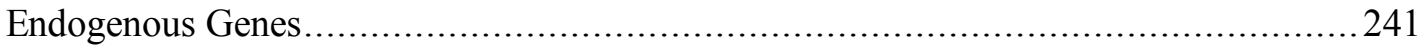

9.3.3 Next-Generation Mutations and Next-Generation Technologies ....................243

9.3.4 The Quest for the Holy Grail: The First Human Germ Cell Mutagen................245

9.4 Concluding Remarks ................................................................. 248

References .....................................................................................................250

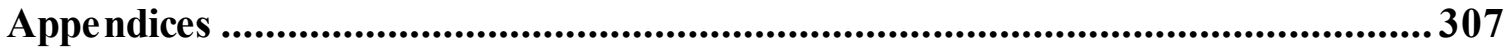

Appendix A Transgenic Rodent Assay for Quantifying Male Germ Cell Mutant

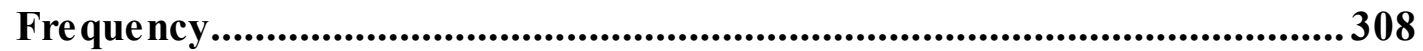

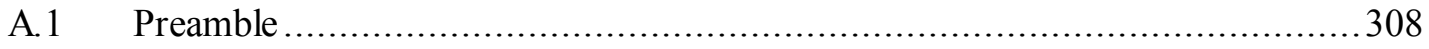

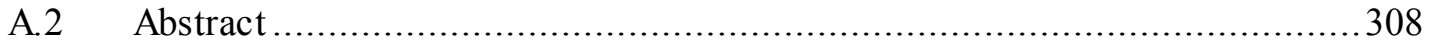

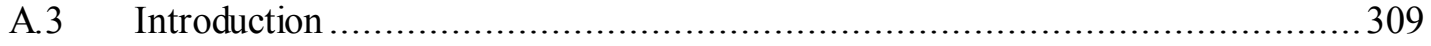

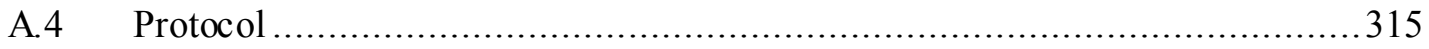

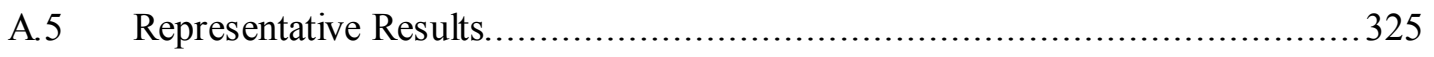

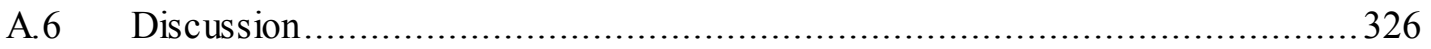

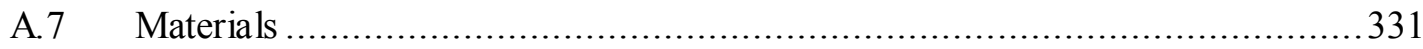




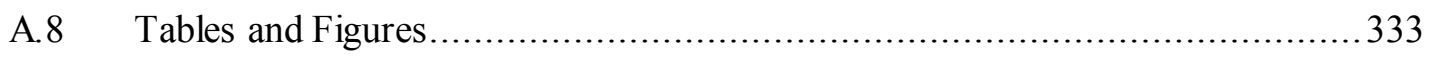

Appendix B In Utero Expos ure to Benzo[a]pyre ne Increases Mutation Burden in the Soma and Sperm of Adult Mice.............................................................342

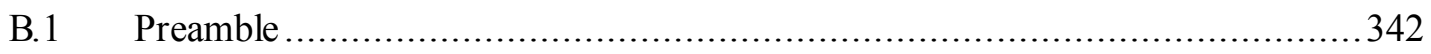

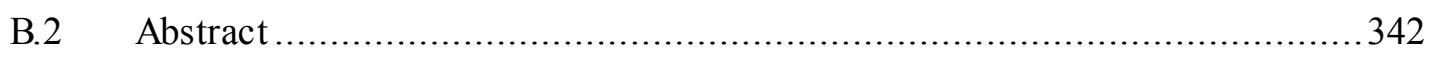

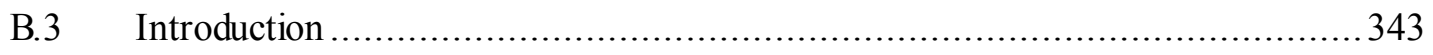

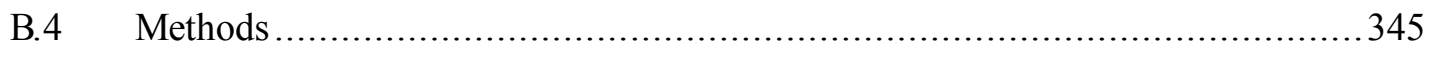

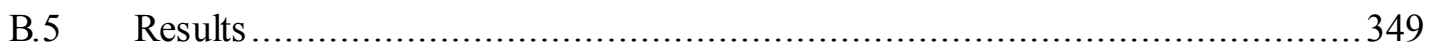

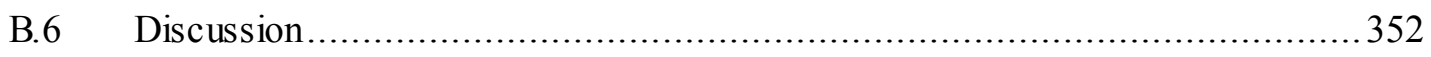

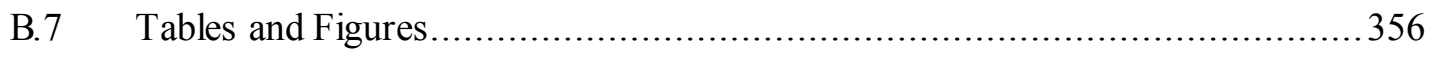

Appendix C Supple mental Mate rial.................................................................366

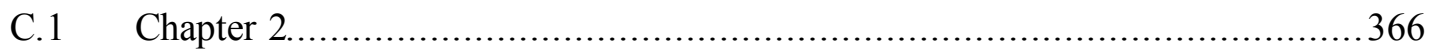

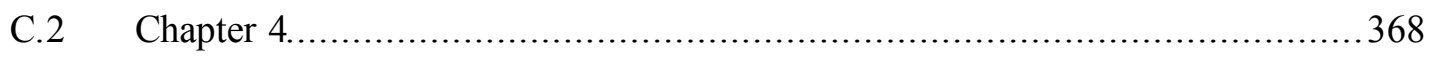

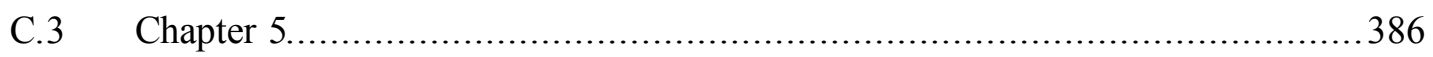

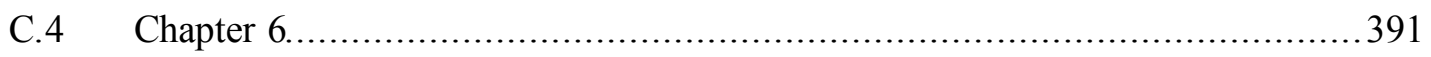

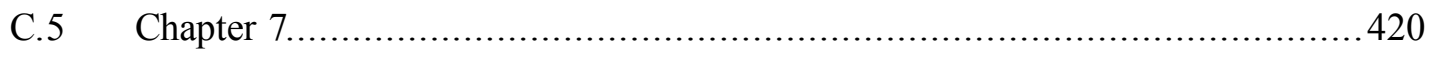

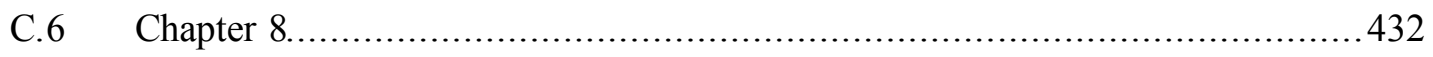




\section{List of Tables}

Table 2.1 Mutation frequencies (with 95\% confidence intervals) ${ }^{\mathrm{a}}$ in the sperm of control and treated animals from different experiments.

Table 3.1 Mutation frequencies in the sperm of mice exposed to 0 (control), 25, 50, or $100 \mathrm{mg} / \mathrm{kg} /$ day BaP.

Table 3.2 Mutation frequencies from multiplex PCR of sperm from control and 100 $\mathrm{mg} / \mathrm{kg} /$ day BaP-treated animals

Table 4.1 Glossary.....

Table 4.2 Summary of all mutations called using three different thresholds. 104

Table 5.1 Cell types and phases of spermatogenesis that are targeted by various experimental designs.

Table 5.2 lacZ mutant frequency in male germ cells of Mutamouse mice exposed to benzo[a]pyrene for 28 days and collected at various sampling times

Table 6.1 lac Z mutant frequency in sperm collected 42 days after a 28 -day exposure to benzo(a)pyrene, and clonal correction.

Table 6.2 Analysis of sequence context of lac $Z$ mutations identified in spermatozoa from the cauda epididymis of male mice exposed to benzo(a)pyrene.

Table 6.3 Analysis of sequence context of lac $Z$ mutations identified in the bone marrow

of male mice exposed to benzo(a)pyrene. 154

Table 7.1 de novo mutations in the offspring of control and BaP-treated animals.

Table 8.1 Summary of studies investigating the relationship between tobacco smoke and germ cell mutagenicity endpoints. 
Table 8.2 Potential intergenerational outcomes of the estimated 7.3 million LOF IDDG

mutations associated with paternal smoking. ................................................. 223 


\section{List of Figures}

Figure 1.1 Schematic diagram of mouse spermatogenesis showing germ cell types and phases that can be targeted by various experimental designs.

Figure 2.1 Variability of locus Mm2.2.1 across thirteen inbred mouse strains and a F1

cross.

Figure 2.2 Examples of capillary eletropherograms of SM-PCR amplifications of Mm2.2.1 in sperm DNA. .50

Figure 2.3 Mutation frequency at locus Mm2.2.1 in the sperm of different control and treated mice

Figure 2.4 Frequency spectrum of Mm2.2.1 mutant size classes in sperm.

Figure 3.1 Examples of capillary electropherograms of multiplex SM-PCR of microsatellites Mm2.2.1 and Mm19.2.3 from sperm DNA. .72

Figure 3.2 Mutation frequency for Mm2.2.1 and Mm19.2.3, analyzed independently, in the sperm of control mice and mice exposed to 25,50 , and $100 \mathrm{mg} \mathrm{BaP} / \mathrm{kg}$. .73 Figure 3.3 Mutation spectrum of (a) Mm2.2.1 (b) Mm19.2.3 in control and BaP-exposed

sperm. .74

Figure 4.1 Brief overview of workflow used in this study. 105

Figure 4.2 Corre lation between observed and expected mutant counts. 106 Figure 4.3 Relative proportion of control and $\mathrm{BaP}$-induced lacZ mutations in bone marrow.

Figure 4.4 Distribution of unique base substitutions across the lac $Z$ transgene for control and $\mathrm{BaP}$ samples. 108 
Figure 4.5 Distribution of unique indels across the lac $Z$ transgene for control and $\mathrm{BaP}$ samples.

Figure 5.1 lacZ mutant frequency dose response in spermatogenic cells exposed to benzo[a]pyrene 130

Figure 5.2 lacZ mutant frequency dose-response models used to determine: A) the breakpoint dose; and B) the slope transition dose for sperm collected 42 days after exposure to benzo[a]pyrene.

Figure 6.1 The type and position of all independent spontaneous (control) and benzo[a]pyrene(BaP)-induced lacZ mutations detected in sperm collected 42 days after the treatment period.

Figure 6.2 The proportion of all independent spontaneous (control) and benzo[a]pyrene (BaP)-induced lacZ mutations detected in sperm collected 42 days after the treatment period.

Figure 6.3 Comparison of the proportion of spontaneous mutation types in the lacZ gene collected from sperm and bone marrow.

Figure 6.4 Comparison of the proportion of benzo[a]pyrene $(\mathrm{BaP})$-induced mutation types in the lac $Z$ gene isolated from sperm and bone marrow.

Figure 7.1 Identification and characterization of de novo CNVs

Figure 7.2 Size and types of de novo CNVs characterized in control and BaP animals. 185

Figure 7.3 Germline mosaic CNVs detected in the BaP exposure group. 186

Figure 7.4 Mutation counts and spectra in control and BaP offspring as measured by WGS 
Figure 7.5 Mechanisms by which DNA adducts become embryonic or de novo mutations

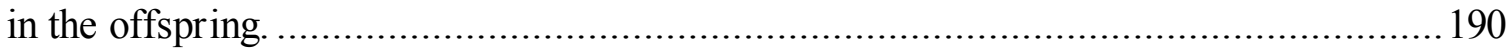

Figure 8.1 Overview of the evidence that tobacco smoke is a germ cell mutagen. ........224 Figure 8.2 Spermatogenesis and different mutations that are prevalent in the different

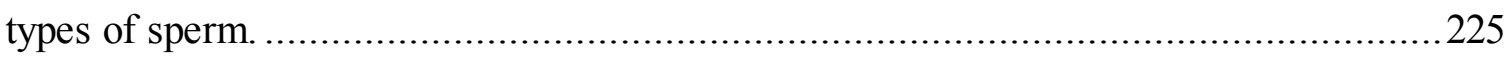




\section{List of Appendices}

Appendix A Transgenic Rodent Assay for Quantifying Male Germ Cell Mutant

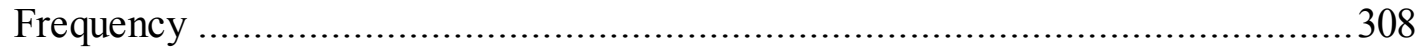

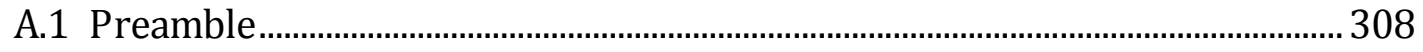

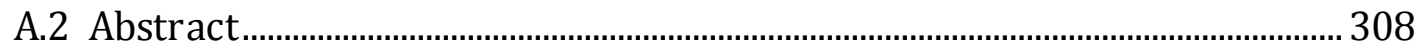

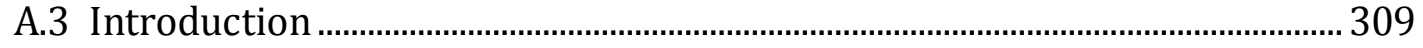

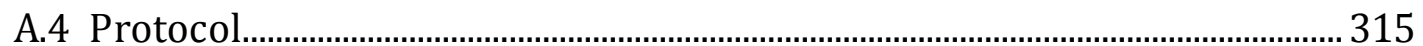

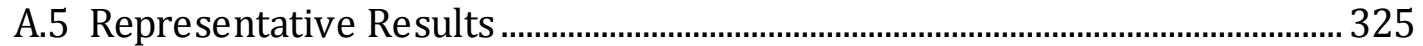

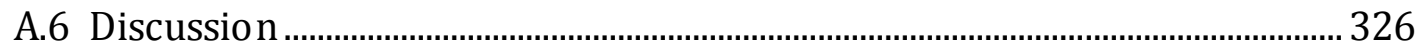

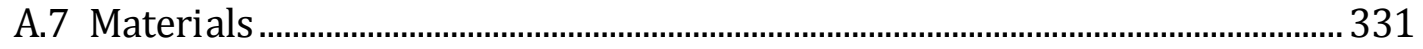

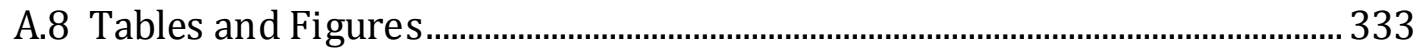

Appendix B In Utero Exposure to Benzo[a]pyrene Increases Mutation Burden in the Soma and Sperm of Adult Mice ............................................................. 342

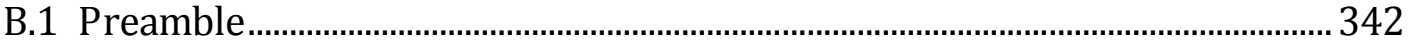

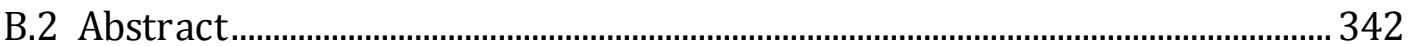

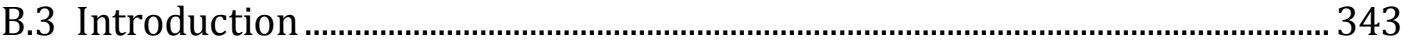

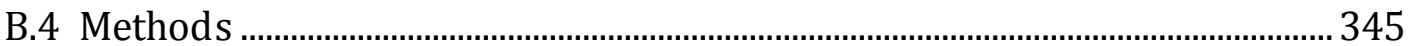

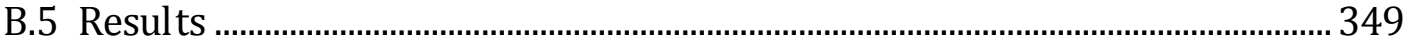

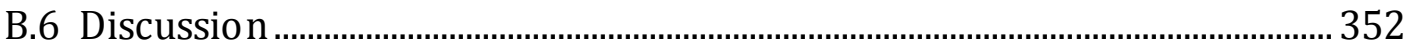

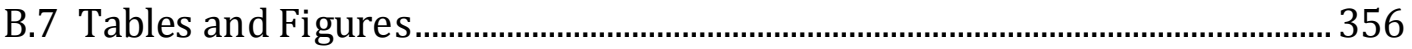

Appendix C Supplemental Material …................................................................ 366

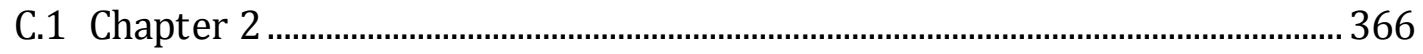

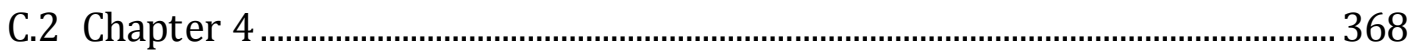




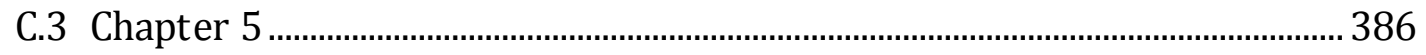

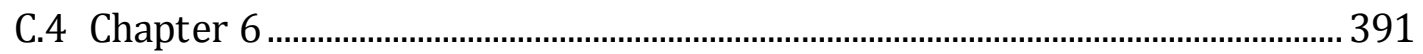

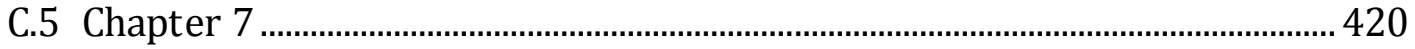

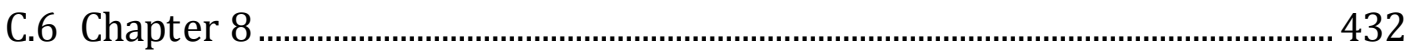




\section{List of Abbreviations}

aCGH Comparative Genomic Hybridization Array

AIC Akaike Information Criterion

BaP Benzo[a]pyrene

BMD Benchmark Dose

BMDS Benchmark Dose Software

BMR Benchmark Response

BPD Breakpoint Dose

B PDE Benzo[a]pyrene 7,8-dihydrodiol-9,10-epoxide

CNV Copy Number Variations

DLT Dominant Lethal Test

E. coli Escherichia coli

em-PCR Emulsion Polymerase Chain Reaction

ENU $\quad N$-ethyl- $N$-nitrosourea

EPA Environmental Protection Agency

ESTR Expanded Simple Tandem Repeat

GEE Generalized Estimating Equations

HRR Homologous Recombination Repair

HTT Heritable Translocation Test

Indel Insertion/Deletion Mutation

OECD Organisation for Economic Co-operation and Development

IARC International Agency for Research on Cancer

LOD Limit of Detection

LRT Likelihood Ratio Test

MALBAC Multiple Annealing and Looping Based Amplification Cycles

MDS Maximum-Depth Sequencing

MMBIR Microhomology-Mediated Break-Induced Replication

MF Mutant Frequency

MV Mendelian Violations

NER Nucleotide Excision Repair

NGS Next-Generation Sequencing 
NOGEL No Observable Genotoxic Effect Level

8-oxo-dG 8-Oxo-deoxyguanosine

PAH Polycyclic Aromatic Hydrocarbon

PFU Plaque-Forming Units

P-Gal Phenyl- $\beta$-D-galactopyranoside

qPCR Quantitative Polymerase Chain Reaction

ROS Reactive Oxygen Species

SLT Specific Locus Test

SM-PCR Single Molecule Polymerase Chain Reaction

SNV Single Nucleotide Variation

STD Slope Transition Dose

TGR Transgenic Rodent

VAF Variant Allele Fraction

WGS Whole Genome Sequencing 


\section{Statement of Contributions}

\section{Chapter 2: Single-molecule PCR analys is of an unstable micros atellite for detecting mutations in sperm of mice exposed to che mical mutagens}

Published in: Mutation Research: Fundamental and Molecular Mechanisms of Mutagenesis, 775:26-32. May 2015.

Authors: Beal, M.A. ${ }^{1,2}$, Rowan-Carroll, A. ${ }^{2}$, Campbell, C. ${ }^{2}$, Williams, A. ${ }^{2}$, Somers, C.M. ${ }^{3}$, Marchetti, F. ${ }^{2}$, Yauk, C.L. ${ }^{2}$

\section{Affiliations:}

${ }^{1}$ Carleton University, Ottawa, Ontario K1S 5B6, Canada; Environmental Health Science and Research Bureau, Healthy Environments and Consumer Safety Branch, Health Canada, Ottawa, Ontario K1A 0K9, Canada.

${ }^{2}$ Environmental Health Science and Research Bureau, Healthy Environments and Consumer Safety Branch, Health Canada, Ottawa, Ontario K1A 0K9, Canada.

${ }^{3}$ University of Regina, Regina, Saskatchewan S4S 0A2, Canada.

DOI: 10.1016/j.mrfmmm.2015.03.010

Overall Data Interpretation and Manuscript Writing Marc Beal

Experimental Design Marc Beal Carole Yauk Francesco Marchetti

Manuscript Preparation Marc Beal Carole Yauk Francesco Marchetti Christopher Somers

PCR Optimization Marc Beal Andrea Rowan-Carroll Caleigh Campbell

Tissue Collection and DNA Extraction Marc Beal

SM-PCR and Mutation Calling Marc Beal Andrea Rowan-Carroll

Statistical Analysis Andrew Williams 
Chapter 3: Dose-response mutation and spectrum analys es re veal similar responses at two micros atellite loci in be nzo[a]pyre ne-exposed mouse spermatogonia

Published in: Mutagenesis, in press. 2017.

Authors: Rowan-Carroll, A. , Beal, M.A. ${ }^{1,2}$,Williams, A. ${ }^{1}$, Marchetti, F. ${ }^{1}$, Yauk, C.L. ${ }^{1}$

Affiliations:

${ }^{1}$ Environmental Health Science and Research Bureau, Healthy Environments and Consumer Safety Branch, Health Canada, Ottawa, Ontario K1A 0K9, Canada.

${ }^{2}$ Carleton University, Ottawa, Ontario K1S 5B6, Canada; Environmental Health Science and Research Bureau, Healthy Environments and Consumer Safety Branch, Health Canada, Ottawa, Ontario K1A 0K9, Canada.

Overall Data Interpretation and Manuscript Writing .......... Andrea Rowan-Carroll

Experimental Design ......................................... Marc Beal

Carole Yauk

Francesco Marchetti

Manuscript Preparation.

Andrea Rowan-Carroll

Marc Beal

Francesco Marchetti

Carole Yauk

Tissue Collection and DNA Extraction

Marc Beal

SM-PCR and Mutation Calling Andrea Rowan-Carroll Marc Beal

Statistical Analysis Andrew Williams 


\section{Chapter 4: Characte rizing Benzo[a]pyre ne-induce d lac $Z$ mutation s pectrum in transgenic mice using next-generation sequencing}

Published in: BMC Genomics, 16:812. October 2015.

Authors: Beal, M.A. ${ }^{1,2}$, Gagné, R. ${ }^{2}$, Williams, A. ${ }^{2}$, Marchetti, F. ${ }^{2}$, Yauk, C.L. ${ }^{2}$

Affiliations:

${ }^{1}$ Carleton University, Ottawa, Ontario K1S 5B6, Canada; Environmental Health Science and Research Bureau, Healthy Environments and Consumer Safety Branch, Health Canada, Ottawa, Ontario K1A 0K9, Canada.

${ }^{2}$ Environmental Health Science and Research Bureau, Healthy Environments and Consumer Safety Branch, Health Canada, Ottawa, Ontario K1A 0K9, Canada.

DOI: $10.1186 / \mathrm{s} 12864-015-2004-4$

Overall Data Interpretation and Manuscript Writing ........... Marc Beal

Experimental Design ................................................... Marc Beal Carole Yauk

Francesco Marchetti

Manuscript Preparation

Marc Beal

Rémi Gagné

Francesco Marchetti

Carole Yauk

Mutation Collection, Library Preparation, and NGS Marc Beal

Bioinformatics Rémi Gagné

Statistical Analysis Marc Beal Andrew Williams 


\section{Chapter 5: Benzo(a)pyre ne is mutage nic in mouse spermatogonial stem cells and dividing spermatogonia}

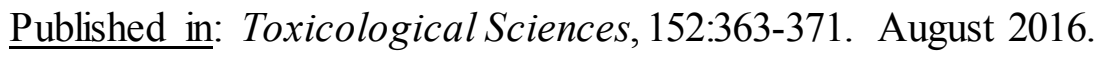

Authors: O’Brien J.M. ${ }^{1}$, Beal, M.A. ${ }^{1,2}$, Yauk, C.L. ${ }^{1}$, Marchetti, F. ${ }^{1}$

\section{Affiliations:}

${ }^{1}$ Environmental Health Science and Research Bureau, Healthy Environments and Consumer Safety Branch, Health Canada, Ottawa, Ontario K1A 0K9, Canada.

${ }^{2}$ Carleton University, Ottawa, Ontario K1S 5B6, Canada; Environmental Health Science and Research Bureau, Healthy Environments and Consumer Safety Branch, Health Canada, Ottawa, Ontario K1A 0K9, Canada.

DOI: $10.1093 /$ toxsci/kfw088

Overall Data Interpretation and Manuscript Writing .......... Jason O'Brien

Experimental Design ............................................ Jason O’Brien Marc Beal Carole Yauk

Francesco Marchetti

Manuscript Preparation Jason O'Brien Marc Beal Carole Yauk Francesco Marchetti

Animal Handling, Exposure, and Tissue Collection Jason O'Brien Marc Beal

Transgenic Rodent Gene Mutation Assay...... Jason O'Brien Marc Beal

Statistical Analysis Jason O’Brien 


\section{Chapter 6: Next ge neration sequencing of be nzo(a)pyre ne-induced lac Z mutants ide ntifies a germ cell-s pe cific mutation spectrum}

Published in: Scientific Reports, 6:36743. November 2016.

Authors: O’Brien J.M. ${ }^{1}$, Beal, M.A. ${ }^{1,2}$, Yauk, C.L. ${ }^{1}$, Marchetti, F. ${ }^{1}$

\section{Affiliations:}

${ }^{1}$ Environmental Health Science and Research Bureau, Healthy Environments and Consumer Safety Branch, Health Canada, Ottawa, Ontario K1A 0K9, Canada.

${ }^{2}$ Carleton University, Ottawa, Ontario K1S 5B6, Canada; Environmental Health Science and Research Bureau, Healthy Environments and Consumer Safety Branch, Health Canada, Ottawa, Ontario K1A 0K9, Canada.

DOI: $10.1038 /$ srep36743

Overall Data Interpretation and Manuscript Writing..... Jason O’Brien

Experimental Design Jason O’Brien Marc Beal Carole Yauk Francesco Marchetti

Manuscript Preparation. Jason O’Brien Marc Beal Carole Yauk Francesco Marchetti

Mutation Collection, Library Preparation, and NGS......... Marc Beal

Data Analysis Marc Beal Jason O'Brien

Statistical Analysis Jason O’Brien 


\section{Chapter 7: Paternal exposure to Benzo(a)pyre ne induces genome-wide mutations in offs pring}

Authors: Beal, M.A. ${ }^{1,2}$, Meier M.J. ${ }^{2}$, Williams, A. ${ }^{2}$, Rowan-Carroll, A. ${ }^{2}$, Gagné, R. ${ }^{2}$, Lindsay, S.J. ${ }^{3}$, Fitzgerald T. ${ }^{3}$, Hurles, M.E. ${ }^{3}$, Marchetti, F. ${ }^{2}$, Yauk, C.L. ${ }^{2}$

Affiliations:

${ }^{1}$ Environmental Health Science and Research Bureau, Healthy Environments and Consumer Safety Branch, Health Canada, Ottawa, Ontario K1A 0K9, Canada.

${ }^{2}$ Carleton University, Ottawa, Ontario K1S 5B6, Canada; Environmental Health Science and Research Bureau, Healthy Environments and Consumer Safety Branch, Health Canada, Ottawa, Ontario K1A 0K9, Canada.

${ }^{3}$ Wellcome Trust Sanger Institute, Wellcome Trust Genome Campus, Hinxton, Cambridge CB10 1SA, United Kingdom.

Overall Data Interpretation and Manuscript Writing.......... Marc Beal

Experimental Design ............................................... Marc Beal

Carole Yauk

Francesco Marchetti

Matthew Hurles

Manuscript Preparation

Marc Beal

Matthew Meier

Carole Yauk

Francesco Marchetti

Animal Handling, Exposure, Tissue Collection

Marc Beal

DNA Extraction

Marc Beal

Microarray Experiments

Marc Beal

Copy Number Variation Detection Andrew Williams

Tomas Fitzgerald

Copy Number Variation Validation.

Marc Beal

Andrea Rowan-Carroll

Whole Genome Sequencing.

Marc Beal

Mutation Detection

Matthew Meier

Mutation Validation

Sarah Lindsay

Statistical Analysis

Marc Beal 


\section{Chapter 8: From Sperm to Offspring: As sessing the Heritable Ge netic}

Consequences of Paternal Smoking and Potential Public Health Impacts

Published in: Mutation Research Reviews, 773:26-50. April 2017.

Authors: Beal, M.A. ${ }^{1,2}$, Yauk, C.L. ${ }^{2}$, Marchetti, F. $^{2}$

\section{Affiliations:}

${ }^{1}$ Carleton University, Ottawa, Ontario K1S 5B6, Canada; Environmental Health Science and Research Bureau, Healthy Environments and Consumer Safety Branch, Health Canada, Ottawa, Ontario K1A 0K9, Canada.

${ }^{2}$ Environmental Health Science and Research Bureau, Healthy Environments and Consumer Safety Branch, Health Canada, Ottawa, Ontario K1A 0K9, Canada.

Project Design Marc Beal Francesco Marchetti Carole Yauk

Data Collection, Interpretation, and Manuscript Writing..... Marc Beal Manuscript Preparation Marc Beal Carole Yauk Francesco Marchetti 


\section{Chapter 1: Introduction}

\subsection{Importance of Studying de novo Mutations in Germ Cells}

There is a growing recognition of the importance of heritable mutations across a broad array of human genetic disease, especially at the population level. This is evident based on the Human Gene Mutation Database (Cooper and Krawczak, 1996; Stenson et al., 2009), which currently reports online more than 190,000 known genetic lesions affecting over 7,000 genes. It has been predicted that the vast majority of coding sequence alterations (70-80\%) are mildly to highly deleterious (Boyko et al., 2008; Fay et al., 2001; Kryukov et al., 2007). Furthermore, recent estimates suggest that developmental disorders caused by newly arising (de novo) mutations have a prevalence of approximately 1 in 213 births (Deciphering Developmental Disorders Study, 2017). Hence, it is becoming increasingly apparent that mutations are a major contributor to the formation of genetic disease (Veltman and Brunner, 2012), and prevention of these mutations is paramount.

A recent example of the effects of germ cell mutations in human populations comes from large-scale sequencing studies that have identified a link between de novo mutations and sporadic neuropsychiatric disorders such as schizophrenia and autism (Girard et al., 2011; Michaelson et al., 2012; O'Roak et al., 2011; Sanders et al., 2012; Xu et al., 2011; Yuen et al., 2015). This relationship is corroborated by DNA microarray studies that show that many of these disorders can be caused by large de novo structural variations in the genome (Cook Jr and Scherer, 2008; Sebat et al., 2007; Tam et al., 2009; Xu et al., 2008). It appears that the incidence of these types of disorders may be on the rise (Atladóttir et al., 2007), yet it remains unclear how much this increase is due to better 
assessment and diagnosis or unknown environmental factors (Rutter, 2005). Recent evidence suggests that advanced paternal age at the time of conception is leading to children inheriting more de novo mutations from their fathers, which in turn increases their chances of having impaired brain development (D'Onofrio et al., 2014; Kong et al., 2012). This phenomenon can be explained in part by the accumulation of mutations in spermatogonial stem cells that propagate throughout a male's life and the possibility that DNA replication fidelity decreases over time (Crow, 1997). However, what is also of concern is that extended exposures to environmental factors may be causing sperm mutations (Aitken et al., 2004), but the extent of this contribution to the development of disorders is often underestimated or not even considered.

Demonstrating that exposure to environmental mutagens is responsible for human genetic disease remains challenging and is a highly understudied area of toxicology. Since the 90- year-old revelation that radiation causes germline mutations in Drosophila (Muller, 1927), researchers have been trying to identify other agents that are germ cell mutagens. To date, there have only been $\sim 40$ agents confirmed to be germ cell mutagens, but most of the evidence comes from rodent studies (DeMarini, 2012). Many of the mutagenic agents are chemicals (Russell, 2004), including anti-cancer drugs (Glen et al., 2008; Witt and Bishop, 1996) administered at large doses. In addition, complex mixtures such as cigarette smoke (Marchetti et al., 2011; Yauk et al., 2007) and air pollution (Somers et al., 2002; Somers et al., 2004; Yauk et al., 2008b) have also been shown to induce germline mutations in rodents. The mutagenicity of these complex mixtures is particularly alarming considering that a large percent of men are exposed to these mixtures daily for prolonged periods prior to reproduction. For example, in humans it has 
been shown that more mutations are passed onto children with increased smoking habit by the father (Linschooten et al., 2013). Despite the accumulative evidence, there are still no established human germ cell mutagens (DeMarini, 2012; Yauk et al., 2013). The lack of any declared human germ cell mutagens is likely due to the technical limitations of existing germ cell mutation tests that are unable to detect rare germline mutation events (Wyrobek et al., 2007). With renewed interest in the field and the adaptation of genomics technologies for detecting de novo mutations, it has been hypothesized that we will finally be able to determine whether human germ cell mutagens exist (Yauk et al., 2013).

\subsection{Spermatogenesis, Fertilization, and Development}

The outcomes of germ cell mutations are very different from somatic mutations. Specifically, germ cell mutations are heritable, meaning they can cause genetic disease in the next generation and can have population level repercussions. In contrast, somatic mutations predominately lead to cancer and will only affect the individual with the mutation. It is often assumed that mutagens induce the same types of mutations in somatic and germ cells. In reality, we know very little regarding differences in germ cell and somatic cell mutagenicity. For example, there exist differences in pharmacokinetics of chemicals to germ cells versus somatic tissues. Also, the blood-testis barrier, formed by tight junctions between Sertoli cells, controls the passage of xenobiotics to germ cells. Indeed, preliminary evidence suggests that there are germ cell specific mutagens such as sidestream tobacco smoke (Marchetti et al., 2011) and N-hydroxymethylacrylamide (Witt et al., 2003). Below I review the fate of a gamete and how the unique properties of 
different germ cells and developmental stages can respond very differently to mutagen exposures (Figure 1.1).

Heritable mutations can occur in many different forms (single nucleotide variations (SNVs), insertions/deletions (indels), rearrangements, etc.) and can be induced at several different stages during development. Gametogenesis is the earliest stage at which a germline mutation can be formed. For females, the proliferative phase of oogenesis occurs during in utero development and the formation of mature eggs occurs during adulthood. Therefore, the majority de novo mutations that can be passed onto the next generation are produced prior to birth in females since replication is one of the primary mechanisms by which mutations are created and fixed in cells. In contrast, males produce sperm via spermatogenesis throughout their post-pubertal lives. Consequently, de novo mutations are more commonly paternal in origin as there are more rounds of DNA replication and cell division (Crow, 2000). Thus, paternal germline exposures are the main focus of this thesis.

The entire duration of spermatogenesis is estimated to last $>70$ days in adult humans (Amann, 2008; Hess and de Franca, 2009) and approximately 49 days in mice (Hess and de Franca, 2009). This process consists of 3 phases: mitotic, meiotic, and postmeiotic. Using a proper study design, the different phases of spermatogenesis can be targeted to test mutagenicity in different germ cells. For example, a 28 day exposure in mice with a 3 day fixation period will target the post-mitotic phase, whereas a 42 day fixation period will target the mitotic phases. Hence, a thorough understanding of spermatogenesis is required to design experiments to properly characterize germ cell 
mutagens, as the same agent can have different qualitative and quantitive mutagenic outcomes depending on which germ cell phases were exposed to the substance.

Spermatogenesis (reviewed in detail in refs. (Hermo et al., 2010; Hess and de Franca, 2009; Oatley and Brinster, 2012)) begins in the seminiferous tubules of the testes with a low number of self-regenerating spermatogonial stem cells that formed in utero. A portion of these mitotic stem cells differentiate into spermatogonia that proliferate through several mitotic divisions where errors during replication may produce SNVs and indels. Stem cells and differentiating spermatogonia are on the "open" side of the bloodtestis barrier and are therefore unprotected from xenobiotic exposures (Yauk et al., 2015a). However, even though the other post-mitotic germ cells are on the "closed" side of the blood-testis barrier, the vast majority of toxicants bypass the barrier and can still damage DNA in these germ cells (Yauk et al., 2015a). Spermatogonia undergo a final round of DNA synthesis and divide to form 2 spermatocytes that enter meiosis. During meiosis, recombination between homologous chromosomes occurs; thus, chromosomal alterations and aneuploidies are more likely to occur in this cell type than SNVs or indels. Spermatocytes undergo 2 rounds of meiotic divisions to produce 4 haploid spermatids. DNA in these post-meiotic spermatids becomes highly condensed when histones are replaced with protamines. After major morphological changes and maturation in the seminiferous tubules, spermatids are released to the epididymis and stored in the cauda as mature spermatozoa prior to ejaculation. DNA repair declines throughout postmeiosis and any DNA lesions occurring in spermatids or spermatozoa will not be repaired until after fertilization (Marchetti and Wyrobek, 2008). DNA damage or mutations in sperm 
may only be transmitted to the offspring if these events have no selective disadvantage to the sperm.

After fertilization, DNA lesions in the paternal genome will be subjected to DNA repair using the oocyte's cellular machinery. This is supported by the observation that females deficient in DNA repair have increased frequencies of zygotes with chromosomal aberrations derived from the paternal genome (Marchetti et al., 2007). Hence, erroneous DNA replication of the paternal genome at sites of DNA damage may lead to any unrepaired lesions being converted into mutations in the early embryo. Considering these mutations occur during the first round of DNA replication and cell cleavage, they will not be present in every cell. For example, replication of a DNA adduct may lead to incorporation of an incorrect nucleotide; however, replication of the opposite strand lacking the adduct will lead to proper replication. Thus, after the first cleavage there will be 2 cells: cell 1 is paternal mutant, maternal wild-type; cell 2 is paternal wildtype, maternal wild-type. This is in contrast to de novo mutations arising in sperm where the mutant copy will be the only copy in haploid spermatids; thus, the mutant will be present in every cell of the developing organism in a 1:1 ratio with the maternal wild-type copy. This information can be used to distinguish de novo mutations from mutations occurring post-zygotically. In theory, the mutant copy should represent $25 \%$ (1/4) of the copies for post-zygotic mutations and $50 \%$ for de novo mutations. Overall, this information can be used to identify mutations that occurred in the earlier phases of spermatogenesis or post-mitotically, allowing for comparisons between pre- and postfertilization mutation fixation, respectively. 
Mutations can also be induced in the later stages of embryo development, independent of a direct germ cell exposure, leading to a variety of altered genomes throughout an individual (mosaicism). Recent genome-scale studies have revealed that seemingly normal tissues have higher levels of mosaicism than expected (Campbell et al., 2015; Erickson, 2010; Rahbari et al., 2015). Mosaic mutations can occur throughout an individual's life, but the most sensitive window is during in utero development as earlier mutations will have more time for clonal expansion. Mutations induced during these stages of development by environmental factors remain understudied compared to germline exposures.

As a whole, this thesis aimed to examine mutations induced at all developmental stages (spermatogenesis, fertilization, embryo/fetal development), with a primary focus on mutations arising in spermatogenesis. Chapters 2-3 and 5-7 focus on mutations arising during spermatogenesis in adult rodents, Chapter 7 on mutation induction in the early embryo, and Appendix B investigates the effects of an in utero chemical exposure on fetal mosaicism and reproductive health.

\subsection{His torical Overview of Me thods Used to Study Germ Cell Mutagenicity}

Traditional germ cell mutation tests have been very beneficial in our understanding of the relationship between environment and germline mutation. Classic biologists first used pedigree studies beginning with Drosophila melanogaster and then moving onto mice. These studies involved visual inspection of the progeny for changes in specific phenotypes to estimate a mutation rate. As new technologies became available researchers moved more towards the analysis of mutations in the germ cells directly. 
Most of our understanding of germ cell mutation comes from the male germline, because actively dividing sperm are more sensitive to mutation induction compared to nonreplicating oocytes (Crow, 2000), and because sperm are both abundant and accessible. Although each of the existing methods have allowed for tremendous strides in identifying germ cell mutagens, each of the methods have their limitations, and some are either performed less frequently or not at all (Yauk et al., 2015a).

\subsubsection{The Specific Locus Test}

The earliest method used to investigate germline mutations was the specific locus test (SLT) (Russell, 2004; Russell, 1951). The SLT used a specific mouse strain referred to as the $T$ (test) stock. These mice were homozygous for 7 different recessive viable mutations with recognizable traits such as changes in coat colour and ear morphology (Davis and Justice, 1998). When wild-type males were crossed with $T$-stock females, most of the offspring would be heterozygous and therefore, have the wild-type phenotypes. However, loss-of-function mutations in any of the 7 genes could be observed and scored, allowing for a mutation rate to be determined. Thus, the benefit of this method was that there was proof that mutations induced in the sperm were transmissible to the offspring. Using the SLT, researchers were able to identify 20 germ cell mutagens (Russell, 2004) and improve our understanding of the sensitive stages of spermatogenesis.

The main problem with SLT studies was that because the mutation rates of these loci were $10^{-5}$, incredibly large sample sizes were required to test an agent for mutagenicity. In addition, historical controls were often used as no mutations were 
detected in concurrent control mice. It was recommended that at least $12,000-18,000$ mice were used per experimental group (Russell et al., 1981). With these kinds of sample sizes, one could imagine that every study was a Herculean endeavour. Although it is difficult to pinpoint exactly how many animals were used throughout the long history of the SLT, it can be estimated that approximately $>10$ million offspring (Singer et al., 2006) were used based on counts of historical (Russell et al., 1981) or concurrent controls, and experimental groups. Thus, these studies were very expensive, timeconsuming, and likely would be considered unethical by today's standards. For these reasons, the $T$-stock is no longer maintained and the SLT is no longer performed.

\subsubsection{The Dominant Lethal Test}

Another early and important test that has been widely used for studying in vivo germ cell mutations is the dominant lethal test (DLT). Early observations that a reduction in litter size may not be due to reduced fertility per se (Brenneke, 1937), but rather due to cytological abnormalities, lead to the idea that "dominant lethal" mutations could be inhibiting embryo survival during implantation (Bateman, 1958). Another important observation was that measuring lethality of early implants was a more sensitive predictor of mutagenicity than measuring the loss of embryos at a more advanced stage (Bateman, 1966). These crucial considerations lead to the development of the DLT.

Since the inception of the DLT it has become the most frequently used germ cell mutation test, leading to the development of a test guideline (OECD, 2013) in 1984 by the OECD. In these studies, male mice or rats are exposed to the suspected mutagen and mated with virgin females every week ( 8 weeks in mice) to examine a specific phase of 
spermatogenesis. Alternatively, mice can be exposed for an entire round of spermatogenesis and then mated with females. Females are euthanized on gestation day 13 and the uteri are examined to calculate the number of implants, live or dead embryos, and corpora lutea. Post-implantation loss is calculated by the ratio of dead to total implants while pre-implantation loss is determined as the difference in numbers of corpora lutea and implants. A higher level of pre- or post-implantation loss in the treatment group is indicative that the agent being tested induces dominant lethal mutations and that it is mutagenic.

Although the DLT is still used today (E.g., (Shvets and Kovalchuk, 2015)), it has many disadvantages that have reduced its frequency of use. The major limitation of the DLT is that it does not measure heritable mutations as the mutations lead to embryo death (Yauk et al., 2015a). Furthermore, the cause of embryo loss is difficult to ascertain without further investigation. Although it is supported that chromosomal damage (Marchetti and Wyrobek, 2005) and to a lesser extent gene mutations are the main cause of embryo death, the possibility of teratogenicity or cytotoxicity cannot be ruled out (Yauk et al., 2015a). Consequently, the DLT is more sensitive at detecting clastogens that can induce post-meiotic lesions (Singer et al., 2006), while mutagens that induce premeiotic mutations may go undetected (Russell and Matter, 1980). Lastly, the high background rate of embryo death (5-10\%) limits the DLT's ability to detect weak mutagens (Russell and Matter, 1980), and increases the sample size required to detect mutation induction. Considering that the OECD guideline recommends that 40-50 females are bred per mating interval, and multiple mating intervals are often used, the number of animals per study ranges between hundreds to thousands. Thus, similar to the 
SLT, the large sample sizes required for the DLT makes these studies too expensive, time-consuming, and impractical for routine application.

\subsubsection{The Heritable Translocation Test}

The Heritable Translocation Test (HTT) detects translocations passed on through the male germline. The HTT is similar to DLT in that it detects lethal mutations that lead to early embryo death. Early work done in the 1930's showed that exposure of males to mutagens resulted in semi-sterile F1 progeny, and later cytological analysis revealed that the semi-sterility was a result of unbalanced chromosomes caused by translocations (Generoso et al., 1980). These observations lead to the development of the HTT and a subsequent OECD test guideline (OECD, 1986). Like the other traditional germ-cell mutagenicity tests, the HTT involves exposing males to an agent and then mating them. A key difference is that F1 males are then mated and the litter sizes are examined to see if the exposure group had fewer F2 pups per litter. Meiotic cells at diakinesis from the F1 males are then examined for the presence of translocations to confirm the cause of the semi-sterility. In addition, the uterine contents of F1 male breeding partners can be analyzed for translocations. Heritable translocations are a type of DNA damage strongly associated with human diseases; thus, the HTT provided a means to identify an important class of mutagens (Marchetti and Wyrobek, 2005).

Similar to the other traditional germ-cell tests, the HTT has drawbacks that make using this assay unfavourable. The primary problem is that heritable translocations occur at low frequencies. The OECD guideline recommends $500 \mathrm{~F} 1$ males per dose group alone (OECD, 1986). Factoring in the F0 and F2 generation, a HTT study would require 
thousands of mice. In addition, cytological or genetic analysis is also required to validate the cause of the semi-sterility, making this assay more time consuming. Lastly, the HTT detects clastogenic agents exclusively, and therefore, agents with a very specific mutational fingerprint would test negative using this assay. Due to these limitations, the HTT is seldom performed.

\subsubsection{Tandem Repeat Mutation Analys is}

Perhaps the most sensitive approach for studying germ cell mutagenesis is to analyze tandem repeat sequences, such as microsatellites (short repeats) or minisatellites (long repeats), for mutations. The discovery of DNA fingerprinting of tandem repeats in the mid 1980's (Jeffreys et al, 1985a; Jeffreys et al., 1985b) revolutionized the field of genetic analysis (forensics, paternity testing, etc.) (Jobling and Gill, 2004; Primorac and Schanfield, 2000), but also provided a novel and easier means for identifying mutations in family pedigrees (Dubrova et al., 1993). The original method involves comparing allele sizes of tandem repeat DNA loci between parents and their progeny on a Southern blot gel. Novel alleles in the progeny are a result of parental alleles changing in size by 1 or more repeat units and are scored as mutation events. Tandem repeats are prone to having high rates of mutation because of their propensity to gain or lose whole repeat units during replication or recombination.

Human studies have relied on minisatellites to measure mutation induction in populations exposed to mutagenic agents. Most of this work has focused on the effects of accidental radiation exposure on the germline. Specifically, individuals exposed during the Chernobyl disaster were shown to have an increased rate of minisatellite mutations 
through family pedigree analysis (Dubrova et al., 1996; Dubrova et al., 2002b).

Furthermore, proximity to nuclear contamination sites has also been associated with increased rates of germline mutation (Dubrova et al., 2002a; Dubrova et al., 2006). In contrast, atomic bomb survivors show no increase in mutation rates at minisatellite loci (Kodaira et al., 1995). These discrepancies between minisatellite studies have sparked fierce debates (Goldstein and Stawkowski, 2015), and thus additional technologies are required to understand germ cell mutagenesis in humans.

Mouse studies use what are known as Expanded Simple Tandem Repeats (ESTRs) (Bois et al., 1998; Gibbs et al., 1993; Kelly et al., 1989). ESTRs are repeat sequences with short repeat units (like microsatellites) that are arranged in tandem arrays that can be extremely long (like minisatellites). Mutations at these loci occur more frequently than at coding loci, such as the loci screened in the SLT, and the instability of these sequences makes them highly sensitive to mutagen exposure. Therefore, the sample sizes required for statistical analysis are greatly reduced.

To further reduce the number of animals needed for analysis, more recent studies have examined mutation frequencies of tandem repeat loci directly in sperm using singlemolecule PCR (SM-PCR) (Yauk et al., 2002; Yauk et al., 2007; Yauk et al., 2008b). ESTRs specifically have been used to identify and characterize several germ cell mutagens such as ionizing radiation (Dubrova et al., 1993; Somers, 2006), air pollution (Somers et al., 2002; Somers et al., 2004; Yauk et al., 2008b), tobacco smoke (Marchetti et al., 2011; Yauk et al., 2007), anti-cancer drugs (Glen et al., 2008), and various chemicals (Hedenskog et al., 1997; Liu et al., 2009; Vilariño-Güell et al., 2003). Nutrient deficiencies, such as a diet low in folic acid (folate is required for nucleotide synthesis 
and DNA methylation), have also been shown to increase mutation formation at ESTR loci (Swayne et al, 2012). Studies using ESTRs have also shown that mutation rates can be increased when certain DNA repair genes are knocked out (Barber et al., 2004; Yamauchi et al., 2002). Furthermore, ESTRs have been used to demonstrate that mutagen exposure can cause trans-generational effects (Dubrova et al., 2000; Glen and Dubrova, 2012). Thus, ESTRs are sensitive markers for identifying mutagens that pose a risk to germ cells.

Although ESTRs and minisatellites have greatly improved our understanding of germ cell mutations, studies using these loci continue to be limited by several challenges. The technical challenges of long tandem repeats include: 1) imprecise mutation detection due to poor size-resolution on agarose gels, 2) technically difficult and problematic protocols that impede standardization among labs, and 3) low-throughput mutation detection because the large size of the repeat arrays require Southern blotting and probing (Somers, 2006). Furthermore, mutation induction at large tandem repeat DNA loci results from an unknown, indirect mechanism as opposed to direct DNA damage at the loci (Yauk, 2004). This indirect nature of tandem repeat DNA mutation induction makes it difficult to generalize the findings at these loci to other loci in the genome (Somers and Cooper, 2009). Lastly, ESTRs have only been characterized in mice so far (Bois et al., 1998; Gibbs et al., 1993; Kelly et al., 1989), and thus, it is difficult to use ESTR mutation induction in mice as a model for mutation induction in other species.

As an alternative to ESTRs, researchers have explored using microsatellites to quantify germline mutation rates. Typically, microsatellite mutation frequencies tend to be much lower than those of ESTRs (Beal et al., 2012a); however, some loci have been 
identified in different species that have comparable mutation frequencies (Ellegren et al., 1997; Tsyusko et al., 2007). Furthermore, as microsatellites are ubiquitous in eukaryotic genomes, multiple loci can be examined for mutations within a sample to improve the sensitivity of the assay. For example, the largest scale study examined 2,477 microsatellite loci in 85,289 humans to detect 2,058 germline mutations (Sun et al., 2012). By analyzing multiple loci, the researchers were able to confirm that older males pass more microsatellite mutations on to their children (paternal age effect). Mutation detection using microsatellites instead of ESTRs offers several advantages. Most importantly, microsatellite mutations can be detected using capillary electrophoresis on a standard sequencing instrument because microsatellite sequences are much shorter in length than ESTRs. Mutation detection on a sequencer is not only more accurate and precise, but it can also be performed with higher throughput. In addition, there are known clinical consequences of microsatellite mutations (Mirkin, 2007), making microsatellites a more useful marker for human health risk assessment. Several studies have used microsatellites to examine how germline mutation induction can be caused by exogenous factors (Anmarkrud et al., 2011; Beal et al., 2012a; Costa et al., 2011; da Cruz et al., 2008; Ellegren et al., 1997; Furitsu et al., 2005; King et al., 2014; Kodaira et al., 2010; Kovalchuk et al., 2000; Satoh et al., 1996; Slebos et al., 2004; Sun et al., 2012; Tsyusko et al., 2007; Tsyusko et al., 2011) and endogenous factors, such as genotype (Baker et al., 1995). Despite the clear benefits of microsatellites, these loci lack the sensitivity of ESTRs to detect mutation induction using the family pedigree approach (Beal et al., 2012a). For this reason, I modified the SM-PCR method (Yauk et al., 2002) to detect sperm mutations at microsatellite loci following chemical exposures (Chapters 2 and 3). 
SM-PCR amplification of microsatellites has the potential to be a new higher-throughput method for detecting mutation induction in germ cells.

\subsubsection{Transgenic Rodent Gene Mutation Assays}

Another contemporary germ cell test used to detect mutations is the transgenic rodent gene mutation (TGR) assay (reviewed in (Lambert et al., 2005)). This assay was first reported by (Gossen et al., 1989), and there have been several modifications since (Lambert et al., 2005). TGR assays use transgenic mice that carry a bacterial transgene, such as $\operatorname{lac} Z$, to detect mutations. After the mice are exposed to a test agent, the transgene can be isolated from any tissue, packaged into bacteriophage particles, and then the phages are infected into specific strains of Escherichia coli (E. coli) cells. Mutations in the transgene are detected by the appearance of plaques that can only develop on specific media (i.e., positive selection). This assay has been used to investigate more than 150 different exposure conditions, in several different tissues (Lambert et al., 2005), and has established OECD guidelines for mutagenicity testing (OECD, 2011). Furthermore, several studies have demonstrated the efficacy of this assay in detecting germ cell mutation induction (Ashby, 1995; Ashby et al., 1997; Douglas et al., 1994; Douglas et al., 1995; O’Brien et al., 2013; O'Brien et al., 2014; O'Brien et al., 2015; Olsen et al., 2010; Verhofstad et al., 2011; Vijg and Douglas, 1996; Xu et al., 2014).

The TGR assay offers a lot of promise in detecting germ cell mutagens. One of the main advantages of the TGR assay is that it detects mutations in germ cells directly, eliminating the demands of screening progeny. The OECD guideline only requires 5 mice per exposure group, making several different analyses (doses, stage of spermatogenesis, 
etc.) per agent feasible. For example, the TGR assay has been used to investigate the trans-generational effects of mutagen exposure on the germline (O'Brien et al., 2013), an undertaking that would be very difficult to do using classical tests. Another major advantage of the TGR assay is that mutant transgenes can be recovered and sequenced, providing mechanistic insight into the mode of action of the mutagen. Determining the mutation spectra of compounds is especially important for supporting the mutagenic effect of weak mutagens. Lastly, the TGR assay is sensitive, reproducible, and is not technically challenging, making it a suitable tool for studying germ cell mutagenesis.

There are some perceived drawbacks of the TGR assay for studying germ cell mutations. The most apparent disadvantage is that the assay requires specific transgenic rodents and these animals can be expensive. Another problem with this assay is that most mouse models, with the exception of the gpt delta mouse, are unable to detect large deletions that may be produced by clastogens (Lambert et al., 2005). Finally, there is still debate over whether mutations in the transgene are a good proxy for mutations in the rest of the genome (endogenous loci). It has been noted that the transgene is heavily methylated, and because it is not expressed is not subject to transcription-coupled repair (Ikehata et al., 2000). Furthermore, there is evidence that mutation accumulation over the duration of mutagen exposure differs between endogenous and exogenous genes (Cosentino and Heddle, 2000). Thus, more direct comparisons of results observed in the transgene and endogenous loci (e.g., whole genome sequencing) are required to address if mutation induction in the mouse genome is comparable to mutation induction in the transgene. In this thesis, the TGR assay is adapted for detecting germ cell mutations (Appendix A and Chapter 5). Moreover, a next-generation sequencing (NGS) approach is 
developed to characterize mutants produced by TGR assays (Chapter 4), and this approach is applied to study sperm mutations (Chapter 6).

\subsection{Whole Genome Analys es of Ge rmline Mutation}

The fundamental issue with contemporary germline mutation tests is the assumption that an increased frequency of specific mutation types (e.g., tandem repeat mutations), or mutations at certain markers (e.g., transgene), is characteristic of what is going on in the rest of the genome. The correlation between the different germ cell mutation assays (Singer et al., 2006) may be indicative of a genome-wide effect; however, no direct comparisons have been made. That is because until recently this was not possible (Wyrobek et al., 2007); only with the development of high-throughput genomics technologies can this be done. There has been a growing interest in adapting new genomics tools to quantify germline mutation induction, but with some reservations over the cost and technical limitations (Beal et al., 2012b; Singer and Yauk, 2010; Yauk et al., 2015a; Yauk et al., 2013). However, now that the costs have declined and the methods have been improved, the time is ripe to use these technologies to study germline mutagenesis. These technologies are poised to offer unprecedented insight into the genome-wide effects of mutagen exposure on the germline, and confirm the existence of human germ-cell mutagens.

\subsubsection{Copy Number Variations}

One possible approach to study genome-wide effects of a germ-cell mutagen is to use Comparative Genomic Hybridization arrays (aCGH) arrays to look for Copy Number 
Variations (CNVs). CNVs are alterations in the number of copies of a large section of DNA (section can either be deleted or duplicated). CNVs range in size from kilobases to megabases and make up a large portion of eukaryotic genomes (Stankiewicz and Lupski, 2010). Detection of CNVs has become more high-throughput and accurate as a result of the development of aCGH. In these experiments, DNA from 2 different samples (test and reference) are labelled with different fluorescent dyes and are added to the glass slide of the array in equal amounts. The samples then compete for hybridization to the thousands of oligonuc leotide probes on the array. The fluorescence intensity is compared between samples at each probe to detect deletions or duplications in the test sample. Using the robust aCGH approach, researchers can detect CNVs in the kilobase range and can characterize the genome architecture of individuals.

CNVs have the potential to be an ideal marker for germline mutation analyses (Singer and Yauk, 2010) because they have high de novo mutation rates $(0.5$ - 3\% (Adewoye et al., 2015; Conrad et al., 2009; Itsara et al., 2010; Sebat et al., 2007; Xu et al., 2008) and they are amenable to standardized mutation detection using aCGH (Zhang et al., 2009). Moreover, CNVs are associated with several disorders such as Crohn's disease (McCarroll et al., 2008), obesity (Bochukova et al., 2009), neurological disorders (Castellani et al., 2014; Cook Jr and Scherer, 2008; O’Dushlaine et al., 2014; Olson et al., 2014; Pinto et al., 2010; Sebat et al., 2007; Tam et al., 2009; Xu et al., 2008), cognitive impairment (Friedman et al., 2006), and cancer (Shlien and Malkin, 2009; Shlien and Malkin, 2010). Hence, the induction of de novo CNVs has a direct health consequence. CNVs arise in part due to errors during DNA repair and replication (Gu et al., 2008; Stankiewicz and Lupski, 2010); thus, agents that induce de novo CNVs are likely causing 
DNA lesions that invoke DNA repair processes or interfere with DNA replication in germ cells. For example, it has been demonstrated that radiation, a strong inducer of double strand breaks, can induce de novo CNVs in the offspring of exposed male mice (Adewoye et al., 2015). Furthermore, in vitro studies have shown that replication stress, caused by chemical inhibitors of replication, leads to the formation of CNVs (Arlt et al., 2009; Arlt et al., 2011; Arlt et al., 2012). Using aCGH to detect CNVs offers promise as a powerful new method for quantifying germline mutation induction, and in this thesis I used aCGH to detect de novo CNVs induced by a chemical exposure (Chapter 7).

\subsubsection{Next-Generation Se que ncing of Whole Genomes}

In order to fully understand how a mutagen affects the germline it is necessary to sequence whole genomes in family pedigrees to look for de novo mutations in the offspring of exposed parents. With NGS, genomes can now be sequenced rapidly and at a reduced cost (Metzker, 2009; Snyder et al., 2010). Since the release of the first commercial platforms, whole genome sequencing (WGS) has become routine and thousands of human genomes have now been sequenced (1000 Genomes Project Consortium, 2015; Genome of the Netherlands Consortium, 2014). NGS has also recently been used to sequence family pedigrees to quantify baseline germline mutation rates in humans (Conrad et al., 2011; Francioli et al., 2015; Kloosterman et al., 2015; Kong et al, 2012; Rahbari et al., 2015; Roach et al., 2010; Wong et al., 2016), and thus the proof of principle of using NGS to quantify germline mutation rates already exists. For example, the large-scale study by Kong et al. (2012) sequenced 78 families (234 genomes) with 30 -fold coverage to establish that paternal age is a strong determinant of germline 
mutation rate. This finding was supported by an even larger study that sequenced 250 families to identify $>10,000$ de novo SNVs (Francioli et al., 2015) and more than 300 de novo structural variations including indels, retrotransposon insertions, and other interchromosomal events (Kloosterman et al., 2015). What is remarkable about these studies is that the researchers were able to eliminate thousands of false-positives by applying stringent bioinformatics filters and targeted re-sequencing to identify the rare de novo mutation events. With the increased sensitivity NGS offers, it has the potential to be the most informative tool for understanding heritable mutations.

Despite the excitement surrounding NGS (Yauk et al., 2013), few experiments have harnessed the power of NGS to explore de novo mutation induction. The first study to capitalize on the advantages of NGS for this purpose sequenced mutagenized lines of Caenorhabditis elegans to identify mutation events (Flibotte et al., 2010). Animals exposed to ethyl methanesulfonate, $N$-ethyl- $N$-nitrosourea (ENU), or ultraviolet trimethylpsoralen were self-crossed to the $\mathrm{F} 7$ generation to drive mutations to homozygosity. This allowed for the characterization of the mutation spectra of each mutagen and provided the first evidence that mutagen exposures cause heritable genomewide mutations. However, the study was limited because it used an invertebrate model, it took several generations before the mutations were detected, and only homozygous mutations were analyzed. Only one study has attempted to detect de novo mutation induction following endogenous mutagen exposure in a single generation family pedigree (Adewoye et al., 2015). In that study, male mice were irradiated with 3 Gy of X-rays and mated with females 2-4 days after dosing to produce offspring that developed from exposed post-meiotic spermatids. Whole genome sequencing using NGS of exposed and 
control families demonstrated that the radiation exposure induced indels and multinucleotide base substitutions, but not SNVs. Thus, it is clear that NGS can characterize the types and locations of induced mutation events, and even provide predictions as to how harmful they may be. NGS will no doubt revolutionize the field of germ cell mutagenesis; however, the power of NGS has not yet been sufficiently tested on chemical mutagens. For my thesis, I explored this technology to fully characterize the effects of a chemical mutagen exposure on the germline and compared the inherited de novo mutations in offspring with those detected in sperm (Chapter 7).

\subsection{Test compound Benzo(a)pyrene as a Model For Environmental Expos ures}

In my thesis, I studied the mutagenic effects of benzo[a]pyrene $(\mathrm{BaP})$ on the germline as a model for other chemical mutagens. $\mathrm{BaP}$ is a polycyclic aromatic hydrocarbon $(\mathrm{PAH})$ and common environmental pollutant formed during incomplete combustion or pyrolysis of organics (IARC, 2012b). The main sources of BaP exposures in humans include cigarette smoke (case study presented in Chapter 8), smoked meats, motor vehicle exhaust, industrial air emissions, occupational exposures (e.g., aluminum production), and natural phenomena such as forest fires (IARC, 2010). BaP is a known human carcinogen (Group 1) and somatic/germ cell mutagen, but it is only mutagenic following metabolism. BaP is metabolized by phase-I and phase-II enzymes to form several different compounds, but the carcinogenesis of $\mathrm{BaP}$ is explained mainly by the formation of diolepoxides and radical cations (IARC, 2012b). In the cytosol, BaP binds to the aryl hydrocarbon receptor, and this complex translocates to the nucleus to activate transcription of cytochrome P450s. Specifically, metabolism is carried out by CYP1A1, 
CYP1B1, and epoxide hydrolase to convert $\mathrm{BaP}$ to its most reactive form benzo[a]pyrene 7,8-dihydrodiol-9,10-epoxide (BPDE) (Xue and Warshawsky, 2005). BPDE is capable of forming multiple DNA adducts, but the most stable adduct involves the 2-amino group $\left(\mathrm{N}^{2}\right)$ of guanine (Xue and Warshawsky, 2005). These adducts are often repaired by nucleotide excision repair (NER) when incorporated into the DNA (Verhofstad et al., 2011); however, error-prone translesion synthesis during replication can lead to mutations (Avkin et al., 2004). The most common BaP-associated mutation is G:C $\rightarrow$ T:A transversions, but other mutational events can occur (Mass et al., 1993; Shane et al, 2000).

There is growing evidence that $\mathrm{BaP}$ crosses the blood-testis barrier and impairs sperm function and quality. For example, sub-chronic oral exposure to $100 \mathrm{mg} / \mathrm{kg}$ of BaP in mice for 30 or 60 days is associated with impaired sperm motility, vitality, morphology, and sperm concentration (Jeng and Bocca, 2013; Jeng et al., 2013). There is even evidence that some of these effects on sperm parameters in mice may be transgenerational and carry on to the F2 generation (Mohamed et al., 2010). In addition to impaired sperm parameters, $\mathrm{BaP}$ can cause DNA damage in sperm. The same sub-chronic $\mathrm{BaP}$ exposures associated with impaired sperm parameters also showed dose-dependent increases in DNA damage (Jeng and Bocca, 2013; Jeng et al., 2013). Furthermore, Big Blue ${ }^{\circledR}$ mice that received 3 intraperitoneal (i.p.) injections of $50 \mathrm{mg} / \mathrm{kg}$ of $\mathrm{BaP}$ had elevated BPDE- $\mathrm{N}^{2}$-dG adducts in spermatozoa (Olsen et al., 2010). Thus, BaP causes DNA damage and adducts in sperm, indicative that it is mutagenic to male germ cells.

Studies using the TGR assay have investigated if $\mathrm{BaP}$ induces mutations in sperm. The first study by Olsen et al., 2010 showed that the BPDE- $\mathrm{N}^{2}$-dG adducts formed during 
$\mathrm{BaP}$ injections were associated with an increased mutant frequency (MF) of the $c I I$ transgene in spermatogonial stem cells. Sequencing of those mutants revealed a mutational spectrum consistent with $\mathrm{BaP}$ exposure in somatic cells. Similarly, another study demonstrated that pre-pubertal, pubertal, and adult transgenic mice receiving i.p. injections of $300 \mathrm{mg} / \mathrm{kg}$ all had increased mutant frequencies in meiotic pachytene spermatocytes (Xu et al., 2014). Interestingly, the early-life exposures resulted in larger increases of mutation induction in the lacI transgene compared to the adult exposure, suggesting that younger individuals may be more sensitive to genotoxic exposures. In addition, subchronic oral exposures $(3 \times 13 \mathrm{mg} / \mathrm{kg} \times 6$ weeks $)$ increased the MF of the lacZ transgene in spermatogonial stem cells (Verhofstad et al., 2011). Thus, all the studies that used the TGR assay reported that $\mathrm{BaP}$ is capable of increasing mutant frequencies in different sperm types.

Other methods have explored the heritable effects of $\mathrm{BaP}$ exposure on the germline. For example, pedigree analysis of ESTRs has shown that male mice deficient in NER pass on more tandem repeat mutations to their offspring when exposed to BaP (Godschalk et al., 2015). Interestingly, wild-type mice exposed to BaP did not pass on more mutations compared to controls, demonstrating the importance of NER in minimizing tandem repeat mutation formation. Moreover, traditional assays have shown that high doses of $\mathrm{BaP}$ exposure in male mice in the week before fertilization lead to dominant lethal mutations (Generoso et al., 1982; Shukla and Taneja, 2001), but both the SLT and HTT gave negative results (Russell et al., 1981). Gene expression analysis of in vitro fertilization embryos from sperm of $\mathrm{BaP}$-treated mice showed upregulation of genes involved in DNA transcription, DNA damage response, cell cycle regulation, chromatin 
modification, oxidation-reduction processes, apoptosis, and embryo development (Brevik et al., 2012). Together, these results indicate that BaP exposure causes DNA damage and mutations in sperm that persist as far as early embryonic development. However, genome-wide analyses of family pedigrees are required to understand the full spectrum of heritable mutations induced by $\mathrm{BaP}$ and the potential consequences of these mutations to offspring health.

\subsection{Thes is Hypothesis and Study Design}

My main hypothesis for this thesis was that mutagens, such as $\mathrm{BaP}$, induce de novo germ cell mutations that can be detected in the offspring of exposed male mice using genomics technologies. To test this hypothesis, I designed a study to characterize and quantifiy mutations in both the sperm and offspring of $\mathrm{BaP}$-exposed males.

Specifically, I modified traditional approaches to make them amenable to the thesis work. These modified approaches were used to investigate the number and types of mutations in sperm, and genomics technologies were used to study the offspring for genome-wide effects. Furthermore, I explored the potential impacts of an increase in heritable mutations using paternal smoking as one source of $\mathrm{BaP}$ exposure. For this purpose, I reviewed the literature to estimate an effect size and model a global population burden of genetic disease that might result from paternal smoking (Chapter 8).

The study for this thesis used 2 groups of 30 MutaMouse males. One group served as controls while the other group was exposed to $100 \mathrm{mg} / \mathrm{kg}$ of $\mathrm{BaP}$ per day for 28 days (administration time recommended by OECD). This dose was chosen for the purpose of demonstrating proof of concept for each method. The dose is several orders of 
magnitude greater than a highly exposed individual and is therefore not reflective of a normal physiological exposure. However, humans are exposed throughout their life to low doses of $\mathrm{BaP}$ and other mutagenic PAHs. Three groups of 6 controls and 6 treated mice were euthanized 3, 42 and 70 days post-exposure, respectively, and sperm were collected for the analysis of mutations using the TGR assay. These timings correspond to sperm that were mature sperm, dividing spermatogonia, and stem cells at the end of the exposure period, respectively. Sperm DNA from the 42 day time-point were also analyzed for the induction of tandem repeat mutations using SM-PCR. Analyses of sperm from different time-points allows for comparisons to be made between pre-meiotic and post-mitotic mutation induction. The remaining 12 males in each group were mated with 4 different C57BL females 3, 42, and 70 days post-exposure. Analysis of offspring developing from sperm exposed during different phases of spermatogenesis allows for comparisons to be made between mutation fixation before and after fertilization. Offspring were euthanized after weaning (approximately 6 weeks after birth). The following tissues were collected from all mice for analyses: bone marrow, liver, spleen, brain, cauda epididymis and seminiferous tubules. Family pedigrees were analyzed using aCGH and NGS for the induction of CNVs and all mutation types, respectively.

Overall, using new genomics tools (aCGH and NGS) to search for de novo mutations in the offspring of exposed mice, and modified existing methods (SM-PCR and TGR) to search for mutations in sperm will: (i) provide further information into how mutagens affect germ cell and offspring DNA; (ii) support and provide insight into the relevance of existing methods for germ cell mutation analysis; and, (iii) facilitate the development of new approaches to identify hazards posed to germ cells and offspring. 
Studies that use genomics tools to detect germline mutation induction following an exogenous exposure are still in their early stages (Adewoye et al., 2015), and successful application of the tools used in my thesis will pave the way for other researchers interested in these types of studies. Finally, risk assessments that use heritable mutation as the endpoint of concern are rare. My research demonstrates the population level effects of germ cell mutagenesis and makes a strong case for considering heritable mutation as an endpoint of regulatory concern.

\subsection{Thes is Objectives}

Overarching objectives:

(a) To determine whether paternal exposure to $\mathrm{BaP}$ increases mutation frequencies and alters mutation spectrum in sperm.

Chapters 2, 3, 5, 6, and Appendix B analyze sperm for BaP mutation induction. The methods developed for this purpose are described in Appendix A and Chapter 4.

(b) To determine whether paternal exposure to $\mathrm{BaP}$ increases mutation frequencies and alters mutation spectrum in the offspring. This objective is addressed in Chapter 7.

(c) To assess pre-fertilization versus post-fertilization mutation fixation.

Pre-fertilization effects in sperm are examined in Chapters 2, 3, and 5. A comparison of pre- and post-fertilization mutation fixation is conducted in Chapter 7. 
(d) To assess the utility of whole genome approaches in identifying heritable mutations. The results from Chapters 2, 3, 5, and 6 are compared against the results from Chapter 7 to meet this objective.

Specific objectives:

(e) Detect microsatellite mutations in sperm using SM-PCR (Chapters 2 and 3).

(f) Develop a method to characterize mutation spectra of TGR assay mutants using NGS (Chapter 4).

(g) Detect germline mutation induction in sperm using the TGR assay (Chapter 5 and Appendices A and B).

(h) Characterize the mutation spectrum of $\mathrm{BaP}$ in sperm (Chapter 6).

(i) Detect de novo $\mathrm{CNV}$ induction in the offspring of exposed males following $\mathrm{BaP}$ exposure using aCGH (Chapter 7).

(j) Detect the induction of de novo SNVs and indels across the entire genome using NGS technologies (Chapter 7).

(k) Explore a case study on tobacco smoke as a common environmental source of BaP exposure, characterize its potential as a human germ cell mutagen, and model the socioeconomic impacts of this using intellectual disability as an example (Chapter 8).

Overall, the objective for my thesis is to elucidate the mechanisms of inherited mutagenesis and assist in making recommendations on how germ cell mutagen hazard assessment should be undertaken in the future. 


\subsection{Tables and Figures}

\begin{tabular}{|c|c|c|c|c|c|}
\hline Phase & \multicolumn{2}{|c|}{ Mitosis } & \multirow{2}{*}{$\begin{array}{c}\text { Meiosis } \\
\text { Spermatocyte }\end{array}$} & \multirow{2}{*}{$\frac{\text { Post-Meiosis }}{\text { Spermatid }}$} & \multirow{2}{*}{$\begin{array}{c}\text { Maturation } \\
\text { Sperm } \\
\end{array}$} \\
\hline Germ Cell & Stem Cells & Spermatogonia & & & \\
\hline Processes & \multicolumn{2}{|c|}{ DNA Synthesis } & & \multicolumn{2}{|c|}{ Declining Repair } \\
\hline \multirow{2}{*}{$\begin{array}{r}\text { Mutation Type } \\
\text { Location }\end{array}$} & \multicolumn{2}{|c|}{ Point Mutations, Insertions, Deletions } & $\begin{array}{c}\text { Aneuploidies, } \\
\text { Recurrent CNVs } \\
\end{array}$ & \multicolumn{2}{|c|}{$\begin{array}{l}\text { Chromosomal Abnormalities, DNA Adducts, } \\
\text { Strand Breaks }\end{array}$} \\
\hline & \multicolumn{4}{|c|}{ Testis } & Epididymis \\
\hline Days to Mat & uration 4 & & & 14 & 7 \\
\hline
\end{tabular}

$28+42$

$28+70$

Figure 1.1 Schematic diagram of mouse spermatogenesis showing germ cell types and phases that can be targeted by various

experimental designs. The boxes at the bottom indicate the exposure regiment (duration of exposure $=28$ days) and the dashed lines indicate the days between the last exposure and when the sperm are collected for analsyis (fixation period $=3,42$, or 70 days). Figure is modified from ref. (O'Brien et al., 2014). 


\section{Chapter 2: Single-Molecule PCR Analysis of an Unstable Microsatellite for Detecting Mutations in Sperm of Mice Exposed to Chemical}

Mutagens

Modified from: Beal, M.A., Rowan-Carroll, A., Campbell, C., Williams, A., Somers, C.M., Marchetti, F., Yauk, C.L. 2015. Mutation Research: Fundamental and Molecular Mechanisms of Mutagenesis, 775:26-32.

\subsection{Preamble}

One of the main thesis objectives was to determine if $\mathrm{BaP}$ increases mutation frequencies in sperm. For this purpose, we developed SM-PCR of microsatellites as a novel method for detecting $\mathrm{BaP}$ mutations in sperm. As a positive control, ENU treated animals were also tested for microsatellite mutations in sperm. Understanding how a chemical induces sperm mutations is necessary to make predictions on the types of mutation events that will occur genome-wide in the offspring.

\subsection{Abstract}

SM-PCR analysis of long and repetitive DNA sequences, known as ESTRs, has been the most efficient method for studying germline mutation induction in endogenous sequences to date. However, the long length of these sequences makes mutation detection imprecise and labourious, and they have been characterized only in mice. Here, we explore the use of unstable microsatellite sequences that can be typed with high precision by capillary electrophoresis as alternative loci for detecting germline mutations. We 
screened 24 microsatellite loci across inbred mouse strains and identified Mm2.2.1 as the most polymorphic microsatellite locus. We then optimized SM-PCR of Mm2.2.1 to detect mutations in sperm. SM-PCR analysis of sperm from untreated B6C3F1 and MutaMouse samples revealed mutation frequencies that are consistent with rates derived from family pedigree analysis $\left(\sim 5 \times 10^{-3}\right)$. To determine whether this locus can be used to detect chemically induced germline mutations, MutaMouse males were exposed by oral gavage to a single dose of $100 \mathrm{mg} / \mathrm{kg}$ of ENU or to $100 \mathrm{mg} / \mathrm{kg}$ of $\mathrm{BaP}$ for 28 days alongside vehicle treated controls. Sperm were collected 10 weeks post-ENU exposure to sample sperm exposed as spermatogonial stem cells and 6 weeks post-BaP exposure to sample sperm that were dividing spermatogonia when the exposure was terminated. Both treatments resulted in a significant (approximately 2-fold) increase in mutation frequency in sperm compared to the control animals. The work establishes the utility of this microsatellite for studying mutation induction in the germ cells of mice. Because microsatellites are found in virtually every species, this approach holds promise for other organisms, including humans.

\subsection{Introduction}

There is growing recognition that environmental and lifestyle variables may contribute to increased heritable-mutation burden and adverse effects in the descendants of exposed males (D’Onofrio et al., 2014; Hehir-Kwa et al., 2011; Hurles, 2012; Kong et al., 2012; Linschooten et al., 2013). Applications of high-throughput genomics technologies in human populations are revealing that there are a diversity of genomic changes that can contribute to human disorders, including gene mutations, genomic 
rearrangements (e.g., copy-number variations, and tandem-repeat mutations), and mutations in important regulatory non-coding domains. However, our understanding of how environmental exposures may affect the genetic integrity of the germline is unclear (Beal et al., 2012b; Wyrobek et al., 2007). Identification of agents that can cause increases in germline mutation rates can be difficult due to the rarity of de novo mutations, which occur at an average rate of $1 \times 10^{-8}$ per nucleotide per generation (Campbell and Eichler, 2013). Thus, in order to adequately assess in vivo germline mutation risk following mutagen exposures, an array of sensitive assays that capture a broad range of mutagenic endpoints are required.

In the past 2 decades, sensitive approaches have been proposed to detect increases in induced germ cell mutation frequencies, including the TGR assay and ESTR analysis (Singer et al., 2006; Verhofstad et al., 2008). The TGR assay uses mice that carry a reporter transgene that allows for mutation analysis following viral packaging of the transgene and infection in a bacterial host (Lambert et al., 2005). This readily allows the detection of chemically induced mutations occurring in germ cells or any other tissue (Douglas et al., 1994; Douglas et al., 1995; O’Brien et al., 2013; O'Brien et al., 2015; Vijg and Douglas, 1996). However, this method can be applied only to transgenic animals carrying the reporter gene and is also limited to a few bacterial gene sequences. Thus, more broadly applicable assays for studying germ-cell mutation are required.

ESTRs, short repetitive DNA sequences (4-6 bp) arranged in very long arrays (up to $\sim 20 \mathrm{~kb}$ ) (Yauk, 2004), have also provided a useful tool for studying germ cell mutagenesis because they are highly sensitive to mutagens. Studies using ESTRs have demonstrated increased mutation frequencies in sperm and offspring following exposure 
to radiation (Dubrova et al., 1993), chemicals (Glen et al., 2008; Vilariño-Güell et al., 2003), and complex mixtures (Marchetti et al., 2011; Somers et al., 2002; Yauk et al., 2007). The development of a SM-PCR technique to directly quantify ESTR mutations in sperm of exposed males (rather than in offspring of exposed parents) has reduced greatly the sample sizes required for animal studies (Yauk et al., 2002). Although ESTRs have provided a useful tool to identify potential male germ cell mutagens, analysis of ESTRs requires long agarose gel electrophoresis, Southern blotting, and a scoring technique that relies on measurement by eye and comparison across lanes and blots. Overall, ESTR mutation detection can be subjective, at times technically challenging (if a locus is long), and time consuming. Furthermore, to date ESTRs have been identified only in mice (Bois et al., 1998; Gibbs et al., 1993; Kelly et al., 1989); thus, similar to the TGR assay, these studies are limited to specific models, making it more difficult to extrapolate findings to humans. Enhanced approaches that reduce some of these limitations would be of obvious benefit to the assessment of potential germ cell mutagens.

Microsatellites are another class of tandem repeats that can be useful in the study of germ cell mutations. Microsatellites are common in the genomes of most eukaryotes (in contrast with ESTRs, which have only thus far been characterized in mice). Their small size $(2-6$ bp repeats, but with total length $<1 \mathrm{~kb})$ makes mutation detection more precise and amenable to higher-throughput approaches than ESTR analysis. Furthermore, various microsatellite mutations are associated with different diseases (Mirkin, 2007), even when the mutation does not occur in a coding sequence (Mori et al., 2013). Direct identification of de novo germline mutations at microsatellite loci has already been applied in different species (e.g. (Ellegren et al., 1997; Tsyusko et al., 2007)), including 
humans (Furitsu et al., 2005; Sun et al., 2012) and mice (Beal et al., 2012a), through family pedigree analysis. One of the most powerful microsatellite studies to date applied a pedigree approach in 85,289 individuals at 2477 microsatellites to demonstrate older males transmit more mutations to their offspring, confirming that paternal age is a modulating factor in germline mutation rates (Sun et al., 2012). Thus, microsatellite mutations are certainly relevant in humans and factors that can elevate mutation frequencies at these loci need to be identified. However, many studies on mutageninduced increases in microsatellite mutation frequency have thus far had insufficient sample sizes and failed to detect a response (reviewed in (Beal et al., 2012a)). Baker et al. (1995) used monomorphic loci to detect several mutations in the sperm of PMS2 deficient mice, but the loci used had mutation frequencies too low to detect any spontaneous mutations. Beal et al. (2012a) identified a panel of polymorphic microsatellites and used them to screen family pedigrees following paternal irradiation. Although several mutations were detected in control and treated families, there was no evidence for an increase in microsatellite mutations using the pedigree approach. Hence, an approach more sensitive than screening family pedigrees is needed to detect microsatellite mutation induction.

Here we develop and optimize SM-PCR for an unstable microsatellite locus, Mm2.2.1 (Beal et al., 2012a), to detect mutations in germ cells and overcome the limitations of sample sizes with family pedigrees. By screening highly inbred strains for microsatellite polymorphisms, as a likely reflection of high mutation rate, we identify Mm2.2.1 as a sensitive marker for SM-PCR mutation detection. We test the efficacy of this SM-PCR approach by determining mutation frequencies in the sperm of mice 
exposed to ENU, a prototypical germ-cell mutagen tested by both the TGR (Douglas et al., 1995; O’Brien et al., 2013; O'Brien et al., 2014; O'Brien et al., 2015) and ESTR (Dubrova et al., 2008; Swayne et al., 2012; Vilariño-Güell et al., 2003) assays, and BaP, a common environmental pollutant (IARC, 2012b) following spermatogonial exposure. $\mathrm{BaP}$ is an established somatic-cell mutagen (IARC, 2012b), and there is also evidence that $\mathrm{BaP}$ induces germ cell mutations (Olsen et al., 2010; Verhofstad et al., 2011; Xu et al., 2014), but there is currently no evidence that $\mathrm{BaP}$ induces tandem repeat mutations through the male germline. We demonstrate that SM-PCR analysis of unstable microsatellites by capillary electrophoresis can be used to detect changes in germline mutation frequencies arising in chemically treated spermatogonia.

\subsection{Materials and Methods}

\subsubsection{Selection and Prioritization of Micros atellites}

Sixteen trinucleotide microsatellites (Table C1) were selected for preliminary sizing and analysis from the Mus musculus genome. Selection was based on core sequence and repeat length, using the online Eukaryotic Microsatellite Database (Aishwarya et al., 2007). Additionally, 8 polymorphic tetranucleotide repeats from Beal et al. (2012a) were also selected. BLAST was used to design primers for the trinucleotide repeats, and primers designed previously were used for PCR amplification of the tetranucleotide repeats (Table $\mathrm{C} 1$ ). The primers were used to amplify DNA from a panel of 13 inbred mouse strains (Charles River Laboratories, Wilmington, MA) and a F1 cross to identify polymorphic microsatellites that could be developed for SM-PCR. 


\subsubsection{Spontane ous and Induced Ge rmline Mutation Frequencies}

All animal protocols were conducted under conditions approved by Health Canada's Animal Care Committee. Sperm samples isolated from 6 male B6C3F1 mice were used to determine the spontaneous-mutation frequency. Sperm-mutation frequency at Mm2.2.1 by SM-PCR was compared to mutation frequencies in family pedigrees published previously (Beal et al., 2012a).

We also measured the microsatellite-mutation frequencies of MutaMouse (Gossen et al., 1989) males that were exposed to chemicals. For the first study, treated mice received an acute dose of ENU (100 mg/kg) dissolved in phosphate buffer $(\mathrm{pH} 6,8.71$ $\mathrm{mM} \mathrm{Na}_{2} \mathrm{HPO}_{4}, 58.29 \mathrm{mM} \mathrm{KH} \mathrm{PO}_{4}$ ) via oral gavage, and control animals received phosphate buffer (O'Brien et al., 2014; O'Brien et al., 2015). Cauda epididymides were collected from 3 treated and 3 untreated males 10 weeks post-exposure (sperm exposed as spermatogonial stem cells). A second study was performed in which microsatellite frequencies were measured in MutaMouse males exposed to $100 \mathrm{mg} / \mathrm{kg}$ of BaP. Mice were exposed to BaP dissolved in olive oil for 28 days by oral gavage while concurrent control animals received olive oil by oral gavage. The cauda epididymides were collected from $3 \mathrm{BaP}$-treated and 6 control animals 6 weeks post-exposure (sperm exposed as dividing spermatogonia).

For each experiment, all cauda epididymides were removed from the mice upon necropsy, flash frozen, and stored at $-80{ }^{\circ} \mathrm{C}$. Following SM-PCR analysis, the mutation frequencies of the treated animals were compared to the mutation frequencies in matched controls to test the sensitivity of microsatellite SM-PCR. 


\subsubsection{DNA Isolation and Preparation for Mutation Analysis}

Sperm DNA was prepared following a protocol described previously (Yauk et al., 2002). All DNA manipulations were performed in a laminar flowhood to minimize the risk of contamination. Briefly, cauda epididymides were chopped in $1.5 \mathrm{~mL}$ of phosphate-buffered saline, filtered, and pelleted by centrifugation for $5 \mathrm{~min}$ at 15,000 $\mathrm{rpm}$. The pellet was then re-suspended in $1 \times$ saline-sodium citrate $(\mathrm{SSC} ; 0.15-\mathrm{M} \mathrm{NaCl}$, $0.015-\mathrm{M}$ sodium citrate) and $0.15 \%(\mathrm{w} / \mathrm{v})$ sodium dodecyl sulfate (SDS) to lyse the somatic cells. The lysate was centrifuged and the purified sperm pellet was resuspended in $1 \mathrm{~mL}$ of $0.2 \times \mathrm{SSC}(0.03-\mathrm{M} \mathrm{NaCl}, 0.003-\mathrm{M}$ sodium citrate), $1 \% \mathrm{SDS}, 1 \mathrm{M} 2-$ mercaptoethanol, and digested with $>50 \mathrm{U}$ of proteinase $\mathrm{K}$ (Invitrogen ${ }^{\mathrm{TM}}$, Carlsbad, California) at $37{ }^{\circ} \mathrm{C}$ overnight. Sperm purification was determined using phase-contrast microscopy prior to digestion, and all somatic cells appeared to be removed prior to lysis of sperm cells. DNA was then isolated by phenol/chloroform extraction and re-suspended in 5-mM Tris- $\mathrm{HCl}(\mathrm{pH} 7.5)$. DNA concentrations were determined using nano-drop spectrophotometry (NanoDrop UV-vis spectrophotometry - model number ND-1000, Asheville, NC). Approximately $2 \mu \mathrm{g}$ of each DNA sample was digested overnight at 37 ${ }^{\circ} \mathrm{C}$ with 20 U of HindIII (New England Biolabs, Ipswich, MA). Digested DNA samples were diluted to approximately $1 \mathrm{ng} / \mu \mathrm{L}$ in $5-\mathrm{mM}$ Tris- $\mathrm{HCl}(\mathrm{pH} 7.5)$ and $5 \mathrm{ng} / \mu \mathrm{L}$ herring sperm DNA (Sigma-Aldrich, St. Louis, MO).

\subsubsection{SM-PCR Amplification}

DNA samples were diluted to $10,25,50$, and $100 \mathrm{pg} / \mu \mathrm{L}$ aliquots in herring sperm DNA, and PCRs were carried out for each dilution to determine the concentration that 
gave positive amplifications in $40-60 \%$ of reactions (Poisson analysis shows that this amplification success range equates to approximately 1 amplifiable molecule per positive reaction (Yauk et al., 2002)). If necessary, concentrations were adjusted accordingly for subsequent mutation analysis. The primers used for locus prioritization (Table C1) were also used for SM-PCR analyses. DNA was amplified in $8.9-\mu \mathrm{L}$ reactions using a $10-\mu \mathrm{M}$ fluorescently tagged forward primer and an unlabelled reverse primer (Table C1). Each PCR was done using Roche High Fidelity PCR System Reagents (Roche \# 04738276 001). Within each reaction, the following volume for each of the kit reagents were used: $1 \mu \mathrm{L} 10 \times$ PCR buffer, $1 \mu \mathrm{L}$ dNTPs, $1 \mu \mathrm{L}$ forward primer, $1 \mu \mathrm{L}$ reverse primer, $1.2 \mu \mathrm{L}$ $\mathrm{MgCl} 2$ stock solution, $1.1 \mu \mathrm{L}$ PCR-safe water, and $0.3 \mu \mathrm{L}$ enzyme mix. In addition, each reaction contained $2.5 \mu \mathrm{L}$ 4-M Betaine (Sigma) and $1 \mu \mathrm{L}$ sperm DNA (concentration that gives a product in $40-60 \%$ of amplifications; $~ 10 \mathrm{pg} / \mu \mathrm{L})$. SM-PCR amplification conditions were optimized, and the thermal-cycle program was $94{ }^{\circ} \mathrm{C}$ for $120 \mathrm{~s} ; 38$ cycles of $94{ }^{\circ} \mathrm{C}$ for $30 \mathrm{~s}, 57^{\circ} \mathrm{C}$ (variable depending on locus) for $30 \mathrm{~s}, 70{ }^{\circ} \mathrm{C}$ for $120 \mathrm{~s}$; final extension at $70{ }^{\circ} \mathrm{C}$ for $30 \mathrm{~min}$.

\subsubsection{Mutation Detection}

For mutation analysis, PCRs were carried out in 96-well plates with control and treatment plates run in parallel. To detect mutations, amplicons were analyzed with single base-pair resolution using an internal size standard (GeneScan ${ }^{\mathrm{TM}} 500$ LIZ®) on an Applied Biosystems 3130×1 DNA sequencer (Life Technologies ${ }^{\mathrm{TM}}$, Carlsbad, CA). A set of scoring criteria was used to ensure that PCR artifacts were not scored as mutations. Specifically, in order for an allele to be considered mutant, the novel allele needed to 
have resulted in a shift of 1 or more whole repeat units, and the -1 repeat unit stutter band needed to be present. In addition, no trace of the wild-type allele could co-occur with a mutant band; if the wild-type progenitor was present, the additional peak was presumed to be the result of an artifact resulting from replication slippage during the early rounds of PCR. As a result of the stringent scoring criteria used, our mutation estimates are likely conservative. Putative mutants were analyzed on the sequencer an additional time to confirm that they were true mutations and that the appearance of a mutation was not due to electric current problems during electrophoresis. To prevent clonal expansions from biasing results, a specific change in microsatellite length (e.g., +1 repeat unit) was counted only once even if it was found several times in a sample because it was presumed to represent a single mutation event. If the mutation was seen in a second sample, it was counted twice (once for each sample).

\subsubsection{Statistical Analyses}

Statistical analyses were based on procedures published previously (Swayne et al., 2012; Yauk et al., 2008b; Yauk et al., 2008a). Briefly, a Poisson distribution was used to estimate the number of progenitor PCR template molecules (haploid sperm genomes) determined from the number of positive/negative PCR wells and the number of wells used for PCR. Assuming a Poisson distribution for the error, generalized estimating equations (GEE) (Liang and Zeger, 1986; Prentice and Zhao, 1991) were used to estimate microsatellite-mutation frequencies. Pairwise comparisons of mutation frequencies were made between control and exposed samples to detect mutation induction $(\mathrm{P}<0.05)$. 
Comparison of the mutation spectra was done using Pearson's Chi-squared test with Monte Carlo simulation.

\subsection{Results}

\subsubsection{Selection and Prioritization of Micros atellites}

PCR conditions for 24 microsatellite loci (17-111 repeat units; Table C1) were optimized, and a panel of 14 different mouse strains was screened for the presence of heterozygosity or multiple alleles as an indicator of instability. Locus Mm2.2.1 (mutation frequency $=0.005 \%$ (Beal et al., 2012a) ) showed the most variation among the inbred mouse samples (Figure 2.1) and had strong amplification without background noise. Therefore, Mm2.2.1 was selected as the primary locus for SM-PCR mutation detection.

\subsubsection{Spontane ous and Induced Germline Mutation Frequencies}

Sperm from $6 \mathrm{~B} 6 \mathrm{C} 3 \mathrm{~F} 1$ samples were screened for microsatellite mutations to establish the spontaneous-mutation frequency of locus Mm2.2.1 in sperm (example mutation: Figure 2.2). This was done to determine if SM-PCR-mutation frequencies are consistent with those measured in family pedigrees (Beal et al., 2012a). The genotype of each sample consisted of 2 alleles that varied in size. The long allele was between 362 and $370 \mathrm{bp}$ and is likely of C57BL/6 descent, based on the length of the C57BL/6J reference sequence (366 bp; (AGAT) $\left.)_{17} \ldots(\text { AGAT) })_{18}\right)($ NT_039206.8). The shorter allele was between 295 and 299 bp and is likely inherited from the $\mathrm{C} 3 \mathrm{H} / \mathrm{He}$ line (Figure 2.1). Across the 6 samples, there were 6 independent mutations that appeared in the complete absence of any peak for the wild-type progenitor allele. With a total of 1354 (1283-1427) 
progenitors, the mutation frequency was $4.3 \times 10^{-3}\left(2.2-8.6 \times 10^{-3}\right)$. This is consistent with the mutation frequency of Mm2.2.1 measured in Swiss-Webster (allele sizes: 292$308 \mathrm{bp})$ family pedigrees: $5.0 \times 10^{-3}\left(1.0-14.9 \times 10^{-3}\right)($ Beal et al., 2012a). The same number of mutations occurred in the small alleles ( 3 deletions) as the large alleles ( 2 deletions, 1 gain).

In order to investigate response to mutagen exposure, sperm samples from ENU and BaP-treated MutaMouse males were subjected to SM-PCR, and the mutation frequencies in Mm2.2.1 were compared against matched controls. Genotyping of all MutaMouse males revealed that all samples were homozygous with a $291 \mathrm{bp}$ allele. The spontaneous mutation frequency of Mm2.2.1 in the MutaMouse was $2.38 \times 10^{-3}(1.57-$

$\left.3.63 \times 10^{-3}\right) . \mathrm{Mm} 2.2 .1$ sperm mutation frequencies were greater in males exposed to ENU (2.4-fold; $\mathrm{P}=0.0001)$ and $\mathrm{BaP}(2.3$-fold; $\mathrm{P}=0.012$ ) (Figure 2.3; Table 2.1). Although there were several large deletions (but no large gains), the majority of mutations detected in the MutaMouse samples were single-repeat changes (Figure 2.4). All of the mutations at the tetranucleotide repeat locus were multiples of $4 \mathrm{bp}$, indicating that the mutations were true repeat length polymorphisms and not false-positives.

\subsection{Discussion}

In this study, we screened a panel of microsatellites across inbred strains to identify potentially unstable loci. The microsatellite exhibiting the most variability across these strains, Mm2.2.1, was presumed to be the most unstable. Conditions were then optimized for SM-PCR of Mm2.2.1 (Figure 2.1) to determine both spontaneous and induced mutation frequencies. We demonstrate that the mutation frequency of $\mathrm{Mm} 2.2 .1$ 
in B6C3F1 measured using SM-PCR is highly concordant with its mutation frequency in family pedigrees (Beal et al., 2012a). This consistency suggests that mutations detected in sperm are true de novo mutations rather than artifacts. SM-PCR was then used to determine whether increases in germ-cell mutation occur following treatment with chemicals that are known to cause mutations in spermatogonia. We show a 2.3- and 2.4fold increase in the mutation frequencies induced in the spermatogonia of mice exposed to $\mathrm{BaP}$ and ENU, respectively, relative to control mice. These results demonstrate SMPCR can be used to detect chemically induced mutations in sperm.

The 2.4-fold increase in microsatellite mutation induction compared to control mice detected in the sperm samples 10 weeks after oral exposure to $100 \mathrm{mg} / \mathrm{kg}$ of ENU was expected based on previous studies investigating the effects of ENU on sperm at ESTR loci. Multiple studies have shown that ENU is able to induce repeat mutations in ESTRs in both sperm and offspring of exposed male mice (Dubrova et al., 2008; Swayne et al., 2012; Vilariño-Güell et al., 2003). Thus, the concordance between our findings in microsatellites and previous studies on ESTRs suggests that microsatellites are a useful marker for detecting mutations that occur following exposure to spermatogonial stem cells.

$\mathrm{BaP}$ is a model mutagen that has been studied extensively in somatic cells (IARC, 2012b), but the mutagenic effects of $\mathrm{BaP}$ on male germ cells are less understood. To date, no study has explored the effects of $\mathrm{BaP}$ on tandem repeat mutations in sperm. However, embryonic mouse cells in culture exposed to $\mathrm{BaP}$ for a prolonged period of time exhibit increases in ESTR mutations (Polyzos et al., 2006). Moreover, recent studies have demonstrated that $\mathrm{BaP}$ can impair functional parameters in sperm, induce DNA strand 
breaks in sperm, and increase the proportion of apoptotic germ cells (Jeng et al., 2013; Jeng and Bocca, 2013). Furthermore, studies using the TGR assay have revealed that BaP induces point mutations in different germ cells (Olsen et al., 2010; Verhofstad et al, 2011; Xu et al., 2014). In this study, we demonstrate that mice treated with $100 \mathrm{mg} / \mathrm{kg}$ of $\mathrm{BaP}$ exhibit mutation induction at a microsatellite locus in spermatogonia and that the magnitude of the effect is similar to that observed with an acute exposure to ENU. The mutagenic effects of ENU are likely permanent as the treatment induced microsatellite mutations directly in stem cells; however, more work would need to be done to determine if $\mathrm{BaP}$ induces microsatellite mutations in stem cells.

Comparison of ENU-mutagenesis using tandem repeat loci versus the TGR assay at the same dose shows that the TGR assay consistently reports a higher fold increase in mutation frequency following ENU exposure (O'Brien et al., 2013; O'Brien et al., 2014; Swayne et al., 2012; Vilariño-Güell et al., 2003). It has been demonstrated using ENU that the mutation response of ESTRs in sperm appears to plateau at a 3-fold increase (Vilariño-Güell et al, 2003), whereas ENU-induced point mutations plateau at a 5-fold increase (Douglas et al., 1995; O'Brien et al., 2014). Similarly, we found that the mutation induction observed at the microsatellite locus following both the ENU (2.4-fold) and $\mathrm{BaP}$ (2.3-fold) exposures were lower than the induced lac $Z$ mutation frequency measured using the TGR assay ( $\sim 5$-fold) in the same samples (ENU published in (O'Brien et al., 2014; O'Brien et al., 2015); BaP unpublished data).

This may be attributed to the different mutational mechanisms that impact these different sequence contexts. ENU is an alkylating agent that forms mutagenic DNA adducts in pre-meiotic germ cells, specifically O4-ethyl thymine, O2-ethyl thymine, and 
O6-ethyl guanine (Beranek, 1990; Shelby and R Tindall, 1997). Insufficient repair of these lesions prior to replication leads to an increase in point mutations, mainly A:T $\rightarrow$ G:C transitions and A:T $\rightarrow$ T:A transversions in transgenic reporter genes (Douglas et al., 1995). $\mathrm{BaP}$ is metabolized to its active form, BPDE, and forms DNA adducts leading to base substitutions predominantly at guanine (Shane et al., 2000). Thus, these lesions cause large increases in base substitutions in reporter transgenes.

In contrast, the mechanisms causing tandem-repeat mutations are not well understood. Evidence suggests that these mutations arise via an indirect mechanism caused by genomic instability as opposed to direct damage within the repeat itself (Dubrova et al., 1998; Dubrova et al., 2000; Somers, 2006; Yauk et al., 2002; Yauk, 2004). The model for ESTR mutation induction (Barber et al., 2004; Yauk, 2004) speculates that lesions in the DNA result in cell-cycle pausing; if a polymerase is replicating an ESTR at that moment, then the pausing can result in the formation of secondary structures. Upon resumption of DNA replication, the secondary structures can lead to the formation of deletions or duplications of repeat units if not repaired. It has been demonstrated that knocking out mismatch-repair genes, which are important for repairing DNA secondary structures, can lead to an increase in microsatellite mutation frequency in mice (Baker et al., 1995). It is possible that the chemically induced microsatellite mutations observed in this study may also occur by this mechanism. It is also conceivable that this process would saturate at lower levels of adducts (or DNA damage) than point mutations. Additional work is required to understand the mechanisms leading to chemically induced microsatellite mutations. 
We note that there are several important considerations when designing an SMPCR experiment using microsatellites. First, it is imperative that the chosen model organism is genotyped before the experiment is initiated to ensure optimal sensitivity of the assay. If the model is heterozygous for 2 alleles that are similar in size, the ability to detect mutations is reduced. For example, Swiss-Webster mice had a microsatellite locus with allele sizes of 262 and $266 \mathrm{bp}$, and thus gains at the 262-bp allele and deletions at the 266-bp allele would not be detected (Beal et al., 2012a). Considering that mutations are rare events, it is important to reduce the number of false-negatives. Heterozygous samples are acceptable as long as the 2 alleles are sufficiently different in size, as with the $\mathrm{B} 6 \mathrm{C} 3 \mathrm{~F} 1$ samples. Second, repeat length is a determinant of mutation rate (Primmer et al., 1996). For example, spontaneous microsatellite mutation frequency was higher in the sperm of $\mathrm{B} 6 \mathrm{C} 3 \mathrm{~F} 1$ samples than MutaMouse samples, likely due to longer allele lengths. Thus, longer allele sizes may be preferable for SM-PCR. Lastly, the number of progenitor molecules per group that will be screened needs to be considered carefully. If too few molecules are screened per sample, there will be missed mutations. In contrast, if too many molecules are screened, the mutation frequency will be diluted because the number of unique, non-clonally expanded mutations per sample is limited to primarily single repeat changes at each locus (Figure 2.4). Simulations based on the empirical data from this study estimate that approximately 300 molecules per sample is sufficient to detect a 2 -fold change in mutation frequency with $80 \%$ power. However, the desirable number of molecules will vary with the sample size (number of biological replicates), the number of loci screened, the agent's mutagenicity, the background mutation frequency and spectrum of each locus, and the number of alleles at each locus. All of these considerations should 
be taken into account if analysis of microsatellite mutation is to be applied to outbred study populations, including humans.

In summary, we have optimized an approach for SM-PCR analysis of mutations at an unstable microsatellite locus and used it to measure increases in germline-mutation frequencies following ENU and BaP exposures in a mouse model. There are several advantages to this approach. First, mutation detection can be done in vivo in any mouse strain. Furthermore, because this method detects mutations in sperm rather than in offspring, the number of animals required is greatly reduced, but the sample size is greatly improved allowing for increased statistical power. The reduced statistical power of analyzing family pedigrees may be why Beal et al. (2012a) did not see a mutagenic response with ionizing radiation; thus, retesting of irradiation with SM-PCR at locus Mm2.2.1 would be valuable. Another benefit of this method is that it can be adapted readily to study mutation induction in humans using polymorphic microsatellites identified previously (e.g. (Furitsu et al., 2005; Sun et al., 2012)). Lastly, analysis by capillary electrophoresis (because microsatellites are short relative to ESTRs) allows for more precise, standardized, and higher throughput mutation detection than is possible for ESTRs. PCR amplification of these much shorter microsatellite loci is less difficult technically than the long GC-rich ESTR alleles used historically. Future work with this method will focus on developing multiplex SM-PCR amplification to allow for mutation detection simultaneously at multiple loci, which would increase power and sensitivity. Analyzing multiple loci will provide insight into how different microsatellite loci (that differ in length, sequence content, genomic position, etc.) can have varying mutagenic responses. We also recommend that additional dose-response studies and analysis of 
other mutagens be conducted to establish fully the sensitivity of the assay for germ-cell mutation analysis. This method can be applied potentially to environmental biomonitoring using sentinel species and to human populations to assess the effects of variables that influence heritable mutagenesis (Armour et al., 1999; Dubrova et al., 2002a; Dubrova et al., 2002b; Dubrova et al., 2006; Kong et al., 2012; Linschooten et al., 2013; May et al., 2000; Sun et al., 2012; Tawn et al., 2011; Zheng et al., 2000). Our new method expands our ability to detect mutations in germ cells, but we must consider the diverse types of mutation mechanisms and continue to expand the mutational landscape that we can capture in toxicological screening (Yauk et al., 2015a). 


\subsection{Tables and Figures}

Table 2.1 Mutation frequencies (with $95 \%$ confidence intervals) ${ }^{\mathrm{a}}$ in the sperm of control and treated animals from different experiments.

\begin{tabular}{lllllll}
\hline Experiment & $\begin{array}{l}\text { No. of } \\
\text { samples }\end{array}$ & $\begin{array}{l}\text { No. of } \\
\text { mutations }\end{array}$ & $\begin{array}{l}\text { No. of } \\
\text { progenitors }\end{array}$ & $\begin{array}{l}\text { Mutation } \\
\text { Frequency } \\
\left(\times 10^{-3}\right)\end{array}$ & $\begin{array}{l}\text { Ratio } \\
\text { exposed / } \\
\text { control }\end{array}$ & $\begin{array}{l}\text { P- } \\
\text { value }^{\mathrm{b}}\end{array}$ \\
\hline Control $^{\mathrm{c}}$ & 9 & 9 & $\begin{array}{l}3587 \\
(3470-3705)\end{array}$ & $\begin{array}{l}2.38 \\
(1.57-3.63)\end{array}$ & & \\
& & & 1629 & 5.52 & 2.31 & $\mathbf{0 . 0 1 2}$ \\
$\mathrm{BaP}$ & 3 & 9 & $(1550-1709)$ & $\begin{array}{l}(3.35-9.10) \\
(1.21-4.45)\end{array}$ & \\
$100 \mathrm{mg} / \mathrm{kg}$ & & & 1043 & $\begin{array}{l}5.66 \\
(5.19-6.16)\end{array}$ & $\begin{array}{l}2.37 \\
(1.53-3.67)\end{array}$ & $\mathbf{0 . 0 0 0 1}$ \\
$\mathrm{ENU}$ & 3 & 6 & $(981-1107)$ & & & \\
$100 \mathrm{mg} / \mathrm{kg}$ & & & & & &
\end{tabular}

${ }^{\mathrm{a}}$ Lower and upper 95\% CIs given for the number of progenitor molecules, mutation frequencies, and ratios.

${ }^{\mathrm{b}}$ Statistical significance shown in bold. P-values were generated using generalized estimating equations.

${ }^{c}$ There were no statistically significant differences between control animals from the different experiments; thus the results were combined. 


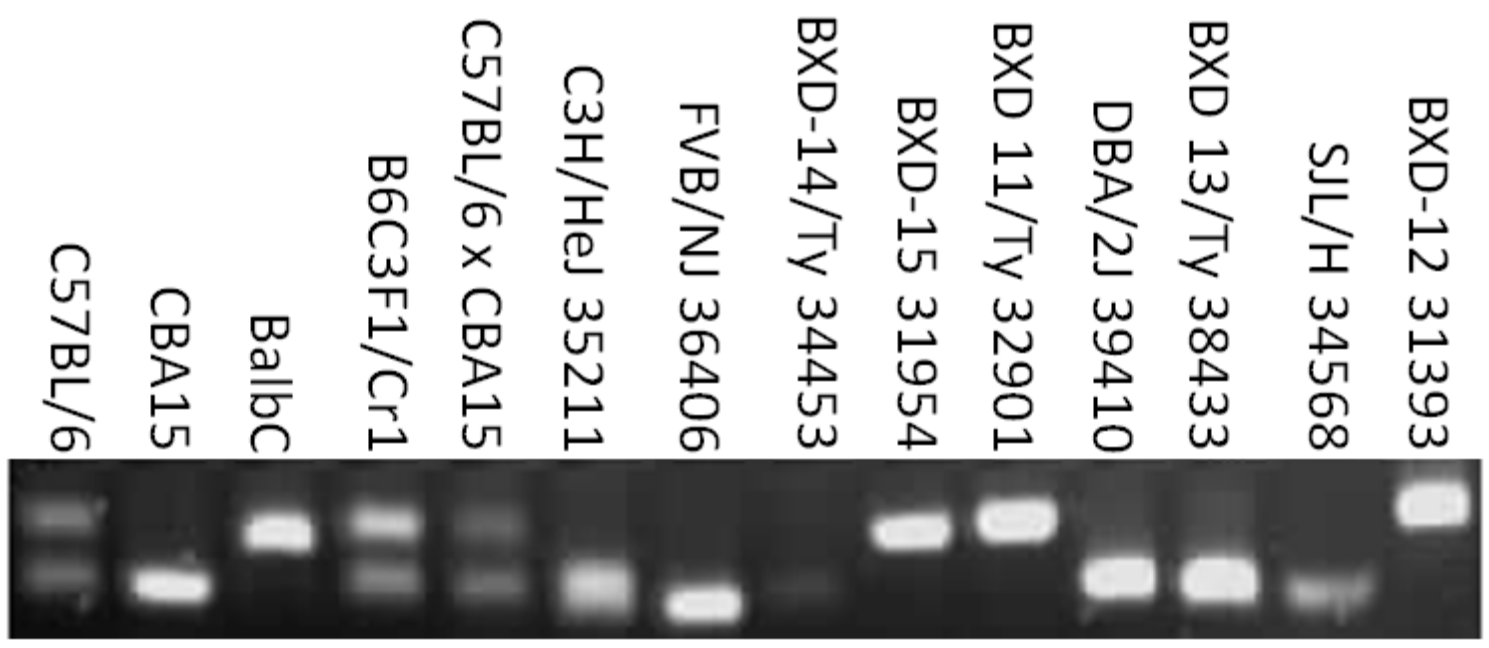

Figure 2.1 Variability of locus Mm2.2.1 across thirteen inbred mouse strains and a F1 cross. The largest allele is approximately $100 \mathrm{bp}(\sim 25$ repeat units) larger than the smallest allele. 


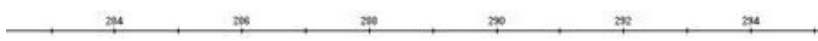

\section{$291 \mathrm{bp}$}

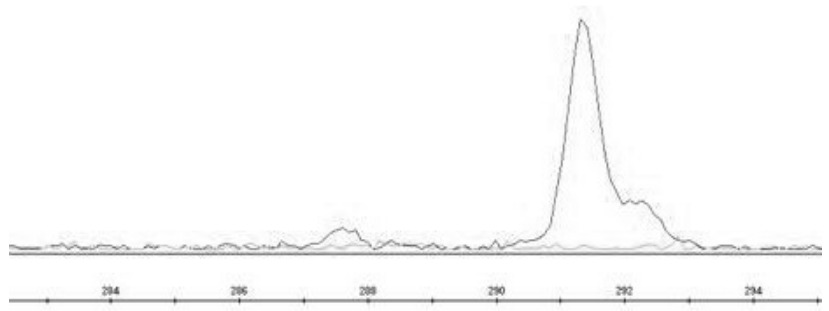

\section{7 bp}

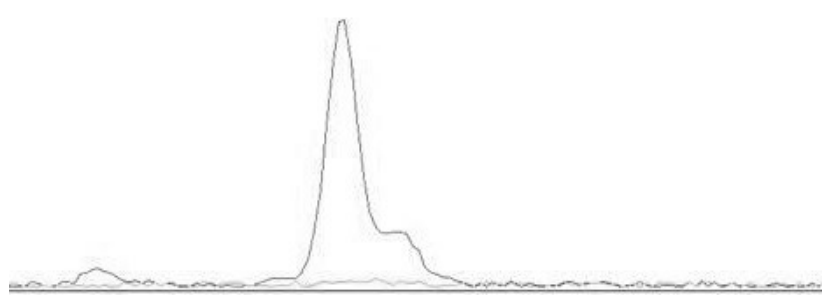

Figure 2.2 Examples of capillary eletropherograms of SM-PCR amplifications of Mm2.2.1 in sperm DNA. The top electropherogram shows the wild-type MutaMouse allele (291 bp), and the bottom electropherogram shows an example of a -1-unit repeatdeletion mutation $(287 \mathrm{bp})$. 


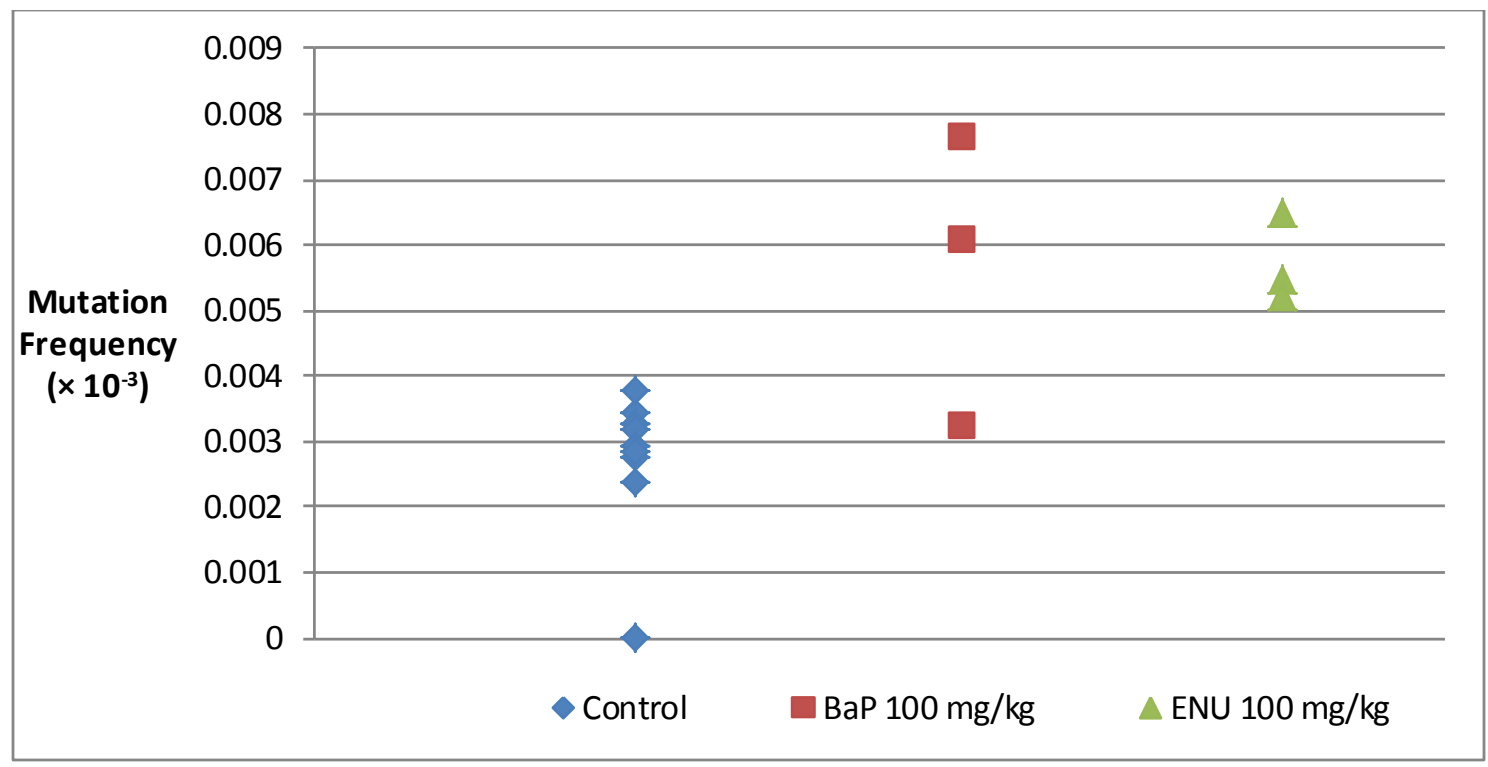

Figure 2.3 Mutation frequency at locus Mm2.2.1 in the sperm of different control and treated mice. 


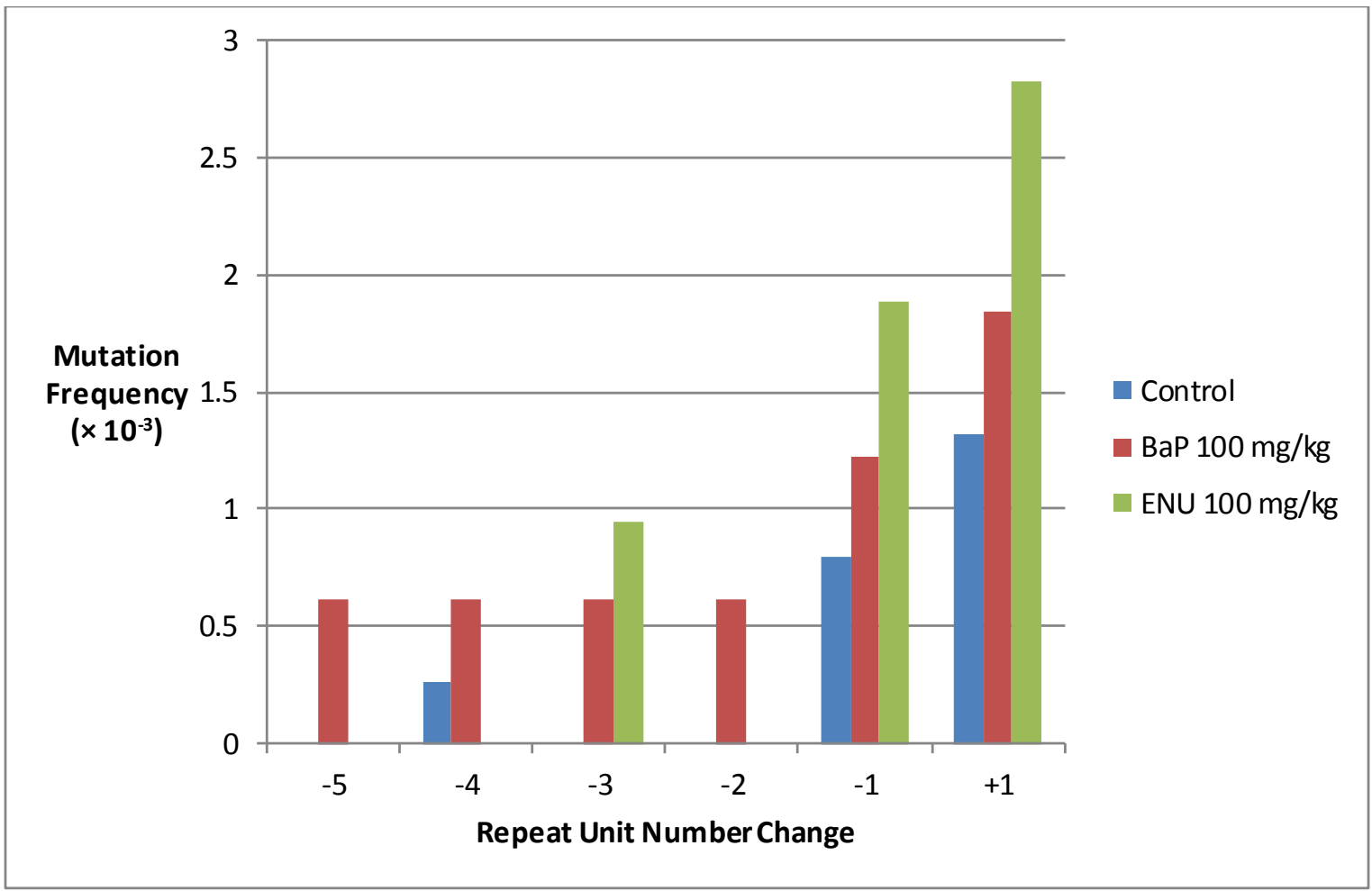

Figure 2.4 Frequency spectrum of Mm2.2.1 mutant size classes in sperm. Most of the mutations observed were single-repeat changes. There were no statistical differences in mutation spectra between treatment groups. 


\section{Chapter 3: Dose-Response Mutation and Spectrum Analyses Reveal Similar Responses at Two Microsatellite Loci in Benzo[a]pyrene- Exposed Mouse Spermatogonia}

Modifie d from: Rowan-Carroll, A., Beal, M.A., Williams, A., Marchetti, F., Yauk, C.L. Mutagenesis.In press. 2017.

\subsection{Preamble}

The results of Chapter 2 demonstrate that high dose BaP exposures cause microsatellite mutations in dividing spermatogonia. To further test the sensitivity of SMPCR, sperm from mice treated with lower doses of BaP were tested for microsatellite mutations. Moreover, to increase the assay sensitivity, an additional microsatellite locus was incorporated into a multiplexed PCR to simultaneously score mutations for 2 different microsatellites. The results of this chapter indicate that the assay sensitivity was improved by the addition of another locus and that microsatellites may be more sensitive markers of mutation induction than other genetic endpoints used for germ cell mutation analysis.

\subsection{Abstract}

Identifying chemical exposures that can cause germline mutations is important as these mutations can be inherited, impacting both individual and population health. However, germline mutations are extremely rare and difficult to detect. Chemically induced germline mutations can be detected through analysis of highly unstable tandem 
repeat DNA. We recently developed a SM-PCR approach to quantify mutations at a mouse microsatellite locus (Mm2.2.1) in sperm for such purposes. In the present study, we refine this approach through the combined analysis of mouse microsatellites $\mathrm{Mm} 2.2 .1$ and $\mathrm{Mm19.2.3.} \mathrm{Mice} \mathrm{were} \mathrm{exposed} \mathrm{to} 0,25,50$, or $100 \mathrm{mg} / \mathrm{kg} /$ day BaP by oral gavage for 28 days and sperm sampled 42 days after the end of exposure to measure effects on dividing spermatogonia. DNA was diluted to a single genome per PCR well for amplification of microsatellites in singleplex and multiplex reactions, and alleles were sized to identify mutations using capillary electrophoresis. Analysis of 300-500 molecules per animal at both microsatellite loci, when tested individually, showed a $\sim 2$ fold increase in mutations relative to the controls at both the 50 and $100 \mathrm{mg} / \mathrm{kg} /$ day $\mathrm{BaP}$ doses. Multiplex SM-PCR revealed similar increases in mutation frequencies in both microsatellites. Comparison with results from a previous $l a c Z$ mutation assay conducted on the same mice revealed that although microsatellite mutations are a sensitive tool for detecting changes in mutation frequencies at lower doses, they appear to be saturable and thus have a reduced dynamic range. These results confirm that $\mathrm{BaP}$ is a male germ cell mutagen that broadly impacts tandem repeat DNA. Likewise, addition of a second hypervariable microsatellite increases the sensitivity of this assay.

\subsection{Introduction}

Regulatory agencies worldwide acknowledge the importance of evaluating chemicals for the potential to cause harm to the next generation as a result of germ cell mutations (Eastmond et al., 2009; Yauk et al., 2015a). However, the study of germline mutagenesis has been hampered by a lack of tools to efficiently quantify the ability of an 
agent to cause heritable mutations. As a result, regulatory agencies have primarily relied on analysis of mutagenicity in somatic tissues to assess genetic damage, and assumed that decisions based on these assessments would protect from potential germ cell mutagenicity as well (Singer and Yauk, 2010; Yauk et al., 2013).

For over 2 decades an important approach to study germ cell mutagenicity has been the analysis of hypervariable ESTRs in mouse sperm genomes, or in pedigrees, to detect length change mutations (i.e., gain/loss of repeat units). The high mutation rates at these loci make the detection of germ cell and heritable mutations feasible in relatively small sample sizes (Yauk et al., 2015a). Sperm analysis is the preferred approach as it greatly reduces the number of animals required in the experiments over measuring mutations arising in offspring (Yauk et al., 2002). The method requires SM-PCR amplification of long GC-rich ESTR regions, agarose gel electrophoresis on $40 \mathrm{~cm}$ long gels, Southern blotting followed by radioactive labelling of probes for blot hybridization, and visualization of repeat length changes (Yauk et al., 2002). The protocol is technically challenging and can be time consuming because of the length of some alleles. In addition, scoring can be subjective and only gains or losses of at least $20 \mathrm{bp}$ can be reliably scored. Nevertheless, application of this method has revealed a variety of environmental variables that influence both germ cell and inherited tandem repeat mutations (e.g., radiation (Yauk et al., 2002), air pollution (Yauk et al., 2008b), cigarette smoke (Marchetti et al., 2011; Yauk et al., 2007), anti-cancer drugs (Glen et al., 2008), and folate deficiency (Swayne et al., 2012)). The study of ESTRs has likewise revealed that the most sensitive window of exposure for the male germline is during the spermatogonial phase (Vilariño-Güell et al., 2003). As germ cells mature from stem cells 
into mature sperm, they undergo several rounds of DNA replication, which is required to permanently fix mutations.

Direct relevance to humans of ESTR mutations is unclear because they have only been characterized in mice to date (Bois et al., 1998; Gibbs et al., 1993; Kelly et al., 1989). Conversely, microsatellites are $2-5 \mathrm{bp}$ simple tandem repetitive sequences that range from $10 \mathrm{bp}-1 \mathrm{~kb}$ in length (Yauk and Polyzos, 2005) that are found in all eukaryotic genomes. These long stretches of repetitive sequences are inherently prone to errors during DNA replication (Barber et al., 2004; Yauk, 2004). As such, they represent excellent candidates for mutation analysis. In addition, microsatellite mutations are associated with many human diseases including: frontotemporal lobar degeneration, amyotrophic lateral sclerosis, fragile $\mathrm{X}$ syndrome, fragile $\mathrm{X}$ mental retardation, spinocerebellar ataxia, myotonic dystrophy 1, spinocerebellar ataxia 12, and Huntington's-disease-like-2 (Mirkin, 2007; Mori et al., 2013). Thus, they are potentially more directly relevant to human genetic disease than ESTRs.

In previous work, a variety of microsatellites were screened in several inbred strains of mice to identify highly polymorphic loci (Beal et al., 2012a), and a SM-PCR methodology was then developed to study induced germ cell mutations for the unstable locus Mm2.2.1 (Beal et al., 2015b). Although our microsatellite analysis method also relies on SM-PCR, the approach is less technically challenging than ESTR analysis because Mm2.2.1 is short $(<300 \mathrm{bp})$ and its allele length can be rapidly and precisely scored using capillary gel electrophoresis. SM-PCR analysis revealed a greater than 2fold increase in mutation frequencies in Mm2.2.1 in mouse sperm following exposure to 2 established germ cell mutagens, ENU and BaP (Beal et al., 2015b). In the present study, 
we expand our methodology to assess response in Mm19.2.3, a second variable microsatellite. Mm19.2.3 is a tetramer found on chromosome 19 and is approximately 200-300 bp in length, depending on rodent strain (Beal et al., 2012a). The locus repeat motif (GATA $)_{11}(\text { GACA })_{15}(\text { GATA })_{17}$ GATTGG CAGATG(GACA $)_{3}$ is a complicated series of repetitive elements separated by a short stretch of non-repetitive nucleotides, and was identified from analysis of the reference C57B1/6J genome (build 37.2; $\mathrm{NCBI}$ ) (Beal et al., 2012a). In this study, we examined sperm from male mice exposed orally to 0, 25, 50, or $100 \mathrm{mg} / \mathrm{kg} /$ day $\mathrm{BaP}$ for 28 days and sampled 42 days post-exposure (to assay exposures that targeted spermatogonial stem cells and dividing spermatogonia). Using these samples we explore the sensitivity of mutation detection individually for these two loci, the effects of lower doses of $\mathrm{BaP}$, and the use of a multiplex SM-PCR approach.

\subsection{Materials and Methods}

\subsubsection{Selection of Micros ate llites}

Microsatellite loci Mm2.2.1 and Mm19.2.3 were chosen from among a panel of previously identified Mus musculus microsatellites (Beal et al., 2012a). Selection of these microsatellites was based on the screening of multiple mouse microsatellite sequences in inbred mouse strains to identify loci that showed variability in length.

We used the NCBI Basic Local Alignment Search Tool (BLAST) as well as the University of California Santa Cruz Genome Browser website tools to query $50 \mathrm{~kb}$ upstream and downstream of the Mm2.2.1 and Mm19.2.3 microsatellites to examine the genomic context surrounding the microsatellites. Secondary structural analysis of the 
genomic sequences surrounding both loci were conducted using the search engine tools from Integrated DNA Technologies (https://www.idtdna.com) and the Predict a Secondary Structure Web Server (rna.urmc.rochester.edu/RNAstructureWeb/Servers/Predict1/Predict1.html) secondary RNA (DNA application) structure predictor tool.

\subsubsection{Animal Treatment and Sample Collection}

All animal protocols were approved by the Health Canada Ottawa Animal Care Committee. Mature 8-week old MutaMouse males were exposed by oral gavage to 0, 25, 50 , or $100 \mathrm{mg} / \mathrm{kg} /$ day BaP dissolved in olive oil (volume $=5 \mu \mathrm{l} / \mathrm{g}$ ) daily for 28 days $(\mathrm{n}=$ 6, 4, 4 and 4 for the 4 dose groups, respectively). Forty-two days after the last exposure mice were euthanized by cervical dislocation under isofluorane anaesthesia. Cauda epididymides were immediately removed, flash frozen, and stored at $-80{ }^{\circ} \mathrm{C}$ until use. Mice from the control and $100 \mathrm{mg} / \mathrm{kg} /$ day $\mathrm{BaP}$ groups were used in our previous work on the microsatellite locus Mm2.2.1 (Beal et al., 2015b) and TGR assay (O'Brien et al., 2016a).

\subsubsection{DNA Isolation and SM-PCR Mutation Analysis}

DNA from mouse sperm was prepared using the protocol described previously (Beal et al., 2015b). In order to minimize the possibility of contamination, DNA manipulations were performed in a sterilized laminar flowhood. Briefly, cauda epididymides were thawed and chopped up in $1.5 \mathrm{~mL}$ of phosphate-buffered saline to liberate sperm, then filtered through a mesh, and pelleted by centrifugation for $5 \mathrm{~min}$ at 
$15,000 \mathrm{rpm}$. The pellet, containing both somatic and sperm cells, was re-suspended in 1 $\mathrm{ml}$ of $1 \mathrm{X}$ saline-sodium citrate (SSC; $0.15 \mathrm{M} \mathrm{NaCl}, 0.015 \mathrm{M}$ sodium citrate) and $15 \mu \mathrm{l}$ of $10 \%$ sodium dodecyl sulfate (SDS) to lyse any remaining somatic cells. The lysate was centrifuged and the purified sperm pellet was re-suspended in $940 \mu \mathrm{l}$ of $0.2 \mathrm{X} \mathrm{SSC}(0.03$ $\mathrm{M} \mathrm{NaCl}, 0.003 \mathrm{M}$ sodium citrate), $100 \mu \mathrm{l}$ of $10 \% \mathrm{SDS}, 120 \mu \mathrm{l}$ of 2-mercaptoethanol, and digested with $20 \mu \mathrm{l}$ of $20 \mathrm{mg} / \mathrm{ml}$ proteinase $\mathrm{K}$ (InvitrogenTM, Carlsbad, California) at $37 \circ \mathrm{C}$ overnight. Sperm DNA was then isolated using phenol/chloroform extraction and finally re-suspended in $50 \mu \mathrm{l}$ of Tris EDTA, TE-4 (0.1 M Tris pH 7.6, 0.002 M EDTA pH8.0). A nano-drop spectrophotometry (NanoDrop UV-vis spectrophotometry - model number ND-1000, Asheville, NC) was used to determine final DNA concentrations.

\subsubsection{SM-PCR Amplification}

DNA samples $(2 \mu \mathrm{g})$ were digested overnight at $37{ }^{\circ} \mathrm{C}$ with $20 \mathrm{U}$ of HindIII (New England Biolabs, Ipswich, MA). The digested samples were serially diluted to concentrations of $1 \mathrm{ng} / \mu \mathrm{l}, 100 \mathrm{pg} / \mu \mathrm{l}, 50 \mathrm{pg} / \mu \mathrm{l}, 25 \mathrm{pg} / \mu \mathrm{l}$, and $10 \mathrm{pg} / \mu \mathrm{l}$ in water. PCR was carried out on each dilution in order to determine the concentration at which a $40-60 \%$ positive PCR rate was achieved. Poisson distribution analysis shows that amplification success ranging between $40-60 \%$ equates to approximately 1 amplifiable molecule per reaction (Yauk et al., 2002).

Primers for loci Mm2.2.1 and Mm19.2.3, developed by Beal et al. (2012a), were fluorescently tagged with 6-fluorescein amidite (6-FAM) (Integrated DNA technologies):

\subsubsection{FORWARD: FAM-GGCTACTGGGTATCAAACAGG,}

\subsubsection{REVERSE: TGAAGGGTATTGGAGTTCAGTC,}




\subsubsection{FORWARD: FAM-TCAAAAGCACCTTTCAGTTCTTC,}

\subsubsection{REVERSE: CTAGAAGACCTTTGGTCTCAC.}

For analysis of individual loci, DNA was amplified in $10 \mu \mathrm{l}$ reactions using 10 $\mu \mathrm{M}$ fluorescently tagged forward primer and $10 \mu \mathrm{M}$ unlabelled reverse primers. Each 10 $\mu \mathrm{l}$ PCR contained: $5 \mu \mathrm{l}$ of Norgen $2 \mathrm{X}$ PCR master mix, $1 \mu \mathrm{l}$ of $10 \mu \mathrm{M}$ forward primer (Mm2.2.1 or Mm19.2.3), $1 \mu \mathrm{l}$ of $10 \mu \mathrm{M}(\mathrm{Mm} 2.2 .1$ or Mm19.2.3) reverse, $3 \mu \mathrm{l}$ of water, and $1 \mu \mathrm{l}$ of DNA template at the desired concentration (the concentration yielding product in $40-60 \%$ of the amplifications; generally this was approximately $10 \mathrm{pg} / \mu \mathrm{L}$ based on spectrophotometer readings).

For multiplex reactions, $10 \mu \mathrm{M}$ of $\mathrm{Mm19.2.3}$ forward and reverse primers were combined with $10 \mu \mathrm{M}$ of Mm2.21 forward and reverse primers in the same reaction tube. Each $10 \mu \mathrm{l}$ reaction contained: $5 \mu \mathrm{l}$ of Norgen $2 \mathrm{X}$ PCR master mix, $0.5 \mu \mathrm{l}$ of $10 \mu \mathrm{M}$ Mm2.2.1 forward primer, $0.5 \mu \mathrm{l}$ of $10 \mu \mathrm{M} \mathrm{Mm2.2.1} \mathrm{reverse} \mathrm{primer,} 0.5 \mu \mathrm{l}$ of $10 \mu \mathrm{M}$ Mm19.2.3 forward primer, $0.5 \mu \mathrm{l}$ of $10 \mu \mathrm{M}$ Mm19.2.3 reverse primer, $3 \mu \mathrm{l}$ of water, and $1 \mu l$ of DNA template at the desired concentration.

For Mm19.2.3 the following amplification protocol was used for SM-PCR: $94{ }^{\circ} \mathrm{C}$ for $120 \mathrm{~s} ; 38$ cycles of $94{ }^{\circ} \mathrm{C}$ for $30 \mathrm{~s}, 53^{\circ} \mathrm{C}$ for $30 \mathrm{~s}, 70{ }^{\circ} \mathrm{C}$ for $120 \mathrm{~s}$; final extension at 70 ${ }^{\circ} \mathrm{C}$ for $30 \mathrm{~min}$. The annealing temperature was increased to $57^{\circ} \mathrm{C}$ for $\mathrm{Mm} 2.2 .1$. An annealing temperature of $55^{\circ} \mathrm{C}$ was used in the multiplex reactions, all other parameters where kept the same.

\subsubsection{Mutation Detection}


PCR amplifications were carried out in 96-well plates. Amplicons were analyzed on an Applied Biosystems 3130 XL DNA sequencer (Life Technologies TM, Carlsbad, CA) with internal size standard (GeneScanTM500 LIZ®) using the FragmentAnalysis50_POP7 run model to determine size. The same scoring criteria used by Beal et al. (2015b) were applied. Briefly, for an amplicon to be called a mutation it needed to have a size change that was equivalent to 1 or more whole repeat unit (i.e., 4 bp and multiples thereof) from the wild-type allele. For a mutant to be considered legitimate, no trace of the wild-type allele could be present in the same reaction. In addition, a stutter band or an N-1 band (a hallmark of repetitive sequence PCR) should be present (Figure 3.1 - see small peak before large allele). If the wild-type progenitor band was present in the same amplicon reaction as a mutant allele, the mutant alle le was presumed to have resulted from replication error/slippage at some stage of the PCR, and thus was the result of a PCR artifact. Specific microsatellite length change mutations were counted only once per sample, even if the same mutation reoccurred multiple times in the same sample (i.e., 2 identically sized mutations in the same sample would only be counted as a single mutation event). Therefore, only non-clonally expanded mutations were measured.

\subsubsection{Statistical Analyses}

Statistical analyses were carried out as described previously (Swayne et al., 2012; Yauk et al., 2008b; Yauk, 2004). Briefly, a Poisson distribution was used to estimate the number of progenitor PCR molecules from the number of positive/negative PCR wells and the number of wells used for PCR. Assuming a Poisson distribution for the error, GEEs were used to estimate microsatellite mutation frequencies (Liang and Zeger, 1986; 
Yauk et al., 2008a). Pairwise comparisons of mutation frequencies were made between control and exposed samples to detect induced mutations. Changes in mutation frequencies were considered statistically significant if $\mathrm{P}<0.05$. Mutation spectrum analysis was conducted using the likelihood ratio statistic as described in (Piegorsch and Bailer, 1994) using R (R Core Team, 2013). P-values for this test statistic were estimated using the Monte Carlo approximation (Holm, 1979; Piegorsch and Bailer, 1994).

\subsection{Results}

\subsubsection{General Fe atures of Mm2.2.1 and Mm19.2.3}

Genotyping of MutaMouse male sperm revealed that the mice in the present study were homozygous at both Mm2.2.1 (291 bp) and Mm19.2.3 (265 bp) loci. Figure 3.1 illustrates a multiplex reaction of $\mathrm{Mm} 2.2 .1$ and Mm19.2.3 showing the wild type genotype (panel A), and deletion mutations (panels B and C), detected by capillary electrophoresis for both loci. The spontaneous mutation frequencies in the control $(0$ $\mathrm{mg} / \mathrm{kg} /$ day) animals were $1.88 \times 10^{-3}$ for locus $\mathrm{Mm} 2.2 .1$ and $3.71 \times 10^{-3}$ for locus Mm19.2.3 (Table 3.1).

\subsubsection{Induced Germline Mutation Frequencies}

To explore the response of both microsatellite loci to a chemical mutagen, sperm from $\mathrm{BaP}$-treated male mice were analyzed by SM-PCR, and mutation frequencies at Mm2.2.1 and Mm19.2.3 were compared with matched controls. Each locus was first analyzed independently (Table 3.1, Figure 3.2), then data from both loci were analyzed together to examine the dose effect (Table 3.1). While mice exposed to the $25 \mathrm{mg} / \mathrm{kg} / \mathrm{day}$ 
dose of $\mathrm{BaP}$ had a marginally significant increase in mutations $(1.9$-fold increase; $\mathrm{P}=$ 0.0532), mice exposed to 50 and $100 \mathrm{mg} / \mathrm{kg} / \mathrm{day} \mathrm{BaP}$ had significant 2.7 -fold $(\mathrm{P}=$ 0.0052) and 3.1-fold $(\mathrm{P}=0.0048)$ increases in mutation frequencies relative to matched controls for locus Mm2.2.1. Likewise, at locus Mm19.2.3, the $25 \mathrm{mg} / \mathrm{kg} /$ day dose did not cause an increase in mutations relative to controls ( 0.6 -fold change relative to controls; $\mathrm{P}$ $=0.5242)$; however, statistically significant increases of 2.6 -fold $(\mathrm{P}=0.0068)$ and 2.3 fold $(\mathrm{P}=0.0142)$ above controls were found for the 50 and $100 \mathrm{mg} / \mathrm{kg} /$ day dose groups, respectively (Table 3.1). Combining the loci (Table 3.1) revealed significant increases in mutation frequencies at the $50 \mathrm{mg} / \mathrm{kg} /$ day dose $(2.6$-fold; $\mathrm{P}=0.0001)$ and the 100 $\mathrm{mg} / \mathrm{kg} /$ day dose $(2.7$-fold; $\mathrm{P}=0.0002)$ relative to controls.

Multiplex PCR was performed using sperm from the control and $100 \mathrm{mg} / \mathrm{kg} / \mathrm{day}$ BaP dose groups ( $\mathrm{N}=3$ per group) (Table 3.2). In the multiplex reactions, the spontaneous mutation frequency of Mm2.2.1 was $2.17 \times 10^{-3}$, which was highly consistent with the results of independent analyses. The spontaneous mutation frequency of Mm19.2.3 in the multiplex reactions was $1.92 \times 10^{-3}$ which is slightly lower, but not significantly different, from the independent analysis $(\mathrm{P}=0.3950$; GEE analysis using only the controls and the $100 \mathrm{mg} / \mathrm{kg} /$ day data). Exposure to $100 \mathrm{mg} / \mathrm{kg} /$ day $\mathrm{BaP}$ over 28 days resulted in statistically significant 2.9 -fold $(\mathrm{P}<0.0001)$ and 3.9 -fold $(\mathrm{P}=0.005)$ increases over control samples for Mm2.2.1 and Mm19.2.3, respectively, within the multiplex reaction. Combined analysis of Mm2.2.1 and Mm19.2.3 within the multiplex experiment revealed a significant 3.3 -fold increase in mutation frequency relative to controls for $\mathrm{BaP}$ treated sperm $(\mathrm{P}<0.0001)$ (Table 3.2). 


\subsubsection{Mutation Spectrum Analysis}

The majority of mutations detected at both loci were single-repeat changes; however, several large mutations were observed in the mutation spectra for both Mm2.2.1 and Mm19.2.3 (Figure 3.3). Analysis of the mutation spectra over all doses did not reveal significant differences between loci Mm2.2.1 and Mm19.2.3 ( $\mathrm{P}=0.1848)$. Likelihood ratio test (LRT) (Holm, 1979) of control versus treated spectra also did not reveal statically significant alterations in mutation spectra for either loci for any the dose examined (Figure 3.3).

\subsection{Discussion}

In this study, we expand on our previous work to assess the utility of SM-PCR analysis of microsatellite mutations to identify germ cell mutagens. We used $\mathrm{BaP}$ as our prototype chemical for this work. $\mathrm{BaP}$ is produced from the incomplete burning of fossil fuels and is a ubiquitous well-known environmental mutagen that is classified by the International Agency for Research on Cancer (IARC) as a Group 1 carcinogen (International Agency for Research on Cancer and International Agency for Research on Cancer, 1987). Sources of human exposure to $\mathrm{BaP}$ include cigarette smoke, the burning of coal or wood for heating, cooked foods, and vehicle exhaust (Bostrom et al., 2002; Conney et al., 1994; World Health Organization, 1983). BaP is metabolized to a diol epoxide that can form covalent adducts with DNA which in turn lead to mutations (Hargis et al., 2010). Other reactive metabolites, such as reaction oxygen species and superoxides produced during BaP metabolism, are also mutagenic (Griffiths et al., 2000; Valavanidis et al., 2009). 
We demonstrate that 28-day repeat dose exposure of spermatogonial stem cells and dividing spermatogonia to 50 and $100 \mathrm{mg} / \mathrm{kg} \mathrm{BaP} /$ day results in an increase in mutations in 2 microsatellites: Mm2.2.1 and 19.2.3. A combined analysis of both loci improved sensitivity and power demonstrating the advantages of analyzing data from multiple microsatellites together. The response appears to be saturable, as there was no clear dose-response increase above $50 \mathrm{mg} / \mathrm{kg} /$ day for either of the loci. The $\sim 2$ - to 3-fold increase in mutation frequency seen across all experiments is similar to what has been observed for ESTR mutations arising in the offspring of male mice exposed to BaP. These results demonstrate consistency in the response of different tandem repeats to $\mathrm{BaP}$, and supporting that repeat mutations found in germ cells are transmitted to offspring (Verhofstad et al., 2011; Yauk et al., 2008b). Therefore, our study and the work of others (Godschalk et al., 2015; O'Brien et al., 2016a; Olsen et al., 2010; Verhofstad et al., 2011; White et al., 1999; Xu et al., 2014) confirm that, in addition to causing somatic cell mutations and cancer, $\mathrm{BaP}$ causes mutations in male germ cells.

We developed a multiplex PCR approach to improve the sensitivity, and reduce the time and cost associated with the microsatellite assay. Multiplexing led to decreases in the P-values obtained, and thus increased our ability to detect a chemical's potential mutagenic effects on the germline. Minor differences in mutation frequencies were observed between singleplex and multiplex results (Table 3.1 vs. Table 3.2). Differences between experiments may occur because a single mutation event in a given population can significantly impact the measured mutation frequency. Thus, although small differences in mutation frequencies may be expected by chance between experiments, trends in mutation frequencies remain consistent. Indeed, the $95 \%$ confidence interval of 
the singleplex and multiplex overlapped in all cases, and mutation frequencies were not significantly different $(\mathrm{P}=0.3950)$ from each other.

Mutation rates at tandem repeats are influenced by multiple factors including genomic structure, sequence, and location (Schlötterer and Pemberton, 1998). Therefore, we explored genomic context surrounding the 2 loci. Mm2.2.1 is located on chromosome 2 and has a tetranucleotide repeat of (AGAT) ${ }_{17}$ GATAGCTAGATGATA GGT(AGAT) $)_{18}$. Mm 2.2.1 is not transcribed or in direct proximity to any transcribed genes, although a query of the genome $50 \mathrm{~kb}$ up and downstream of locus revealed the presence of the Tas $2 \mathrm{r}$ 134 gene (Taste receptor 2, member 134) (approximately $12 \mathrm{~kb}$ upstream of Mm2.2.1) and the Rps8 gene (ribosomal protein S8) (approximately $50 \mathrm{~kb}$ upstream). Mm 19.2.3 has a complicated tetranucleotide repeat, and is located on a non-transcribed region of chromosome 19. A query $50 \mathrm{~Kb}$ up and downstream of Mm19.2.3 failed to identify any transcribed genes. The GC content of the 2 loci are similar (37.7\% versus $36.0 \% \mathrm{GC}$ content for Mm2.2.1 and Mm19.2.3, respectively). Structural analysis suggests that Mm2.2.1 forms 12 different hairpin loops, whereas Mm19.2.3 may form 20 different secondary structures. Thus, the 2 loci generally share similar features and mutation rates are not likely to be greatly influenced by the genomic context surrounding them.

The above similarities are consistent with the observation that there were no significant differences in mutation spectra between the 2 loci within control samples, between treated and control mice, or between the dose groups. Similar mutation signatures suggest that the 2 microsatellite loci mutate via a common mechanism both during normal cellular replication and division, and following challenge with a mutagen forming bulky DNA adducts. Moreover, the mechanism by which the BaP exposure 
increases the mutation frequency at both loci is likely the same or similar to the endogenous mechanism by which mutations occur at these sites. Within our study, the mutation spectra for both $\mathrm{Mm}$ 2.2.1 and Mm19.2.3 even after BaP exposure was predominated by small changes. Although we found some large deletions (e.g., 40 bp) and gains (e.g., 64 bp), there were no shifts in these spectra as a result of $\mathrm{BaP}$ exposure. The lack of effects of $\mathrm{BaP}$ on microsatellite mutation spectrum supports that induced mutations occur indirectly through enhancement of processes that are already occurring in the cell as has been suggested previously (Dubrova et al., 1998; Dubrova et al., 2000; Somers, 2006; Yauk et al., 2002; Yauk, 2004).

The mechanism through which $\mathrm{BaP}$ and other mutagens induce microsatellite mutations is unclear, although replication-based processes are involved. Knockout mice deficient for the mismatch-repair gene PMS2 (Post-meiotic segregation 2), which is required for repair of secondary DNA structure formation during DNA replication, have increased microsatellite mutation frequencies relative to controls (Baker et al., 1995) suggesting that the formation of secondary structures during replication contribute to increases in microsatellite mutation frequencies. Secondary DNA structures may also occur when cells are exposed to genotoxic agents that induce cell cycle checkpoints to pause DNA replication for repair. During pauses in replication, microsatellite sequences may form secondary structures, and the DNA template can thus become misaligned, resulting in gains or deletions of repeat units upon subsequent replication initiation (Barber et al., 2004; Yauk, 2004). It follows that if a chemical is capable of inducing DNA damage, an increase in replication errors of highly repetitive sequences might also be observed. We speculate that this process would be saturable (as is observed in this 
study) because it would be limited by the probability that replication is occurring at the locus, and the on/off nature of a cell cycle checkpoint. Similarly saturated responses at high doses have been observed in previous studies on germ cell tandem repeat mutations following mouse exposures to a variety of chemicals (Vilariño-Güell et al., 2003). Thus, our work supports the hypothesis that induced tandem repeat mutations arise via indirect mechanisms related to replication errors that may result either from genomic instability and/or pauses in cell cycle following DNA damage as proposed previously (Dubrova et al., 1998; Dubrova et al., 2000; Somers, 2006; Yauk et al., 2002; Yauk, 2004).

O'Brien et al. (2016a) used the TGR assay to examine mutation frequencies in the lac $Z$ transgene of sperm from the same animals as those used in our study. Using the lac $Z$ assay, no increases in mutation frequencies were observed at the $25 \mathrm{mg} / \mathrm{kg} / \mathrm{day}$ dose, a marginally significant 1.7 -fold increase in $\mathrm{MF}$ was reported at $50 \mathrm{mg} / \mathrm{kg} /$ day $(\mathrm{P}=0.0738)$ dose, and a significant 4.2-fold increase was found relative to controls at the 100 $\mathrm{mg} / \mathrm{kg} /$ day $(\mathrm{P}<0.0001)$ dose (O'Brien et al., 2016a). Our analysis of the $25 \mathrm{mg} / \mathrm{kg} / \mathrm{day}$ dose likewise did not reveal a significant increase in mutation frequency over that of controls; however, significant increases were observed starting at the $50 \mathrm{mg} / \mathrm{kg} /$ day dose (Table 3.1; Figure 3.2). These findings suggest that the microsatellite SM-PCR technique provides a sensitive tool for detecting changes in mutation frequencies at lower doses. However, it should be noted that a larger fold change in the lacZ MF was found at the $100 \mathrm{mg} / \mathrm{kg}$ /day dose; as described above, microsatellite mutation rates appear to be saturable and thus have a reduced dynamic range. Overall, the combined results of lacZ and microsatellite mutations in sperm confirm that exposure to $\mathrm{BaP}$ is mutagenic to 
mouse spermatogonia inducing a diverse array of genetic alterations and thus poses a heritable genetic hazard.

\subsection{Conclusions}

We developed a sperm SM-PCR approach to measure mutations in 2 unstable tetranucleotide microsatellites in the mouse genome following exposure of spermatogonia to BaP. We explored response in singleplex and multiplex PCR reactions and showed increased mutation frequencies relative to controls at both loci consistent with results from previous studies. The multiplex PCR approach may provide a slightly higher sensitivity than the singleplex approach; however, the scoring of multiplex gel electropherograms proved challenging. In addition, achieving the (40-60\%) positive to negative ratio of amplicons needed for the SM-PCR Poisson distribution at both the Mm2.2.1 and Mm19.2.3 loci in a single reaction was difficult, and did not greatly improve efficiencies. Moving forward, we propose continued refinement of the multiplex SM-PCR microsatellite technique and suggest using different fluorophores for each primer to aid in the differentiation of alleles in multiplex PCR. PCRs could also be run individually and combined post amplification to be resolved in a single sequencing run in order to improve resolution and processing time. An added benefit of sperm microsatellite analysis is that this technique can be developed for application in any species, including humans. Overall, we confirm that $\mathrm{BaP}$ is a male germ cell mutagen that may pose a risk for transmission of heritable mutations. 


\subsection{Tables and Figures}

Table 3.1 Mutation frequencies in the sperm of mice exposed to 0 (control), 25, 50, or $100 \mathrm{mg} / \mathrm{kg} /$ day BaP.

\begin{tabular}{|c|c|c|c|c|c|c|c|}
\hline By locus & Locus & $\begin{array}{c}\text { No. of } \\
\text { samples }\end{array}$ & $\begin{array}{c}\text { No. of } \\
\text { mutations }\end{array}$ & No. of progenitors ${ }^{\mathrm{a}}$ & $\begin{array}{l}\text { Mutation frequency }{ }^{\mathrm{a}} \\
\qquad\left(\times 10^{-3}\right)\end{array}$ & $\begin{array}{c}\text { Ratio } \\
\text { exposed/control }{ }^{\mathrm{a}}\end{array}$ & $\begin{array}{c}\mathrm{P}- \\
\text { value }^{\mathrm{b}}\end{array}$ \\
\hline Control & 2.2 .1 & 7 & 7 & $3598(3481-3716)$ & $1.88(1.04-3.40)$ & & \\
\hline $25 \mathrm{mg} / \mathrm{kg}$ & & 4 & 7 & $1886(1801-1971)$ & $3.62(2.67-4.91)$ & $1.9(1.0-3.7)$ & 0.0532 \\
\hline $50 \mathrm{mg} / \mathrm{kg}$ & & 4 & 9 & $1760(1678-1843)$ & $5.07(3.51-7.33)$ & $2.7(1.3-5.4)$ & 0.0052 \\
\hline $100 \mathrm{mg} / \mathrm{kg}$ & & 4 & 13 & $2202(2110-2294)$ & $5.90(3.47-10.04)$ & $3.1(1.4-6.9)$ & 0.0048 \\
\hline Control & 19.2 .3 & 7 & 12 & $3127(3018-3237)$ & $3.71(2.03-6.78)$ & & \\
\hline $25 \mathrm{mg} / \mathrm{kg}$ & & 3 & 4 & $1534(1458-1611)$ & $2.18(0.48-9.91)$ & $0.6(0.1-3.0)$ & 0.5242 \\
\hline $50 \mathrm{mg} \mathrm{kg}$ & & 4 & 19 & $1941(1855-2027)$ & $9.55(6.91-13.2)$ & $2.6(1.3-5.1)$ & 0.0068 \\
\hline $100 \mathrm{mg} / \mathrm{kg}$ & & 4 & 14 & $1550(1473-1628)$ & $8.53(6.44-11.29)$ & $2.3(1.2-4.5)$ & 0.0142 \\
\hline $\begin{array}{l}\text { Combined } \\
\text { loci }\end{array}$ & & $\begin{array}{c}\text { No. of } \\
\text { samples }\end{array}$ & $\begin{array}{c}\text { No. of } \\
\text { mutations }\end{array}$ & No. of progenitors ${ }^{\mathrm{a}}$ & $\begin{array}{l}\text { Mutation frequency }{ }^{\mathrm{a}} \\
\qquad\left(\times 10^{-3}\right)\end{array}$ & $\begin{array}{c}\text { Ratio } \\
\text { exposed/control }^{\text {a }}\end{array}$ & $\begin{array}{c}\mathrm{P}- \\
\text { value }^{\mathrm{b}}\end{array}$ \\
\hline Control & & 7 & 19 & $6718(6558-6879)$ & $2.64(1.73-4.03)$ & & \\
\hline $25 \mathrm{mg} / \mathrm{kg}$ & & 4 & 11 & $3399(3285-3514)$ & $2.81(1.30-6.08)$ & $1.1(0.4-2.6)$ & 0.8883 \\
\hline $50 \mathrm{mg} / \mathrm{kg}$ & & 4 & 28 & $3696(3577-3816)$ & $6.96(5.44-8.90)$ & $2.6(1.6-4.3)$ & 0.0001 \\
\hline $100 \mathrm{mg} / \mathrm{kg}$ & & 4 & 27 & $3651(3533-3770)$ & $7.09(5.27-9.54)$ & $2.7(1.6-4.5)$ & 0.0002 \\
\hline
\end{tabular}

${ }^{a}$ Lower and upper 95\% CIs given for the number of progenitor molecules, mutation frequencies, and ratios.

${ }^{\mathrm{b}} \mathrm{P}$-values were generated using GEEs. 
Table 3.2 Mutation frequencies from multiplex PCR of sperm from control and 100 $\mathrm{mg} / \mathrm{kg} /$ day BaP-treated animals.

\begin{tabular}{|c|c|c|c|c|c|c|c|}
\hline By locus & Locus & $\begin{array}{c}\text { No. of } \\
\text { samples }\end{array}$ & $\begin{array}{c}\text { No. of } \\
\text { mutations }\end{array}$ & No. of progenitors ${ }^{a}$ & $\begin{array}{c}\text { Mutation } \\
\text { frequency }^{\mathrm{a}}\left(\times 10^{-3}\right)\end{array}$ & $\begin{array}{c}\text { Ratio } \\
\text { exposed/control }^{\text {a }}\end{array}$ & P-value ${ }^{b}$ \\
\hline Control & 2.2 .1 & 3 & 4 & $1840(1756-1924)$ & $2.17(1.95-2.43)$ & & \\
\hline $100 \mathrm{mg} / \mathrm{kg}$ & & 3 & 9 & $1374(1302-1447)$ & $6.24(4.63-8.40)$ & $2.9(2.1-3.9)$ & $<0.0001$ \\
\hline Control & 19.2 .3 & 3 & 3 & $1559(1482-1637)$ & $1.92(0.78-4.73)$ & & \\
\hline $100 \mathrm{mg} / \mathrm{kg}$ & & 3 & 10 & $1326(1255-1398)$ & $7.43(5.73-9.64)$ & $3.9(1.5-9.9)$ & 0.005 \\
\hline $\begin{array}{l}\text { Combined } \\
\text { loci }\end{array}$ & & $\begin{array}{c}\text { No. of } \\
\text { samples }\end{array}$ & $\begin{array}{c}\text { No. of } \\
\text { mutations }\end{array}$ & No. of progenitors ${ }^{a}$ & $\begin{array}{c}\text { Mutation } \\
\text { frequency }^{\mathrm{a}}\left(\times 10^{-3}\right)\end{array}$ & $\begin{array}{c}\text { Ratio } \\
\text { exposed/control }^{\text {a }}\end{array}$ & P-value ${ }^{b}$ \\
\hline Control & & 3 & 7 & $3386(3272-3500)$ & $2.04(1.30-3.22)$ & & \\
\hline $100 \mathrm{mg} / \mathrm{kg}$ & & 3 & 19 & $2700(2598-2802)$ & $6.81(5.59-8.30)$ & $3.3(2.0-5.5)$ & $<0.0001$ \\
\hline
\end{tabular}

${ }^{\mathrm{a}}$ Lower and upper $95 \%$ CIs given for the number of progenitor molecules, mutation frequencies, and ratios.

${ }^{\mathrm{b}} \mathrm{P}$-values were generated using GEEs. 


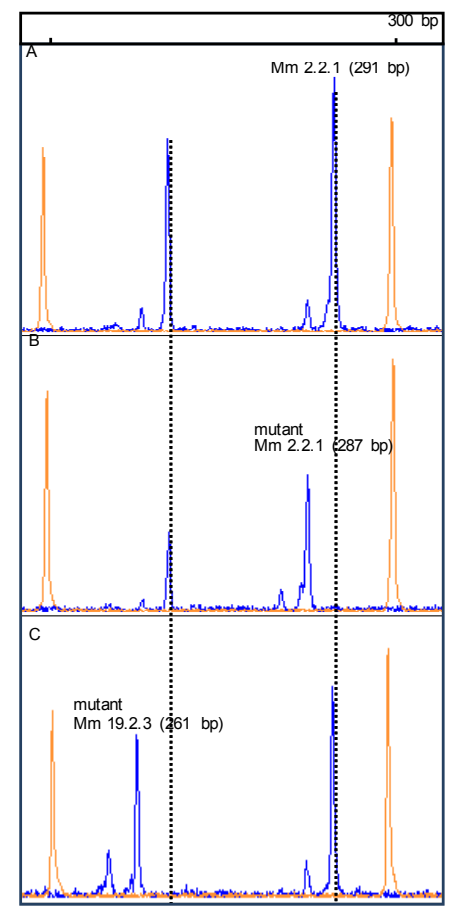

Figure 3.1 Examples of capillary electropherograms of multiplex SM-PCR of microsatellites Mm2.2.1 and Mm19.2.3 from sperm DNA. Standard peaks at 246 bp and $300 \mathrm{bp}$ shown in orange. A) Electropherogram showing the wild-type Mm2.2.1 and Mm19.2.3 alleles (291 bp and $265 \mathrm{bp}$ ). B) Electropherogram of Mm2.2.1 allele with a 1unit repeat-deletion mutation (287 bp). C) Electropherogram of Mm19.2.3 allele with a 1unit repeat-deletion mutation (261 bp). 


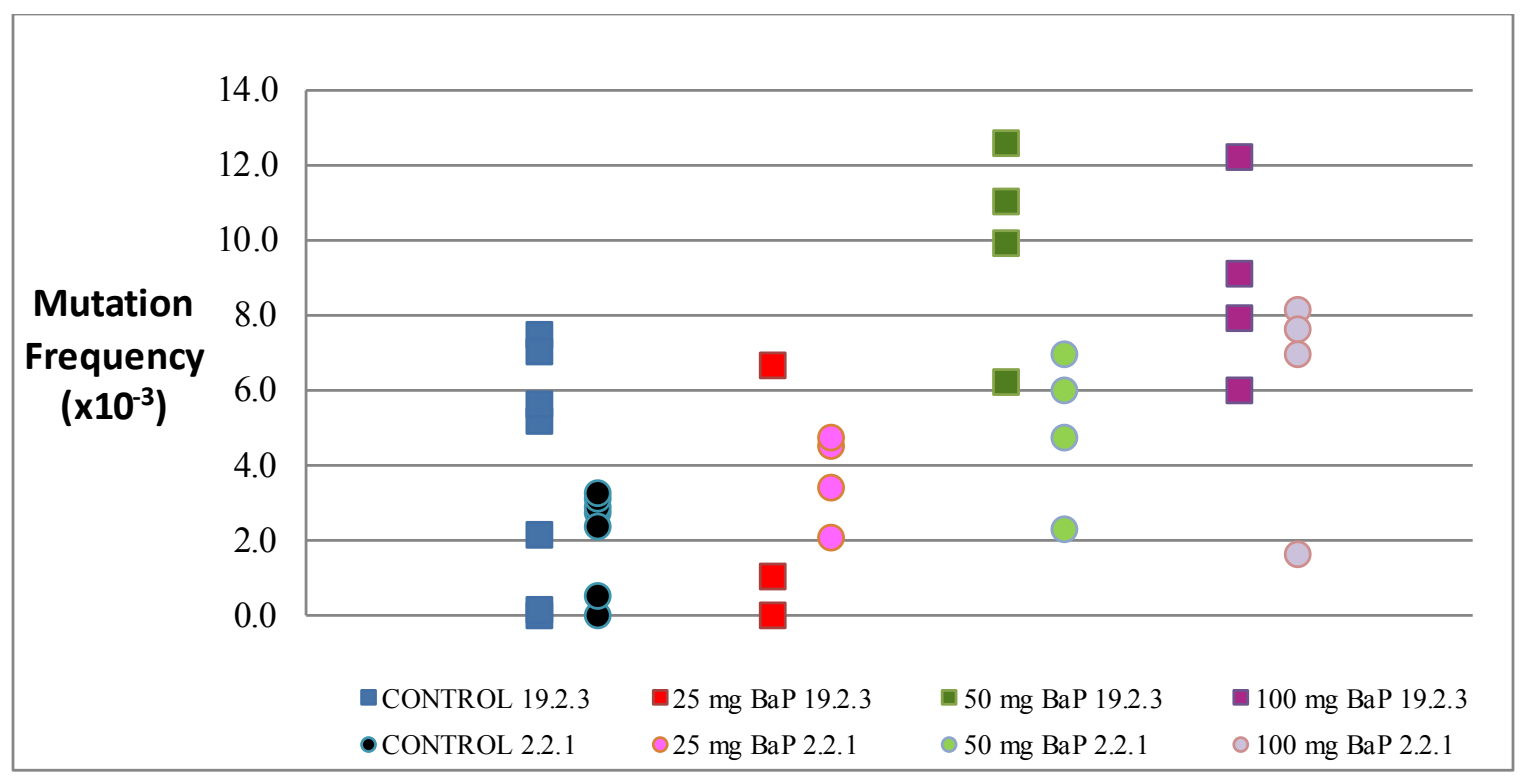

Figure 3.2 Mutation frequency for Mm2.2.1 and Mm19.2.3, analyzed independently, in the sperm of control mice and mice exposed to 25,50 , and $100 \mathrm{mg} \mathrm{BaP} / \mathrm{kg}$. 

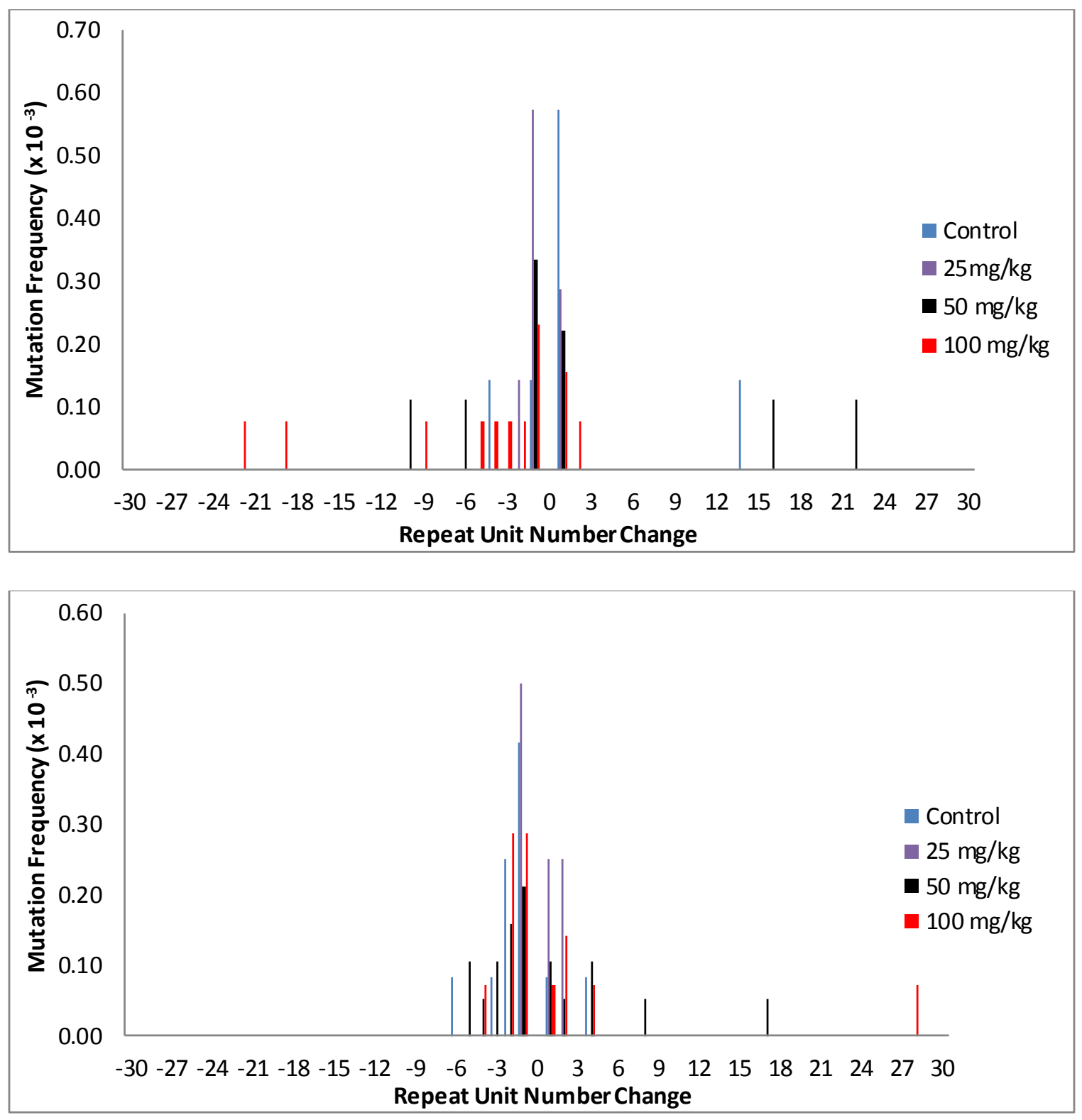

Figure 3.3 Mutation spectrum of (a) Mm2.2.1 (b) Mm19.2.3 in control and BaP-exposed sperm. 


\section{Chapter 4: Characterizing Benzo [a]pyrene-Induced lacZ Mutation Spectrum in Transgenic Mice Using Next-Generation Sequencing}

Modified from: Beal, M.A., Gagné, R., Williams, A., Marchetti, F., Yauk, C.L. 2015. BMC Genomics, 16:812.

\subsection{Preamble}

A main thesis objective was to compare the mutation signatures of $\mathrm{BaP}$ in both the sperm and offspring. However, there was no suitable method for achieving this objective. Thus, for this chapter, I developed a NGS approach to characterize TGR assay mutants. To demonstrate proof of principle, the mutation spectra of bone marrow mutants were characterized. This was done because there were thousands of TGR assay mutants from control and BaP-treated animals available from bone marrow, enabling an analysis of the sensitivity and power of the method.

\subsection{Abstract}

The TGR assay provides an efficient approach to identify mutagenic agents in vivo. A major advantage of this assay is that mutant reporter transgenes can be sequenced to provide information on the mode of action of a mutagen and to identify clonally expanded mutations. However, conventional DNA sequence analysis is labourious and expensive for long transgenes, such as lacZ(3096 bp), and is not normally implemented in routine screening. We developed a high-throughput NGS approach to simultaneously sequence large numbers of barcoded mutant lac $Z$ transgenes from different animals. We 
collected 3872 mutants derived from the bone marrow DNA of 6 MutaMouse males exposed to the well-established mutagen $\mathrm{BaP}$ and 6 solvent-exposed controls. Mutants within animal samples were pooled, barcoded, and then sequenced using NGS. We identified 1652 mutant sequences from 1006 independent mutations that underwent clonal expansion. This deep sequencing analysis of mutation spectrum demonstrated that $\mathrm{BaP}$ causes primarily guanine transversions (e.g. G:C $\rightarrow \mathrm{T}: \mathrm{A}$ ), which is highly consistent with previous studies employing Sanger sequencing. Furthermore, we identified novel mutational hotspots in the lacZ transgene that were previously uncharacterized by Sanger sequencing. Deep sequencing also allowed for an unprecedented ability to correct for clonal expansion events, improving the sensitivity of the mutation reporter assay by $50 \%$. These results demonstrate that the high-throughput nature and reduced costs offered by NGS provide a sensitive and fast approach for elucidating and comparing mutagenic mechanisms of various agents among tissues and enabling improved evaluation of genotoxins.

\subsection{Background}

Transgenic mutation reporter rodent models, such as MutaMouse (Gossen et al., 1989), enable the detection of spontaneous and induced mutations in vivo (reviewed in ref. (Lambert et al., 2005)) and the identification of chemical mutagens. These transgenic rodents carry neutral reporter transgenes that can be easily recovered and detected in a bacterial host when mutated. Studies using the TGR assay are very efficient and commonly used. The OECD has recently established guidelines for mutagenicity testing using this assay (OECD, 2011). One major advantage of the TGR assay is that mutant 
transgenes can be sequenced to provide mechanistic insight into spontaneous and induced mutagenesis. DNA sequence analysis of mutations offers several major benefits in genetic toxicology: 1) mutation spectrum identifies the target nucleotides and types of mutations providing information on the mutagen's mode of action; 2) it can be used to correct for clonal expansion of mutant transgenes for a more accurate calculation of mutation frequency (versus mutant frequency); 3) it allows for a full characterization of mutational hotspots across a specific locus; 4) it permits a comparison of mechanisms of mutagenesis operating in different target tissues; and 5) differences in mutation spectra between treated and control groups can further support a mutagenic effect, especially for weak mutagens. Thus, sequence analysis provides the TGR assay with a depth of information that is not available in most genotoxicity assays.

One of the most commonly used reporter transgenes is the bacterial lacZ gene because there is a simple, efficient, and robust positive selection method for identifying lacZmutations in E. coli (Vijg and Douglas, 1996). Mutant detection is conducted by extracting DNA from the tissues of exposed lacZ-carrying transgenic rodents, packaging of the lacZ transgene into bacteriophage and infecting host E. coli. Under selective conditions, only phage particles with mutated lacZ genes will be able to form plaques. MF is calculated as the proportion of mutant plaques versus total plaques under nonselective conditions. The lac $Z$ coding sequence is the longest of the mutation reporter transgenes (3096 bp) and thus has many target nucleotide positions where mutations can induce the mutant phenotype, making it a very sensitive reporter. However, the length of the lac $Z$ gene poses challenges for sequence analysis of mutants. For this reason, few studies have sequenced the lac $Z$ transgene to characterize mutation spectra following 
mutagen challenge (Douglas et al., 1994; Douglas et al., 1995; Douglas et al., 1996; Hakura et al., 2000; Ikehata et al., 2000; Ono et al., 2000; Vijg and Douglas, 1996). Sequencing lac $Z$ requires a complementation assay to narrow down the mutation location, followed by several Sanger sequencing reactions of the section containing the mutant (Douglas et al., 1994; Douglas et al., 1995; Douglas et al., 1996; Hakura et al., 2000). Therefore, the number of mutants that can be sequenced in an experiment is limited and most studies generally only sequence a few hundred mutants in total across their experimental groups. The majority of studies conducting mutation spectrum analysis use different and much shorter mutation reporter genes (cII, 294 bp; gpt, 456 bp; lacI, $1080 \mathrm{bp}$ ) because they are small enough to be sequenced using Sanger sequencing.

NGS had been used to target and sequence pools of $c I I$ mutants from Big Blue ${ }^{\circledR}$ mice (Besaratinia et al., 2012). These authors pooled $150 \mathrm{cII}$ mutants per sample and then sequenced 1776 mutations using 454 pyrosequencing to characterize the mutation spectra of ultraviolet radiation, 4-aminobiphenyl, and second-hand smoke, demonstrating the power of using NGS to sequence reporter genes. However, the short $c I I$ transgene is not as sensitive to induced mutations as longer genes and is therefore used much less frequently for routine screening (only $\sim 2 \%$ of studies using Big Blue ${ }^{\circledR}$ or MutaMouse actually measure mutations in the $c I I$ gene (Lambert et al., 2005)). With the advent of NGS, targeted sequencing of large fragments is now possible (Harismendy et al., 2009), and with the high-throughput nature of this technology, thousands of long fragments, such as the lacZ gene, can be sequenced in a single run. Thus, NGS can be adapted to identify mutations within longer reporter genes enabling the routine incorporation of mutation spectrum analysis into the standard TGR assay. 
Here we developed a high-throughput and cost-effective approach that uses NGS to characterize lacZmutation spectra in the MutaMouse model. We collected lacZ mutants from the bone marrow of control and $\mathrm{BaP}$ treated mice. $\mathrm{BaP}$ is a model mutagen known to form bulky DNA adducts with guanine bases, inducing primarily G:C $\rightarrow \mathrm{T}: \mathrm{A}$ transversions (DeMarini et al., 2001; Fernandes et al., 1998; Granville et al., 2003; Hakura et al., 2000; Moriya et al., 1996; Yu et al., 2002). Barcoding these individual mutant plaques would allow for the sequence from each plaque to be followed independently. However, this approach would not be any more efficient or cost-effective than Sanger sequencing individual mutants (Sanger: PCR amplification of multiple amplicons for sequencing vs. NGS: building a library). In the present work, we pooled mutants from each animal in a mixture, barcoded the mixture, and sequenced all the mutants in that mixture at the same time under the same barcode using an Ion Proton ${ }^{\mathrm{TM}}$ sequencer. This approach is much more efficient but does not allow us to track the mutations within a single plaque. Rather than assigning mutants to a specific plaque, mutations are identified within the pool and considered together as representative of the mouse sample. Using this approach, we enriched and sequenced an unprecedented 3872 lac $\mathrm{Z}$ mutant plaques from control and $\mathrm{BaP}$-exposed samples in a single sequencing run and established the BaP-induced mutation spectrum with unparalleled precision. We developed an analytical pipeline to reduce false positive mutants (PCR and sequencing artifacts) that includes the use of technical replicates and stringent filtering approaches. We applied our pipeline to determine the degree of clonal expansions and make adjustments to determine mutation frequency within our population of mutants for each mouse, to precisely characterize spontaneous and $\mathrm{BaP}$-induced mutation spectrum, and to 
determine the mutational hotspots across the lacZlocus in both control and BaP-exposed mice.

\subsection{Methods}

\subsubsection{Animal Treatment}

Six MutaMouse males (approximately 15 weeks of age; strain 40.6 carrying 29.0 \pm 4.0 copies of lac $Z$ on chromosome 3 (Shwed et al., 2010)) were exposed daily to BaP dissolved in olive oil by oral gavage for 28 days (100 mg/kg body weight/day). An additional 6 males received olive oil and served as the vehicle controls. Three days after the last treatment, mice were anaesthetized with isofluorane and euthanized via cervical dislocation. Bone marrow was isolated from the mice by flushing the femurs with $1 \mathrm{X}$ phosphate-buffered saline. After brief centrifugation and supernatant removal, the pellet was flash-frozen in liquid nitrogen and then stored at $-80^{\circ} \mathrm{C}$. All animal procedures were carried out under conditions approved by the Health Canada Ottawa Animal Care Committee.

\subsubsection{MutaMouse lacZ Mutation Analys is}

Bone marrow was thawed, digested in $5 \mathrm{~mL}$ of lysis buffer $(10 \mathrm{mM}$ Tris- $\mathrm{HCl}, \mathrm{pH}$ 7.6, $10 \mathrm{mM}$ ethylenediaminetetraacetic acid (EDTA), $100 \mathrm{mM} \mathrm{NaCl}, 1 \%$ sodium dodecyl sulfate (w/v), $1 \mathrm{mg} / \mathrm{mL}$ Proteinase $\mathrm{K})$, and incubated at $37^{\circ} \mathrm{C}$ overnight with gentle shaking. Genomic DNA was then isolated using a phenol/chloroform extraction procedure described previously (Douglas et al., 1994; Vijg and Douglas, 1996). DNA was dissolved in $100 \mu \mathrm{L}$ of TE buffer (10 mM Tris $\mathrm{pH} 7.6,1 \mathrm{mM}$ EDTA) and stored at 4 
${ }^{\circ} \mathrm{C}$ for several days before use. $\mathrm{Lac} Z$ transgene mutants present in the genomic DNA were identified using the Phenyl- $\beta$-D-galactopyranoside (P-gal) positive selection assay (Vijg and Douglas, 1996). Briefly, the $\lambda$ gt10lacZ construct was packaged into phage particles through the cohesive ends (cos sites) using the Transpack ${ }^{\mathrm{TM}}$ lambda packaging system (Agilent, Santa Clara, CA). Packaged phage particles were subsequently mixed with the host bacterium (E. coli lacZ-, galE-, recA-, pAA119 with galT and galK) (Gossen et al., 1992) in order to infect the E. coli with the lacZ construct. The E. coli were then plated on minimal medium containing $0.3 \%(\mathrm{w} / \mathrm{v}) \mathrm{P}$-gal and incubated overnight at $37^{\circ} \mathrm{C}$. P-gal is toxic to galE- strains that express a functional copy of lacZ, and thus, only E. coli receiving a mutated copy of the lacZ transcript will form plaques on the P-Gal medium. Packaged phage particles were concurrently plated on titre plates without P-Gal to determine the total plaque-forming units (PFU). The lacZ mutant frequencies were then calculated by determining the ratio of mutant PFU to total PFU. The experimental design and lacZ mutation analysis used in this study followed OECD guidelines (OECD, 2011). For more information on the lacZ mutation assay, there is a recently published video describing the assay and procedural steps used here (O'Brien et al., 2014).

\subsection{3 lacZ Mutant Plaque Collection and Preparation for Next-Generation}

\section{Sequencing}

The procedure used by Besaratinia et al. (2012) to amplify cII mutant plaques was adapted here for $l a c Z$ mutant plaques. Figure 4.1 shows a brief overview of the workflow used in this experiment. After the lacZ positive selection assay was performed, lac $Z$ 
mutant plaques from the bone marrow of each animal were collected using a transfer pipet and pooled together into microtubes containing autoclaved milliQ sterile water ( 0.3 mutants $/ \mu \mathrm{L} ; 1$ sample per tube). All of the mutants from a single animal were pooled together in a single microtube. Thus, mutations were not assigned to individual plaques; rather, all mutations within an individual mouse were identified by alignment of each read against the $l a c Z$ gene. For a negative control, wild-type plaques with no expected mutations were collected and processed for sequencing. Microtubes containing pooled plaques were boiled for $5 \mathrm{~min}$ and then centrifuged at $18,000 \mathrm{~g}$ for $5 \mathrm{~min}$. The supernatant was immediately transferred to a new microtube for storage, and $10 \mu \mathrm{L}$ of the supernatant was transferred to another microtube for PCR. To control for the introduction of PCR errors, each pool of mutants was amplified in 2 separate reactions (i.e., 2 technical replicates per DNA pool). As the 2 replicates contain the same mutant DNA, it is expected that the same mutation should be present in both technical replicate sequences. The $30 \mu \mathrm{L}$ PCR mastermix contained a final concentration of $1 \mathrm{X}$ PCR buffer, 1X Q solution, $200 \mathrm{nM}$ of each primer, $50 \mu \mathrm{M}$ dNTP, and 2.5 Units Taq DNA polymerase (Qiagen, Valencia, CA). The PCR primers used for amplification were custom designed using flanking DNA outside of the lacZ gene [GenBank:J01636.1]. The forward and reverse primers were GGCTTTACACTTTATGCTTC and ACATAATGGATTTCCTTACG, respectively. Amplification of the $l a c Z$ gene was carried out using the following thermocycle program: $95^{\circ} \mathrm{C}$ for $3 \mathrm{~min} ; 30$ cycles of $95^{\circ} \mathrm{C}$ for $45 \mathrm{~s}, 50{ }^{\circ} \mathrm{C}$ for $1 \mathrm{~min}, 72{ }^{\circ} \mathrm{C}$ for $4 \mathrm{~min}$; final extension at $72{ }^{\circ} \mathrm{C}$ for $7 \mathrm{~min}$. PCR products were purified using the QIAquick PCR purification kit (Qiagen) prior to library preparation. 


\subsubsection{Ion Proton ${ }^{\mathrm{TM}}$ Sequencing}

NGS of the lacZ mutant products was done in-house using an Ion Proton ${ }^{\mathrm{TM}}$ sequencer (Life Technologies, Carlsbad, CA). The Ion Xpress ${ }^{\mathrm{TM}}$ Plus Library Kits and AB Library Builder ${ }^{\mathrm{TM}}$ (Ion Xpress Plus Library Protocol v. 1.00) were used to prepare a library for each animal's pool of mutants from purified PCR products (for a total of 24 libraries). The procedure outlined in the user's manual was followed with the exception of a 10 min custom shearing time and the replacement of Agencourt ${ }^{\circledR}$ AMPure ${ }^{\circledR}$ XP Reagent with SPRIselect ${ }^{\circledR}$ Reagent (Beckman Coulter, Brea, CA). The P1 Adapter and barcoded A adapters provided in the Ion Xpress ${ }^{\mathrm{TM}}$ Barcode Adapter kits were ligated to the ends of the fragmented libraries (i.e., each PCR product received a unique barcode). Gel electrophoresis of the libraries was performed on E-Gel® SizeSelect ${ }^{\mathrm{TM}} 2 \%$ Agarose Gel using the $\mathrm{E}-\mathrm{Ge}{ }^{\circledR} \mathrm{iBase}^{\mathrm{TM}}$ and visualized using the $\mathrm{E}-\mathrm{Ge}{ }^{\circledR}$ Safe Imager ${ }^{\mathrm{TM}}$ transilluminator. Fragments that were between $\sim 175$ and $\sim 225$ bp were excised from the gel and purified using the Invitrogen ${ }^{\mathrm{TM}}$ PureLink ${ }^{\circledR}$ Quick Gel Extraction Kit. Libraries were then amplified using the Library Amplification Primer Mix (Ion Plus Fragment Library Adapters kit) and Platinum ${ }^{\circledR}$ PCR SuperMix High Fidelity. Each amplified library was quantified/qua lified using the Agilent ${ }^{\circledR}$ High Sensitivity DNA Kit and Agilent ${ }^{\circledR}$ Bioanalyzer ${ }^{\circledR} 2100$ Instrument. Aliquots of each library were pooled together for a total final concentration of $16 \mathrm{pM}$. Emulsion PCR (em-PCR) of the pooled libraries was carried out using Ion Sphere ${ }^{\mathrm{TM}}$ particles on the Ion OneTouch ${ }^{\mathrm{TM}} 2$ system. The Ion OneTouch ${ }^{\mathrm{TM}}$ ES was used to enrich for particles containing em-PCR products and the Qubit ${ }^{\circledR}$ 2.0 Fluorometer was used to assess the quality of enrichment. Enriched particles 
were deposited into the wells of an Ion P1 $1^{\mathrm{TM}}$ chip (version 1) and sequenced by semiconductor sequencing (Rothberg et al., 2011).

\subsubsection{Bioinformatics}

The Proton ${ }^{\mathrm{TM}}$ Torrent Server version 3.6.2 interpreted the sequencing data and generated FASTQ files for each barcoded library. Reads were trimmed to remove low quality base calls and aligned to the reference sequence [GenBank: J01636.1] using Bowtie2 (Langmead and Salzberg, 2012) (Settings: “- local” mode with "- verysensitive-local"). The lacZgene in our MutaMouse colony has 4 variations (Hakura et al., 2000) relative to the reference coding sequence [GenBank:V00296.1] (Kalnins et al., 1983) that were corrected for.

Following alignment, a pileup format was prepared for each sample using SAMtools (Li et al., 2009a). From the sample pileups, the number of reads that were wild-type or polymorphic (base substitutions/indels) was determined for each nucleotide position of the lac $Z$ gene. At each nucleotide position, a proportion of reads is expected to carry a technical artifact (e.g., PCR artifacts and sequencing noise) for each possible mutation type (referred to as the false mutation proportion: see glossary in Table 4.1). However, these false mutation calls are consistent across samples (Figure C1) and thus the false mutation proportion of each mutation type can be estimated individually at each nucleotide position. In order to prevent outlier libraries from influencing the background reading, false mutation proportions were estimated by calculating the median rate of occurrence of each mutation type across all samples for each nucleotide position. The data were then normalized by subtracting the false mutation proportion from the total 
mutation proportion to determine the true mutation proportion at each nucleotide position (Figure C2; see glossary for clarification). Due to homopolymer sequencing errors, the false mutation proportion for some indels can be much higher than false mutation proportions for base substitutions. To minimize false indel calls, indels appearing with a false mutation proportion higher than the highest false mutation proportion for base substitution calls were ignored.

Variants that remained following the false mutation proportion subtraction were considered mutations if the true mutation proportion was above threshold values determined based on the number of mutant plaques recovered from each sample and used in the sequencing (Table C2). For example, if a mutant pool for an individual animal consisted of 100 plaques, a single mutation should be present in approximately $1 \%$ of the sequenced reads ( $1 \%$ of reads would have the variant while $99 \%$ would have the reference nucleotide). To determine the threshold that should be set for identifying true mutations, the data were first analyzed using 3 different thresholds to optimize the sensitivity of the assay. The thresholds were set at: 1) the exact proportion for the mutation expected; 2) $75 \%$ of the expected proportion; and 3) $50 \%$ of the expected proportion. Using the previous example, the thresholds would be $0.01,0.0075$, and 0.005 , respectively. In addition, in order for a mutation to be counted it needed to be present at values above the threshold in both technical replicates.

The average between the true mutation proportions of each technical replicate was used to provide a rough estimation of the total number of each type of mutation. For example, a base substitution present at a frequency of 0.10 would be counted as 10 mutations in a sample that had a minimum threshold of 0.01 . These values were used to 
determine the distribution of mutations across the lac $\mathrm{Z}$ gene for both the $\mathrm{BaP}$-treated and control groups and identify mutation hotspots. In contrast, only independent mutations were used to determine the lac $Z$ mutation spectra for both groups.

Finally, we examined the sequence sites of each specific mutation identified in the lacZ gene. The DNA codon table was used to determine whether base substitution changes observed in the coding sequence would result in amino acid changes in the protein. Based on this analysis, mutations were classified as nonsense, missense or silent.

\subsubsection{Statistical Analysis}

Statistical analyses were performed using R programming language to compare mutant frequencies and mutation spectra between control and treated animals ( $\mathrm{R}$ Core Team, 2013). The lac Z mutant frequencies in the bone marrow of control and BaP-treated animals were compared by Poisson Regression. Statistical comparisons of mutation types between control and treated groups were done using Pearson's chi-squared test of independence.

Mutations that occurred more than once in the same animal were considered to be derived from clonal expansion (see Table 4.1) from a single 'independent' event. The formula of [(total mutations - independent mutations)/total mutations] was used to estimate clonality. To test the method's ability to estimate clonality, 8 mutant plaques from different animals (each mutation identified by Sanger sequencing) were subcloned and then mixed together in different proportions (Table C3). One of these mixtures contained different sized plaques to see whether plaque size affected clonality estimation. As an additional control, 100 random mutants from 100 different animals were pooled 
together to estimate the ability to identify unique mutations that were not expected to be clonally amplified. The observed proportions recovered by sequencing were then compared with the expected proportions of each mutant mixture. A Fitting Linear Models algorithm (Chambers, 1992; Wilkinson and Rogers, 1973) in R was used to estimate the limit of detection (LOD) for accurately quantifying mutant count using NGS. The algorithm searches for the LOD with the lowest squared error using 1000 iterations with decreasing step size. The model estimated that the LOD was 4 mutants (horizontal blue line in Figure 4.2) and thus mutants with an observed count of 4 or less were set to a count of one. Above the LOD, the relationship between expected and observed mutant count is linear $(\mathrm{P}=0.4249)$. Thus, using the LOD/Linear model we adjusted the count of each mutant in the BaP experiment for clonality measurements with the formula:

Adjusted Mutant Count $=($ Observed Count - Intercept $) /$ Slope for mutant counts $>4$ where the $\mathrm{y}$ intercept $=0.3123$ and the slope $=1.1363$. Clonality was then calculated based on the adjusted mutant counts. For example, a count of 3 mutations was adjusted to 1 mutation and a count of 50 mutations was adjusted to 44 mutations.

\subsection{Results}

\subsection{1 lacZ Mutant Frequency}

As expected, bone marrow lacZ mutant frequencies were significantly higher in BaP-treated mice $\left(701.7 \pm 49.3 \times 10^{-5}\right)$ than control mice $\left(8.6 \pm 2.0 \times 10^{-5}\right)(\mathrm{P}<0.0001)$. This increase in MF is consistent with previous results (Lemieux et al., 2011). However, the spontaneous MF was higher than expected based on historical controls (example: (Lemieux et al., 2011)), mostly due to animal Control20, which had a MF of $17.8 \times 10^{-5}$. 
This animal was included in the study to test the method's ability to detect clonal expansion.

\subsubsection{Sequencing Output and Read Coverage}

Sequencing of the pooled libraries using 1 Ion P $1^{\mathrm{TM}}$ chip (version 1) gave $87,666,405$ reads of $95 \mathrm{bp}$ mean length for a total of $8.3 \mathrm{~Gb}$. The number of usable reads was reduced to $66 \%$ after removal of polyclonal Ion Sphere ${ }^{\mathrm{TM}}$ particles $(29 \%)$ obtained from the pooled library. Thus, the average nucleotide coverage per library was $9.1 \pm 0.4$ $\times 10^{4}$. The starts and ends of the reads across the gene appeared to be uniform (Figure C3).

\subsubsection{False Mutation Proportion Subtraction and Threshold Comparis ons}

An analysis of our data using conventional approaches revealed that most existing algorithms or variant callers were not equipped to detect rare mutations in pooled samples. Thus, we explored the use of a false mutation proportion subtraction methodology that we developed in-house. False mutation proportion refers to the sequencing artifacts that occur by chance at each nucleotide position. We use the term 'proportion' here to differentiate the proportion of reads carrying errors (background noise) from reads with true mutations. The false mutation proportions (see Table 4.1 for specific definition) for base substitutions ranged from $1.70 \times 10^{-5}$ to $2.15 \times 10^{-2}$ (median: $4.25 \times 10^{-3}$ ), and from $1.64 \times 10^{-5}$ to $4.64 \times 10^{-1}$ (median: $7.52 \times 10^{-3}$ ) for indels (Figure C4). As the number of indel technical errors increase, the ability to reliably call mutations diminishes. Thus, indels were only called if the false mutation proportion was less than 
$2.15 \times 10^{-2}$. Indels occurring at sites with a false mutation proportion greater than this were not included in the analysis. In the final analysis, this resulted in the removal of 21 out of 65 insertions (3/6 control, 18/59 BaP) and 99 out of 297 deletions (31/53 control, 68/244 BaP).

After correcting for the false mutation proportion, we explored mutation calling using 3 different thresholds for optimization (Table C2). With the stringent threshold (i.e., the exact proportion expected), 152 and 854 independent mutations were recovered in both technical replicates for the control and $\mathrm{BaP}$ groups respectively (Table 4.2). The total number of mutations (unadjusted by LOD/Linear model) recovered out of 872 and 3000 mutant plaques from control and BaP-treated samples were 583 (67\%) and 2077 (69\%), respectively. Applying the 2 less stringent thresholds increased the number of mutations called from 2660 to 2912 and 3282, respectively; however, the discrepancies between technical replicates greatly increased. Thus, all downstream analyses were done on mutations called using the most stringent threshold. As expected, applying the most stringent threshold resulted in no mutation calls for the wild-type plaques.

\subsubsection{Detection of Clonal Expansion}

We created 5 control libraries (Table C3) in which the number of mutations was known in order to determine the ability of our NGS approach to accurately predict the number of copies of each mutation. In addition, a library of 100 random mutants was sequenced to determine our ability to detect unique mutations. For each mutation detected, we divided the true mutation proportion by the expected mutation proportion for a single mutation (threshold) to estimate the number of copies of that mutation in any 
given sample. In the library of 100 random mutants there were 49 independent mutants detected with a total count of 102 recurrent mutations, which is a higher proportion of recurrent mutations than was expected (Douglas et al., 1994). It is possible that some of the recurrent mutations occurred at mutation hotspots. For example, 5 independent mutants and 15 recurrent mutations were detected at 4 of 5 known mutation hotspots (Ikehata et al., 2000). However, it is more likely that some mutants were not detected while the count of the detected mutants was overestimated. When the expected mutant count was compared with the observed mutant count in the 5 control libraries (Figure 4.2), we found that the count of each mutant was overestimated. Therefore, a LOD/linear model was developed to adjust the recurrent mutation counts of each independent mutation. Based on the model, the total mutation count for the library containing 100 random plaques was estimated to be 55 mutations. The mutant count estimation for the experimental libraries was 1652 mutant sequences (411 control, $1241 \mathrm{BaP}$ ) from 1006 independent mutations. A total of 917 mutants in the experimental libraries fell below the LOD, indicating a relatively small proportion of mutants underwent clonal expansion. The adjusted average clonality in the control and BaP samples was 43 and $31 \%$ respectively (Table $\mathrm{C} 4$ ), which is comparable to what was measured at the $c I I$ locus in the same mice (O'Brien, unpublished) and in other studies (Lambert et al., 2005). After adjusting for clonality, the lacZ mutation frequencies were $4.0 \pm 1.0 \times 10^{-5}$ and $484.0 \pm$ $34.9 \times 10^{-5}$ for control and BaP mice, respectively. The clonality adjustment revealed that the raw control MF was elevated in part by the presence of 1 outlier animal with extensive clonal expansion (86\%). After correcting for clonal expansion, the mutation frequency of this animal was in line with the mutation frequency of the rest of the 
animals (within 1 standard deviation of the mean; Table C4). The model for adjusting clonality showed that $\mathrm{BaP}$ induction was 121 -fold higher than control.

\subsection{5 lacZ Mutation Spectra and Dis tribution}

The $\mathrm{BaP}$ mutation spectrum in bone marrow had significant differences from the control spectrum (Figure 4.3). The proportions of transitions, transversions, and indels were $0.35,0.49$, and 0.16 in the control and $0.14,0.61$, and 0.25 in the $\mathrm{BaP}$ animals, respectively. The proportion of G:C $\rightarrow$ T:A and G:C $\rightarrow \mathrm{C}: \mathrm{G}$ transversions was significantly increased in $\mathrm{BaP}$-treated animals relative to controls $(\mathrm{P}<0.05)$. In addition, the proportion of $\mathrm{G}: \mathrm{C} \rightarrow \mathrm{A}: \mathrm{T}$ transitions was reduced $(\mathrm{P}<0.0001)$ following $\mathrm{BaP}$ treatment. These findings are consistent with the lacI mutation spectrum in BaP-treated mouse liver (Shane et al., 2000) (Figure C5). There were no significant differences in the proportion of the other mutation types between control and $\mathrm{BaP}$-treated groups. When comparing the absolute frequencies of each mutation type, $\mathrm{BaP}$ exposure increased all mutation types above control except for A:T $\rightarrow$ G:C transitions (Figure C6). To further test the accuracy of our NGS data, we compared the control and BaP-induced bone marrow lacZ mutation spectrum with published mutation spectra for transgene reporters from control and BaP-treated mice. The comparisons of control (Figure C7) and BaPinduced (Figure C8) spectra were consistent with the published data using Sanger sequencing, demonstrating that our NGS approach accurately identifies mutation spectra.

Plotting the locations of the independent (Figure 4.4) and recurrent (Figure C9) base substitutions along the lac $Z$ transgene to identify mutation hotspots demonstrated that the majority of induced and spontaneous mutations were at G:C base pairs (Table 
C5). Within the lac $Z$ transgene there are 12 short $\mathrm{CpG}$ sequences $\geq 5$ nucleotides long. At these $\mathrm{CpG}$ sites there were 5 control point mutations $(1 \mathrm{G}: \mathrm{C} \rightarrow \mathrm{A}: \mathrm{T}, 3 \mathrm{G}: \mathrm{C} \rightarrow \mathrm{T}: \mathrm{A}, 1$ G:C $\rightarrow$ C:G) and $36 \mathrm{BaP}$ point mutations $(1 \mathrm{G}: \mathrm{C} \rightarrow$ A:T, 24 G:C $\rightarrow$ T:A, 11 G:C $\rightarrow$ C:G). All nucleotide positions that had more than 2 independent mutations were at G:C sites in both control and BaP groups. Previously identified mutation hotspots in the lacZ gene using Sanger sequencing, based on $\sim 500$ mutations (Douglas et al., 1994; Douglas et al., 1995; Douglas et al., 1996; Hakura et al., 2000; Ikehata et al., 2000; Ono et al., 2000), were also hotspots in our dataset (Figure C10). In all previous studies, there were 67 nucleotide positions with more than 1 mutation, and $51(76 \%)$ of these positions had a mutation in our dataset. We found mutations at all of the nucleotide positions that had more than 4 mutations across the previously published mutation hotspots. In the NGS data, there were 29 hotspots with more than 4 independent mutations across the different animals; of these nucleotide positions only $2(7 \%)$ were not identified using Sanger sequencing in previous studies.

The majority of indels that we observed were gains/losses of single nucleotides. There were 34 indels (of 242 in total) that involved 2 nucleotides: $32 / 198$ deletions (3/22 control, 29/176 BaP); and 2/44 insertions (0/3 control, 2/41 BaP). Detecting indels was not as reliable as detecting base substitutions due to difficulty in sequencing homopolymer regions; sequencing accuracy of homopolymers falls to $97 \%$ at homopolymer stretches of 5 or more base pairs (Rothberg et al., 2011). In the lacZ gene, there are 591 short homopolymers (462 2-mononucleotide repeats; 97 3-mononucleotide repeats; and $32 \geq 4$-mononucleotide repeats). This means that $1353 \mathrm{bp}$ (44\%) of the lacZ gene are within a short homopolymer. To test for a homopolymer bias, indels were 
plotted across the lac Z gene (Fig 4.5, Figure C11). The numbers of mutations occurring inside or adjacent to homopolymer stretches $\geq 3$ bp long were: 2 out of 3 control insertions, 5 out 22 control deletions, 7 out of $41 \mathrm{BaP}$ insertions, and 33 out of $176 \mathrm{BaP}$ deletions (Figure 4.3). Furthermore, only $10(31 \%)$ of the 32 longest homopolymers in the lacZ gene had mutations within or adjacent to the homopolymers. Thus, the false mutation proportion subtraction accounted for many sequencing artifacts occurring around homopolymer regions.

Using the coding sequence of lacZ, we identified the amino acid changes that would result from each mutation. This analysis revealed that the proportion of missense, nonsense, and silent mutations was 39,59 , and $2 \%$ in the control, and 50, 44, and $7 \%$ in the $\mathrm{BaP}$ group, respectively. The silent mutations that were identified by NGS are likely "hitchhiker" mutations that occurred in the same plaque as a phenotype-inducing mutation. Sanger sequencing of mutant plaques shows that the proportion of plaques with more than 1 mutation (synonymous or non-synonymous) can be as high as $11 \%$, and the proportion of plaques with mutations separated by hundreds of nucleotides is around 3\% (Hakura et al., 2000). Also, the proportion of silent mutations observed here is consistent with the proportion of silent mutations detected using Sanger sequencing $(<6 \%)$ (Douglas et al., 1995; Douglas et al., 1996; Hakura et al., 2000; Ikehata et al., 2000; Shane et al., 2000). The number of missense, nonsense, and silent mutations that occurred more than once at a nucleotide position was 10,7 , and $0(20,9$, and $0 \%)$ for the control, and 55, 62, and $4(18,22$, and $8 \%)$ for the $\mathrm{BaP}$, respectively. Taking both groups into account there were 66,81 , and 4 positions where there was more than 1 missense, nonsense, and silent 
mutation, respectively. The low number of recurring silent mutations is as expected and reinforces the reliability of the data.

We also performed simulations, using random mutant sampling, to determine the minimum number of mutants per samples that would be sufficient to achieve a consistent mutation spectrum in the BaP group. In this simulation, mutations were sampled from 375, 348, 364, 360, and $299 \mathrm{BaP}$ mutants $(150,142,161,138$, and $139 \mathrm{BaP}$ mutations) in 5 of the 6 treated animals, respectively. One animal had to be excluded because some of the mutation types did not occur frequently enough for accurate simulations. There were no significant differences in the mutation spectra of the 5 remaining animals $(P=0.3)$. As shown in Figure C12, the results of our simulations demonstrated that sequencing approximately 100 mutants from a mouse provides an $80 \%$ power to obtain the true mutation spectrum for that individual.

\subsection{Discussion}

We developed a simple, cost-effective, and high-throughput method to characterize the mutation spectrum of a transgenic mutation reporter locus in rodents using targeted NGS. This method can be adapted for any mutation reporter model using any NGS platform. In addition, the data analysis is simple and non-subjective. Using our conservative approach, we identified 1006 independent mutations within the lacZ transgene allowing us to characterize the spontaneous and $\mathrm{BaP}$-induced mutation spectra with unparalleled precision. As expected, $\mathrm{BaP}$ exposure induced primarily $\mathrm{G}: \mathrm{C} \rightarrow \mathrm{T}: \mathrm{A}$ transversions. Our approach also allowed us to identify 1652 total mutations (including recurrent mutations that occurred more than once per mouse), permitting clonal 
expansion correction and making the assay 50\% more sensitive in detecting increases in mutation frequencies. Furthermore, comparisons of our NGS results with previous results obtained using Sanger sequencing show that the mutation spectra (Figure 4.3), mutation hotspots (Figure C10), and amino acid changes were as expected, demonstrating the robustness of our approach.

Characterization of the mutation spectra is important for identifying which sites are targeted by chemicals to elucidate mutagenic mechanism (e.g., what types of adducts are formed, faulty DNA repair processes, etc.). We investigated the mutation signature of $\mathrm{BaP}$ because it is a model carcinogen and its metabolic activation is well understood (Baird et al., 2005; Miller and Ramos, 2001). BaP is metabolized by cytochrome P450 enzymes and epoxide hydrolase to mutagenic metabolites including BPDE (Gelboin, 1980; Miller and Ramos, 2001; Singh et al., 2007). BPDE forms DNA adducts with the 2-amino group (N2) of guanine, and to a lesser extent with N6 and N4 of adenine and cytosine, respectively (Gelboin, 1980; Singh et al., 2007; Wang et al., 2003). BPDE also produces reactive oxygen species (ROS) through the spontaneous rearrangement of the epoxides or redox cycling, which also react with DNA leading to oxidative DNA damage, such as 8-oxo-deoxyguanosine (8-oxo-dG) (Singh et al., 2007). These BaP damaging events explain the high proportion of guanine base substitutions, specifically $G$ $\rightarrow \mathrm{T}$ and $\mathrm{G} \rightarrow \mathrm{C}$, seen here in bone marrow. In total, $89 \%$ of $\mathrm{BaP}$-induced mutations occurred at G:C nucleotides and all of the $\mathrm{BaP}$-associated mutation hotspots were at $\mathrm{G}: \mathrm{C}$ nucleotides. However, the refined mutation spectra obtained from sequencing thousands of mutants demonstrated that absolute mutation frequencies of all mutation types were elevated relative to the control with the exception of $\mathrm{A}: \mathrm{T} \rightarrow \mathrm{G}: \mathrm{C}$ transitions. Thus, $\mathrm{BaP}$ is 
a potent mutagen capable of inducing transversions at any nucleotide and transitions at G:C nucleotides.

One of the main reasons for sequencing mutants is to account for clonal expansion (OECD, 2011). This can cause a tissue sample to have a higher MF than the rest of the samples within a group and falsely over-represents independent mutation events. To correct for clonal expansion, a subset of the mutant plaques from the tissue sample is sequenced to identify recurrent mutations arising from 1 progenitor cell that may be inflating the MF. Recurrent mutations are only counted as 1 mutation and the MF is adjusted accordingly (to reflect the true mutation frequency). Plotting the mutation distribution of all animals revealed potential clonal expansion events. Using the LOD/Linear model the corrected clonality estimates were $43 \%$ and $31 \%$ in the control and exposed groups respectively. Correcting for clonality revealed that the fold increase in mutation frequency following $\mathrm{BaP}$ treatment was $\sim 120$-fold. This suggests that although NGS may overestimate the clonality of a tissue sample, the overestimation is consistent across samples and therefore a relative clonality comparison between groups can be achieved.

It should also be noted that the number of mutants sequenced can influence the clonal expansion estimation. In this study we sequenced a large number of mutants unmatched by any other study, and for this reason we may be seeing higher levels of clonality. Simulations show that had only 25 mutations been sequenced per animal (more typical of past experiments using Sanger sequencing (Thybaud et al., 2003)), the clonality would have remained at $43 \%$ in the control group, but would have been reduced to $9 \%$ in treated samples. The differences in clonality between actual and simulated measurements 
highlight the need to use caution when comparing clonality results determined by sequencing large versus small numbers of mutants. The lower clonality observed in the treated group can be explained by the presence of thousands of $\mathrm{BaP}$-induced mutations that are reducing the chance of randomly sampling spontaneous mutations that were clonally expanded during mouse development.

The need to account for clonality was clearly demonstrated by the presence of 1 outlier control animal with a high MF in our study (Table C4). Because of this outlier, which had $86 \%$ clonality, our control group had a mean MF that was higher than expected based on previous studies (Lambert et al., 2005). However, after correcting for clonal expansion there was less variability in mutation frequencies within each group. Furthermore, the adjusted spontaneous mutation frequency was consistent with historical values for bone marrow from our laboratory (Lemieux et al., 2011; O'Brien et al., 2013). Finally, correcting for clonal expansion demonstrated that the mutation induction caused by $\mathrm{BaP}$ was underestimated by $50 \%$ in the standard non-sequenced based approach. Had a weaker mutagen been tested, the TGR assay may have given a false-negative result without adjustment of clonal expansion. These examples show the added value of sequencing to measure clonality when interpreting the results of the TGR assay and demonstrate that our method can be used to correct for clonal expansion events at the lacZ locus to improve the reliability of reporter gene data.

There are notable strengths in our approach. First, our method uses a simple false mutation proportion subtraction to minimize sequencing artifacts and alignment errors. It is known that different NGS platforms and alignment tools have biases that can introduce false positives (Beal et al., 2012b; Farrer et al., 2013; Koboldt et al., 2010; Snyder et al., 
2010). For example, the accuracy of semi-conductor sequencing decreases with increased homopolymer length (Rothberg et al., 2011), as is seen with pyrosequencing (Huse et al,, 2007; Margulies et al., 2005). However, these biases are consistent between the samples at each nucleotide position (Figure C1) and thus can be corrected (Figure C2). After applying the correction, we were able to identify independent mutations along the lac $Z$ gene and approximate the number of times each mutation occurred. Second, we used technical replicates (2 PCR amplifications) for each sample as a strategy to minimize the number of false-positives. This was done to account for errors that occurred during PCR as a result of Taq replication errors or thermal DNA damage (Pienaar et al., 2006; Schmitt et al., 2012). Using technical replicates does not reduce the throughput of the sequencing, and only marginally increases the cost of the experiment. Although this approach is very conservative and reduces the number of mutations called (i.e., we identified 1652 mutants from 3872 plaques), it is currently needed to eliminate falsepositives, especially for indels, and accurately call mutations (Table 4.2). Thus, we strongly recommend that technical replicates be used with this method to control for PCR-based errors, allowing for accurate characterization of mutation spectra. Given the significant increases in the number of mutants that can be sequenced relative to Sanger sequencing, our stringent criteria for calling mutations does not reduce the power of the approach; indeed, we have sequenced an unprecedented number of lac $Z$ mutations within a single experiment. This method was designed to be modular; therefore, researchers can explore the use of other bioinformatics and statistical tools that best fit their data. Before setting up this protocol in a laboratory, it is recommended that experiments be conducted using positive and negative controls to optimize parameters as we have done here. 
The number of mutants that should be pooled per barcode must be considered carefully in the experimental design. As the number of mutants increase, the threshold to call a mutation decreases and the chance of identifying an artifact as a mutation increases. In addition, having too many mutants pooled per barcode will decrease the detection sensitivity of the NGS method. Contrarily, having too few mutants will not provide an accurate mutation spectrum. In order to approximate the number of mutants that should be pooled per barcode, we performed multiple data simulations based on the available results. These simulations show that sampling around 100 mutants per animal is sufficient to achieve reliable mutation spectra with a power of $80 \%$, and therefore, pooling approximately 170 mutants per barcode (assuming a mutant recovery of $\sim 60 \%$ ) should be more than enough for any sequencing application. If sequencing of more mutations per sample is desired, additional DNA barcodes can be used. Another consideration is that the short length of the reads hinders the detection of large indels or complex mutations (multiple mutations occurring across the same DNA molecule). However, read lengths for the different platforms continue to increase (Liu et al., 2012) and may eventually increase to a point where complex mutations can be detected with a standard library.

Our method was designed using DNA barcoding to allow for cost- and timeeffective analysis of lacZ mutation spectra. We estimate that the cost to sequence 3872 mutants using Sanger sequencing would be 20 -fold higher than using NGS in reagent costs alone. Furthermore, this sequencing can be done in a week using NGS, while it would take at least 25 times as long using Sanger sequencing. If more mutants were sequenced, whether they were from the same samples or different experiments, the 
difference in cost and time would be greater. As demonstrated, there is an added bonus of sequencing large numbers of mutants: 1) mutation spectra can be characterized with high precision; 2) novel mutational hotspots can be identified (important for understanding an agent's mode of action); and 3) silent mutations present on a mutated transgene, which are normally undetected, can be characterized. Considering that each barcoded library was sequenced with an average coverage of 91,000x (182,000x coverage per sample), several more samples could have been sequenced, making this approach highly efficient and amenable to the simultaneous sequencing of mutant plaques from different tissues and/or chemical agents. Furthermore, the cost and time to do these experiments will decline further as the throughput of NGS increases.

\subsection{Conclusions}

In summary, we developed a high-throughput method for targeted-sequencing of long mutant reporter genes, specifically the lac $Z$ transgene. The mutation spectra determined using the NGS analytical process are highly concordant with results using Sanger sequencing, demonstrating the accuracy of the methodology. After the animal exposure and tissue sample collection is complete, a whole experiment, from the beginning of the TGR assay to the end of the data analysis, can be done in less than 3 weeks using our newly established data analysis. The benefit of this method is that it can sequence as many mutants as the TGR assay can produce. Thus, this method has the potential to sequence hundreds to thousands of mutants from different tissues, exposure groups, and doses simultaneously. Despite the apparently low recovery of mutants by NGS due to the reduced sensitivity of calling mutations from pooled DNA, the rapid 
characterization of large numbers of mutants provides a more accurate measure of mutation spectra and clonal expansion. Although the method is applied to sequencing lac $Z$ mutants, this approach can be modified to identify mutations in any reporter gene used in genetic toxicity testing. With the higher throughput and reduced cost offered by NGS, mutations induced by drugs or chemicals can now be more routinely assessed. Understanding the mode of action of different mutagenic agents enables improved regulation of genotoxins and ultimately a reduction in harmful diseases such as cancer. 


\subsection{Tables and Figures}

Table 4.1 Glossary.

Mutant - A plaque, grown on P-Gal media, that contains a loss of function mutation in the $l a c Z$ transgene.

Mutation - The change in DNA sequence (point mutation, indel) that knocks out the function of the lac $Z$ transgene. There may also be silent mutations that can co-occur on the same lacZ copy as a loss of function mutation ("hitchhiker mutations").

Clonal Expansion - When a cell containing a mutation produces daughter cells containing that same mutation, propagating it throughout the tissue.

False Mutation Proportion - At each nucleotide position, a proportion of the reads may contain a technical artifact and this proportion is consistent across samples. For example, if at position 25 , the median frequency of $\mathrm{G} \rightarrow \mathrm{A}$ substitutions across all samples was 1 in every 1000 reads, the false mutation proportion would be 0.001 .

Total Mutation Proportion - At each nucleotide position, this is the mutation proportion for each mutation type within a single sample. For example, if sample 3 had a $\mathrm{G} \rightarrow \mathrm{A}$ substitutions at position 25 in $0.5 \%$ of reads, the total mutation proportion would be 0.005 .

True Mutation Proportion - The true mutation proportion of each mutation type at each position is determined by subtracting the false mutation proportion from the total mutation proportion. In the above example the true mutation proportion would be 0.004 . This value is used to determine mutation counts.

Independent Mutations - Mutations that appear multiple times in a same sample due to clonal expansion are only counted as 1 mutation per sample. For example, if sample 1 
had $5 \mathrm{G} \rightarrow$ A substitutions at position 25 , this would be called a single independent mutation at position 25 .

Recurrent Mutations - If a mutation occurs more than once within a sample it is considered recurrent as is likely the result of clonal expansion. In the previous example there are $5 \mathrm{G} \rightarrow \mathrm{A}$ recurrent mutations at position 25. 
Table 4.2 Summary of all mutations called using three different thresholds.

\begin{tabular}{|c|c|c|c|c|c|c|c|c|c|c|}
\cline { 3 - 11 } \multicolumn{1}{c|}{} & \multicolumn{2}{c|}{ Independent Mutations } & \multicolumn{2}{c|}{ Total Mutations } & \multicolumn{2}{c|}{${ }^{\mathrm{a}}$ Mutants Removed } \\
\cline { 2 - 11 } \multicolumn{1}{c|}{} & Threshold & ${ }^{\mathrm{b}}$ Base Subs. & ${ }^{\mathrm{c}}$ Ins & ${ }^{\mathrm{d}}$ Dels & Base Subs. & Ins & Dels & Base Subs. & Ins & Dels \\
\hline Control & Stringent & 127 & 3 & 22 & 542 & 7 & 34 & 14 & 101 & 117 \\
& Medium & 159 & 7 & 27 & 574 & 7 & 39 & 18 & 193 & 223 \\
& Low & 190 & 12 & 36 & 605 & 16 & 48 & 55 & 426 & 420 \\
\hline $\mathrm{BaP}$ & Stringent & 637 & 41 & 176 & 1583 & 66 & 428 & 108 & 750 & 577 \\
& Medium & 767 & 60 & 239 & 1714 & 85 & 493 & 183 & 1139 & 818 \\
& Low & 921 & 125 & 338 & 1869 & 151 & 593 & 374 & 1846 & 1284 \\
\hline
\end{tabular}

${ }^{a}$ Mutants removed refer to the number of variants that passed the threshold in 1 technical replicate but not the other.

${ }^{\mathrm{b}}$ Base substitutions

${ }^{\mathrm{c}}$ Insertions

${ }^{\mathrm{d}}$ Deletions 
Protocol
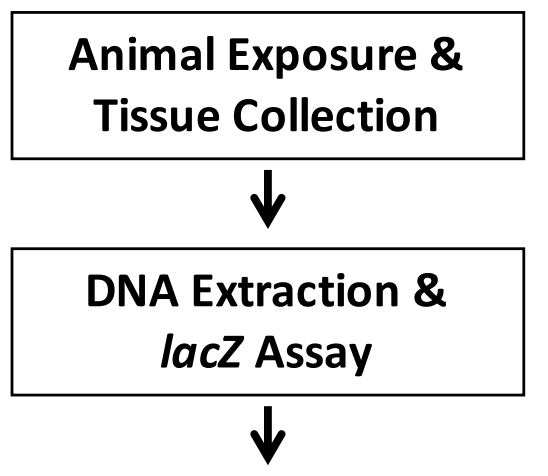
Collect \& Pool Mutants Within Animal

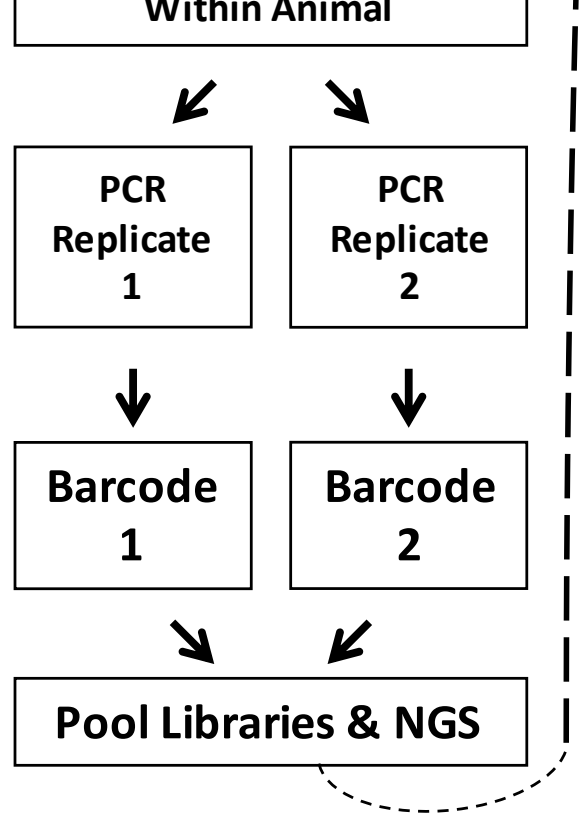

Data Analysis

Calculate Total Mutation Proportions of All Mutation Types at All Positions<smiles>C1C2CC12</smiles>

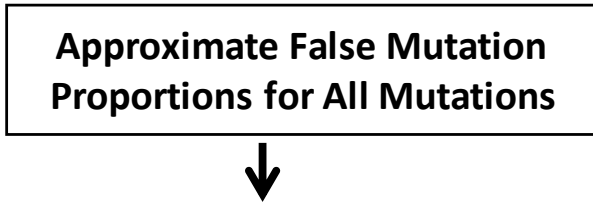

Total Mutation Proportion False Mutation Proportion = True Mutation Proportion

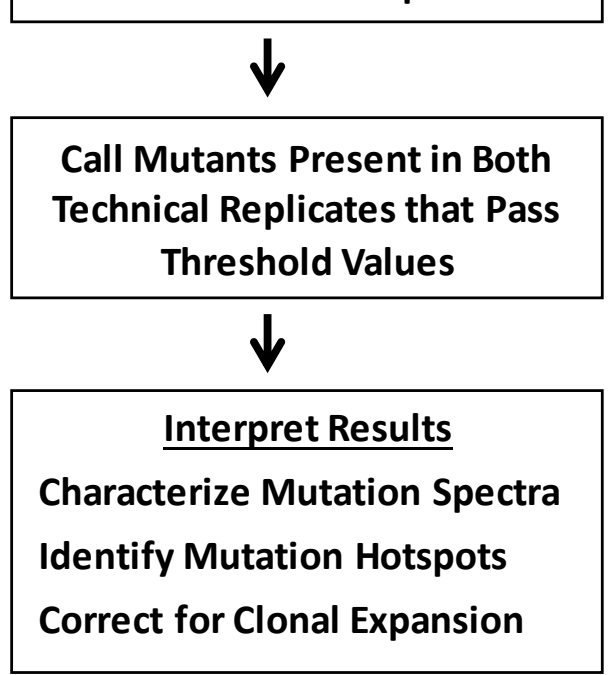

Figure 4.1 Brief overview of workflow used in this study. 


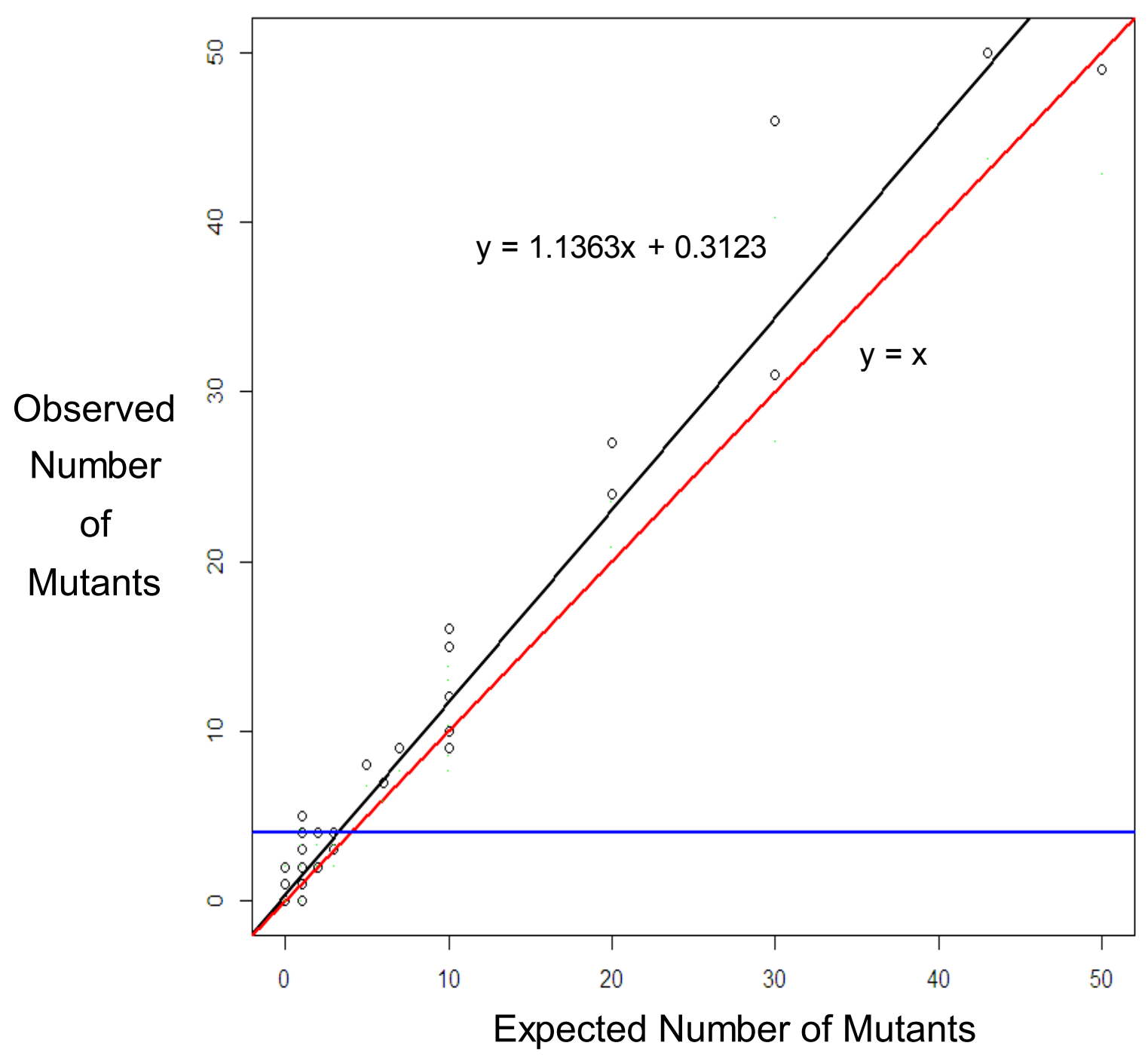

Figure 4.2 Correlation between observed and expected mutant counts. The red line shows a 1:1 ratio and the black line shows the true relationship between expected and observed mutant count. The plot shows that mutants with low counts cannot be accurately quantified. This results in a non-linear relationship between expected and observed mutant count $(\mathrm{P}<0.0001)$. Hence, a LOD/linear model was used to adjust the total mutation counts of each independent mutation. 


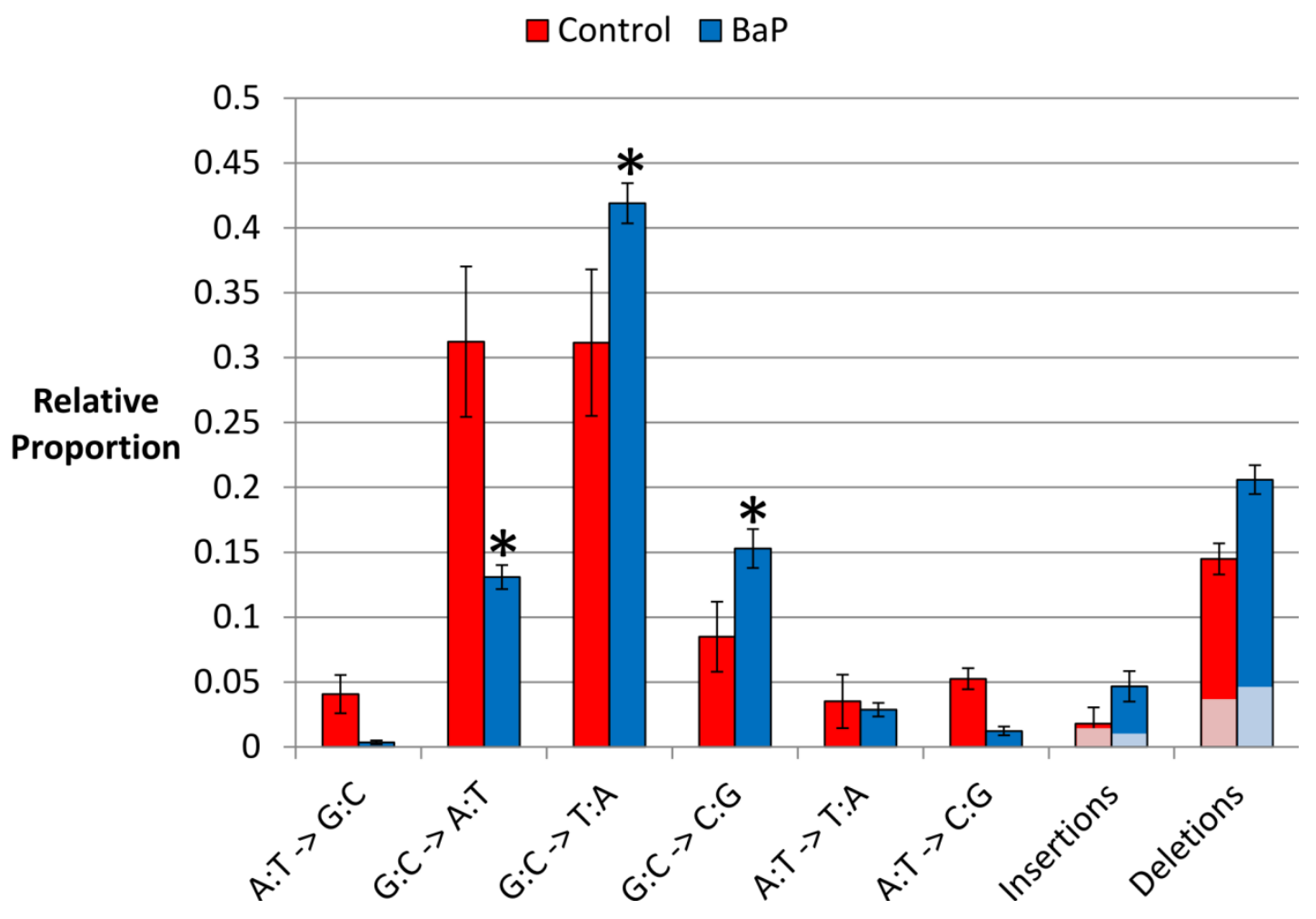

Figure 4.3 Relative proportion of control and $\mathrm{BaP}$-induced lacZ mutations in bone marrow. Only independent mutations were considered. Mutation types with asterisks are significantly different between control and treated groups. The light-colored boxes within indels represent the fraction of indels that was inside or next to homopolymer stretches $\geq$ 3 bp long. The error bars represent the standard error between samples. 
Control $\square \mathrm{BaP}$

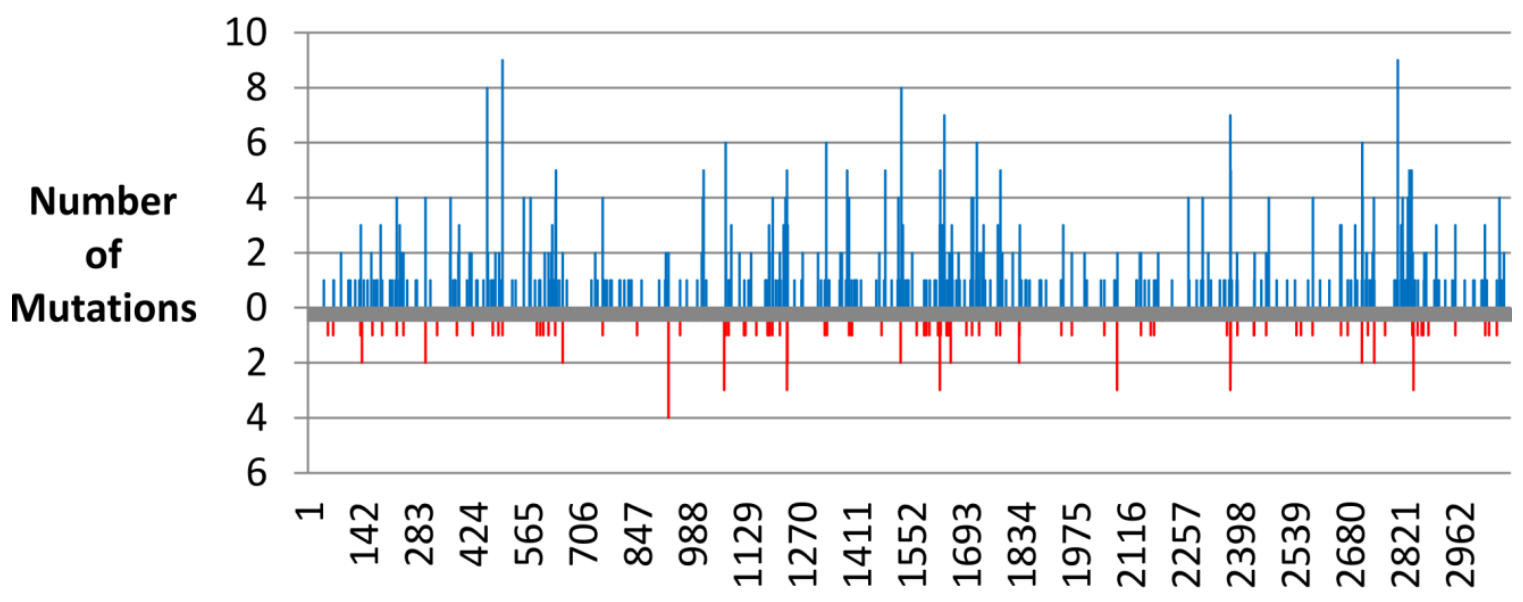

Nucleotide Position

Figure 4.4 Distribution of unique base substitutions across the lac $Z$ transgene for control and $\mathrm{BaP}$ samples. $\mathrm{BaP}$ base substitutions are on the positive $\mathrm{y}$-axis and control substitutions are on the negative y-axis. 
Control Insertions Control Deletions $\square$ BaP Insertions BaP Deletions

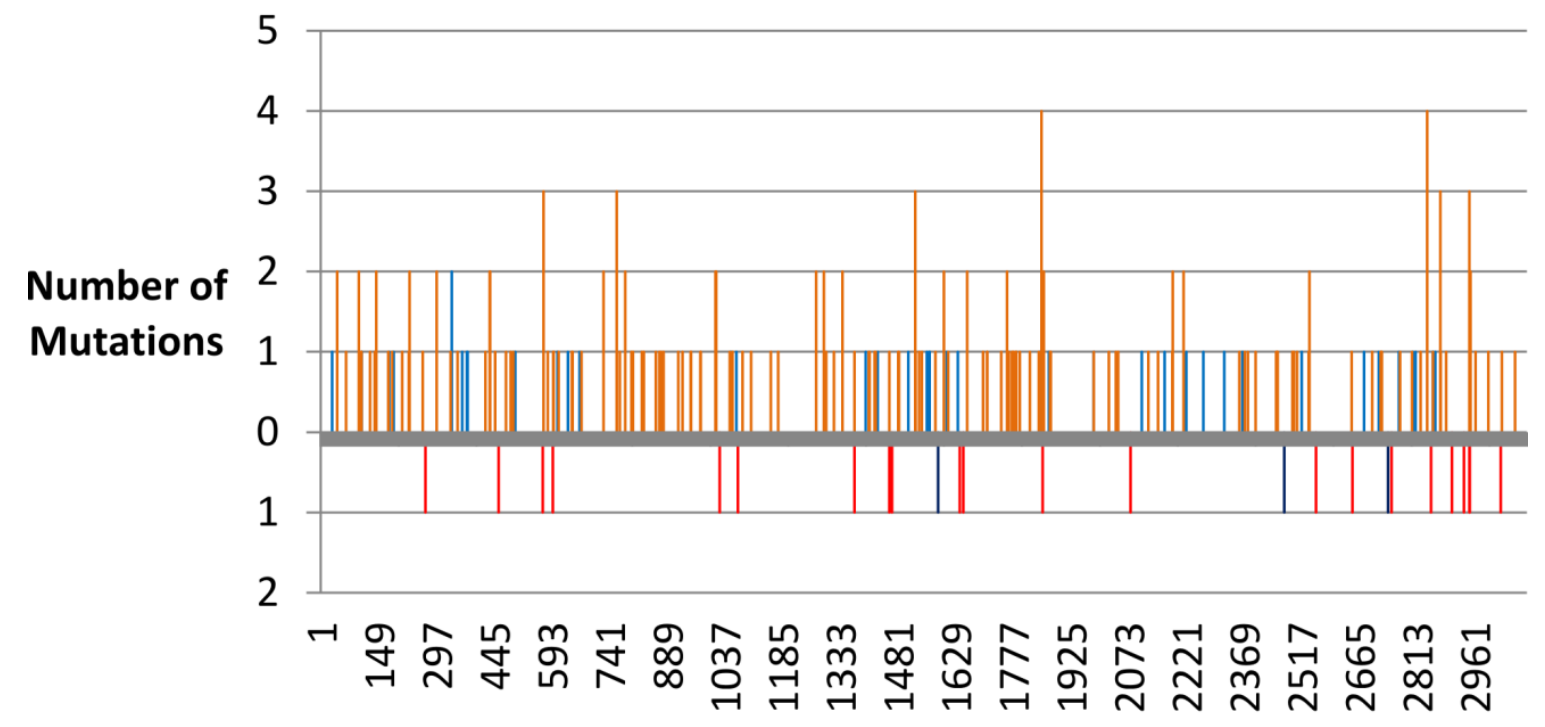

\section{Nucleotide Position}

Figure 4.5 Distribution of unique indels across the lac $Z$ transgene for control and $\mathrm{BaP}$ samples. $\mathrm{BaP}$ indels are on the positive $\mathrm{y}$-axis and control indels are on the negative $\mathrm{y}$ axis. 


\section{Chapter 5: Benzo(a)pyrene is Mutagenic in Mouse Spermatogonial Stem Cells and Dividing Spermatogonia}

Modifie d from: O’Brien, J.M., Beal, M.A., Yauk, C.L., Marchetti, F. 2016.

Toxicological Sciences, 152:363-371.

\subsection{Preamble}

The purpose of this chapter was to measure the mutant frequencies induced in sperm by $\mathrm{BaP}$ using the TGR assay. This was done in order to make comparisons between the mutations rates measured by whole genome approaches and the mutant frequencies observed in sperm. This comparison is important as it is not known if there will be a strong correlation between mutant frequencies in sperm and mutation rates in offspring. For example, mutation rates may be higher in the offspring due to the detection of silent non-functional mutations, or lower due to selection against sperm carrying mutations.

\subsection{Abstract}

Although many environmental agents are established male germ cell mutagens, few are known to induce mutations in spermatogonial stem cells. Stem cell mutations are of great concern because they result in a permanent increase in the number mutations carried in sperm. We investigated mutation induction during mouse spermatogenesis following exposure to BaP. MutaMouse males were given $0,12.5,25,50$, or $100 \mathrm{mg} / \mathrm{kg}$ bw/day BaP for 28 days by oral gavage. Germ cells were collected from the cauda 
epididymis and seminiferous tubules 3 days after exposure and from cauda epididymis 42 and 70 days after exposure. This design enabled targeted investigation of effects on postspermatogonia, dividing spermatogonia and spermatogonial stem cells, respectively. BaP increased lacZ MF in cauda sperm after exposure of dividing spermatogonia (4.2-fold at highest dose, $\mathrm{p}<0.01)$ and spermatogonial stem cells $(2.1$-fold at highest dose, $\mathrm{p}<0.01$ ). No significant increases in MF were detected in cauda sperm or seminiferous tubule cells collected 3 days post-exposure. Dose-response modelling suggested that the mutational response in male germ cells to $\mathrm{BaP}$ is sub-linear at low doses. Our results demonstrate that oral exposure to $\mathrm{BaP}$ causes spermatogonial stem cell mutations, that different phases of spermatogenesis exhibit varying sensitivities to $\mathrm{BaP}$, with dividing spermatogonia representing a window of peak sensitivity, and that sampling spermatogenic cells from the seminiferous tubules at earlier time-points may underestimate germ cell mutagenicity. This information is critical to optimize the use of the international test guideline for TGR assays for detecting germ cell mutagens.

\subsection{Introduction}

Exposure to environmental agents can cause de novo mutations in the germline, leading to concerns over the genetic health of future generations (recently reviewed in (DeMarini, 2012)). Extensive evidence suggests that the majority of de novo mutations arise in the male germline (Conrad et al., 2011; Crow, 2000; Kong et al., 2012; Rahbari et al., 2015). Thus, there is a need to characterize the mutagenicity of environmental agents in male germ cells. The OECD Test Guideline for TGR assay (TG 488) is an internationally endorsed method (OECD, 2011) that can be used to quantify induced 
mutations in somatic tissues and male germ cells (Lambert et al., 2005; O'Brien et al., 2014; Yauk et al., 2015a). Of critical importance is the ability to detect mutations in spermatogonial stem cells because these cells can permanently and continuously produce sperm that carry mutations.

In the mouse, mutations fixed in spermatogonial stem cells can be detected in sperm collected from the cauda epididymis at least 49 days after exposure to a mutagen (O'Brien et al., 2014). This provides sufficient time for exposed stem cells to replicate and daughter cells to progress through spermatogenesis before they are collected as mature sperm. In practice, longer sampling times (i.e., 70 days) are preferable to account for pharmacokinetic distribution of the mutagen after an exposure, and to allow stem cells to go through several rounds of replication while being exposed. Although stem cells are critical for detecting permanent effects, differentiated spermatogonia may be more sensitive to mutagens, as they divide more rapidly than stem cells (de Rooij, 2001). Mutations fixed in dividing spermatogonia can be detected in cauda sperm 35-42 days after they are induced. Thus, adjusting the sampling time between mutagen exposure and sperm collection allows the detection of induced mutations in specific germ cell populations.

The sampling times required to detect spermatogonial stem cell mutations in sperm are longer than what is generally recommended for measuring somatic mutation using the TGR assay. TG488 recommends a 28-day exposure period followed by a 3-day sampling time $(28+3)$ for measuring mutations in somatic tissues (OECD, 2011). Cauda sperm collected using this sampling time are derived from cells that were in the later phases of spermatogenesis during the exposure period. As germ cells progress through 
spermatogenesis they progressively lose their DNA repair capacity, and no longer replicate DNA (Baarends et al., 2001; Gunes et al., 2015; Marchetti and Wyrobek, 2008; Olsen et al., 2005; Xu et al., 2005). Since errors in these processes are the primary mechanism of mutation fixation, post-meiotic germ cells are not sensitive to the induction of mutations that can be detected with the TGR assay, although they represent a sensitive window for the induction of lesions that may result in adverse effects after fertilization (Marchetti and Wyrobek, 2005). As a possible compromise, TG488 recommends collecting germ cells at the 3-day sampling time from the seminiferous tubules. However, cells from the seminiferous tubules represent a mixed population not adequately exposed as stem cells and are enriched for post-meiotic germ cells. Therefore, it is presently unknown whether the analysis of cells from seminiferous tubules provides the same sensitivity to detect germ cell mutagens as the analysis of sperm derived from exposed stem cells or dividing spermatogonia.

The mutagenic activity of $\mathrm{BaP}$, a ubiquitous environmental pollutant and Group 1 human carcinogen, has been extensively characterized for somatic tissues (IARC, 2012b; Moffat et al., 2015). BaP mutagenesis is less well-characterized in germ cells. BaP exposure via intraperitoneal (i.p.) injection induced dominant lethal mutations in meiotic and post-meiotic spermatogenic cells, but not heritable translocations (Generoso et al., 1982). Results for the SLT were negative or inconclusive for pre- and postspermatogonial germ cells after i.p. injection (Russell et al., 1981). Recent studies using TGR models have reported increases in the mutant frequencies (MF) of lacZ(Verhofstad et al., 2011) and $c I I$ reporter genes (Olsen et al., 2010) in mouse sperm collected 42 and 119 days after oral exposure to $\mathrm{BaP}$, respectively. The latter increase was statistically significant 
compared with concurrent solvent-treated controls, but not significantly distinguishable from the MF in naïve animals. Thus, although $\mathrm{BaP}$ does induce mutations in male germ cells, the susceptibility of adult spermatogonial stem cells to $\mathrm{BaP}$-induced mutation remains unclear.

Here, we characterize the dose response in germ cells of male transgenic mice (MutaMouse) exposed subchronically to $\mathrm{BaP}$ across various phases of spermatogenesis. We also compared the response in germ cells collected from the seminiferous tubules using a $28+3$ experimental design with the response in cauda sperm collected at later sampling times to scrutinize the sensitivity of this design for detecting germ cell mutagenicity. Our results clarify the phase-specific sensitivity of male germ cells to the mutagenicity of $\mathrm{BaP}$ after subchronic oral exposure and provide important information on the ability to detect germ cell mutation at different sampling times.

\subsection{Mate rials and Methods}

\subsubsection{Exposures and Tissue Collection}

All protocols involving animal use were approved by the Health Canada Ottawa Animal Care Committee. Experiments were designed based on the OECD guideline TG488 (OECD, 2011) and conducted as described previously (O'Brien et al., 2014). We used MutaMouse transgenic mice, which are derived from a DBA2 and BALB/c cross (originally obtained from Covance Laboratories, ltd., and maintained at in-house colony of for $>25$ years), and harbour $\sim 29$ tandem copies of a recombinant $\lambda$ gt10 phage vector on each copy of chromosome 3 (Shwed et al., 2010). The recombinant phage vector contains a mutation-reporting E. coli lacZ gene. Male MutaMouse mice (8-10 weeks old, 
$\mathrm{n}=5-6$ per treated group, $8-12$ for controls) were orally exposed to an olive oil vehicle control or $12.5,25,50$, or $100 \mathrm{mg} / \mathrm{kg}$ bw BaP (Sigma-Aldrich, Oakville, ON, Canada) dissolved in olive oil for 28 consecutive days via gavage (volume $=5 \mu / \mathrm{g} \mathrm{bw}$ ). Mice were euthanized by cervical dislocation under isofluorane anaesthesia 3,42 , or 70 days after the end of the exposure to assess effects on post-spermatogonia, dividing spermatogonia, and spermatogonial stem cells, respectively. Control groups comprised about 10 animals to provide a robust spontaneous MF especially for the seminiferous tubules, which had not been previously examined in our lab.

Cauda epididymides were collected for all 3 time-points, flash frozen in liquid nitrogen, and stored at $-80^{\circ} \mathrm{C}$. Sperm were later collected from the cauda as described previously (O'Brien et al., 2014). Briefly, cauda were defrosted, minced, and suspended in cold phosphate buffered saline. The suspension was filtered through a size 80 mesh filter (\#S3770, Sigma-Aldrich). Non-germ cells in the filtrate were lysed with sodium dodecyl sulfate and the unlysed sperm were collected by centrifugation. During method development, analysis by microscopy did not reveal any contaminating somatic cells following this lysis step (Yauk et al, 2002). Seminiferous tubules cells were extracted from freshly excised testes only for the 3-day post-exposure time-point as described previously (O'Brien et al., 2014). Briefly, the epithelial capsule was removed from the testes to release the seminiferous tubules. Germ cells were extracted from the seminiferous tubules by squeezing the tubules in phosphate buffered saline using a tissue roller. Tubules were removed from the suspension by gravity sedimentation, and the remaining cells in suspension were collected by centrifugation, flash frozen in liquid nitrogen, and stored at $-80^{\circ} \mathrm{C}$. Table 5.1 shows the type of germ cells targeted and the 
phases of spermatogenesis the target germ cells passed through during exposure for each time-point.

\subsection{2 lacZ Mutation Assay}

Genomic DNA was isolated from cauda sperm and seminiferous tubule cells as described previously (O'Brien et al., 2014). Germ cells were digested with proteinase K and $\beta$ - mercaptoethanol (cauda sperm only). Genomic DNA was extracted using phenol/chloroform. The extracted DNA was concentrated by ethanol precipitation and stored at $4{ }^{\circ} \mathrm{C}$ in Tris-EDTA buffer, $\mathrm{pH} 8$, then quantified using a NanoDrop spectrophotometer (Thermo Scientific).

The lacZ MF was determined in genomic DNA isolated from germ cells as previously described (O'Brien et al., 2014). Briefly, $\lambda$ gt10 phage vectors were recovered from $~ 1-4 \mu \mathrm{g}$ of DNA using Transpack Packaging Extract kits (Agilent Technologies, Mississauga, ON, Canada) according to the manufacturer's instructions. The lac $Z^{-}$ $\operatorname{lgalE}{ }^{-} E$. coli were infected with the recovered phage and grown on agar containing $0.3 \% \mathrm{P}-\mathrm{Gal}$ to detect mutants, or on agar without $\mathrm{P}-\mathrm{Gal}$ to determine the total number of PFU. A minimum of 125,000 total PFU were scored for each animal (average $=390,000$ PFU). When necessary, plaque counts from multiple assays per animal, including some instances of reactions with zero mutant counts, were combined to achieve this minimal total PFU only if replicates did not fail a binomial LRT $(\mathrm{P}<0.05)$.

The MF was calculated by dividing the number of mutant plaques by the total PFU count. The proportion of $\mathrm{BaP}$ induced mutants to total PFU was compared to the proportion in the control group by logistic regression using the glm function in $\mathrm{R}$ ( $\mathrm{R}$ Core 
Team, 2013) with a quasibinomial error distribution to account for overdispersion in the data. The resulting p-values were adjusted for multiple comparisons using a Bonferroni correction. Outlier samples ( 4 out of 137 samples: the cauda and tubules of 1 animal from the $28+325 \mathrm{mg} /$ day had unusually high $\mathrm{MF}$, as did 1 sample from the 42-day 25 $\mathrm{mg} / \mathrm{kg} /$ day group and 1 sample from the 70-day control group) were removed from the analysis if their MF were significantly different from the other samples in the same dose group based on the above logistic regression. The highest dose of $\mathrm{BaP}$ that did not induce a statistically significant $(\mathrm{P}<0.05)$ change in $\mathrm{MF}$ over controls was identified as the no observable genotoxic effect level (NOGEL).

\subsubsection{Dose-Response Modelling}

Characterization of the dose response was conducted following established approaches (Gollapudi et al., 2013; Johnson et al., 2014; O'Brien et al., 2015). This analysis was only performed for time-points that displayed a significant increasing trend in MF with dose, based on the Cochrane Armitage test for trend. These data sets met the minimum recommended number of dose groups for this type of analysis, which is 5 groups including controls (Johnson et al., 2014). Dose response modelling was performed using the U.S. Environmental Protection Agency's (EPA) Benchmark Dose Software (BMDS, v2.6.0.1). The BMDS variance LRT was used to select between a homogeneous or non-constant variance model. A dose-response model was then selected from the linear, quadratic, exponential, and power models based on the lowest Akaike Information Criterion (AIC). An additional goodness-of-fit LRT was used to assess the model fit. The benchmark response (BMR) was set to $10 \%$ of the control group above the estimated 
response of the model at dose $=$ zero in order to determine the benchmark dose (BMD10) and its lower 95\% confidence limit (BMDL10).

The “drsmooth" R package (Johnson et al., 2014) was used to assess additional characteristics of the dose-response relationship. We used the "linearity before cut-off dose" (lbcd) function to test whether the slope of the dose response before the identified NOGEL was significantly greater than zero $(\mathrm{P}<0.05)$. The "segmented" function was used to identify the dose at which the slope of a fitted bilinear model becomes greater than zero (the breakpoint dose, BPD) and its lower 95\% confidence limit (BPDL). The "smooth" function was used to identify the point at which the slope of a fitted smoothing regression spline first became significantly greater than zero (the slope transition dose, STD) and its lower 95\% confidence limit (STDL).

\subsection{Results}

\subsubsection{BaP Exposures and lacZ Mutant Frequencies in Germ Cells}

MutaMouse males were treated by oral gavage with an olive oil vehicle control or with $\mathrm{BaP}$ (up to $100 \mathrm{mg} / \mathrm{kg}$ bw/day) for 28 consecutive days. Germ cells were collected from the cauda epididymis 3,42 or 70 days after treatment, or from the seminiferous tubules 3 days after treatment. BaP treatment did not have a significant effect on testicular mass relative to body weight (data not shown). The lacZ MFs in BaP-exposed germ cells collected at various times are summarized in Table 5.2 and shown graphically in Figure 5.1. Plaque counts for each individual animal are shown in Table C6. The lacZ MF in cauda sperm collected 3 days after exposure was not significantly different from control levels for any dose group. The MFs in germ cells from the seminiferous tubules 
of testes collected at this time-point reached a maximum average of $6.4 \times 10^{-5}(2.7$-fold above concurrent controls) in the $100 \mathrm{mg} / \mathrm{kg}$ bw/day dose group. However, this increase in MF was not statistically significant $(\mathrm{P}=0.35)$ compared to the control group due to the high variation in the treated group. Sperm collected from cauda 42 days post-exposure had a significant dose-dependent increase in MF that reached $13.1 \times 10^{-5}$ (4.2-fold above controls) at $100 \mathrm{mg} / \mathrm{kg}$ bw/day. The other doses did not cause significant increases in MF at this time-point. Sperm collected from the cauda 70 days after exposure showed significant increases in both the 50 and $100 \mathrm{mg} / \mathrm{kg}$ bw/day dose groups, achieving a maximum induced MF of $5.3 \times 10^{-5}$ (2.1-fold above controls) in the high dose group. At $100 \mathrm{mg} / \mathrm{kg}$ bw/day, the MF at 42 days was significantly higher $(\mathrm{P}<0.01)$ than all other time-points, including 70 days. Thus, $\mathrm{BaP}$ induced significant increases in $\mathrm{MF}$ in sperm exposed as dividing spermatogononia and spermatogonial stem cells, whereas no statistically significant increase was observed in cauda sperm cells or cells collected from seminiferous tubules 3 days post-exposure.

\subsubsection{Dose-Response Modelling}

Highly significant trends for increasing MF with dose were observed for all timepoints (Cochrane Armitage test for trend, $\mathrm{p}<0.001$ ), except for the $28+3$ cauda sperm (P $=0.922$ ). As such, the $28+3$ cauda sperm data were omitted from further dose-response analysis. Dose-response data for all other data sets were modelled using U.S. EPA BMDS software. Data were fit to 4 different dose-response models (linear, quadratic, exponential, or power models) each with 2 different variance models (constant or nonconstant variance) for a total of 8 possible models. The best fitting model was selected for 
each time-point based on the lowest AIC score. The models selected for each time-point are shown graphically in Figure 5.1. Goodness-of-fit LRT p-values are shown in Table C7. Only the data from the 42 day time-point had poor fit $(\mathrm{P}<0.05)$ for either variance model. This was not improved after applying a log or square-root transformation, which are often recommended (Gollapudi et al., 2013) (data not shown). Since none of the variance models in BMDS had ideal fits $(\mathrm{P}>0.1)$ for this data set, we used the constant variance model because it produced the lowest AIC value and best model fit $p$-value. The selected dose-response models showed acceptable fit $(\mathrm{P}>0.1)$ for all data sets. BMDs based on a $10 \%$ response were derived using the best fit models and are shown graphically in Figure 5.1, and with their 95\% lower confidence limits in Table C7. We also tested whether the slope of the dose response was significantly different from zero up to and including the NOGEL. Although no single dose group has a response that was significantly greater than controls for the tubules at $28+3$, a statistically significant trend was observed, that was best described by the exponential model (and therefore not linear up to the NOGEL of $100 \mathrm{mg} / \mathrm{kg}$ bw/day), with a BMD10 of $10.4 \mathrm{mg} / \mathrm{kg}$ bw/day. Sperm from the 42-day time-point were best described by a polynomial dose response, with a BMD10 of $16.7 \mathrm{mg} / \mathrm{kg}$ bw/day. The slope of this dose response was not significantly different than zero up to the NOGEL of $50 \mathrm{mg} / \mathrm{kg}$ bw/day, although only marginally so (P $=0.053$ ). The lac $Z M F$ in the 70-day group was best described by an exponential dose response. The highest BMD10 (43.0 mg/kg bw/day) was found for this post-exposure time-point. The slope of this dose response was also not significantly different than zero up to the NOGEL of $25 \mathrm{mg} / \mathrm{kg}$ bw/day $(\mathrm{P}=0.752)$. A significant $\mathrm{BPD}$ and $\mathrm{STD}$ was only detected for the day 42 sperm data (shown graphically in Figure 5.2, and with their 95\% 
lower confidence limits in Table C7). These values were $34.1 \mathrm{mg} / \mathrm{kg}$ bw/day and 22.1 $\mathrm{mg} / \mathrm{kg}$ bw/day, respectively. The slopes of lacZ MF response in all 3 time-points at which dose-dependent increases were observed (28+3 tubules, 42-day and 70 day sperm) were not significantly different than zero up to and including $25 \mathrm{mg} / \mathrm{kg}$ bw/day $(\mathrm{P}>0.05)$. In summary, sperm derived from exposed dividing spermatogonia (42 days post-exposure) were more sensitive to $\mathrm{BaP}$-induced mutation following subchronic oral exposure compared to sperm from exposed spermatogonial stem cells (based on BMD analysis). Despite having no single dose with a significantly elevated MF (based on logistic regression), a significant trend was observed (based on the Cochrane Armitage test) in germ cells from the $28+3$ seminiferous tubules that was comparable to the trends observed in cauda sperm. Collectively, the dose-response analyses from all 3 of these data sets suggest that the mutational response in male germ cells to $\mathrm{BaP}$ is sub-linear in the low dose range.

\subsection{Discussion}

We used the lacZ mutation assay to characterize the dose response of various phases of spermatogenesis in transgenic mice exposed subchronically to BaP. Our results demonstrate that $\mathrm{BaP}$ induces mutations in spermatogonial stem cells of adult mice, but that the strongest mutational effect is observed in dividing spermatogonia. Comparison of the response in germ cells collected from the seminiferous tubules 3 days after a 28 day exposure (MF ranging from 2.4 to $6.4 \times 10^{-5}$ ) with that in cauda sperm collected 70 days after exposure (ranging from 2.5 to $5.3 \times 10^{-5}$ ) shows that, although comparable MFs 
were obtained in the 2 systems, statistically significant effects were observed only in cauda sperm.

Our results strengthen the existing body of evidence that $\mathrm{BaP}$ is a spermatogonial stem cell mutagen in mice. We observed a dose-dependent increase in lacZ MF that was statistically significant at the 2 top dose groups $(50 \mathrm{mg} / \mathrm{kg}$ bw/day and $100 \mathrm{mg} / \mathrm{kg}$ bw/day) in sperm collected 70 days after the end of the 28-day exposure. One study previously reported an increased MF in sperm of mice collected 119 days after 3 daily intraperitoneal (i.p.) exposures of $50 \mathrm{mg} / \mathrm{kg}$ bw BaP (150 mg/kg bw total) (Olsen et al., 2010). The stem cell effect was statistically significant when compared with concurrent solvent-treated controls, but not when compared to naïve animals. More definitive stem cell effects were observed in pre-pubescent mice exposed to BaP (Xu et al., 2014); increased lacI MFs were observed in isolated pachytene spermatocytes, round spermatids, and spermatozoa collected 35 days after juvenile mice ( 7 days old) were exposed to a single i.p. injection of up to $300 \mathrm{mg} / \mathrm{kg} / \mathrm{bw} \mathrm{BaP}$. This effect was less apparent in older mice (25 and 60 days old) using a similar experimental design, where significant effects were only detected in pachytene spermatocytes at the highest dose. These findings and the results from the present study collectively demonstrate that $\mathrm{BaP}$ is a spermatogonial stem cell mutagen in mice and that exposure to this chemical can permanently increase the production of mutated sperm throughout the reproductive life of the animal.

Dividing spermatogonia represent the most sensitive spermatogenic population to $\mathrm{BaP}$-induced mutations following oral exposure. In fact, the MF for the highest BaP dose was significantly higher than that observed after stem cell exposure $(\mathrm{P}<0.01)$. An 
elevated MF was also detected at $50 \mathrm{mg} / \mathrm{kg}$ bw/day, but this was only marginally significant $(\mathrm{P}=0.074)$, likely due to the unusually high variability in the control group at this time-point (Table 5.2). The strong MF response observed at 42 days post-exposure correlates with the higher rate of division in dividing spermatogonia relative to spermatogonial stem cells (de Rooij, 2001). In addition, it has been shown that there is approximately a week delay in the formation of $\mathrm{BaP}$-induced $\mathrm{DNA}$ adducts in sperm after an oral exposure (Verhofstad et al., 2010). Therefore, BaP may continue to be delivered to the testes several days after the final oral dose and additional mutations may have been produced during the last week of active spermatogonial divisions before the cells commit to meiosis. Dividing spermatogonia represent a sensitive window for induction for other types of genomic alterations such as tandem repeat mutations (Beal et al., 2015b; Somers, 2006). Therefore, dividing spermatogonia may be the most useful spermatogenic population to determine whether a chemical is a germ cell mutagen.

Important data gaps exist on whether the analysis of cells from seminiferous tubules at the time-point commonly used for detecting mutations in somatic tissues (i.e., $28+3$ ) provides the same sensitivity to detect germ cell mutagens as the analysis of sperm derived from exposed spermatogonia (Yauk et al., 2015a). As expected, we observed no change in the lacZMF in cauda sperm collected at this time-point (Figure 5.1), which represent germ cells that were spermatocytes or spermatids during the exposure period. These cell types do not synthesize DNA and lose their DNA repair capacity, processes that are required to fix mutations (Marchetti and Wyrobek, 2008). They are also on the lumen ("closed") side of the blood-testes barrier, which can differentially affect the distribution of toxicants to the various germ cell phases (Yauk et al., 2015a). Conversely, 
the analysis of germ cells from seminiferous tubules at the $28+3$ time-point targets a mixture of cells with varying capacity for DNA synthesis and repair during exposure (Marchetti and Wyrobek, 2008; Olsen et al., 2005; Xu et al., 2005). The MF obtained from seminiferous tubule cells were comparable with those observed in sperm collected 70 days after the end of exposure (Table 5.2) and showed an apparent dose response (Figure 5.1). However, no statistically significant increase above controls was found. Similarly, Verhofstad et al. (2011) detected a significant increase in plasmid-born lacZ MF in sperm collected 42 days after BaP exposure, but not in the testes at this same timepoint. The apparent signal suppression in the testes is likely due to the mixed nature of the exposed and sampled cell population, which contains germ cells from various phases of spermatogenesis that are enriched for post-meiotic germ cells, and a small amount of somatic (Leydig and Sertoli) cells. These non-target cells may dilute the mutational effect, thereby decreasing assay sensitivity. Therefore, simultaneous ly measuring induced mutations in somatic tissues and germ cells at $28+3$ may not represent an optimal approach for detecting germ cell mutagens, especially weak mutagens. A time-point that would allow more cells exposed as dividing spermatogonia to be collected (e.g., $28+28$ ) may provide a stronger signal in the tubules.

MF data from the TGR mutation assay often suffer from overdispersion (i.e., greater variability than predicted by the theoretically binomial error distribution), which can negatively impact assay sensitivity. One of the primary causes of this increased variability is that the clonal expansion of mutations in some samples is greater than in others. This is of particular concern for control samples, where recovered mutations may have occurred before the experimental time-frame. Overdispersion can be accounted for, 
to a limited degree, during statistical analysis (for example, by assuming a quasi-binomial error distribution). Alternatively, assay sensitivity can be greatly improved by correcting the MF for clonality, which can only be done by sequencing the recovered mutants to quantify the degree of clonal expansion in each sample. Previously, such an endeavour would be rather costly and time-consuming, especially for the large lacZ gene. However, technological advances have made sequencing much more amendable to regulatory testing, and may soon make routine clonal correction of TGR MF data more practical (Beal et al., 2015a; Besaratinia et al., 2012).

Dose-response modelling showed that the BMD10 was lower in dividing spermatogonia than spermatogonial stem cells. Our result supports the previous observation that actively dividing germ cells are more susceptible to BaP-induced mutations than spermatogonial stem cells. These BMDs are higher $(10.4-43.0 \mathrm{mg} / \mathrm{kg}$ bw/day in germ cells, present study) than what has been reported for mutation in somatic tissues $(0.5-7.2 \mathrm{mg} / \mathrm{kg}$ bw/day, (Moffat et al., 2015)). The BMD10 of germ cells collected from the tubules was very similar to the BMD10 of dividing spermatogonia. However, caution should be used when interpreting the BMD10 derived from tubule cells, considering the mixed nature of the target cell population and the fact that significant effects were not detected at any dose tested. Differences in the pharmacokinetic distribution of $\mathrm{BaP}$ and its metabolites in the testes and somatic tissues may contribute to the variation in response. BPDE-DNA adducts are detected in somatic tissues and testes within days of exposure (Lemieux et al., 2011; Olsen et al., 2010; Verhofstad et al., 2010). However, no studies have compared adduct levels between these tissues after the typical OECD-recommended 28-day exposure regimen. Such 
information would help clarify how much of the $\mathrm{BaP}$ metabolites reach the testes relative to other tissues, enabling comparison of mutagenic efficiency in these cell types. It is thought that germ cells in general may have superior resistance to spontaneous and environmentally induced mutation (Murphey et al., 2013), which may also have contributed to the reduced levels of $\mathrm{BaP}$-induced mutation in sperm.

The findings from dose-response modelling suggest a sub-linear dose response for the induction of mutations by $\mathrm{BaP}$ after spermatogonial stem cell exposure. In fact, slope analysis in the low dose region of the curves indicated that the mutagenic effect of $\mathrm{BaP}$ did not have a slope significantly greater than zero at doses below $25 \mathrm{mg} / \mathrm{kg}$ bw/day for all cell types targeted. A significant BPD and STD were also identified at the 42-day time-point, further supporting a sublinear response. There is increasing evidence for sublinear dose responses for DNA reactive mutagens, such as ethyl methanesulfonate, and ENU in both somatic tissues and germ cells (Bryce et al., 2010; Doak et al., 2007; Gocke and Müller, 2009; Lynch et al., 2011; O'Brien et al., 2015; Pottenger et al., 2009). This is indicative of a saturable repair response, where at lower doses the DNA damage is repaired, but repair processes are overwhelmed as doses increase (Julien et al., 2009; Yauk et al., 2015b). Since BaP induces mutations primarily through DNA adductforming metabolites, it is possible that the sub-linear dose response is attributable to a saturable repair mechanism for BaP adducts. Data with knockout mice suggest that NER is essential to BaP-induced mutation (Verhofstad et al., 2011) and that NER activity varies among spermatogenic cell types (Jansen et al., 2001; Xu et al., 2005).

In conclusion, we demonstrated that oral exposure to BaP causes spermatogonial stem cell mutations in mice and that dividing spermatogonia represent a window of peak 
sensitivity for the induction of mutations. Evaluation of MFs in cells from the cauda and seminiferous tubules at the time-point recommended in the OECD test guideline for measuring mutations in somatic tissues $(28+3)$ may not be sufficiently sensitive to detect germ cell mutagens, suggesting that data derived from this design be interpreted with caution. We advise that a later time-point after the exposure (e.g., 28 days for tubules, 42 days for cauda sperm) provides a more sensitive measure of germ cell mutagenic response for chemical assessment. 


\subsection{Tables and Figures}

Table 5.1 Cell types and phases of spermatogenesis that are targeted by various experimental designs.

\begin{tabular}{|c|c|c|c|c|c|}
\hline $\begin{array}{l}\text { Sampling } \\
\text { time } \\
\text { (days) }\end{array}$ & Tissue & $\begin{array}{l}\text { Phase at } \\
\text { beginning of } \\
\text { exposure }\end{array}$ & $\begin{array}{c}\text { Phases passed } \\
\text { during } \\
\text { exposure }\end{array}$ & $\begin{array}{c}\text { Phases passed } \\
\text { after } \\
\text { exposure }\end{array}$ & $\begin{array}{c}\text { Collected cell } \\
\text { types }\end{array}$ \\
\hline \multirow[t]{2}{*}{3} & cauda & spermatocyte & $\begin{array}{l}\text { spermatocyte } \\
\text { spermatid } \\
\text { sperm }\end{array}$ & sperm & sperm \\
\hline & tubules & $\begin{array}{c}\text { stem cell } \\
\text { spermatogonia } \\
\text { spermatocyte }\end{array}$ & $\begin{array}{c}\text { stem cell } \\
\text { spermatogonia } \\
\text { spermatocyte } \\
\text { spermatid }\end{array}$ & $\begin{array}{c}\text { stem cell } \\
\text { spermatogonia } \\
\text { spermatocyte } \\
\text { spermatid }\end{array}$ & $\begin{array}{c}+ \text { spermatogonia } \\
++ \text { spermatocyte } \\
+++ \text { spermatids } \\
+++ \text { sperm }\end{array}$ \\
\hline 42 & cauda & stem cell & $\begin{array}{c}\text { stem cell } \\
\text { spermatogonia }\end{array}$ & $\begin{array}{l}\text { spermatogonia } \\
\text { spermatocyte } \\
\text { spermatid } \\
\text { sperm }\end{array}$ & sperm \\
\hline 70 & cauda & stem cell & stem cell & $\begin{array}{l}\text { stem cell } \\
\text { spermatogonia } \\
\text { spermatocyte } \\
\text { spermatid } \\
\text { sperm }\end{array}$ & sperm \\
\hline
\end{tabular}

+ indicates the relative proportion of cell types when a mixture is collected 
Table 5.2 lacZ mutant frequency in male germ cells of Mutamouse mice exposed to benzo[a]pyrene for 28 days and collected at various sampling times.

\begin{tabular}{|c|c|c|c|c|c|c|c|c|}
\hline $\begin{array}{c}\text { Sampling } \\
\text { time }\end{array}$ & Tissue & $\begin{array}{c}\text { Dose } \\
\text { (mg/kg/day) }\end{array}$ & $\mathbf{n}$ & $\begin{array}{c}\# \\
\text { mutants }\end{array}$ & \# PFU & $\begin{array}{l}\text { Average } \\
\mathrm{MF} \times 10^{-5}\end{array}$ & $\mathbf{S D}^{\mathbf{a}}$ & p-value \\
\hline \multirow[t]{5}{*}{3} & cauda & 0 & 12 & 138 & 4446661 & 3.1 & 1.7 & - \\
\hline & & 12.5 & 5 & 14 & 1066397 & 1.4 & 0.9 & 0.0987 \\
\hline & & 25 & 4 & 24 & 913653 & 2.6 & 0.9 & 1.0000 \\
\hline & & 50 & 5 & 33 & 1101683 & 3.1 & 1.3 & 1.0000 \\
\hline & & 100 & 5 & 87 & 3669186 & 2.5 & 0.7 & 0.5539 \\
\hline \multirow[t]{5}{*}{3} & tubules & 0 & 8 & 51 & 2194254 & 2.4 & 1.1 & - \\
\hline & & 12.5 & 5 & 52 & 1452400 & 3.4 & 1.7 & 1.0000 \\
\hline & & 25 & 5 & 38 & 1449087 & 2.6 & 1.2 & 1.0000 \\
\hline & & 50 & 6 & 85 & 2173052 & 3.9 & 1.5 & 0.6645 \\
\hline & & 100 & 6 & 102 & 2341201 & 6.4 & 4.0 & 0.3501 \\
\hline \multirow[t]{5}{*}{42} & cauda & 0 & 11 & 102 & 3716402 & 3.1 & 2.5 & - \\
\hline & & 12.5 & 6 & 67 & 2007879 & 3.3 & 0.8 & 1.0000 \\
\hline & & 25 & 5 & 30 & 1239188 & 2.4 & 0.6 & 1.0000 \\
\hline & & 50 & 6 & 104 & 1937802 & 5.4 & 2.5 & 0.0738 \\
\hline & & 100 & 5 & 447 & 3523564 & 13.1 & 3.4 & $<0.0001$ \\
\hline \multirow[t]{5}{*}{70} & cauda & 0 & 9 & 130 & 5118604 & 2.5 & 0.7 & - \\
\hline & & 12.5 & 6 & 75 & 3095980 & 2.7 & 1.1 & 1.0000 \\
\hline & & 25 & 5 & 65 & 2261681 & 2.3 & 1.2 & 1.0000 \\
\hline & & 50 & 5 & 86 & 1842379 & 4.7 & 1.2 & 0.0045 \\
\hline & & 100 & 5 & 186 & 3598943 & 5.3 & 1.2 & 0.0001 \\
\hline
\end{tabular}

${ }^{\mathrm{a}}$ standard deviation (SD) based on the arithmetic mean of individual animals PFU: plaque forming units

Bold p-values indicate statistical significance $(\mathrm{P}<0.05)$ 

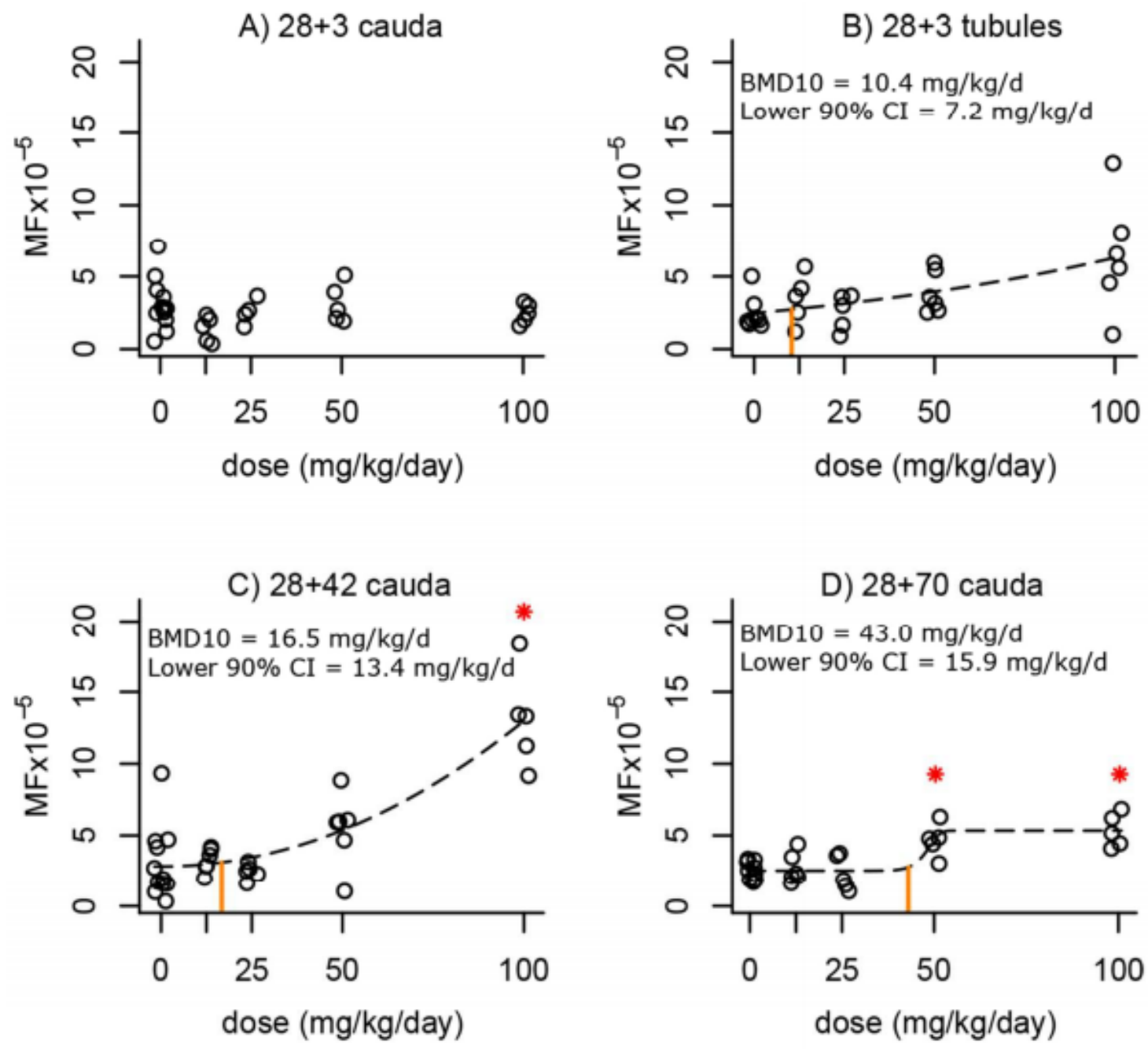

-- - Dose-response model $\mathrm{BMD}_{10} \quad * \mathrm{p}<0.05$

Figure 5.1 lacZ mutant frequency dose response in spermatogenic cells exposed to benzo[a]pyrene: A) sperm collected from the cauda epididymis 3 days after a 28 day exposure; B) germ cells collected from the seminiferous tubules 3 days after exposure; C) sperm collected from the cauda epididymis 42 days after exposure; and D) sperm collected from the cauda epididymis 70 days after exposure. 

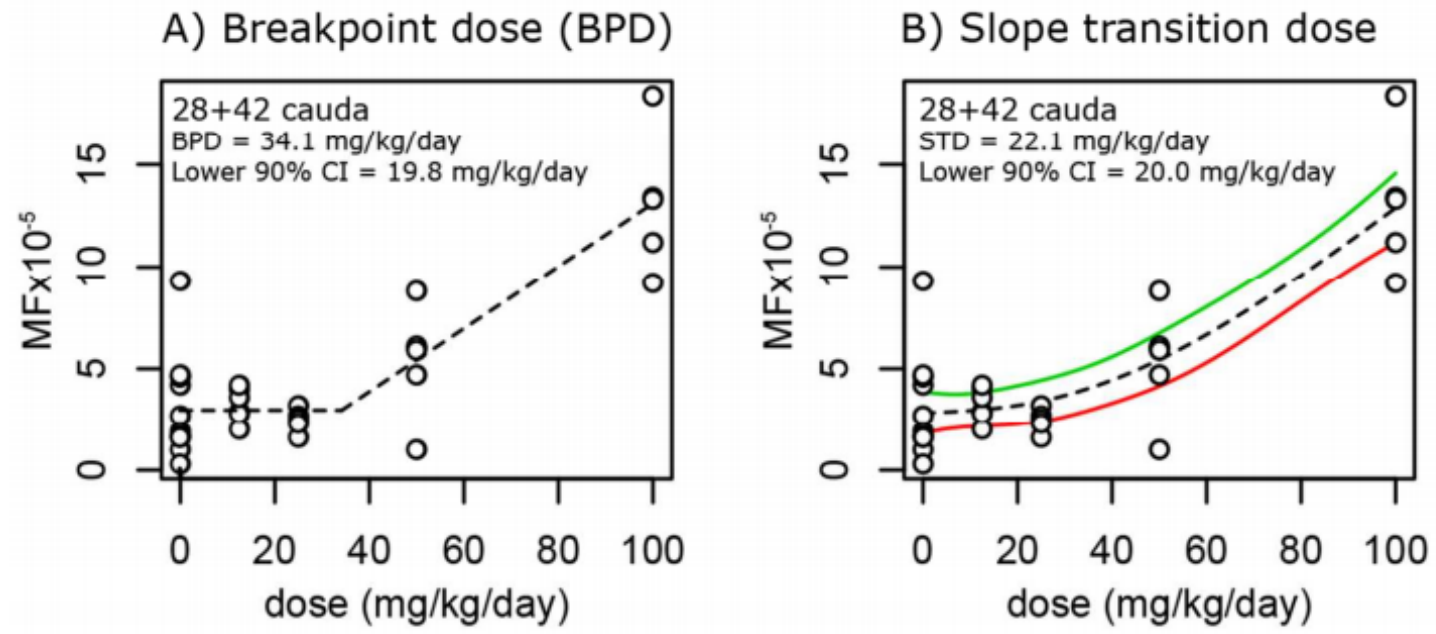

- - dose-response model

upper $95 \%$ confidence bound

lower $95 \%$ confidence bound

Figure 5.2 lacZ mutant frequency dose-response models used to determine: A) the breakpoint dose; and B) the slope transition dose for sperm collected 42 days after exposure to benzo[a]pyrene. 


\section{Chapter 6: Next Generation Sequencing of Benzo(a)pyrene-Induced lacZ Mutants Identifies a Germ Cell-Specific Mutation Spectrum}

Modifie d from: O’Brien, J.M., Beal, M.A., Yauk, C.L., Marchetti, F. 2016. Scientific Reports, 6:36743.

\subsection{Preamble}

The method developed in Chapter 4 is used here to characterize the mutation spectrum of $\mathrm{BaP}$ in sperm. This is important for determining how mutations induced in sperm compare to those inherited by progeny as detected by whole genome sequencing. Another important question addressed in this chapter is whether chemical exposures affect germ cells differently than somatic cells. Differences in mutation spectra between somatic and germ cells would suggest that different mutational mechanism may be operating in these cell types. This is important for clarifying whether data derived from somatic cell testing is predictive of mutagenic effects in germ cells.

\subsection{Abstract}

De novo mutations are implicated in a variety of genetic diseases and arise primarily in the male germline. We investigated whether male germ cells have unique mechanisms for spontaneous or chemically induced mutation relative to somatic cells using the MutaMouse model. We sequenced lac $Z$ transgenes recovered from sperm 42 days after a 28 -day exposure to $\mathrm{BaP}(100 \mathrm{mg} / \mathrm{kg} / \mathrm{day})$ to assess mutations arising in dividing spermatogonia. BaP caused a 3.4-fold increase in lacZ MF over controls which 
increased to 4.1-fold after correcting for clonality. We then used next generation sequencing to compare the spontaneous and $\mathrm{BaP}$-induced mutation spectra in sperm and bone marrow. The spontaneous spectrum in sperm had significantly more G:C to A:T transitions and fewer mutations at A:T basepairs than bone marrow. BaP predominantly induced G:C to T:A transversions in both cell types, and both were enriched for mutations at $\mathrm{CpG}$ dinucleotides. However, $\mathrm{BaP}$ induced significantly more deletions in sperm, but more $\mathrm{G}: \mathrm{C}$ to $\mathrm{A}: \mathrm{T}$ transitions and $\mathrm{G}: \mathrm{C}$ to $\mathrm{C}: \mathrm{G}$ transversions in bone marrow. Differences in error-prone translesion DNA synthesis polymerases may underlie the observed spectrum differences between sperm and bone marrow. These findings suggest that mutations in sperm can arise via mechanisms that are unique to male germ cells.

\subsection{Introduction}

Over long periods of time, germline mutation is a primary driving force of evolution. However, short term increases in mutation rates can lead to unwanted increases in the incidence of genetic disease and cancer. Recent studies using next generation sequencing confirm that the male germline is the primary source of de novo mutations (i.e., genetic alterations in an offspring that are not present in the parents) (Conrad et al., 2011; Crow, 2000; Kong et al., 2012; Rahbari et al., 2015). These efforts have focused primarily on the rate of male-mediated inherited mutations, but few have explored what environmental factors are responsible for differences among males. Thus, environmental variables that contribute to de novo mutations remain poorly characterized. 
Elevated mutagenicity in the male germline is believed to be due to the continuous replication of spermatogonial stem cells (Crow, 2000). Indeed, the number of mutations inherited by offspring is proportional to paternal age at the time of conception, supporting the hypothesis that the male germline mutation rate is directly correlated with the number of spermatogonial cell divisions (Kong et al., 2012; Rahbari et al., 2015). In addition, there are several mechanistic factors, some of which are unique to males, that may contribute to germline specific mutation (Gregoire et al., 2013): a) male germ cells progressively lose DNA repair capacity as they advance through spermatogenesis (Marchetti and Wyrobek, 2008; Olsen et al., 2005); b) the chromatin remodeling required for the replacement of histone proteins with protamines during spermiogenesis (Rathke et al., 2014) may promote additional opportunities for misrepair of endogenous strand breaks (Gouraud et al., 2013); c) the process of meiosis provides opportunities to affect nucleotide composition that are unique to germ cells; and, d) post-meiotic haploid germ cells cannot rely on sister chromatids as templates for homology-based repair mechanisms (Baarends et al, 2001). Thus, there are multiple mechanisms unique to male germ cells by which mutations can arise. In addition to influencing mutation rate, these factors may also contribute to the generation of different types of mutation (ie, mutation spectrum) in male germ cells relative to other cell types. It stands to reason, then, that mutagenic chemicals may induce mutations via germ cell-specific mechanisms, resulting in a unique spectrum of mutations relative to other cell types. However, this has never been clearly demonstrated.

The TGR assay is an internationally supported method for testing the in vivo mutagenicity of chemicals (OECD, 2011). The assay uses rodents that harbour mutation- 
reporting transgenes in their genomes. The transgenes are easily recovered, from virtually any tissue, and screened for induced mutations. TGR models offer a practical, fast and efficient means to assess the mutagenicity of chemicals in germ cells and compare the responses with somatic tissues (Douglas et al., 1995; O'Brien et al., 2014; Olsen et al., 2010; Verhofstad et al., 2011). One of the many benefits of the TGR mutation assay is that the recovered transgenes can be sequenced to provide additional data on the types of mutations induced, which can be used for developing and supporting mechanistic hypotheses. Previously, sequencing data were limited to very low copy numbers of the smaller $c I I$ transgene due to technical restrictions. However, when coupled with next generation sequencing (NGS) technologies, the TGR assay can be used to generate a high-resolution mutation spectrum of the larger lac $Z$ transgene, providing coverage of a much greater numbers of mutants (Beal et al., 2015a; Besaratinia et al., 2012). Thus, NGS-lac $Z$ analysis allows the detection of subtle differences in mutation spectra that may otherwise be missed when analyzing a smaller sample of mutants.

$\mathrm{BaP}$ is a ubiquitous environmental pollutant and Group 1 human carcinogen (IARC, 2012b) that causes both somatic and male germline mutations (Generoso et al, 1982; Olsen et al., 2010; Verhofstad et al., 2011; Xu et al., 2014). The mechanisms of $\mathrm{BaP}$ mutagenicity have been well characterized and reviewed for somatic tissues (IARC, 2012b; Moffat et al., 2015). The primary mechanism is the faulty replication of unrepaired DNA adducts formed by a BaP metabolite, BPDE. BPDE adducts occur predominantly at the $\mathrm{N}^{2}$ position of guanosine, resulting in an increased frequency of G:C to T:A transversions (Cheng et al., 1989; Eisenstadt et al., 1982; Lagerqvist et al., 2008). Several other DNA-reactive by-products of $\mathrm{BaP}$ metabolism, such as $\mathrm{BaP}$ radical cations 
and ROS, also cause varying degrees of other types of mutations. The BaP mutation spectrum is less well characterized in germ cells. While $\mathrm{BaP}$ can induce mutations during various phases of spermatogenesis, including stem cells, dividing spermatogonia appear to be the most sensitive (Generoso et al., 1982; O'Brien et al., 2016a; Olsen et al., 2010; Russell et al., 1981; Verhofstad et al., 2011; Xu et al., 2014). The characteristic G:C to $\mathrm{T}$ :A transversion also appears to be the primary $\mathrm{BaP}$-induced mutation detected in sperm (Olsen et al., 2010). However, a high resolution mutation spectrum has not yet been generated to assess the effect of $\mathrm{BaP}$, or any other chemical, on all mutation types in male germ cells.

We hypothesize that mutations can arise via mechanisms that are unique to male germ cells. A testable prediction of this hypothesis is that somatic cells and male germ cells will yield distinct spectra of mutation types both before and after exposure to a chemical mutagen. Here, we use the TGR mutation assay coupled with NGS to produce high-resolution spectra of mutation types in the germ cells of untreated and $\mathrm{BaP}$-exposed male mice. We then compare these to the spontaneous and $\mathrm{BaP}$-induced mutation spectra in bone marrow (Beal et al., 2015a) and speculate on the possible mechanisms responsible for any spectral discrepancies between these tissues. This study provides important evidence for elucidating whether or not there are germ cell-specific mechanisms for spontaneous and $\mathrm{BaP}$-induced mutation.

\subsection{Materials and Methods}

\subsubsection{Animal Exposures and Tissue Collection}


All protocols involving animal use were approved by the Health Canada Ottawa Animal Care Committee. Experiments were conducted following the recommendations in OECD guideline TG488 (OECD, 2011). Briefly, MutaMouse males (8-10 weeks old) were orally exposed to an olive oil vehicle control $(\mathrm{n}=6)$ or $100 \mathrm{mg} / \mathrm{kg}$ bw $\mathrm{BaP}(\mathrm{n}=5)$ dissolved in olive oil for 28 consecutive days via gavage. Mice were euthanized by cervical dislocation under isofluorane anaesthesia 42 days after the end of the exposure period. Cauda epididymides were collected, flash frozen in liquid nitrogen, and stored at $80{ }^{\circ} \mathrm{C}$. Sperm were later collected from the cauda as described previously (O'Brien et al., 2014).

\subsection{2 lacZ Mutation Assay}

Genomic DNA was isolated from spermatozoa from the cauda epididymis by digestion with proteinase $\mathrm{K}$ and $\beta$-mercaptoethanol, followed by phenol/chloroform extraction, and ethanol precipitation as described previously (O'Brien et al., 2014). The lacZMF was determined in genomic DNA as previously described (O'Brien et al., 2014). Briefly, viral transgene vectors were recovered from $\sim 1-4 \mu \mathrm{g}$ of DNA using Transpack Packaging Extract kits (Agilent Technologies, Mississauga, ON, Canada) according to the manufacturer's instructions. E. Coli (lacZ-/galE-) were infected with the recovered viral vectors and grown on agar containing $0.3 \% \mathrm{P}-\mathrm{Gal}$ to detect mutants, or on agar without P-Gal to determine the total number of PFU. A minimum of 125,000 total PFU were scored for each animal. MF was calculated as the number of mutant plaques divided by the total PFU count. Statistical differences in MF between treatment groups were 
determined by logistic regression using the glm function in R (R Core Team, 2013) with a quasibinomial error distribution to account for overdispersion of the data.

\subsubsection{Mutant Sequencing}

Mutant plaques recovered from spermatozoa from the cauda epididymis were collected for sequencing. Sequencing was performed using an Ion Proton ${ }^{\mathrm{TM}}$ sequencer (Life Technologies, Carlsbad, CA) as described previously (Beal et al., 2015a). Briefly, for each animal, mutant plaques were pooled into a microcentrifuge tube containing sterile water (average of 144 plaques per animal). Tubes were boiled to melt the agar and an aliquot of the suspension was used as template for a 30-cycle PCR amplification of the lacZ gene. To control for PCR errors, each sample was amplified and sequenced in duplicate. The PCR products were purified using QIAquick PCR purification kit (Qiagen). Libraries were prepared from each purified PCR sample using Ion Xpress ${ }^{\mathrm{TM}}$ Plus kits and an AB Library Builder ${ }^{\mathrm{TM}}$ (Ion Xpress Plus Library Protocol v. 1.00) following the manufacturer's directions with the following exceptions: we used a custom shearing time of 10 minutes and replaced the Agencourt ${ }^{\circledR}$ AMPure ${ }^{\circledR}$ XP Reagent with SPRIselect ${ }^{\circledR}$ Reagent (Beckman Coulter, Brea, CA). The ends of each library were ligated with P1 adapters and a unique barcode A adapter from the Xpress ${ }^{\mathrm{TM}}$ barcode adapter kit. Fragments between $\sim 175-225$ bp were selected from each library using an EGel® Safe Imager ${ }^{\mathrm{TM}}$, purified using the Invitrogen ${ }^{\mathrm{TM}}$ PureLink ${ }^{\circledR}$ Quick Gel Extraction Kit, and amplified using the Library Amplification Primer Mix (Ion Plus Fragment Library Adapters kit) and High Fidelity Platinum ${ }^{\circledR}$ PCR SuperMix. Barcoded libraries were pooled together to a final total concentration of $8 \mathrm{pM}$. Pooled libraries were 
amplified by em-PCR using Ion Sphere ${ }^{\mathrm{TM}}$ particles on an Ion OneTouch ${ }^{\mathrm{TM}} 2$ system. Particles containing PCR products were enriched using an Ion OneTouch ${ }^{\mathrm{TM}}$ ES. Enriched particles were loaded onto an Ion P1 $1^{\mathrm{TM}}$ chip (version 1) and sequenced.

Bioinformatic analysis of sequence data was conducted as described previously (Beal et al., 2015a). Briefly, raw sequence data were interpreted using a Proton ${ }^{\mathrm{TM}}$ Torrent Server (version 3.6.2). Reads were trimmed and aligned to the reference $l a c Z$ sequence (GenBank ID: J01636.1) using Bowtie2 (Langmead and Salzberg, 2012). Alignment pileup was performed using SAMtools (Li et al., 2009a). The proportion of reads containing base-substitutions or indels relative to the reference sequence was determined from the pileup, adjusting for the false mutation proportion (which estimates PCR and sequencing errors) (Beal et al., 2015a). Indels with a false mutation proportion higher than the highest false mutation proportion for base substitution calls were ignored to reduce false indel calls that result from homopolymer sequencing errors. The mutationcalling-threshold was conservatively set based on the number of plaques sequenced for each animal as follows: mutation-calling-threshold $=1$ /\#plaques sequenced for that animal (e.g., if 100 plaques were sequenced, the required threshold would be at least 1 read with the mutation $/ 100$ plaques sequenced $=0.01$ ). Deviations from the reference sequence were called as mutations (corrected for false mutation proportion) if they were above the mutation-calling-threshold in both technical replicates. Mutations that occurred more than once per animal were considered to originate by clonal expansion from a single independent event. The number of clones for each mutation per animal was determined by dividing the average corrected proportion of deviant reads between technical replicates by the mutation-calling-threshold. This value was then adjusted using 
a LOD/linear model to account for the imprecise quantification of clonality near the LOD, as described previously (Beal et al., 2015a).

Only the independent mutations were used to determine the mutation spectra for each treatment group. Significant differences in mutation spectra, when considering both nucleotide position and mutation type, were determined using a Monte Carlo generated $X^{2}$ distribution (Roff and Bentzen, 1989). Significant differences in mutation spectra when considering only mutation type were determined using a Fisher's exact test. To determine which specific types of mutations were significantly different between the control and treatment groups, a Fisher's exact test was performed on $2 \times 2$ sub-tables for each mutation type, followed by a Bonferroni correction for multiple comparisons. Each sub-table comprised a count of the mutation type of interest and a pooled count of all other mutation types in each treatment groups.

\subsection{Results}

\subsection{1 lacZ Mutant Fre quency}

The animals used in the present study are a subset from a previously reported study (O’Brien et al., 2016a). MutaMouse males were exposed to a vehicle control (olive oil, $\mathrm{n}=5)$ or $100 \mathrm{mg} / \mathrm{kg}$ bw/day $\mathrm{BaP}(\mathrm{n}=6)$ by oral gavage for 28 consecutive days. This dose is on the higher end of the dose-response curve, and is not necessarily representative of an environmentally relevant exposure. We selected this dose due to its high mutagenicity in germ cells (O'Brien et al., 2016a), which allowed us to produce a high resolution spectrum of $\mathrm{BaP}$-induced mutations. Spermatozoa from the cauda epididymis were collected 42-days after exposure to target cells that were dividing spermatogonia at 
the end of the exposure period (O'Brien et al., 2014). A positive selection assay was used to recover DNA harbouring mutated copies of the $l a c Z$ gene from the spermatozoa (in the form of viral plaques grown on a lawn of host bacteria). The lacZ MF in the BaP group $\left(\mathrm{MF}=13.1 \times 10^{-5}\right)$ was 3.4-fold higher $(\mathrm{P}<0.001)$ than in controls $\left(\mathrm{MF}=3.9 \times 10^{-5}\right)$, as shown in Table 6.1.

\subsubsection{Sequencing and Clonality}

We used NGS to sequence a total of 1591 lacZ mutant plaques and 200 titre plaques (assumed wild-type) derived from spermatozoa from the cauda epididymis in 5 control (578 plaques) and 6 BaP-treated animals (1013 plaques). Sequencing generated $80,327,390$ reads with an average read-length of 129 bp resulting in a total of $9.2 \times 10^{9}$ high quality base calls $(\geq Q 20)$. Alignment to the reference sequence resulted in an average depth of coverage per copy of $l a c Z$ sequenced of approximately1600X $[9.2 \mathrm{Gbp} \div$ $(1591+200)$ copies of $l a c Z \div 3096$ bp (length of sequenced portion of lac $Z$ )]. A total of 1117 mutants were identified (393 in controls and 724 in the $\mathrm{BaP}$ group) resulting in $70 \%$ recovery of the original input (1117 recovered mutants $\div 1591$ input mutants). Mutations that occurred more than once per animal were considered to have originated from a single independent event via clonal expansion. Using the LOD/linear model (Beal et al., 2015a) to correct for clonal expansion we identified 427 independent mutations (131 in controls and 296 in the BaP-exposed group). The average clonality in the germ cells of BaPexposed animals $(24.8 \%)$ was lower than in control animals (41\%). This difference, however, was not statistically significant $(\mathrm{P}=0.122$, t-test), owing largely to the high variability in clonality among samples. The lacZMFs were then corrected for the varying 
degrees of clonality (Table 6.1). Clonal correction increased the relative fold change in mutational response between controls and $\mathrm{BaP}$-treated samples (fold change $=3.4$ before; fold change $=4.1$, after correction), and reduced the variability in the $\mathrm{BaP}$ group (standard deviation $= \pm 3.4(26 \%)$ before; standard deviation $= \pm 1.0(11 \%)$ after correction).

\subsubsection{BaP-Induced Mutation Spectrum in Germ Cells}

The type, position, and frequency of independent mutations in the control and $\mathrm{BaP}$ groups are shown graphically along the $l a c Z$ gene in Figure 6.1 and described in detail in Table C8. One large 899 bp deletion, which was confirmed by Sanger sequencing, was identified in 1 of the control animals. This large deletion was omitted from downstream mutation spectrum analysis. All other reported deletions are 1 or $2 \mathrm{bp}$ in length. The lac $Z$ mutation spectrum in the sperm of BaP-exposed animals showed significant differences $(\mathrm{P}=0.01)$ with respect to the control group when compared using a Monte Carlo generated $X^{2}$ distribution. The proportional spectra of mutation types for each treatment group are shown in Figure 6.2. The most frequent types of mutation in the control group were G:C to A:T transitions (57\%), followed by G:C to T:A transversions $(22 \%)$. The proportional spectrum of mutation types in the BaP group was significantly different compared with the control group ( $\mathrm{P}<0.001$; Fisher's exact test). A multiple comparison of the individual mutation types showed that the most significant differences were the decreased proportion of G:C to A:T transitions (from $57 \%$ in controls to $13 \%$ in the $\mathrm{BaP}$ group), and the elevated proportion of $\mathrm{G}: \mathrm{C}$ to $\mathrm{T}: \mathrm{A}$ transversions (from $23 \%$ to $39 \%$ ) and deletions (from $7 \%$ to $29 \%$ ) in the $\mathrm{BaP}$-induced mutation spectrum. When the spectra were corrected for differences in MF (by multiplying the proportion of mutation 
types by the MF of each group (Figure C13), it was apparent that $\mathrm{BaP}$ exposure increased the frequency of all mutation types compared to background levels except for G:C to A:T transitions.

To analyze the influence of the sequence context on mutation formation, we compared the proportion of mutations that occurred at G:C or A:T base-pairs in each treatment group, and checked whether the nucleotide position of each mutation occurred within or adjacent to a nucleotide homopolymer repeat or at areas with 1 or more $\mathrm{CpG}$ dinucleotides (Table C9, summarized in Table 6.2). These results show that, in addition to the overall increase in the proportion of $\mathrm{G}: \mathrm{C}$ to $\mathrm{T}: \mathrm{A}$ transversions, there was also a statistically significant increase $(\mathrm{P}<0.05$, Fisher's exact test $)$ in the proportion of mutations that occurred at A:T nucleotides, from $3.8 \%$ in the control group to $18.2 \%$ in the $\mathrm{BaP}$ group. We also detected a marginally significant $(\mathrm{P}<0.1)$ increase in the proportion of all mutations that occurred at $\mathrm{CpG}$ dinucleotides in the $\mathrm{BaP}$ group. When divided into mutation types, it became apparent that the increased proportion of $\mathrm{BaP}$ induced mutations at A:T nucleotides was predominantly due to deletions: $43 \%$ of the deletions that occurred in BaP-treated animals were at A:T nucleotides. However, this increased proportion was not significantly greater than the control group. The elevated proportion of mutations at $\mathrm{CpG}$ dinucleotides in the $\mathrm{BaP}$ group appeared to be mostly G:C to T:A and G:C to $\mathrm{C}: \mathrm{G}$ transversions, which occurred significantly more frequently than in controls by approximately $30 \%$. The occurrence of homopolymers did not have an obvious impact on the different types of mutations across treatment groups.

Several positions along the lacZ gene were mutated in more than 1 animal (Figure 6.1, Table C10) indicating possible hotspots. A position was considered a hotspot if a 
mutation occurred there in more than 1 animal per dose-group. Eighteen hotspots were identified in mice from the control group. Nearly half $(43 \%)$ of the mutations in the control group occurred at these hotspots. All of the hotspots identified in the control group occurred at $\mathrm{G}: \mathrm{C}$ basepairs, at which the majority of mutations were $\mathrm{G}: \mathrm{C}$ to $\mathrm{A}: \mathrm{T}$ transitions. None of the control hotspots had deletions or insertions. In the BaP treatment group, we identified 48 hotspots. As was observed in control mice, $43 \%$ of all independent mutations identified in the $\mathrm{BaP}$-treated animals occurred at these hotspots. Most of the hotspots in the exposed group were also at G:C basepairs; however, around $11 \%$ of the hotspots occurred at A:T basepairs. Approximately $56 \%$ of the mutations in hotspots of $\mathrm{BaP}$-exposed mice were $\mathrm{G}: \mathrm{C}$ to $\mathrm{T}: \mathrm{A}$ transversions, and $21 \%$ were deletions, most of which occurred at G:C basepairs. Between both groups, a total of 63 unique hotspots were identified. Six of these had mutations only in the control group, 36 had mutations only in the BaP group, and 21 of the identified hotspots had mutations in both control and BaP-exposed groups. Finally, we determined the codon and amino acid changes caused by each mutation (Table C11). Similar proportions of premature stop codons (56.4\% vs $48.2 \%)$ and missense mutations (43.6\% vs $46.2 \%)$ were observed in control and BaP-treated mice, respectively. No silent mutations were observed in controls, while they represented $5.6 \%$ of the mutations induced by $\mathrm{BaP}$ (but this was not significantly higher than controls). These silent mutations likely represent "hitch-hiker" mutations that co-occurred with other mutations that were detectable by the positive selection assay (Beal et al., 2015a).

\subsubsection{Comparison of Sperm and Bone Marrow Mutation Spectra}


We compared the control and $\mathrm{BaP}$-induced mutation spectra in sperm to the control and induced spectra in bone marrow published previously (Beal et al., 2015a) (Figure 6.3 and Table 6.3). The background mutation spectrum in sperm was largely similar to the background spectrum in bone marrow (Figure 6.3). However, there were 2 key differences: sperm had significantly $(\mathrm{P}<0.001)$ more $\mathrm{G}: \mathrm{C}$ to $\mathrm{A}: \mathrm{T}$ transitions $(57 \%$ in sperm vs $32 \%$ in bone marrow $)$, and significantly $(\mathrm{P}<0.001)$ less mutations at A:T basepairs $(3.8 \%$ in sperm vs $18.8 \%$ in bone marrow) (Table 6.3$)$. Because the magnitude of the BaP-induced response was much greater in bone marrow $\left(\mathrm{MF}=701.7 \times 10^{-5}\right.$ in bone marrow; $\mathrm{MF}=13.1 \times 10^{-5}$ in sperm), $\mathrm{BaP}$-induced mutation spectra in sperm and bone marrow were corrected for differences in background mutation spectrum and for differences in the BaP-induced mutation frequency (Figure 6.4 and Table 6.3). G:C to $\mathrm{T}$ :A transversions were the most common mutation following $\mathrm{BaP}$ exposure in both tissues, and were induced at nearly identical proportions in both cell types. BaP induced a significantly greater proportion of $\mathrm{G}: \mathrm{C}$ to $\mathrm{A}: \mathrm{T}$ transitions and $\mathrm{G}: \mathrm{C}$ to $\mathrm{C}: \mathrm{G}$ transversions in bone marrow, but a greater proportion of deletions in sperm. Thus, the mutation spectra in sperm and bone marrow show differences before and after $\mathrm{BaP}$ exposure.

\subsection{Discussion}

We use the TGR mutation assay to recover mutated lacZ genes from spermatozoa from the cauda epididymis of untreated and BaP-exposed male mice. Using NGS we generated the most detailed spontaneous and $\mathrm{BaP}$-induced mutation spectrum in sperm to date (Figures 6.1 and 6.2), which provided insights into the mechanistic underpinnings of the spontaneous and induced mutations in male germ cells. We detected the characteristic 
$\mathrm{BaP}$ signature of increased $\mathrm{G}: \mathrm{C}$ to $\mathrm{T}: \mathrm{A}$ transversions in sperm, but also identified several significant differences in the mutation spectrum of sperm compared to the bone marrow of similarly treated animals (Figures 6.3 and 6.4). These results suggest that the mutagenic mechanisms operating in germ cells may differ from those in somatic tissues.

One of the major benefits of sequencing mutant genes recovered from the TGR assay is the ability to correct for clonal expansion (Beal et al., 2015a) that can artificially inflate the observed MF. In the present study, clonality was lower in the BaP-treatment group $(24.8 \%)$ compared with controls $(41 \%)$. Correcting for clonality had the effect of increasing the fold-change of the mutational effect from 3.4- to 4.1-fold (Table 6.1) and reduced the relative inter-individual variability within the BaP group (the standard deviation was reduced from $26 \%$ to $10.5 \%$, Table 6.1 ). Thus, clonal correction improved the TGR assay's ability to detect statistically significant effects.

Using NGS we sequenced $\sim 5$-fold more mutants than the combined number of mutants sequenced from all previous studies that have recovered transgenes (cII or lacZ) from germ cells (Douglas et al., 1994; Douglas et al., 1995; Olsen et al., 2010). We found that spontaneous mutations occurred almost entirely at G:C basepairs ( $96.2 \%$, Table 6.2 ), which is significantly greater than what would be expected by chance $(\mathrm{P}<0.001$, Fisher's Exact) given that the entire $l a c Z$ sequence consists of $57 \%$ G:C base-pairs. Consistent with previous reports using traditional Sanger sequencing (Douglas et al., 1995), the predominant forms of spontaneous mutations were G:C to A:T transitions followed by G:C to T:A transversions. The bias for spontaneous G:C to A:T transitions was believed to arise primarily from the deamination of methylated cytosine (Griffiths, 2005). However, this mechanism does not explain the G:C to A:T bias in non- $\mathrm{CpG}$ regions (Fu 
et al., 2011), nor does it explain the overall G:C to A:T bias in species with little to no methylation (Capuano et al., 2014). In the present study, 56\% (985/1754) of all G:C basepairs in the lacZ sequence are in $\mathrm{CpG}$ dinucleotides, but only $49 \%$ (37/75) of the spontaneous G:C transition mutations occurred at $\mathrm{CpGs}$, which is not significantly different from chance ( $P=0.287$, Fisher's exact). This is not consistent with cytosine deamination as the primary mechanism for spontaneous $\mathrm{G}: \mathrm{C}$ transition mutations, given that all of the CpGs of the lac Z transgene are known to be heavily methylated in all tissues, including the testes (Ikehata et al., 2000; Lambert et al., 2005). A similar lack of selection for $\mathrm{CpG}$ sites was also observed in the lacI and $c I I$ transgenes of Big Blue rats and mice (Monroe et al., 2001), leading the authors to conclude mechanisms other than spontaneous deamination of methylcytosine were driving spontaneous mutation.

Other proposed mechanisms for the G:C to A:T spontaneous mutation bias include DNA damage from ROS (Cooke et al., 2003), or G:C tautomerization caused by proton transfer across the base-pair hydrogen bonds (Cerón-Carrasco and Jacquemin, 2015; Fu et al., 2011). In the present study, there was a marginally significant enrichment $(\mathrm{P}=0.066)$ in the proportion of $\mathrm{G}: \mathrm{C}$ to $\mathrm{A}: \mathrm{T}$ transitions that occurred at dipyrimidine sites. In fact, $81 \%(61 / 75)$ of spontaneous transitions occurred at dipyrimidine sites, whereas only $71 \%(1235 / 1737)$ of all G:Cs in the lacZ gene are in a dipyrimidine context. These results suggest that the occurrence of dipyrimidines may be an important factor in the observed spontaneous mutation spectrum, especially for spontaneous G:C to A:T transitions.

Spontaneous G:C to T:A transversions are likely associated with damage from ROS produced by aerobic metabolism (Griffiths, 2005). One of the most common forms 
of ROS damage is the oxidation of guanosine into 8-oxo-dG, which mispairs with adenosine causing high levels of spontaneous G:C to T:A transversions. As 8-oxo-dG is readily detectable in the testes and sperm of mice (Attia et al., 2013; Chabory et al., 2009; Khan et al., 2011), we speculate that this mechanism is responsible for the high proportion of $\mathrm{G}: \mathrm{C}$ to $\mathrm{T}: \mathrm{A}$ transversions in the spontaneous mutation spectrum in sperm.

The spectrum of spontaneous mutations in sperm was comparable to that observed in bone marrow (Figure 6.3). As in sperm, CpG frequency did not influence the occurrence of G:C to A:T transitions. Also as in sperm, 80\% (36/45) of the G:C to A:T mutations in bone marrow occurred at dipyrimidines. However, this observation was not statistically significant for bone marrow due to the few number of G:C to A:T transitions recovered from this tissue $(\mathrm{P}=0.24)$. In fact, germ cells had a significantly higher proportion of $\mathrm{G}: \mathrm{C}$ to $\mathrm{A}: \mathrm{T}$ transitions $(\mathrm{P}<0.001)$ than bone marrow, suggesting that a dipyrimidine-based mechanism of spontaneous mutation may be more predominant in sperm. All other mutation types in the spontaneous mutation spectra, including G:C to $\mathrm{T}$ :A transversions, had similar proportions (Figure 6.3) and sequence context (Table 6.2 and Table 6.3) among the 2 cell types. Overall, these results show that subtle differences exist in the spontaneous mutations that occur in sperm versus bone marrow.

BaP's mutagenic mode of action in germ cells is generally assumed to be similar to somatic tissues. $\mathrm{BaP}$ is metabolized by cytochrome $\mathrm{P} 450$ s and epoxide hydrolase to form the predominant mutagenic metabolite BPDE, which forms DNA adducts on N2 of guanosine causing G:C to T:A transversions during replication (Gelboin, 1980; Miller and Ramos, 2001; Singh et al., 2007). In the present study, the BaP-induced mutation spectrum in sperm was dominated by $\mathrm{G}: \mathrm{C}$ to $\mathrm{T}: \mathrm{A}$ transversions (Figure 6.1 and 6.2), 
occurring primarily at $\mathrm{CpG}$ dinucleotides (Table 6.2). This is consistent with a BPDE mode of action, since methylated CpGs are the preferential target for BPDE adduct formation (Weisenberger and Romano, 1999; Yoon et al., 2001). Similarly, Olsen et al. (2010) reported an increased proportion of $\mathrm{G}: \mathrm{C}$ to $\mathrm{T}: \mathrm{A}$ transversions after sequencing $~ 50$ mutant $c I I$ genes from spermatozoa of $\mathrm{BaP}$-treated mice. $\mathrm{BaP}$ also induces mutations via several other minor mechanisms. BPDE forms adducts at N6 of adenosine and N4 of cytosine, but at a lower frequency than with guanosine. BaP metabolism also results in the production of ROS species that can form various types of DNA damage, including 8oxo-dG (Valavanidis et al., 2009), and other secondary adducts that can contribute to the mutagenic profile of $\mathrm{BaP}$. These various mechanisms are likely to be at least partially responsible for the more modest increase in other mutation types.

The BaP-induced mutation spectrum in germ cells and bone marrow shared many characteristics (Figure 6.4). The predominant mutation type in both cell types was the $\mathrm{G}: \mathrm{C}$ to $\mathrm{T}: \mathrm{A}$ transversion, and both spectra were enriched for mutations at $\mathrm{CpG}$ dinucleotides (Table 6.2 and 6.3), which is the typical $\mathrm{BaP}$ mutation signature. These results suggest that the major mechanism for $\mathrm{BaP}$-induced mutation in both sperm and bone marrow is via BPDE adducts on guanosine bases. However, there were also some notable differences: sperm from BaP-exposed mice had proportionally fewer G:C to A:T transitions and $\mathrm{G}: \mathrm{C}$ to $\mathrm{C}: \mathrm{G}$ transversions than bone marrow, and a greater proportion of deletions.

Indels are most often associated with replication errors due to polymerase slippage across homopolymeric sequences. BPDE-DNA adducts may increase the chance of slippage. In fact, all identified deletion hotspots occurred at homopolymeric regions, 
indicating they are important for BaP-induced deletions. However, only $\sim 25 \%$ and $22 \%$ of $\mathrm{BaP}$-induced deletions were associated with homopolymers, in sperm and bone marrow respectively, indicating that homopolymer slippage is not the primary mechanism responsible for the preponderance of deletions in sperm. We suggest that error-prone translesion DNA synthesis polymerase polymerase kappa (polK), and perhaps other Y-family polymerases (Guo et al., 2009; Sale et al., 2012; Yang, 2014), are likely involved. These polymerases have large active sites that can accommodate bulky lesions on the template strand, thus permitting synthesis through damaged regions. Due to their tolerant active sites, strand slippage and erroneous base-pairing are more frequent compared to normal polymerases and occur independent of the presence of homopolymer repeats (Kokoska et al., 2002). The Y-family polymerases is involved in the repair of BaP adducts (Avkin et al., 2004) and have a mechanism that would result in elevated deletions in non-polymer regions, as well as increased rates of all possible base substitutions. Further, their elevated expression in the testes (Guo et al., 2005; Guo et al., 2009; Ogi et al., 2001) may explain the greater proportion of homopolymer-independent deletions in sperm observed in the present study.

In conclusion, analysis of high-resolution mutational spectra by NGS showed that there is overlap in BaP's mutagenic mode of action between germ cells and bone marrow, although some notable differences were observed. Differential distribution of $\mathrm{BaP}$ metabolites between tissues (Ramesh et al., 2002) cannot be discounted as another mechanism contributing to the observed differences in the mutation spectra between germ cells and somatic cells. However, our results point to potential differences in DNA repair mechanisms operating on $\mathrm{BaP}$-induced damage in the 2 cell types. Our results are 
in accordance with the testable prediction that male germ cells will have a divergent mutation spectra in untreated and mutagen-exposed animals compared with somatic tissues. Thus, these findings support the hypothesis that mutations in sperm can arise via mutational mechanisms that are unique to male germ cells. 


\subsection{Tables and Figures}

Table 6.1 lacZ mutant frequency in sperm collected 42 days after a 28 -day exposure to benzo(a)pyrene, and clonal correction.

\begin{tabular}{|c|c|c|c|c|c|c|c|c|c|c|c|}
\hline \multirow[b]{2}{*}{$\begin{array}{c}\text { Dose } \\
\text { (mg/kg } \\
\text { bw/day) }\end{array}$} & \multirow[b]{2}{*}{$\underset{\text { ID }}{\text { Animal }}$} & \multicolumn{4}{|c|}{ Raw data } & \multirow[b]{2}{*}{$\begin{array}{c}\% \\
\text { clonality }\end{array}$} & \multirow[b]{2}{*}{$\begin{array}{c}\text { Average } \\
\% \\
\text { clonality } \\
\end{array}$} & \multicolumn{4}{|c|}{ Clonal correction } \\
\hline & & $M F \times 10^{-5}$ & $\begin{array}{l}\text { Average } \\
M F \times 10^{-5}\end{array}$ & SD & FC & & & $\begin{array}{l}\text { Corrected } \\
\mathrm{MF} \times 10^{-5}\end{array}$ & $\begin{array}{l}\text { Average } \\
M F \times 10^{-5}\end{array}$ & SD & FC \\
\hline \multirow[t]{6}{*}{0} & 1 & 1.6 & \multirow[t]{6}{*}{3.9} & \multirow[t]{6}{*}{$\begin{array}{c}1.8 \\
(46.2 \%)\end{array}$} & \multirow[t]{6}{*}{-} & 56.8 & \multirow[t]{6}{*}{41.0} & 0.7 & \multirow[t]{6}{*}{2.3} & \multirow[t]{6}{*}{$1.2(52.2 \%)$} & \multirow[t]{6}{*}{-} \\
\hline & 2 & 4.6 & & & & 14.3 & & 3.9 & & & \\
\hline & 3 & 4.7 & & & & 58.5 & & 2.0 & & & \\
\hline & 4 & 2.5 & & & & 40.6 & & 1.5 & & & \\
\hline & 5 & 3.3 & & & & 26.8 & & 2.4 & & & \\
\hline & 6 & 6.7 & & & & 49 & & 3.4 & & & \\
\hline \multirow[t]{5}{*}{100} & 7 & 13.4 & \multirow[t]{5}{*}{13.1} & \multirow[t]{5}{*}{$\begin{array}{c}3.4 \\
(26.0 \%)\end{array}$} & \multirow[t]{5}{*}{3.4} & 27.8 & \multirow[t]{5}{*}{24.8} & 9.7 & \multirow[t]{5}{*}{9.5} & \multirow[t]{5}{*}{$1.0(10.5 \%)$} & \multirow[t]{5}{*}{4.1} \\
\hline & 8 & 13.3 & & & & 35.2 & & 8.6 & & & \\
\hline & 9 & 11.2 & & & & 17.2 & & 9.3 & & & \\
\hline & 10 & 9.2 & & & & 4.8 & & 8.8 & & & \\
\hline & 11 & 18.4 & & & & 39.1 & & 11.2 & & & \\
\hline
\end{tabular}

SD: standard deviation

FC: fold change 
Table 6.2 Analysis of sequence context of lac $Z$ mutations identified in spermatozoa from the cauda epididymis of male mice exposed to benzo(a)pyrene.

\begin{tabular}{|c|c|c|c|c|c|c|}
\hline & \multicolumn{2}{|c|}{ ALL MUTATIONS } & \multicolumn{2}{|c|}{ Ins ertions } & \multicolumn{2}{|c|}{ Deletions } \\
\hline & Control & $\mathrm{BaP}$ & Control & $\mathrm{BaP}$ & Control & $\mathrm{BaP}$ \\
\hline \# Mutations & 131 & 296 & 3 & 13 & 11 & 86 \\
\hline $\mathrm{A}: \mathrm{T}$ & $5(3.8 \%)$ & $54(18.2 \%)$ & $1(33.3 \%)$ & $3(23.1 \%)$ & $2(18.2 \%)$ & $37(43 \%)$ \\
\hline G:C & $125(96.2 \%)$ & $242(81.8 \%)$ & $2(66.7 \%)$ & $10(76.9 \%)$ & $9(81.8 \%)$ & $49(57 \%)$ \\
\hline $\mathrm{CpG}$ & $60(46.2 \%)$ & $\underline{168(56.8 \%)}$ & $0(0 \%)$ & $6(46.2 \%)$ & $7(63.6 \%)$ & $43(50 \%)$ \\
\hline \multirow[t]{3}{*}{ Homopolymer } & $27(20.8 \%)$ & $71(24 \%)$ & $1(33.3 \%)$ & $4(30.8 \%)$ & $2(18.2 \%)$ & $22(25.6 \%)$ \\
\hline & \multicolumn{2}{|c|}{$\mathrm{GC}->\mathrm{AT}$} & \multicolumn{2}{|c|}{ GC->TA } & \multicolumn{2}{|c|}{$\mathrm{GC}->\mathrm{CG}$} \\
\hline & Control & $\mathrm{BaP}$ & Control & $\mathrm{BaP}$ & Control & $\mathrm{BaP}$ \\
\hline \# Mutations & 75 & 39 & 28 & 117 & 11 & 27 \\
\hline $\mathrm{CpG}$ & $37(49.3 \%)$ & $20(51.3 \%)$ & $10(35.7 \%)$ & $75(64.1 \%)$ & $6(54.5 \%)$ & $24(88.9 \%)$ \\
\hline \multirow[t]{3}{*}{ Homopolymer } & $14(18.7 \%)$ & $7(17.9 \%)$ & $5(17.9 \%)$ & $28(23.9 \%)$ & $4(36.4 \%)$ & $5(18.5 \%)$ \\
\hline & \multicolumn{2}{|c|}{$\mathrm{AT}->\mathrm{GC}$} & \multicolumn{2}{|c|}{ AT $->$ TA } & \multicolumn{2}{|c|}{$\mathrm{AT}->\mathrm{CG}$} \\
\hline & Control & $\mathrm{BaP}$ & Control & $\mathrm{BaP}$ & Control & $\mathrm{BaP}$ \\
\hline \# Mutations & 1 & 4 & 1 & 9 & 0 & 1 \\
\hline $\mathrm{CpG}$ & $0(0 \%)$ & $0(0 \%)$ & $0(0 \%)$ & $0(0 \%)$ & 0 & $0(0 \%)$ \\
\hline Homopolymer & $0(0 \%)$ & $1(25 \%)$ & $1(100 \%)$ & $4(44.4 \%)$ & 0 & $0(0 \%)$ \\
\hline
\end{tabular}

Bold indicates $\mathrm{P}<0.05$ relative to controls

Underline indicates $\mathrm{P}<0.1$ 
Table 6.3 Analysis of sequence context of lacZ mutations identified in the bone marrow of male mice exposed to benzo(a)pyrene. These data are derived from previously published results (Beal et al., 2015a).

\begin{tabular}{|c|c|c|c|c|c|c|}
\hline & \multicolumn{2}{|c|}{ ALL MUTATIONS } & \multicolumn{2}{|c|}{ Insertion } & \multicolumn{2}{|c|}{ Deletion } \\
\hline & control & $\mathrm{BaP}$ & control & $\mathrm{BaP}$ & control & $\mathrm{BaP}$ \\
\hline \# Mutations & 144 & 802 & 3 & 27 & 17 & 152 \\
\hline $\mathrm{A}: \mathrm{T}$ & $27(18.8 \%)$ & $108(13.5 \%)$ & $1(33.3 \%)$ & $8(29.6 \%)$ & $7(41.2 \%)$ & $62(40.8 \%)$ \\
\hline G:C & $117(81.2 \%)$ & $694(86.5 \%)$ & $2(66.7 \%)$ & $19(70.4 \%)$ & $10(58.8 \%)$ & $90(59.2 \%)$ \\
\hline $\mathrm{CpG}$ & $69(47.9 \%)$ & $470(58.6 \%)$ & $2(66.7 \%)$ & $8(29.6 \%)$ & $9(52.9 \%)$ & $81(53.3 \%)$ \\
\hline \multirow[t]{3}{*}{ Homopoly } & $31(21.5 \%)$ & $156(19.5 \%)$ & $2(66.7 \%)$ & $9(33.3 \%)$ & $2(11.8 \%)$ & $34(22.4 \%)$ \\
\hline & \multicolumn{2}{|c|}{$\mathrm{GC}->\mathrm{AT}$} & \multicolumn{2}{|c|}{$\mathrm{GC}->\mathrm{TA}$} & \multicolumn{2}{|c|}{$\mathrm{GC}->\mathrm{CG}$} \\
\hline & control & $\mathrm{BaP}$ & control & $\mathrm{BaP}$ & control & $\mathrm{BaP}$ \\
\hline \# Mutations & 45 & 111 & 47 & 345 & 13 & 129 \\
\hline $\mathrm{CpG}$ & $24(53.3 \%)$ & $45(40.5 \%)$ & $27(57.4 \%)$ & $230(66.7 \%)$ & $7(53.8 \%)$ & $106(82.2 \%)$ \\
\hline \multirow[t]{3}{*}{ Homopoly } & $13(28.9 \%)$ & $21(18.9 \%)$ & $7(14.9 \%)$ & $56(16.2 \%)$ & $2(15.4 \%)$ & $25(19.4 \%)$ \\
\hline & \multicolumn{2}{|c|}{$\mathrm{AT}->\mathrm{GC}$} & \multicolumn{2}{|c|}{ AT $->$ TA } & \multicolumn{2}{|c|}{ AT $->C G$} \\
\hline & control & $\mathrm{BaP}$ & control & $\mathrm{BaP}$ & control & $\mathrm{BaP}$ \\
\hline \# Mutations & 6 & 3 & 5 & 25 & 8 & 10 \\
\hline $\mathrm{CpG}$ & $0(0 \%)$ & $0(0 \%)$ & $0(0 \%)$ & $0(0 \%)$ & $0(0 \%)$ & $0(0 \%)$ \\
\hline Homopoly & $1(16.7 \%)$ & $0(0 \%)$ & $1(20 \%)$ & $6(24 \%)$ & $3(37.5 \%)$ & $5(50 \%)$ \\
\hline
\end{tabular}

Bold indicates $\mathrm{P}<0.05$ relative to controls 


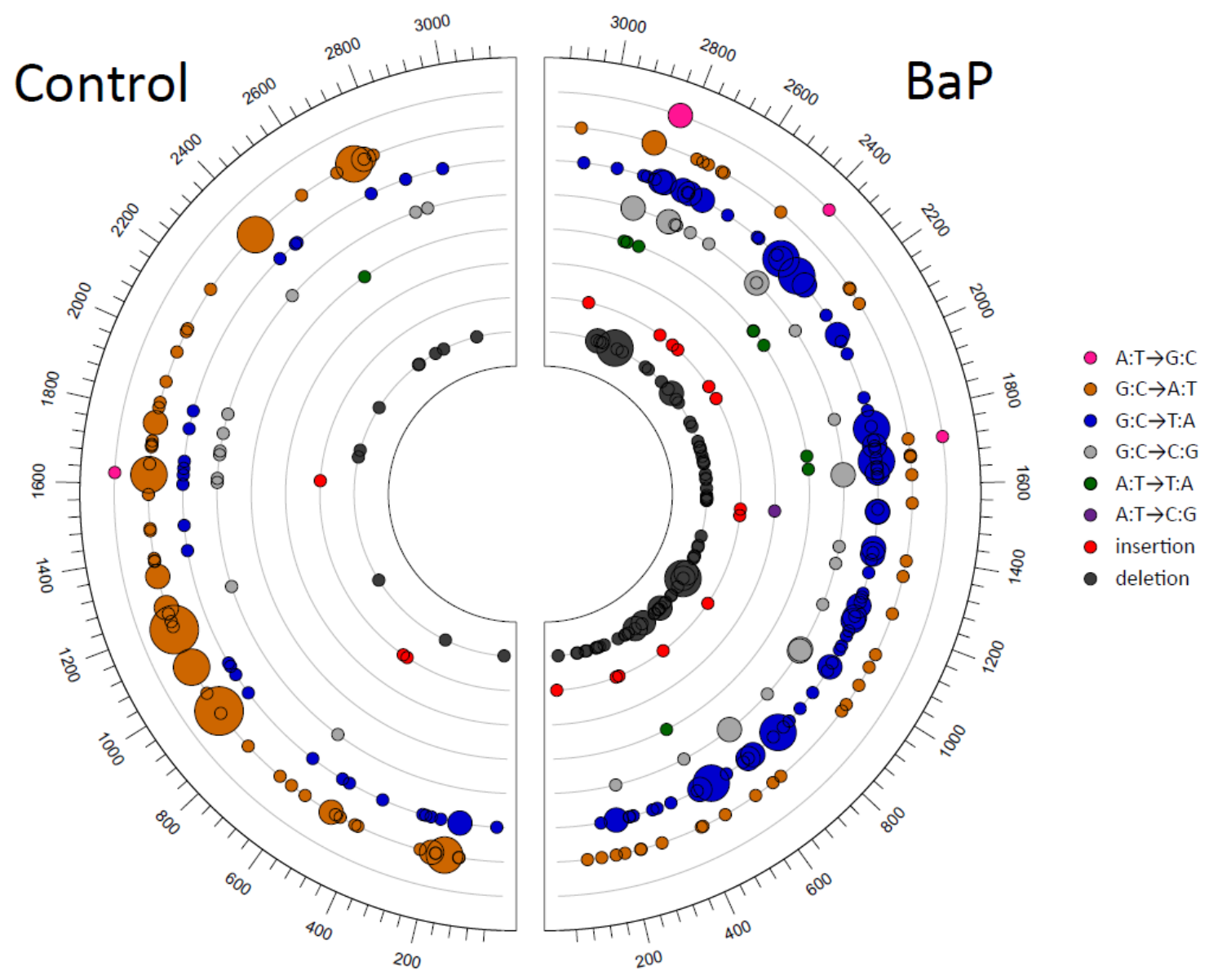

Figure 6.1 The type and position of all independent spontaneous (control) and benzo[a]pyrene(BaP)-induced lacZ mutations detected in sperm collected 42 days after the treatment period. Circle size is proportional to the number of times a mutation was detected in different animals. 


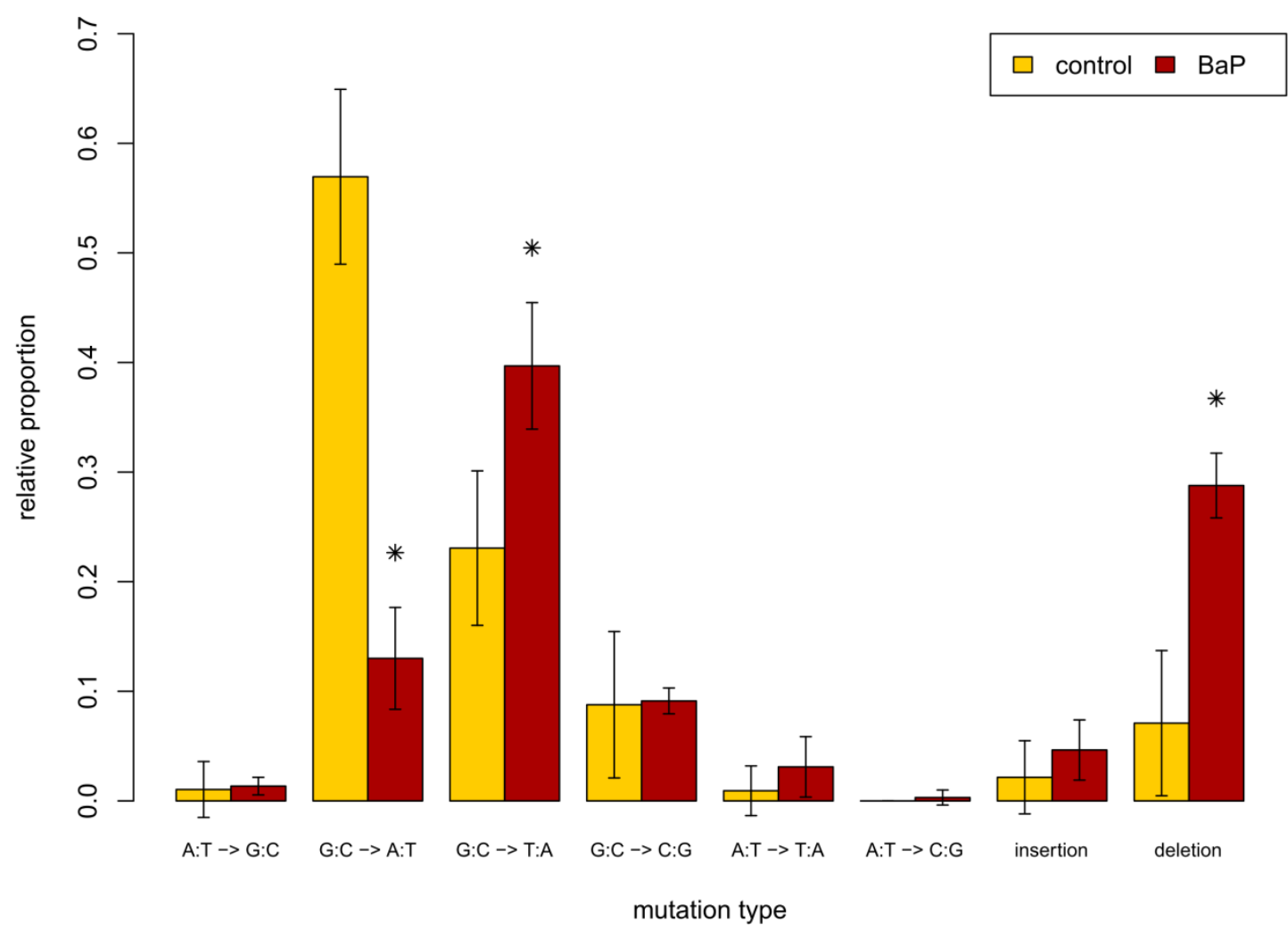

Figure 6.2 The proportion of all independent spontaneous (control) and benzo[a]pyrene (BaP)-induced lac $Z$ mutations detected in sperm collected 42 days after the treatment period. * indicates significant differences $(\mathrm{P}<0.05)$ between control and $\mathrm{BaP}$ groups. 


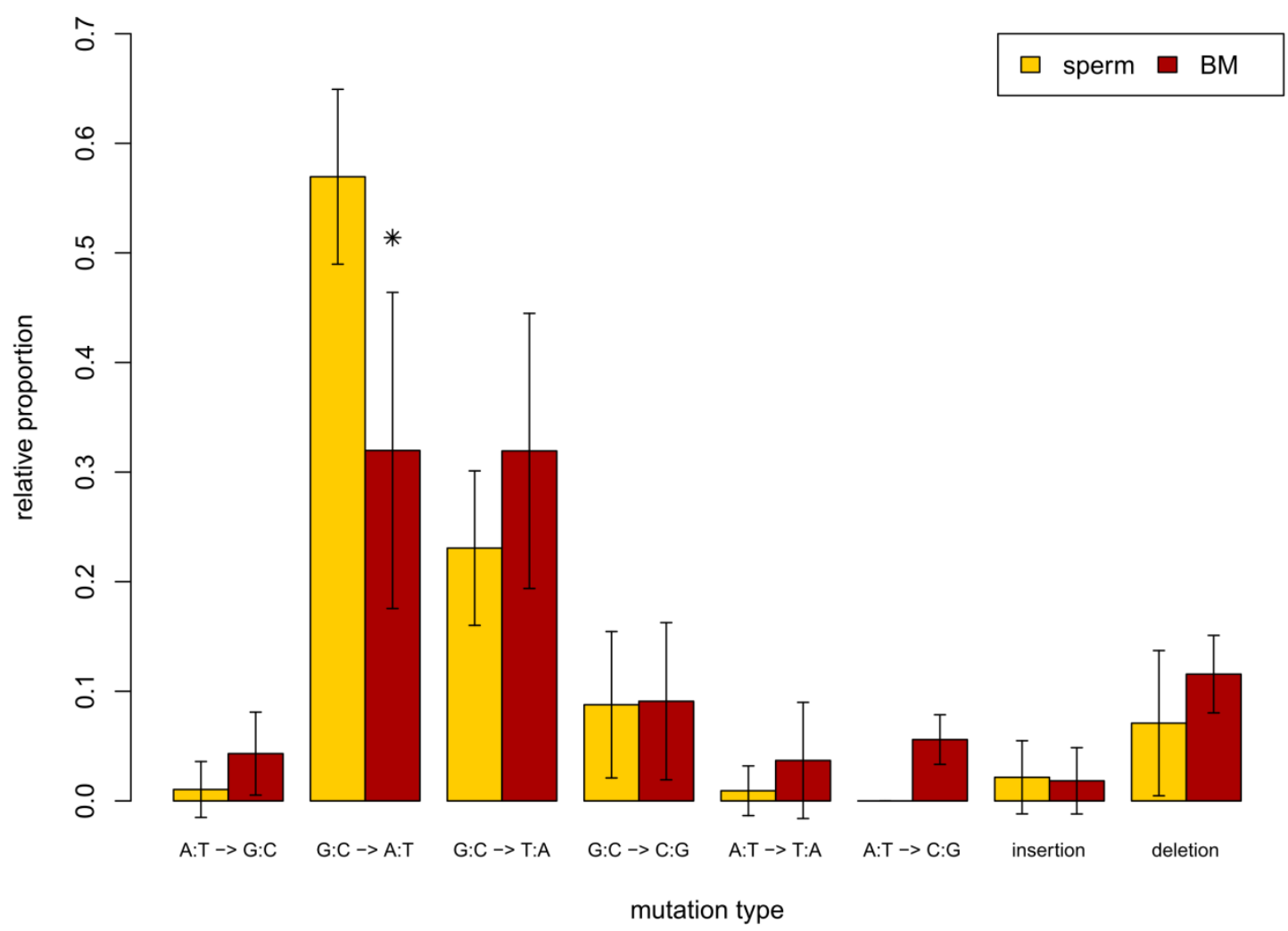

Figure 6.3 Comparison of the proportion of spontaneous mutation types in the lac $Z$ gene collected from sperm and bone marrow. * indicates significant differences $(\mathrm{P}<0.05)$ between tissues. Bone marrow data are from a previously published study (Beal et al., 2015a). 


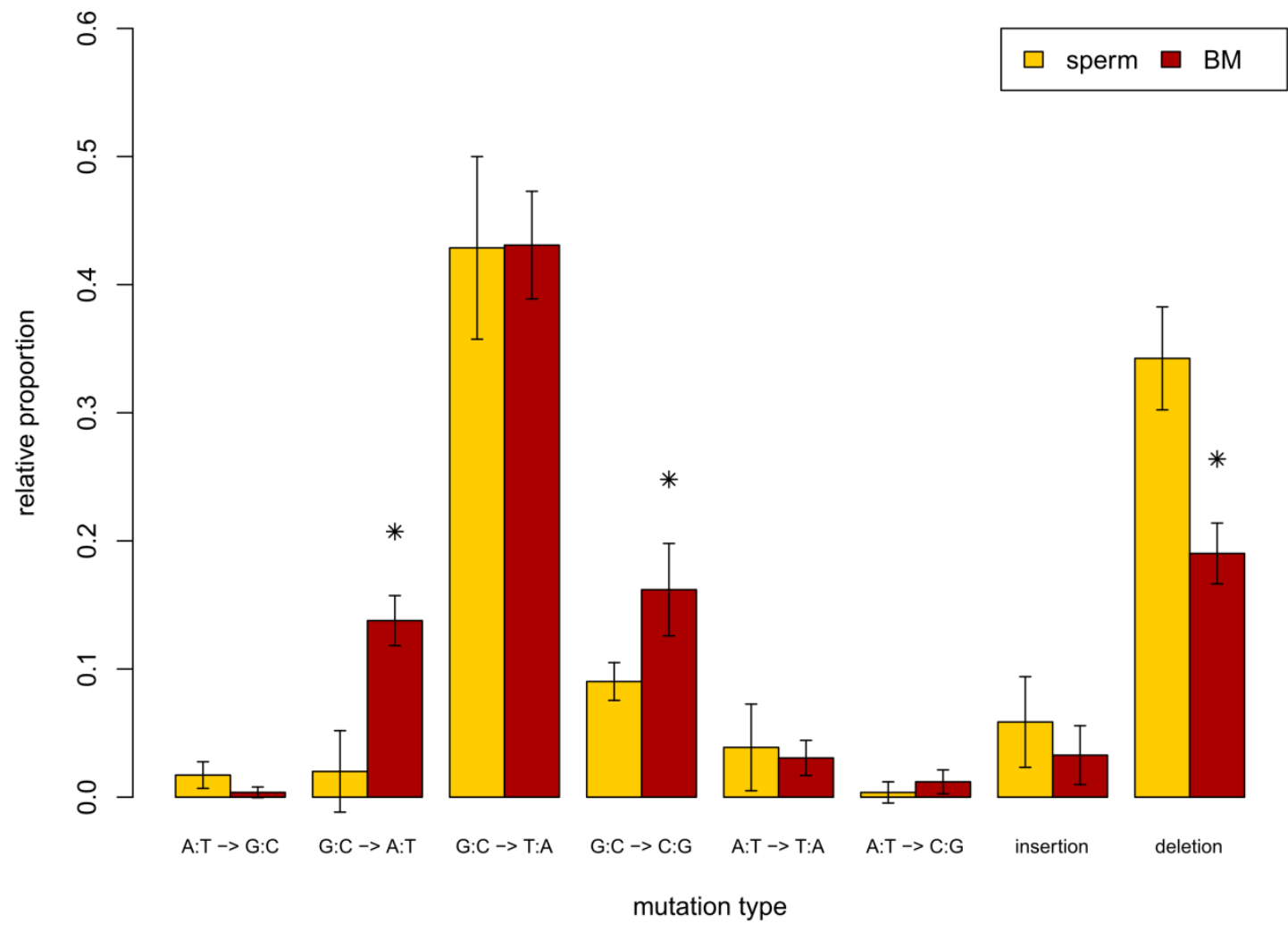

Figure 6.4 Comparison of the proportion of benzo[a]pyrene (BaP)-induced mutation types in the lacZ gene isolated from sperm and bone marrow. * indicates significant differences $(\mathrm{P}<0.05)$ between tissues. Bone marrow data are from a previously published study (Beal et al., 2015a). 


\section{Chapter 7: Paternal Exposure to Benzo(a)pyrene Induces Genome- Wide Mutations in Offspring}

\subsection{Preamble}

The main focus of this thesis was to assess the utility of whole genome approaches (aCGH and NGS) in identifying and quantifying chemically-induced heritable mutations. The results obtained from analyzing the genomes of offspring for de novo mutations are reported and discussed in this chapter. The $\mathrm{BaP}$ exposure used in this chapter to examine offspring for mutations is the same exposure that was used previously to examine mutations in sperm. Thus, direct comparisons can be made between existing germ cell mutation assays and genomics technologies. These comparisons allow preliminary assessment of the sensitivity of the genomic approaches and also demonstrate the utility of existing mutation assays.

\subsection{Abstract}

Understanding the effects of environmental exposures on germline mutation rates has been a decades-long pursuit in the field of genetics. The emergence of whole genome approaches has provided an unprecedented opportunity to discover rare mutation events and understand their consequences. Whole genome mutation analysis has predominantly focused on understanding the effects of environmental exposures on somatic mutation rates/spectra and implications to cancer. In this study, we harness the power of NGS and $\mathrm{CGH}$ arrays to investigate whether exposure of male mice to $\mathrm{BaP}$, a common environmental pollutant, causes genome-wide mutations in their offspring. We previously reported that mice exposed to $100 \mathrm{mg} / \mathrm{kg}$ of $\mathrm{BaP}$ have elevated mutation frequencies in a 
transgenic reporter gene and in endogenous microsatellite loci in their sperm. Here, we demonstrate that offspring developing from sperm exposed during the post-mitotic (meiotic and post-meiotic) or pre-meiotic phases of spermatogenesis have more heritable mutations than control animals, specifically, large duplications and SNVs. For SNVs there was an overall 2-fold increase in mutation frequency $(\mathrm{P}<0.01)$, although the number of $\mathrm{BaP}$-associated $\mathrm{SNV}$ s varied substantially among animals. This foundational study underlines the need to investigate other environmental exposures, especially in humans, to protect future generations from potential genetic hazards.

\subsection{Introduction}

There is a growing recognition of the importance of heritable mutations across a broad array of human genetic disease, especially at the population level. However, germline mutations are rare $\left(10^{-8}\right.$ per nucleotide) (Campbell and Eichler, 2013) and studying these mutation events has been difficult historically because of technological limitations. Consequently, there are no accepted human germ cell mutagens, although many rodent germ cell mutagens have been identified (DeMarini, 2012). Recently, advancements in genomics technologies, specifically aCGH and NGS, have allowed de novo mutation rates and spectra to be studied at the genome-wide level (Beal et al., 2012b; Yauk et al., 2015a). These technologies have been applied to confirm previous observations that paternal age is a strong determinant of de novo mutation rate, with older males passing on more mutations to their children (Francioli et al., 2015; Kong et al., 2012). Hence, paternal age can now be considered a human germ cell mutagen. 
Many paternal mutations are assumed to occur spontaneously during DNA replication in self-renewing spermatogonia. As a result, the contributions of paternal environmental exposures to mutation formation are likely underestimated. Pedigree studies in mice have demonstrated that exposures to DNA damaging agents lead to the transmission of induced de novo mutations (Russell, 2004). New whole genome approaches in animal models support the hypothesis that paternal environmental exposures contribute to heritable mutagenesis. For example, Adewoye et al. (2015) demonstrated using aCGH and NGS that paternal radiation exposure induces large deletions and clustered mutations (multisite mutations) in the genomes of their offspring. Thus, it is clear that physical DNA damaging agents can induce genome-wide mutations through the germline, but the genomic consequences of chemical exposures are unknown. To address this, we applied genomics tools to study the consequences of paternal exposure to $\mathrm{BaP}$, a ubiquitous environmental pollutant, on the mouse germline. We previously showed that the same exposure induces a 4-fold increase in mutation frequency in a transgenic reporter gene in mouse sperm (O'Brien et al., 2016a). Here, we make the first direct comparison between the spectrum and frequencies of mutations measured in exposed germ cells versus mutations found in the offspring to demonstrate the heritability of environmentally induced germ cell mutations.

\subsection{Materials and Methods}

\subsubsection{Benzo(a)pyre ne Exposure and Mouse Breeding}

All animal procedures were conducted according to conditions approved by the Health Canada Ottawa Animal Care Committee. The BaP exposure was conducted in 
parallel with the exposure done in previous studies (Beal et al., 2015a; Beal et al., 2015b; O'Brien et al., 2016a; O'Brien et al, 2016b). Twelve MutaMouse males (BALB/C × DBA/2) (Gossen et al., 1989), approximately 15 weeks of age, were exposed daily to BaP (Sigma-Aldrich, St. Louis, Missouri) dissolved in olive oil via oral gavage (approximately $150 \mu \mathrm{L}$ ) for 28 days ( $100 \mathrm{mg} / \mathrm{kg}$ body weight/day). Twelve concurrent males received olive oil and served as negative controls. Three days after the last treatment all males were mated with $4 \mathrm{C} 57 \mathrm{~B} / / 6 \mathrm{~J}$ females each over a 2 week period to produce offspring derived from germ cells exposed during the post-mitotic phase of spermatogenesis. This exposure window covered most of meiosis and the entirety of spermiogenesis (post-meiosis). Breeding was repeated 42 days after the last treatment with 4 new C57B1/6J females per male to produce offspring from germ cells exposed during the pre-meiotic phase of spermatogenesis. The offspring produced from each mating were weaned at approximately 3 weeks. Three weeks later, litters and dams were anaesthetized with isoflourane and euthanized via cervical dislocation. Sires were euthanized after tissue samples from the offspring were collected. From each animal the liver, spleen, and brain were collected and stored at $-80^{\circ} \mathrm{C}$.

\subsubsection{Comparative Genomic Hybridization and CNV Detection}

Agilent SurePrint G3 Mouse CGH arrays with 1M probes (G4838A; Agilent Technologies, Santa Clara, California) were used to detect de novo CNVs in the litters produced from mating MutaMouse sires with $\mathrm{C} 57 \mathrm{~B} / 6 \mathrm{~J}$ dams. The manufacturer's instructions were followed for each aCGH experiment (Agilent Oligonucleotide ArrayBased CGH for Genomic DNA Analysis; version 7.3). Specifically, high molecular 
weight DNA was extracted from the liver of each animal using the DNeasy Blood \& Tissue Kit (Qiagen, Venlo, Limburg, Netherlands). Two of the BaP-treated males exhibited high degrees of somatic mosaicism in their liver, apparent by the presence of several false-positive CNV calls in the offspring, and therefore brain DNA was used instead. One $\mu \mathrm{g}$ of offspring DNA was labelled with Cy5 dye (5190-3400 SureTag DNA Labeling Kit). Five hundred ng of DNA from each respective parent were mixed together and labelled with $\mathrm{Cy} 3$ dye. As positive controls, and to identify existing strain differences, 2 additional arrays were used to compare MutaMouse with C57BL/6J DNA. DNA labelled with $\mathrm{Cy} 3$ and $\mathrm{Cy} 5$ were mixed together for hybridization onto the $\mathrm{CGH}$ array. The hybridized slides were scanned using an Agilent $\mathrm{C}$ scanner and Feature Extraction v10.5 was used to extract the data. Following Feature Extraction, signal intensities (Cy3 and Cy5) were background subtracted and then normalized (Lowess normalization using Rank invariant probes). Non-uniform features (outlier probes with signals influenced by array position) were removed from analysis. The algorithms previously used by Adewoye et al. (2015) were applied to detect autosomal CNVs as genomic segments with parent vs. offspring fluorescent signal $\log 2$ ratios above or below threshold values as described previously (Conrad et al., 2009). Specifically, de novo CNV duplications/deletions were called if they had an absolute $\mid \log 2$ ratio $\mid>0.4$ (1.32-fold higher signal) and the $\mathrm{CNV}$ segment was a minimum of 5 consecutive altered probes. Probes had an average spacing of approximately 3,000 bp; therefore, this approach detected CNVS that were at least approximately $15,000 \mathrm{bp}$ in size. Only unique CNVs (i.e., observed once only) were considered to eliminate multi-alle lic CNVs that existed in the parents and were unlikely to be de novo mutations. Lastly, CNVs were identified as 
germline mosaic when they were present in a portion of the siblings from the same sire but not in any offspring from a different sire. A total of 29 de novo CNVs (27 unique, 2 mosaic) passed the threshold criteria and were tested for validation.

\subsubsection{CNV Validation}

Real-time PCR using TaqMan ${ }^{\circledR}$ Copy Number Assays that targeted the CNV regions were analyzed using a CFX96 ${ }^{\mathrm{TM}}$ Real-Time System (Bio-Rad, Hercules, California) to validate putative de novo CNVs. The mouse transferrin receptor gene (Tfrc) was used as a reference and analyzed simultaneously with the CNV targeting assays. Family quartets (sire, dam, offspring with $\mathrm{CNV}$, offspring without $\mathrm{CNV}$ ) were analyzed together and each tissue was analyzed with at least 4 technical replicates. The same DNA used in the arrays from the liver (endoderm) was used for validation. DNA from the brain (ectoderm) or spleen (mesoderm) was also analyzed for the presence of the $\mathrm{CNV}$ in the offspring to confirm that the CNV was germline in origin and not a result of somatic mosaicism. CNVs that adhered to Mendelian inheritance patterns or had a copy number change of $<0.5$ copies were considered as false-positives.

\subsubsection{Identifying CNV B reakpoint and Parental Origin}

Mate-pair libraries were built for each animal carrying a CNV (Table C12) using the Nextera mate pair library prep kit (Illumina ${ }^{\circledR}$, San Diego, California). Each library was sequenced using a NextSeq 500 (Illumina ${ }^{\circledR}$ ) sequencer and approximately $20 \%$ of a high-output flow cell. Following alignment to the mm10 genome using bwa (version 0.7.12), the regions identified by aCGH were visually inspected using the Integrative 
Genomics Viewer (Robinson et al., 2011) to pinpoint the CNV breakpoint sequence and elucidate the mechanism for formation. The sequence data were also compared against the MutaMouse and C57BL/6J haplotype information to characterize the parental origin of each CNV. Loss of the allele in deletions or higher proportion of the allele in duplications would indicate that the $\mathrm{CNV}$ originated in a germ cell of the parent carrying that allele.

\subsubsection{Whole Genome Sequencing}

Two of the families from each group used for array analysis were chosen at random for WGS. Each family consisted of a sire, dam, and 2 offspring (1 male, 1 female). DNA was extracted from the Spleen using the DNeasy Blood \& Tissue Kit (Qiagen, Venlo, Limburg, Netherlands) and NGS libraries were built using NEBNext ${ }^{\circledR}$ Fast DNA Library Prep Set for Ion Torrent ${ }^{\mathrm{TM}}$ (New England Biolabs, Ipswich, Massachusetts) according to manufacturer's instructions. NGS was performed in-house using the Ion Chef ${ }^{\mathrm{TM}}$ and Ion Proton ${ }^{\mathrm{TM}}$ instruments (Thermo Fisher Scientific, Waltham, Massachusetts). Each genome was sequenced to an average autosomal coverage of approximately $31 \mathrm{x}$.

\subsection{6 de novo Mutation Calling and Filtering}

Sequence data were aligned to the GRCm38/mm10 reference genome using the Torrent Mapper, and the Torrent Variant Caller was used to call variants (Torrent Suite version 5.0.2). Genomic variant call files ( $\mathrm{gVCFs}$ ) were merged and Mendelian violations (MVs) between offspring and parents were called and filtered to eliminate false-positive 
calls. Specifically, the loci \pm 10 bp of each putative MV were examined for allelic depth using samtools (v. 1.3). MVs were kept if they met the following criteria: the variant allele fraction (VAF) for the variant in the parents was $<5 \%$, VAF in the offspring was $>$ $25 \%$, the fraction of reads with mapping quality of zero did not exceed 0.1 , read depth in the parents was at least 5, read depth in the offspring was at least 10, and the Fisher's exact test for strand bias had a P-value $>0.05$. Variants occurring within repeat sequences and other related elements were removed from further analysis. A total of 1402 variant calls passed filtering (average of 117 per animal) and were selected for validation (Table C13).

\subsubsection{Cross-Platform Targe te d Re-sequencing Validation}

Mutations were validated by Agilent Sure Select Target Enrichment as done in ref. (Lindsay et al., 2016). Bait design was successful for 1333 of the 1402 putative variants (Table C13). After enrichment, all variants were multiplexed and sequenced to approximately 600x coverage on one lane of an Illumina HiSeq V4 (75 bp paired-end reads). Duplicates were removed and in-house scripts were used to quantify read counts at each candidate site to determine if the variant was a true de novo, inherited, or a false positive based on parental and child VAFs (Figure C14). The Integrative Genomics Viewer (Robinson et al, 2011) was used to manually inspect all confirmed mutations. Further filtering was applied to eliminate mosaic mutations (mutations called in siblings or present in parents with VAFs above 1\%) and mutations that occurred during embryogenesis (VAF in offspring $<39 \%$; Figure C15). 


\subsubsection{Mutation Frequency Es timation}

Bedtools (Quinlan and Hall, 2010) was used to estimate the percent of autosomal nucleotides in the diploid mouse genome (4.7 GB) (Chinwalla et al., 2002) covered by NGS reads ( $\geq 10 \mathrm{x}$ coverage). Approximately $93 \%$ of the genome (4.4 GB) was covered by NGS reads in each animal. Repetitive DNA sequence regions, according to Repeatmasker (Smit et al., 1996), were removed (45\% of diploid genome) leaving 2.4 GB of the autosomal diploid genome where mutations could be detected with approximately 30x coverage. True de novo mutations with a VAF $>39 \%$ in the autosomes were divided by the number of autosomal nucleotides to estimate mutation frequency.

\subsubsection{Statis tical Analyses}

One-tailed Fisher's exact tests were used to compare CNV events between control and $\mathrm{BaP}$ animals. Assuming a Poisson distribution for the error, GEEs were used to analyze SNV/indel data. The modified Wald-method was used to measure $95 \%$ confidence intervals for CNV data and the Poisson distribution was used for SNV data.

\subsection{Results and Discussion}

We investigated the effects of paternal $\mathrm{BaP}$ exposure on genome-wide heritable mutation frequencies and spectra in the unexposed offspring. Twelve MutaMouse males received $100 \mathrm{mg} / \mathrm{kg}$ of $\mathrm{BaP}$ via oral gavage for 28 days and twelve males received olive oil as negative control. The males were mated with $4 \mathrm{C} 57 \mathrm{Bl} / 6 \mathrm{~J}$ females each at 2 different time points. The distinct genetic backgrounds of the parents were chosen to 
allow for the parental origin of mutations to be determined. Matings occurred 3 and 42 days after the last exposure to investigate offspring developing from sperm exposed during the post-mitotic phases of spermatogenesis (spermatocytes and spermatids) and before meiosis (dividing spermatogonia), respectively (Yauk et al., 2015a). This was done to compare de novo mutations originating in the sperm themselves during spermatogenesis (42 day mating time point) versus those most likely originating in the egg after fertilization ( 3 day mating time point). Germ cells exposed during the postmeiotic phase lack the DNA synthesis and repair capabilities required to fix premutational lesions into mutations, and thus, no detectable level of mutation induction occurs in these cells (O'Brien et al., 2016a). However, it has been postulated that if sperm with damaged DNA were to fertilize the oocyte, replication of unrepaired lesions in sperm DNA during the first cellular divisions would convert those lesions into mutations in the developing embryo (Yauk et al, 2015a). Thus, we hypothesized that the BaP exposure would lead to increases in the number of induced mutations in the offspring at both mating time-points. In previous work, we showed that this same exposure caused a 4-fold increase in mutation frequency in the lac $Z$ transgene of sperm exposed as spermatogonia, but not as spermatids (O’Brien et al., 2016a; O'Brien et al., 2016b).

Two approaches were used to quantify and characterize genome-wide mutations occurring before and after fertilization. For the first approach, CGH arrays were used to identify de novo CNVs (Figure 7.1). In total, 100, 83, and 98 offspring were analyzed for de novo $\mathrm{CNVs}$ in control, post-mitotic, and pre-meiotic groups, respectively. All CNVs were validated using real-time PCR and by mate-pair NGS (Figure 7.1). The second approach involved whole genome sequencing of family quartets for the identification of 
all detectable mutation events. The whole genomes of 2 control families 42 days after the last oral gavage, and 2 treated families from both mating periods, were sequenced. Thus, a sample size of 4 offspring per treatment group was studied. The $2 \mathrm{BaP}$-treated sires used for mating in the pre-meiotic group were also used for mating in the post-mitotic group. Putative mutations were cross-validated using Illumina ${ }^{\circledR}$ sequencing. Broadly, the WGS and CNV results of this study were used to: (1) determine whether paternal BaP exposure causes increases in heritable mutations in offspring; (2) explore whether the presence of post-mitotic lesions in sperm are converted into mutations in the offspring after fertilization; and (3) compare pre-meiotic SNV/indel mutation induction between sperm and offspring.

\subsection{1 de novo CNVs}

Stringent filters and validation measures were performed to identify true de novo CNV events. Out of the initial call-set of 29 CNVs from the arrays, 12 were eliminated (6 false-positives, 6 inherited from parent). In 4 of the 6 false-positives, the relative PCR amplification of mutant liver DNA compared to parental DNA was concordant with array data; however, the absolute copy number change was less than $<0.5$ copies. Thus, these CNVs were more likely to be somatic mosaics that arose during early embryogenesis as opposed to in the germ cells (Lupski, 2013; Meier et al., 2017). One additional CNV failed to amplify after testing multiple assays. This CNV was likely a somatic mosaic based on the array results. The remaining $16 \mathrm{CNVs}$ were considered de novo (14 CNVs) or germline mosaic events (2 CNVs). In all cases where SNP information was available, the $\mathrm{CNV}$ was determined to have arisen from the paternal genome (6 of $16 \mathrm{CNVs}$ ). 
However, there were 2 deletions $(\log 2$ ratio of $\geq-0.75)$ where neither parental allele dropped out, indicating that the CNVs were somatic events occurring after fertilization. Thus, in order for a deletion to be called as a true de novo mutation the $\log 2$ ratio needed to be $<-0.75$. This resulted in a final set of $9 \mathrm{CNVs}$ being confirmed as de novo and 2 CNVs confirmed as germline mosaics (Table C12).

The spontaneous de novo deletion CNV frequency observed in our control mice $(2 \% ; 2 / 100)$ was comparable to previous estimates in the mouse (1\%) (Adewoye et al., 2015). The frequency of deletion CNVs in the treated group was $0.5-1 \%(1 / 83,0 / 98$; 1/181 total), and hence, there was no evidence for deletion induction (Table 7.1). In contrast, there was an enrichment of non-recurrent duplication CNVs in the treated group $(4 / 83,2 / 98 ; 6 / 181$ total) relative to the control animals $(0 / 100$; Figure 7.2 ; Table 7.1; Table C12). This result did not reach statistical significance; however, the spontaneous frequency of duplications appears to be rare compared to deletions, as none were detected in the 93 control animals from ref. (Adewoye et al., 2015) or in the 100 animals in this study. With the controls from the 2 studies combined, the duplication induction reaches statistical significance (Fisher's exact $\mathrm{P}=0.03$ ). These duplication events have potential phenotypic consequences as they can disrupt gene dosage and homeostasis. Furthermore, a duplicated sequence can be inserted within a gene and thus disrupt that gene's function (Table C12). Therefore, it is important to understand the nature of these BaP-induced duplications (tandem vs inserted) and to understand the mechanism for their formation.

We used sequencing to characterize the breakpoints of the CNVs. We found that 3 of the 6 duplication CNVs were insertions at other locations in the genome (different chromosomes). Two of these insertion CNVs were present in the same animal (Figure 
C16A), but on separate chromosomes (4 and 10), and hence, the total number of duplications was counted as 5 (Table 7.1). Sequencing revealed that the $2 \mathrm{CNVs}$ were joined together as an insertion into a long interspersed nuclear element (LINE: L1Md_T). The exact location of this insertion could not be determined due to the repetitive structure and abundancy of this LINE. The other insertion (Figure C16B) involved a region from chromosome 16 being copied into a satellite sequence (GSAT_MM) on another chromosome (most likely chromosome 2 based on the $97 \%$ sequence identity at the breakpoint). Remarkably, both breakpoints had nucleotide similarity to the satellite sequence indicating that normal replication of the satellite resumed after insertion of the chromosome 16 donor sequence. The other 3 duplication CNVs occurred in tandem (Figure C16C-D). One of the tandem duplications was a complex event involving a $28 \mathrm{bp}$ insertion from a region $16 \mathrm{~kb}$ upstream from the nearest breakpoint (Figure $\mathrm{C} 16 \mathrm{C}$ ). The orientation of the discordant mate-pairs also signified that this event was associated with an inversion, further highlighting the complexity of this mutation and the region where it occurred. Considering that the arrays are limited to detecting large CNVs associated with low complexity DNA (repetitive sequences), and CNV breaks were found within satellite DNA, it is probable that several CNVs of moderate size occurred in repetitive regions of the genome and were undetected.

All of the de novo CNVs with detectable breakpoints appear to have been mediated by overlaps consisting of short microhomologies of $1-6$ bp between sequence breakpoints (Table C12). These events are similar to the CNVs detected in irradiated human fibroblasts where 1-8 bp homologies were observed at most breakpoint junctions (Arlt et al., 2014). The CNVs with microhomology preclude involvement of homologous 
recombination repair (HRR) because the sequence homology required for HRR is $>200$ bp (Liskay et al., 1987). The model that best explains these mutation events is microhomology-mediated break-induced replication (MMBIR) (Hastings et al., 2009a; Hastings et al., 2009b), which is an alternative DNA repair pathway invoked to repair double-strand DNA breaks.

The results of this study and previous observations (Agam et al., 2010) suggest that deletions occur more commonly in the mouse germline and that duplication CNVs form infrequently in the absence of replication fork collapse near DNA damage. In this experiment, the source of fork collapse was likely a result of BPDE, a BaP metabolite that forms bulky DNA adducts (Bi et al., 2005). An excess of collapsed replication forks in the presence of BaP damage may lead to Rad51 depletion, inhibiting strand invasion into the homologous sequence required for HHR (Hastings et al., 2009a), leading to prefertilization CNVs in spermatogonia. Likewise, mature sperm lack homologous chromosomes to guide repair, leading to post-fertilization CNVs in the developing embryo. In lieu of a homologous template, the collapsed fork invades an active replication fork within physical proximity using the microhomology as a primer. This hypothesis is supported by previous observations using a bacterial model investigating BPDE adducts (Kokontis et al., 1988). Specifically, DNA segments containing BPDE adducts were more likely to be deleted or replaced with an alternative sequence ( $>42 \mathrm{bp}$ ) than segments without adducts (no alterations observed). Similar to the CNVs detected here, the breakpoints of the alterations showed microhomology (most often $2 \mathrm{bp)}$ ) and the alterations occurred independent of $\operatorname{Rec} A(\operatorname{Rad} 51$ homolog). Taken together, these 
findings suggest that BPDE adducts lead to fork collapse and instability, favouring MMBIR which copies DNA from another source at the site of fork collapse.

It is important to note the limitations of the de novo CNV analysis. The offspring used in this study came from 11 and 8 sires in the control and BaP groups, respectively. Due to the low number of meioses analyzed for CNVs in each sire, it is not possible to use the sire as the biological unit of measurement for CNV frequency because of the rarity of these events. Therefore, each meiosis was treated as an independent event and CNV frequencies were estimated for exposure group rather than for individual sires. Moreover, parental origin could only be assigned for 3 of the 8 de novo CNVs. Of these, all were paternal in origin. Thus, when comparing CNV frequencies between control and $\mathrm{BaP}$ animals, the assumption was made that all de novo $\mathrm{CNV}$ s came from the paternal genome. For this study inbred mice were used and there should have been no differences in CNV susceptibility. This is supported by the observation that the de novo CNVs were detected in multiple litters from different sires. In order to determine CNV frequencies for individual sires, single sperm analyses may need to be performed. Thus, further work is required to better understand the relationship between DNA adducts and CNV duplication/insertion frequencies.

\subsubsection{Germline and Somatic Mosaic CNVs}

Mosaicism, the presence of genetically unique cell populations in an organism, is beginning to be recognized as a contributing source for genetic disease (Campbell et al., 2014; Zlotogora, 1998). Mosaicism is produced from mutations that arise postzygotically and are distributed to different tissues in different proportions. We aimed to 
investigate variants in offspring that were not considered as unique de novo CNVs but rather germline mosaic CNVs in the parents (i.e., parental germ cells carry CNVs in a subpopulation of germ cells that were passed onto a portion of the siblings). We observed 2 instances of germline mosaic large deletions in the pre-meiotic $\mathrm{BaP}$ exposure group (Table C12). The first CNV was present in 2 male littermates and the second was present in 3 female littermates. The parental origin of the deletions could not be determined due to the lack of SNPs within the deletions. However, each deletion only appeared in 1 of the sires' 4 litters from separate dams (Figure 7.3), indicating that the deletion was likely maternal in origin. Interestingly, these deletions had a different mutation signature at the breakpoint than the de novo CNVs. Instead of microhomology at the breakpoint, there was a single nucleotide insertion, which is characteristic of non-homologous end-joining. This difference is intriguing and suggests that further investigations to determine if there are different mechanisms by which CNVs are generated during spermatogenesis and oogenesis are warranted.

In addition to germline mosaics, we also observed a high number of somatic mosaic deletion events in the offspring (Table C12). Based on quantitative PCR (qPCR) and aCGH alone, the somatic deletions originally appeared to be de novo events with a copy number of 1.19-1.37. However, the parental origin of these deletions could not be established by mate-pair sequencing due to the presence of both parental alleles within the deletion loci. The presence of both alleles suggests that the deletions occurred postzygotically and the deletions were thus characterized as somatic mosaics accordingly. Among the different groups, more deletions were somatic mosaics ( 5 total: 2 control, 3 $\mathrm{BaP}$ ) than de novo (3 total: 2 control, $1 \mathrm{BaP}$ ). The number of somatic events is likely 
higher because of the high degree of stringency of the algorithms and filters used to call CNVs. For example, there were 3 additional deletions confirmed to have a copy number of 1.5-1.6 by qPCR that were eliminated by filtering (results not shown). Overall, it appears that CNVs, especially large deletions, occur spontaneously at high rates during development. Single-cell analyses will be required to measure the true extent of CNV mosaicism and to further determine the contribution of environmental influences during in utero development.

\subsection{3 de novo SNVs and Indels}

Whole genome sequencing of family pedigrees followed by targeted resequencing of variants determined that 184 variants were true mutations (average 18 per $\mathrm{BaP}$ animal; 10 per control; Table C13; Figure C14). In total, 127 validated variants were de novo (average 12 per $\mathrm{BaP}$ animal; 7 per control; Table $\mathrm{C} 13$ ), 45 were early embryonic (average 2 per BaP animal, 1 per control; Table C14), 6 mutations were germline mosaics in the parents ( 3 mutations shared by 2 siblings), and 6 mutations were somatic mosaics in the parents. The spontaneous de novo mutation frequency for the mice used in this study was $2.9 \pm 0.8 \times 10^{-9}$, which is lower than other estimates using different

technologies (Adewoye et al., 2015; Lindsay et al., 2016) but is within range. The proportion of mosaic/embryonic mutations for SNVs/indels was approximately $25 \%$, which is highly consistent with previous measurements in mice with a larger sample size (Lindsay et al., 2016). Three de novo and 3 embryonic mutations were identified as nonsilent mutations within genes by Ensembl Variant Effect Predictor (McLaren et al., 2016) (4 treated; 2 control; Table C15). Haplotype phasing of the mutations, where possible, 
indicated that most mutations were paternal in origin (15 paternal, 3 maternal; Table C16).

Overall, the number of de novo mutations passed onto offspring was marginally higher in the fathers treated with $\mathrm{BaP}$ (45 mutations in 4 post-mitotic animals, 54 mutations in 4 pre-meiotic animals) compared with olive oil-treated controls (28 mutations in 4 animals) (1.6- to 1.9 -fold; $\mathrm{P}=0.057)$. However, $\mathrm{BaP}$ exposure elevated the total number of mutations (i.e., both embryonic and de novo) per offspring by 1.8-fold $(\mathrm{P}=0.009 ;$ Table 7.1; Figure 7.4; $68+67 \mathrm{BaP}$ vs 37 control mutations $)$. Taken together there is strong evidence that $\mathrm{BaP}$-induced sperm mutations were heritable and genomewide.

A large portion (6/8) of offspring in the $\mathrm{BaP}$-exposed group had a higher number of mutations than the mean number of mutations in controls. Moreover, there was a large degree of variation within the $\mathrm{BaP}$-treated group, with 4 of the mice being greatly affected by the exposure and the other 4 mice having relatively lower numbers of mutations (within range of control mutations). Analyzing the mutation spectra of the offspring with the highest numbers of mutations in the BaP group (i.e., the 4 mice above the mean of the exposed group: Figure 7.4) revealed that the extra mutations in these highly affected animals match the expected mutation profile of BaP (i.e., increased frequency of G:C $\rightarrow$ T:A, G:C $\rightarrow \mathrm{C}: \mathrm{G})$ observed in the sperm and bone marrow of exposed males (Fisher's Exact $\mathrm{P}=0.0002$ ) (Beal et al., 2015a; O'Brien et al., 2016b). Furthermore, there was also an increase in the number of G:C $\rightarrow$ A:T mutations in the affected $\mathrm{BaP}$ animals (Figure 7.4). We have shown previously that proportionally $\mathrm{G}: \mathrm{C} \rightarrow$ A:T mutations are lower in the cells of BaP-treated animals, but the absolute mutation 
frequency is increased (Beal et al., 2015a). These findings provide strong support that the additional mutation burden is due to the presence of the bulky BPDE adducts at guanine nucleotides, confirming the effects of $\mathrm{BaP}$ on both de novo and embryonic mutation induction. We estimate, based on previous measurements of BaP adducts (Verhofstad et al., 2010), that the dose and frequency of the exposure would lead to approximately 1000 bulky DNA adducts per genome. The detection of the additional BaP-type mutations indicates that several of these adducts elude DNA repair. Furthermore, the observation that only some animals were affected suggests that $\mathrm{BaP}$, and similar xenobiotics, are not evenly distributed among the germ cell population during exposure. Alternatively, different cells may respond differently to exposures, possibly through adaptive upregulation of DNA repair genes (Christmann et al., 2016). Hence, only a certain percent of animals would have $\mathrm{BaP}$-associated insertions/duplications or show an overall increase in genome-wide mutation count.

There was a high degree of variability in the number of de novo vs embryonic mutations that each animal in the $\mathrm{BaP}$ group received (Figure 7.4). This is because $\mathrm{BaP}$ adduct fixation can occur through different mechanisms that lead to different VAFs for each mutation: $\sim 0.25$ for embryonic mutations and 0.5 for de novo mutations (Figure 7.5). The timing and kinetics of the $\mathrm{BaP}$ exposure during spermatogenesis will dictate if the mutation fixation will occur before or after fertilization. For example, if the adduct fixation leads to a mutation prior to meiosis in the parental gamete, the mutation will have a VAF of 0.5 in the developing embryo and will be called as de novo. If the adduct is formed after meiosis, when there is no DNA replication and repair is declining, the resulting mutation will arise in the first embryonic division and will appear as embryonic 
with a VAF of 0.25 . However, segregation and distribution of the mosaic mutation could lead to varying VAFs in the animal and its adult tissues. Previous studies have shown that 3 cells from the 64-cell stage go on to form the entire animal (Markert and Petters, 1978). In these 3 cells, there would be 6 haploid genomes total ( 3 paternal, 3 maternal). Therefore, the VAFs for embryonic events could potentially be 0.17 (1/6), $0.33(2 / 6)$, or $0.5(3 / 6)$ when there are 1, 2, or 3 mutant cells that form the animal, respectively (Figure 7.5). These findings highlight the need to control for embryonic mutations when studying germline exposures in order to capture the full extent of the DNA damage and increase the power for detecting mutation induction.

Most of the highly affected animals in the BaP group (3/4) developed from sperm exposed during the post-mitotic phases of spermatogenesis. This further supports the hypothesis that DNA damage occurring in later phases of spermatogenesis can lead to mutation formation in the developing embryo. Interestingly, the animal with the most embryonic mutations (32CM1) had 12 BaP-type mutations in total with evidence of some clustered mutations. Specifically, this mouse had 3 G:C $\rightarrow$ T:A mutations (VAF: 0.180.21 ) within a $45 \mathrm{~kb}$ window on chromosome 12 and $2 \mathrm{G:C} \rightarrow \mathrm{T}: \mathrm{A}$ mutations (VAF: 0.31-0.35) within a $438 \mathrm{~kb}$ window on chromosome 17. Considering that the average spacing of mutations across all animals was $13 \mathrm{Mb}$, it is unlikely that these mutations occurred close together by chance alone (Besenbacher et al, 2016). Furthermore, the mutation signature of these events indicates that they were likely a result of $\mathrm{BaP}$ adducts, and the similarities in VAFs indicate that the mutations formed concurrently as that genomic region was replicated during early embryonic development. Previous observations suggest that the earliest cell divisions during embryogenesis have the 
highest spontaneous mutation rates compared to any other germline stage (Harland et al., 2016; Lindsay et al., 2016). The evidence presented here also suggests that these early stages represent a sensitive window by which DNA damage leads to genetic mosaicism in the developing organism.

The overall increase in mutation count in the BaP-exposed offspring was unexpectedly lower than the increase in mutation frequency observed in sperm following the exact same exposure (2-fold vs. 4-fold, respectively) (O'Brien et al., 2016a; O'Brien et al., 2016b). There are a few possibilities that could explain this: 1) The small number of meioses analyzed in the pedigree study versus the sperm work (4 meioses per group for pedigree study, $>100,000$ per group in sperm study) may lead to lower accuracy in the pedigree approach. 2) Negative selection against germ cells and embryos with the highest amounts of DNA damage decreased the number of detectable mutations in $\mathrm{BaP}$ offspring. 3) Mutation induction in the transgene analyzed in sperm does not provide an accurate representation for the mutation induction in the rest of the genome. This could be due to sequence or region biases that make this transgene a mutational hotspot. 4) A disproportionate amount of $\mathrm{BaP}$ mutations were missed compared to control using the WGS approach. This would most likely be due to the high probability of BPDE adducts forming in regions rich in CG nucleotides and are therefore difficult to sequence. However, a combination of technical issues with sequencing and stringent bioinformatics filtering cannot be ruled out. For example, indels accounted for $33 \%$ of the BaP mutations detected in sperm (O'Brien et al., 2016b), and only a few indels were detected in the genomes of the offspring. Based on the functional mutations observed in sperm, we estimated the total number of expected mutations in the genomes of each mouse from 
control and BaP-treated sires. In total, there were approximately 60 mutations detected in 11.5 Gb and 300 mutations in $10.9 \mathrm{~Gb}$ for control and BaP sperm, respectively. Assuming random distribution of mutations, there should be approximately 32 and 170 mutations per diploid genome in control and BaP mice, respectively. By this logic, 29\% of possible control mutations were detected by WGS but only $10 \%$ were detected in the $\mathrm{BaP}$ group. Thus, although we have shown that $\mathrm{BaP}$ induces genome-wide mutations, the discrepancy between expected and observed number of mutations suggests that the full extent of the damage induced by $\mathrm{BaP}$ remains uncertain. In the regions where mutations could be detected, specifically genic regions (Table C15), the 2-fold increase in mutation frequency could still negatively impact the health of the affected individual.

Similar to CNV analyses, there were limitations that prevented the estimation of mutation rate for each sire. Specifically, the work was somewhat confounded by litter, as multiple offspring per male were sampled. However, there was a large degree of variability between siblings, and there were no apparent litter effects. Indeed, there were instances where some offspring had extremely high numbers of mutations, and others were unaffected. Moreover, the males used were all inbred mice. Therefore, there are unlikely to be biologically relevant genetic confounders that would lead to differences in susceptibility to BaP between fathers. Thus, for the purposes of this study each offspring was analyzed independently. Although the measured increase in mutations per genome following $\mathrm{BaP}$ exposure shows a compelling biological response, future work is needed to understand the effects of chemical exposures on mutation rates.

\subsection{Conclusions}


We demonstrated that sub-chronic 28 day high-dose $\mathrm{BaP}$ exposure increases the frequency of SNVs and large duplications in affected individuals. The research demonstrates the promise offered by applying whole genome approaches to the study of heritable mutagenesis. Using our approach, we were able to detect mutations originating as DNA damage in mature sperm that are undetected by existing sperm assays (O'Brien et al., 2016a; O'Brien et al., 2014). The results confirm that mature sperm represent a sensitive window of spermatogenesis by which exposure to DNA damaging agents can lead to mutations in the embryo. A major finding from this study was that many of the heritable mutations are mosaics. Thus, caution is required in interpreting exposure-related mutations to ensure that the damage caused by the exposure is not underestimated through application of filtering approaches that eliminate such mutation events. Considering that men are exposed to low doses of $\mathrm{BaP}$ throughout their life (occupational exposure, tobacco smoke, etc.), there are potential human health considerations for this work. Men need to be aware that their lifestyle, long before family planning as well as in the weeks leading in to conception, is important for the future health of their children. 


\subsection{Tables and Figures}

Table 7.1 de novo mutations in the offspring of control and $\mathrm{BaP}$-treated animals.

\begin{tabular}{|c|c|c|c|c|c|c|}
\hline Mutation Type & Group & $\begin{array}{c}\text { Sample } \\
\text { Size }\end{array}$ & $\begin{array}{l}\text { Number of } \\
\text { Mutations }\end{array}$ & Mutations Per Offspring $(95 \% \mathrm{Cl})$ & $\begin{array}{l}\text { Ratio to } \\
\text { Control }^{1}\end{array}$ & $\begin{array}{c}\text { P- } \\
\text { Value }\end{array}$ \\
\hline \multirow[t]{4}{*}{ Large Deletions } & Control & 100 & 2 & $0.0200(0.0011-0.0744)$ & - & \\
\hline & Post-meiotic & 83 & 1 & $0.0120(0.0001-0.0716)$ & 0.60 & \\
\hline & Pre-meiotic & 98 & 0 & $0.0000(0.0000-0.0453)$ & 0.00 & \\
\hline & All BaP & 181 & 1 & $0.0055(0.0001-0.0338)$ & 0.27 & 0.289 \\
\hline \multirow[t]{4}{*}{ Duplications/Insertions } & Control & 100 & 0 & $0.0000(0.0000-0.0444)$ & - & \\
\hline & Post-meiotic & 83 & 3 & $0.0361(0.0080-0.1053)$ & 2.37 & \\
\hline & Pre-meiotic & 98 & 2 & $0.0204(0.0011-0.0759)$ & 1.71 & \\
\hline & All BaP & 181 & 5 & $0.0276(0.0101-0.0648)$ & 1.46 & 0.109 \\
\hline \multirow[t]{4}{*}{ de novo SNVs/Indels } & Control & 4 & 28 & $7.0000(4.6515-10.1170)$ & - & \\
\hline & Post-meiotic & 4 & 45 & $11.2500(8.2058-15.0534)$ & 1.61 & \\
\hline & Pre-meiotic & 4 & 54 & $13.5000(10.1416$ - 17.6146) & 1.93 & \\
\hline & All BaP & 8 & 99 & $12.3750(10.0578-15.0661)$ & 1.77 & 0.057 \\
\hline \multirow[t]{4}{*}{ Embryonic SNVs/Indels } & Control & 4 & 9 & $2.2500(1.0300-4.2700)$ & - & \\
\hline & Post-meiotic & 4 & 23 & $5.7500(3.6500-8.6250)$ & 2.56 & \\
\hline & Pre-meiotic & 4 & 13 & $3.2500(1.7300-5.5575)$ & 1.44 & \\
\hline & All BaP & 8 & 36 & $4.5000(3.1500-6.2250)$ & 2.00 & 0.109 \\
\hline \multirow[t]{4}{*}{ Total SNVs/Indels } & Control & 4 & 37 & $9.2500(6.5250-12.7500)$ & - & \\
\hline & Post-meiotic & 4 & 68 & $17.0000(13.2000-21.5500)$ & 1.84 & \\
\hline & Pre-meiotic & 4 & 67 & 16.7500 (12.9750 - 21.2750) & 1.81 & \\
\hline & All BaP & 8 & 135 & $16.8750(14.1250-20.0000)$ & 1.82 & 0.009 \\
\hline
\end{tabular}

${ }^{1}$ In the cases where the ratio was undefined (control rate $=0$ ) the upper limits of the $95 \%$ CI were used to estimate the ratio. 


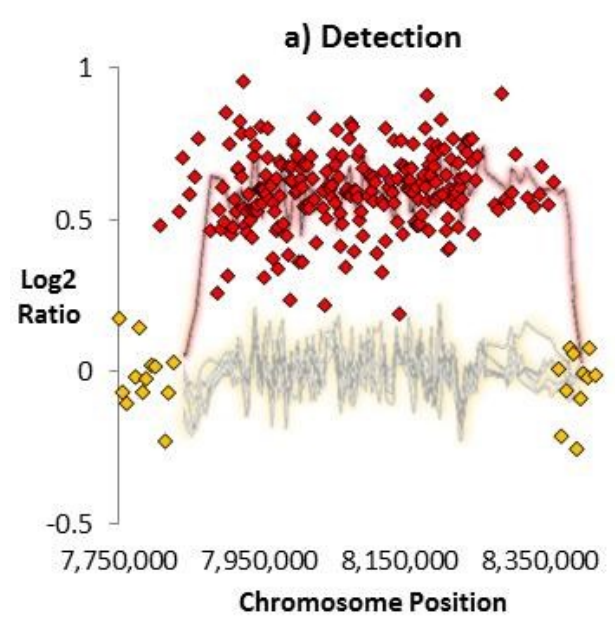

c) Parental Origin

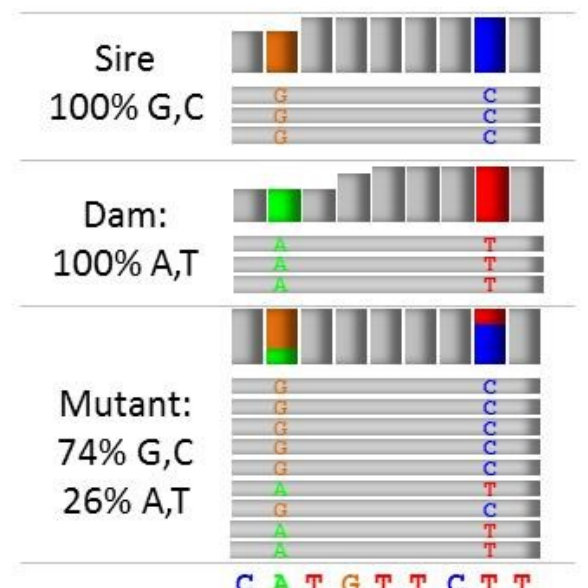

b) Validation

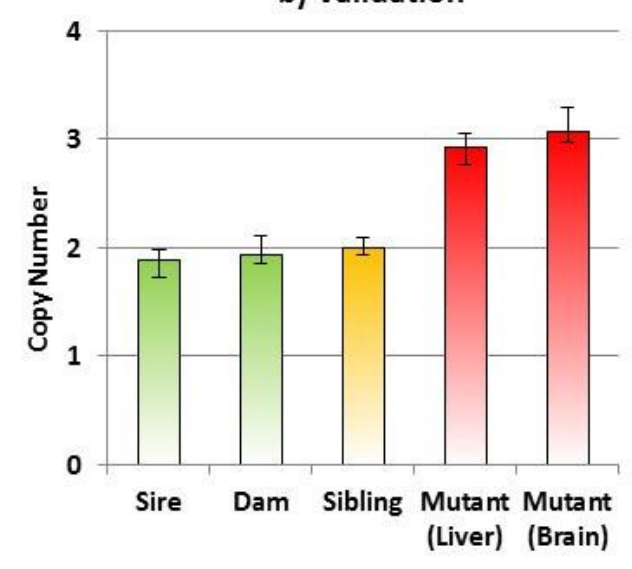

d) Breakpoint

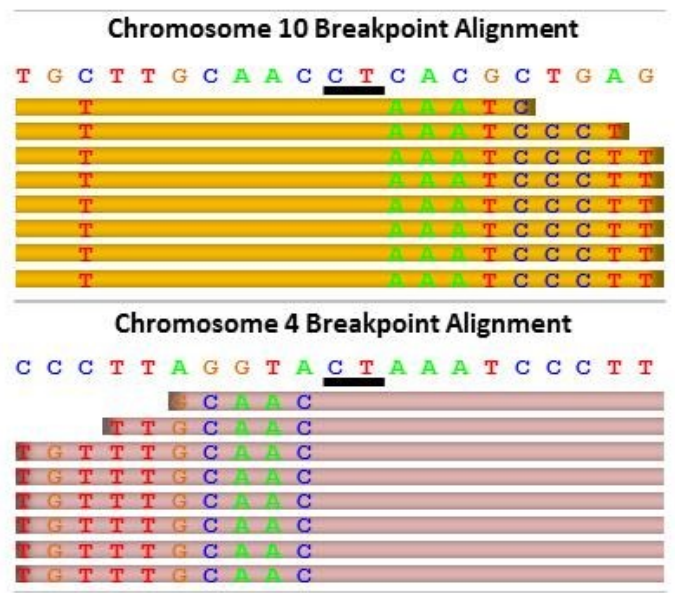

Figure 7.1 Identification and characterization of de novo CNVs. (a) Example of a 533-kb duplication. Red diamonds show the DNA probes with higher offspring signal (elevated Cy5 at CNV locus) and the yellow diamonds show the DNA probes where offspring DNA is the same quantity as the parental DNA. The red glowing line shows the average $\log 2$ ratio $(0.61)$ for the mouse with a $\mathrm{CNV}$ while the yellow glowing lines show the average $\log 2$ ratio for the 6 litter mates. The $\log 2$ ratio of 0 indicates no change in copy number. (b) The copy number measured by qPCR determined that the putative CNV was a true de novo event because it was present in multiple tissues with a copy number of 3 , 
and the CNV was not present in the parents or sibling. (c) Mate-pair sequencing revealed that the CNV came from the sire because the paternal SNPs were present at a higher allele fraction within the CNV locus. (d) Mate-pair sequencing also determined the breakpoint locations by mapping discordant mate-pairs against the genome (mapping at chromosome 10 in orange and 4 in pink). Split reads mapping to both chromosome locations simultaneously allowed for the breakpoint sequence to be determined. In this example, the CNV was facilitated by microhomology of 2 base pairs (CT). 


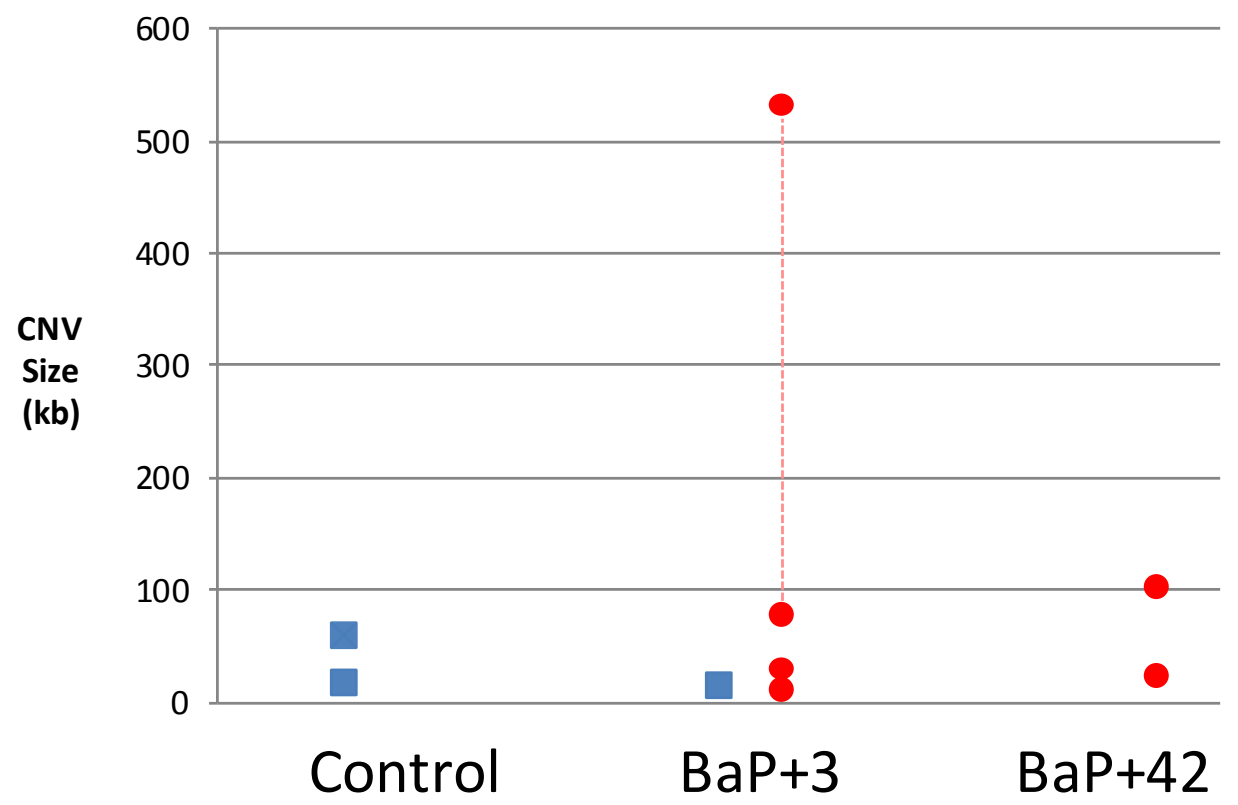

Figure 7.2 Size and types of de novo $\mathrm{CNVs}$ characterized in control and $\mathrm{BaP}$ animals.

Blue squares indicate deletions and red circles indicate duplications or insertions. The red dotted line signifies that the 2 insertion CNVs are from the same animal and were derived from the same mutation event. 

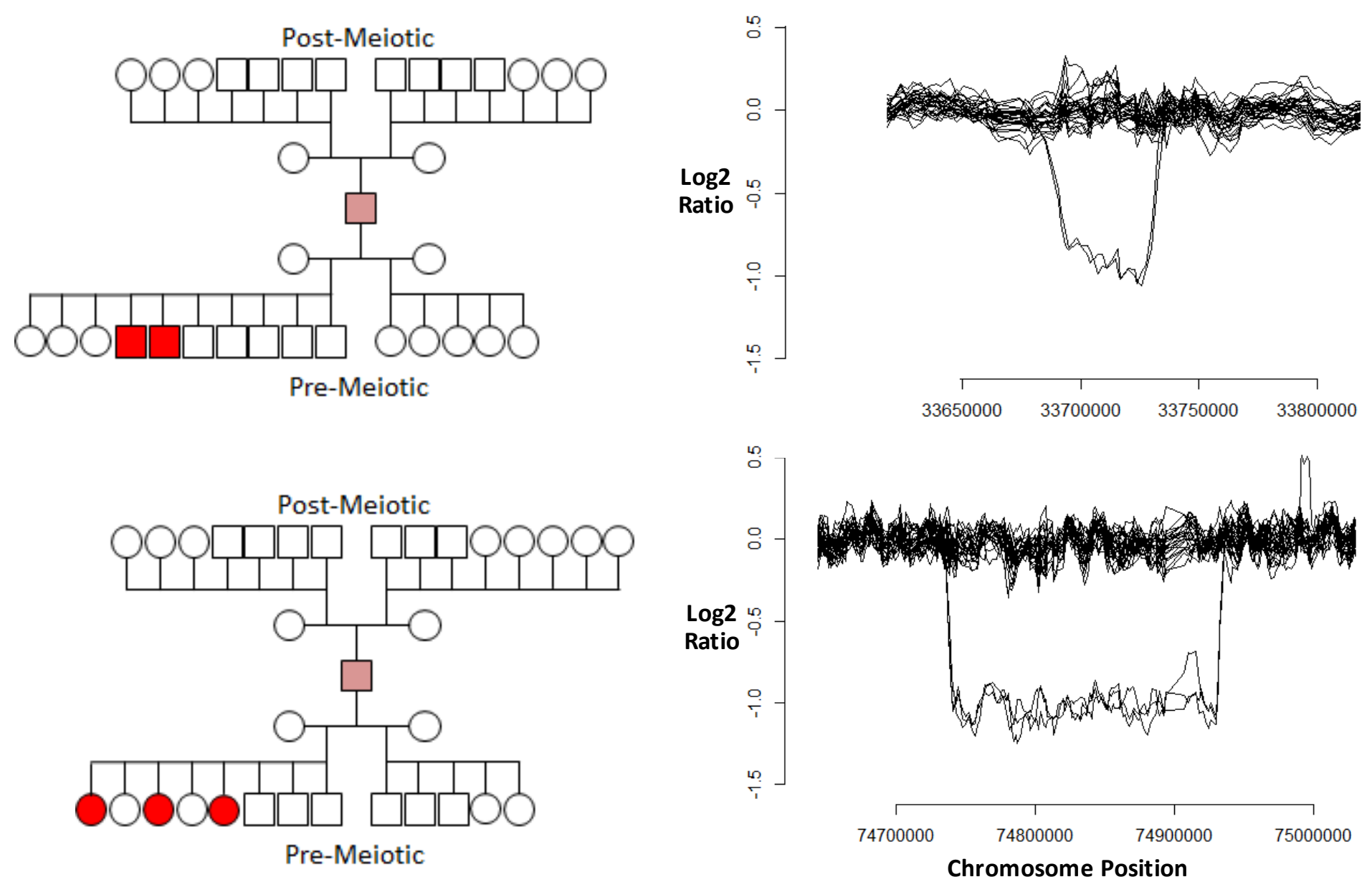

Figure 7.3 Germline mosaic CNVs detected in the BaP exposure group. The pink square indicates the sire that was exposed to BaP.

The bright red circles and squares indicate female and male offspring that inherited the CNV. The parental origin of the mosaic CNVs could not be determined because there were no unique SNPs in the locus. 

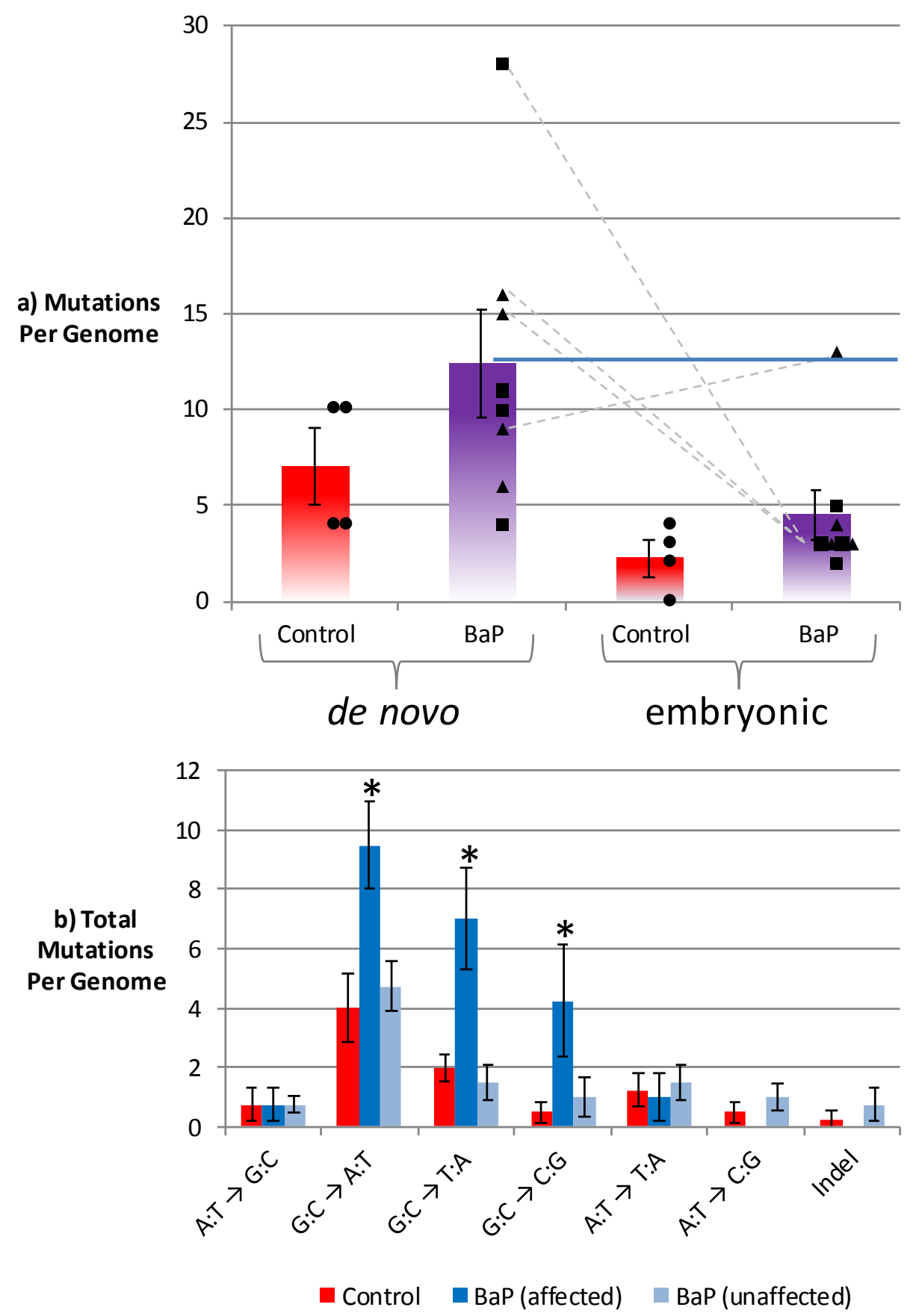

Figure 7.4 Mutation counts and spectra in control and BaP offspring as measured by WGS. A) Each circle, triangle, and square represents 1 offspring from the control, postmeiotic, and pre-meiotic groups, respectively. The dashed lines connect the embryonic 
and de novo mutations for the respective animals. For analytical purposes, we called the offspring above the dark blue line (above the mean of the $\mathrm{BaP}$-exposed group) as "affected" by BaP, and those below "unaffected." B) Separating the BaP offspring into 2 groups (affected, and unaffected) shows that the additional mutations in the affected mice match the mutation fingerprint of $\mathrm{BaP}(\mathrm{G}: \mathrm{C} \rightarrow \mathrm{T}: \mathrm{A}, \mathrm{G}: \mathrm{C} \rightarrow \mathrm{C}: \mathrm{G}$; Table C13; Table C14; Generalized estimating equations $\mathrm{P} \leq 0.0007)$. Furthermore, there is an increase in $\mathrm{G}: \mathrm{C}$ $\rightarrow$ A:T mutations. In our previous analyses, we observed a decrease in proportion of this mutation type, but the absolute mutation frequency is increased (Beal et al., 2015a; O'Brien et al., 2016b). Overall, the mutations induced by BaP occur at guanine nucleotides as expected. 


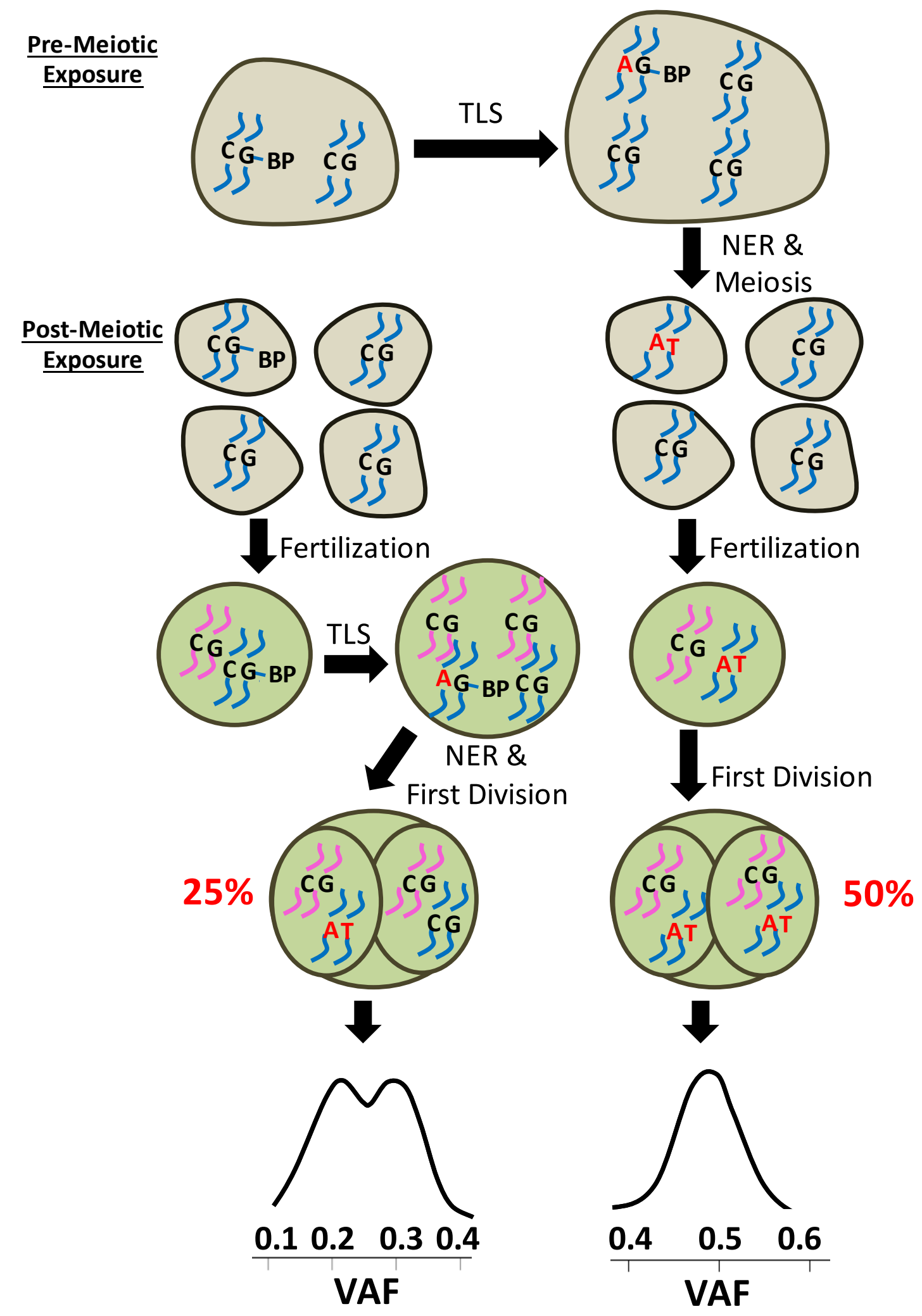


Figure 7.5 Mechanisms by which DNA adducts become embryonic or de novo mutations in the offspring. A pre-meiotic exposure to $\mathrm{BaP}$ can lead to BPDE adducts (G-BP) in dividing spermatogonia. Translesion synthesis (TLS) results in error-prone replication and mismatch pairing. On the right, NER will remove the bulky adduct and subsequent replication will lead to a mutation. If the sperm carrying that mutation fertilizes the egg the resulting embryo will have a paternal mutation with a VAF of 0.5 . This matches the VAFs for de novo mutations detected in the offspring (bottom density plot). On the left, a new adduct is induced post-meiosis and the sperm carrying the adduct can fertilize the egg. Similar to dividing spermatogonia, TLS and NER in the embryo will convert the adduct to a mutation. Due to the semi-conservative nature of replication, the resulting embryo will have both the mutation and wild-type sequence from the sire. Therefore, the VAF for the mutation will be 0.25 in the early embryo. However, previous studies have shown that only 3 cells from the inner cell mass of the embryo go on to form the entire animal (Markert and Petters, 1978), resulting in VAFs of approximately $0.2,0.3$, or 0.5 in the animal. This is consistent with the VAFs for embryonic mutations detected in the offspring (as shown in density plot). 


\title{
Chapter 8: From Sperm to Offspring: Assessing the Heritable Genetic Consequences of Paternal Smoking and Potential Public Health Impacts
}

\author{
Modifie d from: Beal, M.A., Yauk, C.L., Marchetti, F. 2017. Mutation Research
} Reviews, 773:26-50.

\subsection{Preamble}

$\mathrm{BaP}$ is a common environmental pollutant present in many environmental mixtures. This chapter presents a case study on the germ cell consequences of smoking tobacco, as it is among the most common routes of $\mathrm{BaP}$ exposure. This was done to emphasize that the $\mathrm{BaP}$-induced mutations detected in this thesis are relevant to human health. Furthermore, this chapter demonstrates that on a population level a slight increase in de novo mutation rate due to germ cell mutagens has staggering consequences in terms of disease burden and socio-economic costs.

\subsection{Abstract}

Background: Individuals who smoke generally do so with the knowledge of potential consequences to their own health. What is rarely considered are the effects of smoking on their future children.

Objectives: The objective of this work was to review the scientific literature on the effects of paternal smoking on sperm and assess the consequences to offspring. Methods: A literature search identified over 200 studies with relevant data in humans and animal models. The available data were reviewed to assess the weight of evidence that 
tobacco smoke is a human germ cell mutagen and estimate effect sizes. The results were used to model the potential increase in genetic disease burden in offspring caused by paternal smoking, with specific focus on aneuploid syndromes and intellectual disability, and the socioeconomic impacts of such an effect.

Discussion: Growing evidence suggests that tobacco smoking is associated with impaired male fertility, and increases in DNA damage, aneuploidies, and mutations in sperm. Studies support that these effects are heritable and adversely impact the offspring. At the population level, our model estimates that millions of smoking-induced mutations in sperm may be transmitted to offspring each generation and significantly contribute to the global burden of intellectual disability. Furthermore, paternal smoking is estimated to contribute to 1.3 million extra cases of aneuploid pregnancies per generation. Conclusion: The available evidence makes a compelling case that tobacco smoke is a human germ cell mutagen with serious public health implications.

\subsection{Introduction}

Smoking is a major societal health problem. Tobacco smoke contains more than 4000 compounds, of which at least 70 have been identified as carcinogenic (Hecht, 2003; Hecht, 2012; IARC, 2012a; US Department of Health and Human Services, 2010). Although the consequences of smoking are well characterized, the vast majority of studies have focused on effects in the smoker (Vineis et al., 2004), or trans-placental effects on the fetus in mothers who smoke (Hackshaw et al., 2011). However, there is also strong evidence that paternal preconception exposure to tobacco smoke can cause 
intergenerational effects through the germline (Secretan et al., 2009). This effect of paternal smoking is predominantly overlooked (Aitken et al., 2004).

Inherited germline mutations may have devastating impacts in the affected offspring and significant socio-economic repercussions at the population level. It is well established from animal studies that environmental exposures can increase mutation frequencies in germ cells (DeMarini, 2012). Accordingly, the presence of potential germ cell mutagens in the environment, such as tobacco smoke, could be contributing to the occurrence of sporadic genetic diseases in human populations through the induction of de novo mutations. Whole genome sequencing has enabled the unprecedented analysis of the de novo mutations that contribute to a variety of genetic diseases. For example, recent large-scale sequencing projects have revealed that there is a strong association between de novo germline mutations and sporadic neuropsychiatric disorders including schizophrenia and autism (Girard et al., 2011; Michaelson et al., 2012; O'Roak et al., 2011; Sanders et al., 2012; Xu et al., 2011; Yuen et al., 2015). There is also evidence that the number of de novo mutations in germ cells increases with paternal age (Kong et al., 2012; Rahbari et al., 2015; Sun et al., 2012). However, thus far there has been no effort to harness genomic tools to understand the environmental mediators of such de novo mutations in human cohorts.

The mutagenic effects of tobacco smoking on germ cells are not well characterized. In contrast, it is well established that tobacco smoke induces DNA damage and mutations in somatic cells (reviewed in (DeMarini, 2004; Husgafvel-Pursiainen, 2004)). Indeed, approximately $90 \%$ of lung cancers are attributed to tobacco smoking (Kiyosawa et al., 1990). Tobacco smoke was first suggested as a possible germ cell 
mutagen almost 4 decades ago (Bridges et al., 1979). Since that time, many studies have demonstrated the mutagenic effects of tobacco smoke on the germline in humans and in rodent models. In the most recent IARC monograph on tobacco smoke (IARC, 2012a) it was stated that "data suggest that smoking is likely a germ cell mutagen in humans." However, there is currently no governing agency that declares agents as human germ cell mutagens (DeMarini, 2012). It has been argued that an IARC-type of assessment may be suitable for identifying germ cell mutagens (DeMarini, 2012). Such an assessment would have important implications because it is estimated that approximately 800 million men worldwide smoke (Eriksen et al., 2012) and even more may be exposed to second-hand and third-hand smoke.

In this comprehensive review, we present the evidence that first-hand smoking affects sperm function and induces heritable genetic changes in male germ cells (Figure 8.1), and discuss the impact of this genetic damage on phenotypic consequences in the offspring. To meet this objective, a Scopus search (last retrieved June 30, 2016) was conducted on Life/Health Science articles using the criteria: "Tobacco" OR "Cigarette" OR "Smoke” OR "Smoking” AND "Sperm.” Additional searches were performed for data poor retrievals such as "Paternal Smoking" AND "Miscarriage" OR "Congenital" OR "Perinatal Mortality." Relevant data regarding the germ cell mutagenicity of smoking were extracted from over 200 manuscripts from approximately 1,000 manuscripts in the search. No restriction on date was applied and manuscripts dating back to 1974 were included in the analysis. Manuscripts were only included if they contained information on the relevant consequences of smoking and an effect size could be estimated for that consequence. Furthermore, manuscripts written in a language that could not be translated 
or interpreted by the authors were not included. In this case, the review only contained data from manuscripts written in English, French, Spanish, or German. The data are summarized in Table 8.1 and all of the data are presented in their entirety in Tables C17C19. Specifically, Table C17 summarizes tobacco effects on sperm parameters, Table C18 summarizes animal studies investigating tobacco, while all the human evidence that tobacco smoke is a germ cell mutagen are summarized in Table C19. In addition, manuscripts with relevant information that did not fit into any of the tables are also discussed. Finally, we used the extracted information to evaluate the potential socioeconomic ramifications of tobacco-induced mutations and show that modest increases in global germline mutation rates caused by smoking have severe consequences.

\subsection{Human Spermatogenes is}

Spermatogenesis is estimated to last $>70$ days in adult humans (Amann, 2008; Hess and de Franca, 2009) and consists of 3 phases: mitotic, meiotic, and post-meiotic. Spermatogenesis begins in the seminiferous tubules of the testes with a low number of self-regenerating spermatogonial stem cells (Figure 8.2). A portion of these mitotic stem cells differentiate into spermatogonia that proliferate through mitosis and are sensitive to replicative errors such as SNVs and indels. Spermatogonia undergo a final round of DNA synthesis and divide to form 2 spermatocytes that enter meiosis. During meiosis, recombination between homologous chromosomes occurs; thus, chromosomal alterations and aneuploidies are more likely to occur in this cell type than SNVs or indels. Spermatocytes undergo 2 rounds of meiotic divisions to produce 4 haploid spermatids. DNA in these post-meiotic spermatids becomes highly condensed when histones are 
replaced with protamines. After major morphological changes and maturation in the seminiferous tubules, spermatids are released to the epididymis and stored in the cauda as mature spermatozoa prior to ejaculation. DNA repair declines throughout spermatogenesis and any DNA lesions occurring in spermatids or spermatozoa will carry over into fertilization. A more detailed description of spermatogenesis can be found in Refs. (Hermo et al., 2010; Hess and de Franca, 2009; Oatley and Brinster, 2012).

Throughout a male's reproductive life spermatogenesis can be greatly impaired by external perturbations (Beal and Somers, 2013). Men continually produce sperm as they age and during this time the different germ cell types can be adversely affected by toxicant exposure. There is a common misconception that germ cells are protected from toxicants by the blood-testis barrier formed by tight junctions between Sertoli cells (Smith and Braun, 2012) (Figure 8.2). However, stem cells and differentiating spermatogonia are on the open side of the barrier and are therefore unprotected (Yauk et al., 2015a). Exposure and genotoxicity to stem cells is of particular concern, as genetic alterations in these cells will be permanent, leading to an accumulation of mutations in the germ cell population that can be passed onto the next generation. Although the other germ cell types are on the closed side of the barrier, the vast majority of toxicants can bypass the barrier and reach germ cells (Yauk et al., 2015a). Therefore, the blood-testis barrier is permeable to many xenobiotics leaving sensitive spermatogenic cells open to some exposure. This permeation of toxicants is troublesome because DNA repair declines during the final phases of spermatogenesis and induced DNA damage can persist in sperm leading to subfertility or genetic alterations in the zygote (Marchetti and Wyrobek, 2008). 
There is growing but controversial evidence that male fertility is declining (Rolland et al., 2013), and environmental factors may be associated with this decline. Environmental exposures can affect male fertility by 1) causing a decline in semen parameters such as sperm count, motility etc., and/or 2) damaging the genetic integrity of the germ cells. In the following sections, we will present and discuss the evidence that tobacco smoking is affecting both of these aspects of male germ cell function.

\subsection{Male Smoking and Fertility}

Infertility is a common issue in couples attempting to conceive, with $15 \%$ of couples presenting infertility, half of which are due to male factors (Poongothai et al, 2009). It is estimated that nearly $7 \%$ of men are either subfertile or infertile, and genetic abnormalities account for only $\sim 15 \%$ of these cases (Lotti and Maggi, 2015). Whether tobacco smoke and other environmental factors contribute to the development of male infertility is an area of active research. Approximately 60 studies report that smoking impairs seminal parameters (Table 8.1, C17, and C18) and thus tobacco smoking is considered a male infertility risk factor (reviewed in (Mostafa, 2010; Vine, 1996)). Specifically, epidemiology studies demonstrate, using microscopy (World Health Organization, 1999) or computer assisted sperm analysis, that the effects of cigarette smoke (or chewing tobacco (Said et al., 2005)) include diminished semen volume, sperm motility, count, morphology, etc. (Li et al., 2011; Mostafa, 2010). The reported effects are modest, with reductions in sperm concentrations averaging $13-22 \%$ among studies (Practice Committee of the American Society for Reproductive Medicine, 2012; Vine et

al., 1994), and are observed mostly in cohorts with healthy smoking subjects (Marinelli et 
al., 2004). Nevertheless, these modest reductions in sperm quality are an indication that tobacco smoke components bypass the blood-testis barrier and exert toxic effects in sperm.

Despite the acknowledgement of smoking as a male infertility risk factor, the relationship between smoking and fertility in humans is not clear as discrepancies exist between studies (Table 8.1 ). For example, approximately $50 \%$ of the data entries for total sperm count show no effect (Table C17). However, there are $\sim 25$ studies reporting large impairments ( $>50 \%$ change) in sperm parameters (Table C17). Rubes et al. 1988 analyzed the sperm of healthy 18-year old military registrants in the Czech Republic and, by controlling for age and other confounding variables, found a 2.2-fold decrease in sperm concentration in smokers. Fold change here and throughout the review refers to the relative difference between smokers and controls. In addition, these impaired sperm parameters were associated with an increased frequency of aneuploid sperm (discussed in a later section). Yu et al. 2013 showed a 1.7-fold decrease in sperm concentration for smokers. Interestingly, within this study, there was no difference observed between smokers and non-smokers with the wild-type $N R F 2$ genotype (which confers protection against oxidative damage (Ma, 2013)). In contrast, a smoking-associated reduction in sperm count was detected when the analysis was limited to men carrying the rs6721961 TT functional polymorphism of NRF2. This suggests that reductions in sperm count and fertility may be related to oxidative damage and apoptosis caused by cigarette smoke (Opuwari and Henkel, 2016) (discussed in more detail below).

It is important to note that many of the studies with no effect either do not report smoking behaviours of study participants or fail to include heavy smokers $(\geq 20$ 
cigarettes/day). In the studies that do show an effect, it is most often only significant in the heavy smoker category. Thus, there are dose-dependent decreases in functional sperm parameters with increasing smoking behaviour. Interestingly, this dose relationship may reach saturation at approximately 20 cigarettes/day as Lewin et al. 1991 saw no further impairment to sperm concentration in the heaviest of smokers (40-80 cigarettes/day; 3060 pack-years). Hence, the degree to which an individual smokes is an important consideration when studying the effects of smoking on fertility and the germline.

In addition to impaired sperm quality, male smoking reduces pregnancy success rates by up to $44 \%$ among couples undergoing in vitro fertilization (Joesbury et al., 1998; Zitzmann et al., 2003). Also, male smoking is associated with delayed conception in couples planning to have children. For example, 1 study showed that men who smoke have lower odds of conceiving by 6 months than non-smokers, with heavy smokers ( $\geq 20$ cigarettes per day) being less likely to conceive even after 12 months (Hull et al., 2000). It has not yet been determined if conception impairment in humans is due to maternal second-hand smoke exposure (Neal et al., 2005) or pre-zygotic genetic DNA damage in sperm that hinders embryo development (Joesbury et al., 1998); however, studies that use biomarkers to control for maternal second-hand tobacco smoke exposure may be able to address this issue.

Animal studies (Table 8.1 and C18) have been useful in differentiating between the contributions of paternal and maternal smoke exposure as well as eliminating other confounding factors. For example, rodents exposed to tobacco smoke for 2-10 weeks have impaired sperm parameters, including a 1.2-1.7 fold reduction in sperm count and 1.0-3.3 fold increase in morphologically abnormal sperm (Abdul-Ghani et al., 2014; 
Chen and Jiang, 2015; La Maestra et al., 2015; Sankako et al., 2012; Sankako et al., 2013; Yamamoto et al., 1998). These impaired sperm parameters correspond with 1.1-4.5 fold reduction in fertility (Kapawa et al., 2004; Yamamoto et al., 1998) and a delayed embryo development rate (Kapawa et al., 2004; Polyzos et al., 2009). Apart from rodent studies, 1 study measured sperm function in 4 rhesus monkeys exposed to environmental tobacco smoke, but the researchers did not observe a treatment effect with the small sample size used (Hung et al., 2009). However, the results of rodent studies show consistent evidence that tobacco smoke is damaging to sperm parameters and fertility (Table 8.1).

Overall, it is clear that smoking tobacco, or at least heavy smoking, can have detrimental effects on sperm quality and male fertility. The mechanisms underlying this pathophysiology are likely associated with the cytotoxicity of tobacco smoke. However, these smoking associated fertility problems may also be a result of DNA damage in sperm that reduces the number of viable sperm through apoptosis (Belcheva et al., 2004; Collodel et al., 2009; El-Melegy and Ali, 2011). Thus, the tobacco-associated impairment in fertility may be indicative of the genotoxic effects smoking can have in sperm.

\subsection{Evide nce that tobacco s moke induces DNA damage in sperm}

Genotoxic agents induce a variety of genetic damage in sperm including DNA adducts, cross-links, single- or double-strand breaks, chromosomal aberrations, and aneuploidies. Failure to properly repair damaged DNA in sperm leads to de novo mutations, which can be passed onto the next generation. Mutation types range from SNVs and indels to large-scale structural variation. Whole genome sequencing studies of 
family pedigrees are showing that on average humans carry approximately 60 de novo point mutations (Kong et al., 2012; Rahbari et al., 2015) and 0-3 indels (Kloosterman et al., 2015). The majority of these mutations arise in the male germline and the number passed on increases with paternal age (Francioli et al., 2015; Kong et al., 2012; Rahbari et al., 2015; Sun et al., 2012). Similarly, structural chromosomal alterations with nonrecurrent breakpoints also have a paternal origin bias (Hehir-Kwa et al., 2011), but occur less frequently with an incidence of $\leq 3 \%$ per live births (Adewoye et al, 2015; Conrad et al., 2009; Itsara et al., 2010; Sebat et al., 2007; Xu et al., 2008). It is possible that the frequency of structural variation is actually higher in germ cells because many of these events will not be viable and therefore go undetected. Overall, the degree to which exposure to genotoxic agents influences variation in germline mutation rates is unknown. As will be apparent in this section, components of tobacco smoke have access to germ cells where they cause most of the types of DNA damage (Table C18 and C19) that are known to be causative of different types of mutations.

Different constituents of tobacco smoke cross the blood-testis barrier (Pacifici et al., 1995; Pacifici et al., 1993; Vine et al., 1993; Zenzes et al., 1999a), thereby allowing DNA damaging agents access to the genomes of germ cells at all phases of spermatogenesis. Exposure to mutagenic PAHs following smoking is evident by increases in the formation of DNA adducts in sperm. Antibody staining for BPDE adducts (one of the main adducts formed by tobacco smoke) (Perrin et al., 2011; Zenzes et al., 1999a) and ${ }^{32} \mathrm{P}$-postlabelling (Horak et al., 2003) reveals a 1.2-7.6 fold increase in bulky DNA adduct formation in the sperm of men who smoke. Smoking also increases the amount of 8-oxo-dG lesions by 1.2-5.2 fold in sperm (Fraga et al., 1996; Hammadeh 
et al., 2010; Ji et al., 2013; Kumar et al., 2015; Shen et al., 1999; Shen et al., 1997). In support of these findings, men who smoke have higher levels of oxidative stress (Fariello et al., 2012; Gautam et al., 2015; Ghaffari and Rostami, 2012; Hamad et al., 2014; Hammadeh et al., 2010; Kiziler et al., 2007; Opuwari and Henkel, 2016) and ROS (Gautam et al., 2015; Hammadeh et al., 2010; Kiziler et al., 2007; Kumar et al., 2015; Saleh et al., 2002; Taha et al., 2014; Taha et al., 2012) in sperm. Furthermore, smoking reduces levels of key antioxidants and impairs cellular defence against oxidative damage (Elshal et al., 2009; Fraga et al., 1996; Kiziler et al., 2007; Mostafa et al., 2006; Pasqualotto et al., 2008; Viloria et al., 2010; Zhang et al., 2000), indirectly contributing to oxidative stress and DNA damage. These DNA lesions caused by tobacco smoke can lead to mutations if not properly repaired pre- and/or post-fertilization.

Tobacco smoke has also been linked to DNA strand breaks as demonstrated using several methods to assess DNA fragmentation in sperm (Andrabi, 2007). The majority of human studies $(63 \%)$ that used the TUNEL assay (terminal deoxynucleotidyl transferase dUTP nick end labeling) (Gavrieli et al., 1992) show that the sperm of smokers have 1.24.3 times the amount of DNA fragmentation of non-smokers (Aydin et al., 2013; Hammadeh et al., 2010; Potts et al., 1999; Sepaniak et al., 2006; Sun et al., 1997). The majority of results (75\%) obtained using the comet assay in humans and rodents (Antoniassi et al., 2016; Garcia et al., 2012; La Maestra et al., 2015; Ostling and Johanson, 1984; Polyzos et al., 2009; Singh et al., 1988) are concordant with these findings, indicating that smoking increases DNA damage in humans by 1.3-5.0 fold (Fariello et al., 2012; Linschooten et al., 2011) and 1.1-9.2 in rodents (Garcia et al., 2012; La Maestra et al., 2015; Polyzos et al., 2009). Furthermore, DNA integrity may be 
compromised in smokers relative to non-smokers (Elshal et al., 2009; Gautam et al., 2015; Kumar et al., 2015) as measured by the Sperm Chromatin Structure Assay (SCSA) (Evenson et al., 1980; Evenson et al., 1999), but several studies do not support this observation (Bojar et al., 2013; Hsu et al., 2006; Jurewicz et al., 2013; Rubes et al., 1998; Rybar et al., 2009; Smit et al., 2010; Spano et al., 1998). A more recent method for studying DNA fragmentation called the Sperm Chromatin Dispersion test (Fernandez et al., 2003) has also been used to show that smoking increases DNA fragmentation 1.1-2.1 fold (Anifandis et al., 2014; Omran et al., 2013; Viloria et al., 2007). A variety of other studies using additional methods support that DNA fragmentation is increased in the sperm of smokers (Cui et al., 2016; El-Melegy and Ali, 2011; Mitra et al., 2012; Taha et al., 2014; Taha et al., 2012; Tawadrous et al., 2011). Therefore, the available data provide clear evidence that tobacco smoking induces DNA strand breaks in sperm.

The evidence that smoking increases DNA damage suggests that smoking may cause chromosomal aberrations in germ cells. However, only a single study has so far examined the sperm of smokers for chromosomal aberrations. Specifically, Lähdetie et al. 1986 examined round spermatids of smoking and non-smoking men for the presence of micronuclei, but the number of round spermatids that could be visually inspected was low and no statistical increase in smokers was detected. Repeating this experiment with modern techniques (such as flow cytometry) and larger sample sizes may help to determine whether tobacco smoke increases the incidence of micronuclei in sperm as it has been demonstrated in somatic cells (Bonassi et al., 2003; Stoichev et al., 1993). It is critical to note that the DNA lesions in sperm produced by tobacco smoke may persist to fertilization when the sperm are still viable. For example, 1 study found that the embryos 
formed from fertilization with sperm from smoking men have higher levels of DNA adducts (Zenzes et al., 1999b), consistent with what was seen in sperm (Zenzes et al., 1999a). This result suggests that smoking may be able to cause DNA lesions that can be transmitted to the zygote where they have the potential to disrupt embryonic viability or induce mutations if not properly repaired by the egg.

\subsection{Evide nce that tobacco s moke induces ane uploidy in spe rm}

Aneuploidy is the presence of an incorrect chromosome number, and these events represent 1 of the most common types of heritable chromosomal abnormalities, occurring in approximately $0.3 \%$ of live births and $5-25 \%$ of human zygotes (Hassold and Hunt, 2001). Aneuploidies are more commonly a result of increased maternal age (Hassold et al., 2007). However, the paternal contribution to sex chromosome aneuploidies is substantial (nearly 50\%) (Hassold and Hunt, 2001) and there is growing evidence for the existence of aneuploidy-inducing agents (aneugens) that affect male germ cells (Robbins et al., 2005; Wyrobek et al., 2005). As aneuploidies disrupt gene dosage and homeostasis, most aneuploidies are lethal and will lead to either spontaneous abortions or stillbirths (Hassold and Hunt, 2001). However, aneuploidies of the sex chromosomes and some autosomal trisomies, mostly 13, 18, and 21 are compatible with life. These viable aneuploidies are associated with serious developmental disorders.

The development of Fluorescence in situ Hybridization (FISH) techniques (Levsky and Singer, 2003) has allowed the investigation of environmental and occupational factors that affect aneuploidy in sperm (reviewed in (Wyrobek et al., 2005)). Using these techniques, tobacco smoke has been demonstrated to induce aneuploidy in 
male germ cells (Faure et al., 2007; Härkönen et al., 1999; Naccarati et al., 2003; Pereira et al., 2014; Robbins et al., 1997; Rubes et al., 1998; Shi et al., 2001). For example, Pereira et al. (2014) found that the sperm of smokers are twice as likely to have an extra chromosome 3, and Härkönen et al. (1999) found that smoking is associated with a 1.3fold increase in chromosome 1 and 7 disomies in sperm. More importantly, several studies have investigated whether tobacco smoking induced aneuploidy for those chromosomes that are compatible with life. The results showed that smoking increases the incidence of all of these aneuploidies in sperm. Specifically, smoking increases the risk of disomy 13 (Shi et al., 2001), 18 (Robbins et al., 1997) and 21 (Faure et al., 2007) in sperm by 3.0-, 1.6-, and 2.2-fold, respectively. In addition, sperm with multiple sex chromosomes, including $\mathrm{X}-\mathrm{X}, \mathrm{Y}-\mathrm{Y}$, and $\mathrm{X}-\mathrm{Y}$ disomies, are more likely to occur in smokers (Naccaratiet al., 2003; Pereira et al., 2014; Robbins et al., 1997; Rubes et al., 1998). Overall, the current evidence supports that tobacco smoke is not only a germ cell mutagen, but an aneugen as well.

The consistent aneugenic effect of tobacco smoking for the chromosomes that have been investigated by sperm FISH suggests that it likely induces aneuploidy for all 22 autosomal chromosomes and the sex chromosomes. Based on the available data, smoking men have an approximate 2 -fold increase in total aneuploid sperm. It is estimated that $\sim 0.15 \%$ of live births have an autosomal trisomy (Hassold et al., 1996) and 5-10\% of these are of paternal origin (Hassold and Hunt, 2001), which translates to $\sim 0.01 \%$ of live births with paternally-derived autosomal aneuploidies. Studies in rodents have shown little evidence for selection against aneuploid sperm at fertilization (Cattanach and Moseley, 1973; Epstein and Travis, 1979; Ford, 1972; Marchetti et al., 
1999); therefore, an increased frequency in aneuploid sperm is expected to proportionally increase the rate of aneuploid conceptuses of paternal origin. The sperm FISH data suggest that $\sim 0.02 \%$ of children born to men who smoke would carry an abnormal number of autosomal chromosomes. If smokers fathered an average of 2 children each this could in theory lead to approximately 160,000 extra cases of children with autosomal aneuploidy globally $(800$ million men smoke $\times 2$ children $/$ man $\times 0.01 \%$ extra aneuploidies/child). By the same logic, paternal smoking could lead to an extra 1.2 million cases of sex chromosome aneuploidies as these aneuploidies are present in $0.15 \%$ of live births (Hassold et al., 1996) and approximately half are paternal in origin. Therefore, the aneugenic activity of tobacco smoking poses a severe hazard, similar to the hazard posed by advancing maternal age, to the health of the next generation that should not be overlooked.

\subsection{Evidence that tobacco smoke induces germline mutations}

The incidence of de novo mutations in human populations and the corresponding contribution to disease development is becoming increasingly apparent. For example, the Human Gene Mutation Database (Cooper and Krawczak, 1996; Stenson et al., 2009) now reports over 170,000 mutation entries in more than 7000 genes. Animal studies provide convincing evidence that tobacco smoke exposure directly induces germline mutations and therefore smoking may be a contributing factor to the incidence of inherited genetic disease. The earliest study by Stoichev et al. (1993) determined that mice exposed to either 8 or 16 cigarettes for 8 weeks passed on more lethal mutations when mated to unexposed females. More recently, Yauk et al. (2007) found that exposure of mice to the 
mainstream tobacco smoke of 2 cigarettes/day for 6 and 12 weeks causes 1.4- and 1.7fold increases in mutation frequencies in exposed spermatogonial stem cells at ESTRs, respectively. The data from this paper and from work on other air pollutants (Yauk et al., 2008b) support that longer periods of exposure lead to accumulating mutations in spermatogonial stem cells. Marchetti et al. (2011) found that mouse spermatogonial stem cells exposed to the mainstream tobacco smoke of 3 and 16 cigarettes/day for 2 weeks exhibit 2.6- and 3.1-fold increases in mutation frequency, respectively. Furthermore, Marchetti et al. (2011) showed that exposure to sidestream tobacco smoke, the major component of second-hand smoke, from 3 or 16 cigarettes per day causes a 3.7- and 2.1fold increase in spermatogonial stem cell mutations at tandem repeat loci, respectively. In addition to the work with rodents, a small study in humans showed that men who smoked in the 6 months before pregnancy are 4 times more likely to pass on a tandem repeat minisatellite mutation to their children than non-smokers (Linschooten et al., 2013). Although this result was marginally significant and requires replication using a larger cohort, it supports the idea that tobacco-induced mutations detected in sperm are being passed onto the offspring. Dividing the families into different groups: non-smokers $(5.3 \%$ of children inherited a mutation), irregular smokers (19\% had a mutation), and daily smokers (33\% had a mutation), suggested that the effects observed in humans are dosedependent.

Most of these studies used non-coding tandem repeat DNA markers to quantify germline mutation induction because these loci are highly mutable sites. However, there is growing evidence that mutations in other tandem repeat loci associated with genes can have negative phenotypic consequences (Armour, 2006; Mirkin, 2007; Mori et al., 2013). 
In addition, exposures that cause tandem repeat mutations are also known to induce other types of mutations (i.e., chromosomal abnormalities, point mutations). Therefore, tandem repeat mutations can be viewed as biomarkers of genetic insult in germ cells that can be transmitted to progeny. It is likely that tobacco smoke exposure is also inducing other types of mutations in germ cells (i.e., base substitutions, indels, etc.) and research using new genomics tools to measure these endpoints in smokers and their offspring is a clear priority.

\subsection{Evide nce that tobacco smoke induces genome ins tability}

Recent evidence suggests that tobacco smoke causes genomic instability through the germline. Genomic instability is defined as a cell lineage having higher mutation frequencies than normal. Laubenthal et al. (2012) found that paternal preconception smoking is a significant predictor for DNA damage in the cord blood of children. Specifically, the cord blood of children whose fathers smoked has higher levels of singlestrand and double-strand breaks than children of non-smokers (Laubenthal et al., 2012). The role of second-hand smoke exposure on this genomic instability remains unclear. However, the results of this study provide the first evidence that tobacco smoke may be inducing genetic changes in the sperm leading to genomic instability that persists after fetal development.

It is possible that the genetic instability described above may be due to smokinginduced epigenetic changes. There is evidence that that tobacco smoke alters microRNA expression in sperm (Marczylo et al., 2012) and methylation patterns in other cell types (Besingi and Johansson, 2014; Guida et al., 2015; Joubert et al, 2012; Shenker et al., 
2013; Steenaard et al., 2015; Zeilinger et al., 2013), some of which can even persist after smoking cessation (Guida et al., 2015). Moreover, in utero smoking alters methylation patterns in the cord blood of newborns (Joubert et al., 2012). There is also evidence that epigenetic changes may be responsible for subtle changes in the offspring that are not immediately apparent until later in life. Specifically, Axelsson et al. (2013) observed that men born to a smoking father and non-smoking mother have a $46 \%$ reduction in total sperm count. In addition, boys whose fathers smoked at an early age have been observed to have high Body Mass Indices (BMI) (Pembrey et al., 2006). An 8-year follow-up of the same cohort showed that this increased BMI association becomes more pronounced with age (Northstone et al., 2014). The effect of epigenetic changes in sperm on offspring is a rapidly growing area of research and further investigations into epigenetic-mediated mechanisms of tobacco smoking-induced instability on the germline are warranted.

\subsection{Preconception paternal smoking and potential phe notypic conse quences}

There is growing evidence that preconception paternal smoking may be a risk factor for different childhood diseases. Ongoing research has specifically investigated whether there is an association between male smoking patterns before conception and the development of cancer in their children (reviewed in (Chang, 2009)). Several casecontrol studies have found that pre-conception paternal smoking increases the odds of children developing different types of cancers including acute lymphoblastic leukemia (Chang et al., 2006; Ji et al., 1997; John etal., 1991; Milne et al., 2012; Rudant et al., 2008; Shu et al., 1996; Sorahan et al., 2001), acute myeloid leukemia (Chang et al., 2006; Rudant et al., 2008), non-Hodgkin's lymphoma (Rudant et al., 2008), rhabdomyosarcoma 
(Grufferman et al., 1982), central nervous system tumours (McCredie et al., 1994;

Plichart et al., 2008), and hepatoblastoma (significant when both parents smoked prior to conception) (Pang et al., 2003) (Table 8.1). Furthermore, a few studies analyzed cases with non-specific cancer types and found a significant association between paternal smoking and cancer risk in the children (Edraki and Rambod, 2011; Ji et al., 1997; John et al., 1991). In contrast, several studies show only a weak or no association between specific cancer types in offspring and paternal smoking (Brondum et al., 1999; Gold et al., 1993; MacArthur et al., 2008; Magnani et al., 1990; Menegaux et al., 2007; Ruckart et al., 2013; Schüz et al., 1999). Thus, we emphasize that the link between paternal smoking and childhood cancer is still controversial. However, 4 separate meta-analyses have been conducted and show that paternal smoking significantly increases the risk of childhood cancer development (Boffetta et al., 2000; Lee et al., 2009; Liu et al., 2011; Milne et al., 2012). Furthermore, a special IARC report examined the results from different studies and deemed the evidence sufficient to suggest a causal link between paternal smoking and childhood cancers (Secretan et al., 2009). Overall, there is suggestive evidence that tobacco smoke is causing genetic changes through the germline that may make children more susceptible to developing cancer.

In addition to the work linking paternal smoking and childhood cancer, studies have also identified an association between paternal smoking and congenital malformations (Table 8.1). The earliest study in this field by Mau and Netter (1974) found that the frequency of major congenital malformations increases with heavier paternal smoking patterns. This work has been supported by other studies that show a link between paternal smoking and several birth defects that can be genetically influenced 
(Cardy et al., 2007; Cresci et al., 2011; Deng et al., 2013; Hearey et al., 1984; Krapels et al., 2006; Kučienė and Dulskiené, 2010; Kurahashi et al., 2005; Mau and Netter, 1974; Palma et al., 2013; Pierik et al., 2004; Savitz et al., 1991; Steinberger et al., 2002; Sun et al., 2013; van Rooij et al., 2010; Wasserman et al., 1996; Zhang et al., 1992). Many of the epidemiological studies examining congenital malformation aetiologies only have modest odds ratios, are limited by confounding variables, and require further corroboration (Savitz, 2003). However, the consistency between studies (70\%) of a link between paternal smoking and congenital defects is alarming and supports the notion that tobacco smoke-induced mutations in sperm are heritable.

There is also a weak link between periconception paternal smoking and pregnancy loss. The majority of studies $(75 \%)$ factoring paternal smoking into spontaneous abortion risk do not find a correlation (Table 8.1). However, the study by Venners et al. (2004) demonstrates that non-smoking women whose husbands are heavy smokers ( $\geq 20$ cigarettes/day) have an increased risk of early/total pregnancy loss and a slightly longer time to clinical pregnancy compared to women married to non-smokers. A major advantage of the study by Venners et al. (2004) was that they measured daily urinary human chorionic gonadotropin to detect early pregnancy losses before the women knew they were pregnant. Similar to this finding, Firns et al. (2015) showed that male smoking increases the risk of pregnancy loss following in vitro fertilization, with a $2.9 \%$ increase in risk for every additional $1 \mathrm{mg}$ of nicotine intake. Also, Blanco-Muñoz et al. (2009) demonstrated that spontaneous abortion risk is increased when both the mother and father smoke. Lastly, there is evidence that paternal smoking is associated with antepartum fetal death (Zhang and Cai, 1992) and perinatal mortality (Mau and Netter, 
1974). An explanation for the discrepancies between studies investigating pregnancy loss is that heavy DNA damage in the paternal haplotype will lead to early loss, whereas lighter DNA damage will lead to a viable embryo carrying mutations that may later lead to phenotypic consequences. In conclusion, there is suggestive evidence that tobacco smoke causes non-neutral genetic changes in the germline that can have phenotypic consequences for the children that inherit these mutations.

\subsection{Evidence that tobacco smoke components induce germline mutations}

As summarized in the previous sections, there is cumulative evidence that tobacco smoke as a complex mixture is capable of inducing germ cell mutations. There is also compelling evidence that some of the constituents of tobacco smoke alone are capable of inducing germline mutations. A full summary of all the data linking tobacco smoke constituents with reduced sperm quality is beyond the scope of this paper. Here we highlight some of the evidence that individual components of tobacco smoke are alone capable of damaging the germline.

Most of the evidence comes from investigations of $\mathrm{BaP}$, a PAH present at a level of 1-50 ng per cigarette (IARC Working Group on the Evaluation of Carcinogenic Risks to Humans, 2004). Studies in animals show that high doses of BaP impair sperm functional parameters (Jeng et al., 2013; Jeng and Bocca, 2013) and induce point mutations (O'Brien et al., 2016a; Olsen et al., 2010; Verhofstad et al., 2011; Xu et al., 2014) and tandem repeat mutations (Beal et al., 2015b; Godschalk et al., 2015) in germ cells. The main type of mutation induced by $\mathrm{BaP}$ in sperm is a $\mathrm{G}: \mathrm{C} \rightarrow \mathrm{T}: \mathrm{A}$ transverison (O’Brien et al., 2016b; Olsen et al., 2010), which is consistent with the mutational 
fingerprint of tobacco smoke (Pfeifer et al., 2002). Furthermore, Generoso et al. (1982) demonstrated that there is a higher incidence of embryonic death when female mice are mated with male mice exposed to $\mathrm{BaP}$, suggesting that $\mathrm{BaP}$ induces premutational lesions that lead to "dominant lethal mutations" in the developing embryo. Based on this evidence, $\mathrm{BaP}$ is likely a main contributor to the mutagenicity of tobacco smoke.

There is evidence that other tobacco smoke components have negative effect on sperm. For example, volatile hydrocarbons such as 1,3-butadiene (20-40 $\mu \mathrm{g} /$ cigarette) and benzene (12-50 $\mu \mathrm{g} /$ cigarette) (IARC Working Group on the Evaluation of Carcinogenic Risks to Humans, 2004) are detrimental to sperm. Exposure of male mice to 1,3-butadiene induces micronuclei in spermatids (Xiao and Tates, 1995) and dominant lethal mutations in developing embryos (Adler et al., 1994; Brinkworth et al., 1998). Men occupationally exposed to benzene have a higher proportion of sperm aneuploidies (Xing et al., 2010) and chromosomal abnormalities (Marchetti et al., 2012). Moreover, sperm incubated with catechol, a benzene metabolite and tobacco constituent, have reduced motility and viability (Mandani et al., 2013). Studies investigating other organic compounds found in tobacco smoke, including formaldehyde (10-30 $\mu \mathrm{g} /$ cigarette), ethylene oxide, and acrylonitrile, demonstrate that these chemicals impair sperm quality (Mori et al., 1991; Odeigah, 1997; Tandon et al., 1988; Xu et al., 2003) and cause different forms of DNA damage (Xu et al., 2003) that lead to dominant lethal mutations (Generoso et al., 1986; Generoso et al., 1990; Odeigah, 1997). In addition, tobacco smoke contains several inorganic compounds that may be mutagenic to sperm. For example, cadmium is present in tobacco smoke and direct exposures to cadmium impairs sperm 
quality (Benoff et al., 2009; Li et al., 2015) and increases DNA fragmentation (Oliveira et al., 2009).

Thus, the germ cell mutations induced by smoking are likely the product of the complex mixture of mutagens found in tobacco smoke. There are many chemicals in tobacco smoke that are individually weakly mutagenic, but prolonged exposure to these chemicals as a complex mixture may be additively, or even synergistically, contributing to mutation induction associated with tobacco smoke.

\subsection{Genetic dise ase and socio-economic burden of s moke exposure}

The main genetic disease burden for smoking individuals is lung cancer. There are approximately 100,000 new lung cancer cases in men per year in the United States alone (Siegel et al., 2013) and 90\% are attributable to smoking (Kiyosawa et al., 1990). It is estimated that it costs $\$ 100,000$ annually per patient to treat lung cancer (Arrieta et al., 2015). Hence, there are enormous costs to the healthcare system to treat affected individuals. In this review, we have presented compelling arguments that smoking can damage sperm DNA and affect the health of smokers' offspring. Based on the reviewed data, we can make assumptions on the genetic disease burden of paternal smoking and hence its estimated impact on the healthcare system.

We selected intellectual disability (ID) as a model to estimate the disease burden of paternal tobacco smoking because genes associated with ID make up the largest target for mutation formation of any disorder, and there is a broad range of phenotypes associated with mutations in these genes (Van Bokhoven, 2011). Furthermore, considerable research has been done to create a list of known ID disease genes (IDDG); 
currently, over 700 IDDGs have been identified, with a 30:70 proportion of dominant and recessive genes (Vissers et al., 2015). At a population level, a modest 25\% increase in mutations that match the molecular signature of tobacco smoke in lung cancers $(\mathrm{G}: \mathrm{C} \rightarrow$ T:A, indels) (Alexandrov and Stratton, 2014; Anna et al., 2009) would result in an extra 2.4 million non-silent mutations in IDDG (Vissers et al., 2015) worldwide per generation (see supplemental information in section C.6.2 for details and Table 8.2 for estimates of mutations based on $50 \%$ and $100 \%$ increases). The estimated number of non-silent mutations is based on the assumption that each smoking male would have on average 2 children and that the spontaneous rate of each mutation matching the molecular signature of tobacco smoke is $1 \times 10^{-9}$ (Kloosterman et al., 2015; Kong et al., 2012). Depending on the type of each non-silent mutation (start lost, stop gained, frameshift, etc.), as predicted by Variant Effect Predictor (McLaren et al., 2010), the vast majority (1.9 million) of these mutations are expected to lead to loss-of-function (LOF) in their respective IDDGs each generation. Taking into account the IDDG locations and sizes, approximately 1.7 and 0.2 million tobacco-induced de novo mutations would be on the autosomes and the $\mathrm{X}$ chromosome, respectively.

The impact that these mutations may have on intergenerational health outcomes is dependent on the inheritance pattern and whether they occur on the autosomal chromosomes or on the X chromosome (Table 8.2). About 60,000 of 0.2 million tobaccoinduced de novo mutations on the $\mathrm{X}$ chromosome inherited by $\mathrm{F} 1$ females will be dominant and lead to the disease phenotype. Furthermore, expression of the recessive mutation when the functional copy has been silenced because of X-inactivation may lead to moderate penetrance of the disease phenotype. In the F2 generation, half of the male 
progeny of the F1 females who will reproduce will inherit the X-chromosome mutation leading to the full disease phenotype. Thus, the consequences of the mutations induced in the sperm of smokers can appear beyond the F1 generation.

For the IDDGs on autosomes, a $25 \%$ increase in mutation rate will potentially lead to 510,000 dominant mutations with a disease phenotype in the F1 (30\% of 1.7 million). The rest of the recessive autosomal mutations will contribute to the genetic load of the population. If an existing mutation is present in the same disease gene as a recessive de novo mutation, then compound heterozygosity will lead to the disease phenotype. In all of these cases, the F2 generation will inherit F0 mutations plus the additional burden of de novo mutations induced in the F1smoking fathers. Thus, the odds of compound heterozygosity and disease phenotypes are expected to further increase with every additional generation that continues to smoke.

The estimated number of non-silent mutations in IDDG genes worldwide per generation is based on the assumption that the same pattern of mutations observed in lung cancers of smokers is induced in germ cells. Recent data show that the pattern of mutations in murine germ cells after exposure to $\mathrm{BaP}$, a mutagenic constituent of tobacco smoke, has subtle differences from that observed in somatic tissues (O'Brien et al., 2016b). If the same is also true for tobacco smoke, the number or spectrum of mutations that are expected in IDDGs may vary slightly. In addition, our model did not account for any pleiotropic consequences of the mutations, or consider how many mutations could be embryonic lethal (Table 8.1). Nevertheless, even if a smaller effect size is used in the calculation to account for these uncertainties, such as $10 \%$, which is below the level of detection of most current mutation assays, the consequences of paternal smoking on 
IDDG mutations at the population level would still be staggering. It is important to note that de novo mutations in IDDGs may occur spontaneously as a consequence of replication errors or may be pre-existing in the parents. Therefore, although children with ID occur independently of tobacco smoking, paternal smoking may exacerbate the effects of these spontaneous mutations through epistatic interactions. Finally, ID is just a single example of an intergenerational disease outcome. Globally, de novo mutations have been recently shown to contribute to developmental disorders in about 400,000 births per year (Deciphering Developmental Disorders Study, 2017). Thus, paternal smoking could be contributing to many other forms of disease.

In addition to the emotional costs that cannot be quantified, it is tempting to speculate on the associated socio-economic costs of the global genetic disease burden of smoking on the next generation. This can be challenging because of economic differences across countries (e.g., differences in annual income, cost of living, etc.), as well as the hidden costs associated with mental health (education, criminal justice, etc.) (World Health Organization, 2003). As an example, we have considered the lifespan costs of individuals with either autism spectrum disorder or mental retardation, which are estimated conservatively at approximately 1 million US dollars (Buescher et al., 2014; Honeycutt et al., 2003). This estimate is based on costs in the United States and United Kingdom, which would be high compared to developing countries where individuals would not obtain specialized treatment. However, assuming that each affected individual will receive the same standard of care for ID, paternal smoking and the extra dominant mutations could in theory be costing the healthcare system approximately 570 billion dollars extra per generation for ID gene mutations alone. Considering only the top 50 
developed countries ranked by Human Development Index $(15 \%$ of the global population), where treatment is most probable, the healthcare costs would be approximately 86 billion dollars per generation. These costs may be greatly underestimated as the economic burden of ID is forecasted to rise (Leigh and $\mathrm{Du}, 2015$ ).

Although this model is speculative, these estimates provide a glimpse of the magnitude of the potential impact of tobacco-induced mutations and their socio-economic consequences. Moreover, all of these consequences are in addition to the impact of increases in aneuploidy conceptuses (as discussed previously) and other diseases not considered here. The sooner the germline consequences of tobacco smoking are acknowledged, the sooner the public can be educated, and global efforts can specifically target reduction or elimination of smoking in people prior to reproduction.

\subsection{Future research needs}

There are no established human germ cell mutagens despite decades of research showing that there are many rodent germ cell mutagens (DeMarini, 2012; Yauk et al., 2013). This is likely due to the ineffective experimental designs and technologies applied to this research question. However, existing NGS platforms now provide an opportunity to assess germ cell genetic hazards directly in human families. Of particular importance is precisely quantifying the extent to which tobacco smoke contributes to mutations in human spermatogonial stem cells. If tobacco smoke induces stem cell mutations to the same extent seen in rodent studies, there may be no minimum cessation period prior to conception that can prevent the formation and inheritance of de novo mutations. In this case, with every additional year of smoking there will be an accumulation of extra 
mutations that will persist throughout the male's life, increasing the odds of genetic disease in descendants. Furthermore, in utero exposures have been shown to induce mutations in primordial germ cells (Meier et al., 2017); thus, multi-generational studies are of critical importance to understand how smoke exposure during pregnancy affects a mother's grandchildren. It is also critical that work is done to address the full mutational fingerprint that smoking may have across the genome in sperm and offspring to understand the underlying mechanisms by which tobacco smoke induces germ cell mutations. This kind of analysis will be useful in determining which components in tobacco are causing mutations throughout the genome.

Now that high-throughput NGS has been developed, pedigree analysis of human cohorts can be performed to address these issues (Beal et al., 2012b; Yauk et al., 2015a). Hundreds of genomes have already been sequenced, many of which are families, and de novo mutations have been identified (1000 Genomes Project Consortium, 2015; Conrad et al., 2011; Genome of the Netherlands Consortium, 2014; Kong et al., 2012; Roach et al., 2010; Yuen et al., 2015). Acquisition of epidemiological data that include parental smoking status and history, among many confounding factors, could be used to determine the relationship between smoking and de novo mutation rate. Furthermore, additional studies could be carried out that focus on whole genome sequencing of families with a smoking father or rodent models (as in (Adewoye et al., 2015)) where the sire has been exposed to mainstream tobacco smoke. Power calculations, based on previous estimates of mutation rate and variation (Kong et al., 2012) demonstrate that a modest smoking effect size of 1.25 would only require 3 families per group at a power of 0.95 . An 
analysis of this type would provide the most convincing evidence that smoking causes heritable intergenerational mutations.

\subsection{Conclusions}

Our review clearly demonstrates that smoking is detrimental to sperm quality. Moreover, our review confirms that tobacco smoke can damage sperm DNA leading to mutations and aneuploidy. Mounting evidence supports that this damage in sperm leads to adverse genetic effects in the resulting embryo and offspring. This evidence is particularly strong in heavy smokers. However, data gaps exist as to the full extent of the heritability of sperm DNA damage and mutations. As described above, human pedigree analyses using whole genome approaches will provide the most informative information regarding the germ cell mutagenicity of smoking. Ultimately, definitively establishing tobacco smoke as a human germ cell mutagen will require changes in policies to minimize exposure to prospective parents from an early age in order to protect population health. Moreover, it will be critical to educate smokers that immediate abstinence from smoking, well in advance of reproduction, is the only way to decrease the risk of passing on harmful mutations to their children. 


\subsection{Tables and Figures}

Table 8.1 Summary of studies investigating the relationship between tobacco smoke and germ cell mutagenicity endpoints.

\begin{tabular}{|c|c|c|c|c|c|}
\hline Category & Endpoint & Animal & Studies $^{1}$ & $\begin{array}{c}\text { Studies } \\
\text { with } \\
\text { Effect }^{2}\end{array}$ & $\begin{array}{l}\text { Effect } \\
\text { Size }\end{array}$ \\
\hline \multirow[t]{13}{*}{$\begin{array}{c}\text { Fertility } \\
\text { Parameters }\end{array}$} & $\begin{array}{c}\text { Sperm } \\
\text { Count/Concentration }\end{array}$ & Human & 113 & 48 & $1.0-2.9$ \\
\hline & & Rodent & 8 & 6 & $1.2-1.7$ \\
\hline & & Monkey & 1 & 0 & - \\
\hline & Sperm Morphology & Human & 94 & 36 & $1.0-14.0$ \\
\hline & & Rodent & 5 & 5 & $1.0-3.3$ \\
\hline & Nonviable Sperm & Human & 25 & 12 & $1.0-4.0$ \\
\hline & & Rodent & 3 & 2 & 1.0-1.1 \\
\hline & & Monkey & 1 & 0 & - \\
\hline & Impaired Spermatogenesis & Rodent & 1 & 1 & 11.0 \\
\hline & $\begin{array}{l}\text { Altered Gene or Protein } \\
\text { Expression in Sperm }\end{array}$ & Human & 5 & 5 & 1.3-3.2 \\
\hline & & Rodent & 1 & 1 & 1.5 \\
\hline & Fertilization Rate/Fertility & Human & 3 & 1 & $1.7-1.8$ \\
\hline & & Rodent & 3 & 3 & $1.1-4.5$ \\
\hline \multirow[t]{5}{*}{ DNA Damage } & Sperm DNA Adducts & Human & 5 & 3 & $1.2-7.6$ \\
\hline & Sperm Oxidative Stress & Human & 14 & 12 & $1.2-5.2$ \\
\hline & & Rodent & 4 & 4 & $1.3-2.3$ \\
\hline & $\begin{array}{c}\text { Sperm Reactive Oxygen } \\
\text { Species }\end{array}$ & Human & 8 & 7 & $1.2-19.7$ \\
\hline & & Rodent & 1 & 1 & 1.3 \\
\hline \multirow[t]{9}{*}{$\begin{array}{l}\text { Chromosomal } \\
\text { Effects }\end{array}$} & $\begin{array}{c}\text { Sperm DNA Fragmentation } \\
\text { (TUNEL/TdTa) }\end{array}$ & Human & 8 & 5 & $1.2-4.3$ \\
\hline & & Monkey & 1 & 0 & - \\
\hline & $\begin{array}{c}\text { Sperm DNA Fragmentation } \\
\text { (Comet) }\end{array}$ & Human & 5 & 3 & $1.3-5.0$ \\
\hline & & Rodent & 3 & 3 & 1.1-9.2 \\
\hline & $\begin{array}{l}\text { Sperm DNA Fragmentation } \\
\text { (SCSA) }\end{array}$ & Human & 10 & 3 & $1.2-2.0$ \\
\hline & $\begin{array}{c}\text { Sperm DNA Fragmentation } \\
\text { (Other) }\end{array}$ & Human & 10 & 10 & $1.1-12.0$ \\
\hline & Sperm Micron uclei & Human & 1 & 0 & - \\
\hline & $\begin{array}{l}\text { Sperm Abnormal DNA } \\
\text { Condensation Pattern }\end{array}$ & Human & 2 & 1 & 1.2 \\
\hline & Sperm Aneuploidy & Human & 9 & 7 & $1.2-4.0$ \\
\hline Germline & Mutations in Children & Human & 1 & 1 & 4.0 \\
\hline
\end{tabular}




\begin{tabular}{lccccc}
\hline Mutagenicity & & & & & \\
& Sperm Mutations & Rodent & 3 & 3 & $1.4-3.1$ \\
\hline \multirow{2}{*}{ Consequences } & Cord Blood DNA Damage & Human & 1 & 1 & $1.2-1.5$ \\
& Childhood Cancer Risk & Human & 19 & 12 & $1.2-3.8$ \\
& Congenital Malformations & Human & 23 & 16 & $1.2-13.1$ \\
& Perinatal Mortality & Human & 1 & 1 & 1.5 \\
& Spontaneous Abortion & Human & 12 & 3 & $1.4-2.9$ \\
\hline
\end{tabular}

${ }^{1}$ References are included in Tables C18-C20.

${ }^{2}$ Study demonstrated a statistically significant result or an increased odds/risk ratio. 
Table 8.2 Potential intergenerational outcomes of the estimated 7.3 million LOF IDDG mutations associated with paternal smoking. Methodological details in footnote*.

\begin{tabular}{|c|c|c|c|}
\hline & \multicolumn{3}{|c|}{ Number of F1 Mutations } \\
\hline $\begin{array}{c}\text { Inhe ritance } \\
\text { Patte rn }\end{array}$ & $25 \%$ Increase & $50 \%$ Increase & $100 \%$ Incre ase \\
\hline $\begin{array}{c}\text { Autosomal } \\
\text { Dominant }\end{array}$ & 510,000 & $1,020,000$ & $2,040,000$ \\
\hline $\begin{array}{l}\text { Autosomal } \\
\text { Recessive }\end{array}$ & $1,190,000$ & $2,380,000$ & $4,760,000$ \\
\hline $\begin{array}{c}\text { X-linked } \\
\text { Dominant } \\
\text { (Female) }\end{array}$ & 60,000 & 120,000 & 240,000 \\
\hline $\begin{array}{l}\text { X-linked } \\
\text { Recessive } \\
\text { (Female) }\end{array}$ & 140,000 & 280,000 & 560,000 \\
\hline $\begin{array}{l}\text { X-linked } \\
\text { (Male) }\end{array}$ & \multicolumn{3}{|c|}{0 in F1; Would not appear until F2 generation } \\
\hline Total & $1,900,000$ & $3,800,000$ & $7,600,000$ \\
\hline
\end{tabular}

*Three scenarios are considered where the mutation types that can be induced by tobacco smoke are increased $25 \%, 50 \%$, or $100 \%$ in the children of smokers. In the $25 \%$ increase scenario, there are 1.9 million LOF mutations predicted based on the type of mutations (start lost, stop gained, frameshift, etc.) among the estimated 2.4 million non-silent mutations. The numbers in the table represent the number of dominant vs recessive mutations out of 1.9 million based on the number of dominant $(\sim 30 \%)$ or recessive $(\sim 70 \%)$ genes in the IDDG panel (Vissers et al., 2015). LOF mutations in dominant genes would lead to full penetrance of the dominant phenotype. Although no X-linked mutations will be present in the F1 males, all the X-linked mutations passed on from F1 females will be dominant in the F2 males. 


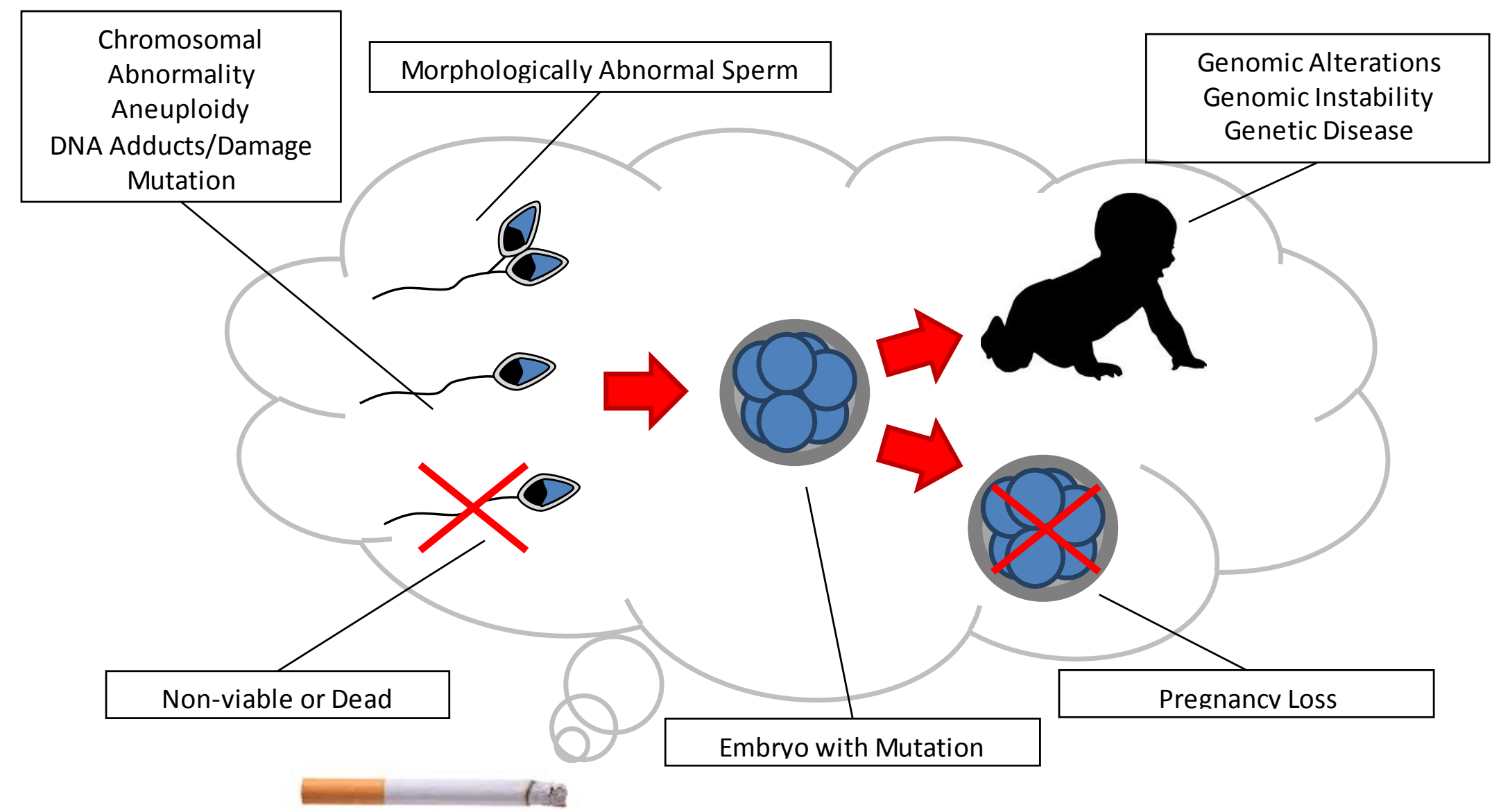

Figure 8.1 Overview of the evidence that tobacco smoke is a germ cell mutagen. 


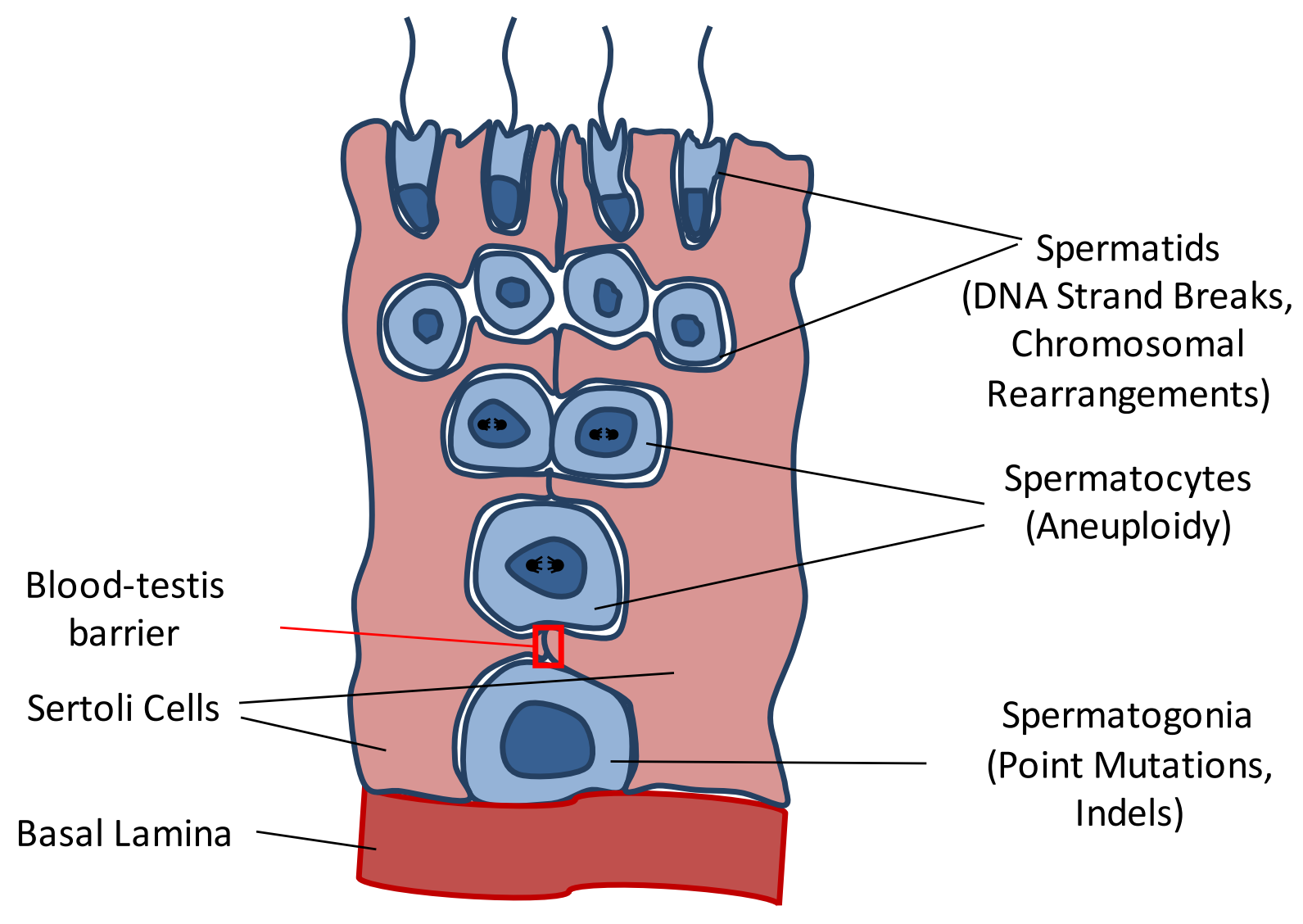

Figure 8.2 Spermatogenesis and different mutations that are prevalent in the different types of sperm. Dividing spermatogonia lay on the "open" side of the blood-testis barrier formed by Sertoli cells. Mutagenic exposures typically increase the frequency of point mutations and indels in spermatogonia. Spermatocytes undergo meiotic divisions and this is the phase where aneuploidies will occur. Haploid spermatids become DNA repair deficient and therefore DNA strand breaks or damaged nucleotides go unrepaired at this stage. This can lead to inviable sperm or the induction of chromosomal rearrangements occurring post-fertilization. 


\section{Chapter 9: Discussion}

\subsection{Thes is Outcomes}

The overall hypothesis of this thesis was that mutagens induce de novo mutations in the germ cells and offspring of exposed males, and that these mutations can be characterized using genomics technologies. Using the common pollutant $\mathrm{BaP}$ as a model mutagen, this thesis demonstrates that paternal exposures increase the number of sperm carrying mutations in tested markers as well as the number of genome-wide mutations in the descendants of the exposed males. The specific outcomes for each objective are discussed here briefly.

\section{Overarching objectives:}

(a) To determine whether paternal exposure to $\mathrm{BaP}$ increases mutation frequencies and alters mutation spectrum in sperm.

The data support that $\mathrm{BaP}$ is a germ cell mutagen. Specifically, the results of Chapters 2 and 3 demonstrate that $\mathrm{BaP}$ induces microsatellite mutations in sperm using SM-PCR. Furthermore, the TGR assay, adapted for germ cell analysis (Appendix A), was used in Chapter 5 to show that $\mathrm{BaP}$ exposure increases the lacZ MF in sperm, especially when dividing spermatogonia are exposed. Using the methodology developed in Chapter 4 (NGS of mutant lacZ plaques), the results of Chapter 6 reveal that BaP induces mainly $\mathrm{G}: \mathrm{C} \rightarrow \mathrm{T}: \mathrm{A}$ and deletion mutations in the lac $Z$ transgene in sperm.

(b) To determine whether paternal exposure to $\mathrm{BaP}$ increases mutation frequencies and alters mutation spectrum in unexposed offspring. 
The results of Chapter 7 demonstrate that paternal $\mathrm{BaP}$ exposure increases germline mutation frequencies and alters the mutation spectrum of the unexposed offspring. Specifically, the NGS results show that $\mathrm{BaP}$ increases the overall number of mutations in offspring by 2-fold when de novo and embryonic mutations are both considered. Induced mutations observed in $\mathrm{BaP}$-treated mice were consistent with the expected $\mathrm{BaP}$ mutation spectrum (guanine substitutions, mainly $\mathrm{G}: \mathrm{C} \rightarrow \mathrm{T}: \mathrm{A}$ ). Moreover, the aCGH results suggest that $\mathrm{BaP}$ exposure increases the frequency of duplication CNVs, but this finding needs to be supported by larger sample sizes.

(c) To assess pre-fertilization versus post-fertilization mutation fixation.

In Chapter 7, the aCGH results identify induced CNVs in both the pre-meiotic (pre-fertilization mutation fixation) and post-mitotic (post-fertilization mutation fixation) groups. The results suggest that the $\mathrm{CNV}$ frequency was higher in the post-mitotic group (0.048 vs. 0.020$)$, but the statistical power was insufficient to draw firm conclusions. Similarly, NGS determined that more animals in the post-mitotic group were affected by $\mathrm{BaP}$ exposure than the pre-meiotic group, but with the small sample sizes there was no statistical significance. Together, the genomics analyses indicate that mutations are being induced both pre- and post-fertilization. In order to determine if post-fertilization mutation fixation is a stronger determinant of mutation formation in the offspring, larger sample sizes will be required to corroborate the suggestive findings observed here.

(d) To assess the utility of whole genome approaches in identifying heritable mutations. 
Comparisons of the endpoints detected by whole genome approaches and existing sperm methods can be made to determine if the mutation types and frequencies detected are similar. In this thesis, the genomics approaches detected the same types of mutations in the offspring that were detected in the sperm. Specifically, G:C $\rightarrow$ T:A mutations were detected in both the sperm and offspring of BaP-treated animals. However, the increase in sperm mutation frequency (4-fold), as detected by the TGR assay, was higher than the increase in offspring mutation frequency (2-fold), as detected by NGS. There are several possible biological explanations for this discrepancy (discussed in Chapter 7). Overall, the comparisons show that all the methods used in this thesis can be effective approaches for identifying heritable mutagenesis when the appropriate study designs and sample sizes are used. There appears to a good correlation between sperm and offspring mutations, with the whole genome approaches providing validation for the heritability of the mutations detected in sperm. Thus, I believe that the sperm methods can serve as effective tools for genetic toxicology testing, while the genomics technologies can be used as powerful tools to investigate other biological questions that cannot be addressed using the sperm methods.

\section{Specific objectives:}

(e) To detect microsatellite mutations in sperm using SM-PCR.

This objective was met in Chapters 2 and 3. The 2 loci tested suggest that microsatellite mutations can be detected at lower doses than point mutations. These results demonstrate that microsatellites are sensitive markers and suitable replacements to ESTRs. As several microsatellites have been characterized in humans (Sun et al., 2012), 
SM-PCR of microsatellites has the potential to serve as a useful approach for studying human mutagenesis.

(f) Develop a method to characterize mutation spectra of TGR assay mutants using NGS.

This objective was met in Chapter 4. The novel method allows for rapid characterization of thousands of mutants produced by the TGR assay. In the proof of principle experiment, we characterized $3 x$ the number of lacZ mutants analyzed in all previous studies from the past 20 years combined. This method also allows for clonal expansion correction, which reduces sample variability and improves the sensitivity. Overall, the depth of data that can be obtained using this new approach will allow for research questions to be addressed that would have otherwise been difficult to answer using previous sequencing methods.

(g) Detect germline mutation induction in sperm using the TGR assay.

This objective was met. Appendix A describes the TGR assay that we refined to study mutations in germ cells. The methods were applied in Chapter 5 to study the effects of $\mathrm{BaP}$ exposure on different phases of spermatogenesis. The results demonstrate that the highest dose of $\mathrm{BaP}$ induces a 4-fold increase in $\mathrm{MF}$ in sperm exposed as dividing spermatogonia. This is the largest induction of sperm mutations in adults by oral gavage that has been observed in our lab. In comparison, Appendix B demonstrates that in utero exposure to lower doses of $\mathrm{BaP}$ eliminates most of the primordial germ cells, and any surviving germ cells have a MF up to 16-fold higher than control. To my knowledge, this 
mutation induction in the germ cells of in utero exposed animals is the highest fold increase ever reported.

(h) Characterize the mutation spectrum of $\mathrm{BaP}$ in sperm.

This objective was met. In Chapter 6 the mutation spectra of control and BaPexposed sperm were characterized for dividing spermatogonia, revealing that $\mathrm{BaP}$ induces more deletions in sperm compared to somatic tissue analyzed in Chapter 4 . Sequencing sperm mutants also demonstrates that there is clonal expansion of both spontaneously occurring and $\mathrm{BaP}$-induced mutations. Clonal expansions occur in stem cells and are biologically relevant because these cells will persist in producing mutant sperm throughout the animals' reproductive lives.

(i) Detect de novo $\mathrm{CNV}$ induction in the offspring of exposed males following $\mathrm{BaP}$ exposure using aCGH.

This objective was met in Chapter 7. The results showed that the number of $d e$ novo deletions is consistent between control and $\mathrm{BaP}$-exposure groups. However, duplication/insertion $\mathrm{CNVs}$ were only found in the $\mathrm{BaP}$ group. $\mathrm{CNV}$ s in which the parental origin could be determined were all confirmed to have arisen in the paternal germline. Sequencing of the CNV breakpoints revealed short regions of microhomology (1-6 bp) shared between both breakpoints, indicative of MMBIR of BaP lesions. I hypothesize that $\mathrm{BaP}$ adducts destabilize DNA replication resulting in the formation of a unique type of CNV. 
(j) Detect the induction of de novo SNVs and indels across the entire genome using NGS technologies.

This objective was met in Chapter 7. NGS identified de novo SNVs and indels across the genome and showed that germline $\mathrm{BaP}$ exposure induces genome-wide mutations in individuals. NGS also identified mutations with VAFs below the level expected for de novo mutations. These mutations were classified as embryonic (postzygotic) events that would not normally be considered as exposure-associated mutations and would be excluded from analysis. However, many of these mutations matched the mutation profile of $\mathrm{BaP}$, and some also occurred as clusters with proximity that is unlikely due to chance. I speculate that these mutations result from post-fertilization mutation fixation of $\mathrm{BaP}$ adducts that persisted in the fertilizing sperm. The work suggests that embryonic mutations can be formed as a consequence of germ cell exposures. Efforts should be made to detect these events along with de novo mutations in regulatory testing. Overall, the mutations observed using NGS in the offspring of BaPtreated males are consistent with mutations detected in sperm, supporting the heritability of induced sperm mutations.

(k) Explore a case study on tobacco smoke as a common environmental source of BaP exposure, characterize its potential as a human germ cell mutagen, and model the socioeconomic impacts of this for intellectual disability as an example.

This objective was met in Chapter 8 . An extensive literature search revealed a large body of evidence that supports the notion that tobacco smoke is a human germ cell mutagen. As $\mathrm{BaP}$ is a main mutagen in tobacco smoke, the BaP-induced mutation events 
detected in this thesis are likely occurring in the germline of smokers. Estimates show that even a modest increase in mutation rates due to smoking can have severe socioeconomic consequences. Thus, further investigations of the genome-wide effects of tobacco smoke exposure in humans are warranted.

\subsection{Advancements in Science and Contribution to Knowle dge}

This thesis asked several biological questions, and developed and applied novel methods to answer these questions. For example, an NGS method was adapted for highthroughput sequencing of mutants produced from the TGR assay. This was necessary to make the first direct comparison between the mutation spectrum of $\mathrm{BaP}$ in sperm and the offspring. Using NGS to analyze both sperm and offspring improved our understanding of inherited mutagenesis by providing evidence that the mutations observed in sperm are being transmitted to the offspring. Another application of the NGS method was to compare mutations between somatic and germ cells. The results of this comparison showed that germ cells have a unique mutation spectrum relative to somatic cells following $\mathrm{BaP}$ exposure. This has important implications for risk assessment because differential responses of germ and somatic cells to mutagens may indicate that somatic cell tests are not predictive of what is happening in germ cells. Furthermore, the NGS/TGR approach allowed for the first assessment of somatic and germline mosaicism induced in utero by a mutagen exposure. Although the methods were not the direct focus of the project, each method played an important part in contributing to scientific knowledge and meeting the objectives of the thesis. In the case of the NGS/TGR method, mutation analysis of all detectable mutats can be done in a matter of days. Thus, mutant 
characterization can be implemented as part of the standard analytical pipeline as opposed to rarely being performed. Given the major findings of this thesis observed using the TGR assay with NGS (germ cell mutations are transmitted to offspring, germ cells respond differently to exposures than somatic cells etc.), this method holds great promise in addressing other biological hypotheses.

Prior to this thesis, the effects of germline exposures on the genome of progeny remained largely unknown. For example, studies investigating the effects of radiation on the germline using tandem repeat markers gave very different results (Goldstein and Stawkowski, 2015). Had genomics approaches been used the results between studies may have been more comparable. Prior to this thesis, only 1 study examined the genome-wide consequences of paternal mutagen exposure (Adewoye et al., 2015). In that study, researchers exposed males to radiation and mated them to produce offspring that developed from irradiated sperm. The results showed that paternal radiation led to an increase in large deletions and small indels in the offspring. Furthermore, the investigators detected elevated rates of events that they classified as multisite mutations. The multisite mutations can be described as multiple variants (SNVs and indels) occurring within a short segment of DNA ( $\leq 15 \mathrm{bp}$ in the paper). The mechanisms for mutation formation were not discussed in detail; however, the results suggest that the radiation induces double-strand breaks in sperm that lead to erroneous repair. Specifically, non-homologous or microhomology-mediated end joining likely lead to multisite mutations or indels, and single strand annealing leads to large deletions (McVey and Lee, 2008). These types of mutations observed with genomics approaches are difficult to detect using most traditional sperm approaches. This is especially true for the 
detection of large deletions using most transgenic rodent models (Lambert et al., 2005). Due to these limitations, the study could not make any direct comparisons between mutations (i.e., large deletions) in offspring and sperm.

The experimental approach used in this thesis was designed to further our understanding of mutation induction in germ cells and their transmission to the offspring. To my knowledge, this is the first study to directly compare point mutations induced in the sperm with heritable mutations induced in the offspring. Hitherto, the only evidence linking DNA damage in sperm to heritable mutations came from comparisons across studies using tandem repeat markers. For example, studies on the effects of alkylating agents show that mutation frequencies in sperm (Swayne et al., 2012; Vilariño-Güell et al., 2003) are comparable to mutation rates in offspring (Dubrova et al., 2008). The results of offspring analysis, using WGS, showed that the same types of mutations induced by $\mathrm{BaP}$ in sperm are observed in the offspring. The frequency of induction (2fold) in the offspring was lower than expected based on TGR assay results in sperm (4fold), but there are several possible biological explanations for this discrepancy (Chapter 7). Overall, the WGS study in this thesis is the first to apply NGS to study chemicallyinduced heritable mutations. Although the sample size used was small, the results provide some support that mutations in sperm may be predictive of what can be expected in the offspring.

The experimental approach of this thesis also used microsatellite mutation induction in sperm as a proxy for CNV formation in offspring. This is because microsatellite mutations and CNVs were hypothesized to be formed through similar mechanisms. The BaP exposure showed a 2-fold increase in microsatellite mutations in 
sperm while the exposure lead to an increase in duplication CNVs, but the CNV result was not significant due to insufficient power. Interestingly, sequencing the CNV breakpoints revealed that there was only 1-6 bp of sequence similarity (microhomology) facilitating the CNV formation. A possible explanation for these results is that the presence of $\mathrm{BaP}$ adducts lead to replication fork pausing or fork collapse, with the former leading to microsatellite mutations and the latter leading to CNVs. Normally, the cellular machinery would re-initiate replication through an available repair pathway. However, the instability caused by the heavy BaP exposure likely oversaturated the cell machinery and hindered its ability to initiate replication using the correct template. As a result, reinitiation of replication was instead aided by microhomology. For CNVs, this consisted of 1-6 bp of a sequence in close physical proximity, including sequences from other chromosomes. In microsatellites, the microhomology consisted of the 4 bp core repeat unit of the microsatellite, leading to indels that were multiples of $4 \mathrm{bp}$ in size. Overall, the comparisons between microsatellite mutations in sperm and CNVs in offspring support the hypothesis that measuring mutations in sperm is indicative of the types and rates of mutations in offspring. Moreover, the results suggest a mechanism by which a very common environmental pollutant might cause $\mathrm{CNVs}$ and contribute to adverse idiopathic disorders.

In this thesis, two post-exposure time points were used for breeding to assess prefertilization versus post-fertilization mutation fixation. The $28+42$ time point was used to capture mutations arising in sperm during pre-meiotic processes (pre-fertilization). The $28+3$ time point was used to capture mutations occuring in the early embryo (postfertilization) from sperm exposed during the post-mitotic phase of spermatogenesis. It 
was anticipated that post-fertilzation events would be abundant after $\mathrm{BaP}$ exposure because previous studies show that the majority of chemicals induce the most DNA damage during post-meiosis (Marchetti and Wyrobek, 2005). The aCGH results showed that duplication CNVs were induced as a consequence of both pre-meiotic and postmitotic exposures. Moreover, several animals had a higher number of mutations per genome in the combined $\mathrm{BaP}$ exposure group (post-mitotic: 3 of 4; pre-meiotic: 1 of 4 ). Combined, these results support the hypothesis that DNA damage in the post-mitotic phase will lead to heritable mutations, even though mutation cannot be observed in the sperm directly using the lac $Z$ assay, and that this phase of spermatogenesis is highly susceptible to mutagen exposure. The detection of post-mitotic mutations induced postzygotically here is important because it demonstrates the need to screen spermatids for DNA damage during testing, and it highlights the need to identify low VAF mutations as these events can be indicative of unrepaired DNA damage in the sperm that is converted into mutations during embryonic development.

Understanding the relevance of the thesis results for human health is an important consideration. For example, several of the mutations detected in the offspring of $\mathrm{BaP}$ exposed animals could have had functional consequences as they occurred within known genes. First, several CNVs detected occurred within or near coding sequences, and many of these were predicted to be associated with possible disease outcomes (Table C12). Second, 4 SNVs (2 missense, 1 nonsense, 1 splice variant) detected in BaP offspring were within protein coding genes and likely disrupted the function of the protein (Table C15). Three of the 4 mutations occurred in genes that have known disease outcomes in humans. Therefore, it is possible that some of these mice with CNVs or SNVs may have 
had observable phenotypic changes associated with the mutations they carried. However, the mice were not monitored for abnormalities in health, behaviour, or appearance. Without careful observation, it is difficult to determine if the mutations would have immediate functional consequences as many mutations would be recessive or moderately penetrant.

The results of this thesis collectively demonstrate that $\mathrm{BaP}$ is a germ cell mutagen that could affect the health of unexposed progeny, but the doses used here were much higher than what a human would be exposed to. However, humans are exposed to several different sources of BaP throughout their lives (air pollution, tobacco smoke, smoked meats, etc.) and to a variety of other environmental pollutants. Thus, I explored tobacco smoke as a case study for $\mathrm{BaP}$ exposure and effects in Chapter 8 . The case study presents substantial evidence that tobacco smoke, as a source of $\mathrm{BaP}$, is mutagenic to male germ cells. This is troublesome as men are exposed daily to not only tobacco smoke, but other sources of $\mathrm{BaP}$ and mutagenic PAHS as well. Furthermore, most men are likely unaware of the potential consequences of these exposures on their future children. It is envisioned that the methods developed in this thesis can be used to further study the consequences of chronic exposure to complex mixtures that include $\mathrm{BaP}$, such as tobacco smoke. The knowledge gained from this thesis can be used as a reminder of the consequences of paternal exposures to germ cell mutagens, and that education and early prevention is important for the protection of the next generation.

Overall, this thesis has major implications for risk assessment. Currently risk assessments for chemical mutagens are based on carcinogenicity - mutation analysis is currently used to determine whether the agent is a possible human carcinogen, and risk 
assessment is centred around lifetime cancer risk of exposure to the substance.

Considering the astonishing socio-economic implications of paternal mutagen exposures (conservatively estimated in Chapter 8), risk assessments may need to more carefully consider the germ cell mutagenicity of agents as well. We still do not know how a risk assessment for germ cell mutagens should be carried out. For example, cancer risk assessments consider a 1 in a million increase in lifetime risk as acceptable; should a similar threshold be used for intergenerational disease risk? As NGS and other new methods are applied to discover human germ cell mutagens and quantify induced inherited mutations, risk assessments for these effects will need to be developed.

\subsection{Future Directions}

\subsubsection{Tandem Repeat Mutations as a Biomarker for Mutagen Expos ure}

Microsatellites are among the most sensitive markers for detecting mutations in the germline, and a major advantage offered by microsatellites is that all organisms have microsatellites throughout their genomes. For example, microsatellites have recently been used to study waterbirds living near a site with elevated pollution levels (King et al, 2014). The results show that the offspring of birds exposed to environmentally relevant levels of PAHs, including BaP, have higher rates of mutations than birds with low exposure levels. Results like this highlight the need to monitor humans exposed to environmental mutagens (e.g., air pollution) for microsatellite mutations, especially considering that our knowledge of their critical roles in genome structure and function are growing (Mirkin, 2007; Mori et al., 2013). 
Few studies have explored using microsatellites as markers for studying human mutagenesis. The most comprehensive study identified 2,058 mutations from 2,477 microsatellites in 85,289 individuals (Sun et al., 2012). With this power, the researchers were able to detect a paternal age effect, with older males passing on more mutations to their children. However, most human microsatellite studies are unable to obtain that kind of power using the family pedigree approach due to insufficient resources or low sample sizes. For example, the studies investigating microsatellite mutations in families exposed to radiation did not observe any effects because the samples sizes and number of loci examined were small (Costa et al., 2011; Furitsu et al., 2005; Kodaira et al., 2010; Satoh et al., 1996; Slebos et al., 2004). SM-PCR analyses of microsatellites can overcome these limitations by examining the sperm of exposed individuals directly. Highly polymorphic loci in humans have already been characterized (Furitsu et al., 2005; Sun et al., 2012), and analyzing only the most informative loci can save on time and resources. Furthermore, analyzing sperm eliminates the need to study the children for mutations, which is beneficial because highly exposed individuals are often rare and may not have many children. Thus, using SM-PCR of microsatellites can serve as a powerful new tool for studying germline mutations in human populations.

Microsatellites and other tandem repeat loci, as markers for studying mutations, are not being used to their full potential; within genomes is an untapped resource of thousands of polymorphic loci yet to be characterized. Considering that mutation frequencies of the 2 microsatellite loci studied in Chapters 2 and 3 were $>10^{-3}$, and thousands of similar microsatellites exist in the genome, there are potentially hundreds of microsatellite mutations being passed onto any individual per generation. Moreover, there 
are large repetitive sequences ( $\mathrm{CpG}$ islands, telomeres, centromeres, heterochromatin, LINES, etc.) that have yet to be investigated for mutations. Therefore, the total number of heritable mutations occurring at these loci each generation remains unknown. One could imagine that thousands of replication slippages are occurring at these loci each generation based on their abundance, length, and low complexity. Thus, fully characterizing all tandem repeat mutations in offspring could be useful in epidemiological studies attempting to identify environmental and lifestyle factors that lead to heritable mutations. For example, with only 2 minisatellite loci, Linschooten et al. 2013 were able to determine that paternal smoking and lower yearly income were associated with increased mutations in children. The results were only marginally significant and could therefore benefit from the added power obtained from characterizing the full tandem repeat mutation profile of the offspring. However, as beneficial as identifying tandem repeat mutations throughout the genome would be, detecting these events remains elusive with current genome sequencing technologies.

The inability to accurately identify microsatellite mutations is mostly due to the short sequence read lengths currently available by most NGS platforms. Sequence reads fail to capture both flanking ends of the microsatellite and the reads cannot map to the reference genome properly. Furthermore, preparation of DNA for sequencing often involves a PCR step that creates stutter noise (PCR product contains incorrect number of repeats due to polymerase slippage). Attempts have been made to develop software, such as lobSTR, that can correctly identify short microsatellite mutations (Gymrek etal., 2012). However, these kinds of tools require sequencing depth much higher than what is required for SNV calling. Moreover, any microsatellite mutations called by the software 
still require validation. As a result, lobSTR was unable to identify true de novo microsatellite mutations in the samples analyzed in Chapter 7 (results not shown). In order to accurately call tandem repeat mutations, long-read and single-molecule sequencing technologies will need to improve. If these improvements in NGS can be achieved, researchers will eventually be able to study mutations being formed in all repetitive regions, including the long and low complexity loci where mutations are most likely to occur. Accurate sequencing of these unstable and repetitive regions could potentially be the most sensitive approach for detecting heritable mutations.

\subsubsection{Understanding the Corre lation Between Mutations in Reporter Transgenes and Endogenous Ge nes}

Transgenic rodent models, such as the MutaMouse, allow for robust mutation detection in any tissue following chemical exposures. Coupling the TGR assay with NGS has further improved the sensitivity of this assay and increased the amount of information that can be gained. In spite of these advantages, TGR assay studies are not routinely used in a risk assessment context and data generated using the assay are not often considered by regulators. This may change now that an OECD test guideline has been established (OECD, 2011). However, there are still some uncertainties about the relevance of mutations within transgenes. Specifically, transgenes are heavily methylated relative to most genes and are not subject to transcription-coupled DNA repair (Ikehata et al., 2000; Lambert et al., 2005). The results of this thesis demonstrate that there is a correlation between lacZ mutations in sperm and SNVs in the genomes of offspring. Hence, the thesis provides support that transgene mutations are a suitable marker for predicting 
heritable mutation induction. However, the mutation induction was higher in sperm than in offspring. Thus, more comparisons are required, with larger sample sizes, to better understand the correlation between transgene mutations in sperm and endogenous genes in offspring.

Detecting mutations in endogenous genes in vivo has been difficult historically due to the rarity of mutations. Thus, few comparisons have been made between mutations using the TGR assay and those in endogenous genes (Cosentino and Heddle, 2000). NGS holds great promise for detecting mutations in any gene of interest in both somatic tissues and germ cells. However, sequencing accuracy has always been too poor to detect low frequency mutations from a pool of cells. Specifically, errors introduced during amplification of target DNA occur at a rate that is magnitudes higher than the true mutation rate. Recent innovations in the way samples are prepared for NGS have now made detecting rare mutations in genes of interest possible. Specifically, a new approach called maximum-depth sequencing (MDS) (Jee et al., 2016) modified the way regions of interest are amplified prior to sequencing and greatly reduced the sequencing error rate. The first study using MDS managed to measure mutation rates in different bacterial genes at rates below $10^{-8}$. The researchers observed that highly transcribed genes had lower mutation rates than genes with low transcription levels. Furthermore, the mutation rates measured matched previously reported estimates of bacterial mutagenesis. Hence, MDS is a promising new method for measuring mutations in endogenous genes of interest.

A comparison of mutation rates in reporter transgenes and endogenous genes using the same methodology is a crucial next step in validating the applicability of TGR 
systems. For example, MDS can be used to measure mutations in a tumour suppressor gene, such as $p 53$, and the $l a c Z$ transgene simultaneously. Such a comparison will determine if there is a strong or weak correlation between mutations induced in reporter transgenes and functional endogenous genes. The fold increase in mutation rate following mutagen exposure may be consistent between reporter genes and transcribed genes, but this needs to be tested. Comparisons between the TGR assay and MDS may also prove the sensitivity of MDS. If MDS in endogenous genes proves to be a sensitive tool, then expensive TGR models will no longer be be necessary. One thing to note is that MDS is currently very expensive, with an estimated cost of $\$ 2000$ per gene per sample. Thus, the TGR assay will continue to be the method of choice for mutation detection. When future innovations reduce the cost and error rate of sequencing, methods such as MDS may be suitable replacements to the TGR assay, but until then, there are many questions to be answered through continued use of the TGR assay.

\subsubsection{Next-Generation Mutations and Next-Generation Technologies}

In the post genome-era tremendous advances have been made that allow for $d e$ novo mutations to be detected between generations. First, there was the development of aCGH, which allowed for high resolution structural variation detection (Solinas-Toldo et al., 1997). Then, in the new millennium, NGS became commercially available and the cost of sequencing genomes dropped from the initial $\$ 3$ billion to now approximately $\$ 1000$ per genome. The applications of these technologies for studying mutations were immediately obvious. However, initial work often focused on studying somatic mutations by comparing tumour vs. wild-type tissues (Ley et al., 2008; Solinas-Toldo et al., 1997). 
Application of technologies for investigating whether environmental exposures increase de novo mutation rate is often delayed. For example, aCGH technology wasn't used to measure mutation rates following an in vivo exposure for 18 years (Adewoye et al, 2015). In addition, NGS technologies have high false-discovery rates that are above the intergenerational germline mutation rate, and therefore it has been difficult to detect subtle changes in mutation rate without extensive validation of mutations. For this reason, only a few research groups have successfully used NGS to directly measure mutation rates in family pedigrees (Francioli et al., 2015; Kong et al., 2012; Rahbari et al., 2015; Yuen et al., 2015). Advances in the ways these technologies detect mutations will be required before routine testing of germ cell mutagens becomes mainstream.

Innovations, which can even be simple modifications to an existing protocol, are anticipated to not only allow for routine application of NGS in germline mutation studies, but will also allow for mutations to be detected in sperm directly at a genome-wide level. For example, researchers developed a whole genome amplification method called MALBAC (Multiple Annealing and Looping Based Amplification Cycles) that can be used for genome analysis of individual cells (Zong et al., 2012). The way MALBAC works is that after a DNA molecule is copied, the new copy forms a loop so that it cannot act as a template. This way, any errors introduced during copying will not be propagated. Using MALBAC, researchers have successfully identified CNVs in individual sperm with a NGS depth of $0.8 x$ (Zong et al., 2012). Thus, this method could be used to analyze the sperm from the sires used in Chapter 7. It is possible that there is negative selection against sperm carrying CNVs, which could have reduced the number of CNVs detected in the offspring of BaP-treated males. MALBAC could be used to test this hypothesis by 
comparing $\mathrm{CNV}$ frequencies in sperm and offspring. MALBAC has also been used to detect SNVs and recombination events in single cells, but at least 3 cells need to be sequenced in order to detect de novo mutations (Lu et al., 2012; Zong et al., 2012). One study has claimed to have measured de novo mutation rates of 8 individual sperm from 1 human (Wang et al., 2012), but further work is required to determine if their approach can detect subtle differences in rates between different individuals. Overall, the field is on the verge of a breakthrough that will enable improved mutation detection that will eventually allow for genome sequences of individual cells to be investigated.

New technologies can also go even further than measuring mutations in individual cells by simultaneously detecting DNA damage events in individual molecules before these events are fixed or lead to mutations. For example, single-molecule NGS, known as Third Generation Sequencing, has been used to detect the incorporation of modified nucleotides, such as 8-oxo-dG (Clark et al., 2011; Song et al., 2012). Specifically, singlemolecule real-time (SMRT $\left.{ }^{\circledR}\right)$ technology uses a single polymerase to sequence a single DNA molecule by synthesis. The time the polymerase spends on a particular nucleotide (pulse width) and time leading up to incorporation of the next nucleotide (interpulse duration) can be measured and used as an indicator of DNA modification. Due to the power of these approaches, application of single molecule and single cell sequencing in germline mutation studies should be a high priority, especially in humans.

\subsubsection{The Quest for the Holy Grail: The First Human Germ Cell Mutagen}

Decades of research have demonstrated the existence of many rodent germ cell mutagens, but none have been officially declared as human germ cell mutagens 
(DeMarini, 2012). This may be due to the fact that human mutation studies have always been limited by a lack of sensitive tools for measuring mutations. In contrast, studies in rodents benefited from the use of several traditional approaches including the SLT, DLT, HTT, transgenic rodent models, and highly unstable ESTRs. Furthermore, mice are generally genotypically homogenous and the experimental environment is strictly controlled. The work of this thesis demonstrates that genomics technologies can be used to detect environmentally induced germline mutations in rodents. Now that proof of principle has been established, these technologies should be used to identify human germ cell mutagens (i.e., sequence families where the father came in contact with a suspected mutagen). These technologies are already better suited for human analyses due to the supporting infrastructure (well-characterized human genome, suite of analytical software, human-specific sequencing facilities, etc.). The information gained in this thesis can be used to inform how future human studies should be performed. For example, power calculations need to consider a high degree of variability in the exposed group, similar to what was observed in the offspring of BaP-exposed males. Analyses of this kind will be crucial in identifying human germ cell mutagens that may be contributing to a high incidence of genetic disorders (Deciphering Developmental Disorders Study, 2017).

There are many suspected human germ cell mutagens that require investigation. For example, the Environmentally Induced Germline Mutation Analysis (ENIGMA) working group developed a list of probable and high priority exposures that should be considered for testing (cigarette smoking, radiation, chemotherapy, hydroxyurea, air pollution) (Yauk et al., 2013). Perhaps one of the most suitable study populations that should be investigated for germline mutagenicity is male smokers because of their high 
prevalence, the wealth of data that smoking damages sperm DNA (Chapter 8), the evidence that it induces germline mutations (Linschooten et al., 2013; Marchetti et al., 2011; Yauk et al., 2007), and the supporting evidence that BaP alone induces heritable mutations. Moreover, the models in Chapter 8 demonstrate that germ cell mutations induced by paternal smoking worldwide may have severe socio-economic consequences. To test the hypothesis that tobacco smoke is a germ cell mutagen, genomics technologies could be applied to analyze the children of smoking males and non-smoking females. To increase the power of the study, some of the single cell approaches discussed in the previous section could be used to analyze the sperm of the heaviest smokers. Moreover, the offspring of men exposed to smoking in utero (i.e., the mens' mothers smoked during pregnancy) should also be assessed as in utero exposures have much greater effects on mutation frequencies than adult exposures (Appendix B). Overall, the experimental design for the first human germ cell mutagen study needs to be carefully considered as it will lay the foundation for identifying other human germ cell mutagens.

In addition to environmental exposures, there may be other factors that increase heritable mutation rates in humans. For example, parental genotypes may have an influence on germline mutation rates, especially when the parents harbour functionaltering SNPs present in key DNA repair genes. Another factor to consider is the effect of assisted reproduction technologies on genome integrity. The health outcomes in in vitro fertilization offspring are still not fully understood (Fauser et al., 2014), and with the exception of studies focused on imprinting disorders there are no data available to determine if assisted reproduction technologies have any effects on the genome. With growing rates of in vitro fertilizations worldwide, this is an important consideration that 
requires attention. Overall, these are just a few examples of environmental exposures and factors that could potentially mediate mutation rates in humans. Considering that there is a large list of potential germ cell mutagens, there is a pressing need to begin characterizing germ cell mutagens for the benefit of population health.

\subsection{Concluding Remarks}

The results of this thesis demonstrate that $\mathrm{BaP}$ is a rodent male germ cell mutagen, inducing guanine base substitutions, small deletions, and large duplications. Furthermore, the evidence presented supports that these mutations are transmitted to the offspring. Thus, other PAHs or mixtures that we are exposed to everyday may be impacting population health, not only by increasing cancer risk, but by increasing the number of heritable mutations passed onto the next-generation. The estimates in Chapter 8 on the genetic disease burden and staggering socio-economic consequences of paternal smoking call for us to revisit how we perform risk assessment for mutagens.

Assessment of genomics technologies revealed that these tools are effective in identifying risk factors that may mediate heritable genetic disease. However, efforts are still required to improve the sensitivity of these methods. Indeed, it is an exciting time to study heritable mutations as the field is rapidly advancing. The development of new tools is allowing for unanswered hypotheses to finally be tested. It is envisioned that the tools explored in this thesis will be used in future germ cell mutagenesis studies.

Overall, the findings of my thesis support the hypothesis that environmental exposures can negatively impact the offspring. The final message is that men should lead 
a healthy life free of harmful exposures to the extent possible, not only for the sake of their own health, but for their unborn children as well. 


\section{ReferencesReference List}

1000 Genomes Project Consortium. (2015). A global reference for human genetic variation. Nature 526, 68-74.

Abdul-Ghani, R., Qazzaz, M., Dabdoub, N., Muhammad, R., and Abdul-Ghani, A. (2014). Studies on cigarette smoke induced oxidative DNA damage and reduced spermatogenesis in rats. J. Environ. Biol. 35,

Adewoye, A.B., Lindsay, S.J., Dubrova, Y.E., and Hurles, M.E. (2015). The genomewide effects of ionizing radiation on mutation induction in the mammalian germline. Nature Communications 6, 6684.

Adler, I., Cao, J., Filser, J., Gassner, P., Kessler, W., Kliesch, U., Neuhäuser-Klaus, A., and Nüsse, M. (1994). Mutagenicity of 1, 3-butadiene inhalation in somatic and germinal cells of mice. Mutation Research/Fundamental and Molecular Mechanisms of Mutagenesis 309, 307-314.

Agam, A., Yalcin, B., Bhomra, A., Cubin, M., Webber, C., Holmes, C., Flint, J., and Mott, R. (2010). Elusive copy number variation in the mouse genome. PLoS One 5, e12839.

Ahmadi, A., and Ng, S. (1999). Fertilizing ability of DNA- damaged spermatozoa. J. Exp. Zool. 284, 696-704.

Ahmadnia, H., Ghanbari, M., Moradi, M.R., and Khaje-Dalouee, M. (2007). Effect of Cigarette Smoke on Spermatogenesis in Rats. Urology Journal 4, 159-163.

Aishwarya, V., Grover, A., and Sharma, P.C. (2007). EuMicroSatdb: a database for microsatellites in the sequenced genomes of eukaryotes. BMC Genomics 8, 225.

Aitken, R.J., Koopman, P., and Lewis, S.E. (2004). Seeds of concern. Nature 432, 48-52.

Al-Bader, A., Omu, A., and Dashti, H. (1999). Chronic cadmium toxicity to sperm of heavy cigarette smokers: immunomodulation by zinc. Arch. Androl. 43, 135-140.

Alexandrov, L.B., and Stratton, M.R. (2014). Mutational signatures: the patterns of somatic mutations hidden in cancer genomes. Curr. Opin. Genet. Dev. 24, 52-60.

Al-Matubsi, H.Y., Kanaan, R.A., Hamdan, F., Salim, M., Oriquat, G.A., and Al Hanbali, O.A. (2011). Smoking practices in Jordanian people and their impact on semen quality and hormonal levels among adult men. Cent. Eur. J. Public Health 19, 54-59.

Al-Turki, H.A. (2015). Effect of smoking on reproductive hormones and semen parameters of infertile Saudi Arabians. Urology Annals 7, 63. 
Al-Turki, H.A. (2016). A 5-year analysis of semen parameters in Saudi Arabian men attending infertility clinics. J. Int. Med. Res. 44, 656-661.

Amann, R.P. (2008). The cycle of the seminiferous epithelium in humans: a need to revisit? J. Androl. 29, 469-487.

Andersen, A., Semczuk, M., and Tabor, A. (1984). Prolactin and Pituitary- Gonadal Function in Cigarette Smoking Infertile Patients. Andrologia 16, 391-396.

Andrabi, S. (2007). Mammalian sperm chromatin structure and assessment of DNA fragmentation. J. Assist. Reprod. Genet. 24, 561-569.

Anifandis, G., Bounartzi, T., Messini, C., Dafopoulos, K., Sotiriou, S., and Messinis, I. (2014). The impact of cigarette smoking and alcohol consumption on sperm parameters and sperm DNA fragmentation (SDF) measured by Halosperm ${ }^{\circledR}$. Arch. Gynecol. Obstet. $290,777-782$.

Anmarkrud, J.A., Kleven, O., Augustin, J., Bentz, K.H., Blomqvist, D., Fernie, K.J., Magrath, M.J., Pärn, H., Quinn, J.S., and Robertson, R.J. (2011). Factors affecting germline mutations in a hypervariable microsatellite: a comparative analysis of six species of swallows (Aves: Hirundinidae). Mutation Research/Fundamental and Molecular Mechanisms of Mutagenesis 708, 37-43.

Anna, L., Holmila, R., Kovacs, K., Gyorffy, E., Gyori, Z., Segesdi, J., Minarovits, J., Soltesz, I., Kostic, S., Csekeo, A., Husgafvel-Pursiainen, K., and Schoket, B. (2009). Relationship between TP53 tumour suppressor gene mutations and smoking-related bulky DNA adducts in a lung cancer study population from Hungary. Mutagenesis 24, 475-480.

Antoniassi, M., Intasqui Lopes, P., Camargo, M., Zylbersztejn, D., Carvalho, V., Cardozo, K., and Bertolla, R. (2016). Analysis of the sperm functional aspects and seminal plasma proteomic profile from male smokers. BJU Int.

Arlt, M.F., Mulle, J.G., Schaibley, V.M., Ragland, R.L., Durkin, S.G., Warren, S.T., and Glover, T.W. (2009). Replication stress induces genome-wide copy number changes in human cells that resemble polymorphic and pathogenic variants. The American Journal of Human Genetics 84, 339-350.

Arlt, M.F., Rajendran, S., Birkeland, S.R., Wilson, T.E., and Glover, T.W. (2014). Copy number variants are produced in response to low- dose ionizing radiation in cultured cells. Environ. Mol. Mutagen. 55, 103-113.

Arlt, M.F., Wilson, T.E., and Glover, T.W. (2012). Replication stress and mechanisms of CNV formation. Curr. Opin. Genet. Dev. 22, 204-210. 
Arlt, M.F., Ozdemir, A.C., Birkeland, S.R., Wilson, T.E., and Glover, T.W. (2011). Hydroxyurea induces de novo copy number variants in human cells. Proc. Natl. Acad. Sci. U. S. A. 108, 17360-17365.

Armour, J.A., Brinkworth, M.H., and Kamischke, A. (1999). Direct analysis by smallpool PCR of MS205 minisatellite mutation rates in sperm after mutagenic therapies. Mutation Research/Genetic Toxicology and Environmental Mutagenesis 445, 73-80.

Armour, J.A.L. (2006). Tandemly repeated DNA: Why should anyone care? Mutation Research/Fundamental and Molecular Mechanisms of Mutagenesis 598, 6-14.

Arrieta, O., Quintana-Carrillo, R.H., Ahumada-Curiel, G., Corona-Cruz, J.F., CorreaAcevedo, E., Zinser-Sierra, J., de la Mata-Moya, D., Mohar-Betancourt, A., MoralesOyarvide, V., and Reynales-Shigematsu, L.M. (2015). Medical care costs incurred by patients with smoking-related non-small cell lung cancer treated at the National Cancer Institute of Mexico. Tobacco Induced Diseases 12, 1.

Aryanpur, M., Tarahomi, M., Sharifi, H., Heydari, G., Hessami, Z., Akhoundi, M., and Masjedi, M.R. (2011). Comparison of spermatozoa quality in male smokers and nonsmokers of Iranian infertile couples. International Journal of Fertility \& Sterility 5, 152.

Ashby, J. (1995). Transgenic germ cell mutation assays: a small collaborative study. Environ. Mol. Mutagen. 25, 1-3.

Ashby, J., Gorelick, N., and Shelby, M. (1997). Mutation assays in male germ cells from transgenic mice: overview of study and conclusions. Mut.Res.-Genetic Toxicology and Environmental Mutagenesis 388, 111-122.

Atladóttir, H.O., Parner, E.T., Schendel, D., Dalsgaard, S., Thomsen, P.H., and Thorsen, P. (2007). Time trends in reported diagnoses of childhood neuropsychiatric disorders: a Danish cohort study. Arch. Pediatr. Adolesc. Med. 161, 193-198.

Attia, S., Ahmad, S., Abd-Ellah, M., Hamada, F., and Bakheet, S. (2013). Germ cell mutagenicity of topoisomerase I inhibitor topotecan detected in the male mouse-dominant lethal study. Food and Chemical Toxicology 62, 470-474.

Autrup, H. (1993). Transplacental transfer of genotoxins and transplacental carcinogenesis. Environ. Health Perspect. 101 Suppl 2, 33-38.

Avkin, S., Goldsmith, M., Velasco-Miguel, S., Geacintov, N., Friedberg, E.C., and Livneh, Z. (2004). Quantitative analysis of translesion DNA synthesis across a benzo[a]pyrene-guanine adduct in mammalian cells: the role of DNA polymerase kappa. J. Biol. Chem. 279, 53298-53305. 
Axelsson, J., Rylander, L., Rignell-Hydbom, A., Silfver, K.Å, Stenqvist, A., and Giwercman, A. (2013). The impact of paternal and maternal smoking on semen quality of adolescent men. PloS One 8 , e66766.

Aydin, M.S., Senturk, G.E., and Ercan, F. (2013). Cryopreservation increases DNA fragmentation in spermatozoa of smokers. Acta Histochem. 115, 394-400.

Baarends, W.M., van der Laan, R., and Grootegoed, J.A. (2001). DNA repair mechanisms and gametogenesis. Reproduction 121, 31-39.

Baird, W.M., Hooven, L.A., and Mahadevan, B. (2005). Carcinogenic polycyclic aromatic hydrocarbon- DNA adducts and mechanism of action. Environ. Mol. Mutagen. $45,106-114$.

Baker, S.M., Bronner, C.E., Zhang, L., Plug, A.W., Robatzek, M., Warren, G., Elliott, E.A., Yu, J., Ashley, T., and Arnheim, N. (1995). Male mice defective in the DNA mismatch repair gene PMS2 exhibit abnormal chromosome synapsis in meiosis. Cell 82, 309-319.

Banerjee, A., Pakrashi, A., Chatterjee, S., Ghosh, S., and Dutta, S. (1993). Semen characteristics of tobacco users in India. Arch. Androl. 30, 35-40.

Barber, R.C., Miccoli, L., van Buul, P.P., Burr, K.L., van Duyn-Goedhart, A., Angulo, J.F., and Dubrova, Y.E. (2004). Germline mutation rates at tandem repeat loci in DNArepair deficient mice. Mutation Research/Fundamental and Molecular Mechanisms of Mutagenesis 554, 287-295.

Bateman, A. (1966). Testing chemicals for mutagenicity in a mammal. Nature 210, 205206.

Bateman, A. (1958). Mutagenic sensitivity of maturing germ cells in the male mouse. Heredity 12, 66.

Beal, M.A., Gagné, R., Williams, A., Marchetti, F., and Yauk, C.L. (2015a). Characterizing Benzo [a] pyrene-induced lacZ mutation spectrum in transgenic mice using next-generation sequencing. BMC Genomics 16, 812.

Beal, M.A., Rowan-Carroll, A., Campbell, C., Williams, A., Somers, C.M., Marchetti, F., and Yauk, C.L. (2015b). Single-molecule PCR analysis of an unstable microsatellite for detecting mutations in sperm of mice exposed to chemical mutagens. Mutation Research/Fundamental and Molecular Mechanisms of Mutagenesis 775, 26-32.

Beal, M.A., and Somers, C.M. (2013). Environment and lifestyle effects. Paternal Influences on Human Reproductive Success 129. 
Beal, M.A., Glenn, T.C., Lance, S.L., and Somers, C.M. (2012a). Characterization of unstable microsatellites in mice: No evidence for germline mutation induction following gamma-radiation exposure. Environ. Mol. Mutagen. 53, 599-607.

Beal, M.A., Glenn, T.C., and Somers, C.M. (2012b). Whole genome sequencing for quantifying germline mutation frequency in humans and model species: Cautious optimism. Mutation Research/Reviews in Mutation Research 750, 96-106.

Belcheva, A., Ivanova-Kicheva, M., Tzvetkova, P., and Marinov, M. (2004). Effects of cigarette smoking on sperm plasma membrane integrity and DNA fragmentation. Int. J. Androl. 27, 296-300.

Benoff, S., Hauser, R., Marmar, J.L., Hurley, I.R., Napolitano, B., and Centola, G.M. (2009). Cadmium concentrations in blood and seminal plasma: correlations with sperm number and motility in three male populations (infertility patients, artificial insemination donors, and unselected volunteers). Mol. Med. 15, 248-262.

Beranek, D.T. (1990). Distribution of methyl and ethyl adducts following alkylation with monofunctional alkylating agents. Mutation Research/Fundamental and Molecular Mechanisms of Mutagenesis 231, 11-30.

Besaratinia, A., Li, H., Yoon, J., Zheng, A., Gao, H., and Tommasi, S. (2012). A highthroughput next-generation sequencing-based method for detecting the mutational fingerprint of carcinogens. Nucleic Acids Res. 40, e116-e116.

Besenbacher, S., Sulem, P., Helgason, A., Helgason, H., Kristjansson, H., Jonasdottir, A., Jonasdottir, A., Magnusson, O.T., Thorsteinsdottir, U., and Masson, G. (2016). Multinucleotide de novo Mutations in Humans. PLoS Genetics 12, e1006315.

Besingi, W., and Johansson, A. (2014). Smoke-related DNA methylation changes in the etiology of human disease. Hum. Mol. Genet. 23, 2290-2297.

Bi, X., Slater, D.M., Ohmori, H., and Vaziri, C. (2005). DNA polymerase kappa is specifically required for recovery from the benzo[a]pyrene-dihydrodiol epoxide (BPDE)induced S-phase checkpoint. J. Biol. Chem. 280, 22343-22355.

Biesecker, L.G., and Spinner, N.B. (2013). A genomic view of mosaicism and human disease. Nature Reviews Genetics 14, 307-320.

Blanco-Muñoz, J., Torres-Sánchez, L., and López-Carrillo, L. (2009). Exposure to maternal and paternal tobacco consumption and risk of spontaneous abortion. Public Health Rep. 124, 317-322.

Bochukova, E.G., Huang, N., Keogh, J., Henning, E., Purmann, C., Blaszczyk, K., Saeed, S., Hamilton-Shield, J., Clayton-Smith, J., and O'Rahilly, S. (2009). Large, rare chromosomal deletions associated with severe early-onset obesity. Nature 463, 666-670. 
Boffetta, P., Trédaniel, J., and Greco, A. (2000). Risk of childhood cancer and adult lung cancer after childhood exposure to passive smoke: A meta-analysis. Environ. Health Perspect. 108, 73.

Bois, P., Williamson, J., Brown, J., Dubrova, Y.E., and Jeffreys, A.J. (1998). A novel unstable mouse VNTR family expanded from SINE B1 elements. Genomics 49, 122-128.

Bojar, I., Witczak, M., and Wdowiak, A. (2013). Biological and environmental conditionings for a sperm DNA fragmentation. Ann. Agric. Environ. Med. 20, 865-868.

Bonassi, S., Neri, M., Lando, C., Ceppi, M., Lin, Y., Chang, W.P., Holland, N., KirschVolders, M., Zeiger, E., and Fenech, M. (2003). Effect of smoking habit on the frequency of micronuclei in human lymphocytes: results from the Human MicroNucleus project. Mutat. Res. 543, 155-166.

Bostrom, C.E., Gerde, P., Hanberg, A., Jernstrom, B., Johansson, C., Kyrklund, T., Rannug, A., Tornqvist, M., Victorin, K., and Westerholm, R. (2002). Cancer risk assessment, indicators, and guidelines for polycyclic aromatic hydrocarbons in the ambient air. Environ. Health Perspect. 110 Suppl 3, 451-488.

Boyko, A.R., Williams on, S.H., Indap, A.R., Degenhardt, J.D., Hernandez, R.D., Lohmueller, K.E., Adams, M.D., Schmidt, S., Sninsky, J.J., and Sunyaev, S.R. (2008). Assessing the evolutionary impact of amino acid mutations in the human genome. PLoS Genetics 4, e1000083.

Brenneke, H. (1937). Strahlenschädigung von Mäuse-und Rattensperma, beobachtet an der Frühentwicklung der Eier. Strahlentherapie 60, 214-238.

Brevik, A., Lindeman, B., Rusnakova, V., Olsen, A.K., Brunborg, G., and Duale, N. (2012). Paternal benzo[a]pyrene exposure affects gene expression in the early developing mouse embryo. Toxicol. Sci. 129, 157-165.

Bridges, B., Clemmesen, J., and Sugimura, T. (1979). ICPEMC publication No. 3 cigarette smoking - Does it carry a genetic risk? Mutation Research/Reviews in Genetic Toxicology 65, 71-81.

Brinkworth, M.H., Anderson, D., Hughes, J.A., Jackson, L.I., Yu, T., and Nieschlag, E. (1998). Genetic effects of 1, 3-butadiene on the mouse testis. Mutation

Research/Fundamental and Molecular Mechanisms of Mutagenesis 397, 67-75.

Brondum, J., Shu, X., Steinbuch, M., Severson, R.K., Potter, J.D., and Robison, L.L. (1999). Parental cigarette smoking and the risk of acute leukemia in children. Cancer 85 , 1380-1388.

Bryce, S.M., Avlasevich, S.L., Bemis, J.C., Phonethepswath, S., and Dertinger, S.D. (2010). Miniaturized flow cytometric in vitro micronucleus assay represents an efficient 
tool for comprehensively characterizing genotoxicity dose-response relationships. Mutation Research/Genetic Toxicology and Environmental Mutagenesis 703, 191-199.

Buescher, A.V., Cidav, Z., Knapp, M., and Mandell, D.S. (2014). Costs of autism spectrum disorders in the United Kingdom and the United States. JAMA Pediatrics 168, 721-728.

Buscariollo, D.L., Fang, X., Greenwood, V., Xue, H., Rivkees, S.A., and Wendler, C.C. (2014). Embryonic caffeine exposure acts via A1 adenosine receptors to alter adult cardiac function and DNA methylation in mice. PloS One 9, e87547.

Campbell, C.D., Chong, J.X., Malig, M., Ko, A., Dumont, B.L., Han, L., Vives, L., O'Roak, B.J., Sudmant, P.H., and Shendure, J. (2012). Estimating the human mutation rate using autozygosity in a founder population. Nat. Genet. 44, 1277-1281.

Campbell, C.D., and Eichler, E.E. (2013). Properties and rates of germline mutations in humans. Trends in Genetics 29, 575-584.

Campbell, I.M., Shaw, C.A., Stankiewicz, P., and Lupski, J.R. (2015). Somatic mosaicism: implications for disease and transmission genetics. Trends in Genetics 31, 382-392.

Campbell, I.M., Yuan, B., Robberecht, C., Pfundt, R., Szafranski, P., McEntagart, M.E., Nagamani, S.C., Erez, A., Bartnik, M., and Wiśniowiecka-Kowalnik, B. (2014). Parental somatic mosaicism is underrecognized and influences recurrence risk of genomic disorders. The American Journal of Human Genetics 95, 173-182.

Capuano, F., Mülleder, M., Kok, R., Blom, H.J., and Ralser, M. (2014). Cytosine DNA methylation is found in Drosophila melanogaster but absent in Saccharomyces cerevisiae, Schizosaccharomyces pombe, and other yeast species. Anal. Chem. 86, 3697-3702.

Cardy, A., Barker, S., Chesney, D., Sharp, L., Maffulli, N., and Miedzybrodzka, Z. (2007). Pedigree analysis and epidemiological features of idiopathic congenital talipes equinovarus in the United Kingdom: a case-control study. BMC Musculoskeletal Disorders 8,1 .

Carmichael, S.L., Shaw, G.M., Yang, W., Lammer, E.J., Zhu, H., and Finnell, R.H. (2004). Limb deficiency defects, MSX1, and exposure to tobacco smoke. American Journal of Medical Genetics Part A 125, 285-289.

Caserta, D., Bordi, G., Di Segni, N., D’Ambrosio, A., Mallozzi, M., and Moscarini, M. (2013). The influence of cigarette smoking on a population of infertile men and women. Arch. Gynecol. Obstet. 287, 813-818. 
Castellani, C.A., Awamleh, Z., Melka, M.G., O'Reilly, R.L., and Singh, S.M. (2014). Copy number variation distribution in six monozygotic twin pairs discordant for schizophrenia. Twin Research and Human Genetics 17, 108-120.

Cattanach, B., and Moseley, H. (1973). Nondisjunction and reduced fertility caused by the tobacco mouse metacentric chromosomes. Cytogenetic and Genome Research 12, 264-287.

Cerón-Carrasco, J.P., and Jacquemin, D. (2015). DNA spontaneous mutation and its role in the evolution of GC-content: assessing the impact of the genetic sequence. Physical Chemistry Chemical Physics 17, 7754-7760.

Chabory, E., Damon, C., Lenoir, A., Kauselmann, G., Kern, H., Zevnik, B., Garrel, C., Saez, F., Cadet, R., Henry-Berger, J., et al. (2009). Epididymis seleno-independent glutathione peroxidase 5 maintains sperm DNA integrity in mice. J. Clin. Invest. 119, 2074-2085.

Chambers, J.M. (1992). Linear models.

Chang, J.S., Selvin, S., Metayer, C., Crouse, V., Golembesky, A., and Buffler, P.A. (2006). Parental smoking and the risk of childhood leukemia. Am. J. Epidemiol. 163, 1091-1100.

Chang, J.S. (2009). Parentalsmoking and childhood leukemia. Methods Mol. Biol. 472, 103-137.

Chatenoud, L., Parazzini, F., Di Cintio, E., Zanconato, G., Benzi, G., Bortolus, R., and La Vecchia, C. (1998). Paternal and maternal smoking habits before conception and during the first trimester: relation to spontaneous abortion. Ann. Epidemiol. 8, 520-526.

Chen, H., and Kuo, C. (2007). Cotinine characterization and quality effect of sperm for smoking and nonsmoking students. Bull. Environ. Contam. Toxicol. 79, 11-14.

Chen, S., and Jiang, W. (2015). Effect of hydrogen injected subcutaneously on testicular tissues of rats exposed to cigarette smoke. International Journal of Clinical and Experimental Medicine 8, 5565.

Chen, X., Xu, W., Miao, M., Zhu, Z., Dai, J., Chen, Z., Fang, P., Wu, J., Nie, D., Wang, L., et al. (2015). Alteration of sperm protein profile induced by cigarette smoking. Acta Biochim. Biophys. Sin. (Shanghai) 47, 504-515.

Chen, Z., Godfrey-Bailey, L., Schiff, I., and Hauser, R. (2004). Impact of seasonal variation, age and smoking status on human semen parameters: The Massachusetts General Hospital experience. J. Exp. Clin. Assist. Reprod. 1, 2. 
Cheng, S.C., Hilton, B.D., Roman, J.M., and Dipple, A. (1989). DNA adducts from carcinogenic and noncarcinogenic enantiomers of benzo [a] pyrenedihydrodiol epoxide. Chem. Res. Toxicol. 2, 334-340.

Chepelev, N.L., Moffat, I.D., Bowers, W.J., and Yauk, C.L. (2015). Neurotoxicity may be an overlooked consequence of benzo [a] pyrene exposure that is relevant to human health risk assessment. Mutation Research/Reviews in Mutation Research 764, 64-89.

Chia, S., Ong, C., and Tsakok, F. (1994). Effects of cigarette smoking on human semen quality. Arch. Androl. 33, 163-168.

Chia, S.E., Tay, S.K., and Lim, S.T. (1998). What constitutes a normal seminal analysis? Semen parameters of 243 fertile men. Hum. Reprod. 13, 3394-3398.

Chinwalla, A.T., Cook, L.L., Delehaunty, K.D., Fewell, G.A., Fulton, L.A., Fulton, R.S., Graves, T.A., Hillier, L.W., Mardis, E.R., and McPherson, J.D. (2002). Initial sequencing and comparative analysis of the mouse genome. Nature 420, 520-562.

Chohan, K.R., and Badawy, S.Z. (2010). Cigarette smoking impairs sperm bioenergetics. International Braz J Urol 36, 60-65.

Christmann, M., Boisseau, C., Kitzinger, R., Berac, C., Allmann, S., Sommer, T., Aasland, D., Kaina, B., and Tomicic, M.T. (2016). Adaptive upregulation of DNA repair genes following benzo (a) pyrene diol epoxide protects against cell death at the expense of mutations. Nucleic Acids Res. 44, 10727-10743.

Cinar, O., Dilbaz, S., Terzioglu, F., Karahalil, B., Yücel, C., Turk, R., Taskin, L., and Kose, S.K. (2014). Does cigarette smoking really have detrimental effects on outcomes of IVF? European Journal of Obstetrics \& Gynecology and Reproductive Biology 174, 106110.

Clark, T.A., Spittle, K.E., Turner, S.W., and Korlach, J. (2011). Direct detection and sequencing of damaged DNA bases. Genome Integrity 2, 1.

Cohen, A., Wilson, S., Trinh, J., and Ye, X. (2015). Detecting somatic mosaicism: considerations and clinical implications. Clin. Genet. 87, 554-562.

Colagar, A.H., Jorsaraee, G., and Marzony, E.T. (2007). Cigarette smoking and the risk of male infertility. Pak J Biol Sci 10, 3870-3874.

Colagar, A.H., Marzony, E.T., and Chaichi, M.J. (2009). Zinc levels in seminal plasma are associated with sperm quality in fertile and infertile men. Nutr. Res. 29, 82-88.

Collodel, G., Capitani, S., Iacoponi, F., Federico, M., Pascarelli, N., and Moretti, E. (2009). Retrospective assessment of potential negative synergistic effects of varicocele and tobacco use on ultrastructural sperm morphology. Urology 74, 794-799. 
Collodel, G., Capitani, S., Pammolli, A., Giannerini, V., Geminiani, M., and Moretti, E. (2010). Semen quality of male idiopathic infertile smokers and nonsmokers: an ultrastructural study. J. Androl. 31, 108-113.

Conney, A.H., Chang, R.L., Jerina, D.M., and Caroline Wei, S. (1994). Studies on the metabolism of benzo [a] pyrene and dose-dependent differences in the mutagenic profile of its ultimate carcinogenic metabolite. Drug Metab. Rev. 26, 125-163.

Conrad, D.F., Keebler, J.E., DePristo, M.A., Lindsay, S.J., Zhang, Y., Casals, F., Idaghdour, Y., Hartl, C.L., Torroja, C., and Garimella, K.V.(2011). Variation in genomewide mutation rates within and between human families. Nature 201, 1.

Conrad, D.F., Pinto, D., Redon, R., Feuk, L., Gokcumen, O., Zhang, Y., Aerts, J., Andrews, T.D., Barnes, C., and Campbell, P. (2009). Origins and functional impact of copy number variation in the human genome. Nature 464, 704-712.

Cook Jr, E.H., and Scherer, S.W. (2008). Copy-number variations associated with neuropsychiatric conditions. Nature 455, 919-923.

Cooke, M.S., Evans, M.D., Dizdaroglu, M., and Lunec, J. (2003). Oxidative DNA damage: mechanisms, mutation, and disease. Faseb J. 17, 1195-1214.

Cooper, D.N., and Krawczak, M. (1996). Human gene mutation database. Hum. Genet. 98, 629-629.

Cosentino, L., and Heddle, J.A. (2000). Differential mutation of transgenic and endogenous loci in vivo. Mutation Research/Fundamental and Molecular Mechanisms of Mutagenesis 454, 1-10.

Costa, E.O., de Melo e Silva, D., de Melo, A.V., Godoy, F.R., Nunes, H.F., Pedrosa, E.R., Flores, B.C., Rodovalho, R.G., da Silva, C.C., and da Cruz, A.D. (2011). The effect of low-dose exposure on germline microsatellite mutation rates in humans accidentally exposed to caesium-137 in Goiania. Mutagenesis 26, 651-655.

Cresci, M., Foffa, I., Ait-Ali, L., Pulignani, S., Gianicolo, E.A.L., Botto, N., Picano, E., and Andreassi, M.G. (2011). Maternal and paternal environmental risk factors, metabolizing GSTM1 and GSTT1 polymorphisms, and congenital heart disease. Am. J. Cardiol. 108, 1625-1631.

Crow, J.F. (2000). The origins, patterns and implications of human spontaneous mutation. Nature Reviews Genetics 1, 40-47.

Crow, J.F. (1997). The high spontaneous mutation rate: is it a health risk? Proc. Natl. Acad. Sci. U. S. A. 94, 8380-8386. 
Cui, X., Jing, X., Wu, X., Wang, Z., and Li, Q. (2016). Potential effect of smoking on semen quality through DNA damage and the downregulation of Chk1 in sperm. Molecular Medicine Reports 14, 753-761.

D’Onofrio, B.M., Rickert, M.E., Frans, E., Kuja-Halkola, R., Almqvist, C., Sjölander, A., Larsson, H., and Lichtenstein, P. (2014). Paternal age at childbearing and offspring psychiatric and academic morbidity. JAMA Psychiatry 71, 432-438.

da Cruz, A.D., de Melo e Silva, Daniela, da Silva, C.C., Nelson, R.J., Ribeiro, L.M., Pedrosa, E.R., Jayme, J.C., and Curado, M.P. (2008). Microsatellite mutations in the offspring of irradiated parents 19 years after the cesium-137 accident. Mutation Research/Genetic Toxicology and Environmental Mutagenesis 652, 175-179.

Davar, R., Sekhavat, L., and Naserzadeh, N. (2012). Semen parameters of non-infertile smoker and non-smoker men. J. Med. Life. 5, 465-468.

Davis, A.P., and Justice, M.J. (1998). An Oak Ridge legacy: the specific locus test and its role in mouse mutagenesis. Genetics 148, 7-12.

De Bantel, A., Fleury- Feith, J., Poirot, C., Berthaut, I., Garcin, C., Landais, P., and Ravel, C. (2014). Simultaneous vitality and DNA- fragmentation measurement in spermatozoa of smokers and non- smokers. Cytometry Part B: Clinical Cytometry

de la Rochebrochard, E., and Thonneau, P. (2002). Paternal age and maternal age are risk factors for miscarriage; results of a multicentre European study. Hum. Reprod. 17, 16491656.

de Rooij, D.G. (2001). Proliferation and differentiation of spermatogonial stem cells. Reproduction 121, 347-354.

De, S. (2011). Somatic mosaicism in healthy human tissues. Trends in Genetics 27, 217223.

Deciphering Developmental Disorders Study,. (2017). Prevalence and architecture of de novo mutations in developmental disorders. Nature

DeMarini, D.M. (2012). Declaring the existence of human germ- cell mutagens. Environ. Mol. Mutagen. 53, 166-172.

DeMarini, D.M. (2004). Genotoxicity of tobacco smoke and tobacco smoke condensate: a review. Mutation Research/Reviews in Mutation Research 567, 447-474.

DeMarini, D.M., Landi, S., Tian, D., Hanley, N.M., Li, X., Hu, F., Roop, B.C., Mass, M.J., Keohavong, P., Gao, W., et al. (2001). Lung tumor KRAS and TP53 mutations in nonsmokers reflect exposure to PAH-rich coal combustion emissions. Cancer Res. 61, 6679-6681. 
Deng, K., Liu, Z., Lin, Y., Mu, D., Chen, X., Li, J., Li, N., Deng, Y., Li, X., and Wang, Y. (2013). Periconceptional paternal smoking and the risk of congenital heart defects: A case- control study. Birth Defects Research Part A: Clinical and Molecular Teratology 97, 210-216.

Doak, S.H., Jenkins, G.J., Johnson, G.E., Quick, E., Parry, E.M., and Parry, J.M. (2007). Mechanistic influences for mutation induction curves after exposure to DNA-reactive carcinogens. Cancer Res. 67, 3904-3911.

Douglas, G.R., Gingerich, J.D., Gossen, J.A., and Barlett, S.A. (1994). Sequence spectra of spontaneous lacZ gene mutations in transgenic mouse somatic and germline tissues. Mutagenesis 9, 451-458.

Douglas, G.R., Jiao, J., Gingerich, J.D., Gossen, J.A., and Soper, L.M. (1995). Temporal and molecular characteristics of mutations induced by ethylnitrosourea in germ cells isolated from seminiferous tubules and in spermatozoa of lac $Z$ transgenic mice.

Proceedings of the National Academy of Sciences 92, 7485-7489.

Douglas, G.R., Jiao, J., Gingerich, J.D., Soper, L.M., and Gossen, J.A. (1996). System issues: Temporal and molecular characteristics of lac Z mutations in somatic tissues of transgenic mice. Environ. Mol. Mutagen. 28, 317-324.

Dubrova, Y.E., Bersimbaev, R.I., Djansugurova, L.B., Tankimanova, M.K., Mamyrbaeva, Z.Z., Mustonen, R., Lindholm, C., Hultén, M., and Salomaa, S. (2002a). Nuclear weapons tests and human germline mutation rate. Science 295, 1037-1037.

Dubrova, Y.E., Grant, G., Chumak, A.A., Stezhka, V.A., and Karakasian, A.N. (2002b). Elevated minisatellite mutation rate in the post-Chernobyl families from Ukraine. The American Journal of Human Genetics 71, 801-809.

Dubrova, Y.E., Hickenbotham, P., Glen, C.D., Monger, K., Wong, H., and Barber, R.C. (2008). Paternal exposure to ethylnitrosourea results in transgenerational genomic instability in mice. Environ. Mol. Mutagen. 49, 308-311.

Dubrova, Y.E., Jeffreys, A.J., and Malashenko, A.M. (1993). Mouse minisatellite mutations induced by ionizing radiation. Nat. Genet. 5, 92-94.

Dubrova, Y.E., Nesterov, V.N., Krouchinsky, N.G., Ostapenko, V.A., Neumann, R., Neil, D.L., and Jeffreys, A.J. (1996). Human minisatellite mutation rate after the Chernobyl accident.

Dubrova, Y.E., Ploshchanskaya, O.G., Kozionova, O.S., and Akleyev, A.V. (2006).

Minisatellite germline mutation rate in the Techa River population. Mutation

Research/Fundamental and Molecular Mechanisms of Mutagenesis 602, 74-82. 
Dubrova, Y.E., Plumb, M., Brown, J., Fennelly, J., Bois, P., Goodhead, D., and Jeffreys, A.J. (1998). Stage specificity, dose response, and doubling dose for mouse minisatellite germ-line mutation induced by acute radiation. Proceedings of the National Academy of Sciences 95, 6251-6255.

Dubrova, Y.E., Plumb, M., Gutierrez, B., Boulton, E., and Jeffreys, A.J. (2000). Genome stability: transgenerational mutation by radiation. Nature 405, 37-37.

Duselis, A.R., and Vrana, P.B. (2007). Harvesting sperm and artificial insemination of mice. J. Vis. Exp. (3):184. doi, 184.

Eastmond, D.A., Hartwig, A., Anderson, D., Anwar, W.A., Cimino, M.C., Dobrev, I., Douglas, G.R., Nohmi, T., Phillips, D.H., and Vickers, C. (2009). Mutagenicity testing for chemical risk assessment: update of the WHO/IPCS Harmonized Scheme. Mutagenesis 24, 341-349.

Edraki, M., and Rambod, M. (2011). Parental smoking and risk of childhood cancer: hospital-based case-control study in Shiraz. Emhj 17,

Edwards, J.L., and Klein, R.E. (1961). Cell renewal in adult mouse tissues. Am. J. Pathol. $38,437-453$.

Eisenstadt, E., Warren, A.J., Porter, J., Atkins, D., and Miller, J.H. (1982). Carcinogenic epoxides of benzo[a]pyrene and cyclopenta[cd]pyrene induce base substitutions via specific transversions. Proc. Natl. Acad. Sci. U. S. A. 79, 1945-1949.

Ellegren, H., Lindgren, G., Primmer, C.R., and Møller, A.P. (1997). Fitness loss and germline mutations in barn swallows breeding in Chernobyl. Nature 389, 593-596.

El-Melegy, N.T., and Ali, M.M. (2011). Apoptotic markers in semen of infertile men: Association with cigarette smoking. International Braz J Urol 37, 495-506.

Elshal, M.F., El-Sayed, I.H., Elsaied, M.A., El-Masry, S.A., and Kumosani, T.A. (2009). Sperm head defects and disturbances in spermatozoal chromatin and DNA integrities in idiopathic infertile subjects: association with cigarette smoking. Clin. Biochem. 42, 589.

Epstein, C.J., and Travis, B. (1979). Preimplantation lethality of monosomy for mouse chromosome 19.

Epstein, S.S. (1973). Use of the dominant-lethal test to detect genetic activity of environmental chemicals. Environ. Health Perspect. 6, 23-26.

Erickson, R.P. (2010). Somatic gene mutation and human disease other than cancer: an update. Mutation Research/Reviews in Mutation Research 705, 96-106. 
Eriksen, M., Mackay, J.L., and Ross, H. (2012). The tobacco atlas American Cancer Society).

Evans, H., Fletcher, J., Torrance, M., and Hargreave, T. (1981). Sperm abnormalities and cigarette smoking. The Lancet 317, 627-629.

Evenson, D.P., Darzynkiewicz, Z., and Melamed, M.R. (1980). Relation of mammalian sperm chromatin heterogeneity to fertility. Science 210, 1131-1133.

Evenson, D.P., Jost, L.K., Marshall, D., Zinaman, M.J., Clegg, E., Purvis, K., de Angelis, P., and Claussen, O.P. (1999). Utility of the sperm chromatin structure assay as a diagnostic and prognostic tool in the human fertility clinic. Hum. Reprod. 14, 1039-1049.

Fariello, R.M., Pariz, J.R., Spaine, D.M., Gozzo, F.C., Pilau, E.J., Fraietta, R., Bertolla, R.P., Andreoni, C., and Cedenho, A.P. (2012). Effect of smoking on the functional aspects of sperm and seminal plasma protein profiles in patients with varicocele. Hum. Reprod. 27, 3140-3149.

Farrer, R.A., Henk, D.A., MacLean, D., Studholme, D.J., and Fisher, M.C. (2013). Using False Discovery Rates to Benchmark SNP-callers in next-generation sequencing projects. Scientific Reports 3,

Faure, A., Aknin - Seifer, I., Frérot, G., Pelletier, R., De Robertis, C., Cans, C., Levy, R., Jimenez, C., Lejeune, H., and Terrier, N. (2007). Predictive factors for an increased risk of sperm aneuploidies in oligo- astheno- teratozoospermic males. Int. J. Androl. 30, 153162.

Fauser, B., Devroey, P., Diedrich, K., Balaban, B., Bonduelle, M., Delemarre-Van De Waal, H., Estella, C., Ezcurra, D., Geraedts, J., and Howles, C. (2014). Health outcomes of children born after IVF/ICSI: a review of current expert opinion and literature. Reproductive Biomedicine Online 28, 162-182.

Fay, J.C., Wyckoff, G.J., and Wu, C. (2001). Positive and negative selection on the human genome. Genetics 158, 1227-1234.

Fernandes, A., Liu, T., Amin, S., Geacintov, N.E., Grollman, A.P., and Moriya, M. (1998). Mutagenic potential of stereoisomeric bay region (+)- and (-)-cis-antibenzo[a]pyrene diol epoxide-N 2-2'-deoxyguanosine adducts in Escherichia coli and simian kidney cells. Biochemistry (N. Y. ) 37, 10164-10172.

Fernandez, J.L., Muriel, L., Rivero, M.T., Goyanes, V., Vazquez, R., and Alvarez, J.G. (2003). The sperm chromatin dispersion test: a simple method for the determination of sperm DNA fragmentation. J. Androl. 24, 59-66.

Firns, S., Cruzat, V.F., Keane, K.N., Joesbury, K.A., Lee, A.H., Newsholme, P., and Yovich, J.L. (2015). The effect of cigarette smoking, alcohol consumption and fruit and 
vegetable consumption on IVF outcomes: a review and presentation of original data. Reproductive Biology and Endocrinology 13, 1-13.

Flibotte, S., Edgley, M.L., Chaudhry, I., Taylor, J., Neil, S.E., Rogula, A., Zapf, R., Hirst, M., Butterfield, Y., Jones, S.J., et al. (2010). Whole-genome profiling of mutagenesis in Caenorhabditis elegans. Genetics 185, 431-441.

Ford, C. (1972). Gross genome unbalance in mouse spermatozoa: does it influence the capacity to fertilize. The Genetics of the Spermatozoon 285-304.

Fowler, P.A., Dora, N.J., McFerran, H., Amezaga, M.R., Miller, D.W., Lea, R.G., Cash, P., McNeilly, A.S., Evans, N.P., Cotinot, C., Sharpe, R.M., and Rhind, S.M. (2008). In utero exposure to low doses of environmental pollutants disrupts fetal ovarian development in sheep. Mol. Hum. Reprod. 14, 269-280.

Fraga, C., Motchnik, P., Wyrobek, A., Rempel, D., and Ames, B. (1996). Smoking and low antioxidant levels increase oxidative damage to sperm DNA. Mutation Research/Fundamental and Molecular Mechanisms of Mutagenesis 351, 199-203.

Francioli, L.C., Polak, P.P., Koren, A., Menelaou, A., Chun, S., Renkens, I., van Duijn, C.M., Swertz, M., Wijmenga, C., and van Ommen, G. (2015). Genome-wide patterns and properties of de novo mutations in humans. Nat. Genet. 47, 822-826.

Friedman, J., Baross, Á, Delaney, A.D., Ally, A., Arbour, L., Asano, J., Bailey, D.K., Barber, S., Birch, P., and Brown-John, M. (2006). Oligonucleotide microarray analysis of genomic imbalance in children with mental retardation. The American Journal of Human Genetics 79, 500-513.

Fu, L., Wang, G., Ma, B., and Zhang, H. (2011). Exploring the common molecular basis for the universal DNA mutation bias: Revival of Löwdin mutation model. Biochem. Biophys. Res. Commun. 409, 367-371.

Furitsu, K., Ryo, H., Yeliseeva, K.G., Thuy, L.T.T., Kawabata, H., Krupnova, E.V., Trusova, V.D., Rzheutsky, V.A., Nakajima, H., and Kartel, N. (2005). Microsatellite mutations show no increases in the children of the Chernobyl liquidators. Mutation Research/Genetic Toxicology and Environmental Mutagenesis 581, 69-82.

Gallagher, J.E., Vine, M.F., Schramm, M.M., Lewtas, J., George, M.H., Hulka, B.S., and Everson, R.B. (1993). 32P-postlabeling analysis of DNA adducts in human sperm cells from smokers and nonsmokers. Cancer Epidemiol. Biomarkers Prev. 2, 581-585.

Garcia, P.C., Piffer, R.C., Gerardin, D.C.C., Sankako, M.K., de Lima, Rodrigo Otávio Alves, and Pereira, O.C.M. (2012). Could zinc prevent reproductive alterations caused by cigarette smoke in male rats? Reproduction, Fertility and Development 24, 559-567. 
Gartner, A., Milstein, S., Ahmed, S., Hodgkin, J., and Hengartner, M.O. (2000). A conserved checkpoint pathway mediates DNA damage-induced apoptosis and cell cycle arrest in C. elegans. Mol. Cell 5, 435-443.

Gaspari, L., Chang, S., Santella, R.M., Garte, S., Pedotti, P., and Taioli, E. (2003). Polycyclic aromatic hydrocarbon-DNA adducts in human sperm as a marker of DNA damage and infertility. Mutation Research/Genetic Toxicology and Environmental Mutagenesis 535, 155-160.

Gautam, S., Chawla, B., Kumar, S.B., Bisht, S., and Dada, R. (2015). Sperm DNA damage in non-familial sporadic heritable retinoblastoma (NFSHRb). Clinical Epidemiology and Global Health 3, S20-S25.

Gavrieli, Y., Sherman, Y., and Ben-Sasson, S.A. (1992). Identification of programmed cell death in situ via specific labeling of nuclear DNA fragmentation. J. Cell Biol. 119, 493-501.

Gelboin, H.V.(1980). Benzo[alpha]pyrene metabolism, activation and carcinogenesis: role and regulation of mixed-function oxidases and related enzymes. Physiol. Rev. 60, 1107-1166.

Generoso, W.M., Bishop, J.B., Gosslee, D.G., Newell, G.W., Sheu, C., and von Halle, E. (1980). Heritable translocation test in mice. Mutation Research/Reviews in Genetic Toxicology 76, 191-215.

Generoso, W., Cain, K., Cornett, C., Cacheiro, N., Hughes, L., Albertini, R., and Casciano, D. (1990). Concentration- response curves for ethylene- oxide- induced heritable translocations and dominant lethal mutations. Environ. Mol. Mutagen. 16, 126131.

Generoso, W., Cain, K., Hellwig, C., and Cacheiro, N. (1982). Lack of association between induction of dominant-lethal mutations and induction of heritable translocations with benzo [a] pyrene in postmeiotic germ cells of male mice. Mutation Research/Fundamental and Molecular Mechanisms of Mutagenesis 94, 155-163.

Generoso, W., Cain, K., Hughes, L., Sega, G., Braden, P., Gosslee, D., and Shelby, M. (1986). Ethylene oxide dose and dose- rate effects in the mouse dominant- lethal test. Environ. Mutagen. 8, 1-7.

Genome of the Netherlands Consortium. (2014). Whole-genome sequence variation, population structure and demographic history of the Dutch population. Nat. Genet. 46, 818-825.

Ghaffari, M.A., and Rostami, M. (2012). Lipid peroxidation and nitric oxide levels in male Smokers' spermatozoa and their relation with sperm motility. Journal of Reproduction \& Infertility 13,81 . 
Ghaffari, M.A., and Rostami, M. (2013). The effect of cigarette smoking on human sperm creatine kinase activity: as an ATP buffering system in sperm. Int. J. Fertil. Steril. $6,258-265$.

Gibbs, M., Collick, A., Kelly, R.G., and Jeffreys, A.J. (1993). A tetranucleotide repeat mouse minisatellite displaying substantial somatic instability during early preimplantation development. Genomics 17, 121-128.

Girard, S.L., Gauthier, J., Noreau, A., Xiong, L., Zhou, S., Jouan, L., Dionne-Laporte, A., Spiegelman, D., Henrion, E., and Diallo, O. (2011). Increased exonic de novo mutation rate in individuals with schizophrenia. Nat. Genet. 43, 860-863.

Glen, C.D., and Dubrova, Y.E. (2012). Exposure to anticancer drugs can result in transgenerational genomic instability in mice. Proceedings of the National Academy of Sciences 109, 2984-2988.

Glen, C.D., Smith, A.G., and Dubrova, Y.E. (2008). Single-molecule PCR analysis of germ line mutation induction by anticancer drugs in mice. Cancer Res. 68, 3630-3636.

Gocke, E., and Müller, L. (2009). In vivo studies in the mouse to define a threshold for the genotoxicity of EMS and ENU. Mutation Research/Genetic Toxicology and Environmental Mutagenesis 678, 101-107.

Godschalk, R.W., Verhofstad, N., Verheijen, M., Yauk, C.L., Linschooten, J.O., van Steeg, H., van Oostrom, C.T., van Benthem, J., and van Schooten, F.J. (2015). Effects of benzo [a] pyrene on mouse germ cells: heritable DNA mutation, testicular cell hypomethylation and their interaction with nucleotide excision repair. Toxicology Research

Gold, E.B., Leviton, A., Lopez, R., Gilles, F.H., Hedley-Whyte, T., Kolonel, L.N., Lyon, J.L., Swanson, G.M., Weiss, N.S., and West, D. (1993). Parental smoking and risk of childhood brain tumors. Am. J. Epidemiol. 137, 620-628.

Goldstein, D.M., and Stawkowski, M.E. (2015). James V. Neel and Yuri E. Dubrova: Cold war debates and the genetic effects of low-dose radiation. Journal of the History of Biology 48, 67-98.

Gollapudi, B., Johnson, G., Hernandez, L., Pottenger, L., Dearfield, K., Jeffrey, A., Julien, E., Kim, J., Lovell, D., and Macgregor, J. (2013). Quantitative approaches for assessing dose-response relationships in genetic toxicology studies. Environ. Mol. Mutagen. 54, 8-18.

Gossen, J., Molijn, A., Douglas, G., and Vijg, J. (1992). Application of galactosesensitive E. coli strains as selective hosts for LacZ-plasmids. Nucleic Acids Res. 20, 3254 . 
Gossen, J.A., De Leeuw, W., Tan, C., Zwarthoff, E.C., Berends, F., Lohman, P., Knook, D.L., and Vijg, J. (1989). Efficient rescue of integrated shuttle vectors from transgenic mice: a model for studying mutations in vivo. Proceedings of the National Academy of Sciences 86, 7971-7975.

Gouraud, A., Brazeau, M., Grégoire, M., Simard, O., Massonneau, J., Arguin, M., and Boissonneault, G. (2013). "Breaking news" from spermatids. Basic and Clinical Andrology 23, 1.

Goverde, H.J., Dekker, H.S., Janssen, H.J., Bastiaans, B.A., Rolland, R., and Zielhuis, G.A. (1995). Semen quality and frequency of smoking and alcohol consumption--an explorative study. Int. J. Fertil. Menopausal Stud. 40, 135-138.

Granville, C.A., Hanley, N.M., Mumford, J.L., and DeMarini, D.M. (2003). Mutation spectra of smoky coal combustion emissions in Salmonella reflect the TP53 and KRAS mutations in lung tumors from smoky coal-exposed individuals. Mutation

Research/Fundamental and Molecular Mechanisms of Mutagenesis 525, 77-83.

Gregoire, M.C., Massonneau, J., Simard, O., Gouraud, A., Brazeau, M.A., Arguin, M., Leduc, F., and Boissonneault, G. (2013). Male-driven de novo mutations in haploid germ cells. Mol. Hum. Reprod. 19, 495-499.

Griffiths, A.J.(2005). An introduction to genetic analysis Macmillan).

Griffiths, A.J., Miller, J.H., Suzuki, D.T., Lewontin, R.C., and Gelbart, W.M. (2000). Spontaneous mutations.

Grufferman, S., Wang, H.H., DeLong, E.R., Kimm, S.Y., Delzell, E.S., and Falletta, J.M. (1982). Environmental factors in the etiology of rhabdomyosarcoma in childhood. J. Natl. Cancer Inst. 68, 107-113.

Gu, W., Zhang, F., and Lupski, J.R. (2008). Mechanisms for human genomic rearrangements. Pathogenetics 1,4 .

Guida, F., Sandanger, T.M., Castagne, R., Campanella, G., Polidoro, S., Palli, D., Krogh, V., Tumino, R., Sacerdote, C., Panico, S., et al. (2015). Dynamics of smoking-induced genome-wide methylation changes with time since smoking cessation. Hum. Mol. Genet. $24,2349-2359$.

Gunes, S., Al-Sadaan, M., and Agarwal, A. (2015). Spermatogenesis, DNA damage and DNA repair mechanisms in male infertility. Reproductive Biomedicine Online 31, 309319.

Guo, C., Gao, T., Confer, N., Velasco-Miguel, S., and Friedberg, E.C. (2005). Multiple PolK (POLK) transcripts in mammalian testis. DNA Repair 4, 397-402. 
Guo, C., Kosarek-Stancel, J.N., Tang, T., and Friedberg, E.C. (2009). Y-family DNA polymerases in mammalian cells. Cellular and Molecular Life Sciences 66, 2363-2381.

Guz, J., Gackowski, D., Foksinski, M., Rozalski, R., Zarakowska, E., Siomek, A., Szpila, A., Kotzbach, M., Kotzbach, R., and Olinski, R. (2013). Comparison of oxidative stress/DNA damage in semen and blood of fertile and infertile men. PLoS One 8, e68490.

Gymrek, M., Golan, D., Rosset, S., and Erlich, Y. (2012). lobSTR: A short tandem repeat profiler for personal genomes. Genome Res. 22, 1154-1162.

Habibi, M., Gilani, S., Hassanzadeh, G., Dargahi, H., Shamsi, A., Choobineh, K., Saiepour, N., and Choobineh, H. (2012). Assessment of socio-demographic characteristics in Infertile men who Referred to Shariati Hospital in Iran. Life Science Journal 9, 5259-5264.

Hackshaw, A., Rodeck, C., and Boniface, S. (2011). Maternal smoking in pregnancy and birth defects: a systematic review based on 173687 malformed cases and 11.7 million controls. Hum. Reprod. Update 17, 589-604.

Hakura, A., Tsutsui, Y., Sonoda, J., Kai, J., Imade, T., Shimada, M., Sugihara, Y., and Mikami, T. (1998). Comparison between in vivo mutagenicity and carcinogenicity in multiple organs by benzo [a] pyrene in the lacZ transgenic mouse (Muta ${ }^{\mathrm{TM}}$ Mouse). Mutation Research/Fundamental and Molecular Mechanisms of Mutagenesis 398, 123130.

Hakura, A., Tsutsui, Y., Sonoda, J., Tsukidate, K., Mikami, T., and Sagami, F. (2000). Comparison of the mutational spectra of the lacZ transgene in four organs of the Muta ${ }^{\mathrm{TM}}$ Mouse treated with benzo [a] pyrene: target organ specificity. Mutation Research/Fundamental and Molecular Mechanisms of Mutagenesis 447, 239-247.

Hamad, M., Shelko, N., Kartarius, S., Montenarh, M., and Hammadeh, M. (2014). Impact of cigarette smoking on histone $(\mathrm{H} 2 \mathrm{~B})$ to protamine ratio in human spermatozoa and its relation to sperm parameters. Andrology 2, 666-677.

Hammadeh, M.E., Hamad, M.F., Montenarh, M., and Fischer-Hammadeh, C. (2010). Protamine contents and $\mathrm{P} 1 / \mathrm{P} 2$ ratio in human spermatozoa from smokers and nonsmokers. Hum. Reprod. 25, 2708-2720.

Handelsman, D.J., Conway, A.J., Boylan, L.M., and Turtle, J.R. (1984). Testicular function in potential sperm donors: normal ranges and the effects of smoking and varicocele. Int. J. Androl. 7, 369-382.

Hargis, J.C., III, H.F.S., Houk, K., and Wheeler, S.E. (2010). Noncovalent Interactions of a Benzo [a] pyrene Diol Epoxide with DNA Base Pairs: Insight into the Formation of Adducts of ( )-B a P DE-2 with DNA. The Journal of Physical Chemistry A 114, 20382044. 
Harismendy, O., Ng, P.C., Strausberg, R.L., Wang, X., Stockwell, T.B., Beeson, K.Y., Schork, N.J., Murray, S.S., Topol, E.J., and Levy, S. (2009). Evaluation of next generation sequencing platforms for population targeted sequencing studies. Genome Biol. 10, R32.

Härkönen, K., Viitanen, T., Larsen, S.B., Bonde, J.P., and Lähdetie, J. (1999). Aneuploidy in sperm and exposure to fungicides and lifestyle factors. Environ. Mol. Mutagen. 34, 39-46.

Harland, C., Charlier, C., Karim, L., Cambisano, N., Deckers, M., Mullaart, E., Coppieters, W., and Georges, M. (2016). Frequency of mosaicism points towards mutation-prone early cleavage cell divisions. bioRxiv 079863.

Hart, R.J., Doherty, D.A., McLachlan, R.I., Walls, M.L., Keelan, J.A., Dickinson, J.E., Skakkebaek, N.E., Norman, R.J., and Handelsman, D.J. (2015). Testicular function in a birth cohort of young men. Hum. Reprod. 30, 2713-2724.

Hassold, T., Abruzzo, M., Adkins, K., Griffin, D., Merrill, M., Millie, E., Saker, D., Shen, J., and Zaragoza, M. (1996). Human aneuploidy: incidence, origin, and etiology. Environ. Mol. Mutagen. 28, 167-175.

Hassold, T., and Hunt, P. (2001). To err (meiotically) is human: the genesis of human aneuploidy. Nature Reviews Genetics 2, 280-291.

Hassold, T., Hall, H., and Hunt, P. (2007). The origin of human aneuploidy: where we have been, where we are going. Hum. Mol. Genet. 16 Spec No. 2, R203-8.

Hastings, P., Ira, G., and Lupski, J.R. (2009a). A microhomology-mediated breakinduced replication model for the origin of human copy number variation. PLoS Genet 5, e1000327.

Hastings, P., Lupski, J.R., Rosenberg, S.M., and Ira, G. (2009b). Mechanisms of change in gene copy number. Nature Reviews Genetics 10, 551-564.

Hearey, C.D., Harris, J.A., Usatin, M.S., Epstein, D.M., Ury, H.K., and Neutra, R.R. (1984). Investigation of a cluster of anencephaly and spina bifida. Am. J. Epidemiol. 120, 559-564.

Hecht, S.S. (2003). Tobacco carcinogens, their biomarkers and tobacco-induced cancer. Nature Reviews Cancer 3, 733-744.

Hecht, S.S. (2012). Research opportunities related to establishing standards for tobacco products under the Family Smoking Prevention and Tobacco Control Act. Nicotine Tob. Res. 14, 18-28. 
Hedenskog, M., Sjögren, M., Cederberg, H., and Rannug, U. (1997). Induction of germline- length mutations at the minisatellites PC- 1 and PC- 2 in male mice exposed to polychlorinated biphenyls and diesel exhaust emissions. Environ. Mol. Mutagen. 30, 254-259.

Hehir-Kwa, J.Y., Rodriguez-Santiago, B., Vissers, L.E., de Leeuw, N., Pfundt, R., Buitelaar, J.K., Perez-Jurado, L.A., and Veltman, J.A. (2011). De novo copy number variants associated with intellectual disability have a paternal origin and age bias. J. Med. Genet. 48, 776-778.

Hellstrom, W.J., Overstreet, J.W., Sikka, S.C., Denne, J., Ahuja, S., Hoover, A.M., Sides, G.D., Cordell, W.H., Harrison, L.M., and Whitaker, J.S. (2006). Semen and sperm reference ranges for men 45 years of age and older. J. Androl. 27, 421-428.

Hermo, L., Pelletier, R., Cyr, D.G., and Smith, C.E. (2010). Surfing the wave, cycle, life history, and genes/proteins expressed by testicular germ cells. Part 1: background to spermatogenesis, spermatogonia, and spermatocytes. Microsc. Res. Tech. 73, 241-278.

Hess, R.A., and de Franca, L.R. (2009). Spermatogenesis and cycle of the seminiferous epithelium. In Molecular Mechanisms in Spermatogenesis, Springer) pp. 1-15.

Holm, S. (1979). A simple sequentially rejective multiple test procedure. Scandinavian Journal of Statistics 65-70.

Holmberg, P.C., and Nurminen, M. (1980). Congenital defects of the central nervous system and occupational factors during pregnancy. A case- referent study. Am. J. Ind. Med. 1, 167-176.

Holzki, G., Gall, H., and Hermann, J. (1991). Cigarette smoking and sperm quality*. Andrologia 23, 141-144.

Honeycutt, A.A., Grosse, S.D., Dunlap, L.J., Schendel, D.E., Chen, H., Brann, E., and al Homsi, G. (2003). Economic costs of mental retardation, cerebral palsy, hearing loss, and vision impairment. Using Survey Data to Study Disability: Results from the National Health Interview Survey on Disability.London, England: Elsevier Science Ltd 207-228.

Horak, S., Polanska, J., and Widlak, P. (2003). Bulky DNA adducts in human sperm: relationship with fertility, semen quality, smoking, and environmental factors. Mutation Research/Genetic Toxicology and Environmental Mutagenesis 537, 53-65.

Hossain, A., Bhaumik, D., Selukar, R., Huff, C., Rizk, B., and Thorneycroft, I. (1997). Assessment of the relationship of sperm morphology with seminal and other clinical conditions of semen donors. Systems Biology in Reproductive Medicine 39, 111-117.

Hsu, P., Chen, I., Pan, C., Wu, K., Pan, M., Chen, J., Chen, C., Chang-Chien, G., Hsu, C., and Liu, C. (2006). Sperm DNA damage correlates with polycyclic aromatic 
hydrocarbons biomarker in coke-oven workers. Int. Arch. Occup. Environ. Health 79, 349-356.

Hull, M.G.R., North, K., Taylor, H., Farrow, A., and Ford, W.C.L. (2000). Delayed conception and active and passive smoking. Fertil. Steril. 74, 725-733.

Hung, P., Froenicke, L., Lin, C.Y., Lyons, L.A., Miller, M.G., Pinkerton, K.E., and VandeVoort, C.A. (2009). Effects of environmental tobacco smoke in vivo on rhesus monkey semen quality, sperm function, and sperm metabolism. Reproductive Toxicology 27, 140-148.

Hurles, M. (2012). Older males beget more mutations. Nat. Genet. 44,

Huse, S.M., Huber, J.A., Morrison, H.G., Sogin, M.L., and Welch, D.M. (2007). Accuracy and quality of massively parallel DNA pyrosequencing. Genome Biol. 8, R143.

Husgafvel-Pursiainen, K. (2004). Genotoxicity of environmental tobacco smoke: a review. Mutat. Res. 567, 427-445.

Hussein, A., AlGadaa, A., ElFaras, M., and ElFiky, M. (2011). Effect of Tobacco Smoking on Semen Quality in Men With Subfertility. Urotoday Int J. 4,

IARC. (2012a). Personal Habits and Indoor Combustions: A Review of Human Carcinogens. IARC Monograph 100E, 43-211.

IARC. (2012b). A review of human carcinogens: chemical agents and related occupations. IARC Monographs on the Evaluation of Carcinogenic Risks to Humans, IARC Monogr. Eval. Carcinog. Risks Hum. 100F, 111-144.

IARC. (2010). Some non-heterocyclic polycyclic aromatic hydrocarbons and some related exposures. IARC Monogr. Eval. Carcinog. Risks Hum. 92, 1-853.

IARC Working Group on the Evaluation of Carcinogenic Risks to Humans. (2004). Tobacco smoke and involuntary smoking Iarc).

Ikehata, H., Takatsu, M., Saito, Y., and Ono, T. (2000). Distribution of spontaneous CpG- associated G: $\mathrm{C} \rightarrow \mathrm{A}: \mathrm{T}$ mutations in the lacZ gene of Muta ${ }^{\mathrm{TM}}$ mice: Effects of $\mathrm{CpG}$ methylation, the sequence context of $\mathrm{CpG}$ sites, and severity of mutations on the activity of the lacZ gene product. Environ. Mol. Mutagen. 36, 301-311.

International Agency for Research on Cancer, and International Agency for Research on Cancer. (1987). Overall evaluations of carcinogenicity: an updating of IARC monographs volumes 1 to 42 IARC Lyon). 
Itsara, A., Wu, H., Smith, J.D., Nickerson, D.A., Romieu, I., London, S.J., and Eichler, E.E. (2010). De novo rates and selection of large copy number variation. Genome Res. $20,1469-1481$.

Jaleel, R., and Khan, A. (2013). Paternal factors in spontaneous first trimester miscarriage.

Jansen, J., Olsen, A.K., Wiger, R., Naegeli, H., de Boer, P., van Der Hoeven, F., Holme, J.A., Brunborg, G., and Mullenders, L. (2001). Nucleotide excision repair in rat male germ cells: low level of repair in intact cells contrasts with high dual incision activity in vitro. Nucleic Acids Res. 29, 1791-1800.

Jee, J., Rasouly, A., Shamovsky, I., Akivis, Y., Steinman, S.R., Mishra, B., and Nudler, E. (2016). Rates and mechanisms of bacterial mutagenesis from maximum-depth sequencing. Nature 534, 693-696.

Jeffreys, A., Wilson, V., and Thein, S. (1985a). Individual-specific 'fingerprints' of human DNA. Nature 316, 76-79.

Jeffreys, A.J., Wilson, V., and Thein, S.L. (1985b). Hypervariable 'minisatellite' regions in human DNA. Nature 314, 67-73.

Jeng, H.A., and Bocca, S.M. (2013). Influence of exposure to benzo [a] pyrene on mice testicular germ cells during spermatogenesis. Journal of Toxicology 2013,

Jeng, H.A., Yordt, D., Davis, S., and Swanson, J.R. (2013). Assessment of alteration of reproductive system in vivo induced by subchronic exposure to benzo (a) pyrene via oral administration. Environ. Toxicol.

Ji, B., Shu, X., Zheng, W., Ying, D., Linet, M.S., Wacholder, S., Gao, Y., and Jin, F. (1997). Paternal cigarette smoking and the risk of childhood cancer among offspring of nonsmoking mothers. J. Natl. Cancer Inst. 89, 238-243.

Ji, G., Yan, L., Liu, W., Qu, J., and Gu, A. (2013). OGG1 Ser326Cys polymorphism interacts with cigarette smoking to increase oxidative DNA damage in human sperm and the risk of male infertility. Toxicol. Lett. 218, 144-149.

Jobling, M.A., and Gill, P. (2004). Encoded evidence: DNA in forensic analysis. Nature Reviews Genetics 5, 739-751.

Joesbury, K., Edirisinghe, W., Phillips, M., and Yovich, J. (1998). Evidence that male smoking affects the likelihood of a pregnancy following IVF treatment: application of the modified cumulative embryo score. Human Reproduction 13, 1506-1513.

John, E.M., Savitz, D.A., and Sandler, D.P. (1991). Prenatal exposure to parents' smoking and childhood cancer. Am. J. Epidemiol. 133, 123-132. 
Johnson, G., Soeteman- Hernández, L., Gollapudi, B., Bodger, O., Dearfield, K., Heflich, R., Hixon, J., Lovell, D., MacGregor, J., and Pottenger, L. (2014). Derivation of point of departure $(\mathrm{PoD})$ estimates in genetic toxicology studies and their potential applications in risk assessment. Environ. Mol. Mutagen. 55, 609-623.

Jong, A., Menkveld, R., Lens, J., Nienhuis, S., and Rhemrev, J. (2014). Effect of alcohol intake and cigarette smoking on sperm parameters and pregnancy. Andrologia 46, 112117.

Joo, K.J., Kwon, Y.W., Myung, S.C., and Kim, T.H. (2012). The effects of smoking and alcohol intake on sperm quality: light and transmission electron microscopy findings. $\mathrm{J}$. Int. Med. Res. 40, 2327-2335.

Joubert, B.R., Håberg, S.E., Nilsen, R.M., Wang, X., Vollset, S.E., Murphy, S.K., Huang, Z., Hoyo, C., Midttun, Ø, and Cupul-Uicab, L.A. (2012). 450K epigenome-wide scan identifies differential DNA methylation in newborns related to maternal smoking during pregnancy. Environ. Health Perspect. 120, 1425.

Julien, E., Boobis, A.R., Olin, S.S., and ILSI Research Foundation Threshold Working Group. (2009). The key events dose-response framework: a cross-disciplinary mode-ofaction based approach to examining dose-response and thresholds. Crit. Rev. Food Sci. Nutr. 49, 682-689.

Jurewicz, J., Radwan, M., Sobala, W., Ligocka, D., Radwan, P., Bochenek, M., and Hanke, W. (2013). Lifestyle and semen quality: role of modifiable risk factors. Systems Biology in Reproductive Medicine 60, 43-51.

Jurewicz, J., Radwan, M., Sobala, W., Polańska, K., Radwan, P., Jakubowski, L., Ulańska, A., and Hanke, W. (2014). The relationship between exposure to air pollution and sperm disomy. Environ. Mol. Mutagen.

Kalnins, A., Otto, K., Rüther, U., and Müller-Hill, B. (1983). Sequence of the lacZ gene of Escherichia coli. Embo J. 2, 593.

Kand'ár, R., Drábková, P., Myslíková, K., and Hampl, R. (2014). Determination of retinol and $\alpha$ - tocopherol in human seminal plasma using an HPLC with UV detection. Andrologia 46, 472-478.

Kapawa, A., Giannakis, D., Tsoukanelis, K., Kanakas, N., Baltogiannis, D., Agapitos, E., Loutradis, D., Miyagawa, I., and Sofikitis, N. (2004). Effects of paternal cigarette smoking on testicular function, sperm fertilizing capacity, embryonic development, and blastocyst capacity for implantation in rats. Andrologia 36, 57-68.

Keck, C., Bramkamp, G., Behre, H.M., Müller, C., Jockenhövel, F., and Nieschlag, E. (1995). Lack of correlation between cadmium in seminal plasma and fertility status of 
nonexposed individuals and two cadmium-exposed patients. Reproductive Toxicology 9, $35-40$.

Kelly, R., Bulfield, G., Collick, A., Gibbs, M., and Jeffreys, A.J. (1989). Characterization of a highly unstable mouse minisatellite locus: evidence for somatic mutation during early development. Genomics 5, 844-856.

Keohavong, P., and Thilly, W.G. (1992). Determination of point mutational spectra of benzo[a]pyrene-diol epoxide in human cells. Environ. Health Perspect. 98, 215-219.

Khademi, A., Alleyassin, A., Safdarian, L., Hamed, E.A., Rabiee, E., and Haghaninezhad, H. (2005). The effects of L-carnitine on sperm parameters in smoker and non-smoker patients with idiopathic sperm abnormalities. J. Assist. Reprod. Genet. 22, 395-399.

Khan, S., Ahmad, T., Parekh, C.V., Trivedi, P.P., Kushwaha, S., and Jena, G. (2011). Investigation on sodium valproate induced germ cell damage, oxidative stress and genotoxicity in male Swiss mice. Reproductive Toxicology 32, 385-394.

King, L.E., de Solla, S.R., Small, J.M., Sverko, E., and Quinn, J. (2014). Microsatellite DNA mutations in Double-crested Cormorants (Phalacrocorax auritus) associated with exposure to PAH-containing industrial air pollution. Environ. Sci. Technol.

Kinsella, R.J., Kahari, A., Haider, S., Zamora, J., Proctor, G., Spudich, G., AlmeidaKing, J., Staines, D., Derwent, P., Kerhornou, A., Kersey, P., and Flicek, P. (2011). Ensembl BioMarts: a hub for data retrieval across taxonomic space. Database (Oxford) 2011, bar030.

Kiyosawa, H., Suko, M., Okudaira, H., Murata, K., Miyamoto, E., Chung, M., Kasai, H., and Nishimura, S. (1990). Cigarette smoking induces formation of 8hydroxydeoxyguanosine, one of the oxidative DNA damages in human peripheral leukocytes. Free Radic. Res. 11, 23-27.

Kiziler, A.R., Aydemir, B., Onaran, I., Alici, B., Ozkara, H., Gulyasar, T., and Akyolcu, M.C. (2007). High levels of cadmium and lead in seminal fluid and blood of smoking men are associated with high oxidative stress and damage in infertile subjects. Biol. Trace Elem. Res. 120, 82-91.

Kloosterman, W.P., Francioli, L.C., Hormozdiari, F., Marschall, T., Hehir-Kwa, J.Y., Abdellaoui, A., Lameijer, E.W., Moed, M.H., Koval, V., Renkens, I., et al. (2015). Characteristics of de novo structural changes in the human genome. Genome Res. 25, 792-801.

Koboldt, D.C., Ding, L., Mardis, E.R., and Wilson, R.K. (2010). Challenges of sequencing human genomes. Briefings in Bioinformatics 11, 484-498. 
Kodaira, M., Ryo, H., Kamada, N., Furukawa, K., Takahashi, N., Nakajima, H., Nomura, T., and Nakamura, N. (2010). No evidence of increased mutation rates at microsatellite loci in offspring of A-bomb survivors. Radiat. Res. 173, 205-213.

Kodaira, M., Satoh, C., Hiyama, K., and Toyama, K. (1995). Lack of effects of atomic bomb radiation on genetic instability of tandem-repetitive elements in human germ cells. Am. J. Hum. Genet. 57, 1275-1283.

Kokontis, J.M., Vaughan, J., Harvey, R.G., and Weiss, S.B. (1988). Illegitimate recombination induced by benzo[a]pyrene diol epoxide in Escherichia coli. Proc. Natl. Acad. Sci. U. S. A. 85, 1043-1046.

Kokoska, R.J., Bebenek, K., Boudsocq, F., Woodgate, R., and Kunkel, T.A. (2002). Low fidelity DNA synthesis by a family DNA polymerase due to misalignment in the active site. J. Biol. Chem. 277, 19633-19638.

Kong, A., Frigge, M.L., Masson, G., Besenbacher, S., Sulem, P., Magnusson, G., Gudjonsson, S.A., Sigurdsson, A., Jonasdottir, A., and Jonasdottir, A. (2012). Rate of de novo mutations and the importance of father's age to disease risk. Nature 488, 471-475.

Kovalchuk, O., Dubrova, Y.E., Arkhipov, A., Hohn, B., and Kovalchuk, I. (2000). Wheat mutation rate after Chernobyl. Nature-London- 583-583.

Krapels, I.P., Raijmakers-Eichhorn, J., Peters, W.H., Roelofs, H.M., Ras, F., and Steegers-Theunissen, R.P. (2008). The I105V polymorphism in glutathione S-transferase $\mathrm{P} 1$, parental smoking and the risk for nonsyndromic cleft lip with or without cleft palate. European Journal of Human Genetics 16, 358-366.

Krapels, I.P.,Zielhuis, G.A., Vroom, F., de Jong- van den Berg, Lolkje, KuijpersJagtman, A., van der Molen, Aebele B Mink, and Steegers- Theunissen, R.P. (2006). Periconceptional health and lifestyle factors of both parents affect the risk of live- born children with orofacial clefts. Birth Defects Research Part A: Clinical and Molecular Teratology 76, 613-620.

Kryukov, G.V., Pennacchio, L.A., and Sunyaev, S.R. (2007). Most rare missense alleles are deleterious in humans: implications for complex disease and association studies. The American Journal of Human Genetics 80, 727-739.

Kučienè, R., and Dulskienė, V. (2010). Parental cigarette smoking and the risk of congenital heart septal defects. Medicina (Kaunas, Lithuania) 46, 635-641.

Kumar, S.B., Chawla, B., Bisht, S., Yadav, R.K., and Dada, R. (2015). Tobacco Use Increases Oxidative DNA Damage in Sperm - Possible Etiology of Childhood Cancer. Asian Pac. J. Cancer. Prev. 16, 6967-6972. 
Kumosani, T., Elshal, M., Al-Jonaid, A., and Abduljabar, H. (2008). The influence of smoking on semen quality, seminal microelements and Ca 2 -ATPase activity among infertile and fertile men. Clin. Biochem. 41, 1199-1203.

Künzle, R., Mueller, M.D., Hänggi, W., Birkhäuser, M.H., Drescher, H., and Bersinger, N.A. (2003). Semen quality of male smokers and nonsmokers in infertile couples. Fertil. Steril. 79, 287-291.

Kunzle, R., Mueller, M.D., Huber, A.W., Drescher, H., and Bersinger, N.A. (2004). Seasonality in human semen quality of smokers and non-smokers: effect of temperature. Asian J. Androl. 6, 243-247.

Kurahashi, N., Kasai, S., Shibata, T., Kakizaki, H., Nonomura, K., Sata, F., and Kishi, R. (2005). Parental and neonatal risk factors for cryptorchidism. Med. Sci. Monit. 11, CR274-283.

La Maestra, S., De Flora, S., and Micale, R.T. (2015). Effect of cigarette smoke on DNA damage, oxidative stress, and morphological alterations in mouse testis and spermatozoa. Int. J. Hyg. Environ. Health 218, 117-122.

Lagerqvist, A., Håkansson, D., Prochazka, G., Lundin, C., Dreij, K., Segerbäck, D., Jernström, B., Törnqvist, M., Seidel, A., and Erixon, K. (2008). Both replication bypass fidelity and repair efficiency influence the yield of mutations per target dose in intact mammalian cells induced by benzo [a] pyrene-diol-epoxide and dibenzo [a, l] pyrenediol-epoxide. DNA Repair 7, 1202-1212.

Lähdetie, J. (1986). Micronucleated spermatids in the seminal fluid of smokers and nonsmokers. Mutation Research/Genetic Toxicology 172, 255-263.

Lambert, I.B., Singer, T.M., Boucher, S.E., and Douglas, G.R. (2005). Detailed review of transgenic rodent mutation assays. Mutation Research/Reviews in Mutation Research $590,1-280$.

Langmead, B., and Salzberg, S.L. (2012). Fast gapped-read alignment with Bowtie 2. Nature Methods 9, 357-359.

Laubenthal, J., Zlobinskaya, O., Poterlowicz, K., Baumgartner, A., Gdula, M.R., Fthenou, E., Keramarou, M., Hepworth, S.J., Kleinjans, J.C., and van Schooten, F. (2012). Cigarette smoke-induced transgenerational alterations in genome stability in cord blood of human F1 offspring. The FASEB Journal 26, 3946-3956.

Laudat, A., Lecourbe, K., and Palluel, A. Paper presented at Annales de Biologie Clinique. 
Lee, K., Ward, M.H., Han, S., Ahn, H.S., Kang, H.J., Choi, H.S., Shin, H.Y., Koo, H., Seo, J., and Choi, J. (2009). Paternal smoking, genetic polymorphis ms in $<$ i CYP1A1 and childhood leukemia risk. Leuk. Res. 33, 250-258.

Leigh, J.P., and Du, J. (2015). Brief report: forecasting the economic burden of autism in 2015 and 2025 in the United States. J. Autism Dev. Disord. 45, 4135-4139.

Lemieux, C.L., Douglas, G.R., Gingerich, J., Phonethepswath, S., Torous, D.K., Dertinger, S.D., Phillips, D.H., Arlt, V.M., and White, P.A. (2011). Simultaneous measurement of benzo [a] pyrene- induced Pig- a and lacZ mutations, micronuclei and dna adducts in mutaTMmouse. Environ. Mol. Mutagen. 52, 756-765.

Levsky, J.M., and Singer, R.H. (2003). Fluorescence in situ hybridization: past, present and future. J. Cell. Sci. 116, 2833-2838.

Lewin, A., Gonen, O., Orvieto, R., and Schenker, J. (1991). Effect of smoking on concentration, motility and zona-free hamster test on human sperm. Arch. Androl. 27, 5154.

Ley, T.J., Mardis, E.R., Ding, L., Fulton, B., McLellan, M.D., Chen, K., Dooling, D., Dunford-Shore, B.H., McGrath, S., and Hickenbotham, M. (2008). DNA sequencing of a cytogenetically normal acute myeloid leukaemia genome. Nature 456, 66-72.

Li, H., Handsaker, B., Wysoker, A., Fennell, T., Ruan, J., Homer, N., Marth, G., Abecasis, G., and Durbin, R. (2009a). The sequence alignment/map format and SAMtools. Bioinformatics 25, 2078-2079.

Li, Y., Lin, H., Li, Y., and Cao, J. (2011). Association between socio-psycho-behavioral factors and male semen quality: systematic review and meta-analyses. Fertil. Steril. 95, 116-123.

Li, Y., Wu, J., Zhou, W., and Gao, E. (2015). Association between environmental exposure to cadmium and human semen quality. Int. J. Environ. Health Res. 1-12.

Li, Y., Lin, H., Ma, M., Li, L., Cai, M., Zhou, N., Han, X., Bao, H., Huang, L., Zhu, C., et al. (2009b). Semen quality of 1346 healthy men, results from the Chongqing area of southwest China. Hum. Reprod. 24, 459-469.

Liang, K., and Zeger, S.L. (1986). Longitudinal data analysis using generalized linear models. Biometrika 73, 13-22.

Lindsay, S.J., Rahbari, R., Kaplanis, J., Keane, T., and Hurles, M. (2016). Striking differences in patterns of germline mutation between mice and humans. bioRxiv 082297.

Linschooten, J.O., Laubenthal, J., Cemeli, E., Baumgartner, A., Anderson, D., Sipinen, V.E., Brunborg, G., Haenen, G.R., Fthenou, E., and Briedé, J.J. (2011). Incomplete 
protection of genetic integrity of mature spermatozoa against oxidative stress. Reproductive Toxicology 32, 106-111.

Linschooten, J.O., Van Schooten, F.J., Baumgartner, A., Cemeli, E., van Delft, J., Anderson, D., and Godschalk, R.W. (2009). Use of spermatozoal mRNA profiles to study gene-environment interactions in human germ cells. Mutation Research/Fundamental and Molecular Mechanisms of Mutagenesis 667, 70-76.

Linschooten, J.O., Verhofstad, N., Gutzkow, K., Olsen, A., Yauk, C., Oligschläger, Y., Brunborg, G., van Schooten, F.J., and Godschalk, R.W.L. (2013). Paternal lifestyle as a potential source of germline mutations transmitted to offspring. The FASEB Journal

Liskay, R.M., Letsou, A., and Stachelek, J.L. (1987). Homology requirement for efficient gene conversion between duplicated chromosomal sequences in mammalian cells. Genetics 115, 161-167.

Liu, L., Li, Y., Li, S., Hu, N., He, Y., Pong, R., Lin, D., Lu, L., and Law, M. (2012). Comparison of next-generation sequencing systems. Journal of Biomedicine and Biotechnology 2012,

Liu, R., Zhang, L., McHale, C.M., and Hammond, S.K. (2011). Paternal smoking and risk of childhood acute lymphoblastic leukemia: systematic review and meta-analysis. Journal of Oncology 2011,

Liu, Y., Zhou, Y., Qiu, W., Zeng, J., Shen, L., Li, A., and Zhou, J. (2009). Exposure to formaldehyde induces heritable DNA mutations in mice. Journal of Toxicology and Environmental Health, Part A 72, 767-773.

Liu, R.Z., Gao, J.C., Zhang, H.G., Wang, R.X., Zhang, Z.H., and Liu, X.Y. (2010). Seminal plasma zinc level may be associated with the effect of cigarette smoking on sperm parameters. J. Int. Med. Res. 38, 923-928.

Lodato, M.A., Woodworth, M.B., Lee, S., Evrony, G.D., Mehta, B.K., Karger, A., Lee, S., Chittenden, T.W., D'Gama, A.M., Cai, X., et al. (2015). Somatic mutation in single human neurons tracks developmental and transcriptional history. Science 350, 94-98.

Loft, S., Kold-Jensen, T., Hjollund, N.H., Giwercman, A., Gyllemborg, J., Ernst, E., Olsen, J., Scheike, T., Poulsen, H.E., and Bonde, J.P. (2003). Oxidative DNA damage in human sperm influences time to pregnancy. Hum. Reprod. 18, 1265-1272.

Lotti, F., Corona, G., Vitale, P., Maseroli, E., Rossi, M., Fino, M.G., and Maggi, M. (2015). Current smoking is associated with lower seminal vesicles and ejaculate volume, despite higher testosterone levels, in male subjects of infertile couples. Hum. Reprod. 30, 590-602. 
Lotti, F., and Maggi, M. (2015). Ultrasound of the male genital tract in relation to male reproductive health. Hum. Reprod. Update 21, 56-83.

Lu, L.J., Disher, R.M., Reddy, M.V., and Randerath, K. (1986). 32P-postlabeling assay in mice of transplacental DNA damage induced by the environmental carcinogens safrole, 4-aminobiphenyl, and benzo(a)pyrene. Cancer Res. 46, 3046-3054.

Lu, S., Zong, C., Fan, W., Yang, M., Li, J., Chapman, A.R., Zhu, P., Hu, X., Xu, L., Yan, L., et al. (2012). Probing meiotic recombination and aneuploidy of single sperm cells by whole-genome sequencing. Science 338, 1627-1630.

Lupski, J.R. (2015). Structural variation mutagenesis of the human genome: impact on disease and evolution. Environ. Mol. Mutagen. 56, 419-436.

Lupski, J.R. (2013). Genome mosaicism--one human, multiple genomes. Science 341, 358-359.

Lynch, A.M., Giddings, A., Custer, L., Gleason, C., Henwood, A., Aylott, M., and Kenny, J. (2011). International Pig- a gene mutation assay trial (Stage III): Results with $\mathrm{N}-$ methyl- N- nitrosourea. Environ. Mol. Mutagen. 52, 699-710.

Ma, Q. (2013). Role of nrf2 in oxidative stress and toxicity. Annu. Rev. Pharmacol. Toxicol. 53, 401-426.

MacArthur, A.C., McBride, M.L., Spinelli, J.J., Tamaro, S., Gallagher, R.P., and Theriault, G. (2008). Risk of childhood leukemia associated with parental smoking and alcohol consumption prior to conception and during pregnancy: the cross-Canada childhood leukemia study. Cancer Causes \& Control 19, 283-295.

MacKenzie, K.M., and Angevine, D.M. (1981). Infertility in mice exposed in utero to benzo(a)pyrene. Biol. Reprod. 24, 183-191.

Maconochie, N., Doyle, P., Prior, S., and Simmons, R. (2007). Risk factors for first trimester miscarriage - results from a UK- population- based case-control study. BJOG: An International Journal of Obstetrics \& Gynaecology 114, 170-186.

Magnani, C., Pastore, G., Luzzatto, L., and Terracini, B. (1990). Parental occupation and other environmental factors in the etiology of leukemias and non-Hodgkin's lymphomas in childhood: a case-control study. Tumori 76, 413.

Mak, V., Jarvi, K., Buckspan, M., Freeman, M., Hechter, S., and Zini, A. (2000). Smoking is associated with the retention of cytoplasm by human spermatozoa. Urology $56,463-466$.

Mandani, P., Desai, K., and Highland, H. (2013). Cytotoxic Effects of Benzene Metabolites on Human Sperm Function: An In Vitro Study. ISRN Toxicology 2013, 
Marchetti, F., Rowan-Carroll, A., Williams, A., Polyzos, A., Berndt-Weis, M.L., and Yauk, C.L. (2011). Sidestream tobacco smoke is a male germ cell mutagen. Proceedings of the National Academy of Sciences 108, 12811-12814.

Marchetti, F., and Wyrobek, A.J. (2008). DNA repair decline during mouse spermiogenesis results in the accumulation of heritable DNA damage. DNA Repair 7, 572-581.

Marchetti, F., and Wyrobek, A.J. (2005). Mechanisms and consequences of paternallytransmitted chromosomal abnormalities. Birth Defects Research Part C: Embryo Today: Reviews 75, 112-129.

Marchetti, F., Eskenazi, B., Weldon, R.H., Li, G., Zhang, L., Rappaport, S.M., Schmid, T.E., Xing, C., Kurtovich, E., and Wyrobek, A.J. (2012). Occupational exposure to benzene and chromosomal structural aberrations in the sperm of Chinese men. Environ. Health Perspect. 120, 229-234.

Marchetti, F., Essers, J., Kanaar, R., and Wyrobek, A.J. (2007). Disruption of maternal DNA repair increases sperm-derived chromosomal aberrations. Proc. Natl. Acad. Sci. U. S. A. $104,17725-17729$.

Marchetti, F., Lowe, X., Bishop, J., and Wyrobek, A.J. (1999). Absence of selection against aneuploid mouse sperm at fertilization. Biol. Reprod. 61, 948-954.

Marczylo, E.L., Amoako, A.A., Konje, J.C., Gant, T.W., and Marczylo, T.H. (2012). Smoking induces differential miRNA expression in human spermatozoa: a potential transgenerational epigenetic concern? Epigenetics 7, 432-439.

Margulies, M., Egholm, M., Altman, W.E., Attiya, S., Bader, J.S., Bemben, L.A., Berka, J., Braverman, M.S., Chen, Y., and Chen, Z. (2005). Genome sequencing in microfabricated high-density picolitre reactors. Nature 437, 376-380.

Marinelli, D., Gaspari, L., Pedotti, P., and Taioli, E. (2004). Mini-review of studies on the effect of smoking and drinking habits on semen parameters. Int. J. Hyg. Environ. Health 207, 185-192.

Markert, C.L., and Petters, R.M. (1978). Manufactured hexaparental mice show that adults are derived from three embyronic cells. Science 202, 56-58.

Martini, A.C., Molina, R.I., Estofán, D., Senestrari, D., de Cuneo, M.F., and Ruiz, R.D. (2004). Effects of alcohol and cigarette consumption on human seminal quality. Fertil. Steril. $82,374-377$.

Mass, M.J., Ross, J.A., Nesnow, S., Jeffers, A.J., Nelson, G., Galati, A.J., and Stoner, G.D. (1993). Ki- ras oncogene mutations in tumors and DNA adducts formed by benz [j] 
aceanthrylene and benzo [a] pyrene in the lungs of strain A/J mice. Mol. Carcinog. 8 , 186-192.

Mau, G., and Netter, P. (1974). Die Auswirkungen des väterlichen Zigarettenkonsums auf die perinatale Sterblichkeit und die Mißbildungshäufigkeit. DMW-Deutsche Medizinische Wochenschrift 99, 1113-1118.

May, C.A., Tamaki, K., Neumann, R., Wilson, G., Zagars, G., Pollack, A., Dubrova, Y.E., Jeffreys, A.J., and Meistrich, M.L. (2000). Minisatellite mutation frequency in human sperm following radiotherapy. Mutation Research/Fundamental and Molecular Mechanisms of Mutagenesis 453, 67-75.

McCallister, M.M., Maguire, M., Ramesh, A., Aimin, Q., Liu, S., Khoshbouei, H., Aschner, M., Ebner, F.F., and Hood, D.B. (2008). Prenatal exposure to benzo (a) pyrene impairs later-life cortical neuronal function. Neurotoxicology 29, 846-854.

McCarroll, S.A., Huett, A., Kuballa, P., Chilewski, S.D., Landry, A., Goyette, P., Zody, M.C., Hall, J.L., Brant, S.R., and Cho, J.H. (2008). Deletion polymorphism upstream of IRGM associated with altered IRGM expression and Crohn's disease. Nat. Genet. 40, 1107-1112.

McCredie, M., Maisonneuve, P., and Boyle, P. (1994). Antenatal risk factors for malignant brain tumours in New South Wales children. International Journal of Cancer $56,6-10$.

McLaren, W., Gil, L., Hunt, S.E., Riat, H.S., Ritchie, G.R., Thormann, A., Flicek, P., and Cunningham, F. (2016). The Ensembl Variant Effect Predictor. Genome Biol. 17, 1.

McLaren, W., Pritchard, B., Rios, D., Chen, Y., Flicek, P., and Cunningham, F. (2010). Deriving the consequences of genomic variants with the Ensembl API and SNP Effect Predictor. Bioinformatics 26, 2069-2070.

McVey, M., and Lee, S.E. (2008). MMEJ repair of double-strand breaks (director's cut): deleted sequences and alternative endings. Trends in Genetics 24, 529-538.

Mehrannia, T. (2007). The effect of cigarette smoking on semen quality of infertile men. Pakistan Journal of Medical Sciences 23, 717.

Meier, M.J., O’Brien, J.M., Beal, M.A., Allan, B., Yauk, C.L., and Marchetti, F. (2017). In Utero Exposure to Benzo [a] Pyrene Increases Mutation Burden in the Soma and Sperm of Adult Mice. Environ. Health Perspect. 125, 82-88.

Menegaux, F., Ripert, M., Hémon, D., and Clavel, J. (2007). Maternal alcohol and coffee drinking, parental smoking and childhood leukaemia: a French population- based casecontrol study. Paediatr. Perinat. Epidemiol. 21, 293-299. 
Meri, Z.B., Irshid, I.B., Migdadi, M., Irshid, A.B., and Mhanna, S.A. (2013). Does cigarette smoking affect seminal fluid parameters? A comparative study. Oman Medical Journal 28, 12.

Merino, G., Lira, S.C., and Martinez-Chequer, J. (1998). Effects of cigarette smoking on semen characteristics of a population in Mexico. Systems Biology in Reproductive Medicine 41, 11-15.

Metzker, M.L. (2009). Sequencing technologies - the next generation. Nature Reviews Genetics 11, 31-46.

Metzler-Guillemain, C., Victorero, G., Lepoivre, C., Bergon, A., Yammine, M., Perrin, J., Sari-Minodier, I., Boulanger, N., Rihet, P., and Nguyen, C. (2015). Sperm mRNAs and microRNAs as candidate markers for the impact of toxicants on human spermatogenesis: an application to tobacco smoking. Systems Biology in Reproductive Medicine 1-11.

Michaelson, J.J., Shi, Y., Gujral, M., Zheng, H., Malhotra, D., Jin, X., Jian, M., Liu, G., Greer, D., and Bhandari, A. (2012). Whole-genome sequencing in autism identifies hot spots for de novo germline mutation. Cell 151, 1431-1442.

Miller, K.P., and Ramos, K.S. (2001). Impact of cellular metabolism on the biological effects of benzo [a] pyrene and related hydrocarbons. Drug Metab. Rev. 33, 1-35.

Milne, E., Greenop, K.R., Scott, R.J., Bailey, H.D., Attia, J., Dalla-Pozza, L., de Klerk, N.H., and Armstrong, B.K. (2012). Parental prenatal smoking and risk of childhood acute lymphoblastic leukemia. Am. J. Epidemiol. 175, 43-53.

Mirkin, S.M. (2007). Expandable DNA repeats and human disease. Nature 447, 932-940.

Mitiku, N., and Baker, J.C. (2007). Genomic analysis of gastrulation and organogenesis in the mouse. Developmental Cell 13,897-907.

Mitra, A., Chakraborty, B., Mukhopadhay, D., Pal, M., Mukherjee, S., Banerjee, S., and Chaudhuri, K. (2012). Effect of smoking on semen quality, FSH, testosterone level, and $\mathrm{CAG}$ repeat length in androgen receptor gene of infertile men in an indian city. Systems Biology in Reproductive Medicine 58, 255-262.

Mocarelli, P., Gerthoux, P.M., Needham, L.L., Patterson Jr, D.G., Limonta, G., Falbo, R., Signorini, S., Bertona, M., Crespi, C., and Sarto, C. (2011). Perinatal exposure to low doses of dioxin can permanently impair human semen quality. Environ. Health Perspect. $119,713$.

Modgil, S., Lahiri, D.K., Sharma, V.L., and Anand, A. (2014). Role of early life exposure and environment on neurodegeneration: implications on brain disorders. Translational Neurodegeneration 3, 1. 
Moein, M.R., Soleimani, M., and Tabibnejad, N. (2009). Reactive oxygen species (ROS) production in seminal fluid correlate with the severity of varicocele in infertile men.

Moffat, I., Chepelev, N.L., Labib, S., Bourdon-Lacombe, J., Kuo, B., Buick, J.K., Lemieux, F., Williams, A., Halappanavar, S., and Malik, A.I. (2015). Comparison of toxicogenomics and traditional approaches to inform mode of action and points of departure in human health risk assessment of benzo [a] pyrene in drinking water. Crit. Rev. Toxicol. 45, 1-43.

Mohamed, M., Sulaiman, S.A., Jaafar, H., and Sirajudeen, K.N.S. (2011). Antioxidant protective effect of honey in cigarette smoke-induced testicular damage in rats. International Journal of Molecular Sciences 12, 5508-5521.

Mohamed, e.A., Song, W.H., Oh, S.A., Park, Y.J., You, Y.A., Lee, S., Choi, J.Y., Kim, Y.J., Jo, I., and Pang, M.G. (2010). The transgenerational impact of benzo(a)pyrene on murine male fertility. Hum. Reprod. 25, 2427-2433.

Monroe, J.J., Manjanatha, M.G., and Skopek, T.R. (2001). Extent of CpG methylation is not proportional to the in vivo spontaneous mutation frequency at transgenic loci in Big Blue $^{\mathrm{TM}}$ rodents. Mutation Research/Fundamental and Molecular Mechanisms of Mutagenesis 476, 1-11.

Moretti, E., Collodel, G., Iacoponi, F., Geminiani, M., Pascarelli, N.A., Campagna, S., Franci, B., and Figura, N. (2011). Detection of obestatin in seminal plasma and its relationship with ghrelin and semen parameters. Fertil. Steril. 95, 2303-2309.

Moretti, E., Collodel, G., Mazzi, L., Campagna, M., Iacoponi, F., and Figura, N. (2014). Resistin, interleukin-6, tumor necrosis factor-alpha, and human semen parameters in the presence of leukocytospermia, smoking habit, and varicocele. Fertil. Steril. 102, 354-360.

Mori, K., Weng, S., Arzberger, T., May, S., Rentzsch, K., Kremmer, E., Schmid, B., Kretzschmar, H.A., Cruts, M., and Van Broeckhoven, C. (2013). The C9orf72 GGGGCC Repeat Is Translated into Aggregating Dipeptide-Repeat Proteins in FTLD/ALS. Science 339, 1335-1338.

Mori, K., Kaido, M., Fujishiro, K., Inoue, N., Koide, O., Hori, H., and Tanaka, I. (1991). Dose dependent effects of inhaled ethylene oxide on spermatogenesis in rats. Br. J. Ind. Med. 48, 270-274.

Moriya, M., Spiegel, S., Fernandes, A., Amin, S., Liu, T., Geacintov, N., and Grollman, A.P. (1996). Fidelity of translesional synthesis past benzo [a] pyrene diol epoxide-2'deoxyguanosine DNA adducts: Marked effects of host cell, sequence context, and chirality. Biochemistry (N. Y. ) 35, 16646-16651. 
Mostafa, T., Tawadrous, G., Roaia, M., Amer, M., Kader, R., and Aziz, A. (2006). Effect of smoking on seminal plasma ascorbic acid in infertile and fertile males. Andrologia 38, 221-224.

Mostafa, T. (2010). Cigarette smoking and male infertility. Journal of Advanced Research 1, 179-186.

Muller, H. (1927). Artificial Transmutation of the Gene. Science 66, 84-87.

Murphey, P., McLean, D.J., McMahan, C.A., Walter, C.A., and McCarrey, J.R. (2013). Enhanced genetic integrity in mouse germ cells. Biol. Reprod. 88, 6.

Naccarati, A., Zanello, A., Landi, S., Consigli, R., and Migliore, L. (2003). Sperm-FISH analysis and human monitoring: a study on workers occupationally exposed to styrene. Mutation Research/Genetic Toxicology and Environmental Mutagenesis 537, 131-140.

Neal, M.S., Hughes, E.G., Holloway, A.C., and Foster, W.G. (2005). Sidestream smoking is equally as damaging as mainstream smoking on IVF outcomes. Human Reproduction $20,2531-2535$.

Nelson, K., and Holmes, L.B. (1989). Malformations due to presumed spontaneous mutations in newborn infants. N. Engl. J. Med. 320, 19-23.

Niwa, O. (2006). Indirect mechanisms of genomic instability and the biological significance of mutations at tandem repeat loci. Mutation Research/Fundamental and Molecular Mechanisms of Mutagenesis 598, 61-72.

Northstone, K., Golding, J., Smith, G.D., Miller, L.L., and Pembrey, M. (2014). Prepubertal start of father's smoking and increased body fat in his sons: further characterisation of paternal transgenerational responses. European Journal of Human Genetics 22, 1382-1386.

Nybo Andersen, A.M., Hansen, K.D., Andersen, P.K., and Davey Smith, G. (2004). Advanced paternal age and risk of fetal death: a cohort study. Am. J. Epidemiol. 160, 1214-1222.

O’Brien, J.M., Beal, M.A., Yauk, C.L., and Marchetti, F. (2016a). Benzo (a) pyrene is mutagenic in mouse spermatogonial stem cells and dividing spermatogonia.

Toxicological Sciences 152, 363-371.

O’Brien, J.M., Beal, M.A., Yauk, C.L., and Marchetti, F. (2016b). Next generation sequencing of benzo (a) pyrene-induced lacZ mutants identifies a germ cell-specific mutation spectrum. Scientific Reports 6, 36743.

O’Brien, J.M., Williams, A., Gingerich, J., Douglas, G.R., Marchetti, F., and Yauk, C.L. (2013). No evidence for transgenerational genomic instability in the F1 or F2 descendants 
of Muta ${ }^{\mathrm{TM}}$ Mouse males exposed to N-ethyl-N-nitrosourea. Mutation Research/Fundamental and Molecular Mechanisms of Mutagenesis

O’Dushlaine, C., Ripke, S., Ruderfer, D.M., Hamilton, S.P., Fava, M., Iosifescu, D.V., Kohane, I.S., Churchill, S.E., Castro, V.M., and Clements, C.C. (2014). Rare copy number variation in treatment-resistant major depressive disorder. Biol. Psychiatry 76, 536-541.

Oatley, J.M., and Brinster, R.L. (2012). The germline stem cell niche unit in mammalian testes. Physiol. Rev. 92, 577-595.

O'Brien, J.M., Beal, M.A., Gingerich, J.D., Soper, L., Douglas, G.R., Yauk, C.L., and Marchetti, F. (2014). Transgenic rodent assay for quantifying male germ cell mutant frequency. JoVE (Journal of Visualized Experiments) e 51576-e51576.

O'Brien, J.M., Walker, M., Sivathayalan, A., Douglas, G.R., Yauk, C.L., and Marchetti, F. (2015). Sublinear response in lacZ mutant frequency of Muta ${ }^{\mathrm{TM}}$ Mouse spermatogonial stem cells after low dose subchronic exposure to N-ethyl-N-nitrosourea. Environ. Mol. Mutagen. 56, 347-355.

Odeigah, P. (1997). Sperm head abnormalities and dominant lethal effects of formaldehyde in albino rats. Mutation Research/Genetic Toxicology and Environmental Mutagenesis $389,141-148$.

OECD. (2013). Test No. 478: Rodent Dominant Lethal Test. OECD Guidelines for Testing of Chemicals.

OECD. (2011). Transgenic Rodent Somatic and Germ Cell Gene Mutation Assays. OECD Guideline for the Testing of Chemicals

\section{OECD. (1986). Test No. 485: Mouse Heritable Translocation Assay, OECD Guidelines for Testing of Chemical Section 4. OECD Guidelines for Testing of Chemicals.}

Ogi, T., Mimura, J., Hikida, M., Fujimoto, H., Fujii- Kuriyama, Y., and Ohmori, H. (2001). Expression of human and mouse genes encoding polk: testis- specific developmental regulation and $\mathrm{AhR}$ - dependent inducible transcription. Genes to Cells 6 , 943-953.

Oh, E., Lee, E., Im, H., Kang, H., Jung, W., Won, N.H., Kim, E., and Sul, D. (2005). Evaluation of immuno-and reproductive toxicities and association between immunotoxicological and genotoxicological parameters in waste incineration workers. Toxicology $210,65-80$.

Oldereid, N., Thomassen, Y., and Purvis, K. (1994). Seminal plasma lead, cadmium and zinc in relation to tobacco consumption. Int. J. Androl. 17, 24-28. 
Oldereid, N.B., Rui, H., Clausen, O.P., and Purvis, K. (1989). Cigarette smoking and human sperm quality assessed by laser-Doppler spectroscopy and DNA flow cytometry. J. Reprod. Fertil. 86, 731-736.

Oliveira, H., Spanò, M., Santos, C., and Pereira, M.d.L. (2009). Adverse effects of cadmium exposure on mouse sperm. Reproductive Toxicology 28, 550-555.

Olsen, A., Andreassen, Å, Singh, R., Wiger, R., Duale, N., Farmer, P.B., and Brunborg, G. (2010). Environmental exposure of the mouse germ line: DNA adducts in spermatozoa and formation of de novo mutations during spermatogenesis. PloS One 5, e11349.

Olsen, A., Lindeman, B., Wiger, R., Duale, N., and Brunborg, G. (2005). How do male germ cells handle DNA damage? Toxicol. Appl. Pharmacol. 207, 521-531.

Olson, H., Shen, Y., Avallone, J., Sheidley, B.R., Pinsky, R., Bergin, A.M., Berry, G.T., Duffy, F.H., Eksioglu, Y., and Harris, D.J. (2014). Copy number variation plays an important role in clinical epilepsy. Ann. Neurol. 75, 943-958.

Omran, H.M., Dashti, M.G., and Bakhiet, M. (2013). Significant Effects of Smoking Habit on Male Fertility. Bahrain Medical Bulletin 35,

Omu, A.E., Dashti, H., Mohammed, A.T., and Mattappallil, A.B. (1998). Cigarette smoking causes impairment of spermatozoal quality: andrological and biochemical evaluation. Medical Principles and Practice 7, 47-53.

Ono, T., Ikehata, H., Nakamura, S., Saito, Y., Hosoi, Y., Takai, Y., Yamada, S., Onodera, J., and Yamamoto, K. (2000). Age-associated increase of spontaneous mutant frequency and molecular nature of mutation in newborn and old lacZ-transgenic mouse. Mutation Research/Fundamental and Molecular Mechanisms of Mutagenesis 447, 165-177.

Opuwari, C.S., and Henkel, R.R. (2016). An update on oxidative damage to spermatozoa and oocytes.

O'Roak, B.J., Deriziotis, P., Lee, C., Vives, L., Schwartz, J.J., Girirajan, S., Karakoc, E., MacKenzie, A.P., Ng, S.B., and Baker, C. (2011). Exome sequencing in sporadic autism spectrum disorders identifies severe de novo mutations. Nat. Genet. 43, 585-589.

Ostling, O., and Johanson, K. (1984). Microelectrophoretic study of radiation-induced DNA damages in individual mammalian cells. Biochem. Biophys. Res. Commun. 123, 291-298.

Ozgur, K., Isikoglu, M., Seleker, M., and Donmez, L. (2005). Semen quality of smoking and non-smoking men in infertile couples in a Turkish population. Arch. Gynecol. Obstet. 271, 109-112. 
Ozyurt, H., Pekmez, H., Parlaktas, B.S., Kus, I., Ozyurt, B., and Sarsilmaz, M. (2006). Oxidative stress in testicular tissues of rats exposed to cigarette smoke and protective effects of caffeic acid phenethyl ester. Asian J. Androl. 8, 189-193.

Pacifici, R., Altieri, I., Gandini, L., Lenzi, A., Passa, A.R., Pichini, S., Rosa, M., Zuccaro, P., and Dondero, F. (1995). Environmental Tobacco-Smoke-Nicotine and Cotinine Concentration in Semen. Environ. Res. 68, 69-72.

Pacifici, R., Altieri, I., Gandini, L., Lenzi, A., Pichini, S., Rosa, M., Zuccaro, P., and Dondero, F. (1993). Nicotine, cotinine, and trans-3-hydroxycotine levels in seminal plasma of smokers: effects on sperm parameters. Ther. Drug Monit. 15, 358-363.

Palma, M., Cook, T., Segura, J., Pecho, A., and Morcuende, J.A. (2013). Descriptive epidemiology of clubfoot in Peru: a clinic-based study. Iowa Orthop. J. 33, 167-171.

Pang, D., McNally, R., and Birch, J. (2003). Parental smoking and childhood cancer: results from the United Kingdom Childhood Cancer Study. Br. J. Cancer 88, 373-381.

Pasqualotto, F.F., Sobreiro, B.P., Hallak, J., Pasqualotto, E.B., and Lucon, A.M. (2006). Cigarette smoking is related to a decrease in semen volume in a population of fertile men. BJU Int. 97, 324-326.

Pasqualotto, F.F., Umezu, F.M., Salvador, M., Borges Jr, E., Sobreiro, B.P., and Pasqualotto, E.B. (2008). Effect of cigarette smoking on antioxidant levels and presence of leukocytospermia in infertile men: a prospective study. Fertil. Steril. 90, 278-283.

Pembrey, M.E., Bygren, L.O., Kaati, G., Edvinsson, S., Northstone, K., Sjöström, M., and Golding, J. (2006). Sex-specific, male-line transgenerational responses in humans. European Journal of Human Genetics 14, 159-166.

Pereira, C.S., Juchniuk de Vozzi, M.S., Dos Santos, S.A., Vasconcelos, M.A., de Paz, C.C., Squire, J.A., and Martelli, L. (2014). Smoking-induced chromosomal segregation anomalies identified by FISH analysis of sperm. Mol. Cytogenet. 7, 58-014-0058-7. eCollection 2014.

Perrin, J., Tassistro, V., Mandon, M., Grillo, J., Botta, A., and Sari-Minodier, I. (2011). Tobacco consumption and benzo (a) pyrene-diol-epoxide-DNA adducts in spermatozoa: in smokers, swim-up procedure selects spermatozoa with decreased DNA damage. Fertil. Steril. 95, 2013-2017.

Pfeifer, G.P., Denissenko, M.F., Olivier, M., Tretyakova, N., Hecht, S.S., and Hainaut, P. (2002). Tobacco smoke carcinogens, DNA damage and p53 mutations in smokingassociated cancers. Oncogene 21, 7435-7451.

Piegorsch, W.W., and Bailer, A.J. (1994). Statistical approaches for analyzing mutational spectra: some recommendations for categorical data. Genetics 136, 403-416. 
Pienaar, E., Theron, M., Nelson, M., and Viljoen, H.J. (2006). A quantitative model of error accumulation during PCR amplification. Computational Biology and Chemistry 30, $102-111$.

Pierik, F.H., Burdorf, A., Deddens, J.A., Juttmann, R.E., and Weber, R.F. (2004). Maternal and paternal risk factors for cryptorchidism and hypospadias: a case-control study in newborn boys. Environ. Health Perspect. 1570-1576.

Pinto, D., Pagnamenta, A.T., Klei, L., Anney, R., Merico, D., Regan, R., Conroy, J., Magalhaes, T.R., Correia, C., and Abrahams, B.S. (2010). Functional impact of global rare copy number variation in autism spectrum disorders. Nature 466, 368-372.

Plichart, M., Menegaux, F., Lacour, B., Hartmann, O., Frappaz, D., Doz, F., Bertozzi, A., Defaschelles, A., Pierre-Kahn, A., and Icher, C. (2008). Parental smoking, maternal alcohol, coffee and tea consumption during pregnancy and childhood malignant central nervous system tumours: the ESCALE study (SFCE). European Journal of Cancer Prevention 17, 376.

Poduri, A., Evrony, G.D., Cai, X., and Walsh, C.A. (2013). Somatic mutation, genomic variation, and neurological disease. Science 341, 1237758.

Polyzos, A., Ernst Schmid, T., Piña-Guzmán, B., Quintanilla-Vega, B., and Marchetti, F. (2009). Differential sensitivity of male germ cells to mainstream and sidestream tobacco smoke in the mouse. Toxicol. Appl. Pharmacol. 237, 298-305.

Polyzos, A., Parfett, C., Healy, C., Douglas, G.R., and Yauk, C.L. (2006). Instability of expanded simple tandem repeats is induced in cell culture by a variety of agents: $\mathrm{N}$ Nitroso-N-ethylurea, benzo(a)pyrene, etoposide and okadaic acid. Mutation Research/Fundamental and Molecular Mechanisms of Mutagenesis 598, 73-84.

Poongothai, J., Gopenath, T., and Manonayaki, S. (2009). Genetics of human male infertility. Singapore Med. J. 50, 336-347.

Pottenger, L.H., Schisler, M.R., Zhang, F., Bartels, M.J., Fontaine, D.D., McFadden, L.G., and Gollapudi, B.B. (2009). Dose-response and operational thresholds/NOAELs for in vitro mutagenic effects from DNA-reactive mutagens, MMS and MNU. Mutation Research/Genetic Toxicology and Environmental Mutagenesis 678, 138-147.

Potts, R., Newbury, C., Smith, G., Notarianni, L., and Jefferies, T. (1999). Sperm chromatin damage associated with male smoking. Mutation Research/Fundamental and Molecular Mechanisms of Mutagenesis 423, 103-111.

Practice Committee of the American Society for Reproductive Medicine. (2012). Smoking and infertility: a committee opinion. Fertil. Steril. 98, 1400-1406. 
Prentice, R.L., and Zhao, L.P. (1991). Estimating equations for parameters in means and covariances of multivariate discrete and continuous responses. Biometrics 825-839.

Primmer, C.R., Ellegren, H., Saino, N., and Møller, A.P. (1996). Directional evolution in germline microsatellite mutations. Nat. Genet. 13, 391-393.

Primorac, D., and Schanfield, M.S. (2000). Application of forensic DNA testing in the legal system. Croat. Med. J. 41, 32-46.

Quinlan, A.R., and Hall, I.M. (2010). BEDTools: a flexible suite of utilities for comparing genomic features. Bioinformatics 26, 841-842.

R Core Team. (2013). R: A Language and Environment for Statistical Computing.R Foundation for Statistical Computing, Vienna, Austria.Version 2.15

Rahbari, R., Wuster, A., Lindsay, S.J., Hardwick, R.J., Alexandrov, L.B., Al Turki, S., Dominiczak, A., Morris, A., Porteous, D., and Smith, B. (2015). Timing, rates and spectra of human germline mutation. Nat. Genet.

Ramesh, A., Hood, D.B., Inyang, F., Greenwood, M., Nyanda, A.M., Archibong, A.E., and Knuckles, M.E. (2002). Comparative metabolism, bioavailability, and toxicokinetics of benzo [a] pyrene in rats after acute oral, inhalation, and intravenous administration. Polycyclic Aromatic Compounds 22, 969-980.

Ramlau-Hansen, C.H., Thulstrup, A.M., Aggerholm, A.S., Jensen, M.S., Toft, G., and Bonde, J.P. (2007). Is smoking a risk factor for decreased semen quality? A crosssectional analysis. Hum. Reprod. 22, 188-196.

Rantala, M., and Koskimies, A. (1987). Semen Quality of Infertile Couples - Comparison between Smokers and Non- Smokers. Andrologia 19, 42-46.

Rathke, C., Baarends, W.M., Awe, S., and Renkawitz-Pohl, R. (2014). Chromatin dynamics during spermiogenesis. Biochimica Et Biophysica Acta (BBA)-Gene Regulatory Mechanisms 1839, 155-168.

Ravnborg, T.L., Jensen, T.K., Andersson, A.M., Toppari, J., Skakkebaek, N.E., and Jorgensen, N. (2011). Prenatal and adult exposures to smoking are associated with adverse effects on reproductive hormones, semen quality, final height and body mass index. Hum. Reprod. 26, 1000-1011.

Rebhan, M., Chalifa-Caspi, V., Prilusky, J., and Lancet, D. (1997). GeneCards:

integrating information about genes, proteins and diseases. Trends in Genetics 13, 163.

Reina Bouvet, B., Vicenta Paparella, C., and Nestor Feldman, R. (2007). Effect of tobacco consumption on the spermatogenesis in males with idiopathic infertility. Arch. Esp. Urol. 60, 273-277. 
Richthoff, J., Elzanaty, S., Rylander, L., Hagmar, L., and Giwercman, A. (2008). Association between tobacco exposure and reproductive parameters in adolescent males. Int. J. Androl. 31, 31-39.

Ritz, C., Ruminski, W., Hougaard, K.S., Wallin, H., Vogel, U., and Yauk, C.L. (2011). Germline mutation rates in mice following in utero exposure to diesel exhaust particles by maternal inhalation. Mutation Research/Fundamental and Molecular Mechanisms of Mutagenesis $712,55-58$.

Roach, J.C., Glusman, G., Smit, A.F., Huff, C.D., Hubley, R., Shannon, P.T., Rowen, L., Pant, K.P., Goodman, N., and Bamshad, M. (2010). Analysis of genetic inheritance in a family quartet by whole-genome sequencing. Science 328, 636-639.

Robbins, W.A., Vine, M.F., Truong, K.Y., and Everson, R.B. (1997). Use of fluorescence in situ hybridization (FISH) to assess effects of smoking, caffeine, and alcohol on aneuploidy load in sperm of healthy men. Environ. Mol. Mutagen. 30, 175-183.

Robbins, W.A., Elashoff, D.A., Xun, L., Jia, J., Li, N., Wu, G., and Wei, F. (2005). Effect of lifestyle exposures on sperm aneuploidy. Cytogenet. Genome Res. 111, 371-377.

Robinson, J.T., Thorvaldsdóttir, H., Winckler, W., Guttman, M., Lander, E.S., Getz, G., and Mesirov, J.P. (2011). Integrative genomics viewer. Nat. Biotechnol. 29, 24-26.

Rodriguez, M., Panda, B.B., and Ficsor, G. (1983). Testes weight reflect ethylnitrosourea induced histopathology in mice. Toxicol. Lett. 17, 77-80.

Roff, D.A., and Bentzen, P. (1989). The statistical analysis of mitochondrial DNA polymorphisms: chi 2 and the problem of small samples. Mol. Biol. Evol. 6, 539-545.

Rolland, M., Le Moal, J., Wagner, V., Royere, D., and De Mouzon, J. (2013). Decline in semen concentration and morphology in a sample of 26,609 men close to general population between 1989 and 2005 in France. Hum. Reprod. 28, 462-470.

Rothberg, J.M., Hinz, W., Rearick, T.M., Schultz, J., Mileski, W., Davey, M., Leamon, J.H., Johnson, K., Milgrew, M.J., and Edwards, M. (2011). An integrated semiconductor device enabling non-optical genome sequencing. Nature 475, 348-352.

Rübe, C.E., Zhang, S., Miebach, N., Fricke, A., and Rübe, C. (2011). Protecting the heritable genome: DNA damage response mechanisms in spermatogonial stem cells. DNA Repair 10, 159-168.

Rubes, J., Lowe, X., Moore II, D., Perreault, S., Slott, V., Evenson, D., Selevan, S.G., and Wyrobek, A.J. (1998). Smoking cigarettes is associated with increased sperm disomy in teenage men. Fertil. Steril. 70, 715-723. 
Ruckart, P.Z., Bove, F.J., and Maslia, M. (2013). Evaluation of exposure to contaminated drinking water and specific birth defects and childhood cancers at Marine Corps Base Camp Lejeune, North Carolina: a case-control study. Environ. Health 12, 10.1186.

Rudant, J., Menegaux, F., Leverger, G., Baruchel, A., Lambilliotte, A., Bertrand, Y., Patte, C., Pacquement, H., Vérité, C., and Robert, A. (2008). Childhood hematopoietic malignancies and parental use of tobacco and alcohol: the ESCALE study (SFCE). Cancer Causes \& Control 19, 1277-1290.

Russell, L., and Russell, W. (1992). Frequency and nature of specific-locus mutations induced in female mice by radiations and chemicals: a review. Mutation Research/Reviews in Genetic Toxicology 296, 107-127.

Russell, L., Selby, P., Von Halle, E., Sheridan, W., and Valcovic, L. (1981). The mouse specific-locus test with agents other than radiations: interpretation of data and recommendations for future work. Mutat. Res. 86, 329.

Russell, L.B. (2004). Effects of male germ-cell stage on the frequency, nature, and spectrum of induced specific-locus mutations in the mouse. Genetica 122, 25-36.

Russell, L.B. (1999). Significance of the perigametic interval as a major source of spontaneous mutations that result in mosaics. Environ. Mol. Mutagen. 34, 16-23.

Russell, L.B., and Matter, B. (1980). Whole-mammal mutagenicity tests: Evaluation of five methods. Mutation Research/Reviews in Genetic Toxicology 75, 279-302.

Russell, L.B., Bangham, J.W., Stelzner, K.F., and Hunsicker, P.R. (1988). High frequency of mosaic mutants produced by N-ethyl-N-nitrosourea exposure of mouse zygotes. Proc. Natl. Acad. Sci. U. S. A. 85, 9167-9170.

Russell, W.L. (1951). X-ray-induced mutations in mice. Cold Spring Harb. Symp. Quant. Biol. 16, 327-336.

Russell, W.L., Hunsicker, P.R., Raymer, G.D., Steele, M.H., Stelzner, K.F., and Thompson, H.M. (1982). Dose-response curve for ethylnitrosourea-induced specificlocus mutations in mouse spermatogonia. Proc. Natl. Acad. Sci. U. S. A. 79, 3589-3591.

Rutter, M. (2005). Incidence of autism spectrum disorders: Changes over time and their meaning*. Acta Paediatrica 94, 2-15.

Rybar, R., Markova, P., Veznik, Z., Faldikova, L., Kunetkova, M., Zajicova, A., Kopecka, V., and Rubes, J. (2009). Sperm chromatin integrity in young men with no experiences of infertility and men from idiopathic infertility couples. Andrologia 41, 141149. 
Saaranen, M., Suonio, S., Kauhanen, O., and Saarikoski, S. (1987). Cigarette smoking and semen quality in men of reproductive age. Andrologia 19, 670-676.

Said, T.M., Ranga, G., and Agarwal, A. (2005). Relationship between semen quality and tobacco chewing in men undergoing infertility evaluation. Fertil. Steril. 84, 649-653.

Salama, N., and El-Sawy, M. (2013). Isolated low follicle stimulating hormone (FSH) in infertile males-a preliminary report. Archivio Italiano Di Urologia E Andrologia 85, 118124.

Sale, J.E., Lehmann, A.R., and Woodgate, R. (2012). Y-family DNA polymerases and their role in tolerance of cellular DNA damage. Nature Reviews Molecular Cell Biology 13, 141-152.

Saleh, R.A., Agarwal, A., Sharma, R.K., Nelson, D.R., and Thomas Jr, A.J. (2002). Effect of cigarette smoking on levels of seminal oxidative stress in infertile men: a prospective study. Fertil. Steril. 78, 491-499.

Sanders, S.J., Murtha, M.T., Gupta, A.R., Murdoch, J.D., Raubeson, M.J., Willsey, A.J., Ercan-Sencicek, A.G., DiLullo, N.M., Parikshak, N.N., and Stein, J.L. (2012). De novo mutations revealed by whole-exome sequencing are strongly associated with autism. Nature 485, 237-241.

Sankako, M.K., Garcia, P.C., Piffer, R.C., Dallaqua, B., Damasceno, D.C., and Pereira, O.C. (2012). Possible mechanism by which zinc protects the testicular function of rats exposed to cigarette smoke. Pharmacological Reports 64, 1537-1546.

Sankako, M.K., Garcia, P.C., Piffer, R.C., and Pereira, O.C.M. (2013). Semen and reproductive parameters during some abstinence periods after cigarette smoke exposure in male rats. Brazilian Archives of Biology and Technology 56, 93-100.

Satoh, C., Takahashi, N., Asakawa, J., Kodaira, M., Kuick, R., Hanash, S.M., and Neel, J.V. (1996). Genetic analysis of children of atomic bomb survivors. Environ. Health Perspect. 104, 511.

Savitz, D.A. (2003). Paternal exposure to known mutagens and health of the offspring: ionizing radiation and tobacco smoke. In Advances in Male Mediated Developmental Toxicity, Springer) pp. 49-57.

Savitz, D.A., Schwingl, P.J., and Keels, M.A. (1991). Influence of paternal age, smoking, and alcohol consumption on congenital anomalies. Teratology 44, 429-440.

Schlötterer, C., and Pemberton, J. (1998). The use of microsatellites for genetic analysis of natural populations - a critical review. In Molecular approaches to ecology and evolution, Springer) pp. 71-86. 
Schmitt, M.W., Kennedy, S.R., Salk, J.J., Fox, E.J., Hiatt, J.B., and Loeb, L.A. (2012). Detection of ultra-rare mutations by next-generation sequencing. Proceedings of the National Academy of Sciences 109, 14508-14513.

Schüz, J., Kaatsch, P., Kaletsch, U., Meinert, R., and Michaelis, J. (1999). Association of childhood cancer with factors related to pregnancy and birth. Int. J. Epidemiol. 28, 631639.

Sebat, J., Lakshmi, B., Malhotra, D., Troge, J., Lese-Martin, C., Walsh, T., Yamrom, B., Yoon, S., Krasnitz, A., and Kendall, J. (2007). Strong association of de novo copy number mutations with autism. Science 316, 445-449.

Secretan, B., Straif, K., Baan, R., Grosse, Y., El Ghissassi, F., Bouvard, V., BenbrahimTallaa, L., Guha, N., Freeman, C., Galichet, L., Cogliano, V., and WHO International Agency for Research on Cancer Monograph Working Group. (2009). A review of human carcinogens--Part E: tobacco, areca nut, alcohol, coal smoke, and salted fish. Lancet Oncol. 10, 1033-1034.

Seidman, D.S., Ever-Hadani, P., and Gale, R. (1990). Effect of maternal smoking and age on congenital anomalies. Obstetrics \& Gynecology 76, 1046-1050.

Selit, I., Basha, M., Maraee, A., El- Naby, S., Nazeef, N., El- Mehrath, R., and Mostafa, T. (2013). Sperm DNA and RNA abnormalities in fertile and oligoasthenoteratozoos permic smokers. Andrologia 45, 35-39.

Sepaniak, S., Forges, T., Gerard, H., Foliguet, B., Bene, M., and Monnier-Barbarino, P. (2006). The influence of cigarette smoking on human sperm quality and DNA fragmentation. Toxicology 223, 54-60.

Sergerie, M., Ouhilal, S., Bissonnette, F., Brodeur, J., and Bleau, G. (2000). Lack of association between smoking and DNA fragmentation in the spermatozoa of normal men. Hum. Reprod. 15, 1314-1321.

Shane, B.S., de Boer, J., Watson, D.E., Haseman, J.K., Glickman, B.W., and Tindall, K.R. (2000). LacI mutation spectra following benzo [a] pyrene treatment of Big Blue ${ }^{\circledR}$ mice. Carcinogenesis 21, 715-725.

Shaw, G.M., Wasserman, C.R., Lammer, E.J., O'Malley, C.D., Murray, J.C., Basart, A.M., and Tolarova, M.M. (1996). Orofacial clefts, parental cigarette smoking, and transforming growth factor-alpha gene variants. Am. J. Hum. Genet. 58, 551-561.

Shelby, M.D., and R Tindall, K. (1997). Mammalian germ cell mutagenicity of ENU, IPMS and MMS, chemicals selected for a transgenic mouse collaborative study. Mutation Research/Genetic Toxicology and Environmental Mutagenesis 388, 99-109. 
Shen, H., Chia, S., and Ong, C. (1999). Evaluation of oxidative DNA damage in human sperm and its association with male infertility. J. Androl. 20, 718-723.

Shen, H.M., Chia, S.E., Ni, Z.Y., New, A.L., Lee, B.L., and Ong, C.N. (1997). Detection of oxidative DNA damage in human sperm and the association with cigarette smoking. Reprod. Toxicol. 11, 675-680.

Shenker, N.S., Polidoro, S., van Veldhoven, K., Sacerdote, C., Ricceri, F., Birrell, M.A., Belvisi, M.G., Brown, R., Vineis, P., and Flanagan, J.M. (2013). Epigenome-wide association study in the European Prospective Investigation into Cancer and Nutrition (EPIC-Turin) identifies novel genetic loci associated with smoking. Hum. Mol. Genet. $22,843-851$.

Shi, Q., Ko, E., Barclay, L., Hoang, T., Rademaker, A., and Martin, R. (2001). Cigarette smoking and aneuploidy in human sperm. Mol. Reprod. Dev. 59, 417-421.

Shlien, A., and Malkin, D. (2010). Copy number variations and cancer susceptibility. Curr. Opin. Oncol. 22, 55-63.

Shlien, A., and Malkin, D. (2009). Copy number variations and cancer. Genome Med 1, 62.

Shu, X., Ross, J.A., Pendergrass, T.W., Reaman, G.H., Lampkin, B., and Robison, L.L. (1996). Parental alcohol consumption, cigarette smoking, and risk of infant leukemia: a Childrens Cancer Group study. J. Natl. Cancer Inst. 88, 24-31.

Shukla, Y., and Taneja, P. (2001). Antimutagenic effect of black tea extract using 'rodent dominant lethal mutation assay'. Toxicology 168, 269-274.

Shvets, L., and Kovalchuk, L.Y. (2015). Gonadotoxic Effects of Technogenic Pollution Factors. Galician Medical Journal 22, 92-95.

Shwed, P.S., Crosthwait, J., Douglas, G.R., and Seligy, V.L. (2010). Characterisation of Muta $^{\mathrm{TM}}$ Mouse $\lambda \mathrm{gt10}$-lacZ transgene: evidence for in vivo rearrangements. Mutagenesis 25, 609-616.

Siegel, R., Naishadham, D., and Jemal, A. (2013). Cancer statistics, 2013. CA: A Cancer Journal for Clinicians 63, 11-30.

Singer, T.M., Lambert, I.B., Williams, A., Douglas, G.R., and Yauk, C.L. (2006). Detection of induced male germline mutation: correlations and comparisons between traditional germline mutation assays, transgenic rodent assays and expanded simple tandem repeat instability assays. Mutation Research/Fundamental and Molecular Mechanisms of Mutagenesis 598, 164-193. 
Singer, T.M., and Yauk, C.L. (2010). Germ cell mutagens: Risk assessment challenges in the 21st century. Environ. Mol. Mutagen. 51, 919-928.

Singh, N.P., McCoy, M.T., Tice, R.R., and Schneider, E.L. (1988). A simple technique for quantitation of low levels of DNA damage in individual cells. Exp. Cell Res. 175, 184-191.

Singh, R., Sram, R.J., Binkova, B., Kalina, I., Popov, T.A., Georgieva, T., Garte, S., Taioli, E., and Farmer, P.B. (2007). The relationship between biomarkers of oxidative DNA damage, polycyclic aromatic hydrocarbon DNA adducts, antioxidant status and genetic susceptibility following exposure to environmental air pollution in humans. Mutation Research/Fundamental and Molecular Mechanisms of Mutagenesis 620, 83-92.

Slebos, R.J., Little, R.E., Umbach, D.M., Antipkin, Y., Zadaorozhnaja, T.D., Mendel, N.A., Sommer, C.A., Conway, K., Parrish, E., and Gulino, S. (2004). Mini-and microsatellite mutations in children from Chernobyl accident cleanup workers. Mutation Research/Genetic Toxicology and Environmental Mutagenesis 559, 143-151.

Smit, A.F., Hubley, R., and Green, P. (1996). RepeatMasker. Published on the Web at Http://Www.Repeatmasker.Org

Smit, M., Romijn, J.C., Wildhagen, M.F., Weber, R.F., and Dohle, G.R. (2010). Sperm chromatin structure is associated with the quality of spermatogenesis in infertile patients. Fertil. Steril. 94, 1748-1752.

Smith, B.E., and Braun, R.E. (2012). Germ cell migration across Sertoli cell tight junctions. Science 338, 798-802.

Snyder, M., Du, J., and Gerstein, M. (2010). Personal genome sequencing: current approaches and challenges. Genes Dev. 24, 423-431.

Sobreiro, B.P., Lucon, A.M., Pasqualotto, F.F., Hallak, J., Athayde, K.S., and Arap, S. (2005). Semen analysis in fertile patients undergoing vasectomy: reference values and variations according to age, length of sexual abstinence, seasonality, smoking habits and caffeine intake. Sao Paulo Medical Journal 123, 161-166.

Sofikitis, N., Miyagawa, I., Dimitriadis, D., Zavos, P., Sikka, S., and Hellstrom, W. (1995). Effects of smoking on testicular function, semen quality and sperm fertilizing capacity. J. Urol. 154, 1030-1034.

Solinas-Toldo, S., Lampel, S., Stilgenbauer, S., Nickolenko, J., Benner, A., Döhner, H., Cremer, T., and Lichter, P. (1997). Matrix- based comparative genomic hybridization: Biochips to screen for genomic imbalances. Genes, Chromosomes and Cancer 20, 399407. 
Somers, C.M. (2006). Expanded simple tandem repeat(ESTR) mutation induction in the male germline: lessons learned from lab mice. Mutation Research/Fundamental and Molecular Mechanisms of Mutagenesis 598, 35-49.

Somers, C.M., and Cooper, D.N. (2009). Air pollution and mutations in the germline: are humans at risk? Hum. Genet. 125, 119-130.

Somers, C.M., McCarry, B.E., Malek, F., and Quinn, J.S. (2004). Reduction of particulate air pollution lowers the risk of heritable mutations in mice. Science 304, 10081010.

Somers, C.M., Yauk, C.L., White, P.A., Parfett, C.L., and Quinn, J.S. (2002). Air pollution induces heritable DNA mutations. Proceedings of the National Academy of Sciences 99, 15904-15907.

Song, C., Clark, T.A., Lu, X., Kislyuk, A., Dai, Q., Turner, S.W., He, C., and Korlach, J. (2012). Sensitive and specific single-molecule sequencing of 5-hydroxymethylcytosine. Nature Methods 9, 75-77.

Sorahan, T., McKinney, P., Mann, J., Lancashire, R., Stiller, C., Birch, J., Dodd, H., and Cartwright, R. (2001). Childhood cancer and parental use of tobacco: findings from the inter-regional epidemiological study of childhood cancer (IRESCC). Br. J. Cancer 84, 141.

Spano, M., Kolstad, A.H., Larsen, S.B., Cordelli, E., Leter, G., Giwercman, A., and Bonde, J.P. (1998). The applicability of the flow cytometric sperm chromatin structure assay in epidemiological studies. Asclepios. Hum. Reprod. 13, 2495-2505.

Spinner, N.B., and Conlin, L.K. Paper presented at American Journal of Medical Genetics Part C: Seminars in Medical Genetics.

Stankiewicz, P., and Lupski, J.R. (2010). Structural variation in the human genome and its role in disease. Annu. Rev. Med. 61, 437-455.

Steenaard, R.V., Ligthart, S., Stolk, L., Peters, M.J., van Meurs, J.B., Uitterlinden, A.G., Hofman, A., Franco, O.H., and Dehghan, A. (2015). Tobacco smoking is associated with methylation of genes related to coronary artery disease. Clinical Epigenetics 7, 1.

Steinberger, E.K., Ferencz, C., and Loffredo, C.A. (2002). Infants with single ventricle: A population- based epidemiological study. Teratology 65, 106-115.

Stenson, P.D., Mort, M., Ball, E.V., Howells, K., Phillips, A.D., Thomas, N., and Cooper, D.N. (2009). The human gene mutation database: 2008 update. Genome Med 1, 13. 
Stoichev, I.I., Todorov, D.K., and Christova, L.T. (1993). Dominant-lethal mutations and micronucleus induction in male $\mathrm{BALB} / \mathrm{c}, \mathrm{BDF} 1$ and $\mathrm{H}$ mice by tobacco smoke. Mutation Research/Genetic Toxicology 319, 285-292.

Stramova, X., and Kandar, R. (2015). Determination of seminal plasma malondialdehyde by high-performance liquid chromatography in smokers and non-smokers. Bratisl. Lek. Listy $116,20-24$.

Sun, J.X., Helgason, A., Masson, G., Ebenesersdóttir, S.S., Li, H., Mallick, S., Gnerre, S., Patterson, N., Kong, A., and Reich, D. (2012). A direct characterization of human mutation based on microsatellites. Nat. Genet. 44, 1161-1165.

Sun, J., Jurisicova, A., and Casper, R.F. (1997). Detection of deoxyribonucle ic acid fragmentation in human sperm: correlation with fertilization in vitro. Biol. Reprod. 56, 602-607.

Sun, T., Tian, H., Wang, C., Yin, P., Zhu, Y., Chen, X., and Tang, Z. (2013). A survey of congenital heart disease and other organic malformations associated with different types of orofacial clefts in Eastern China. PloS One 8, e54726.

Swan, S.H., Brazil, C., Drobnis, E.Z., Liu, F., Kruse, R.L., Hatch, M., Redmon, J.B., Wang, C., Overstreet, J.W., and Study For Future Families Research Group. (2003). Geographic differences in semen quality of fertile U.S. males. Environ. Health Perspect. $111,414-420$.

Swayne, B.G., Kawata, A., Behan, N.A., Williams, A., Wade, M.G., MacFarlane, A.J., and Yauk, C.L. (2012). Investigating the effects of dietary folic acid on sperm count, DNA damage and mutation in Balb/c mice. Mutation Research/Fundamental and Molecular Mechanisms of Mutagenesis 737, 1-7.

Swiger, R.R., Cosentino, L., Shima, N., Bielas, J.H., Cruz-Munoz, W., and Heddle, J.A. (1999). The cII locus in the MutaMouse system. Environ. Mol. Mutagen. 34, 201-207.

Taha, E., Ezz- Aldin, A., Sayed, S., Ghandour, N., and Mostafa, T. (2014). Smoking influence on sperm vitality, DNA fragmentation, reactive oxygen species and zinc in oligoasthenoteratozoospermic men with varicocele. Andrologia 46, 687-691.

Taha, E.A., Ez-Aldin, A.M., Sayed, S.K., Ghandour, N.M., and Mostafa, T. (2012). Effect of smoking on sperm vitality, DNA integrity, seminal oxidative stress, zinc in fertile men. Urology $80,822-825$.

Tam, G.W., Redon, R., Carter, N.P., and Grant, S.G. (2009). The role of DNA copy number variation in schizophrenia. Biol. Psychiatry 66, 1005-1012.

Tandon, R., Saxena, D., Chandra, S., Seth, P., and Srivastava, S. (1988). Testicular effects of acrylonitrile in mice. Toxicol. Lett. 42, 55-63. 
Tang, Y.G., Tang, L.X., Wang, Q.L., Song, G., Jiang, Y.J., Deng, S.M., Jiang, F., and Qin, W.B. (2015). The reference values for semen parameters of 1213 fertile men in Guangdong Province in China. Asian J. Androl. 17, 298-303.

Tareke, E., Rydberg, P., Karlsson, P., Eriksson, S., and Törnqvist, M. (2000). Acrylamide: a cooking carcinogen? Chem. Res. Toxicol. 13, 517-522.

Taskinen, H., Anttila, A., Lindbohm, M., Sallmén, M., and Hemminki, K. (1989). Spontaneous abortions and congenital malformations among the wives of men occupationally exposed to organic solvents. Scand. J. Work Environ. Health 345-352.

Tawadrous, G.A., Aziz, A.A., and Mostafa, T. (2011). Effect of smoking status on seminal parameters and apoptotic markers in infertile men. J. Urol. 186, 1986-1990.

Tawn, E.J., Rees, G.S., Leith, C., Winther, J.F., Curwen, G.B., Stovall, M., Olsen, J.H., Rechnitzer, C., Schroeder, H., and Guldberg, P. (2011). Germline minisatellite mutations in survivors of childhood and young adult cancer treated with radiation. Int. J. Radiat. Biol. 87, 330-340.

Teijon, M.L., Garcia, F., Serra, O., Moragas, M., Rabanal, A., Olivares, R., and Alvarez, J. (2007). Semen quality in a population of volunteers from the province of Barcelona. Reproductive Biomedicine Online 15, 434-444.

Thorne, D., Kilford, J., Hollings, M., Dalrymple, A., Ballantyne, M., Meredith, C., and Dillon, D. (2015). The mutagenic assessment of mainstream cigarette smoke using the Ames assay: A multi-strain approach. Mutation Research/Genetic Toxicology and Environmental Mutagenesis 782, 9-17.

Thybaud, V., Dean, S., Nohmi, T., de Boer, J., Douglas, G.R., Glickman, B.W., Gorelick, N.J., Heddle, J.A., Heflich, R.H., and Lambert, I. (2003). In vivo transgenic mutation assays. Mutation Research/Genetic Toxicology and Environmental Mutagenesis 540, $141-151$.

Tomasetti, C., and Vogelstein, B. (2015). Cancer etiology. Variation in cancer risk among tissues can be explained by the number of stem cell divisions. Science 347, 78-81.

Trummer, H., Habermann, H., Haas, J., and Pummer, K. (2002). The impact of cigarette smoking on human semen parameters and hormones. Hum. Reprod. 17, 1554-1559.

Tsarev, I., Gagonin, V., Giwercman, A., and Erenpreiss, J. (2005). Sperm concentration in Latvian military conscripts as compared with other countries in the Nordic-Baltic area. Int. J. Androl. 28, 208-214.

Tsyusko, O., Glenn, T., Yi, Y., Joice, G., Jones, K., Aizawa, K., Coughlin, D., Zimbrick, J., and Hinton, T. (2011). Differential genetic responses to ionizing irradiation in 
individual families of Japanese medaka, $<\mathrm{i}>$ Oryzias latipes. Mutation Research/Genetic Toxicology and Environmental Mutagenesis 718, 18-23.

Tsyusko, O., Yi, Y., Coughlin, D., Main, D., Podolsky, R., Hinton, T., and Glenn, T. (2007). Radiation-induced untargeted germline mutations in Japanese medaka.

Comparative Biochemistry and Physiology Part C: Toxicology \& Pharmacology 145, 103-110.

Untergasser, A., Cutcutache, I., Koressaar, T., Ye, J., Faircloth, B.C., Remm, M., and Rozen, S.G. (2012). Primer3--new capabilities and interfaces. Nucleic Acids Res. 40, e115.

US Department of Health and Human Services. (2010). How tobacco smoke causes disease: what it means to you. Atlanta: US Department of Health and Human Services, Centers for Disease Control and Prevention. National Center for Chronic Disease Prevention and Health Promotion, Office on Smoking and Health

USEPA. (2011). Recommended Use of Body Weight 3/4 as the Default Method in Derivation of the Oral Reference Dose. Risk Assessment Forum. U. S. Environmental Protection Agency. Washington, DC. Available: Https://Www. Epa. Gov/Sites/Production/Files/2013-09/Documents/Recommended-use-ofbw 34. Pdf

Valavanidis, A., Vlachogianni, T., and Fiotakis, C. (2009). 8-hydroxy-2'-deoxyguanosine (8-OHdG): a critical biomarker of oxidative stress and carcinogenesis. Journal of Environmental Science and Health Part C 27, 120-139.

Van Bokhoven, H. (2011). Genetic and epigenetic networks in intellectual disabilities. Annu. Rev. Genet. 45, 81-104.

van Rooij, I.A., Wijers, C.H., Rieu, P.N., Hendriks, H.S., Brouwers, M.M., Knoers, N.V., de Blaauw, I., and Roeleveld, N. (2010). Maternal and paternal risk factors for anorectal malformations: A Dutch case- control study. Birth Defects Research Part A: Clinical and Molecular Teratology 88, 152-158.

Varshini, J., Srinag, B., Kalthur, G., Krishnamurthy, H., Kumar, P., Rao, S., and Adiga, S. (2012). Poor sperm quality and advancing age are associated with increased sperm DNA damage in infertile men. Andrologia 44, 642-649.

Veltman, J.A., and Brunner, H.G. (2012). De novo mutations in human genetic disease. Nature Reviews Genetics 13, 565-575.

Venners, S.A., Wang, X., Chen, C., Wang, L., Chen, D., Guang, W., Huang, A., Ryan, L., O'Connor, J., and Lasley, B. (2004). Paternal smoking and pregnancy loss: a prospective study using a biomarker of pregnancy. Am. J. Epidemiol. 159, 993-1001. 
Verhofstad, N., van Oostrom, Conny Th M, van Benthem, J., van Schooten, F.J., van Steeg, H., and Godschalk, R.W. (2010). DNA adduct kinetics in reproductive tissues of DNA repair proficient and deficient male mice after oral exposure to benzo (a) pyrene. Environ. Mol. Mutagen. 51, 123-129.

Verhofstad, N., van Oostrom, Conny Th M, Zwart, E., Maas, L.M., van Benthem, J., van Schooten, F.J., van Steeg, H., and Godschalk, R.W. (2011). Evaluation of benzo (a) pyrene-induced gene mutations in male germ cells. Toxicological Sciences 119, 218-223.

Verhofstad, N., Linschooten, J.O., van Benthem, J., Dubrova, Y.E., van Steeg, H., van Schooten, F.J., and Godschalk, R.W. (2008). New methods for assessing male germ line mutations in humans and genetic risks in their offspring. Mutagenesis 23, 241-247.

Vijg, J., and Douglas, G. (1996). Bacteriophage lambda and plasmid lacZ transgenic mice for studying mutations in vivo. Technologies for Detection of DNA Damage and Mutations 391-410.

Vilariño-Güell, C., Smith, A.G., and Dubrova, Y.E. (2003). Germline mutation induction at mouse repeat DNA loci by chemical mutagens. Mutation Research/Fundamental and Molecular Mechanisms of Mutagenesis 526, 63-73.

Viloria, T., Garrido, N., Fernández, J.L., Remohí, J., Pellicer, A., and Meseguer, M. (2007). Sperm selection by swim-up in terms of deoxyribonucleic acid fragmentation as measured by the sperm chromatin dispersion test is altered in heavy smokers. Fertil. Steril. $88,523-525$.

Viloria, T., Meseguer, M., Martínez-Conejero, J.A., O'Connor, J., Remohí, J., Pellicer, A., and Garrido, N. (2010). Cigarette smoking affects specific sperm oxidative defenses but does not cause oxidative DNA damage in infertile men. Fertil. Steril. 94, 631-637.

Viloria, T., Rubio, M.C., Rodrigo, L., Calderon, G., Mercader, A., Mateu, E., Meseguer, M., Remohi, J., and Pellicer, A. (2005). Smoking habits of parents and male: female ratio in spermatozoa and preimplantation embryos. Hum. Reprod. 20, 2517-2522.

Vine, M.F. (1996). Smoking and male reproduction: a review. Int. J. Androl. 19, 323337.

Vine, M.F., Setzer, R.W., Everson, R.B., and Wyrobek, A.J. (1997). Human sperm morphometry and smoking, caffeine, and alcohol consumption. Reproductive Toxicology $11,179-184$.

Vine, M., Margoli, B., Morrison, H., and Hulka, B. (1994). Cigarette smoking and sperm density: a meta-analysis. Fertil. Steril. 61, 35-43. 
Vine, M.F., Hulka, B.S., Margolin, B.H., Truong, Y.K., Hu, P.C., Schramm, M.M., Griffith, J.D., McCann, M., and Everson, R.B. (1993). Cotinine concentrations in semen, urine, and blood of smokers and nonsmokers. Am. J. Public Health 83, 1335-1338.

Vineis, P., Alavanja, M., Buffler, P., Fontham, E., Franceschi, S., Gao, Y.T., Gupta, P.C., Hackshaw, A., Matos, E., Samet, J., et al. (2004). Tobacco and cancer: recent epidemiological evidence. J. Natl. Cancer Inst. 96, 99-106.

Vissers, L.E., Gilissen, C., and Veltman, J.A. (2015). Genetic studies in intellectual disability and related disorders. Nature Reviews Genetics

Vogelstein, B., Papadopoulos, N., Velculescu, V.E., Zhou, S., Diaz, L.A.,Jr, and Kinzler, K.W. (2013). Cancer genome landscapes. Science 339, 1546-1558.

Wallock, L.M., Tamura, T., Mayr, C.A., Johnston, K.E., Ames, B.N., and Jacob, R.A. (2001). Low seminal plasma folate concentrations are associated with low sperm density and count in male smokers and nonsmokers. Fertil. Steril. 75, 252-259.

Wang, J., Fan, H.C., Behr, B., and Quake, S.R. (2012). Genome-wide single-cell analysis of recombination activity and de novo mutation rates in human sperm. Cell 150, 402-412.

Wang, J.J., Marshall, W.D., Frazer, D.G., Law, B., and Lewis, D.M. (2003). Characterization of DNA adducts from lung tissue of asphalt fume-exposed mice by nanoflow liquid chromatography quadrupole time-of-flight mass spectrometry. Anal. Biochem. 322, 79-88.

Wang, S., Wang, X., Chia, S., Shen, H., Song, L., Xing, H., Chen, H., and Ong, C. (2001). A study on occupational exposure to petrochemicals and smoking on seminal quality. J. Androl. 22, 73-78.

Wasserman, C.R., Shaw, G.M., O'Malley, C.D., Tolarova, M.M., and Lammer, E.J. (1996). Parental cigarette smoking and risk for congenital anomalies of the heart, neural tube, or limb. Teratology 53, 261-267.

Wegner, C.C., Clifford, A.L., Jilbert, P.M., Henry, M.A., and Gentry, W.L. (2010). Abnormally high body mass index and tobacco use are associated with poor sperm quality as revealed by reduced sperm binding to hyaluronan-coated slides. Fertil. Steril. 93, 332-334.

Weisenberger, D.J., and Romano, L.J. (1999). Cytosine methylation in a CpG sequence leads to enhanced reactivity with Benzo[a]pyrene diol epoxide that correlates with a conformational change. J. Biol. Chem. 274, 23948-23955.

White, P.A., Robitaille, S., and Rasmussen, J.B. (1999). Heritable reproductive effects of benzo [a] pyrene on the fathead minnow (Pimephales promelas). Environmental Toxicology and Chemistry 18, 1843-1847. 
Wilkinson, G., and Rogers, C. (1973). Symbolic description of factorial models for analysis of variance. Applied Statistics 392-399.

Windham, G.C., Swan, S.H., and Fenster, L. (1992). Parental cigarette smoking and the risk of spontaneous abortion. Am. J. Epidemiol. 135, 1394-1403.

Witt, K.L., and Bishop, J.B. (1996). Mutagenicity of anticancer drugs in mammalian germ cells. Mutation Research/Fundamental and Molecular Mechanisms of Mutagenesis $355,209-234$.

Witt, K.L., Hughes, L.A., Burka, L.T., McFee, A.F., Mathews, J.M., Black, S.L., and Bishop, J.B. (2003). Mouse bone marrow micronucleus test results do not predict the germ cell mutagenicity of $\mathrm{N}$ - hydroxymethylacrylamide in the mouse dominant lethal assay. Environ. Mol. Mutagen. 41, 111-120.

Wong, W.S., Solomon, B.D., Bodian, D.L., Kothiyal, P., Eley, G., Huddleston, K.C., Baker, R., Thach, D.C., Iyer, R.K., and Vockley, J.G. (2016). New observations on maternal age effect on germline de novo mutations. Nature Communications 7 ,

World Health Organization. (2003). Investing in mental health.

World Health Organization. (1999). WHO laboratory manual for the examination of human semen and sperm-cervical mucus interaction Cambridge university press).

World Health Organization. (1983). Polynuclear aromatic compounds, Part 1, chemical, environmental and experimental data. Evaluation of Carcinogenic Risk of Chemicals to Humans 32,

Wu, S., Powers, S., Zhu, W., and Hannun, Y.A. (2016). Substantial contribution of extrinsic risk factors to cancer development. Nature 529, 43-47.

Wyrobek, A.J., Mulvihill, J.J., Wassom, J.S., Malling, H.V., Shelby, M.D., Lewis, S.E., Witt, K.L., Preston, R.J., Perreault, S.D., and Allen, J.W. (2007). Assessing human germ- cell mutagenesis in the Postgenome Era: A celebration of the legacy of William Lawson (Bill) Russell. Environ. Mol. Mutagen. 48, 71-95.

Wyrobek, A.J., Schmid, T.E., and Marchetti, F. (2005). Cross- species sperm- FISH assays for chemical testing and assessing paternal risk for chromosomally abnormal pregnancies. Environ. Mol. Mutagen. 45, 271-283.

Xiao, Y., and Tates, A. (1995). Clastogenic effects of 1, 3- butadiene and its metabolites 1, 2- epoxybutene and 1, 2, 3, 4- diepoxybutane in splenocytes and germ cells of rats and mice in vivo. Environ. Mol. Mutagen. 26, 97-108.

Xing, C., Marchetti, F., Li, G., Weldon, R.H., Kurtovich, E., Young, S., Schmid, T.E., Zhang, L., Rappaport, S., Waidyanatha, S., Wyrobek, A.J., and Eskenazi, B. (2010). 
Benzene exposure near the U.S. permissible limit is associated with sperm aneuploidy. Environ. Health Perspect. 118, 833-839.

Xu, B., Roos, J.L., Dexheimer, P., Boone, B., Plummer, B., Levy, S., Gogos, J.A., and Karayiorgou, M. (2011). Exome sequencing supports a de novo mutational paradigm for schizophrenia. Nat. Genet. 43, 864-868.

Xu, B., Roos, J.L., Levy, S., Van Rensburg, E., Gogos, J.A., and Karayiorgou, M. (2008). Strong association of de novo copy number mutations with sporadic schizophrenia. Nat. Genet. 40, 880-885.

Xu, D., Zhu, Q., Zheng, L., Wang, Q., Shen, H., Deng, L., and Ong, C. (2003). Exposure to acrylonitrile induced DNA strand breakage and sex chromosome aneuploidy in human spermatozoa. Mutation Research/Genetic Toxicology and Environmental Mutagenesis 537, 93-100.

Xu, G., McMahan, C.A., and Walter, C.A. (2014). Early-Life Exposure to Benzo [a] pyrene Increases Mutant Frequency in Spermatogenic Cells in Adulthood. Plos One 9, e87439.

Xu, G., Spivak, G., Mitchell, D.L., Mori, T., McCarrey, J.R., McMahan, C.A., Walter, R.B., Hanawalt, P.C., and Walter, C.A. (2005). Nucleotide excision repair activity varies among murine spermatogenic cell types. Biol. Reprod. 73, 123-130.

Xue, W., and Warshawsky, D. (2005). Metabolic activation of polycyclic and heterocyclic aromatic hydrocarbons and DNA damage: a review. Toxicol. Appl. Pharmacol. 206, 73-93.

Yamamoto, Y., Isoyama, E., Sofikitis, N., and Miyagawa, I. (1998). Effects of smoking on testicular function and fertilizing potential in rats. Urol. Res. 26, 45-48.

Yamauchi, M., Nishimura, M., Tsuji, S., Terada, M., Sasanuma, M., and Shimada, Y. (2002). Effect of SCID mutation on the occurrence of mouse Pc-1 (Ms6-hm) germline mutations. Mutation Research/Fundamental and Molecular Mechanisms of Mutagenesis $503,43-49$.

Yang, W. (2014). An overview of Y-family DNA polymerases and a case study of human DNA polymerase $\eta$. Biochemistry (N. Y. ) 53, 2793-2803.

Yang, H., Chen, Q., Zhou, N., Sun, L., Bao, H., Tan, L., Chen, H., Zhang, G., Ling, X., Huang, L., et al. (2015). Lifestyles Associated With Human Semen Quality: Results From MARHCS Cohort Study in Chongqing, China. Medicine (Baltimore) 94, e1166.

Yauk, C.L. (2004). Advances in the application of germline tandem repeat instability for in situ monitoring. Mutation Research/Reviews in Mutation Research 566, 169-182. 
Yauk, C.L., Aardema, M.J., van Benthem, J., Bishop, J.B., Dearfield, K.L., DeMarini, D.M., Dubrova, Y.E., Honma, M., Lupski, J.R., and Marchetti, F. (2015a). Approaches for identifying germ cell mutagens: Report of the 2013 IWGT workshop on germ cell assays $\_$. Mutation Research/Genetic Toxicology and Environmental Mutagenesis 783, $36-54$.

Yauk, C.L., Berndt, M.L., Williams, A., Rowan-Carroll, A., Douglas, G.R., and Stämpfli, M.R. (2007). Mainstream tobacco smoke causes paternal germ-line DNA mutation. Cancer Res. 67, 5103-5106.

Yauk, C.L., Dubrova, Y.E., Grant, G.R., and Jeffreys, A.J. (2002). A novel single molecule analysis of spontaneous and radiation-induced mutation at a mouse tandem repeat locus. Mutation Research/Fundamental and Molecular Mechanisms of Mutagenesis 500, 147-156.

Yauk, C.L., Lambert, I.B., Meek, M., Douglas, G.R., and Marchetti, F. (2015b). Development of the adverse outcome pathway "alkylation of DNA in male premeiotic germ cells leading to heritable mutations" using the OECD's users' handbook supplement. Environ. Mol. Mutagen. 56, 724-750.

Yauk, C.L., Polyzos, A., Rowan- Carroll, A., Kortubash, I., Williams, A., and Kovalchuk, O. (2008a). Tandem repeat mutation, global DNA methylation, and regulation of DNA methyltransferases in cultured mouse embryonic fibroblast cells chronically exposed to chemicals with different modes of action. Environ. Mol. Mutagen. 49, 26-35.

Yauk, C.L., Argueso, J.L., Auerbach, S.S., Awadalla, P., Davis, S.R., DeMarini, D.M., Douglas, G.R., Dubrova, Y.E., Elespuru, R.K., and Glover, T.W. (2013). Harnessing genomics to identify environmental determinants of heritable disease. Mutation Research/Reviews in Mutation Research

Yauk, C., and Polyzos, A. (2005). Tandem repeat DNA: applications in mutation analysis. The Japanese Environmental Mutagen Society 27, 93-98.

Yauk, C., Polyzos, A., Rowan-Carroll, A., Somers, C.M., Godschalk, R.W., Van Schooten, F.J., Berndt, M.L., Pogribny, I.P., Koturbash, I., and Williams, A. (2008b). Germ-line mutations, DNA damage, and global hypermethylation in mice exposed to particulate air pollution in an urban/industrial location. Proceedings of the National Academy of Sciences 105, 605-610.

Yoon, J.H., Smith, L.E., Feng, Z., Tang, M., Lee, C.S., and Pfeifer, G.P. (2001). Methylated $\mathrm{CpG}$ dinucleotides are the preferential targets for G-to-T transversion mutations induced by benzo[a]pyrene diol epoxide in mammalian cells: similarities with the p53 mutation spectrum in smoking-associated lung cancers. Cancer Res. 61, 71107117. 
Yousefniapasha, Y., Jorsaraei, G., Gholinezhadchari, M., Mahjoub, S., Hajiahmadi, M., and Farsi, M. (2015). Nitric Oxide Levels and Total Antioxidant Capacity in The Seminal Plasma of Infertile Smoking Men. Cell Journal (Yakhteh) 17, 129.

Yu, B., Qi, Y., Liu, D., Gao, X., Chen, H., Bai, C., and Huang, Z. (2014). Cigarette smoking is associated with abnormal histone-to-protamine transition in human sperm. Fertil. Steril. 101, 51-57. e1.

Yu, D., Berlin, J.A., Penning, T.M., and Field, J. (2002). Reactive oxygen species generated by $\mathrm{PAH}$ o-quinones cause change-in-function mutations in p53. Chem. Res. Toxicol. 15, 832-842.

Yu, B., Chen, J., Liu, D., Zhou, H., Xiao, W., Xia, X., and Huang, Z. (2013). Cigarette smoking is associated with human semen quality in synergy with functional NRF2 polymorphisms. Biol. Reprod. 89, 5.

Yuan, P., Okazaki, I., and Kuroki, Y. (1995). Anal atresia: effect of smoking and drinking habits during pregnancy. Jpn. J. Hum. Genet. 40, 327-332.

Yuen, R.K., Thiruvahindrapuram, B., Merico, D., Walker, S., Tammimies, K., Hoang, N., Chrysler, C., Nalpathamkalam, T., Pellecchia, G., and Liu, Y. (2015). Whole-genome sequencing of quartet families with autism spectrum disorder. Nat. Med.

Zavos, P.M., Correa, J.R., Karagounis, C.S., Ahparaki, A., Phoroglou, C., Hicks, C.L., and Zarmakoupis-Zavos, P.N. (1998). An electron microscope study of the axonemal ultrastructure in human spermatozoa from male smokers and nonsmokers. Fertil. Steril. 69, 430-434.

Zeilinger, S., Kühnel, B., Klopp, N., Baurecht, H., Kleinschmidt, A., Gieger, C., Weidinger, S., Lattka, E., Adamski, J., and Peters, A. (2013). Tobacco smoking leads to extensive genome-wide changes in DNA methylation. PloS One 8, e63812.

Zenzes, M.T., Bielecki, R., and Reed, T.E. (1999a). Detection of benzo (a) pyrene diol epoxide-DNA adducts in sperm of men exposed to cigarette smoke. Fertil. Steril. 72, 330-335.

Zenzes, M.T., Puy, L.A., Bielecki, R., and Reed, T.E. (1999b). Detection of benzo [a] pyrene diol epoxide-DNA adducts in embryos from smoking couples: evidence for transmission by spermatozoa. Mol. Hum. Reprod. 5, 125-131.

Zhang, F., Gu, W., Hurles, M.E., and Lupski, J.R. (2009). Copy number variation in human health, disease, and evolution. Annual Review of Genomics and Human Genetics 10, 451-481. 
Zhang, J., Meng, Q., Wang, Q., Zhang, L., Mao, Y., and Sun, Z. (2000). Effect of smoking on semen quality of infertile men in Shandong, China. Asian J. Androl. 2, 143146.

Zhang, J., and Cai, W. (1992). Risk factors associated with antepartum fetal death. Early Hum. Dev. 28, 193-200.

Zhang, J., Savitz, D.A., SCHWINGL, P.J., and Cai, W. (1992). A case-control study of paternal smoking and birth defects. Int. J. Epidemiol. 21, 273-278.

Zhang, Z.H., Zhu, H.B., Li, L.L., Yu, Y., Zhang, H.G., and Liu, R.Z. (2013). Decline of semen quality and increase of leukocytes with cigarette smoking in infertile men. Iranian Journal of Reproductive Medicine 11, 589.

Zhang, M., Zhang, Q.S., Zheng, H.S., Wang, X.Y., Feng, S.Q., Tian, W.J., and Fan, H.T. (2016). Clinical, demographic and psychological characteristics of infertile male smokers in Northeast China. J. Int. Med. Res. 44, 75-80.

Zheng, N., Monckton, D.G., Wilson, G., Hagemeister, F., Chakraborty, R., Connor, T.H., Siciliano, M.J., and Meistrich, M.L. (2000). Frequency of minisatellite repeat number changes at the MS205 locus in human sperm before and after cancer chemotherapy. Environ. Mol. Mutagen. 36, 134-145.

Zitzmann, M., Rolf, C., Nordhoff, V., Schräder, G., Rickert-Föhring, M., Gassner, P., Behre, H.M., Greb, R.R., Kiesel, L., and Nieschlag, E. (2003). Male smokers have a decreased success rate for in vitro fertilization and intracytoplasmic sperm injection. Fertil. Steril. 79, 1550-1554.

Zlotogora, J. (1998). Germ line mosaicism. Hum. Genet. 102, 381-386.

Zong, C., Lu, S., Chapman, A.R., and Xie, X.S. (2012). Genome-wide detection of single-nucleotide and copy-number variations of a single human cell. Science 338, 16221626. 
Appendices 


\section{Appendix A Transgenic Rodent Assay for Quantifying Male Germ Cell Mutant Frequency}

Modified from: O’Brien, J.M., Beal, M.A., Gingerich, J.D., Soper, L., Douglas, G.R., Yauk, C.L., Marchetti, F. Journal of Visualized Experiments, e51576.

\section{A.1 Preamble}

This manuscript describes the method used in this thesis to study germ cell mutations in transgenic mouse models.

\section{A.2 Abstract}

De novo mutations arise mostly in the male germline and may contribute to adverse health outcomes in subsequent generations. Traditional methods for assessing the induction of germ cell mutations require the use of large numbers of animals, making them impractical. As such, germ cell mutagenicity is rarely assessed during chemical testing and risk assessment. Herein, we describe an in vivo male germ cell mutation assay using a transgenic rodent model that is based on a recently approved OECD test guideline. This method uses an in vitro positive selection assay to measure in vivo mutations induced in a transgenic $\lambda \mathrm{gt} 10$ vector bearing a reporter gene directly in the germ cells of exposed males. We further describe how the detection of mutations in the transgene recovered from germ cells can be used to characterize the stage-specific sensitivity of the various spermatogenic cell types to mutagen exposure by controlling 3 experimental parameters: the duration of exposure (administration time), the time 
between exposure and sample collection (sampling time), and the cell population collected for analysis. Because a large number of germ cells can be assayed from a single male, this method has superior sensitivity compared with traditional methods, requires fewer animals, and therefore much less time and resources.

\section{A.3 Introduction}

Sporadic DNA mutations in the germline can lead to reduced reproductive success and, if inherited, may cause genetic disease or heightened predisposition to cancer in the offspring (Ahmadi and Ng, 1999; Crow, 2000; Nelson and Holmes, 1989). Substantial evidence demonstrates that a large proportion of de novo mutations are inherited from the paternal germline (Kong et al., 2012), and that the number of mutations in the offspring is positively correlated with paternal age at the time of conception (Michaelson et al., 2012). The higher proportion of male mutations is believed to be a result of the difference in age during gametogenesis between the sexes, the greater number of spermatogenic cell divisions compared with the number of oogenic cell divisions in the female germline (Crow, 2000), and a progressive decline in DNA repair efficiency with age in males. All of these factors contribute to an increased probability of replication errors in the male germline (Marchetti and Wyrobek, 2008). However, the impact of paternal exposure to environmental factors on the frequency of de novo mutations remains uncertain. Nevertheless, a large number of environmental agents are known to induce germ cell mutations in rodents (Marchetti and Wyrobek, 2005), and there is mounting evidence that some of these agents can also affect the human germline (DeMarini, 2012). Despite these concerns, chemicals are routinely tested 
for their ability to induce mutations in somatic cells for regulatory purposes and it is generally assumed that somatic tests are sufficient to protect the germline. Therefore, chemicals are only rarely assessed for their ability to induce germ cell mutations.

One reason germ cell mutagenicity testing has been largely omitted from the regulatory decision making process is a lack of practical methodologies. Traditional rodent-based methods, such as the dominant lethal (Epstein, 1973) and specific locus (Russell, 1951) tests, estimate germ cell mutation rates by scoring mutant phenotypes in embryos or offspring of exposed parents. These assays require the use of a very large number of animals, time, and resources to acquire statistically meaningful results.

Although several modern methods for quantifying germ cell mutation have recently emerged, many suffer shortcomings in terms of their practicality, efficiency, and biological relevance. For example, repeat length mutations at ESTR loci can be quantified in male germ cells using a single molecule PCR approach (Yauk et al., 2002). However, execution of this method can be technically challenging and labourious, and unlike point mutations, the biological and health significance of changes in the repeat length of the highly unstable ESTR loci remain unclear (Niwa, 2006). Modern whole genome sequencing technologies can provide a wealth of biologically meaningful data when applied to the problem of heritable mutations (Campbell et al., 2012; Kong et al., 2012), but the high cost, high error rates, associated validation required to confirm mutations, and bioinformatics challenges still limit the routine application of this option in a regulatory testing capacity (Beal et al., 2012b).

Herein, we describe a practical method for quantifying induced mutations directly in the germ cells of transgenic male mice. This protocol is described for the transgenic 
MutaMouse model, which has multiple concatenated copies of a recombinant $\lambda$ gt10 phage vector containing an E. coli lacZ reporter gene integrated into both copies of chromosome 3 (Shwed et al., 2010) (Figure A1).

This protocol is also relevant to other transgenic rodent models based on the same principles (BigBlue mouse and rat, or lacZ plasmid mouse, etc.) or slightly different reporter genes (gpt delta mouse and rat, TGR models reviewed in Lambert et al. (2005). This method is based on the TGR mutation assay described in a recently released and revised OECD test guideline (OECD, 2011) and we elaborate upon the special considerations required to accommodate assessment of mutations in the male germline because of the unique characteristics of spermatogenesis. Briefly, the assay involves exposing transgenic male mice to a mutagenic substance, followed by a sampling time where pre-mutational lesions are fixed into stable mutations. At the chosen sampling time, mice are euthanized and germ cells are collected from either the cauda epididymis or the seminiferous tubules. As discussed below, mutagenic effects at different phases of spermatogenesis can be determined by selecting the time between exposure and sample collection. Transgenic inserts, comprising multiple copies of the $\lambda$ phage genome per cell, are isolated from germ cell genomic DNA and packaged into empty $\lambda$ phage capsids creating infectious $\lambda$ phage particles that are then used to infect an $E$. coli host. The infected bacteria are grown on selective media that can distinguish cells containing a vector with a mutated copy of lac $Z$ from cells harbouring wild-type lac $Z$. The mutagenic effect of exposure on the male germline is determined by comparing the frequency of mutant transgenes between control and treated mice (Figure A2, reviewed in Lambert et al. (2005)). A large number of germ cells can be assayed from a single mouse, giving this 
assay superior sensitivity over traditional methods, while reducing the number of animals required. And because no specialized equipment or training is required, this assay provides a practical and efficient option for germ cell mutation testing in most modern toxicology/molecular biology laboratories.

One essential requirement for the effective application of the TGR germ cell mutation assay is an understanding of the spermatogenic cycle (Figure A3). The time for mouse germ cells to progress from stem cells in the seminiferous tubules to spermatogonia, spermatocytes, spermatids, and finally to mature sperm in the epididymis (i.e. spermatogenesis) is approximately 49 days. Mutation can occur at various phases of this cycle and is often compound specific. Two key features that are of particular relevance to mutagenesis in male germ cells are the cessation of DNA synthesis during early meiosis, and the progressive loss of DNA repair capacity (Marchetti and Wyrobek, 2008) during late post-meiosis, 2 processes that are required for the induction and fixation of most mutations.

Because of these unique characteristics of spermatogenesis, there are 3 critical experimental variables for the conduct of the TGR germ cell mutation assay: (1) the test compound administration time; (2) the sampling time; and (3) the selection of the germ cell population to collect for analysis (Figure A3 and Table A1). Administration time is the experimental variable that determines how long target cells are exposed to the test compounds. The length of the administration time can also be used to target exposures to specific cell types or phases of spermatogenesis. For example, a single day administration could be used to determine the effects of an acute exposure on 1 particular cell type. Similarly, exposure can be focused to an entire spermatogenic phase, for example by 
targeting only meiotically dividing spermatocytes, or mitotically dividing spermatogonia using a 2 week administration time and an appropriate sampling time. Chronic and subchronic administration times are used to assess the effects of long term exposure, to ensure sufficient pharmacokinetic distribution of the test compound, or permit sufficient accumulation of mutations from weak mutagens (for example the 28 day administration time recommended in the OECD test guideline).

Sampling time is the critical variable for determining at which phase of spermatogenesis the target cells were in at the time of exposure. The sampling time dictates how much time, and therefore how much further along the spermatogenic cycle, cells pass through after exposure. For example, to investigate effects in stem cell spermatogonia, a sampling time $>49$ days is required if collecting fully matured sperm, or $>42$ days if collecting immature germ cells from the seminiferous tubules, to ensure that all collected cells have had enough time to develop from exposed stems cells. It is important to note that a sampling time of at least 70 days would be preferable to demonstrate a true stem cell effect to provide sufficient time for pharmacokinetic distribution of the toxicant, for elimination of cells exposed at later phases of spermatogenesis, and to account for a period of temporary sterility that may occur $\sim 6$ weeks after exposure to highly mutagenic compounds (Rodriguez et al., 1983). Similarly, a sampling time of 21 days would ensure that sperm collected from the cauda epididymis would have just completed meiosis on the final day of exposure.

Germ cells can be collected as mature sperm from the cauda epididymis, or as a mixture of various spermatogenic cell types from the seminiferous tubules. Mature sperm remain in the cauda for $\sim 3$ days, making it possible to determine with relative accuracy 
the cell type or phase of spermatogenesis from which the sperm originated for any given experimental design. Thus, analysis of cauda sperm permits highly targeted investigations of stage-specific mutational effects. On the other hand, cell suspensions collected from the seminiferous tubules contain a mixture of various germ cell types in different phases of development, and thus offer poorer resolution of the spermatogenic phase in which mutations originated. In addition, cell suspensions recovered from the seminiferous tubules tend to contain an over-representation of spermatids, followed by spermatocytes, and very few spermatogonia and stem cells (these proportions are represented by graduated white bars in Figure A3). Moreover, suspensions prepared from the seminiferous tubules may also contain various somatic cells. Thus, because so many cells types are present, mutational effects can be influenced by a variety of non-target cells. However, collecting samples from seminiferous tubules offers an economical option for simultaneously screening multiple germ cell types, and easy integration of germ cell analysis into the standard OECD test protocol for somatic mutation.

To reiterate, depending on the needs of the investigator, administration time, sampling time and the collected cell population can be adjusted to interrogate the effects of exposure in various cell types and at different phases of spermatogenesis. By carefully selecting these variables, experiments can be designed for targeted mechanistic studies, or for more generalized regulatory testing purposes.

To attain proficiency in the assay, we recommend the use of an acute oral administration of $100 \mathrm{mg} / \mathrm{kg}$ ENU, followed by a 70 day sampling time as positive control. Analysis of cauda sperm thus targets spermatogonial stem cells (Figure A3), which typically exhibit a 4-5 fold increase in MF over controls following this highly 
mutagenic dose of ENU. It should be noted that this dose is known to induce sterility 6 weeks post exposure, thus it may not be a suitable control dose for shorter sampling times. This dose will also produce a detectable increase in MF in most somatic tissues (Lambert et al., 2005). The representative results presented below were generated following an acute +70 exposure regimen using 3 doses of ENU up to and including 100 $\mathrm{mg} / \mathrm{kg}$.

\section{A.4 Protocol}

All protocols involving animal husbandry, maintenance and handling were approved by Health Canada's animal care committee.

\section{Animal Exposures}

1.1. Randomly distribute transgenic male mice (8-12 weeks old) to control groups and treatment groups $(\min =5$ per group). Treat mice with test compound and relevant control by an appropriate exposure route for the selected administration time. Choose the appropriate sampling time according to the spermatogenic cell type of interest (Figure A3).

1.2. After the sampling time, euthanize mice by cervical dislocation under isofluorane anesthesia (or other appropriate method). Carefully draw the testes from an incision in the abdomen or scrotum and excise the cauda epididymides (Figure A4, to view a detailed video of epididymis collection see (Duselis and Vrana, 2007)). Alternatively, collect the testes if analyzing seminiferous tubules cells. Freeze in liquid nitrogen and store at $-80{ }^{\circ} \mathrm{C}$ for later use. 


\section{Isolation and Digestion of Cauda Sperm}

2.1. Defrost cauda epididymis on ice. Transfer thawed cauda to a Petri dish and thoroughly mince with a scalpel or razor blade.

2.2. Add $700 \mu \mathrm{l}$ of room temperature D-PBS to the Petri dish. Using a wide-bore $1,000 \mu \mathrm{l}$ pipette tip, release sperm from the cauda by drawing and releasing the suspension until the D-PBS becomes cloudy with sperm (approximately 10 times). NOTE: To prepare wide-bore pipette tip cut 2-3 $\mathrm{mm}$ from the end of a plastic pipette tip.

2.3. Filter suspension through a stainless steel mesh filter into a fresh $1.5 \mathrm{ml}$ tube. Wash the Petri dish with an additional $700 \mu \mathrm{l}$ of D-PBS and transfer to same $1.5 \mathrm{ml}$ tube through the mesh filter. Remove mesh, place tube on ice.

2.4. Repeat steps 2.1-2.3 for remaining samples.

2.5. Spin samples at $11,000 \times \mathrm{g}$ for $3 \mathrm{~min}$. Carefully decant supernatant. Avoid disturbing the pellet.

2.6. Add $1.0 \mathrm{ml}$ cold $1 \mathrm{x}$ saline sodium citrate (SSC). Vortex until pellet is completely resuspended. This will sometimes take several rounds of vortexing.

2.7. Add $15 \mu \mathrm{l}$ of $10 \%$ SDS. Invert/shake vigorously for $30 \mathrm{sec}$ to disrupt non-sperm cells. Shaking too gently will result in inadequate disruption and the pellet will not form properly in the following step.

2.8. Spin at $11,000 \times \mathrm{g}$ for $2 \mathrm{~min}$. A loose, "fluffy" pellet is indicative of incomplete disruption of somatic cells due to insufficient shaking in step 2.7. If this occurs, simply shake the sample again, and respin until a tight pellet is formed. Carefully decant supernatant. Avoid disturbing the pellet. Briefly spin again and remove remaining supernatant with $200 \mu \mathrm{l}$ pipette. 
2.9. Add $940 \mu \mathrm{l} 0.2 \mathrm{x}$ cold SSC and vortex until pellet is re-suspended. This pellet may be very difficult to re-suspend and may take several rounds of vortexing. Sometimes clumps of sperm are unavoidable.

10. Add $120 \mu \mathrm{l} \beta$-mercaptoethanol, $100 \mu \mathrm{l} 10 \%$ SDS, $20 \mu \mathrm{l}$ 0.5M EDTA, $\mathrm{pH} 8$, and $20 \mu \mathrm{l}$ proteinase $\mathrm{K}$ (60 $\mathrm{mg} / \mathrm{ml}$, prepared fresh). Mix well and digest overnight with rotation at $37{ }^{\circ} \mathrm{C}$. Proceed to phenol/chloroform extraction.

\section{Phenol/chloroform Extraction of DNA from Cauda Sperm}

NOTE: Because the nuclear DNA in late phase spermatids and mature sperm is complexed with protamines, and is highly condensed compared to the DNA of somatic cells, conventional nucleic acid isolation methods will not generate DNA of sufficient yield and purity for the mutation assay to work efficiently. Multiple phenol-chloroform extractions after an aggressive digestion are required to release and purify sperm DNA (based on methods from (Yauk et al., 2002)).

3.1. Transfer sperm cell digest to a $15 \mathrm{ml}$ polypropylene tube.

3.2. Add $2 \mathrm{ml}$ of phenol:chloroform mixture (1:1). Rotate tube at $22 \mathrm{rpm}$ for $3 \mathrm{~min}$. 3.3. Centrifuge at $1,600 \times \mathrm{g}$ for $10 \mathrm{~min}$ and transfer aqueous top layer along with the fuzzy interface layer to a fresh $15 \mathrm{ml}$ tube.

3.4. Repeat steps 3.1.2 and 3.1.3 3x, but changing the rotation times to $3 \mathrm{~min}, 4 \mathrm{~min}$, and 6 min, respectively. On the final repeat, avoid transferring any of the "fuzzy" interface layer. 
3.5. After the 4th extraction, add $70 \mu \mathrm{l}$ of $3 \mathrm{M} \mathrm{NaAc}, \mathrm{pH} 5.2$ per $1 \mathrm{ml}$ of aqueous extract and $2 \mathrm{ml}$ of chloroform:is oamyl alcohol (24:1). Rotate tube at $22 \mathrm{rpm}$ for $12 \mathrm{~min}$.

3.6. Centrifuge at $1,600 \times \mathrm{g}$ for $10 \mathrm{~min}$ and transfer aqueous top layer to a fresh $15 \mathrm{ml}$ tube.

3.7. Precipitate DNA by adding 2 volumes of absolute ethanol and gently rotating the tube on its side with gentle rocking.

3.8. Collect DNA by spooling onto the tip of a heat-sealed pasture pipette. Rinse DNA by swirling the pipet tip in $70 \%$ ethanol and air dry for $5 \mathrm{~min}$.

3.9. After extraction, dissolve DNA precipitate in 40 - $100 \mu \mathrm{l}$ Tris-EDTA buffer, $\mathrm{pH} 8$. Store at $4{ }^{\circ} \mathrm{C}$. Allow the DNA to dissolve at $4{ }^{\circ} \mathrm{C}$ for a minimum of 2 days before proceeding to lac $Z$ mutation assay. If solubility issues are encountered, DNA can be further dissolved at $65{ }^{\circ} \mathrm{C}$ for $15 \mathrm{~min}$ before use. Determine the concentration of the DNA with a spectrophotometre at A260 and ensure that the concentration of the dissolved DNA is between 200-2,000 $\mathrm{ng} / \mu \mathrm{l}$.

\section{Isolation and Digestion of Germ Cells from Seminiferous Tubules}

4.1. If frozen, defrost testis on ice (approximately $1 \mathrm{hr}$ ). Transfer testis to a ground glass plate.

4.2. Hold an end of the testis with a pair of forceps. At the other end of the testis, puncture a hole in the epithelial capsule using another pair of forceps or a pair of dissection scissors (Figure A5A). Squeeze the seminiferous tubules through the puncture and discard the epithelial capsule (Figure A5B).

4.3. Add $500 \mu \mathrm{l}$ of room temperature D-PBS to the decapsulated seminiferous tubules. 
4.4. Angle a tissue roller (silicone rubber tightly fitted over a freely rotating $5 \mathrm{~mm}$ diameter stainless steel tube, or similar apparatus) so that the end is in contact with the plate at an approximate angle of 5-10 (Figure A5C). Without applying any pressure, gently move the roller back and forth across the tubules until they are flattened and the DPBS becomes cloudy with released cells (approximately 5-10x).

4.5. Add another $500 \mu \mathrm{l}$ of D-PBS over the tubules and gently roll over the tubules a few additional times.

4.6. Transfer the cell suspension to a $1.5 \mathrm{ml}$ microfuge tube while minimizing the amount of detached tubules being transferred (Figure A5D).

4.7. Repeat steps 4.5-4.6 to collect more cells if necessary.

4.8. Allow $1-2$ min for any accidentally collected tubules to settle to the bottom of the tube. Transfer the D-PBS to a fresh $1.5 \mathrm{ml}$ tube leaving behind the settled tubules (approximately $100 \mu \mathrm{l}$ of D-PBS). A small aliquot of this suspension can be checked under a microscope (phase contrast) to assess the composition of the cell population. 4.9. Spin down the cells at $11,000 \times \mathrm{g}$ for $30 \mathrm{sec}$. Carefully decant the supernatant without disturbing the pellet. The cell pellet can be frozen at $-80^{\circ} \mathrm{C}$ at this point if needed.

4.10. Thaw cells if required. Transfer to a $15 \mathrm{ml}$ polypropylene tube and re-suspend cells in $5 \mathrm{ml}$ of lysis buffer (10 mM Tris pH 7.6, $10 \mathrm{mM}$ EDTA, $100 \mathrm{mM} \mathrm{NaCl}, 1 \mathrm{mg} / \mathrm{ml}$ proteinase $\mathrm{K}, 1 \% \mathrm{SDS}$ ). Digest overnight in incubator with rotation at $37^{\circ} \mathrm{C}$. 4.11. Proceed to phenol/chloroform extraction. 
NOTE: A less aggressive extraction is used to isolate seminiferous tubules germ cell DNA, since these cell types have not yet progressed through nuclear condensation.

5.1. Add $5 \mathrm{ml}$ of phenol:chloroform mixture (1:1) to overnight seminiferous tubules cell digestion. Rotate tube at $22 \mathrm{rpm}$ for $20 \mathrm{~min}$.

5.2. Centrifuge at $1,600 \times \mathrm{g}$ for $10 \mathrm{~min}$ and transfer aqueous top layer, while avoiding the "fuzzy" interface layer, to a fresh $15 \mathrm{ml}$ tube.

5.3. Add $100 \mu \mathrm{l}$ of $5 \mathrm{M} \mathrm{NaCl}$ per $5 \mathrm{ml}$ aqueous extract (usually $5 \mathrm{ml}$ is recovered)

5.4. Add $5 \mathrm{ml}$ of chloroform:isoamylalcohol (24:1). Rotate tube at $22 \mathrm{rpm}$ for $12 \mathrm{~min}$. 5.5. Centrifuge at $1,600 \times \mathrm{g}$ for $10 \mathrm{~min}$ and transfer aqueous top layer to a fresh $15 \mathrm{ml}$ tube.

5.6. Precipitate DNA by adding 2 volumes of absolute ethanol and gently rotating and inverting tubes.

5.7. Collect DNA by spooling onto the tip of a sealed off pasture pipette. Rinse DNA by swirling the pipet tip in $70 \%$ ethanol and air dry for $5 \mathrm{~min}$.

5.8. Dissolve DNA in 40-100 $\mu$ Tris-EDTA buffer, $\mathrm{pH} 8$. Store at $4{ }^{\circ} \mathrm{C}$. Allow DNA to dissolve at $4{ }^{\circ} \mathrm{C}$ for a minimum of 2 days before proceeding to lacZ mutation assay (see step 3.9). If solubility issues are encountered, DNA can be further dissolved at $65{ }^{\circ} \mathrm{C}$ for 15 min before use. Determine the concentration of the DNA with a spectrophotometre at A260 and ensure that the concentration is between 200 and 2,000 $\mathrm{ng} / \mu \mathrm{l}$.

\section{6. lacZ mutation Assay}


Bacterial or fungal contamination can interfere with packaging efficiency, as well as plaque growth and scoring. It is therefore critical to perform the lacZassay using the appropriate aseptic measures to prevent contamination of the packaging reaction, host culture, and growth media.

\subsection{Day Before Assay: Prepare Bottom Agar and Overnight Culture}

6.1.1. Eight plates ( 4 plates to score mutants, 4 plates to count titer) containing 8 $\mathrm{ml}$ bottom agar each are required per sample (i.e., $64 \mathrm{ml}$ per sample). The bottom agar is identical for both the mutant and titer count plates. Prepare sufficient bottom agar for the number of samples being processed. Aseptically pour into 90 $\mathrm{mm}$ Petri dishes ( $8 \mathrm{ml}$ per dish) and allow agar to solidify. NOTE: Bottom agar plates can be prepared up to 1 week in advance.

6.1.2. In a $50 \mathrm{ml}$ tube add $10 \mathrm{ml} \mathrm{LB}$ broth, $100 \mu \mathrm{l}$ of $20 \%$ maltose solution, $25 \mu \mathrm{l}$ ampicillin $(20 \mathrm{mg} / \mathrm{ml})$ and $20 \mu \mathrm{l}$ kanamycin $(5 \mathrm{mg} / \mathrm{ml})$. Inoculate with $E$. coli (lacZ-/galE-) (Gossen et al., 1992) and grow overnight at $37{ }^{\circ} \mathrm{C}$ with shaking at $240 \mathrm{rpm}$.

\subsubsection{Day 1: Sub-culture Cells}

6.2.1.1. Sub-culture cells by preparing a 1:100 dilution of the overnight culture in fresh LB (no antibiotics). A volume of $8 \mathrm{ml}$ of subculture is needed per sample. Incubate at $37{ }^{\circ} \mathrm{C}$ with shaking at $240 \mathrm{rpm}$ for about $3.5 \mathrm{hr}$ until $\mathrm{OD} 600=1$.

6.2.1.2. When OD600 $=1$, divide cell suspension evenly into $50 \mathrm{ml}$ tubes and centrifuge at $1,300 \times \mathrm{g}$ at $15^{\circ} \mathrm{C}$ for $10 \mathrm{~min}$. Remove supernatant and re-suspend 
cells in half of the original volume (i.e., $4 \mathrm{ml}$ per sample) of LB containing 10 $\mathrm{mM}$ MgSO4. Put cells aside until needed [step 6.2.4.3].

\subsubsection{Day 1: Packaging DNA in Lambda Phage Particles}

6.2.2.1. Warm up a water bath to $30^{\circ} \mathrm{C}$.

6.2.2.2. Using a wide-bore $10 \mu \mathrm{l}$ pipette tip, transfer $4 \mu \mathrm{l}$ DNA to a $1.5 \mathrm{ml}$ tube.

NOTE: This step can be performed a day or more in advance to reduce preparation time.

6.2.2.3. Warm up the first tube (red) from a phage packaging extract kit (1 tube for every 2 samples). Briefly spin to collect extract at bottom of tube.

6.2.2.4. Transfer $4.8 \mu$ of packaging extract from the first tube to the DNA sample and mix by gentle stirring with the pipette tip. Briefly spin down samples in tubes. Incubate for $1.5 \mathrm{hr}$ in $30^{\circ} \mathrm{C}$ water bath.

6.2.2.5. Warm up the second (blue) packaging extract tube ( 1 tube for $\sim 15$ samples). Briefly spin to collect extract at bottom of tube.

6.2.2.6. Transfer $4.8 \mu \mathrm{l}$ of packaging extract from the second tube to the DNA sample and mix by gentle stirring. Briefly spin down samples in tubes. Incubate for an additional $1.5 \mathrm{hr}$ in $30^{\circ} \mathrm{C}$ water bath.

6.2.2.7. Re-suspend packaged phage particles in $500 \mu \mathrm{l}$ SM buffer and mix by rotating for $30 \mathrm{~min}$ at $20 \mathrm{rpm}$.

6.2.2.8. After rotating, briefly vortex samples and centrifuge at $11,000 \times \mathrm{g}$ for 30 sec to collect samples at bottom of tubes. Phage particles are ready for infection [step 6.2.4].

\subsubsection{Day 1: Prepare Top Agar}


6.2.3.1. Prepare separate top agar for the titer plates and mutant selection plates. Each sample requires 4 titer plates, and 4 mutant plates. Each plate requires $8 \mathrm{ml}$ top agar. Add the selective agent P-Gal to the mutant selection top agar only. Prepare both top agars in advance (day of assay) and maintain at $50^{\circ} \mathrm{C}$ before adding $\mathrm{MgSO}_{4}$ to both top agars, and P-Gal to mutant selection agar.

\subsubsection{Day 1: Infecting Cells with Packaged Phage and Plating}

6.2.4.1. Label $250 \mathrm{ml}$ tubes per sample: 1 "mutant" tube per sample and 1 "titer" tube per sample

6.2.4.2. Label 8 agar plates per sample: 4 "mutant" plates per sample and 4 "titer" plates per sample

6.2.4.3. Aliquot $2 \mathrm{ml}$ of resuspended cells [from step 6.2.1.2] to each tube.

6.2.4.4. Add $500 \mu \mathrm{l}$ of packaged phage particles [from step 6.2.2.8] to "mutant"

$50 \mathrm{ml}$ tube (containing cells). Gently mix and allow phage particles to infect cells for $30 \mathrm{~min}$ at room temperature.

6.2.4.5. After $30 \mathrm{~min}$, briefly vortex infected cells and transfer $15 \mu \mathrm{l}$ of infected cells to the appropriate $50 \mathrm{ml}$ "titer" tube (containing cells).

6.2.4.6. To plate titer sample, add $30 \mathrm{ml}$ of warm $\left(50^{\circ} \mathrm{C}\right)$ "titer" top agar (NOT containing P-Gal) to "titer" $50 \mathrm{ml}$ tube. Immediately distribute agar/cell mixture among the 4 "titer" plates ( $\sim 8 \mathrm{ml}$ per plate). Work quickly so that the top agar does not cool in the pipettes, and try not to introduce air bubbles.

6.2.4.7. Plate the "mutant" samples next. Ensure P-Gal is added to "mutant selection" top agar. Add $30 \mathrm{ml}$ of warm "mutant selection" agar (containing P- 
Gal) to "mutant" $50 \mathrm{ml}$ tube. Immediately distribute agar/cell mixture among the 4 "mutant" plates ( $\sim 8 \mathrm{ml}$ per plate)

6.2.4.8. Allow plates to solidify $(\sim 15 \mathrm{~min})$ then invert and incubate at $37^{\circ} \mathrm{C}$ overnight.

\subsection{Day 2: Counting Plaques}

6.3.1. After overnight incubation, count the number of plaques on the mutant and titer plates. For large numbers of plaques, count only a portion of the plate to estimate the total count (e.g., often $1 / 4$ of the titer plate will have between 100-200 plaques). A minimum of 100 plaques should be counted per plate when counting a portion of the plate.

6.3.2. Calculate the number of plaque forming units (PFUs) per $\mu$ l of cells. This is done by dividing the number of plaques on the "titer" plates by the volume of cells plated $(15 \mu \mathrm{l})$.

6.3.3. Use the number of PFUs/ $\mu$ l to estimate the total number of PFUs in the total volume of infected cells plated on the "mutant" plates $(\mathrm{PFUs} / \mu \mathrm{l} *[2 \mathrm{ml}$ cells $+0.5 \mathrm{ml}$ packaged phage particles $-15 \mu \mathrm{l}$ for titer plates]).

6.3.4. Estimate the MF by dividing the total number of mutant plaques counted on the 4 "mutant" plates by the estimated total number of PFUs in the total volume of infected cells determined from the "titer" plates.

6.4. When the spontaneous lacZ MF is in the order of $3 \times 10^{-5}$ in the control group, as it for MutaMouse germ cells, the OECD guideline recommends a minimum of 125,000 to 300,000 non-mutant PFUs per animal be screened for mutation in order to obtain a reliable baseline signal. Other transgenic models may have lower spontaneous $\mathrm{MF}$, in 
which case a greater number of PFUs would be required. A statistical power test can be performed in order to determine the minimum number of PFUs and animals required to obtain the desired resolution. Data from multiple replicates may be pooled to satisfy this minimum PFU requirement, provided they do not produce significantly different mutant frequencies.

\section{Statistics}

The experimental unit for the analysis is the mouse. Data produced from this assay are generally not normally distributed. As such, select the statistical method for analysis based on the distribution characteristic of the data.

NOTE: Standard parametric analyses (e.g., ANOVA) may be employed if appropriate data transformation is applied to equalize the variance of the response across the range of observation. Poisson or binomial regression analyses are often more appropriate. Nonparametric analysis may also be employed. We routinely employ Poisson regression using the generalized linear model procedure (i.e., Proc GENMOD) in SAS v.9.2 (SAS Institute, Cary, NC) as described by Lemieux et al. (2011).

\section{A.5 Representative Results}

With an average plaque count of 200,000 plaques per animal, we typically observe a mean background MF of approximately $2.8 \times 10^{-5}$ in male germ cells with a standard deviation of $1.7 \times 10^{-5}$ in our control groups (based on data from 8 independent experiments). With this plaque count, background level, and variance, dose groups with $n$ $=5$ animals each are sufficient to detect a 2 fold increase in MF with power $>0.8$. 
Results are typically reported in tabular or graphical formats. Table A2 and Figure A6 show representative results from cauda sperm of MutaMouse males ( $n=5$ per group) exposed to a single acute oral dose of $0,25,50$, and $100 \mathrm{mg} / \mathrm{kg}$ ENU followed by a 70 day sampling time. This 70 day period permits the measurement of mutational events that occurred in sperm that were spermatogonial stem cells at the time of exposure (Figure A3). Typical plaque densities on mutant and titer plates are shown in Figure A7. As depicted in Table A2 and Figure A6, acute ENU exposure induced a significant dosedependent increase in the MF of spermatogonial stem cells. The low dose induced a significant 2.6 fold increase over controls, which had a baseline MF of $2.6 \times 10^{-5}$. Maximum induction occurred in the high dose, which elicited a 4.4 fold increase over controls.

An example of this assay performed on seminiferous tubule germ cells can be found in Douglas et al. (1995), where MF was determined in tubule germ cells at various time intervals following a 5 day repeat intraperitoneal injection of $50 \mathrm{mg} / \mathrm{kg}$ ENU. In that study, mutant frequencies in seminiferous cells increased up to 15 days after treatment and remained constant thereafter.

\section{A.6 Discussion}

Compared to traditional methods, the TGR germ cell mutation assay provides a faster, more economical, and more sensitive means of quantifying induced in vivo germ cell mutations. By assessing transgene MF directly in sperm, as opposed to offspring, the number of animals, time and resources required to assess the germline mutagenicity of any single compound is significantly reduced. In terms of sensitivity, we were able to 
detect a significant 2.6 fold increase in spermatogonia stem cell MF following exposure to $25 \mathrm{mg} / \mathrm{kg}$ ENU using only 5 animals per dose group. In contrast, the SLT was unable to detect any significant change in MF at this same dose using $>3,000$ mice in the exposed group and $>500,000$ control mice (Russell et al., 1982).

In addition to its suitability for both mechanistic and regulatory investigations, this method provides an opportunity for comparative studies between somatic and germ line mutation rates. Recent evidence suggests that for some agents germ cell mutations may be induced at lower concentration than required for somatic mutation. For example, prolonged exposure to N-hydroxymethylacrylamide, a metabolite of the food carcinogen acrylamide (Tareke et al., 2000), increases the frequency of dominant lethal germ cell mutations in mice without affecting the micronucleus frequency in red blood cells, a traditional measure of somatic cell cytogenetic damage (Witt et al., 2003). Additionally, exposure of mice to both mainstream and sidestream tobacco smoke causes elevated mutation frequencies at tandem repeat DNA loci in sperm at doses that do not increase blood micronucleus frequency (Marchetti et al., 2011). These findings challenge the assumption that somatic genotoxicity testing is always protective of the germline, and reinforces the demand for a more efficient and cost-effective means to quantify germ cell mutation frequency. However, the evidence for preferential germ cell mutagens is still weak, largely due to the lack of available data for comparing mutation rates in somatic and germ cell tissues. The TGR mutation assay allows parallel testing and comparison of induced mutation rates in multiple tissues using the same transgene. Thus, comparative mutation testing using the TGR assay would help fill data gaps surrounding the possibility of preferential germ cell effects. 
Simultaneous assessment of somatic and germ cell mutation for regulatory testing would also improve efficiency by reducing the number of animals required. The OECD guideline for somatic mutation recommends a 28 day administration time, followed by a 3 day sampling time $(28+3)$. Analysis of cauda sperm may offer poor sensitivity at this time-point, since it targets cells exposed mostly during the spermatocyte and spermatid phases of spermatogenesis (Figure A3). Cells in these phases do not synthesize DNA and progressively lose their capacity for DNA repair (Marchetti and Wyrobek, 2008). Furthermore, sampling cauda sperm at this time-point would fail to detect mutations occurring in spermatogonia and stem cells. Thus, for integration into the $28+3$ design, the OECD guideline recommends collecting germ cells from the seminiferous tubules. This mixed population contains cells derived from DNA synthesis and repair proficient cell types, including stem cells, and are exposed across the majority of spermatogenic phases. However, due to the mixed nature of these cells, seminiferous tubules cell analysis does not provide phase-specific information. Furthermore, there is concern that the presence of non-target cells can influence the observed MF (e.g. false positive germ cell mutagen calls due to contamination of mutated somatic cells, or dilution of a mutated germ cell signal from DNA repair-deficient germ cells). Currently there is insufficient data to conclude whether results from seminiferous tubules cells at $28+3$ offer the same sensitivity and specificity as cauda sperm at later time-points. Our laboratory is currently comparing induced MFs in seminiferous tubules cells and cauda sperm collected after various sampling times to address this point. We note that the OECD guideline suggests an alternative sampling time of 28 days for slowly dividing tissues such as the liver that may also be suitable for germ cell analysis. Nevertheless, available data is still 
insufficient and we are currently unable to recommend a single experimental design for the simultaneous analysis of somatic cells and germ cells using the TGR mutation assay for regulatory testing.

One characteristic of this assay that should be noted is that mutational events are assessed on a non-murine transgene. However, there is ample evidence to suggest that the transgene responds to environmental mutagens in a similar fashion to endogenous genes (Lambert et al., 2005). In addition, because the precise origins of independent mutational events are difficult to resolve, results are generally reported as a mutant frequency (in contrast to a mutation frequency). The actual mutation frequency can be resolved if results are corrected for clonal expansion (i.e. the division and multiplication of a single mutated cell that can contribute to the observed frequency of transgene mutants) by DNA sequencing. Sequencing of the mutant transgenes may be performed to characterize the lac $Z$ mutations, and identify mutants that may be derived from clonal expansion events, although this adds significantly to the time and cost of the analysis. In addition to the lac $Z$ gene, the $\lambda$ gt 10 transgenic vector harbours an alternative temperature-dependent mutation-reporter gene: a variant of the $\lambda$ cII gene, which is shorter (294 bp vs the 3096 bp lacZ, Figure A1) and easier to sequence (Swiger et al., 1999). Sequencing also permits the analysis of the induced mutation spectrum, providing insight into the mutational mechanism of the compound in question. An extreme example of clonal expansion is the occurrence of a "jackpot" mutation (i.e., transgene mutations at a very early stage of an organ's development that contribute to a dramatically elevated MF, sometimes hundreds to thousands of times greater than background). Animals or tissues with a "jackpot" mutation should be removed from the analysis. 
The assay that we have described is broadly applicable to other TGR models such as the BigBlue mouse and rat, and the lacZ plasmid mouse, all of which harbour similar mutation reporter vectors (reviewed in (Lambert et al., 2005)). The vast majority of germ cell studies conducted to date that employ similar methods have focused almost exclusively on well characterized mutagens such as ENU and radiation (reviewed in (Singer et al., 2006)). It is expected that with the recent release of the OECD test guideline for the TGR assay, this assay will be increasingly popular for chemical screening and regulatory evaluation. Incorporation of the TGR germ cell mutation assay into a regulatory testing battery will fill the existing gap by permitting efficient assessment of mutation induction in germ cells (Singer and Yauk, 2010). Moreover, this assay can be used to measure MF in virtually any tissue, providing a suitable means for comparing the relative sensitivities of somatic cells and germ cells to the induction of mutations by environmental agents at identical genetic endpoints. 


\section{A.7 Materials}

\begin{tabular}{|c|c|c|c|}
\hline Name & Company & $\begin{array}{l}\text { Catalog } \\
\text { Number }\end{array}$ & Comments \\
\hline MutaMouse & Covance & - & \\
\hline E. coli (lacZ-/galE-) & Covance & - & $\begin{array}{c}\text { See reference (Gossen et al., } \\
\text { 1992) in manuscript }\end{array}$ \\
\hline Chloroform & Caledon & $3001-2-40$ & \\
\hline $\begin{array}{l}\text { Dulbecco's phosphate- } \\
\text { buffered saline (D-PBS) }\end{array}$ & Gibco & $14190-250$ & \\
\hline $\begin{array}{l}\text { Ethylenediaminetetraacetic } \\
\text { acid (EDTA), } 0.5 \mathrm{M}, \mathrm{pH} 8\end{array}$ & Sigma-Aldrich & $\begin{array}{c}03690 \\
\text { FLUKA }\end{array}$ & \\
\hline Is oamyl alc ohol & Caledon & $2 / 10 / 7900$ & \\
\hline Lennon LB broth base & Invitrogen & $12780-029$ & \\
\hline Stainless steel mesh filter & Sigma-Aldrich & S3770 & \\
\hline $\begin{array}{c}\text { Transpack packaging } \\
\text { extract }\end{array}$ & $\begin{array}{c}\text { Agilent } \\
\text { Technologies }\end{array}$ & 200220 & \\
\hline$\beta$-mercaptoethanol & Sigma-Aldrich & M3148 & \\
\hline $\begin{array}{l}\text { Ethyl alcohol, anhydrous } \\
\text { (EtOH) }\end{array}$ & $\begin{array}{l}\text { Commercial } \\
\text { Alcohols }\end{array}$ & - & \\
\hline Ampicilin & Gibco & $11593-027$ & prepare $20 \mathrm{mg} / \mathrm{ml}$ in $\mathrm{dH}_{2} \mathrm{O}$ \\
\hline Kanamycin & Gibco & $11815-024$ & prepare $5 \mathrm{mg} / \mathrm{ml}$ in $\mathrm{dH}_{2} \mathrm{O}$ \\
\hline Phenol & Invitrogen & $15509-097$ & $\begin{array}{l}\text { Saturate in } 0.1 \mathrm{M} \text { Tris- } \mathrm{HCl} \text { as } \\
\text { per manufacturers direction }\end{array}$ \\
\hline $\begin{array}{c}\text { Phenyl- } \beta-\mathrm{D}- \\
\text { galactopyranoside (P-Gal) }\end{array}$ & Sigma-Aldrich & P6501 & $\begin{array}{l}\text { dissolve } 3 \mathrm{~g} \text { per } 10 \mathrm{ml} \text { of } \\
\text { dimethylformamide }\end{array}$ \\
\hline Proteinase K & Invitrogen & $25530-031$ & $\begin{array}{l}\text { prepare } 60 \mathrm{mg} / \mathrm{ml} \text { solution } \\
\mathrm{dH}_{2} \mathrm{O} \text { just before use, } 20 \mu \mathrm{l} \\
\text { per sample }\end{array}$ \\
\hline Sodium acetate (NaAc) & $\begin{array}{c}\text { Fisher } \\
\text { Scientific }\end{array}$ & ВР333-500 & $\begin{array}{c}\text { prepare } 3 \mathrm{M} \text { solutution, } \mathrm{pH} \\
5.2\end{array}$ \\
\hline $\begin{array}{l}\text { Sodium dodecyl sulfate } \\
\text { (SDS) }\end{array}$ & Sigma-Aldrich & L4390 & prepare $10 \%$ solution in $\mathrm{dH}_{2} \mathrm{O}$ \\
\hline $1 \mathrm{M} \mathrm{MgSO}_{4}$ & & & 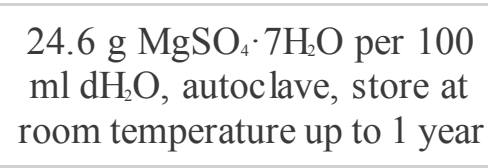 \\
\hline LB Broth & & & 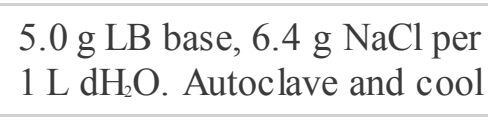 \\
\hline $\begin{array}{l}\text { Saline sodium citrate } \\
\text { (SSC) }\end{array}$ & & & $\begin{array}{l}150 \mathrm{mM} \mathrm{NaCl}, 15 \mathrm{mM} \\
\text { trisodium citrate, } \mathrm{pH} 7.0\end{array}$ \\
\hline
\end{tabular}




\begin{tabular}{|c|c|}
\hline SM Buffer & $\begin{array}{c}5.8 \mathrm{~g} \mathrm{NaCl}, 2.0 \mathrm{~g} \\
\mathrm{MgSO}_{4} \cdot 7 \mathrm{H}_{2} \mathrm{O}, 50 \mathrm{ml} 1 \mathrm{M} \\
\text { Tris- } \mathrm{HCl}(\mathrm{pH} 7.5), 5.0 \mathrm{ml} \text { of } \\
\text { gelatin }(2 \% \mathrm{w} / \mathrm{v}) \text {, per } 1 \mathrm{~L} \\
\mathrm{dH}_{2} \mathrm{O} \text {, autoclave, store at } \\
\text { room temperature up to } 1 \text { year }\end{array}$ \\
\hline TE buffer & $\begin{array}{c}10 \mathrm{mM} \text { Tris, } 1 \mathrm{mM} \text { EDTA, } \\
\text { pH } 8\end{array}$ \\
\hline
\end{tabular}




\section{A.8 Tables and Figures}

Table A.1 Cell types and phases of spermatogenesis that are targeted by the transgenic rodent mutation assay by various experimental designs.

\begin{tabular}{|c|c|c|c|c|}
\hline $\begin{array}{l}\text { Exposure } \\
\text { regimen }\end{array}$ & $\begin{array}{l}\text { Collected } \\
\text { tissue }\end{array}$ & $\begin{array}{c}\text { Collected cell } \\
\text { type (target cell) }\end{array}$ & $\begin{array}{l}\text { Phase of target } \\
\text { cell at the } \\
\text { beginning of } \\
\text { exposure }\end{array}$ & $\begin{array}{c}\text { Phases passed } \\
\text { during } \\
\text { exposure }\end{array}$ \\
\hline Acute +70 & Cauda & Mature sperm & Stem cell & Stem cell \\
\hline $14+21$ & Cauda & Mature sperm & Spermatogonia & Spermatid \\
\hline $14+35$ & Cauda & Mature sperm & Stem cell & Spermatocyte \\
\hline \multirow[t]{5}{*}{$28+3$} & Cauda & Mature sperm & Spermatocyte & $\begin{array}{l}\text { Spermatocyte } \\
\text { Spermatid } \\
\text { Mature sperm }\end{array}$ \\
\hline & Tubules & Stem cell & Stem cell & Stem cell \\
\hline & & Spermatogonia & Spermatogonia & Spermatogonia \\
\hline & & Spermatocyte & & Spermatocyte \\
\hline & & Spermatid & & Spermatid \\
\hline \multirow[t]{2}{*}{$28+49$} & Cauda & Mature sperm & Stem cel & Stem cell \\
\hline & Tubules & $\begin{array}{c}\text { Stem cell } \\
\text { Spermatogonia } \\
\text { Spermatocyte } \\
\text { Spermatid }\end{array}$ & Stem cell & Stem cell \\
\hline
\end{tabular}


Table A.2 The lac $Z$ mutant frequency in cauda sperm of transgenic male mice exposed acutely to ENU when they were spermatogonial stem cells (administration time $=1$ day, sampling time $=70$ days $)$.

\begin{tabular}{|c|c|c|c|c|c|c|c|}
\hline $\begin{array}{l}\text { Dose } \\
\text { group }\end{array}$ & $\underset{\#}{\text { Animal }}$ & $\begin{array}{c}\text { Mutant } \\
\text { PFU }\end{array}$ & $\begin{array}{l}\text { Total } \\
\text { PFU }\end{array}$ & $\begin{array}{c}\text { MF ( } \times \\
\left.10^{-5}\right)\end{array}$ & $\begin{array}{c}\text { Avg } \\
\text { MF ( } \times \\
\left.10^{-5}\right)\end{array}$ & $\begin{array}{l}\text { Fold } \\
\text { change }\end{array}$ & $p$-value \\
\hline \multirow[t]{5}{*}{ Control } & 1 & 5 & 180245 & 2.8 & 2.6 & 1 & - \\
\hline & 2 & 4 & 137835 & 2.9 & & & \\
\hline & 3 & 11 & 385672 & 2.9 & & & \\
\hline & 4 & 2 & 431396 & 0.5 & & & \\
\hline & 5 & 6 & 152413 & 3.9 & & & \\
\hline \multirow[t]{5}{*}{$\begin{array}{c}25 \\
\mathrm{mg} / \mathrm{kg}\end{array}$} & 6 & 17 & 162353 & 10.5 & 6.9 & 2.6 & 0.0002 \\
\hline & 7 & 14 & 150094 & 9.3 & & & \\
\hline & 8 & 4 & 154401 & 2.6 & & & \\
\hline & 9 & 9 & 154401 & 5.8 & & & \\
\hline & 10 & 12 & 196978 & 6.1 & & & \\
\hline \multirow[t]{5}{*}{$\begin{array}{c}50 \\
\mathrm{mg} / \mathrm{kg}\end{array}$} & 11 & 17 & 155727 & 10.9 & 9.3 & 3.6 & $<0.0001$ \\
\hline & 12 & 11 & 135847 & 8.1 & & & \\
\hline & 13 & 25 & 193499 & 12.9 & & & \\
\hline & 14 & 12 & 133859 & 9 & & & \\
\hline & 15 & 14 & 252807 & 5.5 & & & \\
\hline \multirow[t]{5}{*}{$\begin{array}{c}100 \\
\mathrm{mg} / \mathrm{kg}\end{array}$} & 16 & 26 & 170968 & 15.2 & 11.5 & 4.4 & $<0.0001$ \\
\hline & 17 & 28 & 234584 & 11.9 & & & \\
\hline & 18 & 10 & 145289 & 6.9 & & & \\
\hline & 19 & 35 & 292236 & 12 & & & \\
\hline & 20 & 22 & 190848 & 11.5 & & & \\
\hline
\end{tabular}




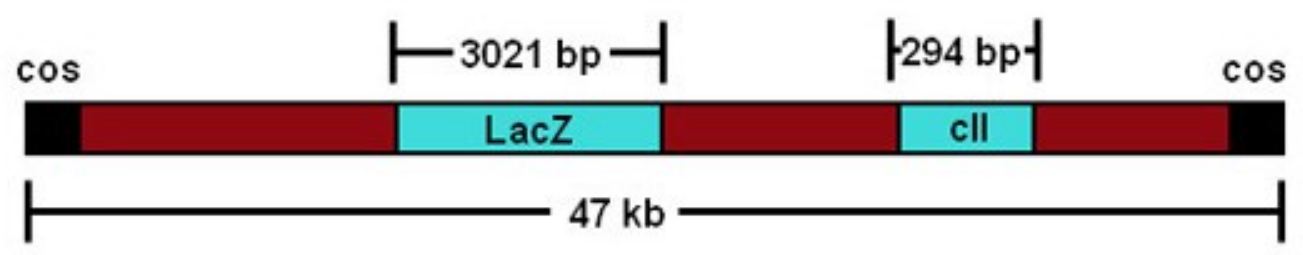

Figure A.1 A schematic representation of the $\lambda \mathrm{gt} 10$ phage construct. 


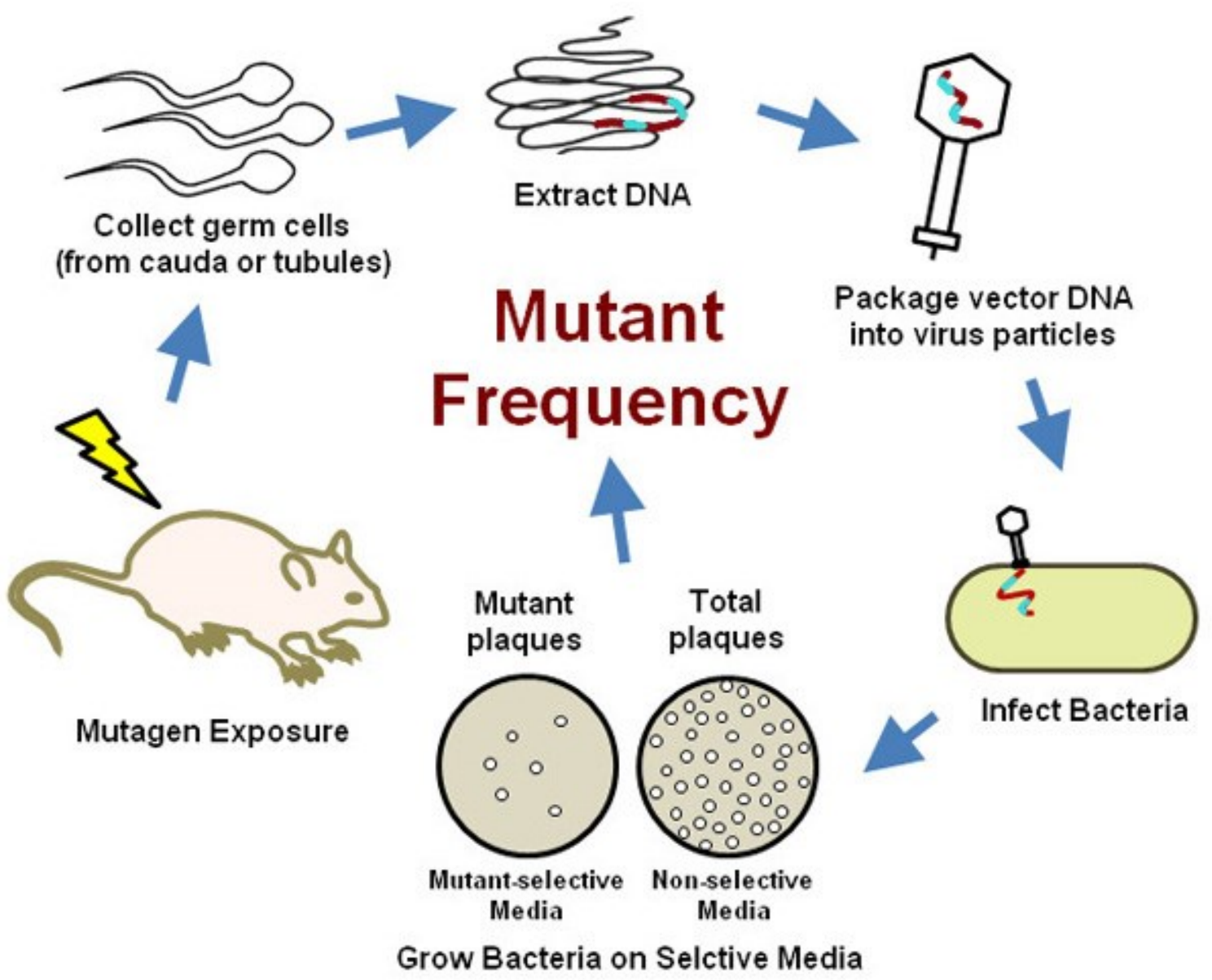

Figure A.2 An outline of the transgenic rodent germ cell mutation assay. 


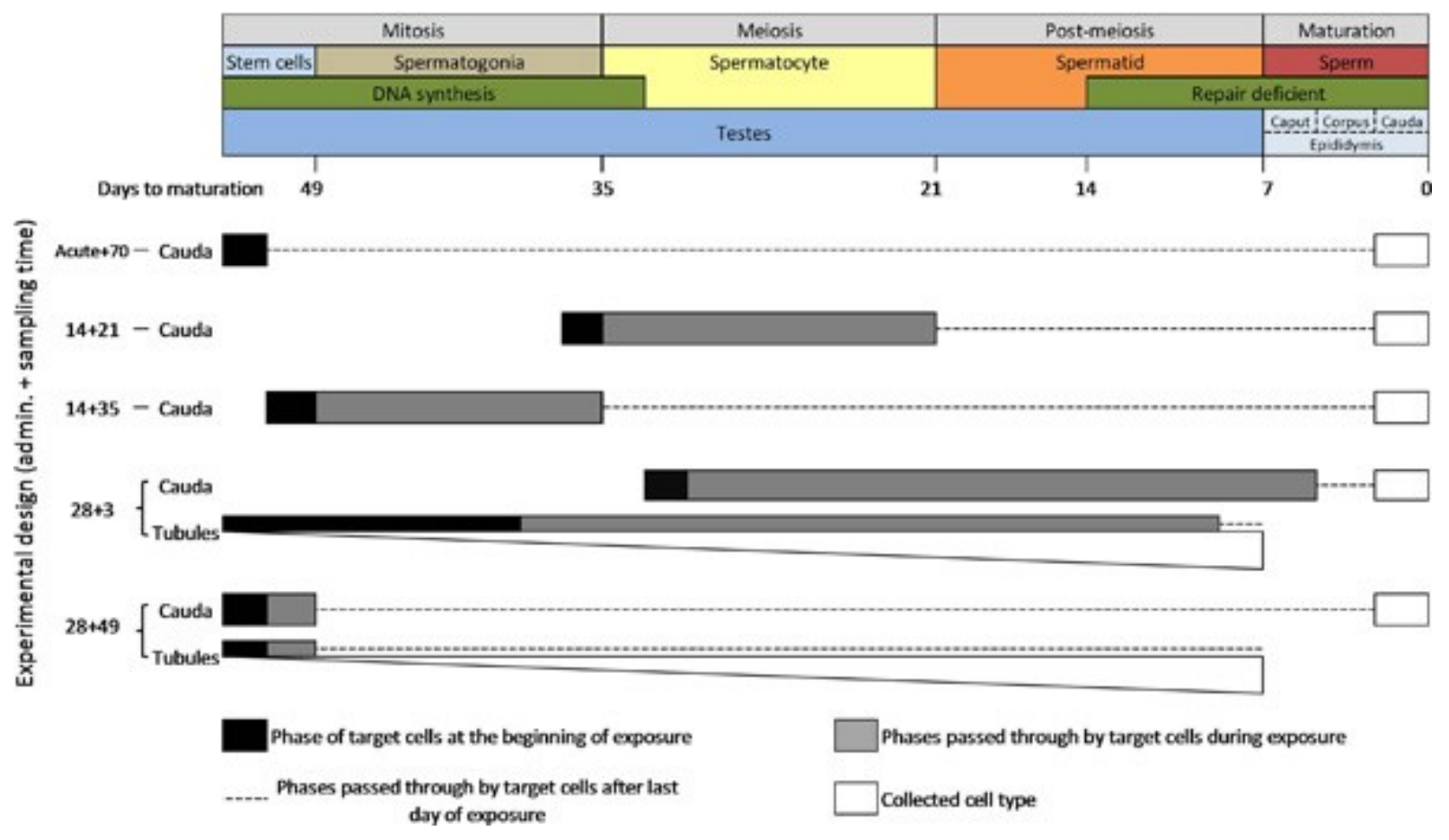

Figure A.3 A schematic diagram of spermatogenesis in the mouse and the cell types and phases that are targeted by the transgenic rodent mutation assay by various experimental designs. NOTE: The graduated white bars represent the relative proportion of cell types present in cell suspensions prepared from the seminiferous tubules (i.e. spermatids $>$ spermatocytes $>$ spermatogonia $>$ stem cells). 

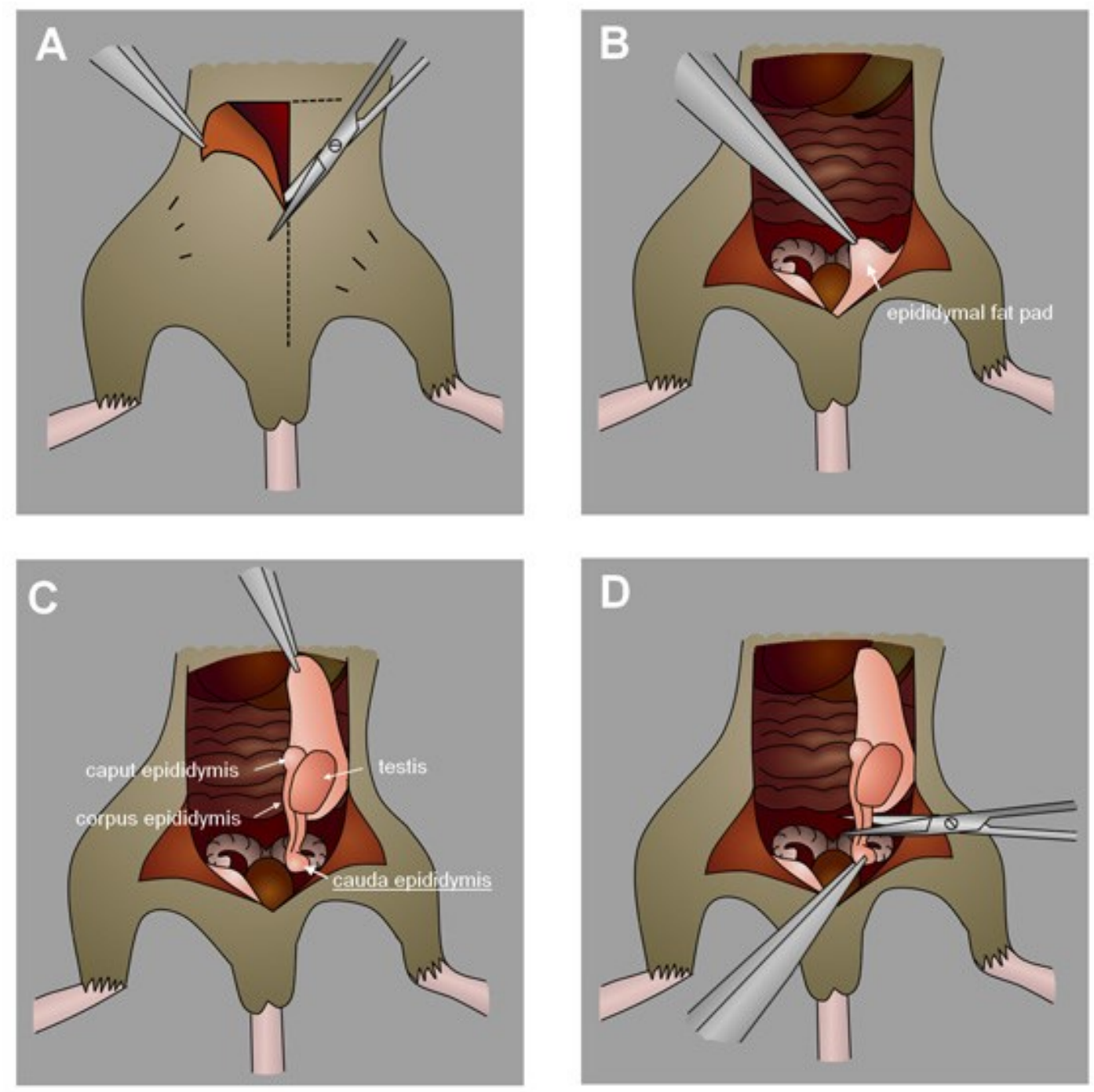

Figure A.4 Collection of the mouse cauda epididymis. A) Make an incision in the abdomen towards the scrotum. B) Identify the epididymal fat pad. C) Gently pull the fat pad to draw out the testes and epididymis. D) Locate and excise the cauda epididymis. 

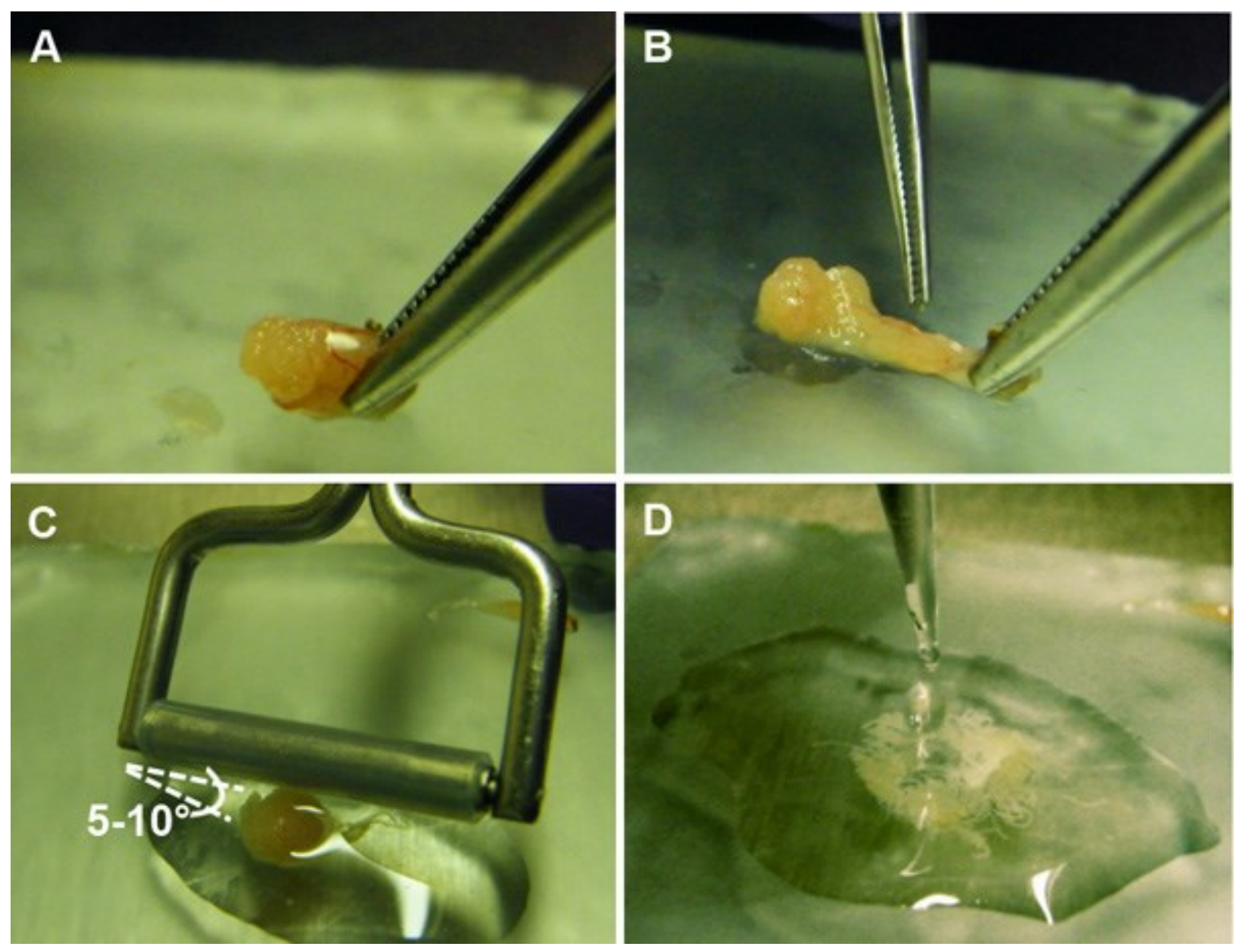

Figure A.5 Preparation of suspension of germ cells from the seminiferous tubules. A) An incision is made in the epithelial capsule of the testis, exposing the seminiferous tubules. B) The seminiferous tubules are squeezed out of the capsule. C) A tissue roller is gently passed over the tubules at a $5-10^{\circ}$ angle to release the germ cells contained within. D) The germ cell suspension is collected for further processing. 


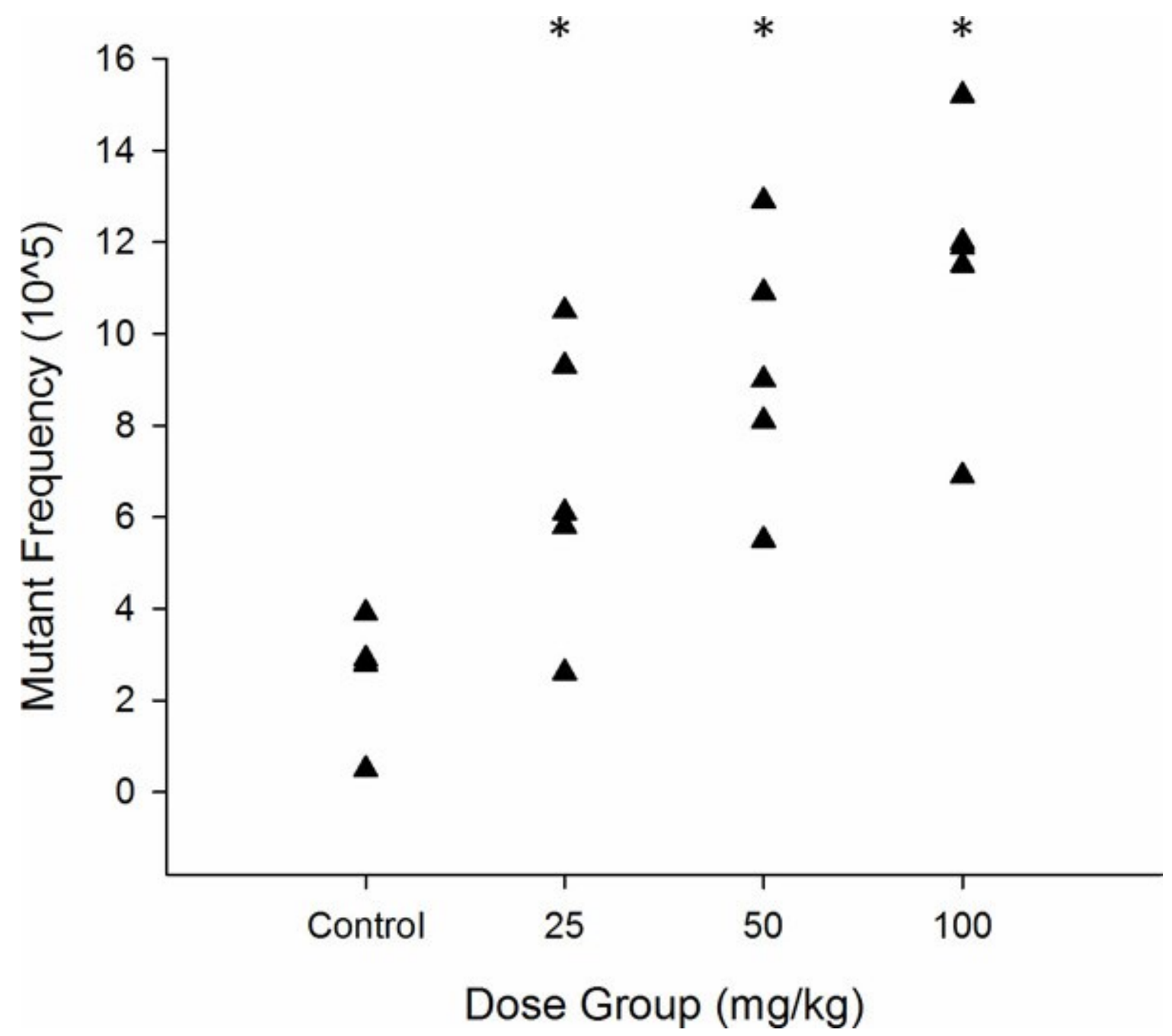

Figure A.6 Graphical representation of lacZ mutant frequency in MutaMouse sperm exposed acutely as stem cells to ENU $(n=5)$. Each triangle represents the MF of 1 animal. ${ }^{*} \mathrm{P}<0.05$ as determined by Poisson regression. 

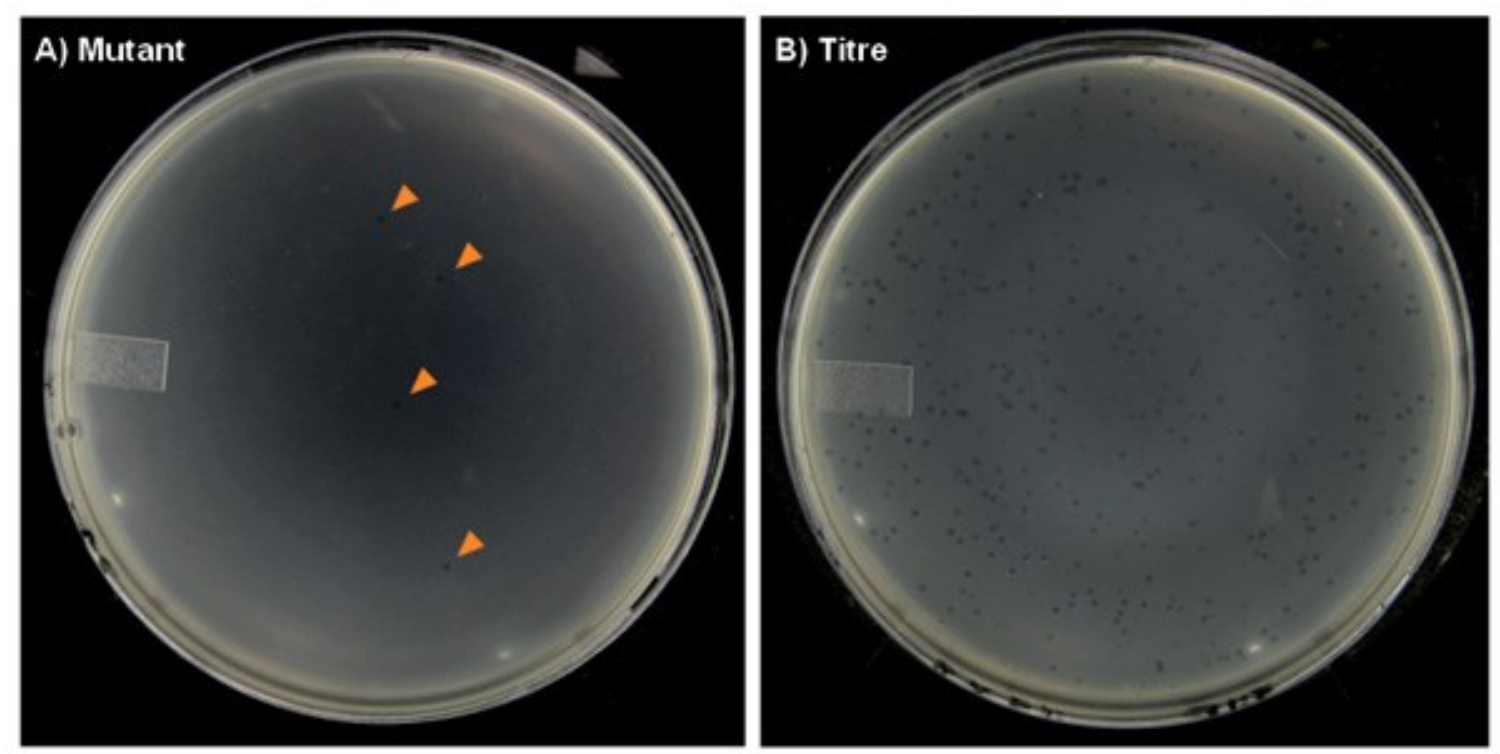

Figure A.7 Representative agar plates from the transgenic rodent mutation assay with plaques formed on a lawn of E. coli host bacteria. A) The "mutant" plates will have very few plaques (marked with arrows), especially in the control dose groups, which will occasionally have zero plaques on some plates. B) The "titer" plates can have hundreds of plaques. 


\section{Appendix B In Utero Exposure to Benzo[a]pyrene Increases Mutation Burden in the Soma and Sperm of Adult Mice}

Modified from: Meier, M.J., O’Brien, J.M., Beal, M.A., Allan, B., Yauk, C.L., Marchetti, F. Environmental Health Perspectives, DOI:10.1289/EHP211.

\section{B.1 Preamble}

In this manuscript, we examined the effects of in utero $\mathrm{BaP}$ exposure on developing embryos, completing the examination of mutations induced at all developmental stages (spermatogenesis, fertilization, embryo/fetal development).

\section{B.2 Abstract}

Mosaicism, the presence of genetically distinct cell populations within an organism, has emerged as an important contributor to disease. Mutational events occurring during embryonic development can cause mosaicism in any tissue, but the influence of environmental factors on levels of mosaicism is unclear. We investigated whether in utero exposure to the widespread environmental mutagen $\mathrm{BaP}$ impacts the burden and distribution of mutations in adult mice. We used the MutaMouse transgenic rodent model to quantify and characterize mutations in the offspring of pregnant mice exposed to $\mathrm{BaP}$ during postconception days 7 through 16 , covering the major period of organogenesis in mice. NGS was then used to determine the spectrum of mutations induced in adult mice that were exposed to BaP during fetal development. Mutation 
frequency was significantly increased in the bone marrow, liver, brain, and sperm of F1 males. Developing embryos accumulated more mutations and exhibited higher proportions of mosaicism than exposed adults, particularly in the brain. Decreased sperm count and motility revealed additional negative impacts on reproductive function of F1 males. We show that in utero exposure to environmental mutagens contributes to somatic and germline mosaicism, permanently impacting both the genetic health of the F1 and the population gene pool.

\section{B.3 Introduction}

Our understanding of human genetic disease is predicated on the idea that most mutations are inherited through the germline. However, mounting evidence suggests that disease-associated genetic changes also arise during embryonic development (Biesecker and Spinner, 2013; Erickson, 2010; Lupski, 2015; Lupski, 2013). These postzygotic events (which may include mutations, large-scale rearrangements, or aneuploidies) produce a varied distribution of altered genomes throughout the individual $-\mathbf{a}$ phenomenon known as mosaicism (De, 2011). Any cell type in the body can accumulate such mutations, including stem cells or primordial germ cell precursors, inducing permanent changes in the individual or their offspring (Campbell et al., 2015; Cohen et al., 2015; Rahbari et al., 2015). Recent genome-scale studies have revealed unexpected levels of mosaicism in seemingly normal tissues (Campbell et al., 2015; Erickson, 2010; Rahbari et al., 2015), and we may still be vastly underestimating the prevalence and health burden of low-level mosaicism (Campbell et al., 2014; Spinner and Conlin, 2014). 
Recent work by Rahbari et al. (2015) exemplifies the prevalence of mutations occurring early during development and their significant contribution to somatic and germline mosaicism in adults. They also provide compelling evidence that germline mutation rates are not constant throughout the lifetime of an organism, and that spontaneous mutation may be more likely during the expansion of male primordial germ cell precursors in embryogenesis than during other developmental stages or post-pubertal spermatogenesis. Although individual differences were observed in the prevalence of germline mosaicism, no studies since the pioneering work of Russell et al. (Russell and Russell, 1992; Russell, 1999; Russell et al., 1988) have investigated whether environmental factors during development alters the induction of mosaic mutations in the germline. This previous work demonstrated, using phenotypic markers in the SLT, that the perigametic interval was a significant source of spontaneous as well as chemical- or radiation-induced mosaic mutations. However, other stages of development, such as fetal growth, have remained uncharacterized with respect to the induction of mosaicism by environmental factors. Mutation assays in general measure both unique and clonally expanded mutations; however, the relative contribution of mosaicism to overall mutation burden is rarely considered in these assays. Moreover, the influence of mutagen exposure during critical developmental stages on the degree of tissue-wide mosaicism remains a significant gap in understanding in the field of genetic toxicology.

In utero exposure to toxicants can cause a range of deleterious health effects in adulthood [e.g., reproductive defects (Fowler et al., 2008; Mocarelli et al., 2011), increased cancer susceptibility (Autrup, 1993), impaired cardiac function (Buscariollo et al., 2014), and neurodegenerative disease (Modgil et al., 2014)]. However, the extent to 
which in utero exposure to environmental chemicals contributes to adult disorders resulting from the induction and distribution of mutations in developing tissues is unknown (Ritz et al., 2011). We used a transgenic rodent model combined with NGS to investigate the effects of in utero exposure to $\mathrm{BaP}$, a common environmental pollutant and human carcinogen produced from a variety of sources, on the burden and distribution of mutations in adults. This study presents the first evidence that fetal exposure of mice to a mutagenic chemical can directly result in an excess burden of mutations and increased mosaicism in both somatic tissues and germ cells of adult F1 mice.

\section{B.4 Methods}

\section{Animal Treatment}

The use of animals in these experiments was approved by the Health Canada Ottawa Animal Care Committee. Animals used in this study were humanely treated with regard to the alleviation of suffering following the guidelines of the Canadian Council on Animal Care. Both male and female MutaMouse mice were obtained from a colony maintained at Health Canada. Males were housed with up to 4 females and every morning females were checked for the presence of vaginal plug as indication of mating. Pregnant mice were dosed with 0, 10, 20, or $40 \mathrm{mg} / \mathrm{kg} / \mathrm{d}$ BaP (Sigma-Aldrich Canada Ltd), dissolved in olive oil (at a volume of $0.15 \mathrm{~mL}$ for $30 \mathrm{~g}$ body weight) and administered on postconception days 7 through 16 by oral gavage (with postconception day 1 indicated by the presence of a vaginal plug). Each pregnant female was housed individually. Pups were weaned at 3 weeks of age and euthanized 10 weeks after birth in accordance with Health Canada's ethical guidelines and tissues collected. The bone marrow, brain, liver, testis, and cauda epididymis (one per mouse) were flash frozen in 
liquid nitrogen immediately following necropsy, and stored at $-80{ }^{\circ} \mathrm{C}$ until DNA extraction was performed.

\section{lacZ Transgene Mutation Assay}

The lacZ TGR assay was performed as previously described, in a manner consistent with OECD Test Guideline 488 (O'Brien et al., 2014; OECD, 2011). DNA was isolated from tissues using pheno//chloroform extraction and packaged into lambda phage (Transpack packaging extract, Agilent Technologies). The packaged reporter constructs were subsequently plated on a lawn of E. coli (galE-) grown on LB media, and positive selection for mutant plaques was performed using P-Gal (Gossen et al., 1992). Mutant plaques were counted, and a subset of plaques were collected in MilliQ water (3 uL per plaque) for sequencing. The dose-response of MF was tested for significance with generalized linear modeling in R using a quasi-Poisson distribution (R Core Team, 2013) as well as dose-response modeling using both the R-package PROAST and BMDS (v. 2.6 from the US-EPA; http://www.epa.gov/ncea/bmds/).

\section{Computer Assisted Sperm Analysis}

Computer assisted sperm analysis (CASA) was performed on an IVOS instrument (Hamilton Thorne Research, USA) using sperm from 1 of the cauda epididymis taken from mice at the time of necropsy. Each cauda was minced into $2.5 \mathrm{~mL}$ of prewarmed M16 or M199 medium (Sigma, USA) for the $10 \mathrm{mg} / \mathrm{kg} / \mathrm{d} \mathrm{BaP}$ or the 20 and $40 \mathrm{mg} / \mathrm{kg} / \mathrm{d} /$ $\mathrm{BaP}$, respectively. After 3 minutes at $37^{\circ} \mathrm{C}$ in $5 \% \mathrm{CO} 2$, a $16 \mu \mathrm{L}$ of a $1: 4$ dilution of sperm was added into the 2 wells of a 2X-CEL $80 \mu \mathrm{m}$ deep chambered slide (Hamilton Thorne Research). At least 10 fields per chamber, automatically selected by the IVOS, 
were imaged with a $4 \mathrm{x}$ objective and analyzed with the IVOS Animal software v14. Setting for CASA analysis were: frame rate, $60 \mathrm{~Hz} ; 30$ frames acquired/samples; minimum contrast, 40; minimum cell size, 3 .

\section{Statistical Analysis}

The P-values for body weight, liver somatic index (LSI), testis somatic index (TSI), and sperm motility metrics were determined using an ANOVA followed by a Bonferroni post-hoc multiple comparison, relative to the control group. Dose-response data from the $l a c Z$ transgene assay was analyzed in $\mathrm{R}$ using the glm function. The number of mutant plaques were compared to dose, setting log (total plaques) as the offset and using the quasi-Poisson distribution family to account for over-dispersion. The resulting p-values were corrected for multiple comparisons using the Bonferroni method. A LRT was used to eliminate outliers between technical replicates (within animals), and subsequently between animals within dose groups. Dose response analysis for all tissues was done using all available models in BMDS v. 2.6, followed by selection of the best fit model using the Akaike information criterion (AIC).

\section{Ion Proton Seque ncing}

Pooled plaques collected from the TGR assay were subjected to PCR following heating at $95{ }^{\circ} \mathrm{C}$ and centrifugation to remove E. coli cellular debris. PCR was performed in duplicate technical replicates for each sample using NEB Phusion DNA Polymerase according to the manufacturer's directions. PCR amplified DNA was purified with the QIAquick PCR purification kit, and then used to create a fragmented DNA library via ligation to P1 adapter and barcoded A adapter, via the NEBNext Ion Kit. The resulting 
libraries were pooled in equimolar quantities after quantification on the Agilent Tapestation D1000. These libraries were used in template preparation on the Ion Chef robot, and sequenced using a P1 chip on the Ion Proton instrument.

\section{Computational Analyses}

Mutations were called as previously described (Beal et al., 2015a). Reads were aligned to the $l a c Z$ sequence from MutaMouse using bowtie 2 with the "very sensitive local" option enabled. Pileups were created using samtools mpileup v 0.1 .19 and mutations were called with a customized $\mathrm{R}$ script (available online at http://usegalaxy.org). Based on the pileups, a proportion of each base call at each position of the lac $Z$ gene was determined for each library (each of which constitutes a different sample of pooled plaques, performed in technical replicates). Putative mutations were filtered based on the criteria that they must be present above the pooled mutation calling threshold (1/\# plaques sequenced) in both technical replicate DNA libraries, and the background rate of the mutation must be less than $2 \%$. For calculating clonal expansion, the count of each mutant was determined by multiplying the percentage of reads containing each unique mutation by the number of plaques sequenced for that animal; since we observed high variability among low numbers of mutant counts, we applied a LOD/linear model to correct the counts (Beal et al., 2015a). This method relies on the conservative assumption that any 2 reads derived from the same biological sample, and possessing an identical mutation within a single tissue, are likely to be the product of clonal expansion rather than independent events. Clonally expanded mutants were then considered to be those with a corrected count greater than 1, while mutants with a 
corrected count of 1 were considered as singletons. Mutation spectra were generated using the counts for each unique mutation type.

\section{B.5 Results}

\section{Mutations in Somatic Tissues After in utero BaP Exposure}

To determine the genetic effects of transplacental exposure to an environmental mutagen, $\mathrm{BaP}$ was administered in olive oil at $0,10,20$, or $40 \mathrm{mg} / \mathrm{kg} /$ day by oral gavage to pregnant MutaMouse females on postconception days 7-16, comprising the period of organogenesis in mice (Mitiku and Baker, 2007). These doses were chosen based on previous literature indicating that such an exposure affected the fertility of the F1 generation (MacKenzie and Angevine, 1981). Neither litter size at birth nor body weight

of the F1 generation (at 10 weeks of age) were significantly affected by BaP administered during pregnancy, indicating that $\mathrm{BaP}$ exposure in the dam did not cause embryo loss or significantly impact postnatal development.

We then measured mutations in 3 somatic tissues derived from each germ layer (ectoderm: brain; mesoderm: bone marrow; endoderm: liver) using the recoverable lacZ reporter transgene within the MutaMouse genome (Lambert et al., 2005). Transplacental $\mathrm{BaP}$ exposure induced dose-dependent increases in mutations in the somatic tissues of $\mathrm{F} 1$ males (Figure B1 and Table B1). At the highest dose, mutant frequencies increased 16, 18 , and 33-fold $(\mathrm{P}<0.0001)$ above controls in bone marrow, brain, and liver, respectively. At the low dose, brain showed a 7-fold increase in $\mathrm{MF}(\mathrm{P}<0.0001)$, liver a 3-fold increase $(\mathrm{P}=0.04)$, and bone marrow was unaffected $(\mathrm{P}=0.13)$. Although $\mathrm{BaP}$ adduct levels are typically elevated in maternal tissues relative to fetal tissues (Lu et al., 1986), in utero exposed liver and brain had 3- and 19-fold higher numbers of mutants per 
mg/kg-bw than adult BaP-exposed tissues, respectively (Figure B2, Table B2).

Conversely, mutagenic response in bone marrow did not differ between in utero and adult exposures. This is likely because all tissues are mitotically active during in utero development and therefore susceptible to mutation fixation; in contrast, adult liver and brain have very low mitotic indices. These results show that the placenta does not shelter the embryo from PAHs and that embryonic development is a highly susceptible window for the induction of somatic mosaicism.

\section{Effects of in utero BaP Exposure on Reproductive Health}

Next, we assessed the effects of in utero exposure to $\mathrm{BaP}$ on the reproductive health of the offspring. Although no effects occurred at $10 \mathrm{mg} / \mathrm{kg} / \mathrm{d}$, we observed doserelated effects on all male reproductive parameters at the other doses. At $40 \mathrm{mg} / \mathrm{kg} / \mathrm{day}$, testis weight decreased by $77 \%$ and sperm concentration decreased $>90 \%$ (Figure B3). Computer assisted sperm analysis indicated that in utero $\mathrm{BaP}$ exposure significantly decreased all of the measured motility parameters (Table B3, Figure B3). Furthermore, in utero exposure to $20 \mathrm{mg} / \mathrm{kg} / \mathrm{d} \mathrm{BaP}$ caused a significant increase $(3$-fold, $\mathrm{P}<0.0001)$ in lacZ mutants in sperm (Figure B4). At $40 \mathrm{mg} / \mathrm{kg} / \mathrm{d}$, so few sperm were present in the cauda epididymis that samples required pooling to obtain sufficient DNA for mutation analysis. These pooled samples had significantly higher mutant frequencies than lower doses and controls (Figure B4; 16-fold, $\mathrm{P}=0.0003)$, and the average MF $\left(46.3 \times 10^{-5}\right)$ was 4-fold higher than that seen in sperm of adult males exposed to $100 \mathrm{mg} / \mathrm{kg} / \mathrm{d}$ ENU (O'Brien et al., 2015), the most potent germ cell mutagen known. This equates to 11-fold higher mutants per mg/kg-bw of $\mathrm{BaP}$ than sperm from exposed adults (O'Brien et al., 2016a). Mutant frequencies in whole testes (Figure B4) closely matched those in sperm. 


\section{Clonal Expansion of Mutations and the Induction of Mosaicism}

We used NGS to determine the mutation spectra induced by transplacental $\mathrm{BaP}$ exposure and assess clonal expansion in both somatic tissues and sperm. We characterized 233 and 648 unique lacZ mutations from controls and BaP-treated tissues, respectively. In all 4 tissues, $\mathrm{BaP}$ exposure increased the proportions of lac $Z$ mutations that were clonally expanded (Figure B5, Table B4). Interestingly, clonality correlates with the adult replication rate of each tissue, with bone marrow and sperm (proxy for spermatogonial stem cells) showing the highest clonality and having higher adult proliferation rates (19\% and 35\% dividing cells, respectively; (Edwards and Klein, 1961)). Conversely, brain and liver showed the lowest clonality, with adult proliferation rates of neurons being negligible $(<0.1 \%$ dividing; (Edwards and Klein, 1961)), and hepatic cells dividing very rarely (0.53\% dividing; (Edwards and Klein, 1961)). This suggests that mutations were induced in stem cells during embryonic development and that the chance of detecting cells carrying the same mutation is affected by the rate of stem cell division in that tissue during adulthood.

Analysis of the mutation spectra provided insights into the mechanism(s) of $\mathrm{BaP}$ mutagenesis in utero and whether these differ among tissues. As BaP mainly induces $\mathrm{G} \rightarrow \mathrm{T}$ transversions via mispairing of adducted DNA (Keohavong and Thilly, 1992), increased proportions of $\mathrm{G} \rightarrow \mathrm{T}$ (as well as $\mathrm{G} \rightarrow \mathrm{C}$ transversions and deletions) were observed (Figure B1). Mutation types were consistent among the 3 somatic tissues and with the mutation spectrum observed in the bone marrow of adult-exposed mice (Beal et al., 2015a). The sperm spectrum was similar except that deletions were not increased (Figure $\mathrm{B} 4)$. Thus, the reactive metabolite of $\mathrm{BaP}(\mathrm{BPDE})$ was likely active through a 
similar genotoxic mechanism in fetal tissues as in adults. The presence of the mutational signature of $\mathrm{BaP}$ also confirms that the increased proportion of lacZ mutations is caused by the reactivity of the chemical itself, and not through induction of genomic instability.

\section{B.6 Discussion}

We demonstrate that $\mathrm{BaP}$ exposure during fetal development causes severe somatic and germ cell consequences in F1 animals, and that tissues originating from all 3 germ layers, as well as the germ cells, are highly mutagenized in adult offspring. Thus, in utero development is a critical window of risk for environmentally induced mutations that can lead to adverse health effects during adult life.

Historically, the discussion of somatic mutations has revolved around their role in tumourigenesis. In light of evidence that the number of lifetime stem cell divisions is a major contributor to cancer formation (Tomasetti and Vogelstein, 2015), any first-hit mutations in critical genes taking place in utero would increase the likelihood for loss of heterozygosity in the affected locus. Because only 3 to 6 mutated driver genes are sufficient to initiate most cancers (Vogelstein et al., 2013), increased numbers of de novo mutations could accelerate cancer progression later in life. Furthermore, extrinsic risk factors such as environmental exposures may contribute 70 to $90 \%$ of lifetime cancer risk, suggesting - in the context of data presented in this study - that variation in developmental exposure to environmental mutagens plays a meaningful role in determining an individual's risk (Wu et al., 2016). However, because mutations arising during in utero development are often observed as widely distributed mosaic variants, they can also result in diseases other than cancer.

Mosaicism in the nervous system is associated with diseases including epilepsy, 
lissencephaly, hemimegalencephaly, early-onset Alzheimer's, autism spectrum disorders, and others (Poduri et al., 2013; Sanders et al., 2012; Yuen et al., 2015). Normal brains also possess distinct cell lineages with genetic variants that originate during development (Lodato et al., 2015). Here, the brains of mice exposed in utero showed a higher mutagenic response at lower doses of $\mathrm{BaP}$ than other tissues $(\mathrm{P}<0.01)$ providing a novel mode of action for neurological effects induced by chemical exposures. We estimate that BaP-induced mutations increase by 1 standard deviation in the brain at $0.68 \mathrm{mg} / \mathrm{kg}-\mathrm{bw}$ for a 10-day exposure (Figure B6). This is a relatively small cumulative dose of 6.8 $\mathrm{mg} / \mathrm{kg}$-bw. In comparison, cancer induction in mice is estimated to occur at $0.8 \mathrm{mg} / \mathrm{kg}$ bw over 2 years (a cumulative dose of $584 \mathrm{mg} / \mathrm{kgbw}$ ) (Moffat et al., 2015). Therefore, mutations in the developing brain are induced by lower cumulative doses of $\mathrm{BaP}$ than those required for adult cancer formation. Coupled with the observation that in utero $\mathrm{BaP}$ exposures as low as $0.3 \mathrm{mg} / \mathrm{kg} / \mathrm{d}$ impaired neuronal activity in rats (McCallister et al., 2008), the developing brain demonstrates unprecedented sensitivity to $\mathrm{BaP}$ (Chepelev et al., 2015).

Developing germ cells differed in their response from developing somatic tissues in 2 major aspects: increased sensitivity to cell killing and mutation spectrum. The reduced sperm count in exposed offspring demonstrates increased sensitivity to BaPinduced apoptosis of primordial germ cell precursors (PGCs) via cellular toxicity, consistent with the framework proposed by (Rübe et al., 2011). This is supported by a study in Caenorhabditis elegans that found somatic cells to be more resistant to DNA damage-induced apoptosis than germ cells (Gartner et al., 2000). Because PGCs were proliferating during the exposure window in this study, PGC toxicity likely reduced the 
pool of germline stem cells in the offspring, and, consequently, drastically reduced sperm count permanently. Furthermore, the BaP-induced mutation spectrum in sperm was significantly different from other tissues (Fisher's exact, Bonferroni adjusted, $\mathrm{P}<0.001$ compared to liver and brain, $\mathrm{P}=0.097$ compared to bone marrow), showing fewer deletions. Germline stem cells are thought to be more effective at repairing DNA damage because the integrity of their genome is vital to the faithful replication of DNA for the organism's descendants (Rübe et al., 2011). It is therefore possible that deletions in sperm were under high selective pressure. These findings suggest that developing germ cells are more susceptible to environmental chemicals than adult spermatogonial stem cells, which may partially explain the higher mutation rate in primordial germ cells observed by Rahbari et al. (2015), and that the health effects of in utero mutagen exposure impact not only the exposed individual, but future generations as well.

Recent research has suggested that a large proportion of de novo mutations in humans are in fact the result of low-level somatic and/or germline mosaicism in the parents (Rahbari et al., 2015). In a similar manner, our study used clonal expansion of independent transgene mutations as a method to quantify mosaicism. Figure B5 shows the relative quantity for each recovered mutation, where the size of the bubble correlates with the number of cells which carried that mutation in a given tissue. The TGR assay positively selects lacZ from cells carrying a mutant copy and enables their relative quantification with NGS; thus, even though the physical distribution of these mutations in situ remains unknown, we are effectively able to interrogate very large numbers of cells that could not be feasibly analyzed with single-cell sequencing based on current costs. Rahbari et al. (2015) suggested that many of these low-level mosaic mutations arose 
spontaneously in the parents during embryogenesis (particularly during expansion of primordial germ cell precursors). Although individual families in their study clearly exhibited different propensities to pass on such mosaic mutations, the underlying cause for the variation remains enigmatic. We propose that a main contributor to variation in mutation burden among individuals is differential exposure to environmental mutagens during development.

The doses used in this study are higher than the levels of $\mathrm{BaP}$ to which most individuals in the population would be exposed. IARC has concluded that dietary exposure to BaP may range as high as $17 \mu \mathrm{g} / \mathrm{person} / \mathrm{day}$, or $0.28 \mu \mathrm{g} / \mathrm{kg} / \mathrm{d}$ for a $60 \mathrm{~kg}$ human (IARC, 2010). This equates to $1.9 \mu \mathrm{g} / \mathrm{kg} / \mathrm{d}$ in mice after an allometric interspecies correction (USEPA, 2011), or 300-fold less than the lowest dose inducing mutations in the brains of developing mice. It should be noted that humans are exposed to complex mixtures of multiple chemicals, through both oral and inhalation routes. Occupational exposures or lifestyle factors may result in highly exposed individuals, and because the pharmacokinetics of these complex mixtures and their interactions with the placenta and fetus are not well known, we believe that further research in this area is warranted.

In summary, this study provides clear evidence that in utero exposure to a common environmental pollutant induces somatic mosaicism in tissues originating from all 3 germ layers and germline mosaicism in the F1 generation. Thus, in utero development represents a sensitive window for the genesis of mosaicism via environmental exposures to chemical mutagens, and the potential health consequences apply not only to the developing organisms, but also to their progeny, which can ultimately influence the genetic health of an entire species. 


\section{B.7 Tables and Figures}

Table B1 Summary of lacZ mutant frequency in somatic and germ tissues of MutaMouse males exposed to BaP in utero during the period of organogenesis.

\begin{tabular}{|c|c|c|c|c|c|c|c|}
\hline Tissue & $\begin{array}{c}\text { Dose } \\
\text { administered } \\
\text { to dam } \\
(\mathrm{mg} / \mathrm{kg} / \mathrm{d}) \\
\end{array}$ & $\begin{array}{c}\text { No of } \\
\text { F1 } \\
\text { animals }\end{array}$ & $\underset{\left(\times 10^{5}\right)}{\mathbf{A v g}^{\mathbf{a}}}$ & $\mathbf{S E}^{\mathbf{b}}$ & P-value ${ }^{c}$ & $\begin{array}{c}\text { Total } \\
\text { Mutants }\end{array}$ & $\begin{array}{c}\text { Total } \\
\text { Plaque } \\
\text { Forming } \\
\text { Units } \\
\end{array}$ \\
\hline \multirow{4}{*}{$\begin{array}{c}\text { Bone } \\
\text { marrow }\end{array}$} & 0 & 16 & 6.2 & 2.19 & - & 169 & $3,148,992$ \\
\hline & 10 & 9 & 13.1 & 4.03 & 0.1323 & 379 & $2,561,041$ \\
\hline & 20 & 18 & 41.6 & 5.46 & $<0.0001$ & 1,874 & $4,205,448$ \\
\hline & 40 & 8 & 98.5 & 14.05 & $<0.0001$ & 1,172 & $1,291,372$ \\
\hline \multirow[t]{4}{*}{ Brain } & 0 & 8 & 3.5 & 0.87 & - & 67 & $2,037,037$ \\
\hline & 10 & 5 & 25.8 & 3.13 & $<0.0001$ & 348 & $1,290,543$ \\
\hline & 20 & 15 & 24.2 & 2.24 & $<0.0001$ & 582 & $2,330,433$ \\
\hline & 40 & 8 & 63.6 & 4.86 & $<0.0001$ & 659 & $1,146,192$ \\
\hline \multirow[t]{4}{*}{ Liver } & 0 & 14 & 3.4 & 1.18 & - & 101 & $2,584,069$ \\
\hline & 10 & 11 & 9.7 & 1.91 & 0.0378 & 252 & $2,533,872$ \\
\hline & 20 & 15 & 45.9 & 3.83 & $<0.0001$ & 1,347 & $2,920,372$ \\
\hline & 40 & 7 & 111.5 & 8.00 & $<0.0001$ & 1,508 & $1,478,244$ \\
\hline \multirow{4}{*}{$\begin{array}{l}\text { Caudal } \\
\text { sperm }\end{array}$} & 0 & 27 & 2.9 & 0.31 & - & 266 & $10,285,084$ \\
\hline & 10 & 17 & 3.8 & 0.43 & 0.1509 & 263 & $7,261,336$ \\
\hline & 20 & 11 & 8.5 & 0.97 & $<0.0001$ & 254 & $3,188,918$ \\
\hline & 40 & 2 & 46.3 & 39.58 & 0.0003 & 7 & 12,922 \\
\hline \multirow{4}{*}{$\begin{array}{l}\text { Whole } \\
\text { testes }\end{array}$} & 0 & 6 & 2.1 & 0.92 & - & 40 & $1,845,468$ \\
\hline & 10 & 4 & 7.2 & 1.77 & 0.0888 & 110 & $1,594,804$ \\
\hline & 20 & 12 & 21.8 & 2.02 & $<0.0001$ & 770 & $3,681,859$ \\
\hline & 40 & 3 & 77.6 & 8.12 & $<0.0001$ & 680 & 862,129 \\
\hline
\end{tabular}

${ }^{\mathrm{a}}$ Arithmetic mean of MF of all animals in group

${ }^{\mathrm{b}}$ Standard error (SE) calculated using the esticon function in the doBy R package

${ }^{\mathrm{c}}$ Generalized linear model in $\mathrm{R}$ with quasi-Poisson distribution and Bonferonni correction 
Table B2 Mutant frequencies expressed per cumulative dose of $\mathrm{BaP}$ administered in this study and in previously exposed adult MutaMouse males. The source of each lacZ mutant frequency data is stated below.

\begin{tabular}{|c|c|c|c|c|}
\hline Tissue & Exposure & $\begin{array}{c}\text { Length of exposure } \\
\text { + time until } \\
\text { euthanasia }\end{array}$ & $\begin{array}{c}\text { Avg. mutants per } \\
\text { mg/kg-bw }\end{array}$ & $\begin{array}{c}\text { Fold difference } \\
\text { between } \text { in utero } \\
\text { and adult BaP } \\
\text { exposure }\end{array}$ \\
\hline Bone marrow & In utero & $10 \mathrm{~d}+10 \mathrm{wk}$ & 0.195 & $\mathbf{0 . 7 5}$ \\
\hline Brain & In utero & $10 \mathrm{~d}+10 \mathrm{wk}$ & 0.179 & $\mathbf{1 9 . 3}$ \\
\hline Liver & In utero & $10 \mathrm{~d}+10 \mathrm{wk}$ & 0.202 & $\mathbf{3 . 2}$ \\
\hline Sperm & In utero & $10 \mathrm{~d}+10 \mathrm{wk}$ & 0.066 & $\mathbf{1 1 . 4}$ \\
\hline Bone marrow & Adult ${ }^{\mathrm{a}, \mathrm{b}, \mathrm{c}}$ & $28 \mathrm{~d}+3 \mathrm{~d}$ & 0.261 & \\
\hline Brain & Adult & $5 \mathrm{~d}+14 \mathrm{~d}$ & 0.009 & \\
\hline Liver & Adult $^{\mathrm{d}}$ & $28 \mathrm{~d}+3 \mathrm{~d}$ & 0.063 & \\
\hline Sperm & Adult $^{\mathrm{a}}$ & $28 \mathrm{~d}+42 \mathrm{~d}$ & 0.006 & \\
\hline
\end{tabular}

a'(O’Brien et al., 2016b)

beal et al., 2015a)

'(Hakura et al., 1998)

d(Lemieux et al., 2011) 
Table B3 In utero exposure to BaP negatively impacts sperm quality in F1 males as measured by computer-assisted sperm analysis.

\begin{tabular}{|c|c|c|c|}
\hline Parameter & \multicolumn{3}{|c|}{ Fraction of control value * } \\
\hline & $\mathbf{1 0} \mathbf{~ m g / k g / d ~}$ & $\mathbf{2 0} \mathbf{~ m g} / \mathbf{k g} / \mathbf{d}$ & $\mathbf{4 0} \mathbf{~ m g} / \mathbf{k g} / \mathbf{d}$ \\
\hline Progressive Motility & 1.02 & $\mathbf{0 . 3 6}$ & $\mathbf{0 . 0 2}$ \\
\hline Path Velocity (VAP) & 1.03 & 0.77 & $\mathbf{0 . 1 9}$ \\
\hline Progressive Velocity (VSL) & 1.04 & 0.85 & $\mathbf{0 . 2 0}$ \\
\hline Track Velocity (VCL) & 1.01 & 0.70 & $\mathbf{0 . 1 8}$ \\
\hline Amplitude Lateral Head (ALH) & 1.03 & $\mathbf{0 . 6 6}$ & $\mathbf{0 . 1 6}$ \\
\hline Beat Cross Frequency (BCF) & 0.98 & 0.90 & $\mathbf{0 . 5 0}$ \\
\hline Straightness (STR) & 1.00 & 1.07 & $\mathbf{0 . 6 4}$ \\
\hline Linearity (LIN) & 1.01 & 1.21 & 0.76 \\
\hline
\end{tabular}

*bold indicates statistical significance of $\mathrm{P}<0.05$ in ANOVA followed by a Bonferroni post-hoc multiple comparison. 
Table B4 Comparison of clonal expansion of lac $Z$ mutants in control mice and mice exposed to $\mathrm{BaP}$ either in utero or as adults.

\begin{tabular}{|c|c|c|c|c|c|c|}
\hline $\begin{array}{l}\text { Tissue } \\
\text { (study) }\end{array}$ & Exposure & $\begin{array}{l}\text { \# unique } \\
\text { mutations }\end{array}$ & $\begin{array}{l}\text { \# clonally } \\
\text { expanded }\end{array}$ & $\begin{array}{l}\% \text { clonally } \\
\text { expanded }\end{array}$ & $\begin{array}{c}\text { Ratio } \\
\text { (BaP:control) }\end{array}$ & $\begin{array}{c}\text { Fisher's exact test } \\
\text { p-value }\end{array}$ \\
\hline \multirow{2}{*}{$\begin{array}{c}\text { Bone } \\
\text { Marrow } \\
\text { (in utero) }\end{array}$} & Control & 50 & 11 & $22 \%$ & \multirow[t]{2}{*}{1.55} & \multirow[t]{2}{*}{0.1171} \\
\hline & Treated & 155 & 53 & $34 \%$ & & \\
\hline \multirow{2}{*}{$\begin{array}{l}\text { Bone } \\
\text { Marrow } \\
\text { (adult) }^{\mathrm{a}}\end{array}$} & Control & 152 & 11 & $8 \%$ & \multirow[t]{2}{*}{1.25} & \multirow[t]{2}{*}{0.5362} \\
\hline & Treated & 854 & 78 & $10 \%$ & & \\
\hline \multirow{2}{*}{$\begin{array}{c}\text { Brain (in } \\
\text { utero) }\end{array}$} & Control & 49 & 2 & $4 \%$ & \multirow[t]{2}{*}{4.27} & \multirow[t]{2}{*}{0.0153} \\
\hline & Treated & 235 & 41 & $17 \%$ & & \\
\hline \multirow{2}{*}{$\begin{array}{c}\text { Caudal } \\
\text { sperm (in } \\
\text { utero) }\end{array}$} & Control & 54 & 8 & $15 \%$ & \multirow[t]{2}{*}{1.97} & \multirow[t]{2}{*}{0.0792} \\
\hline & Treated & 65 & 19 & $29 \%$ & & \\
\hline \multirow{2}{*}{$\begin{array}{l}\text { Caudal } \\
\text { sperm } \\
(\text { adult })^{b}\end{array}$} & Control & 131 & 18 & $16 \%$ & \multirow[t]{2}{*}{0.63} & \multirow[t]{2}{*}{0.1247} \\
\hline & Treated & 295 & 26 & $10 \%$ & & \\
\hline \multirow{2}{*}{$\begin{array}{c}\text { Liver (in } \\
\text { utero) }\end{array}$} & Control & 80 & 3 & $4 \%$ & \multirow[t]{2}{*}{2.07} & \multirow[t]{2}{*}{0.2901} \\
\hline & Treated & 193 & 15 & $8 \%$ & & \\
\hline
\end{tabular}

a(Beal et al., 2015a)

b(O’Brien et al., 2016b) 
A
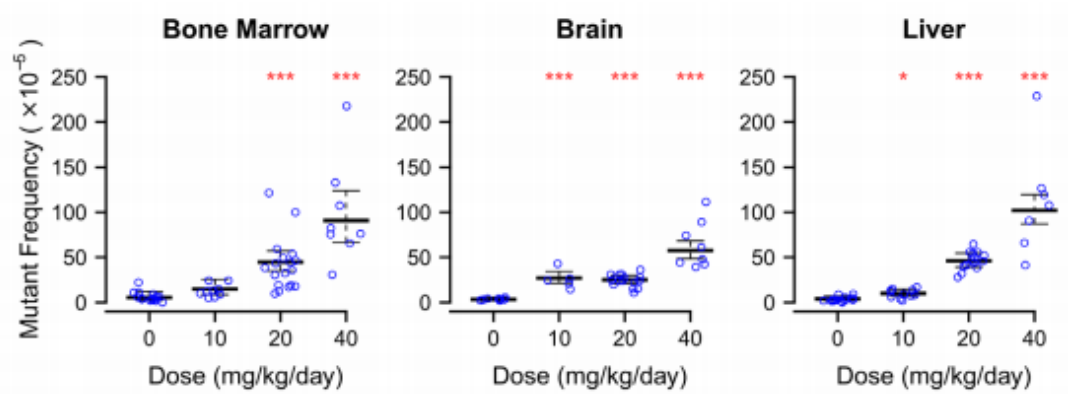

B
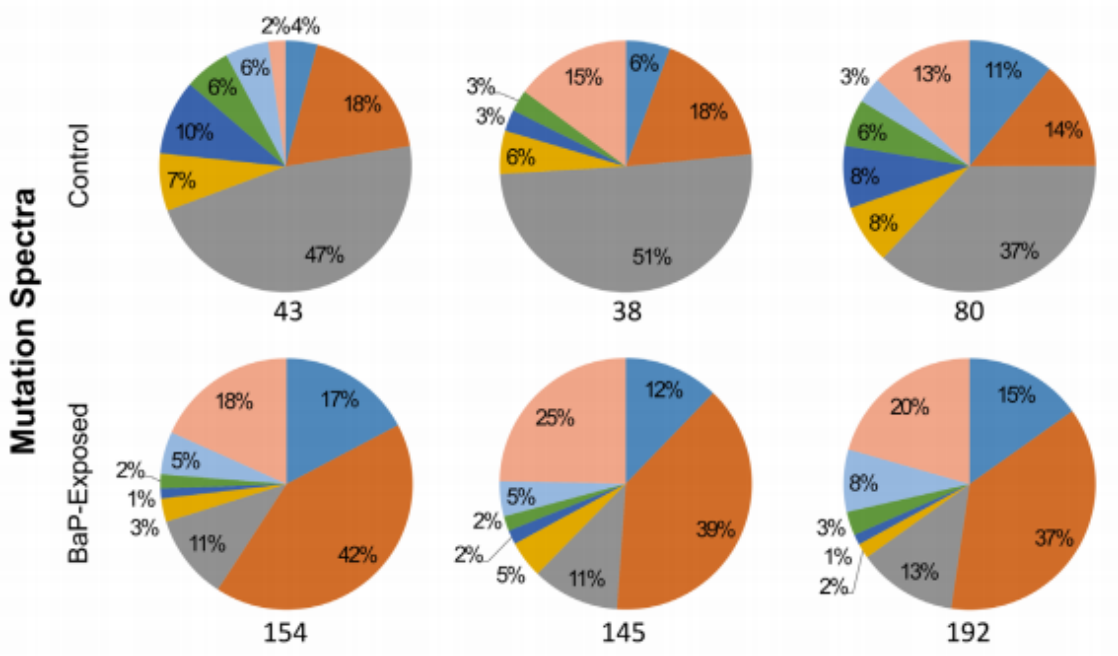

$\mathbf{G} \rightarrow \mathrm{C}$

$G \rightarrow T$

$G \rightarrow A$

$A \rightarrow T$
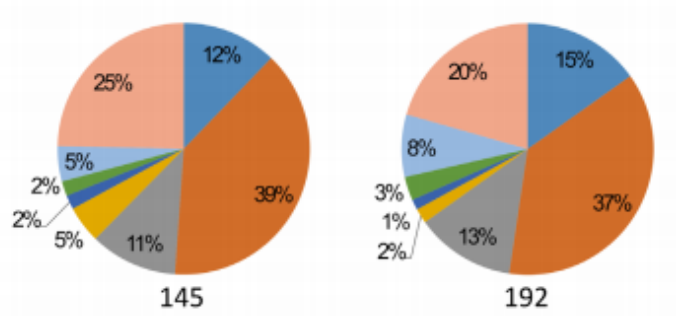

$A \rightarrow G$

$A \rightarrow C$

INS

DEL

Figure B1 Male mice exposed transplacentally to BaP during organogenesis exhibited increased mutant frequencies in multiple somatic tissues. Dams were dosed at 0, 10, 20, or $40 \mathrm{mg} / \mathrm{kg} / \mathrm{d}$, and mutant frequencies (in 10 week old $\mathrm{F} 1$ males) were determined using the lac $Z$ transgene mutation assay. (A) In utero exposure to $\mathrm{BaP}$ induced a significant dose-dependent increase in mutant frequencies in somatic tissues originating from distinct primary germ layers, including the bone marrow (mesoderm), brain (ectoderm), and liver (endoderm). Error bars represent $95 \%$ CI. $* \mathrm{P}<0.05, * * * \mathrm{P}<0.0001$, generalized linear model with quasi-Poisson distribution. (B) NGS of the lacZ reporter gene from animals exposed in utero shows the well-characterized molecular signature of $\mathrm{BaP}$ exposure, visible in the pie charts as an increase in the proportion of $\mathrm{G} \rightarrow \mathrm{T}$ mutations. The number of unique $l a c Z$ mutations sequenced is noted beneath each pie chart. 


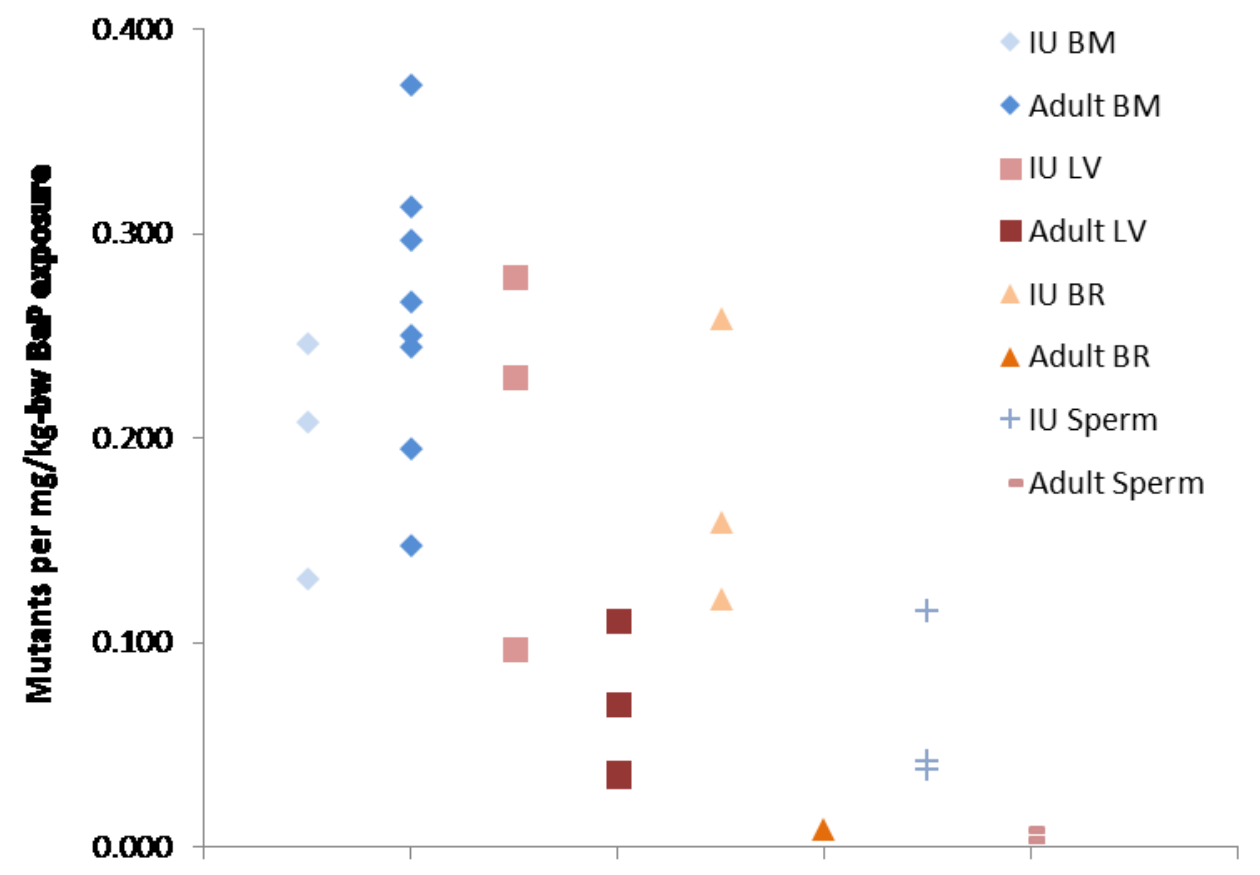

Figure B2 Number of mutations occurring after various animal exposures to $\mathrm{BaP}$ normalized to total quantity of $\mathrm{BaP}$ exposure (see Table B2). Each data point represents the mean value for a different dose group. BM: bone marrow; LV: liver; BR: brain. 

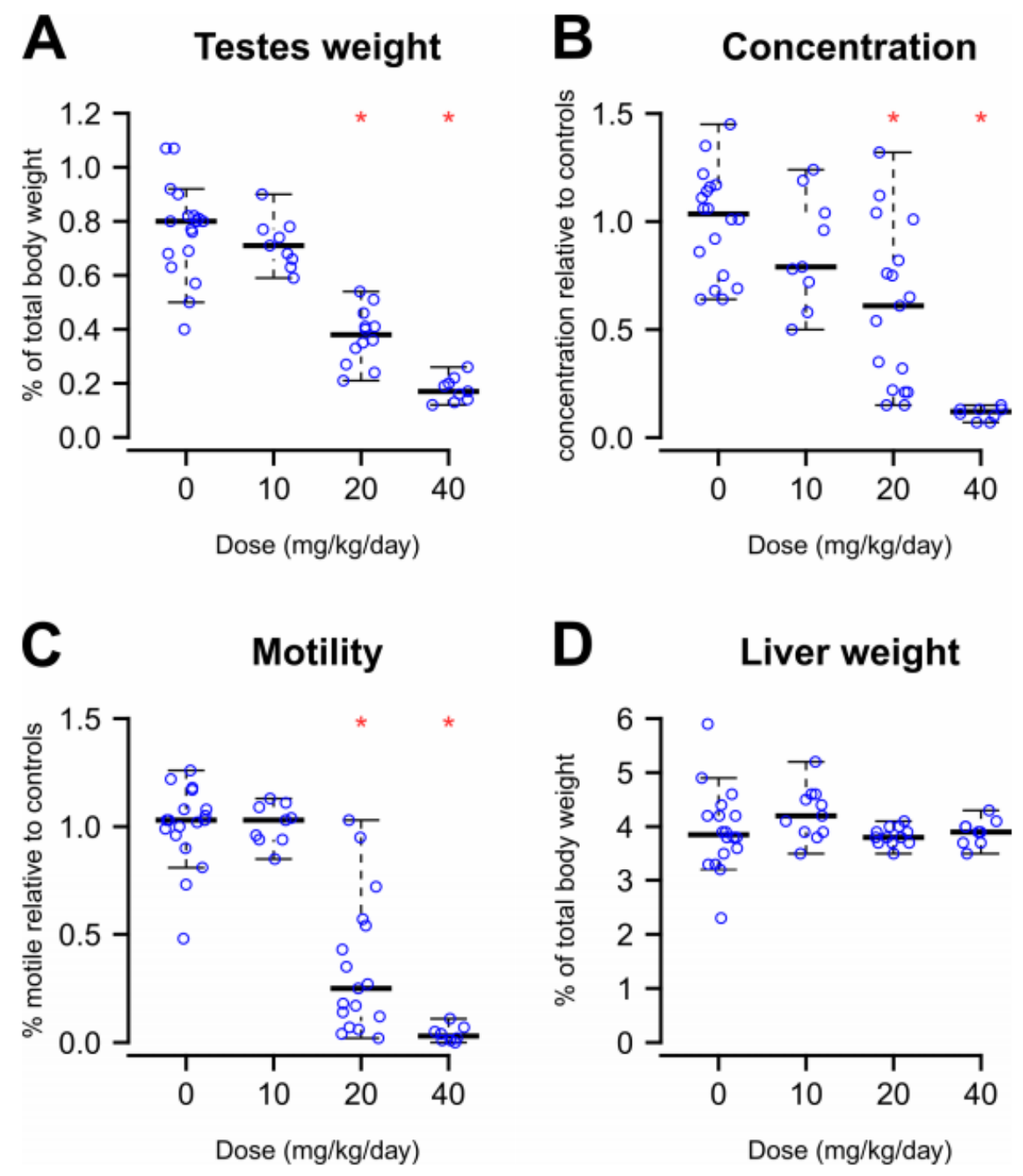

Figure B3 In utero exposure to $\mathrm{BaP}$ significantly decreases multiple reproductive parameters in F1 adult male mice. BaP caused a significant dose-dependent decrease in the testis weight (shown as percentage body weight, A), as well as a drastic decrease in sperm concentration (B) and percentage of motile sperm (C). Liver weight (D) was unaffected. * $\mathrm{P}<0.05$, ANOVA. Error bars represent 95\% CI. 
A

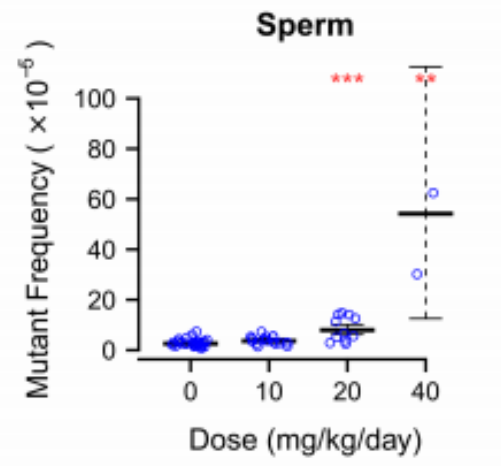

C

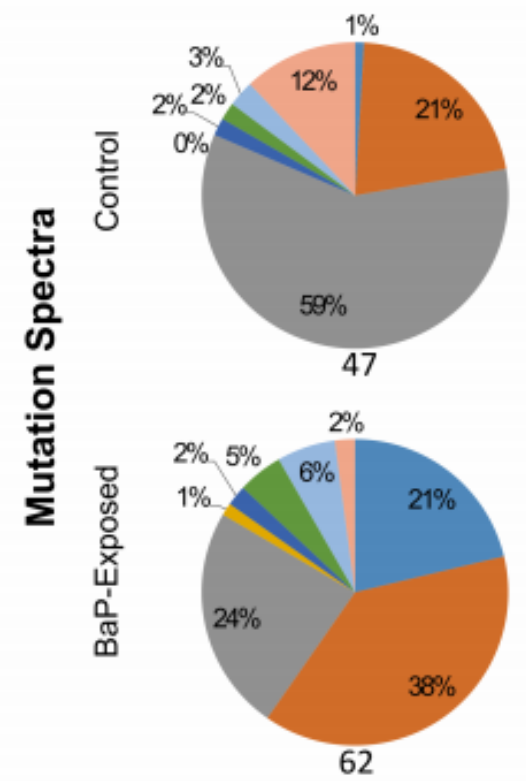

B

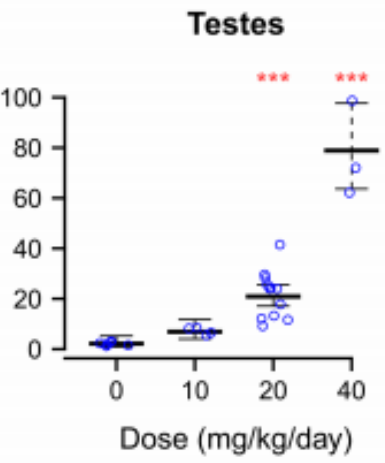

Figure B4 Mutant frequencies were significantly increased in caudal sperm (A) and whole testes (B) of F1 males exposed in utero to 20 and $40 \mathrm{mg} / \mathrm{kg} / \mathrm{d}$ BaP. Mutant frequencies in sperm at $40 \mathrm{mg} / \mathrm{kg} / \mathrm{d}$ were measured by pooling several DNA samples because of extremely low sperm counts. Error bars represent $95 \%$ CI. ** $\mathrm{P}<0.001$, *** $\mathrm{P}$ $<0.0001$, generalized linear model with quasi-Poisson distribution. (C) NGS of the lacZ reporter gene from caudal sperm. BaP exposure (20 and $40 \mathrm{mg} / \mathrm{kg} / \mathrm{d}$ combined) increased the proportion of $\mathrm{G} \rightarrow \mathrm{T}$ and $\mathrm{G} \rightarrow \mathrm{C}$ mutations but had no effect on deletions. The number of unique lacZ mutations sequenced is noted beneath each pie chart. 


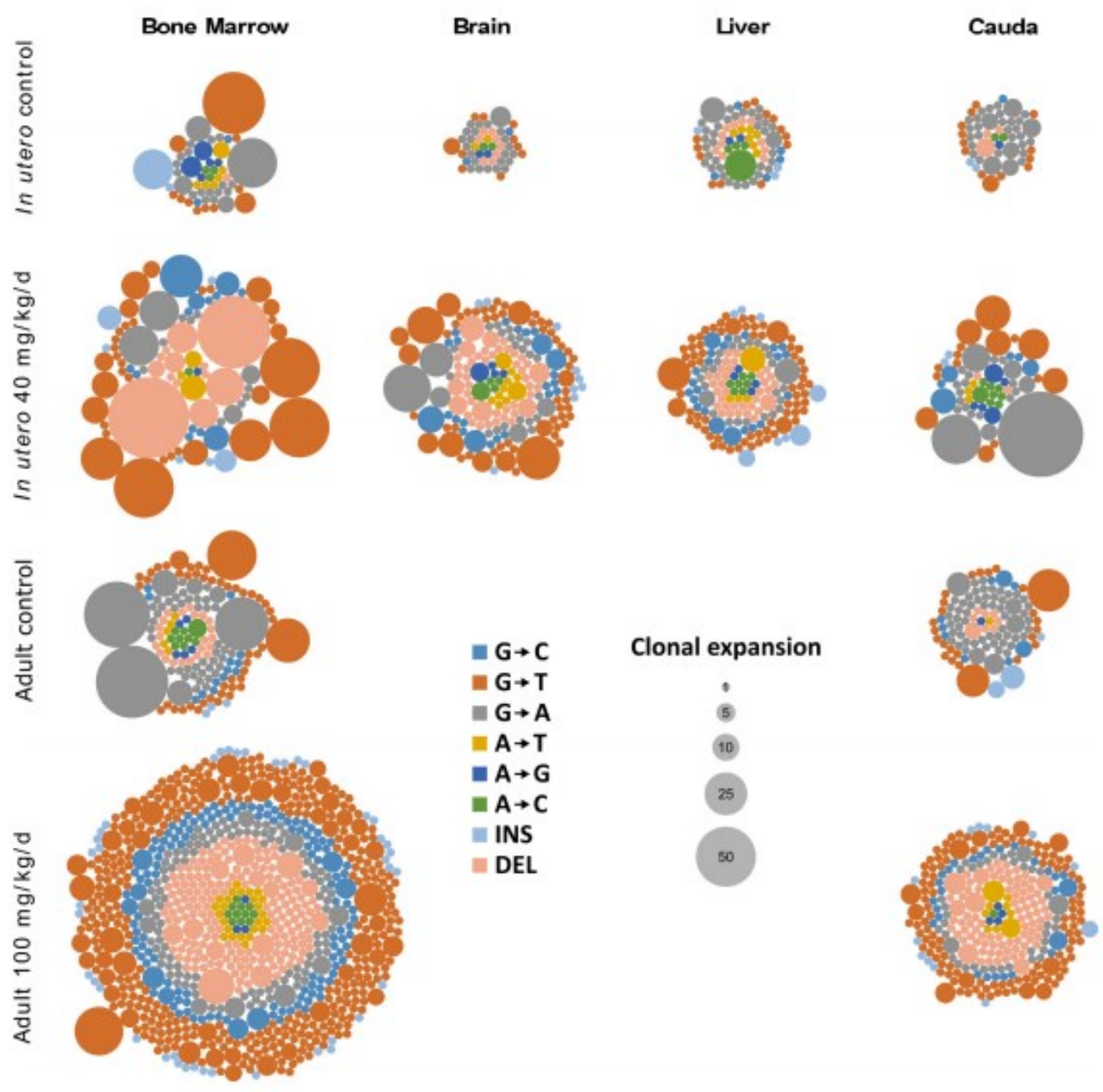

Figure B5. Clonal expansion of the mutations characterized in each somatic tissue and caudal sperm. The proportion of mutations that underwent clonal expansion is consistently higher in animals exposed to $\mathrm{BaP}$ in utero, illustrating the potential for increased mosaicism. As in somatic tissues, the proportion of clonally expanded mutations is higher in $\mathrm{BaP}$-exposed sperm, highlighting the potential for increased germline mosaicism. Compared to exposure in adults (data from (Beal et al., 2015a; O'Brien et al., 2016b)), in utero exposure results in a higher degree of clonal expansion. Each circle within a group corresponds to an independent mutation scored, and the area of each circle represents the number of times that mutation was observed per animal. 


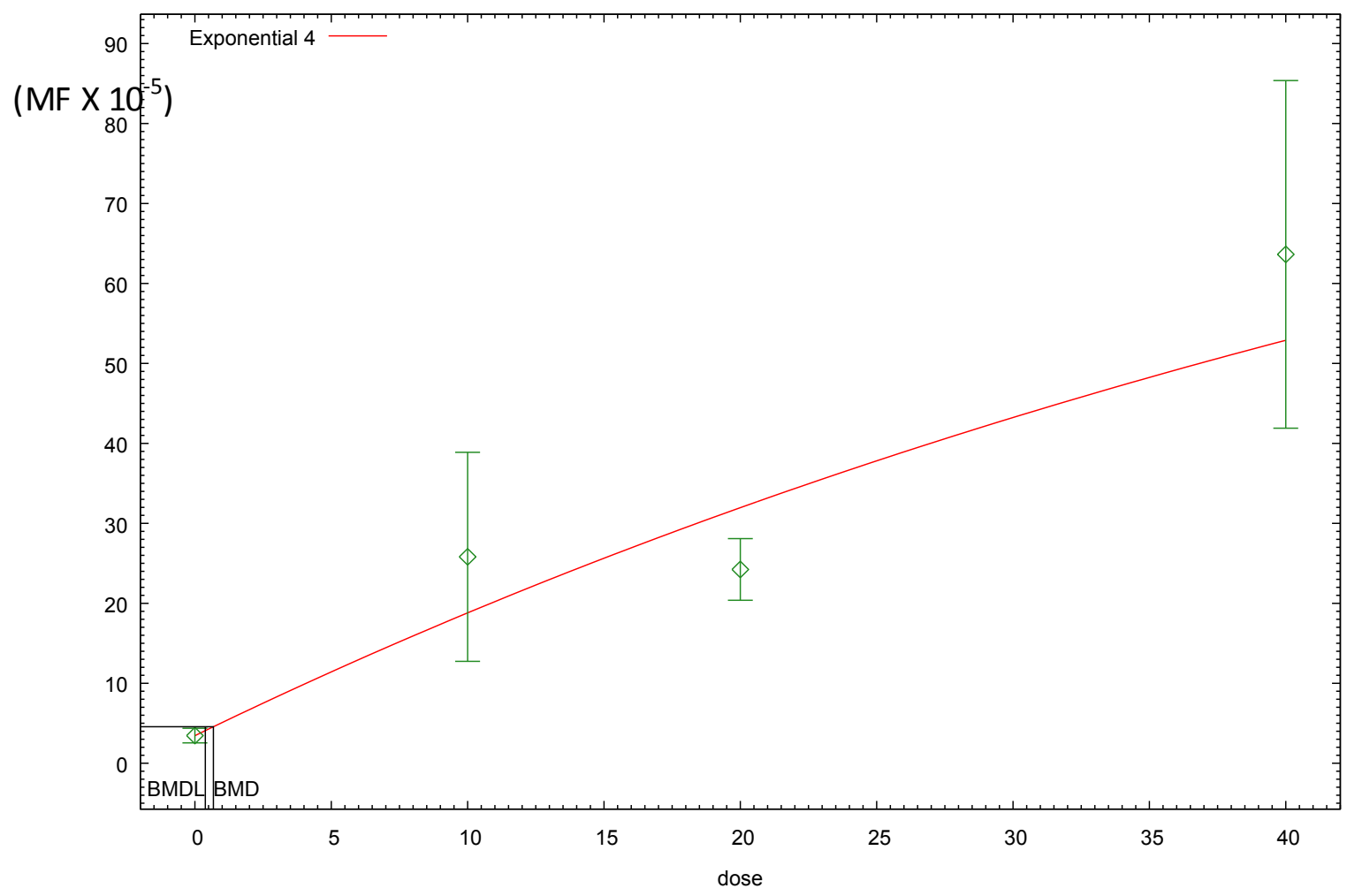

16:02 05/29 2015

Figure B6 Dose-response modeling of the brain of F1 males exposed in utero. The BMDS software v2.6 was used with a BMR of 1 standard deviation with non-constant variance that adequately modelled the data. These parameters were chosen to allow comparison with existing cancer BMD analysis from (Moffat et al., 2015). 


\section{Appendix C Supplemental Mate rial}

\section{C.1 Chapter 2}

Table C1 List of candidate microsatellites for SM-PCR development.

\begin{tabular}{|c|c|c|c|}
\hline Name $^{1}$ & Repeat Unit & Forward Primer & Reverse Primer \\
\hline $\operatorname{Chr3(171)}$ & $(A G G)_{57}$ & TGG AAC ATG ACG & GAG CTT CAG ACC \\
\hline Chr4(177) & $(\mathrm{AAG})_{59}$ & $\begin{array}{c}\text { CAA AGT TGG ACC } \\
\text { TIA GCA GAA }\end{array}$ & $\begin{array}{c}\text { GTG AGT TCC AGG } \\
\text { ACA ACC AG }\end{array}$ \\
\hline Chr4(216) & $(\mathrm{AAG})_{72}$ & $\begin{array}{c}\text { GCT CTG TCA CTG } \\
\text { TAT GTC }\end{array}$ & $\begin{array}{c}\text { СTC TAC ACT TCC TCC } \\
\text { AAT G }\end{array}$ \\
\hline Chr5(159) & $(\mathrm{AAG})_{53}$ & $\begin{array}{c}\text { GCT TCC CTC TCT } \\
\text { GCT TCT CA }\end{array}$ & $\begin{array}{c}\text { GGG AAT TIT GGG } \\
\text { GAT AGC AT }\end{array}$ \\
\hline Chr5(171) & $(\mathrm{AAG})_{57}$ & $\begin{array}{c}\text { GTG ATG GAG TCA } \\
\text { GTG GTT ACA }\end{array}$ & $\begin{array}{c}\text { AGA GAT TTC TAG } \\
\text { ACC AGC CAG }\end{array}$ \\
\hline Chr5(246) & $(\mathrm{AAG})_{82}$ & $\begin{array}{c}\text { CAG GAT ACA CAG } \\
\text { ताT AGT GC }\end{array}$ & $\begin{array}{c}\text { GTC CTG TGA AGG } \\
\text { CTT GAT G }\end{array}$ \\
\hline Chr7(333) & $(\mathrm{AAG})_{111}$ & $\begin{array}{c}\text { AGC CCA ATG TTC } \\
\text { ACA CAC AA }\end{array}$ & $\begin{array}{c}\text { TAG AAT GAA GCC } \\
\text { TGC CAC CT }\end{array}$ \\
\hline Chr8(171) & $(\mathrm{AAG})_{57}$ & $\begin{array}{c}\text { GAG GCA GGC AGA } \\
\text { ता CTG AG }\end{array}$ & $\begin{array}{c}\text { ATG GAC CAG CCTT } \\
\text { GTG AAA C }\end{array}$ \\
\hline Chr9(189) & $(\mathrm{AAG})_{63}$ & $\begin{array}{c}\text { TGA GTT TGA GAC } \\
\text { CAG C }\end{array}$ & $\begin{array}{c}\text { CTG AAG GCT CCA } \\
\text { AGA TAA G }\end{array}$ \\
\hline Chr9(192) & $(\mathrm{AAG})_{64}$ & $\begin{array}{c}\text { ATA GAC ACA TAC } \\
\text { AGC ATA GC }\end{array}$ & $\begin{array}{l}\text { GTG AGT TAA GGT } \\
\text { AAC AAG GTG }\end{array}$ \\
\hline Chr10(186) & $(A A G)_{62}$ & $\begin{array}{c}\text { TCC TAC CTC AGA } \\
\text { GAA GTG }\end{array}$ & $\begin{array}{l}\text { CCT TCT AAT AGT } \\
\text { GCC ATC A }\end{array}$ \\
\hline Chr11(255) & $(\mathrm{AAG})_{85}$ & $\begin{array}{c}\text { CTC ATT AGA TAC } \\
\text { TGC CAC }\end{array}$ & $\begin{array}{c}\text { TCA CTG CCT GTA } \\
\text { ACT CTA }\end{array}$ \\
\hline Chr13(150) & $(A G G)_{50}$ & $\begin{array}{c}\text { GCT TCT CCT TCT } \\
\text { CCT TCT }\end{array}$ & $\begin{array}{c}\text { CCT GAG ACA ATG } \\
\text { TAA CAA GT }\end{array}$ \\
\hline Chr16(165) & $(\mathrm{AAG})_{55}$ & $\begin{array}{c}\text { CAG TTG CAG TIT } \\
\text { GTC CA }\end{array}$ & $\begin{array}{c}\text { TAT GAT ATC CAG } \\
\text { CAG CTG }\end{array}$ \\
\hline Chr16(180) & $(A G G)_{60}$ & $\begin{array}{c}\text { GCA ACA TTA GGT } \\
\text { AGG CAG AA }\end{array}$ & $\begin{array}{c}\text { GGA ATA GGG AAG } \\
\text { GGG ATA GG }\end{array}$ \\
\hline Chr18(156) & $(A G G)_{52}$ & $\begin{array}{c}\text { CGC TAG TGA CAA } \\
\text { GAC AAC }\end{array}$ & $\begin{array}{c}\text { CTA GGA GTT GGT } \\
\text { GAG ACA }\end{array}$ \\
\hline $\mathrm{Mm} 2.2 .1$ & $(\text { AGAT })_{17 \ldots}(\text { AGAT })_{18}$ & $\begin{array}{c}\text { GGC TAC TGG GTA } \\
\text { TCA AAC AGG }\end{array}$ & $\begin{array}{c}\text { TGA AGG GTA TTG } \\
\text { GAG TTC AGT C }\end{array}$ \\
\hline Mm3.2.1 & $\begin{array}{c}(\mathrm{GATA})_{4} \ldots(\mathrm{CAGA})_{4} \ldots(\mathrm{GATA})_{7} \ldots \\
(\mathrm{AGAT})_{11} \ldots(\mathrm{GATA})_{16}\end{array}$ & $\begin{array}{c}\text { AGG TGA CAC TGG } \\
\text { TCA GGG ATC }\end{array}$ & $\begin{array}{l}\text { GCT TGT CTG GAA } \\
\text { GTA GCC AAC }\end{array}$ \\
\hline Mm14.1.1 & $(\mathrm{AGAT})_{17}$ & $\begin{array}{c}\text { ACT GTG AGC ACA } \\
\text { AAT GAG AGG }\end{array}$ & $\begin{array}{l}\text { TGG CTT TAA GTT } \\
\text { CAT GGA TTC C }\end{array}$ \\
\hline Mm10.3.2 & $(\mathrm{AGAT})_{15 \ldots} \ldots(\mathrm{AGAT})_{14.5}$ & $\begin{array}{c}\text { GCC TGT GTT CAG } \\
\text { CGT TCA G }\end{array}$ & $\begin{array}{c}\text { CTG TAA GCC AAG } \\
\text { CTC ATC }\end{array}$ \\
\hline Mm15.3.1 & $(\mathrm{AGAT})_{14 \ldots} \ldots(\mathrm{AGAT})_{14} \ldots(\mathrm{AGAT})_{12}$ & $\begin{array}{c}\text { TAG CAA TAA CAA } \\
\text { GAT GAT GG }\end{array}$ & $\begin{array}{c}\text { AGA GTG TTG GAC } \\
\text { TाT CTC AG }\end{array}$ \\
\hline Mm16.1.2 & $(\mathrm{AGAT})_{17}$ & TाT CAA TCC CTG & GCT GGA AAC AGA \\
\hline
\end{tabular}




\begin{tabular}{|c|c|c|c|} 
& & GAA CCT TTG & ACT CTG GTC \\
Mm7.2.1 & $(\text { AGAT })_{10} \ldots(\text { AGAT })_{10} \ldots(\text { AGAT })_{12}$ & TCA TCA TCT ATC & TCA ATA TCA CTC \\
& & CAA TAC ATC CAT C & AGC TAT AGG \\
6.9487 & $(\text { GAAA })_{39} \ldots(\mathrm{GGGA})_{6}$ & AGC CAC GGC AAA & GCT CCA CT TGT \\
& TGT AAA GAG & GCT GTC AC \\
\hline
\end{tabular}

${ }^{1}$ The trinucleotide repeats are from the Eukaryotic Microsatellite Database (Aishwarya et al., 2007). The trinucleotides are named based on the chromosome location and the length of the repeat. The tetranucleotide repeats are from (Beal et al., 2012a). 


\section{C.2 Chapter 4}

Table C2 Thresholds used to detect mutations from each sample library.

\begin{tabular}{|c|c|c|c|c|}
\hline $\begin{array}{c}\text { Sample } \\
\text { ID }\end{array}$ & $\begin{array}{c}\text { Mutant } \\
\text { Plaques } \\
\text { Sequenced }\end{array}$ & $\begin{array}{c}\text { Stringent } \\
\text { Threshold }\end{array}$ & $\begin{array}{c}\text { Medium } \\
\text { Threshold }\end{array}$ & $\begin{array}{c}\text { Low } \\
\text { Threshold }\end{array}$ \\
\hline Control15 & 125 & ${ }^{\mathrm{a}} 0.0080$ & 0.0060 & 0.0040 \\
\hline Control19 & 185 & 0.0054 & 0.0041 & 0.0027 \\
\hline Control20 & 261 & 0.0038 & 0.0029 & 0.0019 \\
\hline Control22 & 93 & 0.0108 & 0.0081 & 0.0054 \\
\hline Control23 & 128 & 0.0078 & 0.0059 & 0.0039 \\
\hline Control24 & 80 & 0.0125 & 0.0094 & 0.0063 \\
\hline BaP43 & 500 & 0.0020 & 0.0015 & 0.0010 \\
\hline BaP44 & 500 & 0.0020 & 0.0015 & 0.0010 \\
\hline BaP45 & 500 & 0.0020 & 0.0015 & 0.0010 \\
\hline BaP46 & 500 & 0.0020 & 0.0015 & 0.0010 \\
\hline BaP47 & 500 & 0.0020 & 0.0015 & 0.0010 \\
\hline BaP48 & 500 & 0.0020 & 0.0015 & 0.0010 \\
\hline
\end{tabular}
${ }^{\mathrm{a}} 1 / 125=0.008$
${ }^{\mathrm{b}} 0.008 \times 75 \%=0.006$
${ }^{\mathrm{c}} 0.008 \times 50 \%=0.004$ 
Table C3 Comparison of expected and observed mutant count when the number of each mutant plaque was controlled for in each NGS library.

\begin{tabular}{|c|c|c|c|c|c|c|c|c|c|c|c|c|c|c|c|c|}
\hline \multirow[b]{2}{*}{ Library } & \multicolumn{2}{|c|}{$\begin{array}{c}\text { 2413: } \\
\text { Deletion }\end{array}$} & \multicolumn{2}{|c|}{$\begin{array}{c}2286: \\
G \rightarrow A\end{array}$} & \multicolumn{2}{|c|}{$\begin{array}{c}1224: \\
\mathrm{C} \rightarrow \mathrm{A}\end{array}$} & \multicolumn{2}{|c|}{$\begin{array}{l}2374^{\mathrm{a}}: \\
\mathrm{C} \rightarrow \mathrm{A}\end{array}$} & \multicolumn{2}{|c|}{$\begin{array}{c}\text { 491: } \\
\mathrm{G} \rightarrow \mathrm{A}\end{array}$} & \multicolumn{2}{|c|}{$\begin{array}{c}572: \\
\text { Deletion }\end{array}$} & \multicolumn{2}{|c|}{$\begin{array}{l}\text { 1546: } \\
\mathrm{C} \rightarrow \mathrm{T}\end{array}$} & \multirow[t]{2}{*}{ Titre } & \multirow{2}{*}{$\begin{array}{c}\text { Total } \\
\text { Plaques }\end{array}$} \\
\hline & $\mathrm{E}$ & $\mathrm{O}$ & $\mathrm{E}$ & $\mathrm{O}$ & $\mathrm{E}$ & $\mathrm{O}$ & $\mathrm{E}$ & $\mathrm{O}$ & $\mathrm{E}$ & $\mathrm{O}$ & $\mathrm{E}$ & $\mathrm{O}$ & $\mathrm{E}$ & $\mathrm{O}$ & & \\
\hline 1 & 10 & 12 & 10 & 9 & 10 & 10 & 20 & 24 & 10 & 12 & 10 & 15 & 10 & 16 & 0 & 80 \\
\hline 2 & 1 & 1 & 1 & 2 & 2 & 2 & 5 & 8 & 3 & 3 & 3 & 4 & 30 & 46 & 52 & 97 \\
\hline 3 & 5 & 8 & 6 & 7 & 7 & 9 & 43 & 50 & 20 & 27 & 30 & 31 & 50 & 49 & 27 & 188 \\
\hline 4 & 1 & 2 & 1 & 2 & 1 & 2 & 2 & 4 & 1 & 0 & 1 & 1 & 1 & 3 & 90 & 98 \\
\hline $5^{\mathrm{b}}$ & 0 & 0 & 0 & 0 & 0 & 0 & 10 & 11 & 0 & 0 & 10 & 5 & 10 & 20 & 30 & 60 \\
\hline
\end{tabular}

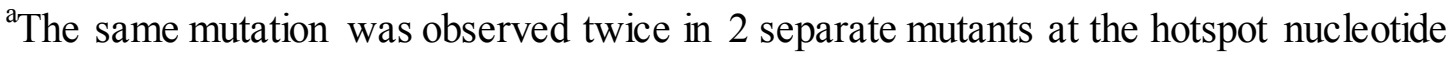
position 2374 .

${ }^{b}$ Different plaque sizes were sequenced for each mutant. Small, medium, and large mutants were sequenced from mutants at positions 572, 2374, and 1546 respectively. 
Table C4 Percent Clonality and Adjusted Mutant Frequencies in Control and BaP-treated samples using the LOD/linear model to adjust mutant counts.

\begin{tabular}{|c|c|c|c|c|c|}
\hline Sample & $\begin{array}{c}\text { Raw MF } \\
\left(\times 10^{-5}\right)\end{array}$ & $\begin{array}{c}\text { Average } \\
\text { Raw MF } \\
\left(\times 10^{-5}\right)\end{array}$ & $\begin{array}{c}\text { Clonal } \\
\text { Expansion }(\%)\end{array}$ & $\begin{array}{c}\text { Adjusted } \\
\text { MF }\left(\times 10^{-5}\right)\end{array}$ & $\begin{array}{c}\text { Average Adjusted } \\
\text { MF } \\
\left(\times 10^{-5}\right)\end{array}$ \\
\hline Control15 & 5.8 & 8.6 & 61.8 & 2.2 & 4.0 \\
Control19 & 9.7 & & 69.2 & 3.0 & \\
Control20 & 17.8 & & 85.5 & 2.6 & \\
Control22 & 3.7 & & 28.1 & 2.7 & \\
Control23 & 6.2 & & 14.8 & 5.3 & \\
Contro124 & 8.3 & & 0 & 8.3 & \\
\hline BaP43 & 590.2 & 701.7 & 33.6 & 391.9 & \\
BaP44 & 725.9 & & 31.4 & 498.0 & \\
BaP45 & 865.2 & & 35.1 & 561.5 & \\
BaP46 & 782.9 & & 31.5 & 536.3 & \\
BaP47 & 538.7 & & 32.4 & 364.2 & \\
BaP48 & 707.3 & & 21.9 & 552.4 & \\
\hline
\end{tabular}


Table C5 Comparison of $\mathrm{BaP}$-induced and spontaneous mutation spectra.

\begin{tabular}{|c|c|c|c|c|c|c|}
\hline \multirow{2}{*}{$\begin{array}{c}\text { Mutated } \\
\text { Nucleotide }\end{array}$} & $\begin{array}{c}|c| \\
\text { Control } \\
\text { Independent } \\
\text { Mutation }^{\mathrm{a}}\end{array}$ & $\begin{array}{c}\geq 2 \\
\text { Independent } \\
\text { Mutations }\end{array}$ & $\begin{array}{c}>2 \\
\text { Independent } \\
\text { Mutations }\end{array}$ & $\begin{array}{c}\geq 1 \\
\text { Independent } \\
\text { Mutation }\end{array}$ & $\begin{array}{c}\text { B } 2 \\
\text { Independent } \\
\text { Mutations }\end{array}$ & $\begin{array}{c}>2 \\
\text { Independent } \\
\text { Mutations }\end{array}$ \\
\hline G:C & $83 \%$ & $88 \%$ & $100 \%$ & $89 \%$ & $98 \%$ & $100 \%$ \\
A:T & $17 \%$ & $12 \%$ & $0 \%$ & $11 \%$ & $2 \%$ & $0 \%$ \\
\hline
\end{tabular}

${ }^{a}$ The majority of mutations occurred at G:C base pairs.

${ }^{\mathrm{b}}$ All the nucleotide positions that had more than 2 independent mutations were at G:C base pairs. 


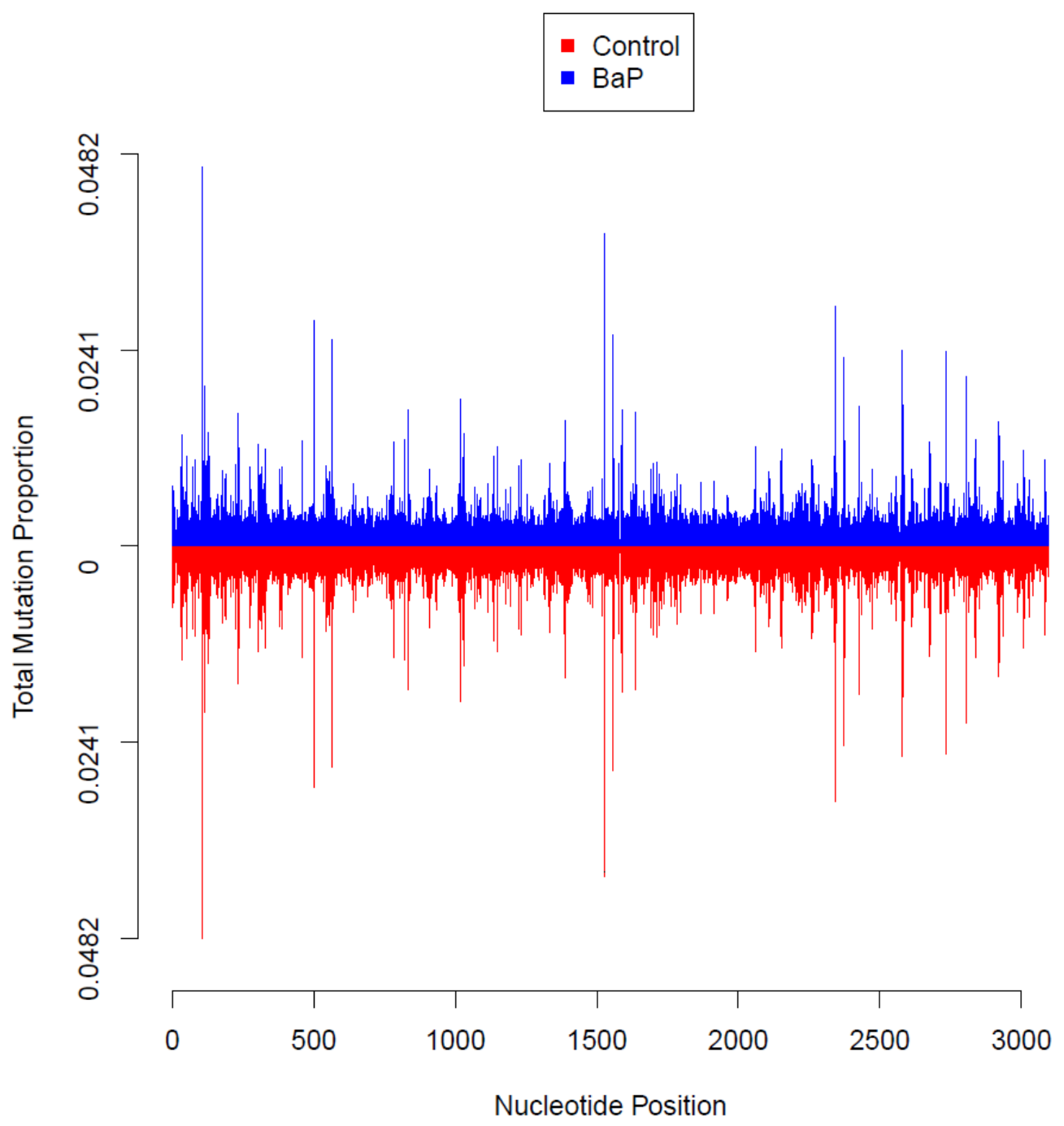

Figure C1 Comparison of mutant read proportions for substitutions at each nucleotide position across all samples. This figure demonstrates that there is symmetry between total mutation proportions of control and treated animals for each nucleotide position. Thus, each nucleotide position had biases (false mutations) that were consistent between control and $\mathrm{BaP}$ samples. It is important to identify the false mutation proportion as we have done to discriminate between background sequencing noise and true mutations. Failure to do so would result in the same false positives (large peaks above) being called in every sample. 
A) Position 41: $G \rightarrow T$
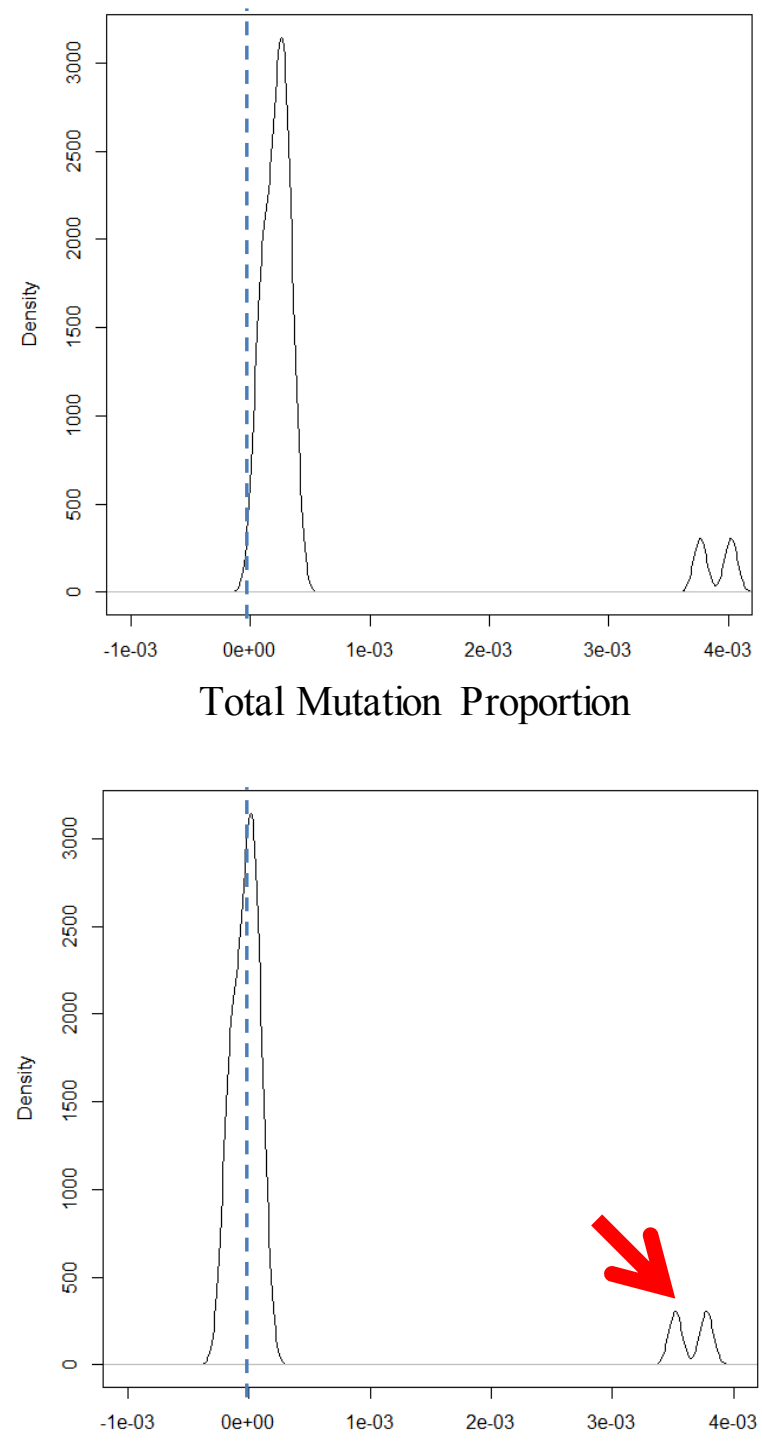

Total Mutation Proportion False Mutation Proportion
B) Position 42: $G \rightarrow T$
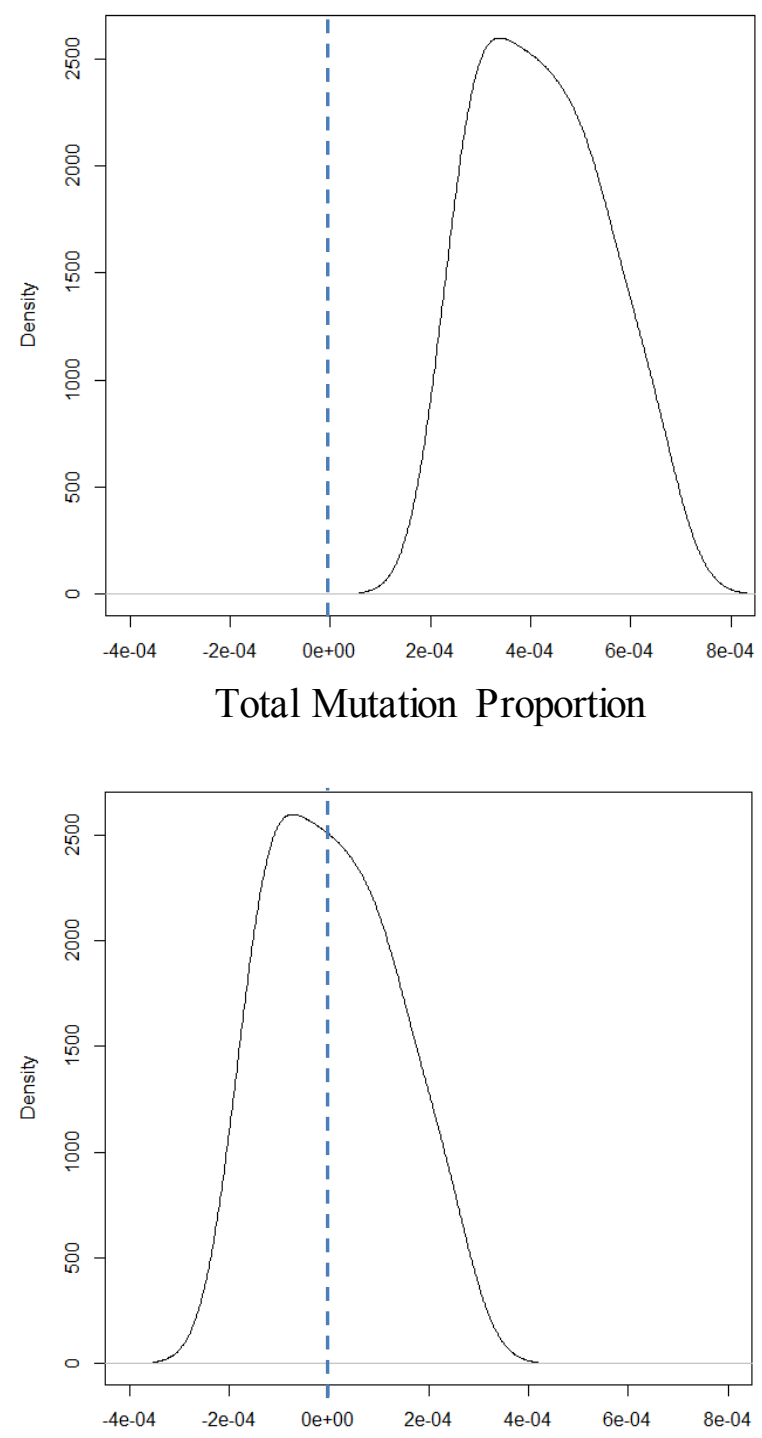

Total Mutation Proportion False Mutation Proportion

Figure C2 Example density graphs (smoothed histograms) for variant calls at 2 different nucleotide positions. (A) Density graph when there is a true mutation (red arrow) present in 1 of the samples. In the top panel, the total proportion of reads carrying the $\mathrm{G} \rightarrow \mathrm{T}$ variant at position 41 across most samples is $2.4 \times 10^{-4}$. This is the false mutation proportion (i.e., sequencing errors). By subtracting this false mutation proportion (bottom 
panel), the true mutation proportion (total mutation proportion - false mutation proportion) for most samples is now 0 (i.e., most samples have no mutations; shown by dotted blue line), except for the 2 peaks on the right of the graph. These peaks represent technical replicates of 1 sample and have true mutation proportions of $3.5 \times 10^{-3}$ and 3.7 $\times 10^{-3}$. Considering that 500 mutant plaques were pooled together for this animal, a true mutation should be present in $1 / 500$ or $2 \times 10^{-3}$ reads. Since both technical replicates have true mutation proportions above this threshold value, the $\mathrm{G} \rightarrow \mathrm{T}$ variant was called as a true mutation. (B) Density graph when there is no mutation in any of the samples. The top panel shows that the total proportion of reads carrying the $\mathrm{G} \rightarrow \mathrm{T}$ variant across all samples is $4.1 \times 10^{-4}$ for position 42 . After applying the false mutation proportion subtraction, all samples cluster around 0 and there is no evidence of a mutation. Note, in the top panel the total mutation proportion for all samples was above 0 without correcting for background. 


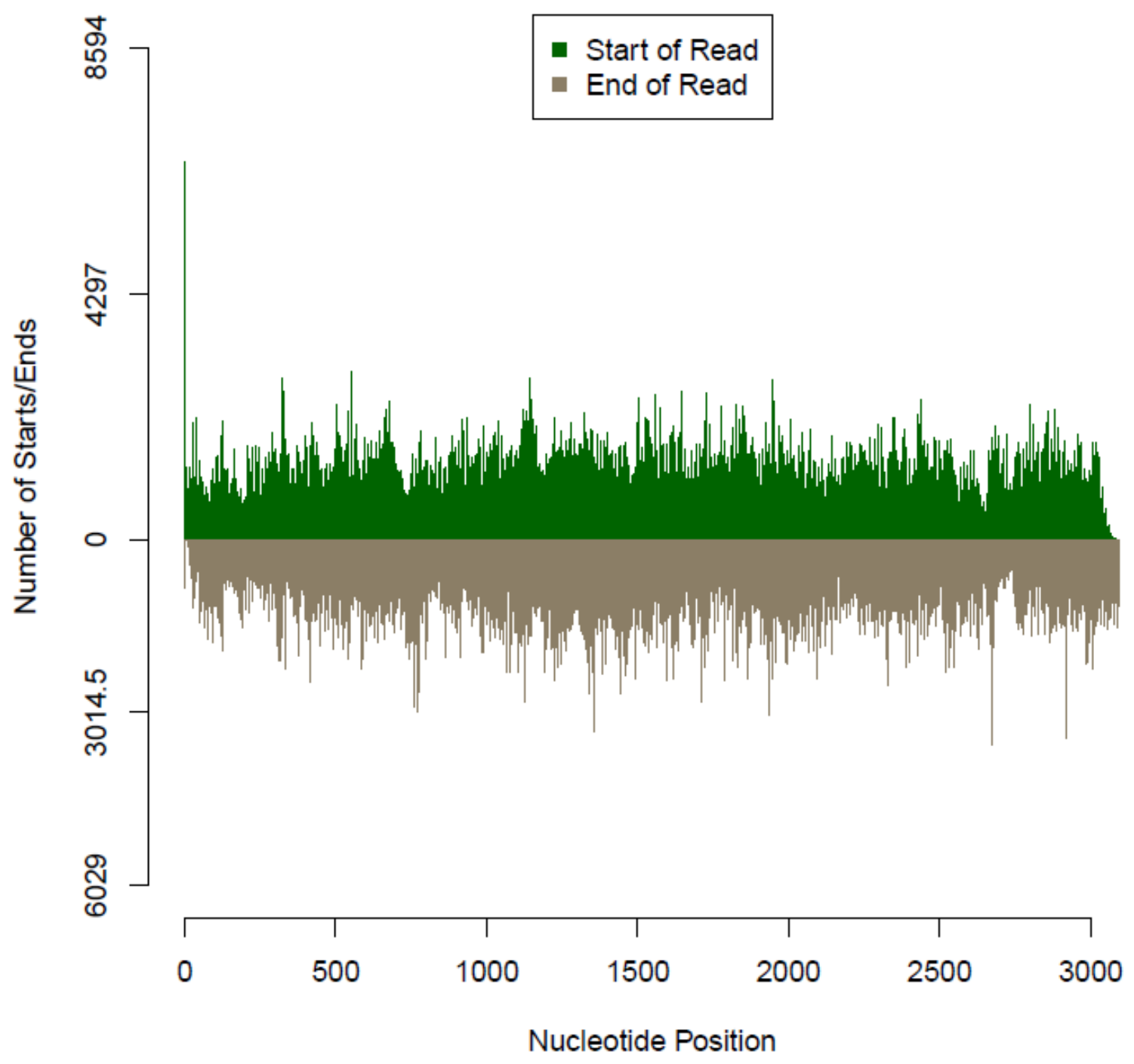

Figure C3 Distribution of read starts and ends. The locations of read starts and ends appear to be uniform across the $l a c Z$ gene. 


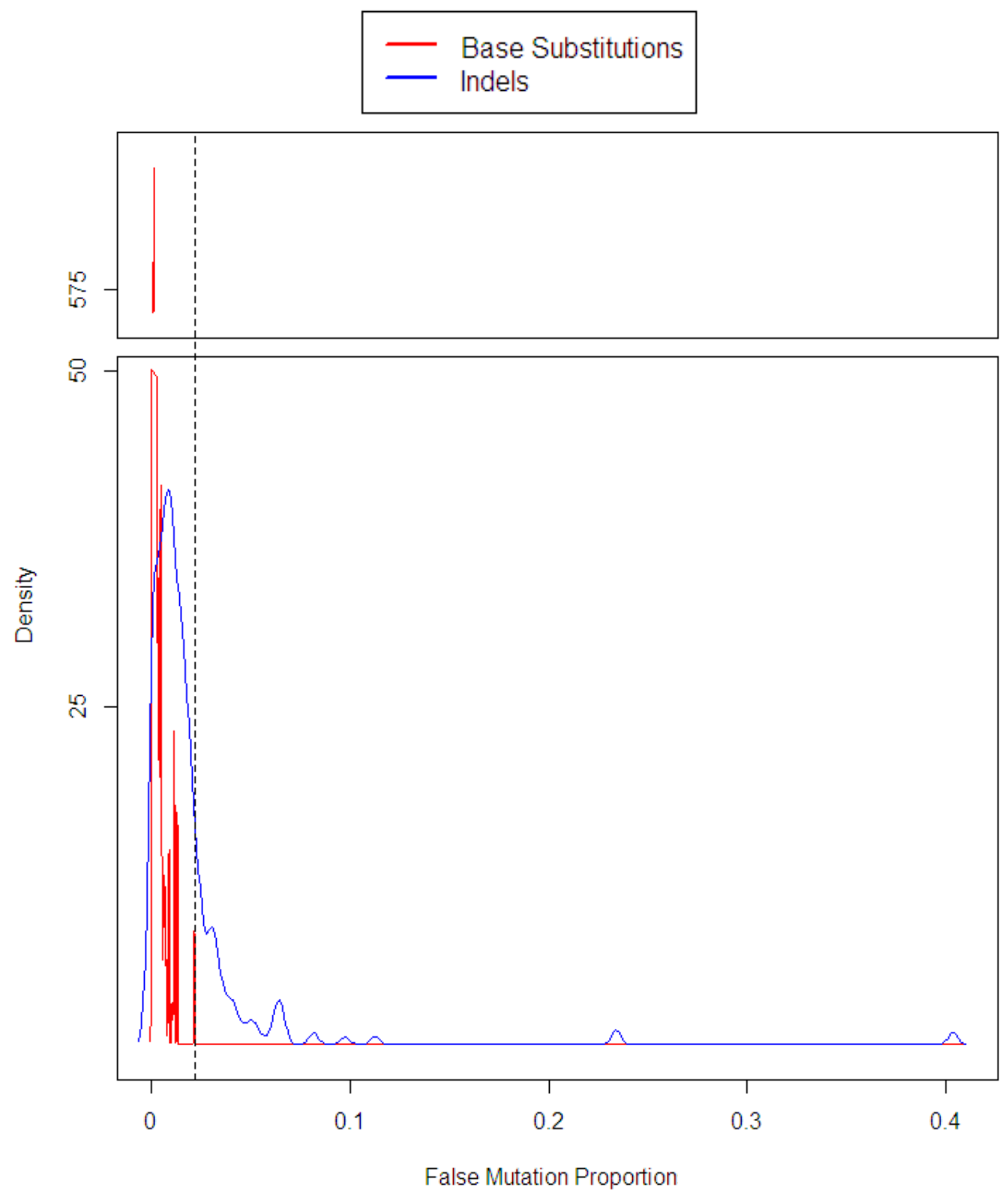

Figure C4 Comparison of the density of false mutation proportions between base substitutions (red) and indels (blue). The highest false mutation proportion where a base substitution was called was $2.15 \times 10^{-2}$ (dotted line). All indel calls at positions with false mutation proportions above $2.15 \times 10^{-2}$ were at error-prone sequencing regions and were therefore ignored. Based on this graph, the indels with high false mutation frequencies were outliers and their removal was necessary. 


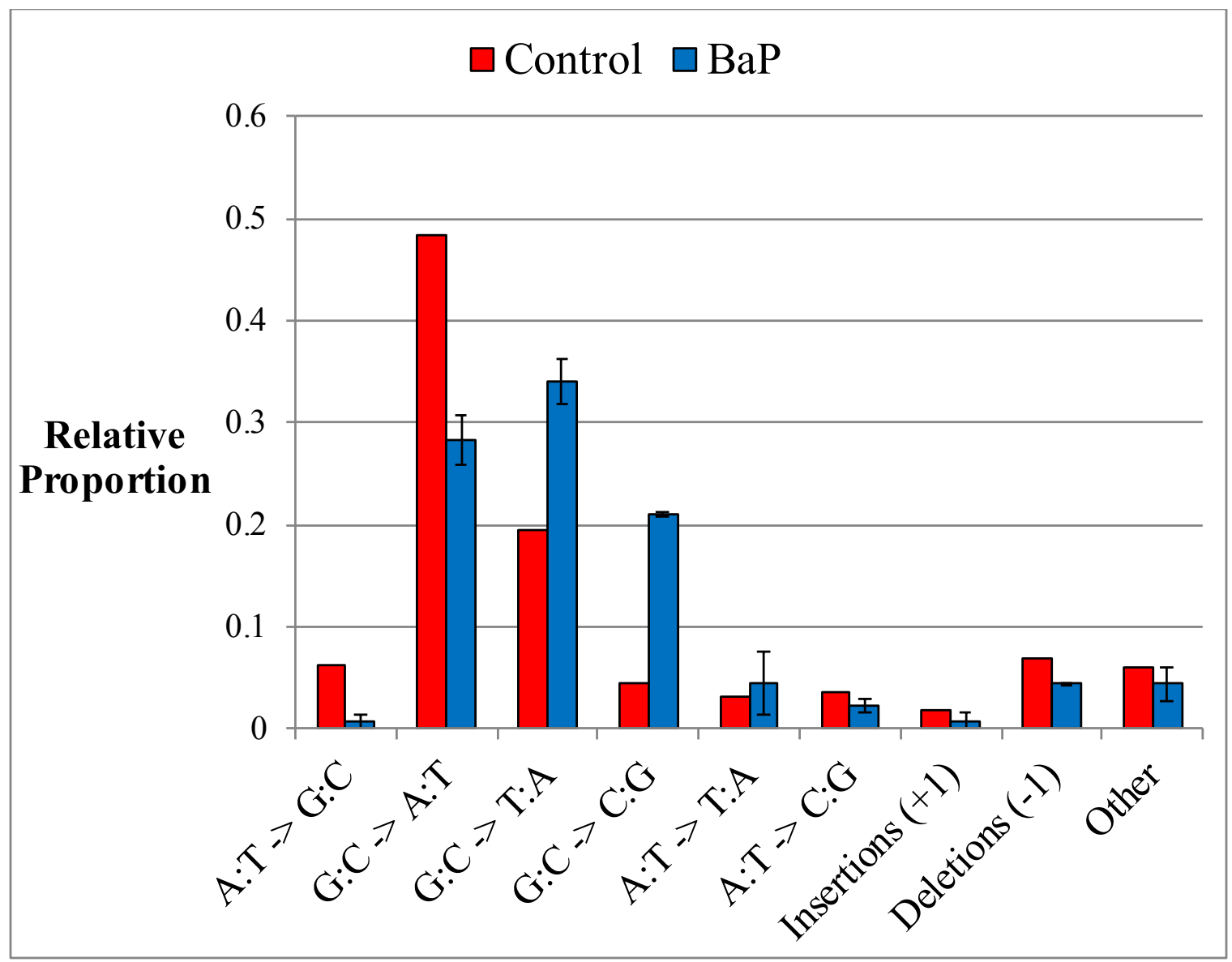

Figure C5 Relative proportion of control and $\mathrm{BaP}$-induced lacI mutations in liver from reference (Shane et al., 2000). The mutation spectra are based on 287 control mutants and $138 \mathrm{BaP}$ mutants (66 from samples collected at hepatectomy, 72 from samples collected at sacrifice). Error bars for the BaP group represent the standard error between the 2 sets of BaP mutants. 


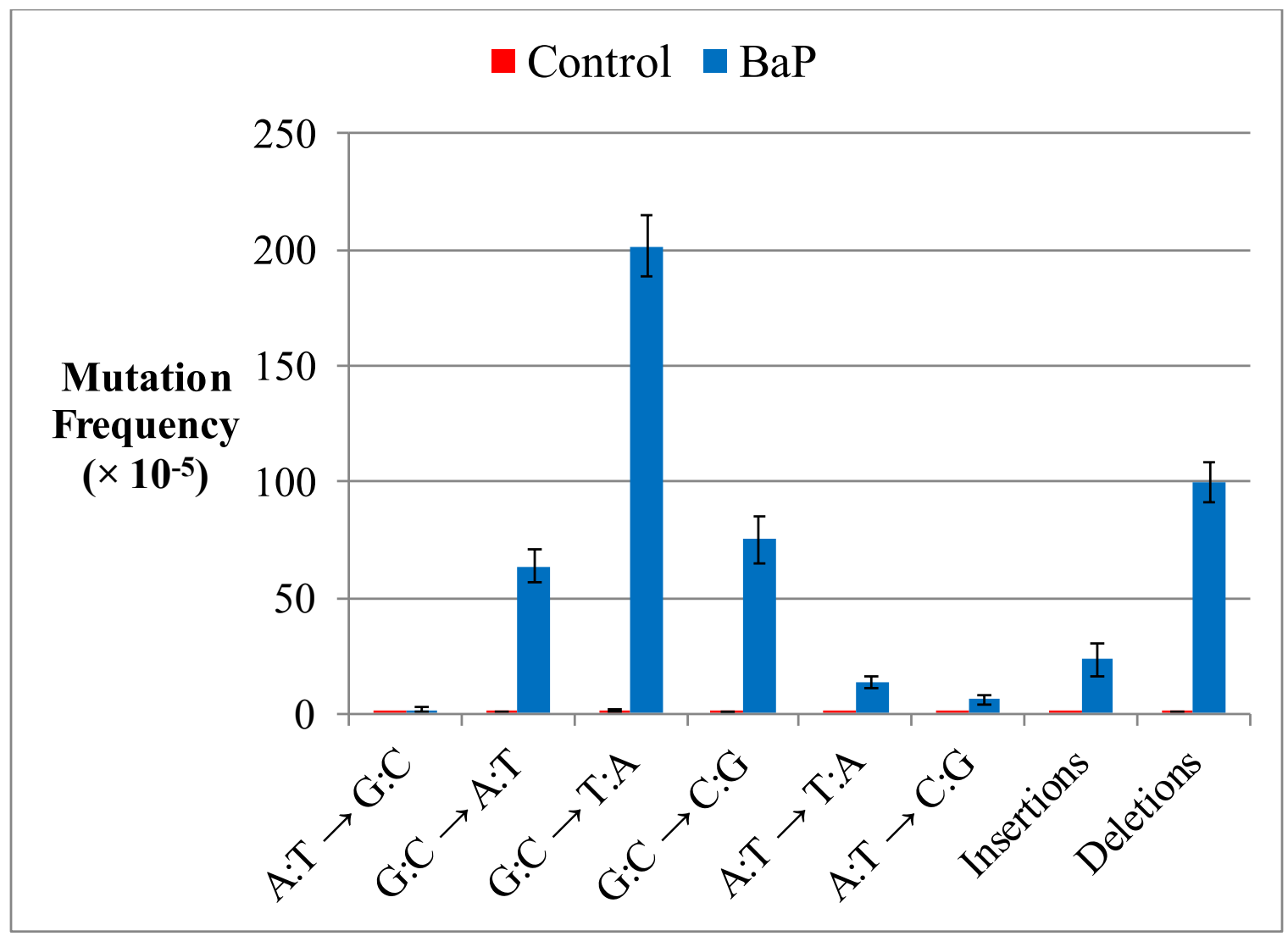

Figure C6 Bone marrow mutation spectra of control and BaP treatments. Only unique, independent mutations were considered and mutant frequencies were corrected for clonal expansion. All mutation types were significantly higher in the $\mathrm{BaP}$ samples (t-test: $\mathrm{P}<$ 0.01) except for A:T $\rightarrow$ G:C transitions. The error bars represent the standard error between samples. 


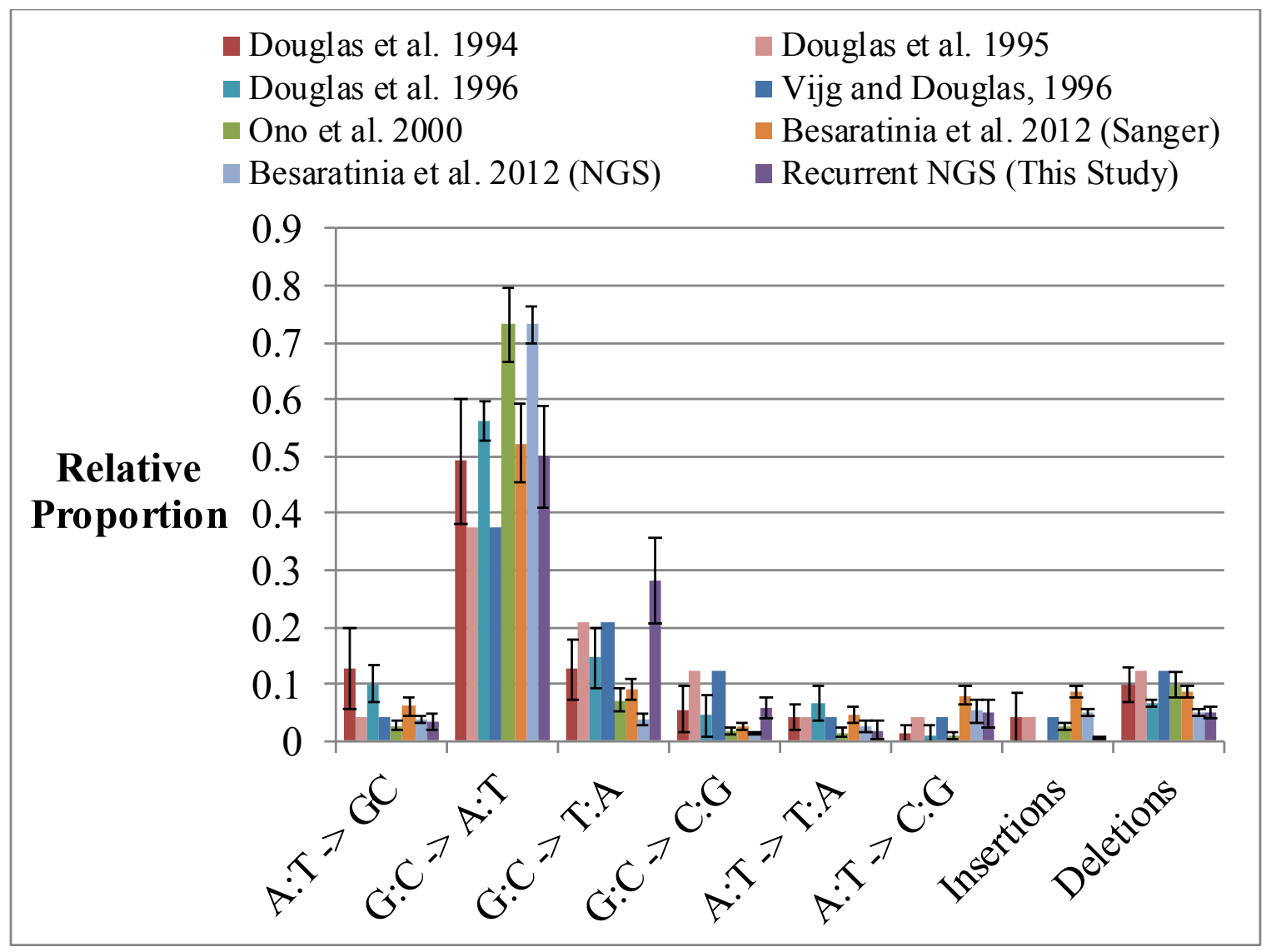

Figure C7 Comparison of spontaneous mutation spectra between our NGS dataset and other published datasets (Besaratinia et al., 2012; Douglas et al., 1994; Douglas et al., 1995; Douglas et al., 1996; Ono et al., 2000; Vijg and Douglas, 1996). The spectrum reported from our NGS dataset is based on total mutation counts to be consistent with the previously published NGS dataset (Besaratinia et al., 2012). Insertions and deletions were combined and reported as "indels" in reference (Besaratinia et al., 2012); in order to include this information in the above graph, the proportion of indels was split evenly between insertions and deletions. The results between our NGS dataset and the other datasets are highly comparable, with G:C $\rightarrow$ A:T transitions being the most common mutation type. The results are based on 71 mutants from 3 tissues (liver, bone marrow, 
germ cells) (Douglas et al., 1994), 24 male germ cell mutants (Douglas et al., 1995), 89 bone marrow/liver mutants (Douglas et al., 1996), 24 germ cell mutants (Vijg and Douglas, 1996), 321 mutants from 6 tissues (spleen, liver, heart, brain, skin, testis) (Ono et al., 2000), 591 lung mutants (Sanger) (Besaratinia et al., 2012), 451 lung mutants (NGS) (Besaratinia et al., 2012), and 572 bone marrow mutants (this study). Error bars are based on standard error between samples (this study), different mutant pools (Besaratinia et al., 2012), or tissues (other studies). 


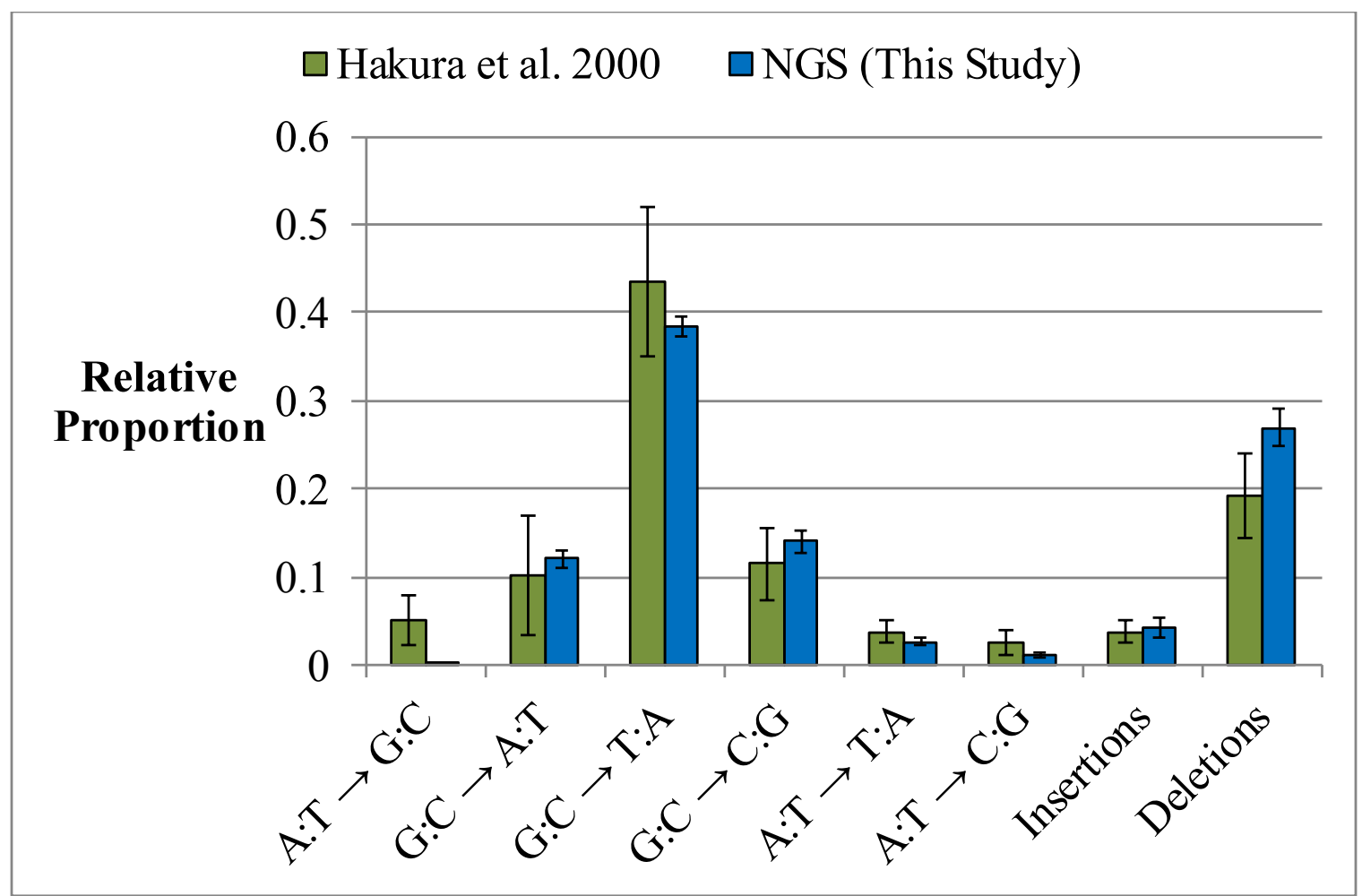

Figure C8 Comparison of $\mathrm{BaP}$-induced mutation spectrum in bone marrow measured using NGS with the mean mutation spectrum measured from 4 tissues (forestomach, spleen, colon, glandular stomach) using Sanger sequencing (Hakura et al., 2000). Error bars are based on standard error between samples (this study) or tissues (Hakura et al., 2000). 


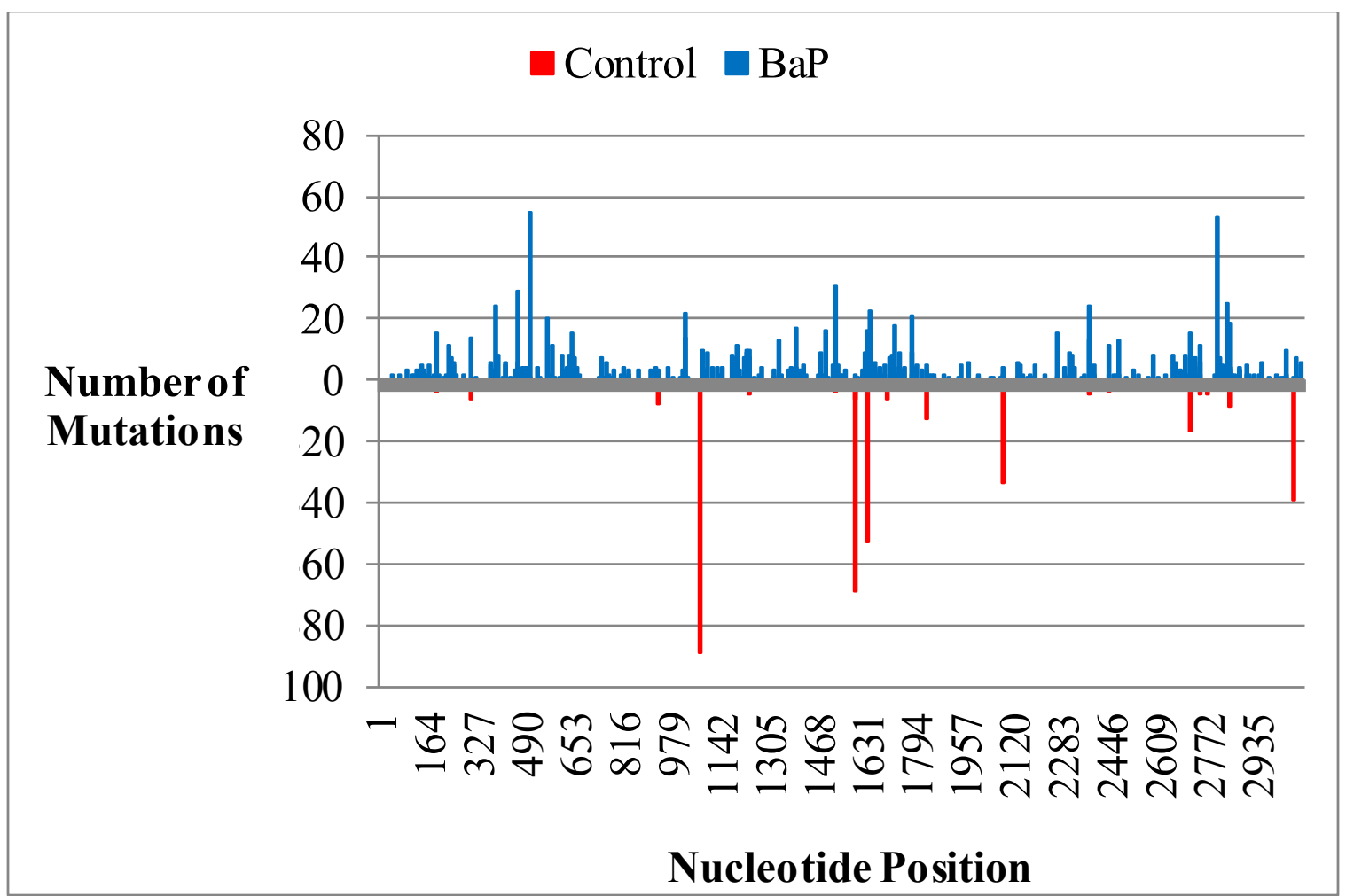

Figure C9 Distribution of non-unique base substitutions across the $l a c Z$ transgene for control and $\mathrm{BaP}$ samples. $\mathrm{BaP}$ base substitutions are on the positive $\mathrm{y}$-axis and control substitutions are on the negative $y$-axis. Nucleotide positions with several mutations are mostly due to clonal expansions. 


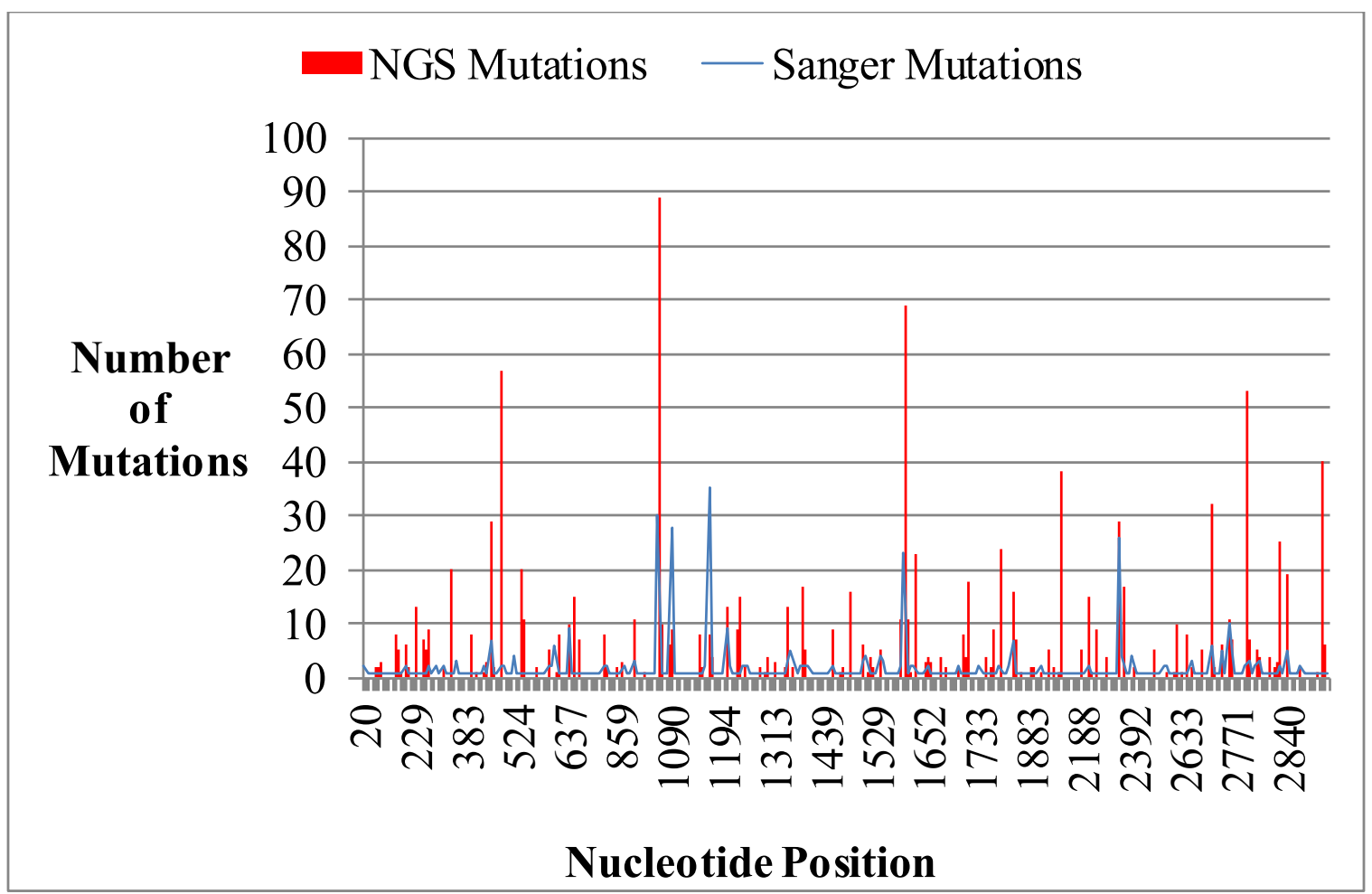

Figure C10 Comparison of lacZ mutation hotspots identified using Sanger sequencing (Douglas et al., 1994; Douglas et al., 1995; Douglas et al., 1996; Hakura et al., 2000;

Ikehata et al., 2000; Ono et al., 2000) with mutations identified using NGS at the same nucleotide positions in the present study. All mutations in the graph correspond to base substitutions. 


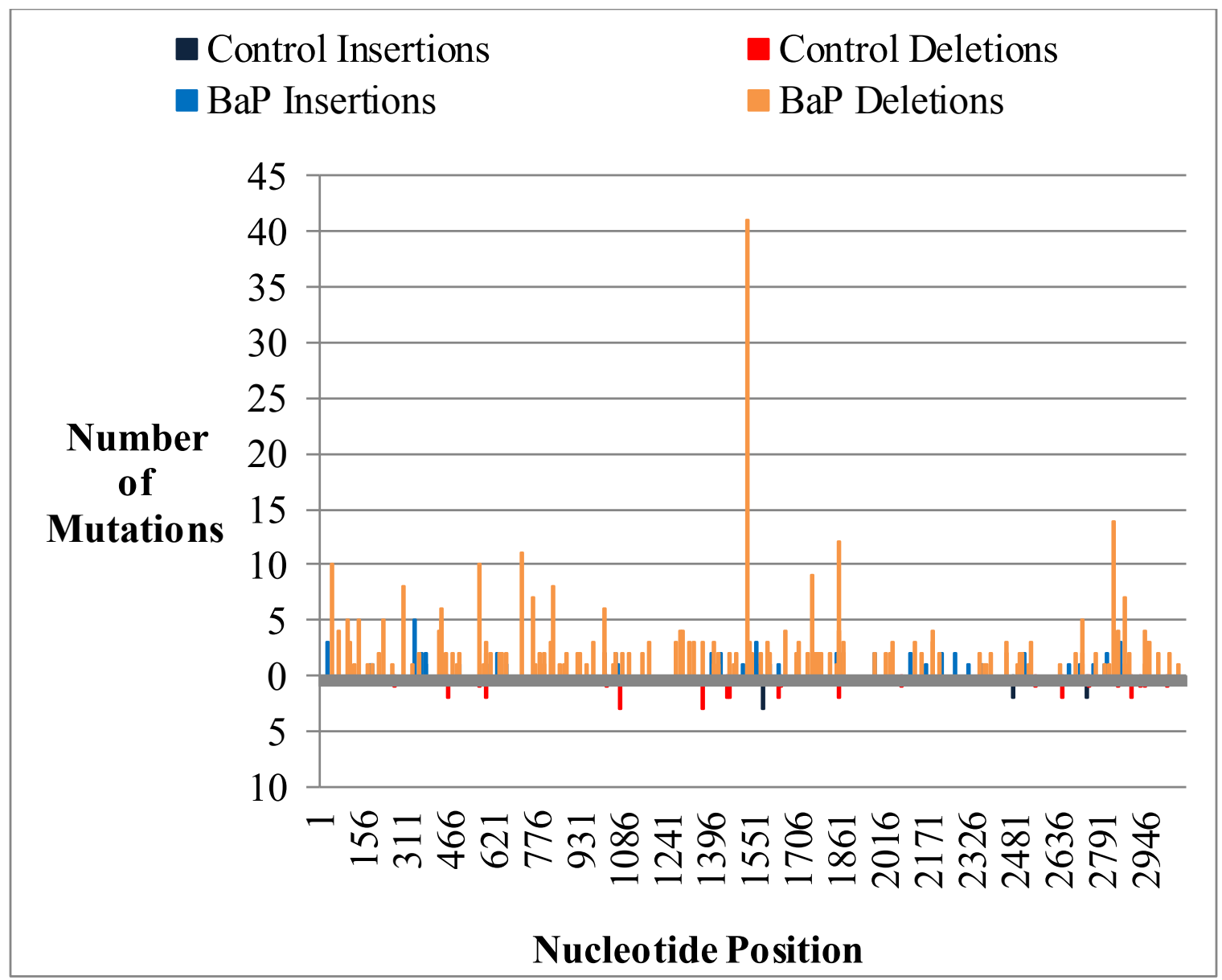

Figure C11 Distribution of non-unique indels across the lac $Z$ transgene for control and $\mathrm{BaP}$ samples. $\mathrm{BaP}$ indels are on the positive $\mathrm{y}$-axis and control indels are on the negative y-axis. The 41 deletions at position 1527 are due to microsatellite deletions of CG at $(\mathrm{CG})_{4}$ in $3 \mathrm{BaP}$ samples. 


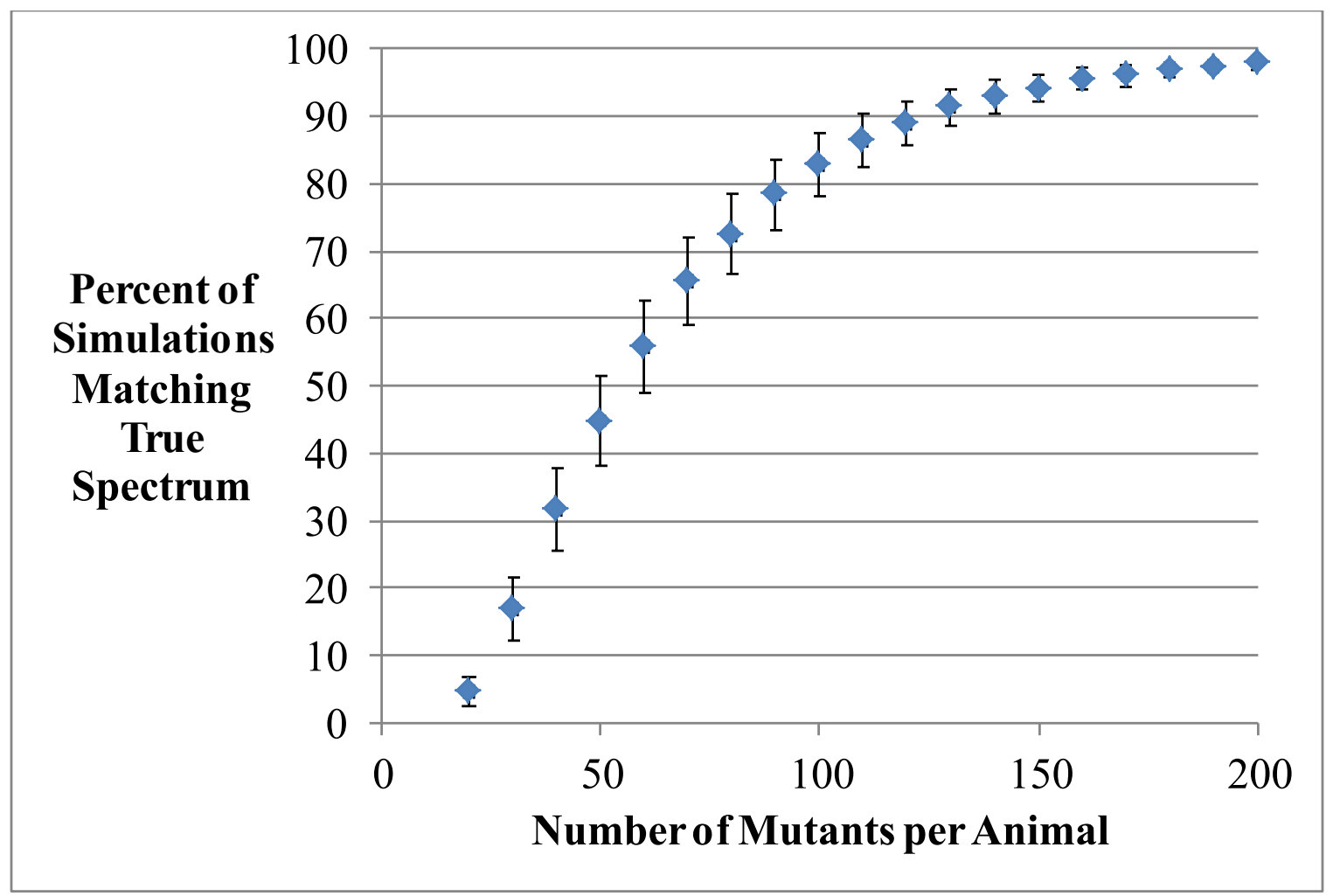

Figure C12 Results of simulations that use random sampling of $\mathrm{BaP}$ mutants to approximate the number of mutants per sample required to achieve a consistent mutation spectrum. The following steps were used: 1) different numbers of mutant plaques were randomly sampled from the available mutants in each BaP-treated animal; 2) the mutation spectrum (transitions, transversions, indels) was then determined using independent mutations that were sampled; and 3) the spectrum generated was compared to the true $\mathrm{BaP}$ spectrum for each animal using Pearson Chi-squared test. The percentage of simulations that gave the same spectrum $(\mathrm{P} \geq 0.05)$ out of 10,000 iterations is plotted on the y-axis, the number of mutants sampled per animal are on the x-axis. Error bars are based on standard error between samples. The saturation curves show that the spectrum consistency has a power of 0.8 at approximately 100 mutants per animal. The spectrum consistency is maximal around 200 mutants per sample. 


\section{C.3 Chapter 5}

Table C6 Mutant plaque and total plaque counts for individual animals.

\begin{tabular}{|c|c|c|c|c|c|c|c|}
\hline Time-point & Tissue & $\begin{array}{c}\text { Animal } \\
\text { ID }\end{array}$ & Dose & Mutants & Plaques & MF & Notes \\
\hline $28+3$ & cauda & 1 & 0 & 2 & 165004 & 1.2 & \\
\hline $28+3$ & cauda & 2 & 0 & 13 & 363804 & 3.6 & \\
\hline $28+3$ & cauda & 3 & 0 & 8 & 315264 & 2.5 & \\
\hline $28+3$ & cauda & 4 & 0 & 9 & 363970 & 2.5 & \\
\hline $28+3$ & cauda & 5 & 0 & 1 & 186541 & 0.5 & \\
\hline $28+3$ & cauda & 6 & 0 & 4 & 198138 & 2 & \\
\hline $28+3$ & cauda & $\mathrm{n} 13$ & 0 & 6 & 147941 & 4.1 & \\
\hline $28+3$ & cauda & n14 & 0 & 19 & 378548 & 5 & \\
\hline $28+3$ & cauda & $\mathrm{n} 15$ & 0 & 17 & 239720 & 7.1 & \\
\hline $28+3$ & cauda & $\mathrm{n} 16$ & 0 & 8 & 288591 & 2.8 & \\
\hline $28+3$ & cauda & $\mathrm{n} 17$ & 0 & 23 & 795200 & 2.9 & \\
\hline $28+3$ & cauda & $\mathrm{n} 18$ & 0 & 28 & 1003940 & 2.8 & \\
\hline $28+3$ & cauda & 8 & 12.5 & 4 & 253470 & 1.6 & \\
\hline $28+3$ & cauda & 9 & 12.5 & 1 & 275007 & 0.4 & \\
\hline $28+3$ & cauda & 10 & 12.5 & 4 & 170306 & 2.3 & \\
\hline $28+3$ & cauda & 11 & 12.5 & 1 & 170637 & 0.6 & \\
\hline $28+3$ & cauda & 12 & 12.5 & 4 & 196977 & 2 & \\
\hline $28+3$ & cauda & 13 & 25 & 4 & 261257 & 1.5 & \\
\hline $28+3$ & cauda & 15 & 25 & 6 & 225638 & 2.7 & \\
\hline $28+3$ & cauda & 16 & 25 & 3 & 125410 & 2.4 & \\
\hline $28+3$ & cauda & 17 & 25 & 55 & 207911 & 26.5 & omitted outlier \\
\hline $28+3$ & cauda & 18 & 25 & 11 & 301348 & 3.7 & \\
\hline $28+3$ & cauda & 20 & 50 & 6 & 283953 & 2.1 & \\
\hline $28+3$ & cauda & 21 & 50 & 10 & 196481 & 5.1 & \\
\hline $28+3$ & cauda & 22 & 50 & 4 & 208408 & 1.9 & \\
\hline $28+3$ & cauda & 23 & 50 & 7 & 259599 & 2.7 & \\
\hline $28+3$ & cauda & 24 & 50 & 6 & 153242 & 3.9 & \\
\hline $28+3$ & cauda & n43 & 100 & 23 & 1151052 & 2 & \\
\hline $28+3$ & cauda & n44 & 100 & 19 & 634172 & 3 & \\
\hline $28+3$ & cauda & $\mathrm{n} 45$ & 100 & 11 & 689836 & 1.6 & \\
\hline $28+3$ & cauda & n46 & 100 & 15 & 609322 & 2.5 & \\
\hline $28+3$ & cauda & $\mathrm{n} 47$ & 100 & 19 & 584804 & 3.2 & \\
\hline $28+3$ & $\begin{array}{c}\text { sem } \\
\text { tubules } \\
\text { sem }\end{array}$ & 1 & 0 & 4 & 243861 & 1.6 & \\
\hline $28+3$ & tubules & 4 & 0 & 7 & 342599 & 2 & \\
\hline $28+3$ & sem & 5 & 0 & 8 & 159371 & 5 & \\
\hline
\end{tabular}


tubules

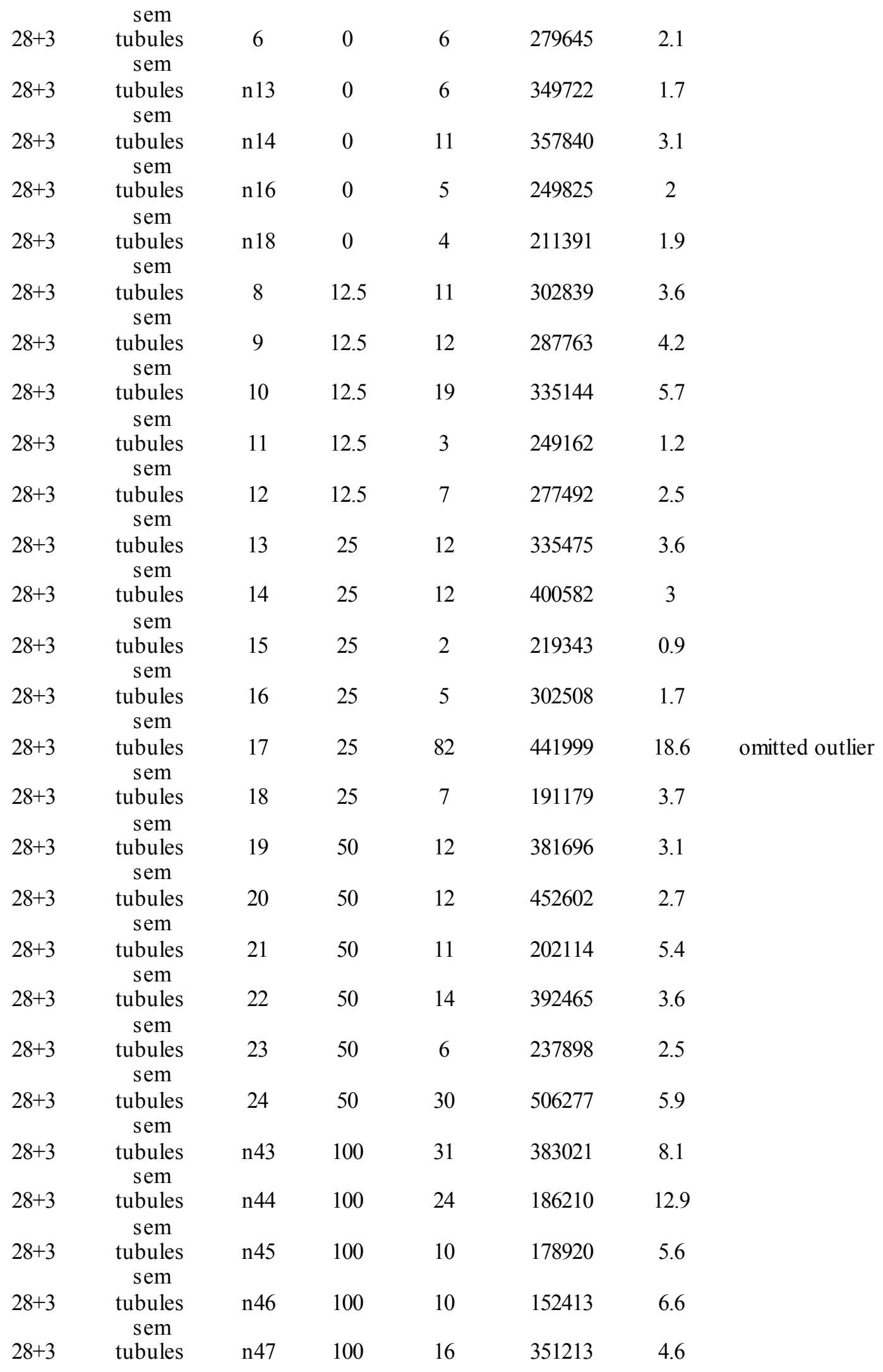




\begin{tabular}{|c|c|c|c|c|c|c|c|}
\hline $28+3$ & $\begin{array}{c}\text { sem } \\
\text { tubules }\end{array}$ & $\mathrm{n} 48$ & 100 & 11 & 1089424 & 1 & \\
\hline $28+42$ & cauda & 25 & 0 & 11 & 406877 & 2.7 & \\
\hline $28+42$ & cauda & 26 & 0 & 4 & 404227 & 1 & \\
\hline $28+42$ & cauda & 27 & 0 & 18 & 192836 & 9.3 & \\
\hline $28+42$ & cauda & 28 & 0 & 1 & 303501 & 0.3 & \\
\hline $28+42$ & cauda & 29 & 0 & 4 & 207415 & 1.9 & \\
\hline $28+42$ & cauda & 30 & 0 & 4 & 235247 & 1.7 & \\
\hline $28+42$ & cauda & $\mathrm{n} 20$ & 0 & 11 & 697126 & 1.6 & \\
\hline $28+42$ & cauda & $\mathrm{n} 21$ & 0 & 19 & 456578 & 4.2 & \\
\hline $28+42$ & cauda & $\mathrm{n} 22$ & 0 & 17 & 369768 & 4.6 & \\
\hline $28+42$ & cauda & $\mathrm{n} 23$ & 0 & 9 & 191345 & 4.7 & \\
\hline $28+42$ & cauda & $\mathrm{n} 24$ & 0 & 4 & 251482 & 1.6 & \\
\hline $28+42$ & cauda & 31 & 12.5 & 26 & 645437 & 4 & \\
\hline $28+42$ & cauda & 32 & 12.5 & 6 & 208077 & 2.9 & \\
\hline $28+42$ & cauda & 33 & 12.5 & 8 & 406877 & 2 & \\
\hline $28+42$ & cauda & 34 & 12.5 & 6 & 212053 & 2.8 & \\
\hline $28+42$ & cauda & 35 & 12.5 & 9 & 248500 & 3.6 & \\
\hline $28+42$ & cauda & 36 & 12.5 & 12 & 286935 & 4.2 & \\
\hline $28+42$ & cauda & 37 & 25 & 10 & 316755 & 3.2 & \\
\hline $28+42$ & cauda & 38 & 25 & 4 & 149763 & 2.7 & \\
\hline $28+42$ & cauda & 39 & 25 & 41 & 798513 & 5.1 & omitted outlier \\
\hline $28+42$ & cauda & 40 & 25 & 5 & 312779 & 1.6 & \\
\hline $28+42$ & cauda & 41 & 25 & 6 & 241211 & 2.5 & \\
\hline $28+42$ & cauda & 42 & 25 & 5 & 218680 & 2.3 & \\
\hline $28+42$ & cauda & 43 & 50 & 2 & 194327 & 1 & \\
\hline $28+42$ & cauda & 44 & 50 & 20 & 335309 & 6 & \\
\hline $28+42$ & cauda & 45 & 50 & 31 & 510253 & 6.1 & \\
\hline $28+42$ & cauda & 46 & 50 & 13 & 147112 & 8.8 & \\
\hline $28+42$ & cauda & 47 & 50 & 24 & 514229 & 4.7 & \\
\hline $28+42$ & cauda & 48 & 50 & 14 & 236572 & 5.9 & \\
\hline $28+42$ & cauda & $\mathrm{n} 49$ & 100 & 61 & 454589 & 13.4 & \\
\hline $28+42$ & cauda & n51 & 100 & 77 & 578508 & 13.3 & \\
\hline $28+42$ & cauda & $\mathrm{n} 52$ & 100 & 101 & 898576 & 11.2 & \\
\hline $28+42$ & cauda & n53 & 100 & 84 & 917131 & 9.2 & \\
\hline $28+42$ & cauda & $\mathrm{n} 54$ & 100 & 124 & 674760 & 18.4 & \\
\hline $28+70$ & cauda & 49 & 0 & 11 & 658525 & 1.7 & \\
\hline $28+70$ & cauda & 50 & 0 & 13 & 573040 & 2.3 & \\
\hline $28+70$ & cauda & 51 & 0 & 12 & 649414 & 1.8 & \\
\hline $28+70$ & cauda & 52 & 0 & 12 & 643947 & 1.9 & \\
\hline $28+70$ & cauda & 53 & 0 & 14 & 442993 & 3.2 & \\
\hline
\end{tabular}




\begin{tabular}{|c|c|c|c|c|c|c|c|}
\hline $28+70$ & cauda & 54 & 0 & 10 & 362479 & 2.8 & \\
\hline $28+70$ & cauda & $\mathrm{n} 26$ & 0 & 18 & 536429 & 3.4 & \\
\hline $28+70$ & cauda & $\mathrm{n} 27$ & 0 & 4 & 157715 & 2.5 & \\
\hline $28+70$ & cauda & $\mathrm{n} 28$ & 0 & 36 & 1094062 & 3.3 & \\
\hline $28+70$ & cauda & $\mathrm{n} 29$ & 0 & 34 & 506775 & 6.7 & omitted outlier \\
\hline $28+70$ & cauda & 55 & 12.5 & 7 & 336635 & 2.1 & \\
\hline $28+70$ & cauda & 56 & 12.5 & 12 & 744174 & 1.6 & \\
\hline $28+70$ & cauda & 57 & 12.5 & 18 & 867266 & 2.1 & \\
\hline $28+70$ & cauda & 58 & 12.5 & 12 & 273184 & 4.4 & \\
\hline $28+70$ & cauda & 59 & 12.5 & 9 & 386501 & 2.3 & \\
\hline $28+70$ & cauda & 60 & 12.5 & 17 & 488220 & 3.5 & \\
\hline $28+70$ & cauda & 61 & 25 & 2 & 134521 & 1.5 & \\
\hline $28+70$ & cauda & 62 & 25 & 22 & 587123 & 3.7 & \\
\hline $28+70$ & cauda & 63 & 25 & 2 & 194161 & 1 & \\
\hline $28+70$ & cauda & 64 & 25 & 29 & 808453 & 3.6 & \\
\hline $28+70$ & cauda & 65 & 25 & 10 & 537423 & 1.9 & \\
\hline $28+70$ & cauda & 68 & 50 & 11 & 174944 & 6.3 & \\
\hline $28+70$ & cauda & 69 & 50 & 7 & 146449 & 4.8 & \\
\hline $28+70$ & cauda & 70 & 50 & 8 & 263078 & 3 & \\
\hline $28+70$ & cauda & 71 & 50 & 10 & 227295 & 4.4 & \\
\hline $28+70$ & cauda & 72 & 50 & 50 & 1030613 & 4.9 & \\
\hline $28+70$ & cauda & n55 & 100 & 30 & 734897 & 4.1 & \\
\hline $28+70$ & cauda & n56 & 100 & 53 & 1029122 & 5.2 & \\
\hline $28+70$ & cauda & $\mathrm{n} 57$ & 100 & 34 & 548025 & 6.2 & \\
\hline $28+70$ & cauda & n58 & 100 & 35 & 789236 & 4.4 & \\
\hline
\end{tabular}


Table C7 Dose-response modelling output.

\begin{tabular}{|c|c|c|c|c|c|c|c|c|c|c|}
\hline $\begin{array}{c}\text { Days } \\
\text { post- } \\
\text { exposure }\end{array}$ & Tissue & $\begin{array}{l}\text { Variance } \\
\text { model }\end{array}$ & $\begin{array}{l}\text { Variance } \\
\text { fit p-value }\end{array}$ & $\begin{array}{c}\text { Dose- } \\
\text { response } \\
\text { model }\end{array}$ & $\begin{array}{c}\text { Goodnes } \\
\text { s-of-fit } \\
\text { p-value } \\
\end{array}$ & $\begin{array}{c}\text { BMD }_{10} \\
\left(\text { BMDL }^{2}\right) \\
{[\mathrm{mg} / \mathrm{kg} / \mathrm{day}]}\end{array}$ & $\begin{array}{c}\text { NOGEL } \\
{[\mathrm{mg} / \mathrm{kg} / \text { day] }}\end{array}$ & $\begin{array}{c}\text { Linear } \\
\leq \text { NOGEL } \\
\text { p-value }\end{array}$ & $\begin{array}{c}\text { BPD (BPDL) } \\
{[\mathrm{mg} / \mathrm{kg} / \text { day] }}\end{array}$ & $\begin{array}{c}\text { STD (STDL) } \\
{[\mathrm{mg} / \mathrm{kg} / \mathrm{day}]}\end{array}$ \\
\hline 3 & tubules & $\begin{array}{c}\text { non- } \\
\text { constant }\end{array}$ & 0.872 & exponential & 0.277 & $10.4(7.2)$ & 100 & 0.001 & no BPD & no STD \\
\hline 42 & cauda & constant & 0.001 & polynomial & 0.726 & $16.5(13.4)$ & 50 & 0.053 & $34.1(19.8)$ & $22.1(20)$ \\
\hline 70 & cauda & constant & 0.586 & exponential & 0.563 & $43.0(15.9)$ & 25 & 0.752 & no BPD & no STD \\
\hline
\end{tabular}

Bold p-values indicate a failed test (i.e. poor model fit) 


\section{C.4 Chapter 6}

Table C8 The position, type, and clone count for each independent lac $Z$ mutation sequenced.

\begin{tabular}{ccccccc} 
treatment & sample & position & reference & mutation type & mutation & $\begin{array}{c}\# \\
\text { clones }\end{array}$ \\
\hline Control & 1 & 125 & G & G:C - A:T & A & 3 \\
Control & 1 & 199 & - & deletion & T & 17 \\
Control & 1 & 229 & G & G:C $>$ T:A & T & 1 \\
Control & 1 & 593 & - & insertion & T & 9 \\
Control & 1 & 928 & G & G:C $>$ A:T & A & 3 \\
Control & 1 & 1187 & C & G:C $>$ A:T & T & 2 \\
Control & 1 & 1234 & G & G:C $>$ A:T & A & 1 \\
Control & 1 & 1252 & G & G:C $>$ A:T & A & 4 \\
Control & 1 & 1252 & G & G:C $>$ C:G & C & 2 \\
Control & 1 & 1628 & A & A:T $>$ G:C & G & 1 \\
Control & 1 & 1652 & G & G:C $>$ T:A & T & 1 \\
Control & 1 & 1773 & G & G:C $>$ A:T & A & 1 \\
Control & 1 & 2041 & C & G:C $>$ A:T & T & 3 \\
Control & 1 & 2375 & G & G:C $>$ T:A & T & 1 \\
Control & 1 & 2713 & G & G:C $>$ A:T & A & 2 \\
Control & 1 & 2953 & G & G:C $>$ T:A & T & 2 \\
\hline Control & 2 & 126 & G & G:C $>$ A:T & A & 4 \\
Control & 2 & 139 & G & G:C $>$ T:A & T & 2 \\
Control & 2 & 246 & C & G:C $>$ T:A & A & 2 \\
Control & 2 & 637 & G & G:C $>$ T:A & T & 2 \\
Control & 2 & 801 & G & G:C $>$ A:T & A & 3 \\
Control & 2 & 928 & G & G:C $>$ A:T & A & 2 \\
Control & 2 & 1072 & C & G:C $>$ A:T & T & 1 \\
Control & 2 & 1187 & C & G:C $>$ A:T & T & 5 \\
Control & 2 & 1477 & G & G:C $>$ T:A & T & 1 \\
Control & 2 & 1627 & G & G:C $>$ A:T & A & 3 \\
Control & 2 & 1850 & G & G:C $>$ C:G & C & 1 \\
Control & 2 & 2315 & C & G:C $>$ C:G & G & 3 \\
Control & 2 & 2374 & C & G:C $>$ A:T & T & 4 \\
Control & 2 & 2557 & A & A:T $>$ T:A & T & 4 \\
Control & 2 & 2659 & C & G:C $>$ A:T & T & 3 \\
Control & 2 & 2772 & G & G:C $>$ A:T & A & 3
\end{tabular}




\begin{tabular}{|c|c|c|c|c|c|c|}
\hline Control & 2 & 2830 & $\mathrm{C}$ & $\mathrm{G}: \mathrm{C}->\mathrm{C}: \mathrm{G}$ & G & 2 \\
\hline Control & 2 & 2873 & G & $\mathrm{G}: \mathrm{C}>\mathrm{C}: \mathrm{G}$ & $\mathrm{C}$ & 2 \\
\hline Control & 3 & 166 & $\mathrm{C}$ & G:C -> A:T & $\mathrm{T}$ & 1 \\
\hline Control & 3 & 192 & $\mathrm{G}$ & G:C $->A: T$ & A & 1 \\
\hline Control & 3 & 387 & $\mathrm{C}$ & G:C -> T:A & A & 4 \\
\hline Control & 3 & 638 & G & G:C -> A:T & A & 3 \\
\hline Control & 3 & 928 & G & G:C $->$ A:T & A & 2 \\
\hline Control & 3 & 1072 & $\mathrm{C}$ & G:C $->\mathrm{A}: \mathrm{T}$ & $\mathrm{T}$ & 2 \\
\hline Control & 3 & 1196 & $\mathrm{C}$ & G:C $->A: T$ & $\mathrm{~T}$ & 4 \\
\hline Control & 3 & 1342 & $\mathrm{C}$ & G:C $->\mathrm{A}: \mathrm{T}$ & $\mathrm{T}$ & 4 \\
\hline Control & 3 & 1603 & G & G:C -> T:A & $\mathrm{T}$ & 1 \\
\hline Control & 3 & 1632 & $\mathrm{C}$ & G:C -> T:A & A & 2 \\
\hline Control & 3 & 1709 & $\mathrm{G}$ & G:C $->A: T$ & A & 4 \\
\hline Control & 3 & 1721 & G & $\mathrm{G}: \mathrm{C}->\mathrm{C}: \mathrm{G}$ & $\mathrm{C}$ & 4 \\
\hline Control & 3 & 1722 & G & G:C -> A:T & A & 3 \\
\hline Control & 3 & 1775 & $\mathrm{C}$ & G:C -> T:A & A & 29 \\
\hline Control & 3 & 1831 & $\mathrm{G}$ & G:C -> T:A & $\mathrm{T}$ & 2 \\
\hline Control & 3 & 2149 & - & deletion & $\mathrm{C}$ & 1 \\
\hline Control & 3 & 2374 & $\mathrm{C}$ & G:C -> A:T & $\mathrm{T}$ & 9 \\
\hline Control & 3 & 2447 & G & G:C -> T:A & $\mathrm{T}$ & 4 \\
\hline Control & 3 & 2648 & - & deletion & G & 1 \\
\hline Control & 3 & 2713 & G & G:C $->\mathrm{A}: \mathrm{T}$ & A & 2 \\
\hline Control & 3 & 2744 & G & $\mathrm{G}: \mathrm{C}->\mathrm{A}: \mathrm{T}$ & A & 3 \\
\hline Control & 3 & 2835 & $\mathrm{C}$ & G:C -> T:A & A & 3 \\
\hline Control & 4 & 166 & $\mathrm{C}$ & G:C -> A:T & $\mathrm{T}$ & 4 \\
\hline Control & 4 & 203 & G & G:C $->\mathrm{A}: \mathrm{T}$ & A & 1 \\
\hline Control & 4 & 236 & G & $\mathrm{G}: \mathrm{C}->\mathrm{A}: \mathrm{T}$ & A & 3 \\
\hline Control & 4 & 476 & $\mathrm{G}$ & G:C $->A: T$ & A & 3 \\
\hline Control & 4 & 501 & $\mathrm{C}$ & G:C -> T:A & A & 2 \\
\hline Control & 4 & 505 & $\mathrm{C}$ & G:C $->\mathrm{A}: \mathrm{T}$ & $\mathrm{T}$ & 6 \\
\hline Control & 4 & 526 & G & G:C -> T:A & $\mathrm{T}$ & 2 \\
\hline Control & 4 & 679 & $\mathrm{C}$ & $\mathrm{G}: \mathrm{C}->\mathrm{A}: \mathrm{T}$ & $\mathrm{T}$ & 2 \\
\hline Control & 4 & 928 & G & G:C $->\mathrm{A}: \mathrm{T}$ & A & 2 \\
\hline Control & 4 & 1001 & - & deletion & $\mathrm{C}$ & 5 \\
\hline Control & 4 & 1030 & G & G:C -> T:A & $\mathrm{T}$ & 2 \\
\hline Control & 4 & 1072 & $\mathrm{C}$ & G:C $->\mathrm{A}: \mathrm{T}$ & $\mathrm{T}$ & 5 \\
\hline Control & 4 & 1187 & $\mathrm{C}$ & G:C $->\mathrm{A}: \mathrm{T}$ & $\mathrm{T}$ & 3 \\
\hline Control & 4 & 1252 & G & G:C -> A:T & A & 1 \\
\hline Control & 4 & 1674 & $\mathrm{C}$ & G:C $>>$ T:A & A & 2 \\
\hline Control & 4 & 1708 & G & G:C $->\mathrm{C}: \mathrm{G}$ & $\mathrm{C}$ & 5 \\
\hline Control & 4 & 1712 & G & G:C -> A:T & A & 1 \\
\hline Control & 4 & 2543 & G & G:C $->\mathrm{A}: \mathrm{T}$ & A & 3 \\
\hline Control & 4 & 2743 & $\mathrm{C}$ & G:C $>>$ A:T & $\mathrm{T}$ & 2 \\
\hline
\end{tabular}




\begin{tabular}{|c|c|c|c|c|c|c|}
\hline Control & 5 & 139 & $\mathrm{G}$ & G:C -> T:A & $\mathrm{T}$ & 3 \\
\hline Control & 5 & 166 & $\mathrm{C}$ & G:C $->A: T$ & $\mathrm{~T}$ & 3 \\
\hline Control & 5 & 191 & G & G:C $->A: T$ & A & 2 \\
\hline Control & 5 & 256 & G & G:C -> T:A & $\mathrm{T}$ & 1 \\
\hline Control & 5 & 428 & - & deletion & $\mathrm{C}$ & 2 \\
\hline Control & 5 & 430 & $\mathrm{C}$ & G:C $->A: T$ & $\mathrm{~T}$ & 1 \\
\hline Control & 5 & 491 & G & G:C $->A: T$ & A & 3 \\
\hline Control & 5 & 505 & $\mathrm{C}$ & G:C $->A: T$ & $\mathrm{~T}$ & 2 \\
\hline Control & 5 & 568 & - & ins ertion & A & 1 \\
\hline Control & 5 & 920 & G & $\mathrm{G}: \mathrm{C}->\mathrm{A}: \mathrm{T}$ & A & 3 \\
\hline Control & 5 & 920 & G & G:C -> T:A & $\mathrm{T}$ & 2 \\
\hline Control & 5 & 1018 & $\mathrm{G}$ & G:C -> T:A & $\mathrm{T}$ & 1 \\
\hline Control & 5 & 1342 & $\mathrm{C}$ & G:C $->A: T$ & $\mathrm{~T}$ & 2 \\
\hline Control & 5 & 1385 & $\mathrm{G}$ & G:C $->A: T$ & A & 7 \\
\hline Control & 5 & 1386 & G & G:C $->A: T$ & A & 2 \\
\hline Control & 5 & 1393 & $\mathrm{G}$ & G:C $->A: T$ & A & 4 \\
\hline Control & 5 & 1471 & $\mathrm{C}$ & G:C $->A: T$ & $\mathrm{~T}$ & 1 \\
\hline Control & 5 & 1613 & $\mathrm{C}$ & G:C $->C: G$ & G & 4 \\
\hline Control & 5 & 1627 & G & $\mathrm{G}: \mathrm{C}->\mathrm{A}: \mathrm{T}$ & A & 2 \\
\hline Control & 5 & 1643 & - & insertion & G & 5 \\
\hline Control & 5 & 1705 & $\mathrm{C}$ & G:C $->A: T$ & $\mathrm{~T}$ & 4 \\
\hline Control & 5 & 1782 & $\mathrm{C}$ & $\mathrm{G}: \mathrm{C}->\mathrm{C}: \mathrm{G}$ & G & 5 \\
\hline Control & 5 & 1809 & - & deletion & G & 3 \\
\hline Control & 5 & 1816 & $\mathrm{C}$ & G:C $->A: T$ & $\mathrm{~T}$ & 3 \\
\hline Control & 5 & 1854 & - & deletion & $\mathrm{C}$ & 1 \\
\hline Control & 5 & 1891 & $\mathrm{C}$ & G:C $->$ A:T & $\mathrm{T}$ & 2 \\
\hline Control & 5 & 2050 & $\mathrm{C}$ & G:C $->A: T$ & $\mathrm{~T}$ & 1 \\
\hline Control & 5 & 2440 & $\mathrm{G}$ & G:C -> T:A & $\mathrm{T}$ & 2 \\
\hline Control & 5 & 2518 & - & deletion & $\mathrm{T}$ & 2 \\
\hline Control & 5 & 2929 & - & deletion & G & 1 \\
\hline Control & 6 & 25 & $\mathrm{G}$ & G:C -> T:A & $\mathrm{T}$ & 6 \\
\hline Control & 6 & 42 & - & deletion & G & 2 \\
\hline Control & 6 & 203 & G & G:C $->A: T$ & A & 8 \\
\hline Control & 6 & 421 & $\mathrm{C}$ & G:C $->$ A:T & $\mathrm{T}$ & 1 \\
\hline Control & 6 & 591 & $\mathrm{G}$ & G:C $->A: T$ & A & 2 \\
\hline Control & 6 & 619 & $\mathrm{G}$ & G:C $->C: G$ & $\mathrm{C}$ & 2 \\
\hline Control & 6 & 988 & $\mathrm{G}$ & $\mathrm{G}: \mathrm{C}->\mathrm{A}: \mathrm{T}$ & A & 1 \\
\hline Control & 6 & 988 & $\mathrm{G}$ & G:C -> T:A & $\mathrm{T}$ & 5 \\
\hline Control & 6 & 1187 & $\mathrm{C}$ & G:C $->A: T$ & $\mathrm{~T}$ & 2 \\
\hline Control & 6 & 1211 & $\mathrm{G}$ & G:C $->A: T$ & A & 1 \\
\hline Control & 6 & 1399 & G & G:C -> T:A & $\mathrm{T}$ & 2 \\
\hline Control & 6 & 1477 & $\mathrm{G}$ & G:C $->$ A:T & A & 5 \\
\hline Control & 6 & 1572 & $\mathrm{G}$ & G:C -> A:T & $\mathrm{A}$ & 1 \\
\hline
\end{tabular}




\begin{tabular}{|c|c|c|c|c|c|c|}
\hline Control & 6 & 1627 & $\mathrm{G}$ & G:C -> A:T & A & 6 \\
\hline Control & 6 & 1627 & $\mathrm{G}$ & G:C $->C: G$ & $\mathrm{C}$ & 2 \\
\hline Control & 6 & 1657 & $\mathrm{G}$ & G:C -> A:T & A & 2 \\
\hline Control & 6 & 1773 & $\mathrm{G}$ & G:C $->$ A:T & A & 3 \\
\hline Control & 6 & 1831 & G & G:C $->A: T$ & A & 2 \\
\hline Control & 6 & 1979 & $\mathrm{G}$ & G:C $->$ A:T & A & 2 \\
\hline Control & 6 & 2177 & $\mathrm{G}$ & G:C -> A:T & A & 3 \\
\hline Control & 6 & 2374 & $\mathrm{C}$ & G:C $->A: T$ & $\mathrm{~T}$ & 3 \\
\hline Control & 6 & 2525 & - & deletion & $\mathrm{TA}$ & 2 \\
\hline Control & 6 & 2706 & - & deletion & $\mathrm{TG}$ & 1 \\
\hline Control & 6 & 2713 & G & G:C $->A: T$ & A & 7 \\
\hline Control & 6 & 2720 & $\mathrm{G}$ & G:C -> T:A & $\mathrm{T}$ & 1 \\
\hline Control & 6 & 2743 & $\mathrm{C}$ & G:C $->$ A:T & $\mathrm{T}$ & 3 \\
\hline $\mathrm{BaP}$ & 7 & 85 & $\mathrm{C}$ & G:C $>>$ A:T & $\mathrm{T}$ & 4 \\
\hline $\mathrm{BaP}$ & 7 & 236 & G & G:C $->A: T$ & A & 2 \\
\hline $\mathrm{BaP}$ & 7 & 241 & $\mathrm{G}$ & G:C -> T:A & $\mathrm{T}$ & 3 \\
\hline $\mathrm{BaP}$ & 7 & 307 & - & deletion & $\mathrm{C}$ & 4 \\
\hline $\mathrm{BaP}$ & 7 & 351 & - & deletion & $\mathrm{C}$ & 3 \\
\hline $\mathrm{BaP}$ & 7 & 363 & - & insertion & A & 2 \\
\hline $\mathrm{BaP}$ & 7 & 495 & - & deletion & G & 1 \\
\hline $\mathrm{BaP}$ & 7 & 519 & - & deletion & $\mathrm{C}$ & 1 \\
\hline $\mathrm{BaP}$ & 7 & 556 & $\mathrm{G}$ & G:C -> T:A & $\mathrm{T}$ & 5 \\
\hline $\mathrm{BaP}$ & 7 & 571 & - & deletion & $\mathrm{C}$ & 4 \\
\hline $\mathrm{BaP}$ & 7 & 572 & - & deletion & G & 2 \\
\hline $\mathrm{BaP}$ & 7 & 656 & $\mathrm{C}$ & G:C -> T:A & A & 2 \\
\hline $\mathrm{BaP}$ & 7 & 725 & - & deletion & $\mathrm{C}$ & 1 \\
\hline $\mathrm{BaP}$ & 7 & 759 & $\mathrm{C}$ & G:C -> T:A & A & 1 \\
\hline $\mathrm{BaP}$ & 7 & 759 & - & deletion & G & 5 \\
\hline $\mathrm{BaP}$ & 7 & 776 & - & deletion & $\mathrm{C}$ & 2 \\
\hline $\mathrm{BaP}$ & 7 & 786 & - & deletion & $\mathrm{AC}$ & 5 \\
\hline $\mathrm{BaP}$ & 7 & 829 & - & deletion & $\mathrm{C}$ & 3 \\
\hline $\mathrm{BaP}$ & 7 & 921 & $\mathrm{G}$ & G:C -> T:A & $\mathrm{T}$ & 2 \\
\hline $\mathrm{BaP}$ & 7 & 1009 & $\mathrm{G}$ & G:C $->C: G$ & $\mathrm{C}$ & 1 \\
\hline $\mathrm{BaP}$ & 7 & 1013 & - & deletion & $\mathrm{C}$ & 3 \\
\hline $\mathrm{BaP}$ & 7 & 1015 & - & deletion & G & 2 \\
\hline $\mathrm{BaP}$ & 7 & 1016 & $\mathrm{G}$ & G:C -> T:A & $\mathrm{T}$ & 2 \\
\hline $\mathrm{BaP}$ & 7 & 1174 & - & deletion & $\mathrm{C}$ & 1 \\
\hline $\mathrm{BaP}$ & 7 & 1330 & $\mathrm{G}$ & G:C -> T:A & $\mathrm{T}$ & 2 \\
\hline $\mathrm{BaP}$ & 7 & 1334 & $\mathrm{G}$ & $\mathrm{G}: \mathrm{C}->\mathrm{C}: \mathrm{G}$ & $\mathrm{C}$ & 2 \\
\hline $\mathrm{BaP}$ & 7 & 1370 & $\mathrm{C}$ & G:C -> T:A & A & 2 \\
\hline $\mathrm{BaP}$ & 7 & 1386 & $\mathrm{G}$ & G:C $->$ A:T & A & 3 \\
\hline $\mathrm{BaP}$ & 7 & 1495 & - & insertion & $\mathrm{T}$ & 3 \\
\hline $\mathrm{BaP}$ & 7 & 1498 & A & A:T -> C:G & $\mathrm{C}$ & 3 \\
\hline
\end{tabular}




\begin{tabular}{|c|c|c|c|c|c|c|}
\hline $\mathrm{BaP}$ & 7 & 1520 & $\mathrm{C}$ & G:C -> T:A & A & 2 \\
\hline $\mathrm{BaP}$ & 7 & 1527 & $\mathrm{C}$ & G:C -> T:A & A & 2 \\
\hline $\mathrm{BaP}$ & 7 & 1553 & - & deletion & $\mathrm{C}$ & 7 \\
\hline $\mathrm{BaP}$ & 7 & 1565 & - & deletion & GA & 2 \\
\hline $\mathrm{BaP}$ & 7 & 1626 & $\mathrm{C}$ & G:C -> T:A & A & 2 \\
\hline $\mathrm{BaP}$ & 7 & 1638 & $\mathrm{C}$ & G:C -> T:A & A & 1 \\
\hline $\mathrm{BaP}$ & 7 & 1639 & G & G:C -> C:G & $\mathrm{C}$ & 2 \\
\hline $\mathrm{BaP}$ & 7 & 1657 & G & G:C -> T:A & $\mathrm{T}$ & 4 \\
\hline $\mathrm{BaP}$ & 7 & 1674 & $\mathrm{C}$ & G:C -> T:A & A & 2 \\
\hline $\mathrm{BaP}$ & 7 & 1678 & $\mathrm{C}$ & G:C $->A: T$ & $\mathrm{~T}$ & 5 \\
\hline $\mathrm{BaP}$ & 7 & 1720 & $\mathrm{~T}$ & $\mathrm{~A}: \mathrm{T}->\mathrm{T}: \mathrm{A}$ & A & 1 \\
\hline $\mathrm{BaP}$ & 7 & 1720 & $\mathrm{~T}$ & $\mathrm{~A}: \mathrm{T}->\mathrm{G}: \mathrm{C}$ & $\mathrm{C}$ & 2 \\
\hline $\mathrm{BaP}$ & 7 & 1721 & G & G:C -> T:A & $\mathrm{T}$ & 2 \\
\hline $\mathrm{BaP}$ & 7 & 1722 & G & G:C -> T:A & $\mathrm{T}$ & 5 \\
\hline $\mathrm{BaP}$ & 7 & 1775 & $\mathrm{C}$ & G:C -> T:A & A & 2 \\
\hline $\mathrm{BaP}$ & 7 & 1871 & - & deletion & G & 2 \\
\hline $\mathrm{BaP}$ & 7 & 2013 & - & deletion & $\mathrm{G}$ & 2 \\
\hline $\mathrm{BaP}$ & 7 & 2017 & G & G:C -> T:A & $\mathrm{T}$ & 1 \\
\hline $\mathrm{BaP}$ & 7 & 2059 & $\mathrm{G}$ & G:C -> T:A & $\mathrm{T}$ & 3 \\
\hline $\mathrm{BaP}$ & 7 & 2083 & G & G:C -> T:A & $\mathrm{T}$ & 4 \\
\hline $\mathrm{BaP}$ & 7 & 2093 & - & insertion & $\mathrm{T}$ & 2 \\
\hline $\mathrm{BaP}$ & 7 & 2130 & $\mathrm{C}$ & $\mathrm{G}: \mathrm{C}->\mathrm{A}: \mathrm{T}$ & $\mathrm{T}$ & 2 \\
\hline $\mathrm{BaP}$ & 7 & 2152 & $\mathrm{G}$ & G:C -> T:A & $\mathrm{T}$ & 1 \\
\hline $\mathrm{BaP}$ & 7 & 2164 & G & G:C -> C:G & $\mathrm{C}$ & 1 \\
\hline $\mathrm{BaP}$ & 7 & 2166 & - & insertion & $\mathrm{T}$ & 2 \\
\hline $\mathrm{BaP}$ & 7 & 2211 & - & deletion & $\mathrm{G}$ & 2 \\
\hline $\mathrm{BaP}$ & 7 & 2303 & $\mathrm{C}$ & G:C -> T:A & A & 3 \\
\hline $\mathrm{BaP}$ & 7 & 2374 & $\mathrm{C}$ & G:C -> C:G & G & 2 \\
\hline $\mathrm{BaP}$ & 7 & 2375 & G & G:C -> T:A & $\mathrm{T}$ & 7 \\
\hline $\mathrm{BaP}$ & 7 & 2721 & - & deletion & $\mathrm{C}$ & 1 \\
\hline $\mathrm{BaP}$ & 7 & 2805 & $\mathrm{C}$ & G:C -> T:A & A & 2 \\
\hline $\mathrm{BaP}$ & 7 & 2807 & - & deletion & $\mathrm{C}$ & 2 \\
\hline $\mathrm{BaP}$ & 7 & 2809 & $\mathrm{~T}$ & A:T -> T:A & A & 2 \\
\hline $\mathrm{BaP}$ & 7 & 2873 & G & G:C $->C: G$ & $\mathrm{C}$ & 1 \\
\hline $\mathrm{BaP}$ & 7 & 2956 & $\mathrm{G}$ & G:C -> T:A & $\mathrm{T}$ & 3 \\
\hline $\mathrm{BaP}$ & 8 & 29 & - & insertion & $\mathrm{C}$ & 1 \\
\hline $\mathrm{BaP}$ & 8 & 125 & $\mathrm{G}$ & G:C $->$ A:T & A & 2 \\
\hline $\mathrm{BaP}$ & 8 & 187 & G & G:C -> T:A & $\mathrm{T}$ & 2 \\
\hline $\mathrm{BaP}$ & 8 & 229 & $\mathrm{G}$ & G:C -> T:A & $\mathrm{T}$ & 2 \\
\hline $\mathrm{BaP}$ & 8 & 319 & - & deletion & $\mathrm{C}$ & 4 \\
\hline $\mathrm{BaP}$ & 8 & 347 & - & insertion & $\mathrm{T}$ & 5 \\
\hline $\mathrm{BaP}$ & 8 & 416 & G & G:C $->A: T$ & A & 3 \\
\hline $\mathrm{BaP}$ & 8 & 461 & $\mathrm{C}$ & G:C $->C: G$ & $\mathrm{G}$ & 6 \\
\hline
\end{tabular}




\begin{tabular}{|c|c|c|c|c|c|c|}
\hline $\mathrm{BaP}$ & 8 & 501 & $\mathrm{C}$ & G:C -> T:A & $\mathrm{A}$ & 7 \\
\hline $\mathrm{BaP}$ & 8 & 619 & G & G:C -> T:A & $\mathrm{T}$ & 2 \\
\hline $\mathrm{BaP}$ & 8 & 629 & - & ins ertion & A & 2 \\
\hline $\mathrm{BaP}$ & 8 & 636 & - & deletion & $\mathrm{G}$ & 3 \\
\hline $\mathrm{BaP}$ & 8 & 679 & $\mathrm{C}$ & G:C $->A: T$ & $\mathrm{~T}$ & 2 \\
\hline $\mathrm{BaP}$ & 8 & 765 & - & deletion & $\mathrm{T}$ & 2 \\
\hline $\mathrm{BaP}$ & 8 & 767 & - & deletion & $\mathrm{G}$ & 2 \\
\hline $\mathrm{BaP}$ & 8 & 820 & - & deletion & $\mathrm{C}$ & 2 \\
\hline $\mathrm{BaP}$ & 8 & 825 & $\mathrm{C}$ & G:C $->C: G$ & $\mathrm{G}$ & 2 \\
\hline $\mathrm{BaP}$ & 8 & 928 & G & G:C $->A: T$ & A & 5 \\
\hline $\mathrm{BaP}$ & 8 & 1002 & $\mathrm{C}$ & G:C -> T:A & A & 2 \\
\hline $\mathrm{BaP}$ & 8 & 1016 & G & G:C $->C: G$ & $\mathrm{C}$ & 2 \\
\hline $\mathrm{BaP}$ & 8 & 1072 & $\mathrm{C}$ & G:C $->$ A:T & $\mathrm{T}$ & 3 \\
\hline $\mathrm{BaP}$ & 8 & 1141 & $\mathrm{G}$ & G:C -> T:A & $\mathrm{T}$ & 2 \\
\hline $\mathrm{BaP}$ & 8 & 1187 & $\mathrm{C}$ & G:C -> T:A & $\mathrm{A}$ & 3 \\
\hline $\mathrm{BaP}$ & 8 & 1187 & $\mathrm{C}$ & $\mathrm{G}: \mathrm{C}->\mathrm{C}: \mathrm{G}$ & $\mathrm{G}$ & 2 \\
\hline $\mathrm{BaP}$ & 8 & 1393 & $\mathrm{G}$ & G:C -> T:A & $\mathrm{T}$ & 1 \\
\hline $\mathrm{BaP}$ & 8 & 1534 & - & deletion & $\mathrm{T}$ & 4 \\
\hline $\mathrm{BaP}$ & 8 & 1542 & - & deletion & $\mathrm{G}$ & 3 \\
\hline $\mathrm{BaP}$ & 8 & 1638 & $\mathrm{C}$ & G:C -> T:A & A & 1 \\
\hline $\mathrm{BaP}$ & 8 & 1674 & $\mathrm{C}$ & G:C -> T:A & A & 1 \\
\hline $\mathrm{BaP}$ & 8 & 1722 & $\mathrm{G}$ & G:C -> T:A & $\mathrm{T}$ & 2 \\
\hline $\mathrm{BaP}$ & 8 & 1775 & $\mathrm{C}$ & G:C -> T:A & $\mathrm{A}$ & 3 \\
\hline $\mathrm{BaP}$ & 8 & 1832 & $\mathrm{G}$ & G:C -> T:A & $\mathrm{T}$ & 4 \\
\hline $\mathrm{BaP}$ & 8 & 2043 & - & deletion & $\mathrm{G}$ & 1 \\
\hline $\mathrm{BaP}$ & 8 & 2182 & $\mathrm{C}$ & $\mathrm{G}: \mathrm{C}->\mathrm{A}: \mathrm{T}$ & $\mathrm{T}$ & 2 \\
\hline $\mathrm{BaP}$ & 8 & 2183 & $\mathrm{~T}$ & $\mathrm{~A}: \mathrm{T}->\mathrm{T}: \mathrm{A}$ & A & 2 \\
\hline $\mathrm{BaP}$ & 8 & 2186 & - & deletion & $\mathrm{G}$ & 2 \\
\hline $\mathrm{BaP}$ & 8 & 2260 & - & deletion & G & 1 \\
\hline $\mathrm{BaP}$ & 8 & 2418 & - & insertion & A & 1 \\
\hline $\mathrm{BaP}$ & 8 & 2466 & $\mathrm{C}$ & G:C -> T:A & A & 2 \\
\hline $\mathrm{BaP}$ & 8 & 2660 & $\mathrm{G}$ & G:C $->C: G$ & $\mathrm{C}$ & 1 \\
\hline $\mathrm{BaP}$ & 8 & 2666 & G & G:C $->A: T$ & A & 1 \\
\hline $\mathrm{BaP}$ & 8 & 2669 & - & deletion & G & 2 \\
\hline $\mathrm{BaP}$ & 8 & 2721 & - & deletion & $\mathrm{C}$ & 5 \\
\hline $\mathrm{BaP}$ & 8 & 2724 & $\mathrm{G}$ & G:C -> T:A & $\mathrm{T}$ & 6 \\
\hline $\mathrm{BaP}$ & 8 & 2817 & $\mathrm{C}$ & G:C -> T:A & $\mathrm{A}$ & 2 \\
\hline $\mathrm{BaP}$ & 8 & 2828 & $\mathrm{~T}$ & $\mathrm{~A}: \mathrm{T}->\mathrm{G}: \mathrm{C}$ & $\mathrm{C}$ & 3 \\
\hline $\mathrm{BaP}$ & 8 & 2844 & - & deletion & GA & 5 \\
\hline $\mathrm{BaP}$ & 9 & 166 & $\mathrm{C}$ & G:C $->A: T$ & $\mathrm{~T}$ & 2 \\
\hline $\mathrm{BaP}$ & 9 & 175 & - & deletion & $\mathrm{G}$ & 3 \\
\hline $\mathrm{BaP}$ & 9 & 191 & G & G:C $->A: T$ & A & 2 \\
\hline $\mathrm{BaP}$ & 9 & 225 & - & deletion & $\mathrm{C}$ & 4 \\
\hline
\end{tabular}




\begin{tabular}{|c|c|c|c|c|c|}
\hline $\mathrm{BaP}$ & 9 & 228 & $\mathrm{G}$ & G:C -> T:A & $\mathrm{T}$ \\
\hline $\mathrm{BaP}$ & 9 & 237 & $\mathrm{G}$ & G:C $>>A: T$ & A \\
\hline $\mathrm{BaP}$ & 9 & 421 & $\mathrm{C}$ & G:C $->\mathrm{A}: \mathrm{T}$ & $\mathrm{T}$ \\
\hline $\mathrm{BaP}$ & 9 & 461 & $\mathrm{C}$ & G:C -> T:A & A \\
\hline $\mathrm{BaP}$ & 9 & 491 & $\mathrm{G}$ & $\mathrm{G}: \mathrm{C}->\mathrm{A}: \mathrm{T}$ & A \\
\hline $\mathrm{BaP}$ & 9 & 501 & - & deletion & G \\
\hline $\mathrm{BaP}$ & 9 & 572 & - & deletion & G \\
\hline $\mathrm{BaP}$ & 9 & 636 & - & deletion & G \\
\hline $\mathrm{BaP}$ & 9 & 637 & $\mathrm{G}$ & G:C -> T:A & $\mathrm{T}$ \\
\hline $\mathrm{BaP}$ & 9 & 638 & $\mathrm{G}$ & G:C -> T:A & $\mathrm{T}$ \\
\hline $\mathrm{BaP}$ & 9 & 647 & $\mathrm{G}$ & G:C $->\mathrm{C}: \mathrm{G}$ & $\mathrm{C}$ \\
\hline $\mathrm{BaP}$ & 9 & 656 & $\mathrm{C}$ & G:C -> T:A & A \\
\hline $\mathrm{BaP}$ & 9 & 759 & $\mathrm{C}$ & G:C -> T:A & A \\
\hline $\mathrm{BaP}$ & 9 & 859 & $\mathrm{G}$ & G:C -> T:A & $\mathrm{T}$ \\
\hline $\mathrm{BaP}$ & 9 & 887 & - & deletion & A \\
\hline $\mathrm{BaP}$ & 9 & 1014 & $\mathrm{C}$ & $\mathrm{G}: \mathrm{C}->\mathrm{A}: \mathrm{T}$ & $\mathrm{T}$ \\
\hline $\mathrm{BaP}$ & 9 & 1015 & - & deletion & G \\
\hline $\mathrm{BaP}$ & 9 & 1016 & $\mathrm{G}$ & $\mathrm{G}: \mathrm{C}->\mathrm{C}: \mathrm{G}$ & $\mathrm{C}$ \\
\hline $\mathrm{BaP}$ & 9 & 1016 & - & deletion & $\mathrm{C}$ \\
\hline $\mathrm{BaP}$ & 9 & 1030 & $\mathrm{G}$ & G:C -> T:A & $\mathrm{T}$ \\
\hline $\mathrm{BaP}$ & 9 & 1053 & - & deletion & G \\
\hline $\mathrm{BaP}$ & 9 & 1076 & $\mathrm{G}$ & G:C -> T:A & $\mathrm{T}$ \\
\hline $\mathrm{BaP}$ & 9 & 1155 & - & deletion & A \\
\hline $\mathrm{BaP}$ & 9 & 1175 & $\mathrm{C}$ & G:C -> T:A & A \\
\hline $\mathrm{BaP}$ & 9 & 1187 & $\mathrm{C}$ & G:C -> T:A & A \\
\hline $\mathrm{BaP}$ & 9 & 1224 & $\mathrm{C}$ & G:C -> T:A & A \\
\hline $\mathrm{BaP}$ & 9 & 1264 & $\mathrm{G}$ & G:C -> T:A & $\mathrm{T}$ \\
\hline $\mathrm{BaP}$ & 9 & 1388 & $\mathrm{C}$ & G:C -> T:A & A \\
\hline $\mathrm{BaP}$ & 9 & 1393 & $\mathrm{G}$ & G:C $>$ C:G & $\mathrm{C}$ \\
\hline $\mathrm{BaP}$ & 9 & 1406 & $\mathrm{G}$ & G:C -> T:A & $\mathrm{T}$ \\
\hline $\mathrm{BaP}$ & 9 & 1461 & - & insertion & $\mathrm{T}$ \\
\hline $\mathrm{BaP}$ & 9 & 1518 & $\mathrm{C}$ & G:C -> T:A & A \\
\hline $\mathrm{BaP}$ & 9 & 1520 & $\mathrm{C}$ & G:C -> T:A & A \\
\hline $\mathrm{BaP}$ & 9 & 1533 & - & deletion & G \\
\hline $\mathrm{BaP}$ & 9 & 1551 & - & deletion & $\mathrm{T}$ \\
\hline $\mathrm{BaP}$ & 9 & 1627 & $\mathrm{G}$ & $\mathrm{G}: \mathrm{C}->\mathrm{A}: \mathrm{T}$ & A \\
\hline $\mathrm{BaP}$ & 9 & 1639 & $\mathrm{G}$ & G:C $->\mathrm{C}: \mathrm{G}$ & $\mathrm{C}$ \\
\hline $\mathrm{BaP}$ & 9 & 1650 & $\mathrm{C}$ & G:C -> T:A & A \\
\hline $\mathrm{BaP}$ & 9 & 1666 & - & deletion & $\mathrm{C}$ \\
\hline $\mathrm{BaP}$ & 9 & 1669 & A & $\mathrm{A}: \mathrm{T}->\mathrm{T}: \mathrm{A}$ & $\mathrm{T}$ \\
\hline $\mathrm{BaP}$ & 9 & 1673 & - & deletion & $\mathrm{C}$ \\
\hline $\mathrm{BaP}$ & 9 & 1677 & $\mathrm{G}$ & G:C $->\mathrm{A}: \mathrm{T}$ & A \\
\hline $\mathrm{BaP}$ & 9 & 1775 & - & deletion & $\mathrm{G}$ \\
\hline
\end{tabular}




\begin{tabular}{|c|c|c|c|c|c|c|}
\hline $\mathrm{BaP}$ & 9 & 1782 & $\mathrm{C}$ & G:C -> T:A & A & 7 \\
\hline $\mathrm{BaP}$ & 9 & 1784 & - & deletion & $\mathrm{C}$ & 2 \\
\hline $\mathrm{BaP}$ & 9 & 1831 & G & G:C -> C:G & $\mathrm{C}$ & 1 \\
\hline $\mathrm{BaP}$ & 9 & 2266 & G & G:C $>$ T:A & $\mathrm{T}$ & 2 \\
\hline $\mathrm{BaP}$ & 9 & 2357 & - & deletion & $\mathrm{GC}$ & 3 \\
\hline $\mathrm{BaP}$ & 9 & 2374 & $\mathrm{C}$ & G:C -> T:A & A & 2 \\
\hline $\mathrm{BaP}$ & 9 & 2375 & G & G:C -> T:A & $\mathrm{T}$ & 2 \\
\hline $\mathrm{BaP}$ & 9 & 2391 & $\mathrm{C}$ & G:C -> T:A & A & 1 \\
\hline $\mathrm{BaP}$ & 9 & 2467 & $\mathrm{C}$ & G:C $->A: T$ & $\mathrm{~T}$ & 3 \\
\hline $\mathrm{BaP}$ & 9 & 2495 & - & deletion & $\mathrm{G}$ & 2 \\
\hline $\mathrm{BaP}$ & 9 & 2586 & $\mathrm{C}$ & G:C -> C:G & G & 4 \\
\hline $\mathrm{BaP}$ & 9 & 2659 & $\mathrm{C}$ & G:C $->A: T$ & $\mathrm{~T}$ & 3 \\
\hline $\mathrm{BaP}$ & 9 & 2706 & - & deletion & $\mathrm{T}$ & 3 \\
\hline $\mathrm{BaP}$ & 9 & 2708 & G & $\mathrm{G}: \mathrm{C}->\mathrm{A}: \mathrm{T}$ & A & 3 \\
\hline $\mathrm{BaP}$ & 9 & 2720 & G & G:C -> T:A & $\mathrm{T}$ & 1 \\
\hline $\mathrm{BaP}$ & 9 & 2721 & - & deletion & $\mathrm{C}$ & 2 \\
\hline $\mathrm{BaP}$ & 9 & 2725 & $\mathrm{C}$ & G:C $->A: T$ & $\mathrm{~T}$ & 2 \\
\hline $\mathrm{BaP}$ & 9 & 2743 & $\mathrm{C}$ & G:C -> T:A & A & 5 \\
\hline $\mathrm{BaP}$ & 9 & 2744 & $\mathrm{G}$ & G:C $->A: T$ & A & 3 \\
\hline $\mathrm{BaP}$ & 9 & 2823 & - & deletion & $\mathrm{G}$ & 2 \\
\hline $\mathrm{BaP}$ & 9 & 2828 & $\mathrm{~T}$ & A:T -> G:C & $\mathrm{C}$ & 2 \\
\hline $\mathrm{BaP}$ & 9 & 2839 & - & deletion & CG & 2 \\
\hline $\mathrm{BaP}$ & 9 & 2856 & $\mathrm{~T}$ & $\mathrm{~A}: \mathrm{T}->\mathrm{T}: \mathrm{A}$ & A & 2 \\
\hline $\mathrm{BaP}$ & 9 & 2871 & G & G:C $->A: T$ & A & 3 \\
\hline $\mathrm{BaP}$ & 9 & 2873 & G & G:C -> C:G & $\mathrm{C}$ & 2 \\
\hline $\mathrm{BaP}$ & 10 & 211 & $\mathrm{G}$ & G:C -> C:G & $\mathrm{C}$ & 1 \\
\hline $\mathrm{BaP}$ & 10 & 235 & - & deletion & $\mathrm{G}$ & 3 \\
\hline $\mathrm{BaP}$ & 10 & 296 & - & deletion & $\mathrm{C}$ & 1 \\
\hline $\mathrm{BaP}$ & 10 & 297 & - & deletion & G & 2 \\
\hline $\mathrm{BaP}$ & 10 & 298 & G & G:C $->A: T$ & A & 1 \\
\hline $\mathrm{BaP}$ & 10 & 318 & $\mathrm{C}$ & G:C -> T:A & A & 2 \\
\hline $\mathrm{BaP}$ & 10 & 363 & $\mathrm{C}$ & G:C -> T:A & A & 1 \\
\hline $\mathrm{BaP}$ & 10 & 447 & - & deletion & $\mathrm{G}$ & 2 \\
\hline $\mathrm{BaP}$ & 10 & 452 & $\mathrm{G}$ & G:C -> T:A & $\mathrm{T}$ & 1 \\
\hline $\mathrm{BaP}$ & 10 & 501 & $\mathrm{C}$ & G:C -> T:A & A & 2 \\
\hline $\mathrm{BaP}$ & 10 & 591 & G & $\mathrm{G}: \mathrm{C}->\mathrm{A}: \mathrm{T}$ & A & 1 \\
\hline $\mathrm{BaP}$ & 10 & 625 & - & deletion & G & 2 \\
\hline $\mathrm{BaP}$ & 10 & 637 & G & G:C -> T:A & $\mathrm{T}$ & 2 \\
\hline $\mathrm{BaP}$ & 10 & 727 & - & deletion & $\mathrm{G}$ & 2 \\
\hline $\mathrm{BaP}$ & 10 & 739 & G & G:C -> T:A & $\mathrm{T}$ & 2 \\
\hline $\mathrm{BaP}$ & 10 & 759 & $\mathrm{C}$ & G:C -> T:A & A & 2 \\
\hline $\mathrm{BaP}$ & 10 & 782 & G & G:C -> T:A & $\mathrm{T}$ & 2 \\
\hline $\mathrm{BaP}$ & 10 & 808 & G & G:C -> T:A & $\mathrm{T}$ & 2 \\
\hline
\end{tabular}




\begin{tabular}{|c|c|c|c|c|c|c|}
\hline $\mathrm{BaP}$ & 10 & 879 & - & deletion & CG & 4 \\
\hline $\mathrm{BaP}$ & 10 & 950 & $\mathrm{C}$ & $\mathrm{G}: \mathrm{C}->\mathrm{A}: \mathrm{T}$ & $\mathrm{T}$ & 2 \\
\hline $\mathrm{BaP}$ & 10 & 950 & - & deletion & G & 4 \\
\hline $\mathrm{BaP}$ & 10 & 1013 & - & deletion & $\mathrm{C}$ & 4 \\
\hline $\mathrm{BaP}$ & 10 & 1090 & G & G:C -> T:A & $\mathrm{T}$ & 2 \\
\hline $\mathrm{BaP}$ & 10 & 1167 & - & deletion & TT & 3 \\
\hline $\mathrm{BaP}$ & 10 & 1224 & $\mathrm{C}$ & G:C -> T:A & A & 2 \\
\hline $\mathrm{BaP}$ & 10 & 1232 & - & deletion & $\mathrm{C}$ & 4 \\
\hline $\mathrm{BaP}$ & 10 & 1234 & G & $\mathrm{G}: \mathrm{C}->\mathrm{A}: \mathrm{T}$ & A & 1 \\
\hline $\mathrm{BaP}$ & 10 & 1235 & G & G:C -> T:A & $\mathrm{T}$ & 4 \\
\hline $\mathrm{BaP}$ & 10 & 1244 & - & deletion & G & 2 \\
\hline $\mathrm{BaP}$ & 10 & 1305 & - & deletion & $\mathrm{CC}$ & 2 \\
\hline $\mathrm{BaP}$ & 10 & 1342 & $\mathrm{C}$ & G:C $->A: T$ & $\mathrm{~T}$ & 1 \\
\hline $\mathrm{BaP}$ & 10 & 1406 & G & G:C -> T:A & $\mathrm{T}$ & 2 \\
\hline $\mathrm{BaP}$ & 10 & 1610 & - & deletion & $\mathrm{C}$ & 4 \\
\hline $\mathrm{BaP}$ & 10 & 1699 & - & deletion & G & 3 \\
\hline $\mathrm{BaP}$ & 10 & 1727 & G & $\mathrm{G}: \mathrm{C}->\mathrm{A}: \mathrm{T}$ & A & 2 \\
\hline $\mathrm{BaP}$ & 10 & 1739 & $\mathrm{C}$ & G:C -> T:A & A & 5 \\
\hline $\mathrm{BaP}$ & 10 & 1766 & - & deletion & $\mathrm{C}$ & 2 \\
\hline $\mathrm{BaP}$ & 10 & 2266 & $\mathrm{G}$ & G:C -> T:A & $\mathrm{T}$ & 2 \\
\hline $\mathrm{BaP}$ & 10 & 2303 & $\mathrm{C}$ & G:C -> T:A & A & 2 \\
\hline $\mathrm{BaP}$ & 10 & 2374 & $\mathrm{C}$ & G:C -> T:A & A & 3 \\
\hline $\mathrm{BaP}$ & 10 & 2374 & $\mathrm{C}$ & G:C $->C: G$ & G & 2 \\
\hline $\mathrm{BaP}$ & 10 & 2376 & $\mathrm{~T}$ & A:T -> G:C & $\mathrm{C}$ & 1 \\
\hline $\mathrm{BaP}$ & 10 & 2455 & - & insertion & $\mathrm{C}$ & 1 \\
\hline $\mathrm{BaP}$ & 10 & 2470 & - & deletion & $\mathrm{C}$ & 2 \\
\hline $\mathrm{BaP}$ & 10 & 2473 & G & G:C -> T:A & $\mathrm{T}$ & 2 \\
\hline $\mathrm{BaP}$ & 10 & 2538 & - & insertion & G & 2 \\
\hline $\mathrm{BaP}$ & 10 & 2586 & $\mathrm{C}$ & G:C -> T:A & A & 1 \\
\hline $\mathrm{BaP}$ & 10 & 2676 & $\mathrm{C}$ & G:C -> T:A & A & 4 \\
\hline $\mathrm{BaP}$ & 10 & 2713 & G & G:C $->C: G$ & $\mathrm{C}$ & 1 \\
\hline $\mathrm{BaP}$ & 10 & 2719 & G & G:C $->C: G$ & $\mathrm{C}$ & 2 \\
\hline $\mathrm{BaP}$ & 10 & 2728 & G & G:C -> T:A & $\mathrm{T}$ & 2 \\
\hline $\mathrm{BaP}$ & 10 & 2743 & $\mathrm{C}$ & G:C -> C:G & G & 1 \\
\hline $\mathrm{BaP}$ & 10 & 2805 & $\mathrm{C}$ & G:C -> T:A & A & 3 \\
\hline $\mathrm{BaP}$ & 10 & 2817 & $\mathrm{C}$ & G:C -> T:A & A & 2 \\
\hline $\mathrm{BaP}$ & 10 & 2835 & $\mathrm{C}$ & G:C -> T:A & A & 4 \\
\hline $\mathrm{BaP}$ & 10 & 2860 & $\mathrm{C}$ & G:C -> T:A & A & 1 \\
\hline $\mathrm{BaP}$ & 10 & 2871 & G & $\mathrm{G}: \mathrm{C}->\mathrm{A}: \mathrm{T}$ & A & 1 \\
\hline $\mathrm{BaP}$ & 10 & 2872 & $\mathrm{C}$ & G:C -> T:A & A & 1 \\
\hline $\mathrm{BaP}$ & 10 & 3078 & G & G:C $->A: T$ & A & 3 \\
\hline $\mathrm{BaP}$ & 11 & 51 & - & deletion & $\mathrm{C}$ & 1 \\
\hline $\mathrm{BaP}$ & 11 & 139 & G & G:C -> T:A & $\mathrm{T}$ & 1 \\
\hline
\end{tabular}




\begin{tabular}{|c|c|c|c|c|c|}
\hline $\mathrm{BaP}$ & 11 & 170 & - & deletion & $\mathrm{G}$ \\
\hline $\mathrm{BaP}$ & 11 & 187 & G & G:C -> T:A & $\mathrm{T}$ \\
\hline $\mathrm{BaP}$ & 11 & 303 & $\mathrm{C}$ & G:C -> T:A & A \\
\hline $\mathrm{BaP}$ & 11 & 455 & $\mathrm{~T}$ & $\mathrm{~A}: \mathrm{T}->\mathrm{T}: \mathrm{A}$ & A \\
\hline $\mathrm{BaP}$ & 11 & 461 & $\mathrm{C}$ & G:C -> T:A & A \\
\hline $\mathrm{BaP}$ & 11 & 501 & $\mathrm{C}$ & G:C -> T:A & A \\
\hline $\mathrm{BaP}$ & 11 & 647 & G & G:C -> C:G & $\mathrm{C}$ \\
\hline $\mathrm{BaP}$ & 11 & 651 & $\mathrm{C}$ & G:C $->$ A:T & $\mathrm{T}$ \\
\hline $\mathrm{BaP}$ & 11 & 776 & - & deletion & $\mathrm{C}$ \\
\hline $\mathrm{BaP}$ & 11 & 970 & - & insertion & $\mathrm{C}$ \\
\hline $\mathrm{BaP}$ & 11 & 1009 & G & G:C -> C:G & $\mathrm{C}$ \\
\hline $\mathrm{BaP}$ & 11 & 1013 & - & deletion & $\mathrm{C}$ \\
\hline $\mathrm{BaP}$ & 11 & 1016 & G & G:C -> T:A & $\mathrm{T}$ \\
\hline $\mathrm{BaP}$ & 11 & 1053 & - & deletion & G \\
\hline $\mathrm{BaP}$ & 11 & 1111 & $\mathrm{C}$ & G:C $->$ A:T & $\mathrm{T}$ \\
\hline $\mathrm{BaP}$ & 11 & 1123 & $\mathrm{G}$ & G:C -> T:A & $\mathrm{T}$ \\
\hline $\mathrm{BaP}$ & 11 & 1175 & $\mathrm{C}$ & G:C -> T:A & A \\
\hline $\mathrm{BaP}$ & 11 & 1189 & $\mathrm{C}$ & G:C -> T:A & A \\
\hline $\mathrm{BaP}$ & 11 & 1252 & $\mathrm{G}$ & G:C -> T:A & $\mathrm{T}$ \\
\hline $\mathrm{BaP}$ & 11 & 1388 & $\mathrm{C}$ & G:C -> T:A & A \\
\hline $\mathrm{BaP}$ & 11 & 1518 & $\mathrm{C}$ & G:C -> T:A & A \\
\hline $\mathrm{BaP}$ & 11 & 1544 & - & deletion & $\mathrm{C}$ \\
\hline $\mathrm{BaP}$ & 11 & 1548 & $\mathrm{G}$ & G:C $->$ A:T & A \\
\hline $\mathrm{BaP}$ & 11 & 1635 & $\mathrm{C}$ & G:C -> T:A & A \\
\hline $\mathrm{BaP}$ & 11 & 1674 & $\mathrm{C}$ & G:C -> T:A & A \\
\hline $\mathrm{BaP}$ & 11 & 1682 & $\mathrm{C}$ & G:C $\rightarrow$ A:T & $\mathrm{T}$ \\
\hline $\mathrm{BaP}$ & 11 & 1683 & $\mathrm{G}$ & G:C -> T:A & $\mathrm{T}$ \\
\hline $\mathrm{BaP}$ & 11 & 1712 & G & G:C -> T:A & $\mathrm{T}$ \\
\hline $\mathrm{BaP}$ & 11 & 1775 & $\mathrm{C}$ & G:C -> T:A & A \\
\hline $\mathrm{BaP}$ & 11 & 1814 & - & deletion & $\mathrm{C}$ \\
\hline $\mathrm{BaP}$ & 11 & 1852 & - & deletion & $\mathrm{CG}$ \\
\hline $\mathrm{BaP}$ & 11 & 1873 & $\mathrm{G}$ & G:C -> T:A & $\mathrm{T}$ \\
\hline $\mathrm{BaP}$ & 11 & 1875 & - & deletion & $\mathrm{G}$ \\
\hline $\mathrm{BaP}$ & 11 & 1912 & - & deletion & $\mathrm{C}$ \\
\hline $\mathrm{BaP}$ & 11 & 2083 & $\mathrm{G}$ & G:C -> T:A & $\mathrm{T}$ \\
\hline $\mathrm{BaP}$ & 11 & 2177 & $\mathrm{G}$ & G:C $->$ A:T & A \\
\hline $\mathrm{BaP}$ & 11 & 2251 & A & A:T -> T:A & $\mathrm{T}$ \\
\hline $\mathrm{BaP}$ & 11 & 2252 & $\mathrm{~T}$ & A:T -> T:A & A \\
\hline $\mathrm{BaP}$ & 11 & 2260 & - & deletion & $\mathrm{G}$ \\
\hline $\mathrm{BaP}$ & 11 & 2294 & - & deletion & $\mathrm{C}$ \\
\hline $\mathrm{BaP}$ & 11 & 2303 & $\mathrm{C}$ & G:C -> T:A & A \\
\hline $\mathrm{BaP}$ & 11 & 2374 & $\mathrm{C}$ & G:C -> T:A & A \\
\hline $\mathrm{BaP}$ & 11 & 2375 & G & $\mathrm{G}: \mathrm{C}->\mathrm{C}: \mathrm{G}$ & $\mathrm{C}$ \\
\hline
\end{tabular}




\begin{tabular}{llllcll}
$\mathrm{BaP}$ & 11 & 2676 & $\mathrm{C}$ & $\mathrm{G}: \mathrm{C}->\mathrm{T}: \mathrm{A}$ & $\mathrm{A}$ & 2 \\
$\mathrm{BaP}$ & 11 & 2720 & $\mathrm{G}$ & $\mathrm{G}: \mathrm{C}->\mathrm{T}: \mathrm{A}$ & $\mathrm{T}$ & 1 \\
$\mathrm{BaP}$ & 11 & 2743 & $\mathrm{C}$ & $\mathrm{G}: \mathrm{C}->\mathrm{T}: \mathrm{A}$ & $\mathrm{A}$ & 2 \\
$\mathrm{BaP}$ & 11 & 2743 & $\mathrm{C}$ & $\mathrm{G}: \mathrm{C}->\mathrm{C}: \mathrm{G}$ & $\mathrm{G}$ & 1 \\
$\mathrm{BaP}$ & 11 & 2839 & - & deletion & $\mathrm{CG}$ & 2 \\
$\mathrm{BaP}$ & 11 & 2869 & $\mathrm{~T}$ & $\mathrm{~A}: \mathrm{T}->\mathrm{T}: \mathrm{A}$ & $\mathrm{A}$ & 1 \\
$\mathrm{BaP}$ & 11 & 2949 & - & insertion & $\mathrm{T}$ & 3 \\
$\mathrm{BaP}$ & 11 & 3060 & $\mathrm{C}$ & G:C $>$ T:A & $\mathrm{A}$ & 2 \\
\hline
\end{tabular}


Table C9 Sequence context analysis of spontaneous or BaP-induced lacZ mutations recovered from sperm 42 days after treatment.

Legend -
pos=position
mutType=mutation Type
\#control=independent occurances in control
group
\#bap=independent occurances in BaP group
mutAA=amino acid change (standard abbreviations) caused by mutation, *:stop mutation, -:indel,
(s):silent
hpLength=length of homopolymer
hpSeq=homopolymer sequence (lower case is nuc in query position)
upHpLength=length of upstream homopolymer
downHpLength=length of downstream homopolymer
adjHpSeq=adjacent sequence if containing homopolyer (lower case is query nuc)
cpgLength=nucleotide length of CpG repeats
cpgSeq=sequence of CpG repeats (lower case is query nuc)

\begin{tabular}{|c|c|c|c|c|c|c|c|c|c|c|c|c|}
\hline pos & ref & mutType & $\begin{array}{c}\# \\
\text { control }\end{array}$ & $\begin{array}{c}\# \\
\text { bap }\end{array}$ & mutAA & hpLength & hpSeq & $\begin{array}{l}\text { upHp } \\
\text { Length }\end{array}$ & $\begin{array}{l}\text { downHp } \\
\text { Length }\end{array}$ & $\begin{array}{c}\text { adjHp } \\
\text { Seq }\end{array}$ & $\begin{array}{c}\text { cpg } \\
\text { Length }\end{array}$ & cpgSeq \\
\hline 25 & $\mathrm{~g}$ & $\mathrm{G}: \mathrm{C}->\mathrm{T}: \mathrm{A}$ & 1 & 0 & $*$ & 2 & $\mathrm{Gg}$ & 0 & 2 & gAA & 0 & \\
\hline 29 & $\mathrm{t}$ & insertion & 0 & 1 & - & 2 & $\mathrm{Tt}$ & 0 & 3 & $\mathrm{tCCC}$ & 0 & \\
\hline 42 & C & deletion & 1 & 0 & - & 4 & $\mathrm{CCCC}$ & 0 & 0 & & 2 & cG \\
\hline 51 & $a$ & deletion & 0 & 1 & - & 0 & & 4 & 0 & TTTTa & 0 & \\
\hline 85 & C & $G: C->A: T$ & 0 & 1 & $*$ & 3 & $\mathrm{CCc}$ & 0 & 2 & CAA & 0 & \\
\hline 125 & g & $G: C->A: T$ & 1 & 1 & $*$ & 2 & gG & 0 & 0 & & 0 & \\
\hline 126 & g & $G: C->A: T$ & 1 & 0 & $*$ & 2 & $\mathrm{Gg}$ & 0 & 0 & & 3 & gCG \\
\hline 139 & g & $\mathrm{G}: \mathrm{C}->\mathrm{T}: \mathrm{A}$ & 2 & 1 & $*$ & 0 & & 2 & 0 & $\mathrm{AAg}$ & 0 & \\
\hline 166 & C & $G: C->A: T$ & 3 & 1 & $*$ & 0 & & 2 & 0 & AAC & 0 & \\
\hline
\end{tabular}




\begin{tabular}{|c|c|c|c|c|c|c|c|c|c|c|c|c|}
\hline 170 & $\mathrm{t}$ & deletion & 0 & 1 & - & 2 & $\mathrm{Tt}$ & 0 & 0 & & 0 & \\
\hline 175 & $\mathrm{a}$ & deletion & 0 & 1 & - & 0 & & 0 & 0 & & 0 & \\
\hline 187 & $\mathrm{~g}$ & $\mathrm{G}: \mathrm{C}->\mathrm{T}: \mathrm{A}$ & 0 & 2 & $*$ & 0 & & 0 & 2 & gAA & 3 & $\mathrm{GCg}$ \\
\hline 191 & $\mathrm{~g}$ & $\mathrm{G}: \mathrm{C}->\mathrm{A}: \mathrm{T}$ & 1 & 1 & $*$ & 2 & gG & 0 & 0 & & 0 & \\
\hline 192 & $\mathrm{~g}$ & $\mathrm{G}: \mathrm{C}->\mathrm{A}: \mathrm{T}$ & 1 & 0 & * & 2 & $\mathrm{Gg}$ & 0 & 0 & & 4 & gCGC \\
\hline 199 & $\mathrm{~g}$ & deletion & 1 & 0 & - & 0 & & 3 & 2 & TTTgCC & 3 & gCC \\
\hline 203 & $\mathrm{~g}$ & $\mathrm{G}: \mathrm{C}->\mathrm{A}: \mathrm{T}$ & 2 & 0 & $*$ & 2 & gG & 0 & 0 & & 0 & \\
\hline 211 & g & $\mathrm{G}: \mathrm{C}->\mathrm{C}: \mathrm{G}$ & 0 & 1 & $P$ & 2 & $\mathrm{Gg}$ & 0 & 0 & & 2 & gC \\
\hline 225 & $\mathrm{~g}$ & deletion & 0 & 1 & - & 0 & & 0 & 2 & gCC & 2 & $\mathrm{gC}$ \\
\hline 228 & $\mathrm{~g}$ & $\mathrm{G}: \mathrm{C}->\mathrm{T}: \mathrm{A}$ & 0 & 1 & $P(s)$ & 2 & gG & 2 & 0 & $\mathrm{CCg}$ & 2 & $\mathrm{Cg}$ \\
\hline 229 & $\mathrm{~g}$ & $\mathrm{G}: \mathrm{C}->\mathrm{T}: \mathrm{A}$ & 1 & 1 & $*$ & 2 & $\mathrm{Gg}$ & 0 & 3 & gAAA & 0 & \\
\hline 235 & $\mathrm{t}$ & deletion & 0 & 1 & - & 0 & & 0 & 2 & $\mathrm{tGG}$ & 0 & \\
\hline 236 & $\mathrm{~g}$ & $\mathrm{G}: \mathrm{C}->\mathrm{A}: \mathrm{T}$ & 1 & 1 & * & 2 & gG & 0 & 0 & & 0 & \\
\hline 237 & $\mathrm{~g}$ & $\mathrm{G}: \mathrm{C}->\mathrm{A}: \mathrm{T}$ & 0 & 1 & $*$ & 2 & Gg & 0 & 0 & & 2 & $\mathrm{gC}$ \\
\hline 241 & g & $\mathrm{G}: \mathrm{C}->\mathrm{T}: \mathrm{A}$ & 0 & 1 & $*$ & 2 & Gg & 0 & 0 & & 0 & \\
\hline 246 & $c$ & $\mathrm{G}: \mathrm{C}->\mathrm{T}: \mathrm{A}$ & 1 & 0 & * & 0 & & 0 & 0 & & 3 & GcG \\
\hline 256 & $\mathrm{~g}$ & $\mathrm{G}: \mathrm{C}->\mathrm{T}: \mathrm{A}$ & 1 & 0 & $*$ & 0 & & 0 & 0 & & 0 & \\
\hline 296 & $a$ & deletion & 0 & 1 & - & 0 & & 0 & 0 & & 0 & \\
\hline 297 & $c$ & deletion & 0 & 1 & - & 0 & & 0 & 2 & cGG & 2 & $c G$ \\
\hline 298 & $\mathrm{~g}$ & $\mathrm{G}: \mathrm{C}->\mathrm{A}: \mathrm{T}$ & 0 & 1 & $S$ & 2 & gG & 0 & 0 & & 2 & $\mathrm{Cg}$ \\
\hline 303 & $c$ & $\mathrm{G}: \mathrm{C}->\mathrm{T}: \mathrm{A}$ & 0 & 1 & * & 0 & & 0 & 0 & & 2 & cG \\
\hline 307 & $\mathrm{~g}$ & deletion & 0 & 1 & - & 0 & & 0 & 0 & & 4 & gCGC \\
\hline 318 & $c$ & $\mathrm{G}: \mathrm{C}->\mathrm{T}: \mathrm{A}$ & 0 & 1 & $*$ & 0 & & 0 & 0 & & 0 & \\
\hline 319 & $\mathrm{a}$ & deletion & 0 & 1 & - & 0 & & 0 & 2 & $\mathrm{aCC}$ & 0 & \\
\hline 347 & $a$ & insertion & 0 & 1 & - & 2 & $\mathrm{Aa}$ & 0 & 0 & & 0 & \\
\hline 351 & $\mathrm{~g}$ & deletion & 0 & 1 & - & 0 & & 2 & 2 & CCgCC & 3 & $\mathrm{CgC}$ \\
\hline 363 & $\mathrm{C}$ & $\mathrm{G}: \mathrm{C}->\mathrm{T}: \mathrm{A}$ & 0 & 1 & $P(s)$ & 3 & $\mathrm{CCc}$ & 0 & 0 & & 0 & \\
\hline 363 & $\mathrm{C}$ & insertion & 0 & 1 & - & 3 & $\mathrm{CCc}$ & 0 & 0 & & 0 & \\
\hline
\end{tabular}




\begin{tabular}{|c|c|c|c|c|c|c|c|c|c|c|c|c|}
\hline 387 & c & $\mathrm{G}: \mathrm{C} \rightarrow \mathrm{T}: \mathrm{A}$ & 1 & 0 & $*$ & 0 & & 0 & 0 & & 0 & \\
\hline 416 & g & $\mathrm{G}: \mathrm{C}->\mathrm{A}: \mathrm{T}$ & 0 & 1 & $*$ & 2 & gG & 0 & 0 & & 0 & \\
\hline 421 & c & $\mathrm{G}: \mathrm{C}->\mathrm{A}: \mathrm{T}$ & 1 & 1 & $*$ & 0 & & 0 & 0 & & 0 & \\
\hline 428 & g & deletion & 1 & 0 & - & 2 & $\mathrm{Gg}$ & 0 & 2 & gCC & 2 & $\mathrm{gC}$ \\
\hline 430 & c & $\mathrm{G}: \mathrm{C} \rightarrow \mathrm{A}: \mathrm{T}$ & 1 & 0 & $*$ & 2 & $\mathrm{Cc}$ & 0 & 0 & & 0 & \\
\hline 447 & $\mathrm{t}$ & deletion & 0 & 1 & - & 5 & TTTTt & 0 & 0 & & 0 & \\
\hline 452 & g & $\mathrm{G}: \mathrm{C}->\mathrm{T}: \mathrm{A}$ & 0 & 1 & $\mathrm{~V}$ & 2 & $\mathrm{Gg}$ & 0 & 0 & & 3 & gCG \\
\hline 455 & $\mathrm{t}$ & $A: T->T: A$ & 0 & 1 & $D$ & 2 & $\mathrm{tT}$ & 0 & 0 & & 0 & \\
\hline 461 & c & $\mathrm{G}: \mathrm{C}->\mathrm{C}: \mathrm{G}$ & 0 & 1 & W & 0 & & 0 & 2 & cGG & 2 & $c G$ \\
\hline 461 & c & $\mathrm{G}: \mathrm{C}->\mathrm{T}: \mathrm{A}$ & 0 & 2 & $*$ & 0 & & 0 & 2 & cGG & 2 & cG \\
\hline 476 & g & $\mathrm{G}: \mathrm{C}->\mathrm{A}: \mathrm{T}$ & 1 & 0 & $*$ & 2 & gG & 0 & 0 & & 0 & \\
\hline 491 & $\mathrm{~g}$ & $\mathrm{G}: \mathrm{C}->\mathrm{A}: \mathrm{T}$ & 1 & 1 & $*$ & 3 & gGG & 0 & 0 & & 0 & \\
\hline 495 & c & deletion & 0 & 1 & - & 0 & & 0 & 2 & cGG & 2 & $c G$ \\
\hline 501 & c & $\mathrm{G}: \mathrm{C}->\mathrm{T}: \mathrm{A}$ & 1 & 3 & $*$ & 0 & & 0 & 2 & cGG & 2 & cG \\
\hline 501 & c & deletion & 0 & 1 & - & 0 & & 0 & 2 & cGG & 2 & cG \\
\hline 505 & c & $\mathrm{G}: \mathrm{C}->\mathrm{A}: \mathrm{T}$ & 2 & 0 & $*$ & 2 & $\mathrm{Cc}$ & 0 & 0 & & 0 & \\
\hline 519 & g & deletion & 0 & 1 & - & 0 & & 3 & 2 & TTTgCC & 2 & $\mathrm{gC}$ \\
\hline 526 & g & $\mathrm{G}: \mathrm{C}->\mathrm{T}: \mathrm{A}$ & 1 & 0 & $*$ & 0 & & 0 & 2 & gAA & 0 & \\
\hline 556 & g & $\mathrm{G}: \mathrm{C}->\mathrm{T}: \mathrm{A}$ & 0 & 1 & $*$ & 2 & gG & 2 & 0 & $\mathrm{CCg}$ & 2 & $\mathrm{Cg}$ \\
\hline 568 & c & insertion & 1 & 0 & - & 2 & Cc & 0 & 0 & & 0 & \\
\hline 571 & g & deletion & 0 & 1 & - & 0 & & 0 & 0 & & 4 & $\mathrm{CgCG}$ \\
\hline 572 & c & deletion & 0 & 2 & - & 0 & & 0 & 2 & cGG & 4 & CGcG \\
\hline 591 & g & $\mathrm{G}: \mathrm{C} \rightarrow \mathrm{A}: \mathrm{T}$ & 1 & 1 & $*$ & 2 & $\mathrm{Gg}$ & 0 & 0 & & 0 & \\
\hline 593 & g & insertion & 1 & 0 & - & 0 & & 0 & 0 & & 0 & \\
\hline 619 & g & $\mathrm{G}: \mathrm{C}->\mathrm{C}: \mathrm{G}$ & 1 & 0 & $\mathrm{H}$ & 2 & $\mathrm{Gg}$ & 0 & 0 & & 0 & \\
\hline 619 & g & $\mathrm{G}: \mathrm{C} \rightarrow \mathrm{T}: \mathrm{A}$ & 0 & 1 & $Y$ & 2 & $\mathrm{Gg}$ & 0 & 0 & & 0 & \\
\hline 625 & $\mathrm{t}$ & deletion & 0 & 1 & - & 0 & & 0 & 2 & $\mathrm{tGG}$ & 0 & \\
\hline 629 & g & insertion & 0 & 1 & - & 2 & $\mathrm{gG}$ & 0 & 0 & & 3 & $\mathrm{GCg}$ \\
\hline
\end{tabular}




\begin{tabular}{|c|c|c|c|c|c|c|c|c|c|c|c|c|}
\hline 636 & c & deletion & 0 & 2 & - & 0 & & 0 & 2 & cGG & 3 & GcG \\
\hline 637 & g & $\mathrm{G}: \mathrm{C}->\mathrm{T}: \mathrm{A}$ & 1 & 2 & $C$ & 2 & gG & 0 & 0 & & 3 & $\mathrm{GCg}$ \\
\hline 638 & g & $G: C->A: T$ & 1 & 0 & D & 2 & $\mathrm{Gg}$ & 0 & 0 & & 2 & $\mathrm{gC}$ \\
\hline 638 & g & $\mathrm{G}: \mathrm{C} \rightarrow \mathrm{T}: \mathrm{A}$ & 0 & 1 & V & 2 & $\mathrm{Gg}$ & 0 & 0 & & 2 & $\mathrm{gC}$ \\
\hline 647 & g & $\mathrm{G}: \mathrm{C}->\mathrm{C}: \mathrm{G}$ & 0 & 2 & $\mathrm{P}$ & 0 & & 2 & 0 & $\mathrm{CCg}$ & 2 & $\mathrm{Cg}$ \\
\hline 651 & c & $\mathrm{G}: \mathrm{C} \rightarrow \mathrm{A}: \mathrm{T}$ & 0 & 1 & $D(s)$ & 0 & & 0 & 0 & & 2 & $c G$ \\
\hline 656 & c & $\mathrm{G}: \mathrm{C}->\mathrm{T}: \mathrm{A}$ & 0 & 2 & $*$ & 0 & & 0 & 0 & & 2 & $c G$ \\
\hline 679 & c & $G: C->A: T$ & 1 & 1 & $*$ & 0 & & 0 & 3 & cAAA & 0 & \\
\hline 725 & g & deletion & 0 & 1 & - & 0 & & 0 & 2 & gCC & 2 & $\mathrm{gC}$ \\
\hline 727 & c & deletion & 0 & 1 & - & 2 & Cc & 0 & 0 & & 5 & cGCGC \\
\hline 739 & g & $\mathrm{G}: \mathrm{C}->\mathrm{T}: \mathrm{A}$ & 0 & 1 & $*$ & 2 & $\mathrm{Gg}$ & 0 & 0 & & 0 & \\
\hline 759 & c & $\mathrm{G}: \mathrm{C}->\mathrm{T}: \mathrm{A}$ & 0 & 3 & $*$ & 0 & & 0 & 2 & cGG & 3 & GcG \\
\hline 759 & c & deletion & 0 & 1 & - & 0 & & 0 & 2 & cGG & 3 & GcG \\
\hline 765 & g & deletion & 0 & 1 & - & 0 & & 0 & 2 & gTT & 0 & \\
\hline 767 & $\mathrm{t}$ & deletion & 0 & 1 & - & 2 & $\mathrm{Tt}$ & 0 & 0 & & 0 & \\
\hline 776 & $a$ & deletion & 0 & 2 & - & 0 & & 0 & 2 & $\mathrm{aCC}$ & 0 & \\
\hline 782 & g & $\mathrm{G}: \mathrm{C}->\mathrm{T}: \mathrm{A}$ & 0 & 1 & $L$ & 3 & gGG & 0 & 0 & & 2 & $\mathrm{Cg}$ \\
\hline 786 & a & deletion & 0 & 1 & - & 2 & $\mathrm{aA}$ & 0 & 0 & & 0 & \\
\hline 801 & g & $\mathrm{G}: \mathrm{C} \rightarrow \mathrm{A}: \mathrm{T}$ & 1 & 0 & $*$ & 2 & $\mathrm{Gg}$ & 0 & 0 & & 2 & $\mathrm{gC}$ \\
\hline 808 & g & $\mathrm{G}: \mathrm{C}->\mathrm{T}: \mathrm{A}$ & 0 & 1 & $*$ & 0 & & 0 & 3 & gAAA & 0 & \\
\hline 820 & g & deletion & 0 & 1 & - & 0 & & 0 & 2 & gCC & 3 & $\mathrm{CgC}$ \\
\hline 825 & c & $\mathrm{G}: \mathrm{C}->\mathrm{C}: \mathrm{G}$ & 0 & 1 & $\mathrm{R}$ & 0 & & 0 & 2 & cGG & 3 & GcG \\
\hline 829 & $a$ & deletion & 0 & 1 & - & 0 & & 0 & 2 & $\mathrm{aCC}$ & 0 & \\
\hline 859 & g & $\mathrm{G}: \mathrm{C}->\mathrm{T}: \mathrm{A}$ & 0 & 1 & $*$ & 0 & & 0 & 0 & & 0 & \\
\hline 879 & $\mathrm{t}$ & deletion & 0 & 1 & - & 0 & & 0 & 0 & & 0 & \\
\hline 887 & c & deletion & 0 & 1 & - & 0 & & 0 & 0 & & 0 & \\
\hline 920 & g & $G: C->A: T$ & 1 & 0 & $*$ & 2 & $g G$ & 0 & 0 & & 0 & \\
\hline 920 & g & $\mathrm{G}: \mathrm{C}->\mathrm{T}: \mathrm{A}$ & 1 & 0 & L & 2 & gG & 0 & 0 & & 0 & \\
\hline
\end{tabular}




\begin{tabular}{|c|c|c|c|c|c|c|c|c|c|c|c|c|}
\hline 921 & $\mathrm{~g}$ & $G: C \rightarrow T: A$ & 0 & 1 & c & 2 & $\mathrm{Gg}$ & 0 & 0 & & 0 & \\
\hline 928 & $\mathrm{~g}$ & $G: C->A: T$ & 4 & 1 & K & 0 & & 2 & 3 & CCgAAA & 2 & $\mathrm{Cg}$ \\
\hline 950 & c & $G: C \rightarrow A: T$ & 0 & 1 & v & 0 & & 0 & 2 & cGG & 3 & GcG \\
\hline 950 & c & deletion & 0 & 1 & - & 0 & & 0 & 2 & cGG & 3 & GcG \\
\hline 970 & g & insertion & 0 & 1 & - & 0 & & 2 & 2 & $\mathrm{CCgCC}$ & 3 & $\operatorname{cgC}$ \\
\hline 988 & $\mathrm{~g}$ & $G: C->A: T$ & 1 & 0 & K & 0 & & 2 & 2 & TTgAA & 0 & \\
\hline 988 & $\mathrm{~g}$ & $G: C->T: A$ & 1 & 0 & * & 0 & & 2 & 2 & TTgAA & 0 & \\
\hline 1001 & $\mathrm{~g}$ & deletion & 1 & 0 & - & 0 & & 0 & 0 & & 3 & gCG \\
\hline 1002 & c & $G: C \rightarrow T: A$ & 0 & 1 & * & 0 & & 0 & 0 & & 3 & GcG \\
\hline 1009 & $\mathrm{~g}$ & $\mathrm{G}: \mathrm{C} \rightarrow \mathrm{C}: \mathrm{G}$ & 0 & 2 & $\mathrm{R}$ & 2 & $g G$ & 0 & 0 & & 2 & $\mathrm{Cg}$ \\
\hline 1013 & $\mathrm{t}$ & deletion & 0 & 3 & - & 3 & $\mathrm{TTt}$ & 0 & 2 & tCC & 0 & \\
\hline 1014 & c & $G: C->A: T$ & 0 & 1 & $F(s)$ & 2 & $\mathrm{cC}$ & 3 & 0 & TTTC & 0 & \\
\hline 1015 & c & deletion & 0 & 2 & - & 2 & Cc & 0 & 0 & & 4 & cGCG \\
\hline 1016 & $\mathrm{~g}$ & $G: C->T: A$ & 0 & 2 & L & 0 & & 2 & 0 & $\mathrm{CCg}$ & 4 & $\mathrm{CgCG}$ \\
\hline 1016 & $\mathrm{~g}$ & $\mathrm{G}: \mathrm{C} \rightarrow \mathrm{C}: \mathrm{G}$ & 0 & 2 & $\mathrm{P}$ & 0 & & 2 & 0 & $\mathrm{CCg}$ & 4 & $\mathrm{CgCG}$ \\
\hline 1016 & $\mathrm{~g}$ & deletion & 0 & 1 & - & 0 & & 2 & 0 & $\mathrm{CCg}$ & 4 & $\mathrm{CgCG}$ \\
\hline 1018 & g & $G: C->T: A$ & 1 & 0 & * & 0 & & 0 & 0 & & 4 & CGCg \\
\hline 1030 & $\mathrm{~g}$ & $\mathrm{G}: \mathrm{C} \rightarrow \mathrm{T}: \mathrm{A}$ & 1 & 1 & $*$ & 0 & & 2 & 4 & TTgAAAA & 0 & \\
\hline 1053 & c & deletion & 0 & 2 & - & 0 & & 2 & 2 & AAcGG & 2 & cG \\
\hline 1072 & c & $G: C->A: T$ & 3 & 1 & * & 0 & & 2 & 0 & TTC & 2 & $c G$ \\
\hline 1076 & $\mathrm{~g}$ & $G: C->T: A$ & 0 & 1 & V & 2 & $\mathrm{Gg}$ & 0 & 0 & & 3 & gCG \\
\hline 1090 & $\mathrm{~g}$ & $G: C->T: A$ & 0 & 1 & * & 0 & & 0 & 0 & & 2 & $\mathrm{Cg}$ \\
\hline 1111 & c & $G: C->A: T$ & 0 & 1 & * & 0 & & 0 & 0 & & 0 & \\
\hline 1123 & g & $G: C->T: A$ & 0 & 1 & $*$ & 0 & & 0 & 0 & & 0 & \\
\hline 1141 & $\mathrm{~g}$ & $G: C->T: A$ & 0 & 1 & Y & 2 & $\mathrm{Gg}$ & 0 & 0 & & 0 & \\
\hline 1155 & $\mathrm{~g}$ & deletion & 0 & 1 & - & 0 & & 0 & 2 & gAA & 0 & \\
\hline 1167 & c & deletion & 0 & 1 & - & 0 & & 2 & 3 & AAcTTT & 0 & \\
\hline 1174 & g & deletion & 0 & 1 & - & 0 & & 0 & 2 & $\mathrm{gCC}$ & 3 & $\operatorname{CgC}$ \\
\hline
\end{tabular}




\begin{tabular}{|c|c|c|c|c|c|c|c|c|c|c|c|c|}
\hline 1175 & c & $\mathrm{G}: \mathrm{C}->\mathrm{T}: \mathrm{A}$ & 0 & 2 & $\mathrm{D}$ & 2 & $c C$ & 0 & 0 & & 3 & CGC \\
\hline 1187 & c & $\mathrm{G}: \mathrm{C}->\mathrm{A}: \mathrm{T}$ & 4 & 0 & $\mathrm{~L}$ & 0 & & 2 & 0 & TTC & 3 & $\mathrm{cGC}$ \\
\hline 1187 & c & $\mathrm{G}: \mathrm{C} \rightarrow \mathrm{T}: \mathrm{A}$ & 0 & 2 & $*$ & 0 & & 2 & 0 & TTC & 3 & $\mathrm{cGC}$ \\
\hline 1187 & c & $\mathrm{G}: \mathrm{C} \rightarrow \mathrm{C}: \mathrm{G}$ & 0 & 1 & W & 0 & & 2 & 0 & TTC & 3 & $\mathrm{cGC}$ \\
\hline 1189 & c & $\mathrm{G}: \mathrm{C}->\mathrm{T}: \mathrm{A}$ & 0 & 1 & $\mathrm{~N}$ & 0 & & 0 & 0 & & 3 & CGc \\
\hline 1196 & c & $\mathrm{G}: \mathrm{C}->\mathrm{A}: \mathrm{T}$ & 1 & 0 & $\mathrm{~L}$ & 2 & Cc & 0 & 0 & & 2 & $c G$ \\
\hline 1211 & g & $\mathrm{G}: \mathrm{C}->\mathrm{A}: \mathrm{T}$ & 1 & 0 & $*$ & 2 & gG & 0 & 0 & & 0 & \\
\hline 1224 & c & $\mathrm{G}: \mathrm{C}->\mathrm{T}: \mathrm{A}$ & 0 & 2 & $*$ & 0 & & 0 & 0 & & 3 & GcG \\
\hline 1232 & a & deletion & 0 & 1 & - & 0 & & 0 & 0 & & 0 & \\
\hline 1234 & g & $\mathrm{G}: \mathrm{C}->\mathrm{A}: \mathrm{T}$ & 1 & 1 & $\mathrm{~S}$ & 2 & gG & 0 & 0 & & 2 & $\mathrm{Cg}$ \\
\hline 1235 & g & $\mathrm{G}: \mathrm{C}->\mathrm{T}: \mathrm{A}$ & 0 & 1 & $\mathrm{~V}$ & 2 & $\mathrm{Gg}$ & 0 & 2 & gCC & 2 & $\mathrm{gC}$ \\
\hline 1244 & $\mathrm{t}$ & deletion & 0 & 1 & - & 0 & & 0 & 2 & $\mathrm{tGG}$ & 0 & \\
\hline 1252 & g & $G: C->A: T$ & 2 & 0 & $\mathrm{~K}$ & 0 & & 0 & 2 & gAA & 0 & \\
\hline 1252 & g & $\mathrm{G}: \mathrm{C}->\mathrm{C}: \mathrm{G}$ & 1 & 0 & $Q$ & 0 & & 0 & 2 & gAA & 0 & \\
\hline 1252 & g & $\mathrm{G}: \mathrm{C} \rightarrow \mathrm{T}: \mathrm{A}$ & 0 & 1 & $*$ & 0 & & 0 & 2 & gAA & 0 & \\
\hline 1264 & g & $\mathrm{G}: \mathrm{C}->\mathrm{T}: \mathrm{A}$ & 0 & 1 & $*$ & 0 & & 2 & 3 & TTgAAA & 0 & \\
\hline 1305 & $\mathrm{t}$ & deletion & 0 & 1 & - & 0 & & 0 & 2 & $\mathrm{tCC}$ & 0 & \\
\hline 1330 & $\mathrm{~g}$ & $\mathrm{G}: \mathrm{C}->\mathrm{T}: \mathrm{A}$ & 0 & 1 & $*$ & 0 & & 0 & 2 & gAA & 3 & $\mathrm{GCg}$ \\
\hline 1334 & $\mathrm{~g}$ & $\mathrm{G}: \mathrm{C}->\mathrm{C}: \mathrm{G}$ & 0 & 1 & $\mathrm{P}$ & 0 & & 0 & 0 & & 4 & $\mathrm{CgCG}$ \\
\hline 1342 & c & $\mathrm{G}: \mathrm{C}->\mathrm{A}: \mathrm{T}$ & 2 & 1 & $*$ & 0 & & 0 & 0 & & 4 & CGcG \\
\hline 1370 & c & $\mathrm{G}: \mathrm{C}->\mathrm{T}: \mathrm{A}$ & 0 & 1 & $Q$ & 3 & $\mathrm{CCC}$ & 0 & 0 & & 2 & $c G$ \\
\hline 1385 & $\mathrm{~g}$ & $G: C->A: T$ & 1 & 0 & $*$ & 2 & gG & 0 & 0 & & 0 & \\
\hline 1386 & $\mathrm{~g}$ & $\mathrm{G}: \mathrm{C}->\mathrm{A}: \mathrm{T}$ & 1 & 1 & $*$ & 2 & $\mathrm{Gg}$ & 0 & 0 & & 0 & \\
\hline 1388 & c & $\mathrm{G}: \mathrm{C}->\mathrm{T}: \mathrm{A}$ & 0 & 2 & $*$ & 0 & & 0 & 0 & & 3 & $\mathrm{cGC}$ \\
\hline 1393 & $\mathrm{~g}$ & $\mathrm{G}: \mathrm{C} \rightarrow \mathrm{A}: \mathrm{T}$ & 1 & 0 & $\mathrm{R}$ & 4 & GgGG & 0 & 0 & & 0 & \\
\hline 1393 & $\mathrm{~g}$ & $\mathrm{G}: \mathrm{C} \rightarrow \mathrm{T}: \mathrm{A}$ & 0 & 1 & W & 4 & GgGG & 0 & 0 & & 0 & \\
\hline 1393 & $\mathrm{~g}$ & $\mathrm{G}: \mathrm{C}->\mathrm{C}: \mathrm{G}$ & 0 & 1 & $\mathrm{R}$ & 4 & GgGG & 0 & 0 & & 0 & \\
\hline 1399 & g & $\mathrm{G}: \mathrm{C}->\mathrm{T}: \mathrm{A}$ & 1 & 0 & $*$ & 0 & & 0 & 2 & gAA & 0 & \\
\hline
\end{tabular}




\begin{tabular}{|c|c|c|c|c|c|c|c|c|c|c|c|c|}
\hline 1406 & g & $\mathrm{G}: \mathrm{C}->\mathrm{T}: \mathrm{A}$ & 0 & 2 & V & 2 & $\mathrm{Gg}$ & 0 & 2 & gCC & 2 & $\mathrm{gC}$ \\
\hline 1461 & c & insertion & 0 & 1 & - & 3 & $\mathrm{CcC}$ & 0 & 0 & & 0 & \\
\hline 1471 & c & $G: C->A: T$ & 1 & 0 & $*$ & 0 & & 0 & 0 & & 2 & Gc \\
\hline 1477 & g & $\mathrm{G}: \mathrm{C}->\mathrm{T}: \mathrm{A}$ & 1 & 0 & $*$ & 0 & & 0 & 2 & gAA & 0 & \\
\hline 1477 & g & $G: C->A: T$ & 1 & 0 & K & 0 & & 0 & 2 & gAA & 0 & \\
\hline 1495 & $a$ & insertion & 0 & 1 & - & 0 & & 0 & 2 & $\mathrm{aCC}$ & 0 & \\
\hline 1498 & $a$ & $A: T->C: G$ & 0 & 1 & $P$ & 0 & & 2 & 0 & $\mathrm{CCa}$ & 0 & \\
\hline 1518 & c & $\mathrm{G}: \mathrm{C}->\mathrm{T}: \mathrm{A}$ & 0 & 2 & $*$ & 3 & $\mathrm{cCC}$ & 0 & 0 & & 2 & $\mathrm{Gc}$ \\
\hline 1520 & c & $\mathrm{G}: \mathrm{C}->\mathrm{T}: \mathrm{A}$ & 0 & 2 & $Q$ & 3 & $\mathrm{CCc}$ & 0 & 0 & & 2 & cG \\
\hline 1527 & c & $\mathrm{G}: \mathrm{C}->\mathrm{T}: \mathrm{A}$ & 0 & 1 & $*$ & 0 & & 0 & 0 & & 8 & cGCGCGCG \\
\hline 1533 & c & deletion & 0 & 1 & - & 0 & & 0 & 0 & & 8 & CGCGCGcG \\
\hline 1534 & g & deletion & 0 & 1 & - & 0 & & 0 & 0 & & 8 & CGCGCGCg \\
\hline 1542 & $a$ & deletion & 0 & 1 & - & 2 & $\mathrm{Aa}$ & 0 & 0 & & 0 & \\
\hline 1544 & $a$ & deletion & 0 & 1 & - & 0 & & 0 & 2 & $\mathrm{aCC}$ & 0 & \\
\hline 1548 & g & $G: C->A: T$ & 0 & 1 & $Q(s)$ & 0 & & 0 & 3 & gCCC & 2 & $\mathrm{gC}$ \\
\hline 1551 & c & deletion & 0 & 1 & - & 3 & $\mathrm{CCc}$ & 0 & 2 & cTT & 0 & \\
\hline 1553 & $\mathrm{t}$ & deletion & 0 & 1 & - & 2 & $\mathrm{Tt}$ & 0 & 3 & $\mathrm{tCCC}$ & 0 & \\
\hline 1565 & c & deletion & 0 & 1 & - & 2 & Cc & 0 & 0 & & 2 & $c G$ \\
\hline 1572 & g & $G: C->A: T$ & 1 & 0 & $*$ & 2 & $\mathrm{Gg}$ & 0 & 0 & & 0 & \\
\hline 1603 & g & $\mathrm{G}: \mathrm{C}->\mathrm{T}: \mathrm{A}$ & 1 & 0 & $*$ & 0 & & 0 & 0 & & 0 & \\
\hline 1610 & g & deletion & 0 & 1 & - & 0 & & 0 & 3 & gCCC & 5 & $\mathrm{CGCgC}$ \\
\hline 1613 & c & $\mathrm{G}: \mathrm{C}->\mathrm{C}: \mathrm{G}$ & 1 & 0 & $\mathrm{R}$ & 3 & $\mathrm{CCC}$ & 0 & 0 & & 3 & cGC \\
\hline 1626 & c & $\mathrm{G}: \mathrm{C}->\mathrm{T}: \mathrm{A}$ & 0 & 1 & $*$ & 0 & & 0 & 0 & & 3 & GcG \\
\hline 1627 & g & $G: C->A: T$ & 3 & 1 & K & 0 & & 0 & 2 & gAA & 3 & $\mathrm{GCg}$ \\
\hline 1627 & g & $\mathrm{G}: \mathrm{C}->\mathrm{C}: \mathrm{G}$ & 1 & 0 & $Q$ & 0 & & 0 & 2 & gAA & 3 & $\mathrm{GCg}$ \\
\hline 1628 & $a$ & $A: T->G: C$ & 1 & 0 & G & 2 & $a A$ & 0 & 0 & & 0 & \\
\hline 1632 & c & $\mathrm{G}: \mathrm{C}->\mathrm{T}: \mathrm{A}$ & 1 & 0 & $*$ & 0 & & 0 & 0 & & 3 & cGC \\
\hline 1635 & c & $\mathrm{G}: \mathrm{C}->\mathrm{T}: \mathrm{A}$ & 0 & 1 & $A(s)$ & 3 & $\mathrm{CcC}$ & 0 & 0 & & 0 & \\
\hline
\end{tabular}




\begin{tabular}{|c|c|c|c|c|c|c|c|c|c|c|c|c|}
\hline 1638 & c & $\mathrm{G}: \mathrm{C}->\mathrm{T}: \mathrm{A}$ & 0 & 2 & $Q$ & 0 & & 0 & 0 & & 4 & cGCG \\
\hline 1639 & $\mathrm{~g}$ & $\mathrm{G}: \mathrm{C}->\mathrm{C}: \mathrm{G}$ & 0 & 2 & $P$ & 0 & & 0 & 0 & & 4 & $\mathrm{CgCG}$ \\
\hline 1643 & $\mathrm{t}$ & insertion & 1 & 0 & - & 0 & & 0 & 3 & tGGG & 0 & \\
\hline 1650 & c & $\mathrm{G}: \mathrm{C}->\mathrm{T}: \mathrm{A}$ & 0 & 1 & K & 0 & & 2 & 0 & AAC & 0 & \\
\hline 1652 & g & $\mathrm{G}: \mathrm{C}->\mathrm{T}: \mathrm{A}$ & 1 & 0 & 1 & 0 & & 0 & 0 & & 0 & \\
\hline 1657 & g & $\mathrm{G}: \mathrm{C} \rightarrow \mathrm{A}: \mathrm{T}$ & 1 & 0 & $S$ & 2 & $\mathrm{gG}$ & 2 & 0 & TTg & 0 & \\
\hline 1657 & g & $\mathrm{G}: \mathrm{C}->\mathrm{T}: \mathrm{A}$ & 0 & 1 & C & 2 & gG & 2 & 0 & TTg & 0 & \\
\hline 1666 & g & deletion & 0 & 1 & - & 0 & & 0 & 0 & & 3 & $\mathrm{CgC}$ \\
\hline 1669 & a & $A: T->T: A$ & 0 & 1 & $*$ & 3 & $\mathrm{aAA}$ & 0 & 0 & & 0 & \\
\hline 1673 & $a$ & deletion & 0 & 1 & - & 0 & & 0 & 0 & & 0 & \\
\hline 1674 & c & $\mathrm{G}: \mathrm{C}->\mathrm{T}: \mathrm{A}$ & 1 & 3 & $*$ & 0 & & 0 & 0 & & 0 & \\
\hline 1677 & g & $\mathrm{G}: \mathrm{C} \rightarrow \mathrm{A}: \mathrm{T}$ & 0 & 1 & $*$ & 2 & $\mathrm{Gg}$ & 0 & 0 & & 2 & $\mathrm{gC}$ \\
\hline 1678 & c & $\mathrm{G}: \mathrm{C}->\mathrm{A}: \mathrm{T}$ & 0 & 1 & $*$ & 0 & & 2 & 0 & GGc & 2 & Gc \\
\hline 1682 & c & $G: C->A: T$ & 0 & 1 & V & 0 & & 2 & 0 & GGc & 3 & GcG \\
\hline 1683 & g & $\mathrm{G}: \mathrm{C}->\mathrm{T}: \mathrm{A}$ & 0 & 1 & $A(s)$ & 0 & & 0 & 3 & gTTT & 3 & $\mathrm{GCg}$ \\
\hline 1699 & c & deletion & 0 & 1 & - & 4 & $\mathrm{CCCC}$ & 0 & 0 & & 2 & cG \\
\hline 1705 & c & $\mathrm{G}: \mathrm{C} \rightarrow \mathrm{A}: \mathrm{T}$ & 1 & 0 & $*$ & 0 & & 0 & 0 & & 0 & \\
\hline 1708 & g & $\mathrm{G}: \mathrm{C}->\mathrm{C}: \mathrm{G}$ & 1 & 0 & $\mathrm{R}$ & 3 & GgG & 0 & 0 & & 0 & \\
\hline 1709 & g & $\mathrm{G}: \mathrm{C} \rightarrow \mathrm{A}: \mathrm{T}$ & 1 & 0 & D & 3 & GGg & 0 & 0 & & 3 & gCG \\
\hline 1712 & g & $\mathrm{G}: \mathrm{C}->\mathrm{A}: \mathrm{T}$ & 1 & 0 & D & 2 & $\mathrm{Gg}$ & 0 & 0 & & 2 & $\mathrm{gC}$ \\
\hline 1712 & g & $\mathrm{G}: \mathrm{C} \rightarrow \mathrm{T}: \mathrm{A}$ & 0 & 1 & V & 2 & $\mathrm{Gg}$ & 0 & 0 & & 2 & $\mathrm{gC}$ \\
\hline 1720 & $\mathrm{t}$ & $A: T->T: A$ & 0 & 1 & $\mathrm{R}$ & 0 & & 0 & 3 & $\mathrm{tGGG}$ & 0 & \\
\hline 1720 & $\mathrm{t}$ & A:T $\rightarrow$ G:C & 0 & 1 & $\mathrm{R}$ & 0 & & 0 & 3 & $\mathrm{tGGG}$ & 0 & \\
\hline 1721 & g & $\mathrm{G}: \mathrm{C}->\mathrm{C}: \mathrm{G}$ & 1 & 0 & $S$ & 3 & gGG & 0 & 0 & & 0 & \\
\hline 1721 & g & $\mathrm{G}: \mathrm{C}->\mathrm{T}: \mathrm{A}$ & 0 & 1 & $\mathrm{~L}$ & 3 & gGG & 0 & 0 & & 0 & \\
\hline 1722 & g & $G: C->A: T$ & 1 & 0 & $*$ & 3 & GgG & 0 & 0 & & 0 & \\
\hline 1722 & g & $\mathrm{G}: \mathrm{C}->\mathrm{T}: \mathrm{A}$ & 0 & 2 & C & 3 & GgG & 0 & 0 & & 0 & \\
\hline 1727 & g & $\mathrm{G}: \mathrm{C}->\mathrm{A}: \mathrm{T}$ & 0 & 1 & $*$ & 3 & gGG & 0 & 0 & & 0 & \\
\hline
\end{tabular}




\begin{tabular}{|c|c|c|c|c|c|c|c|c|c|c|c|c|}
\hline 1739 & c & $\mathrm{G}: \mathrm{C}->\mathrm{T}: \mathrm{A}$ & 0 & 1 & $*$ & 0 & & 0 & 0 & & 3 & $\mathrm{cGC}$ \\
\hline 1766 & $a$ & deletion & 0 & 1 & - & 2 & $\mathrm{Aa}$ & 0 & 3 & accc & 0 & \\
\hline 1773 & g & $\mathrm{G}: \mathrm{C} \rightarrow \mathrm{A}: \mathrm{T}$ & 2 & 0 & $*$ & 2 & $\mathrm{Gg}$ & 0 & 0 & & 0 & \\
\hline 1775 & c & $\mathrm{G}: \mathrm{C}->\mathrm{T}: \mathrm{A}$ & 1 & 3 & $*$ & 0 & & 0 & 2 & cGG & 2 & $c G$ \\
\hline 1775 & c & deletion & 0 & 1 & - & 0 & & 0 & 2 & $\mathrm{cGG}$ & 2 & cG \\
\hline 1782 & c & $\mathrm{G}: \mathrm{C}->\mathrm{C}: \mathrm{G}$ & 1 & 0 & $*$ & 0 & & 0 & 2 & cGG & 2 & $c G$ \\
\hline 1782 & c & $\mathrm{G}: \mathrm{C}->\mathrm{T}: \mathrm{A}$ & 0 & 1 & $*$ & 0 & & 0 & 2 & cGG & 2 & cG \\
\hline 1784 & g & deletion & 0 & 1 & - & 2 & Gg & 0 & 0 & & 3 & gCG \\
\hline 1809 & c & deletion & 1 & 0 & - & 0 & & 2 & 0 & AAc & 2 & $c G$ \\
\hline 1814 & g & deletion & 0 & 1 & - & 0 & & 0 & 2 & gCC & 3 & $\mathrm{CgC}$ \\
\hline 1816 & c & $\mathrm{G}: \mathrm{C} \rightarrow \mathrm{A}: \mathrm{T}$ & 1 & 0 & $*$ & 2 & $\mathrm{Cc}$ & 0 & 0 & & 0 & \\
\hline 1831 & g & $\mathrm{G}: \mathrm{C}->\mathrm{T}: \mathrm{A}$ & 1 & 0 & C & 2 & gG & 0 & 0 & & 2 & $\mathrm{Cg}$ \\
\hline 1831 & g & $\mathrm{G}: \mathrm{C} \rightarrow \mathrm{A}: \mathrm{T}$ & 1 & 0 & $S$ & 2 & gG & 0 & 0 & & 2 & $\mathrm{Cg}$ \\
\hline 1831 & g & $\mathrm{G}: \mathrm{C}->\mathrm{C}: \mathrm{G}$ & 0 & 1 & $\mathrm{R}$ & 2 & gG & 0 & 0 & & 2 & $\mathrm{Cg}$ \\
\hline 1832 & g & $\mathrm{G}: \mathrm{C}->\mathrm{T}: \mathrm{A}$ & 0 & 1 & V & 2 & $\mathrm{Gg}$ & 0 & 0 & & 0 & \\
\hline 1850 & g & $\mathrm{G}: \mathrm{C}->\mathrm{C}: \mathrm{G}$ & 1 & 0 & $P$ & 0 & & 2 & 0 & $\mathrm{CCg}$ & 3 & $\mathrm{CgC}$ \\
\hline 1852 & a & deletion & 0 & 1 & - & 0 & & 0 & 0 & & 0 & \\
\hline 1854 & g & deletion & 1 & 0 & - & 0 & & 0 & 2 & gCC & 3 & $\mathrm{CgC}$ \\
\hline 1871 & c & deletion & 0 & 1 & - & 0 & & 0 & 2 & cGG & 2 & $c G$ \\
\hline 1873 & g & $\mathrm{G}: \mathrm{C}->\mathrm{T}: \mathrm{A}$ & 0 & 1 & $*$ & 2 & $\mathrm{Gg}$ & 0 & 2 & gAA & 0 & \\
\hline 1875 & a & deletion & 0 & 1 & - & 2 & $\mathrm{Aa}$ & 0 & 0 & & 0 & \\
\hline 1891 & c & $\mathrm{G}: \mathrm{C} \rightarrow \mathrm{A}: \mathrm{T}$ & 1 & 0 & $*$ & 0 & & 0 & 0 & & 2 & Gc \\
\hline 1912 & $\mathrm{t}$ & deletion & 0 & 1 & - & 0 & & 0 & 2 & $\mathrm{tCC}$ & 0 & \\
\hline 1979 & $\mathrm{~g}$ & $\mathrm{G}: \mathrm{C} \rightarrow \mathrm{A}: \mathrm{T}$ & 1 & 0 & $*$ & 2 & gG & 0 & 0 & & 0 & \\
\hline 2013 & C & deletion & 0 & 1 & - & 0 & & 0 & 2 & $\mathrm{cGG}$ & 3 & GcG \\
\hline 2017 & $\mathrm{~g}$ & $\mathrm{G}: \mathrm{C}->\mathrm{T}: \mathrm{A}$ & 0 & 1 & $*$ & 0 & & 0 & 2 & gAA & 0 & \\
\hline 2041 & c & $\mathrm{G}: \mathrm{C} \rightarrow \mathrm{A}: \mathrm{T}$ & 1 & 0 & * & 0 & & 0 & 2 & cAA & 0 & \\
\hline 2043 & $a$ & deletion & 0 & 1 & - & 2 & $\mathrm{Aa}$ & 0 & 2 & aGG & 0 & \\
\hline
\end{tabular}




\begin{tabular}{|c|c|c|c|c|c|c|c|c|c|c|c|c|}
\hline 2050 & c & $\mathrm{G}: \mathrm{C} \rightarrow \mathrm{A}: \mathrm{T}$ & 1 & 0 & $*$ & 0 & & 3 & 0 & AAAC & 0 & \\
\hline 2059 & g & $\mathrm{G}: \mathrm{C}->\mathrm{T}: \mathrm{A}$ & 0 & 1 & $*$ & 0 & & 2 & 2 & TTgAA & 0 & \\
\hline 2083 & g & $\mathrm{G}: \mathrm{C}->\mathrm{T}: \mathrm{A}$ & 0 & 2 & $*$ & 2 & $\mathrm{Gg}$ & 0 & 0 & & 0 & \\
\hline 2093 & g & insertion & 0 & 1 & - & 3 & GgG & 0 & 0 & & 0 & \\
\hline 2130 & c & $G: C->A: T$ & 0 & 1 & $N(s)$ & 0 & & 2 & 0 & AAc & 4 & cGCG \\
\hline 2149 & g & deletion & 1 & 0 & - & 0 & & 2 & 2 & $\mathrm{AAgCC}$ & 2 & $\mathrm{gC}$ \\
\hline 2152 & g & $\mathrm{G}: \mathrm{C}->\mathrm{T}: \mathrm{A}$ & 0 & 1 & W & 3 & gGG & 2 & 0 & $\mathrm{CCg}$ & 2 & $\mathrm{Cg}$ \\
\hline 2164 & g & $\mathrm{G}: \mathrm{C}->\mathrm{C}: \mathrm{G}$ & 0 & 1 & $P$ & 0 & & 0 & 2 & gCC & 4 & $\mathrm{GCgC}$ \\
\hline 2166 & c & insertion & 0 & 1 & - & 2 & Cc & 0 & 0 & & 0 & \\
\hline 2177 & g & $\mathrm{G}: \mathrm{C}->\mathrm{A}: \mathrm{T}$ & 1 & 1 & $*$ & 2 & gG & 0 & 0 & & 0 & \\
\hline 2182 & c & $G: C->A: T$ & 0 & 1 & $L(s)$ & 0 & & 0 & 0 & & 0 & \\
\hline 2183 & $\mathrm{t}$ & $A: T->T: A$ & 0 & 1 & $Q$ & 0 & & 0 & 2 & $\mathrm{tGG}$ & 0 & \\
\hline 2186 & c & deletion & 0 & 1 & - & 0 & & 2 & 2 & GGcGG & 3 & GcG \\
\hline 2211 & c & deletion & 0 & 1 & - & 4 & $\mathrm{CCCc}$ & 0 & 0 & & 3 & cGC \\
\hline 2251 & a & $\mathrm{A}: \mathrm{T}->\mathrm{T}: \mathrm{A}$ & 0 & 1 & $\mathrm{~L}$ & 3 & AAa & 0 & 0 & & 0 & \\
\hline 2252 & $\mathrm{t}$ & $A: T->T: A$ & 0 & 1 & K & 0 & & 3 & 2 & AAAtGG & 0 & \\
\hline 2260 & $\mathrm{t}$ & deletion & 0 & 2 & - & 5 & TTTTt & 0 & 0 & & 0 & \\
\hline 2266 & g & $\mathrm{G}: \mathrm{C}->\mathrm{T}: \mathrm{A}$ & 0 & 2 & $*$ & 0 & & 0 & 0 & & 2 & $\mathrm{Cg}$ \\
\hline 2294 & $\mathrm{a}$ & deletion & 0 & 1 & - & 2 & $\mathrm{Aa}$ & 0 & 2 & $\mathrm{aCC}$ & 0 & \\
\hline 2303 & c & $\mathrm{G}: \mathrm{C}->\mathrm{T}: \mathrm{A}$ & 0 & 3 & $*$ & 0 & & 0 & 0 & & 0 & \\
\hline 2315 & c & $\mathrm{G}: \mathrm{C} \rightarrow \mathrm{C}: \mathrm{G}$ & 1 & 0 & $*$ & 0 & & 3 & 0 & TTTC & 0 & \\
\hline 2357 & $\mathrm{t}$ & deletion & 0 & 1 & - & 0 & & 0 & 0 & & 0 & \\
\hline 2374 & c & $\mathrm{G}: \mathrm{C} \rightarrow \mathrm{A}: \mathrm{T}$ & 3 & 0 & C & 3 & $\mathrm{CCc}$ & 0 & 0 & & 2 & $c G$ \\
\hline 2374 & c & $\mathrm{G}: \mathrm{C}->\mathrm{C}: \mathrm{G}$ & 0 & 2 & G & 3 & $\mathrm{CCC}$ & 0 & 0 & & 2 & $c G$ \\
\hline 2374 & c & $\mathrm{G}: \mathrm{C}->\mathrm{T}: \mathrm{A}$ & 0 & 3 & $S$ & 3 & $\mathrm{CCc}$ & 0 & 0 & & 2 & cG \\
\hline 2375 & $\mathrm{~g}$ & $\mathrm{G}: \mathrm{C}->\mathrm{T}: \mathrm{A}$ & 1 & 2 & $\mathrm{~L}$ & 0 & & 3 & 0 & $\mathrm{CCCg}$ & 2 & $\mathrm{Cg}$ \\
\hline 2375 & g & $\mathrm{G}: \mathrm{C}->\mathrm{C}: \mathrm{G}$ & 0 & 1 & $\mathrm{P}$ & 0 & & 3 & 0 & $\mathrm{CCCg}$ & 2 & $\mathrm{Cg}$ \\
\hline 2376 & $\mathrm{t}$ & A:T $\rightarrow$ G:C & 0 & 1 & $R(s)$ & 0 & & 0 & 0 & & 0 & \\
\hline
\end{tabular}




\begin{tabular}{|c|c|c|c|c|c|c|c|c|c|c|c|c|}
\hline 2391 & c & $\mathrm{G}: \mathrm{C} \rightarrow \mathrm{T}: \mathrm{A}$ & 0 & 1 & $\mathrm{~K}$ & 0 & & 2 & 0 & AAC & 2 & $c G$ \\
\hline 2418 & c & insertion & 0 & 1 & - & 0 & & 0 & 0 & & 3 & $\mathrm{CGC}$ \\
\hline 2440 & g & $\mathrm{G}: \mathrm{C}->\mathrm{T}: \mathrm{A}$ & 1 & 0 & $*$ & 0 & & 0 & 2 & gAA & 2 & $\mathrm{Cg}$ \\
\hline 2447 & g & $\mathrm{G}: \mathrm{C}->\mathrm{T}: \mathrm{A}$ & 1 & 0 & $\mathrm{~L}$ & 2 & gG & 0 & 0 & & 0 & \\
\hline 2455 & g & insertion & 0 & 1 & - & 2 & $\mathrm{Gg}$ & 0 & 0 & & 3 & gCG \\
\hline 2466 & c & $\mathrm{G}: \mathrm{C}->\mathrm{T}: \mathrm{A}$ & 0 & 1 & $*$ & 2 & cC & 0 & 0 & & 0 & \\
\hline 2467 & c & $G: C->A: T$ & 0 & 1 & $*$ & 2 & Cc & 0 & 0 & & 0 & \\
\hline 2470 & g & deletion & 0 & 1 & - & 2 & $\mathrm{Gg}$ & 0 & 2 & gCC & 2 & $\mathrm{gC}$ \\
\hline 2473 & g & $\mathrm{G}: \mathrm{C} \rightarrow \mathrm{T}: \mathrm{A}$ & 0 & 1 & $*$ & 0 & & 2 & 2 & CCgAA & 2 & $\mathrm{Cg}$ \\
\hline 2495 & c & deletion & 0 & 1 & - & 0 & & 0 & 2 & cGG & 2 & $c G$ \\
\hline 2518 & g & deletion & 1 & 0 & - & 2 & $\mathrm{Gg}$ & 0 & 0 & & 0 & \\
\hline 2525 & $\mathrm{t}$ & deletion & 1 & 0 & - & 2 & $\mathrm{tT}$ & 0 & 0 & & 0 & \\
\hline 2538 & c & insertion & 0 & 1 & - & 0 & & 0 & 0 & & 4 & cGCG \\
\hline 2543 & g & $\mathrm{G}: \mathrm{C} \rightarrow \mathrm{A}: \mathrm{T}$ & 1 & 0 & $*$ & 2 & gG & 0 & 0 & & 0 & \\
\hline 2557 & $\mathrm{a}$ & $A: T->T: A$ & 1 & 0 & $*$ & 4 & aAAA & 4 & 0 & GGGGa & 0 & \\
\hline 2586 & c & $\mathrm{G}: \mathrm{C}->\mathrm{C}: \mathrm{G}$ & 0 & 1 & $*$ & 2 & $c C$ & 0 & 0 & & 0 & \\
\hline 2586 & c & $\mathrm{G}: \mathrm{C}->\mathrm{T}: \mathrm{A}$ & 0 & 1 & $*$ & 2 & cC & 0 & 0 & & 0 & \\
\hline 2648 & c & deletion & 1 & 0 & - & 2 & $\mathrm{Cc}$ & 0 & 0 & & 3 & cGC \\
\hline 2659 & c & $\mathrm{G}: \mathrm{C} \rightarrow \mathrm{A}: \mathrm{T}$ & 1 & 1 & W & 0 & & 0 & 2 & cGG & 5 & GCGcG \\
\hline 2660 & g & $\mathrm{G}: \mathrm{C}->\mathrm{C}: \mathrm{G}$ & 0 & 1 & $P$ & 2 & gG & 0 & 0 & & 5 & GCGCg \\
\hline 2666 & g & $\mathrm{G}: \mathrm{C} \rightarrow \mathrm{A}: \mathrm{T}$ & 0 & 1 & $D$ & 2 & $\mathrm{Gg}$ & 0 & 2 & gCC & 2 & $\mathrm{gC}$ \\
\hline 2669 & $\mathrm{t}$ & deletion & 0 & 1 & - & 0 & & 2 & 0 & $\mathrm{CCt}$ & 0 & \\
\hline 2676 & c & $\mathrm{G}: \mathrm{C}->\mathrm{T}: \mathrm{A}$ & 0 & 2 & $*$ & 2 & $\mathrm{cC}$ & 0 & 0 & & 2 & Gc \\
\hline 2706 & c & deletion & 1 & 0 & - & 0 & & 3 & 0 & $A A A C$ & 0 & \\
\hline 2706 & c & deletion & 0 & 1 & - & 0 & & 3 & 0 & AAAC & 0 & \\
\hline 2708 & g & $\mathrm{G}: \mathrm{C}->\mathrm{A}: \mathrm{T}$ & 0 & 1 & $*$ & 2 & gG & 0 & 0 & & 0 & \\
\hline 2713 & g & $\mathrm{G}: \mathrm{C}->\mathrm{A}: \mathrm{T}$ & 3 & 0 & $\mathrm{R}$ & 2 & gG & 0 & 0 & & 2 & $\mathrm{Cg}$ \\
\hline 2713 & $\mathrm{~g}$ & $\mathrm{G}: \mathrm{C}->\mathrm{C}: \mathrm{G}$ & 0 & 1 & $\mathrm{R}$ & 2 & gG & 0 & 0 & & 2 & $\mathrm{Cg}$ \\
\hline
\end{tabular}




\begin{tabular}{|c|c|c|c|c|c|c|c|c|c|c|c|c|}
\hline 2719 & $\mathrm{~g}$ & $\mathrm{G}: \mathrm{C}->\mathrm{C}: \mathrm{G}$ & 0 & 1 & $\mathrm{R}$ & 3 & $\mathrm{gGG}$ & 0 & 0 & & 0 & \\
\hline 2720 & $\mathrm{~g}$ & $\mathrm{G}: \mathrm{C}->\mathrm{T}: \mathrm{A}$ & 1 & 2 & V & 3 & GgG & 0 & 0 & & 0 & \\
\hline 2721 & $\mathrm{~g}$ & deletion & 0 & 3 & - & 3 & GGg & 0 & 2 & $\mathrm{gCC}$ & 2 & $\mathrm{gC}$ \\
\hline 2724 & $\mathrm{~g}$ & $\mathrm{G}: \mathrm{C}->\mathrm{T}: \mathrm{A}$ & 0 & 1 & $P(s)$ & 0 & & 2 & 0 & $\mathrm{CCg}$ & 3 & $\operatorname{cgC}$ \\
\hline 2725 & c & $G: C \rightarrow A: T$ & 0 & 1 & * & 0 & & 0 & 2 & CAA & 3 & CGc \\
\hline 2728 & $\mathrm{~g}$ & $\mathrm{G}: \mathrm{C}->\mathrm{T}: \mathrm{A}$ & 0 & 1 & $*$ & 0 & & 2 & 4 & AAgAAAA & 0 & \\
\hline 2743 & c & $G: C \rightarrow A: T$ & 2 & 0 & C & 2 & Cc & 0 & 0 & & 3 & cGC \\
\hline 2743 & c & $\mathrm{G}: \mathrm{C}->\mathrm{T}: \mathrm{A}$ & 0 & 2 & $\mathrm{~S}$ & 2 & Cc & 0 & 0 & & 3 & cGC \\
\hline 2743 & c & $\mathrm{G}: \mathrm{C} \rightarrow \mathrm{C}: \mathrm{G}$ & 0 & 2 & G & 2 & Cc & 0 & 0 & & 3 & cGC \\
\hline 2744 & g & $G: C \rightarrow A: T$ & 1 & 1 & $\mathrm{H}$ & 0 & & 2 & 2 & $\mathrm{CCgCC}$ & 3 & $\operatorname{cgC}$ \\
\hline 2772 & g & $G: C->A: T$ & 1 & 0 & $*$ & 3 & GgG & 0 & 0 & & 0 & \\
\hline 2805 & c & $G: C->T: A$ & 0 & 2 & * & 0 & & 0 & 0 & & 2 & cG \\
\hline 2807 & $t$ & deletion & 0 & 1 & - & 0 & & 0 & 0 & & 0 & \\
\hline 2809 & $\mathrm{t}$ & $A: T->T: A$ & 0 & 1 & 1 & 2 & tT & 0 & 0 & & 0 & \\
\hline 2817 & c & G:C $>$ T:A & 0 & 2 & $\mathrm{R}$ & 0 & & 0 & 0 & & 3 & GcG \\
\hline 2823 & c & deletion & 0 & 1 & - & 0 & & 4 & 2 & AAAAcGG & 2 & cG \\
\hline 2828 & $\mathrm{t}$ & $A: T \rightarrow G: C$ & 0 & 2 & $P$ & 0 & & 0 & 0 & & 0 & \\
\hline 2830 & c & $\mathrm{G}: \mathrm{C} \rightarrow \mathrm{C}: \mathrm{G}$ & 1 & 0 & G & 0 & & 0 & 0 & & 4 & GcGC \\
\hline 2835 & c & $G: C->T: A$ & 1 & 1 & * & 0 & & 0 & 3 & $\mathrm{cGGG}$ & 3 & GcG \\
\hline 2839 & a & deletion & 0 & 2 & - & 0 & & 3 & 0 & GGGa & 0 & \\
\hline 2844 & c & deletion & 0 & 1 & - & 0 & & 0 & 0 & & 6 & CGCGcG \\
\hline 2856 & $\mathrm{t}$ & $A: T->T: A$ & 0 & 1 & * & 0 & & 0 & 2 & $\mathrm{tGG}$ & 0 & \\
\hline 2860 & c & $\mathrm{G}: \mathrm{C}->\mathrm{T}: \mathrm{A}$ & 0 & 1 & $\mathrm{~T}$ & 3 & $\mathrm{CcC}$ & 0 & 0 & & 0 & \\
\hline 2869 & $\mathrm{t}$ & $A: T->T: A$ & 0 & 1 & $\mathrm{R}$ & 0 & & 0 & 2 & $\mathrm{tGG}$ & 0 & \\
\hline 2871 & $\mathrm{~g}$ & $G: C->A: T$ & 0 & 2 & $*$ & 2 & Gg & 0 & 0 & & 5 & gCGCG \\
\hline 2872 & c & $\mathrm{G}: \mathrm{C}->\mathrm{T}: \mathrm{A}$ & 0 & 1 & $\mathrm{~s}$ & 0 & & 2 & 0 & GGc & 5 & GcGCG \\
\hline 2873 & $\mathrm{~g}$ & $\mathrm{G}: \mathrm{C}->\mathrm{C}: \mathrm{G}$ & 1 & 2 & $\mathrm{P}$ & 0 & & 0 & 0 & & 5 & GCgCG \\
\hline 2929 & a & deletion & 1 & 0 & - & 0 & & 2 & 0 & $\mathrm{CCa}$ & 0 & \\
\hline
\end{tabular}




\begin{tabular}{|c|c|c|c|c|c|c|c|c|c|c|c|c|}
\hline 2949 & c & insertion & 0 & 1 & - & 0 & & 0 & 0 & & 4 & cGCG \\
\hline 2953 & $\mathrm{~g}$ & $\mathrm{G}: \mathrm{C}->\mathrm{T}: \mathrm{A}$ & 1 & 0 & $*$ & 2 & $\mathrm{Gg}$ & 0 & 2 & gAA & 0 & \\
\hline 2956 & g & $\mathrm{G}: \mathrm{C}->\mathrm{T}: \mathrm{A}$ & 0 & 1 & $*$ & 0 & & 2 & 2 & AAgAA & 0 & \\
\hline 3060 & C & $\mathrm{G}: \mathrm{C}->\mathrm{T}: \mathrm{A}$ & 0 & 1 & $*$ & 2 & $\mathrm{cC}$ & 0 & 0 & & 0 & \\
\hline 3078 & $\mathrm{~g}$ & $\mathrm{G}: \mathrm{C} \rightarrow \mathrm{A}: \mathrm{T}$ & 0 & 1 & $*$ & 2 & $\mathrm{Gg}$ & 0 & 0 & & 0 & \\
\hline
\end{tabular}


Table $\mathbf{C 1 0}$ The position and frequency of spontaneous and BaP-induced mutations at identified mutation hotspots in lac $Z$ transgenes recovered from sperm 42 days after treatment.

\begin{tabular}{|c|c|c|c|c|c|}
\hline & Control & BaP & $\begin{array}{l}\text { Unique to } \\
\text { Control }\end{array}$ & $\begin{array}{c}\text { Unique to } \\
\text { BaP }\end{array}$ & $\begin{array}{c}\text { Common } \\
\text { (Control, BaP) }\end{array}$ \\
\hline$\% \mathrm{G}: \mathrm{C}$ & 100.0 & 88.9 & 100.0 & 84.0 & $100(100,100)$ \\
\hline \% A:T & 0.0 & 11.1 & 0.0 & 16.0 & $0(0,0)$ \\
\hline$\% \mathrm{G}: \mathrm{C}->\mathrm{AT}$ & 83.0 & 2.6 & 75.0 & 3.7 & $41.6(20.5,60)$ \\
\hline$\%$ G:C $>$ T:A & 12.8 & 55.6 & 25.0 & 49.4 & $40.4(72.7,11.1)$ \\
\hline$\%$ G:C $>$ C:G & 4.3 & 16.2 & 0.0 & 12.3 & $15.7(6.8,24.4)$ \\
\hline$\% A: T->G: C$ & 0.0 & 2.6 & 0.0 & 3.7 & $0(0,0)$ \\
\hline$\% A: T->T: A$ & 0.0 & 0.9 & 0.0 & 1.2 & $0(0,0)$ \\
\hline$\% A: T->C: G$ & 0.0 & 0.0 & 0.0 & 0.0 & $0(0,0)$ \\
\hline$\%$ insertion & 0.0 & 0.9 & 0.0 & 1.2 & $0(0,0)$ \\
\hline$\%$ deletion & 0.0 & 21.4 & 0.0 & 28.4 & $2.2(0,4.4)$ \\
\hline
\end{tabular}

\begin{tabular}{|cccccc|}
\hline position & reference & $\begin{array}{c}\# \\
\text { mutations }\end{array}$ & type & control & BaP \\
\hline 203 & $\mathrm{G}$ & 2 & $\mathrm{G}: \mathrm{C}>\mathrm{A}: \mathrm{T}$ & 2 & 0 \\
\hline 505 & $\mathrm{C}$ & 2 & $\mathrm{G}: \mathrm{C}>\mathrm{A}: \mathrm{T}$ & 2 & 0 \\
\hline 920 & $\mathrm{G}$ & 2 & $\begin{array}{c}\mathrm{G}: \mathrm{C}>\mathrm{T}: \mathrm{A} \\
\mathrm{G}: \mathrm{C}>\mathrm{A}: \mathrm{T}\end{array}$ & 1 & 1 \\
& & & $\mathrm{G}: \mathrm{C}>\mathrm{T}: \mathrm{A}$ & 1 & 0 \\
\hline 988 & $\mathrm{G}$ & 2 & $\mathrm{G}: \mathrm{C}>\mathrm{A}: \mathrm{T}$ & 1 & 0 \\
& & & $\mathrm{G}: \mathrm{C}>\mathrm{T}: \mathrm{A}$ & 1 & 0 \\
\hline 1477 & $\mathrm{G}$ & 2 & $\mathrm{G}: \mathrm{C}>\mathrm{A}: \mathrm{T}$ & 1 & 0 \\
\hline 1773 & $\mathrm{G}$ & 2 & $\mathrm{G}: \mathrm{C}>\mathrm{A}: \mathrm{T}$ & 2 & 0 \\
\hline 187 & $\mathrm{G}$ & 2 & $\mathrm{G}: \mathrm{C}>\mathrm{T}: \mathrm{A}$ & 0 & 2 \\
\hline 363 & $\mathrm{C}$ & 2 & $\mathrm{G}: \mathrm{C}>\mathrm{T}: \mathrm{A}$ & 0 & 1 \\
& & & insertion & 0 & 1 \\
\hline 572 & $\mathrm{C}$ & 2 & $\mathrm{deletion}$ & 0 & 2 \\
\hline 636 & $\mathrm{C}$ & 2 & $\mathrm{deletion}$ & 0 & 2 \\
\hline 647 & $\mathrm{G}$ & 2 & $\mathrm{G}: \mathrm{C}>\mathrm{C}: \mathrm{G}$ & 0 & 2 \\
\hline 656 & $\mathrm{C}$ & 2 & $\mathrm{G}: \mathrm{C}>\mathrm{T}: \mathrm{A}$ & 0 & 2 \\
\hline 776 & $\mathrm{~A}$ & 2 & deletion & 0 & 2 \\
\hline 950 & $\mathrm{C}$ & 2 & $\mathrm{G}: \mathrm{C}>\mathrm{A}: \mathrm{T}$ & 0 & 1 \\
& & & deletion & 0 & 1 \\
\hline 1009 & $\mathrm{G}$ & 2 & $\mathrm{G}: \mathrm{C}>\mathrm{C}: \mathrm{G}$ & 0 & 2 \\
\hline 1015 & $\mathrm{C}$ & 2 & deletion & 0 & 2 \\
\hline
\end{tabular}




\begin{tabular}{|c|c|c|c|c|c|}
\hline 1053 & $\mathrm{C}$ & 2 & deletion & 0 & 2 \\
\hline 1175 & $\mathrm{C}$ & 2 & $\mathrm{G}: \mathrm{C}->\mathrm{T}: \mathrm{A}$ & 0 & 2 \\
\hline 1224 & $\mathrm{C}$ & 2 & $G: C->T: A$ & 0 & 2 \\
\hline 1388 & C & 2 & G:C $\rightarrow \mathrm{T}: A$ & 0 & 2 \\
\hline 1406 & $\mathrm{G}$ & 2 & $G: C \rightarrow T: A$ & 0 & 2 \\
\hline 1518 & $\mathrm{C}$ & 2 & $G: C \rightarrow T: A$ & 0 & 2 \\
\hline 1520 & $\mathrm{C}$ & 2 & $G: C->T: A$ & 0 & 2 \\
\hline 1638 & $C$ & 2 & $G: C \rightarrow T: A$ & 0 & 2 \\
\hline 1639 & $\mathrm{G}$ & 2 & $\mathrm{G}: \mathrm{C}->\mathrm{C}: \mathrm{G}$ & 0 & 2 \\
\hline \multirow[t]{2}{*}{1720} & $\mathrm{~T}$ & 2 & $A: T-P: A$ & 0 & 1 \\
\hline & & & $A: T->G: C$ & 0 & 1 \\
\hline 2083 & G & 2 & $G: C->T: A$ & 0 & 2 \\
\hline 2260 & $\mathrm{~T}$ & 2 & deletion & 0 & 2 \\
\hline 2266 & G & 2 & $G: C \rightarrow T: A$ & 0 & 2 \\
\hline \multirow[t]{2}{*}{2586} & C & 2 & $G: C \rightarrow T: A$ & 0 & 1 \\
\hline & & & $\mathrm{G}: \mathrm{C}->\mathrm{C}: \mathrm{G}$ & 0 & 1 \\
\hline 2676 & C & 2 & $G: C \rightarrow T: A$ & 0 & 2 \\
\hline 2805 & $\mathrm{C}$ & 2 & $\mathrm{G}: \mathrm{C}->\mathrm{T}: \mathrm{A}$ & 0 & 2 \\
\hline 2817 & $\mathrm{C}$ & 2 & $G: C->T: A$ & 0 & 2 \\
\hline 2828 & $\mathrm{~T}$ & 2 & $A: T->G: C$ & 0 & 2 \\
\hline 2839 & $A$ & 2 & deletion & 0 & 2 \\
\hline 2871 & $\mathrm{G}$ & 2 & $G: C \rightarrow A: T$ & 0 & 2 \\
\hline \multirow[t]{2}{*}{461} & $\mathrm{C}$ & 3 & $G: C \rightarrow T: A$ & 0 & 2 \\
\hline & & & $\mathrm{G}: \mathrm{C}->\mathrm{C}: \mathrm{G}$ & 0 & 1 \\
\hline 1013 & $\mathrm{~T}$ & 3 & deletion & 0 & 3 \\
\hline 2303 & $C$ & 3 & $G: C->T: A$ & 0 & 3 \\
\hline 2721 & $\mathrm{G}$ & 3 & deletion & 0 & 3 \\
\hline \multirow[t]{2}{*}{759} & C & 4 & $G: C \rightarrow T: A$ & 0 & 3 \\
\hline & & & deletion & 0 & 1 \\
\hline \multirow[t]{3}{*}{1016} & G & 5 & $G: C \rightarrow T: A$ & 0 & 2 \\
\hline & & & $\mathrm{G}: \mathrm{C}->\mathrm{C}: \mathrm{G}$ & 0 & 2 \\
\hline & & & deletion & 0 & 1 \\
\hline 139 & G & 3 & $\mathrm{G}: \mathrm{C} \rightarrow \mathrm{T}: \mathrm{A}$ & 2 & 1 \\
\hline 637 & G & 3 & $\mathrm{G}: \mathrm{C} \rightarrow \mathrm{T}: \mathrm{A}$ & 1 & 2 \\
\hline 1342 & $\mathrm{C}$ & 3 & $G: C->A: T$ & 2 & 1 \\
\hline \multirow[t]{3}{*}{1393} & G & 3 & $G: C \rightarrow T: A$ & 0 & 1 \\
\hline & & & $G: C \rightarrow A: T$ & 1 & 0 \\
\hline & & & $\mathrm{G}: \mathrm{C}->\mathrm{C}: \mathrm{G}$ & 0 & 1 \\
\hline \multirow[t]{2}{*}{1722} & G & 3 & $\mathrm{G}: \mathrm{C} \rightarrow \mathrm{T}: \mathrm{A}$ & 0 & 2 \\
\hline & & & $\mathrm{G}: \mathrm{C} \rightarrow \mathrm{A}: \mathrm{T}$ & 1 & 0 \\
\hline \multirow[t]{2}{*}{1831} & G & 3 & $G: C->T: A$ & 1 & 0 \\
\hline & & & $G: C \rightarrow A: T$ & 1 & 0 \\
\hline
\end{tabular}




\begin{tabular}{|c|c|c|c|c|c|}
\hline & & & $\mathrm{G}: \mathrm{C}->\mathrm{C}: \mathrm{G}$ & 0 & 1 \\
\hline 2720 & $\mathrm{G}$ & 3 & $\mathrm{G}: \mathrm{C}->\mathrm{T}: \mathrm{A}$ & 1 & 2 \\
\hline 2873 & $\mathrm{G}$ & 3 & $\mathrm{G}: \mathrm{C} \rightarrow \mathrm{C}: \mathrm{G}$ & 1 & 2 \\
\hline 166 & $C$ & 4 & $\mathrm{G}: \mathrm{C}->\mathrm{A}: \mathrm{T}$ & 3 & 1 \\
\hline 1072 & $\mathrm{C}$ & 4 & $\mathrm{G}: \mathrm{C}->\mathrm{A}: \mathrm{T}$ & 3 & 1 \\
\hline \multirow[t]{3}{*}{1252} & $\mathrm{G}$ & 4 & $\mathrm{G}: \mathrm{C}->\mathrm{T}: \mathrm{A}$ & 0 & 1 \\
\hline & & & $\mathrm{G}: \mathrm{C} \rightarrow \mathrm{A}: \mathrm{T}$ & 2 & 0 \\
\hline & & & $\mathrm{G}: \mathrm{C} \rightarrow \mathrm{C}: \mathrm{G}$ & 1 & 0 \\
\hline 1674 & $C$ & 4 & $\mathrm{G}: \mathrm{C}->\mathrm{T}: \mathrm{A}$ & 1 & 3 \\
\hline \multirow[t]{2}{*}{2375} & $\mathrm{G}$ & 4 & $\mathrm{G}: \mathrm{C}->\mathrm{T}: \mathrm{A}$ & 1 & 2 \\
\hline & & & $\mathrm{G}: \mathrm{C} \rightarrow \mathrm{C}: \mathrm{G}$ & 0 & 1 \\
\hline \multirow[t]{2}{*}{2713} & $\mathrm{G}$ & 4 & $\mathrm{G}: \mathrm{C}->\mathrm{A}: \mathrm{T}$ & 3 & 0 \\
\hline & & & $\mathrm{G}: \mathrm{C} \rightarrow \mathrm{C}: \mathrm{G}$ & 0 & 1 \\
\hline \multirow[t]{2}{*}{501} & $\mathrm{C}$ & 5 & $\mathrm{G}: \mathrm{C}->\mathrm{T}: \mathrm{A}$ & 1 & 3 \\
\hline & & & deletion & 0 & 1 \\
\hline 928 & $\mathrm{G}$ & 5 & $\mathrm{G}: \mathrm{C}->\mathrm{A}: \mathrm{T}$ & 4 & 1 \\
\hline \multirow[t]{2}{*}{1627} & $\mathrm{G}$ & 5 & $\mathrm{G}: \mathrm{C} \rightarrow \mathrm{A}: \mathrm{T}$ & 3 & 1 \\
\hline & & & $\mathrm{G}: \mathrm{C} \rightarrow \mathrm{C}: \mathrm{G}$ & 1 & 0 \\
\hline \multirow[t]{2}{*}{1775} & $C$ & 5 & $\mathrm{G}: \mathrm{C}->\mathrm{T}: \mathrm{A}$ & 1 & 3 \\
\hline & & & deletion & 0 & 1 \\
\hline \multirow[t]{3}{*}{2743} & $\mathrm{C}$ & 6 & $\mathrm{G}: \mathrm{C}->\mathrm{T}: \mathrm{A}$ & 0 & 2 \\
\hline & & & $\mathrm{G}: \mathrm{C}->\mathrm{A}: \mathrm{T}$ & 2 & 0 \\
\hline & & & $\mathrm{G}: \mathrm{C} \rightarrow \mathrm{C}: \mathrm{G}$ & 0 & 2 \\
\hline \multirow[t]{3}{*}{1187} & $C$ & 7 & $\mathrm{G}: \mathrm{C}->\mathrm{T}: \mathrm{A}$ & 0 & 2 \\
\hline & & & $\mathrm{G}: \mathrm{C}->\mathrm{A}: \mathrm{T}$ & 4 & 0 \\
\hline & & & $\mathrm{G}: \mathrm{C} \rightarrow \mathrm{C}: \mathrm{G}$ & 0 & 1 \\
\hline \multirow[t]{3}{*}{2374} & $\mathrm{C}$ & 8 & $\mathrm{G}: \mathrm{C}->\mathrm{T}: \mathrm{A}$ & 0 & 3 \\
\hline & & & $\mathrm{G}: \mathrm{C} \rightarrow \mathrm{A}: \mathrm{T}$ & 3 & 0 \\
\hline & & & $\mathrm{G}: \mathrm{C} \rightarrow \mathrm{C}: \mathrm{G}$ & 0 & 2 \\
\hline
\end{tabular}


Table C11 The proportion of spontaneous and $\mathrm{BaP}$-induced base-substitutions that resulted in stop, missense or silent codon mutations in lac $Z$ recovered from sperm 42 days after treatment.

\begin{tabular}{ccc} 
& $\begin{array}{c}\% \\
\text { Control }\end{array}$ & \% BaP \\
\hline stop & 56.4 & 48.2 \\
missense & 43.6 & 46.2 \\
silent & 0 & 5.6
\end{tabular}




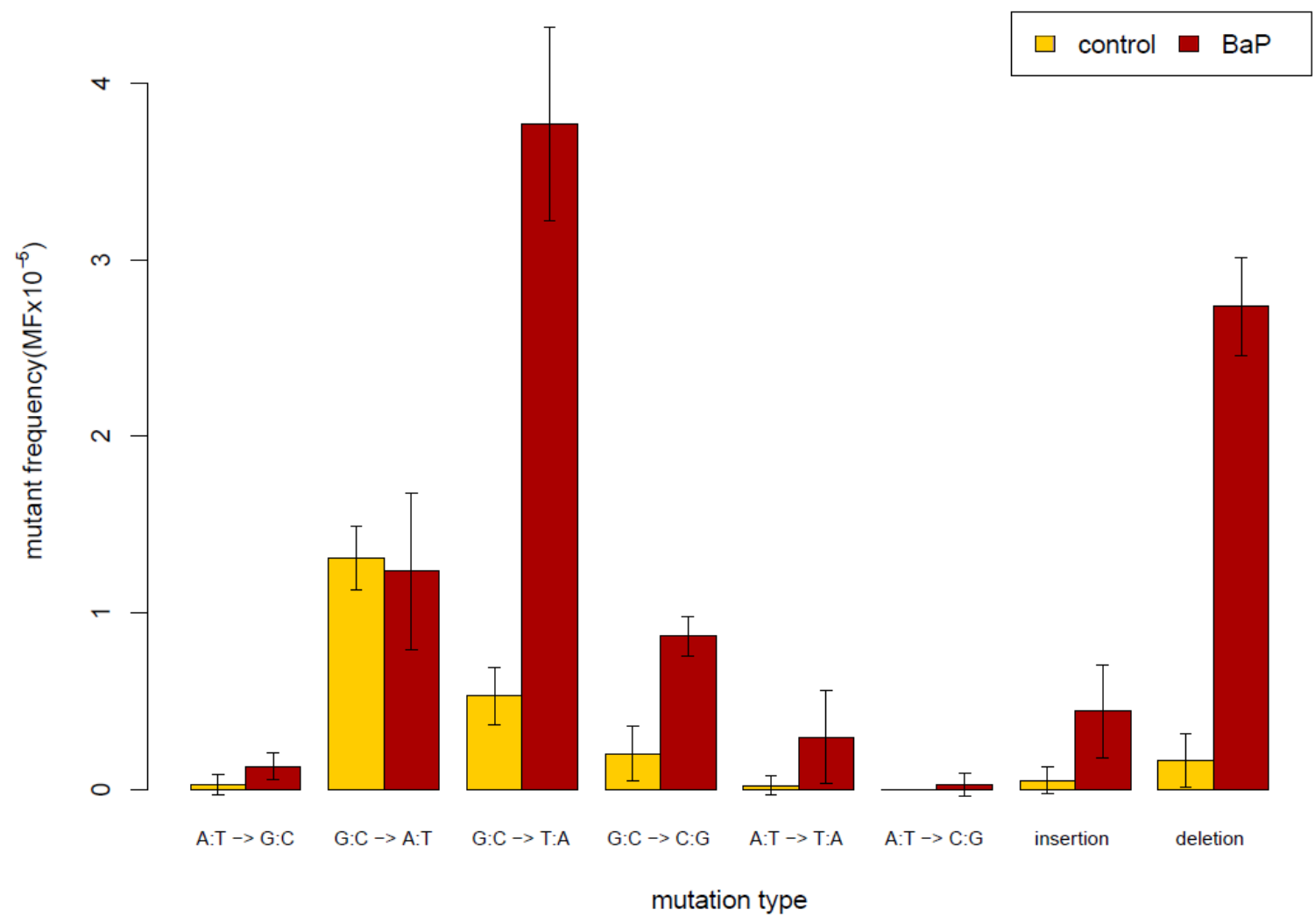

Figure C13 The frequency, by type, of spontaneous or BaP-induced lacZ mutations recovered from sperm 42 days after treatment. The frequency of each mutation type was determined by multiplying the lacZ mutation frequency after clonal correction (Table 1) by the proportion of each mutation type observed (Figure 2). For example, the mutation frequency for BaP after correction was $9.5 \times 10^{-5}$, and the proportion of G:C $\rightarrow$ T:A was 0.40 therefore, the mutation frequency of $\mathrm{G}: \mathrm{C} \rightarrow \mathrm{T}: \mathrm{A}$ in BaP was $3.8 \times 10^{-5}$. 


\section{C.5 Chapter 7}

Table C12 Detailed summary of CNVs detected in this study.

\begin{tabular}{|c|c|c|c|c|c|c|c|c|c|c|c|c|}
\hline Group $^{1}$ & Sample & CNV Type & Origin $^{2}$ & $\begin{array}{l}\text { Break } \\
\text { Homology }\end{array}$ & $\begin{array}{l}\text { Proposed } \\
\text { Mechanism }\end{array}$ & $\begin{array}{c}\text { ProbeC } \\
\text { ount }\end{array}$ & $\begin{array}{l}\text { Probe Location } \\
\text { (mm10) }\end{array}$ & $\begin{array}{l}\text { Size } \\
(\mathbf{k b})^{3}\end{array}$ & $\begin{array}{l}\text { Log2 } \\
\text { Ratio }\end{array}$ & Genes & $\begin{array}{c}\text { Gene } \\
\text { Symbols }\end{array}$ & $\begin{array}{c}\text { Possible } \\
\text { Disease } \\
\text { Outcome (Brief } \\
\text { Description) }\end{array}$ \\
\hline \multirow[t]{2}{*}{ Control } & $5 \mathrm{EF} 2$ & Deletion $^{6}$ & ? & СТСТTT & MMBIR & 12 & $\begin{array}{c}\text { chr5:14061540- } \\
14079968\end{array}$ & 0 & -0.85 & 1 & Sema3e & $\begin{array}{c}\text { Charge } \\
\text { syndrome, } \\
\text { Retinopathy }\end{array}$ \\
\hline & $11 \mathrm{EF} 3$ & Deletion & $P$ & $\begin{array}{c}\text { AAGA + } \\
\text { Deletion of } \\
\text { G }\end{array}$ & MMBIR & 7 & $\begin{array}{c}\text { chr14:106917436- } \\
106976719\end{array}$ & 0 & -1.01 & 0 & - & \\
\hline \multirow[t]{5}{*}{$\begin{array}{l}\text { Post- } \\
\text { Meiotic }\end{array}$} & $32 \mathrm{CF} 2$ & $\begin{array}{l}\text { Gain (Tandem } \\
\text { Duplication + } \\
\text { Insertion }{ }^{7} \text { ) }\end{array}$ & $?$ & AG; TC & MMBIR & 6 & $\begin{array}{c}\text { chr3:96434442- } \\
96446835\end{array}$ & 18 & 0.48 & 1 & BC107364 & \\
\hline & 33DF1 & Deletion & $P$ & GA & MMBIR & 11 & $\begin{array}{c}\text { chr4:147899508- } \\
147914669\end{array}$ & 17 & -0.79 & 2 & $\begin{array}{l}\text { Mfn2 } \\
\text { Plod1 }\end{array}$ & $\begin{array}{l}\text { Charcot-Marie- } \\
\text { Tooth disease, } \\
\text { Neuropathy } \\
\text { Ehlers-Danlos } \\
\text { Syndrome }\end{array}$ \\
\hline & 33DM2 & $\begin{array}{l}\text { Gain (Tandem } \\
\text { Duplication) }\end{array}$ & ? & ACTT & MMBIR & 6 & $\begin{array}{c}\text { chr5:103365011- } \\
103395374\end{array}$ & 39 & 0.56 & 0 & - & \\
\hline & 39AM3 & $\begin{array}{c}\text { Gain } \\
\left(\text { Insertion }{ }^{8}\right)\end{array}$ & $P$ & $\mathrm{CT} ; ?$ & MMBIR & 40 & $\begin{array}{c}\text { chr4:8198042- } \\
8276754\end{array}$ & $\sim 79$ & 0.57 & 1 & Car8 & $\begin{array}{c}\text { Mental } \\
\text { Retardation }\end{array}$ \\
\hline & 39AM3 & $\begin{array}{c}\text { Gain } \\
\left.\text { (Insertion }{ }^{8}\right)\end{array}$ & $P$ & $\mathrm{CT} ; ?$ & MMBIR & 230 & $\begin{array}{c}\text { chr10:8116571- } \\
8649643\end{array}$ & $\sim 533$ & 0.61 & 1 & Ust & $\begin{array}{c}\text { Liver } \\
\text { Inflammatory } \\
\text { Pseudotumor }\end{array}$ \\
\hline \multirow[t]{2}{*}{$\begin{array}{l}\text { Pre- } \\
\text { Meiotic }\end{array}$} & $37 \mathrm{HF} 2$ & $\begin{array}{l}\text { Gain (Tandem } \\
\text { Duplication) }\end{array}$ & ? & TCTTTG & MMBIR & 15 & $\begin{array}{c}\text { chr6:120824505- } \\
120847639\end{array}$ & 20 & 0.47 & 1 & $\mathrm{Bc}|2| 13$ & $\begin{array}{c}\text { Cancer, Cat Eye } \\
\text { Syndrome }\end{array}$ \\
\hline & $32 \mathrm{GM} 3$ & $\begin{array}{c}\text { Gain } \\
\left(\text { Insertion }^{9}\right)\end{array}$ & ? & $A T ;(G) G^{10}$ & MMBIR & 18 & $\begin{array}{c}\text { chr16:9135860- } \\
9238997\end{array}$ & 112 & 0.60 & 0 & - & \\
\hline \multirow[t]{2}{*}{$\begin{array}{l}\text { Pre- } \\
\text { Meiotic }\end{array}$} & $\begin{array}{l}\text { 37HM5, } \\
\text { 37HM6 }\end{array}$ & Deletion & $?$ & $\begin{array}{c}\text { Insertion of } \\
A\end{array}$ & NHEJ & 23 & $\begin{array}{c}\text { chr3:33785808- } \\
33828442\end{array}$ & 39 & -0.88 & 2 & $\begin{array}{l}\text { Ttc14 } \\
\text { Ccdc39 }\end{array}$ & \\
\hline & $\begin{array}{l}33 F F 1 \\
33 F F 3 \\
33 F F 5\end{array}$ & Deletion & $?$ & $\begin{array}{c}\text { AA or } \\
\text { Insertion of } \\
\text { C }\end{array}$ & $\begin{array}{l}\text { MMBIR or } \\
\text { NHEJ }^{11}\end{array}$ & 109 & $\begin{array}{c}\text { chr5:74339797- } \\
74533734\end{array}$ & 196 & -1.03 & 1 & Scfd2 & \\
\hline
\end{tabular}




\begin{tabular}{|c|c|c|c|c|c|c|c|c|c|c|c|c|}
\hline Control & $6 \mathrm{FF} 5$ & $\begin{array}{l}\text { Deletion } \\
\text { (Tandem } \\
\text { Duplication } \\
\text { Removed) }\end{array}$ & $P$ & $\begin{array}{c}\text { None } \\
\text { Detected }\end{array}$ & NAHR & 18 & $\begin{array}{c}\text { chr4 } 62495468- \\
\quad 62522517\end{array}$ & $\sim 27$ & -0.61 & 3 & $\begin{array}{c}\text { Bspry } \\
\text { Hdhd3 } \\
\text { Alad }\end{array}$ & Porphyria \\
\hline & $9 \mathrm{FF} 2$ & Deletion & $?(\mathrm{E})$ & $\mathrm{AC}$ & MMBIR & 31 & $\begin{array}{c}\text { chr3:129439219- } \\
129544739\end{array}$ & 111 & -0.75 & 4 & $\begin{array}{c}\text { AK139522 } \\
\text { AK139614 } \\
\text { Elovl6 } \\
\text { FAE }\end{array}$ & \\
\hline \multirow[t]{2}{*}{$\begin{array}{l}\text { Post- } \\
\text { Meiotic }\end{array}$} & $39 \mathrm{AF} 3$ & Deletion & $?$ & $?$ & $?$ & 5 & $\begin{array}{c}\text { chr4:137054729- } \\
137062737\end{array}$ & $\sim 8$ & -0.58 & 0 & - & \\
\hline & 37AM4 & Deletion & $?$ & $\mathrm{~T}$ & MMBIR & 68 & $\begin{array}{c}\text { chr17:11313356- } \\
11419145\end{array}$ & 110 & -0.57 & 1 & Park2 & $\begin{array}{c}\text { Parkinson's } \\
\text { disease }\end{array}$ \\
\hline $\begin{array}{c}\text { Pre- } \\
\text { Meiotic }\end{array}$ & $33 \mathrm{GF} 1$ & Deletion & $P(E)$ & $?$ & $?$ & 68 & $\begin{array}{c}\text { chr8:16622845- } \\
16729117\end{array}$ & $\sim 106$ & -0.55 & 1 & Csmd1 & $\begin{array}{c}\text { Epilepsy, } \\
\text { Smallpox, } \\
\text { Cancer }\end{array}$ \\
\hline
\end{tabular}

${ }^{1}$ The top 9, middle 2, and bottom 5 CNVs were characterized as de novo, germline mosaic, and embryonic events, respectively.

${ }^{2} \mathrm{P}$ indicates that the $\mathrm{CNV}$ occurred on the paternal chromosome, ? indicates origin was unknown, and $\mathrm{E}$ indicates that the $\mathrm{CNV}$ arose during embryogenesis.

${ }^{3}$ Size based on breakpoint locations found by NGS.

${ }^{4}$ Genes were identified by genome browser.

${ }^{5}$ Possible disease outcomes identified by gene cards (Rebhan et al., 1997).

${ }^{6}$ A single nucleotide deletion of $\mathrm{G}$ was detected 3 bp upstream of deletion break on the same reads that spanned the breakpoint.

${ }^{7}$ Insertion consisted of the sequence (TTTTGTAAGACCCCTAAAGCTGGGCC), originating from DNA $16 \mathrm{~kb}$ upstream of the CNV, within the CNV breakpoint. 
${ }^{8}$ Insertion occurred in Long Interspersed Nuclear Element (L1Md_T, family L1). Both CNVs from 39AM3 are a part of the same event.

${ }^{9}$ Insertion occurred in a satellite (GSAT_MM).

${ }^{10}$ Due to the repetitive nature of the insertion site, it could not be determined if the breakpoint homology was G or GG.

${ }^{11}$ The 2 possible mechanisms for the formation of this CNV are compared in Figure C17. 
Table C13 Overview of mutation candidates and detailed summary of de novo mutations detected in this study.

\begin{tabular}{|c|c|c|c|c|c|c|c|c|c|c|c|c|c|c|c|c|c|}
\hline Group & Sample $^{1}$ & $\begin{array}{l}\text { Mutation } \\
\text { Candidates }\end{array}$ & $\begin{array}{l}\text { Passed } \\
\text { Primer } \\
\text { Design }\end{array}$ & $\mathrm{FP}^{2}$ & $\mathrm{TP}^{3}$ & Mosaic & $E^{4}$ & $\begin{array}{l}\text { Validated } \\
\text { DNM }\end{array}$ & $\begin{array}{l}\text { A:T } \\
\rightarrow \\
\text { G:C }\end{array}$ & $\begin{array}{c}\mathrm{G}: \mathrm{C} \\
\rightarrow \\
\mathrm{A}: \mathrm{T}\end{array}$ & $\begin{array}{c}\mathrm{G}: \mathrm{C} \\
\rightarrow \\
\mathrm{T}: \mathrm{A}\end{array}$ & $\begin{array}{c}\mathrm{G}: \mathrm{C} \\
\rightarrow \\
\mathrm{C}: \mathrm{G}\end{array}$ & $\begin{array}{c}\mathrm{A}: \mathrm{T} \\
\rightarrow \\
\mathrm{T}: \mathrm{A}\end{array}$ & $\begin{array}{l}\mathrm{A}: \mathrm{T} \\
\rightarrow \\
\mathrm{C}: \mathrm{G}\end{array}$ & Indel & $\begin{array}{c}\text { Non- } \\
\text { BaP } \\
\text { Type }\end{array}$ & $\begin{array}{l}\mathrm{BaP} \\
\text { Type }\end{array}$ \\
\hline \multirow[t]{4}{*}{ Control } & $3 \mathrm{HF} 1$ & 162 & 157 & 151 & 6 & 0 & 2 & 4 & 1 & 2 & 1 & 0 & 0 & 0 & 0 & 3 & 1 \\
\hline & 3HM1 & 61 & 59 & 48 & 11 & 1 & 0 & 10 & 2 & 3 & 2 & 0 & 2 & 1 & 0 & 8 & 2 \\
\hline & 4HF1 & 120 & 111 & 95 & 16 & 2 & 4 & 10 & 0 & 6 & 2 & 0 & 1 & 0 & 1 & 8 & 2 \\
\hline & 4HM1 & 49 & 46 & 38 & 8 & 1 & 3 & 4 & 0 & 2 & 1 & 0 & 1 & 0 & 0 & 3 & 1 \\
\hline \multirow{4}{*}{$\begin{array}{l}\text { Post- } \\
\text { Meiotic }\end{array}$} & $32 \mathrm{CF} 1$ & 125 & 109 & 90 & 19 & 1 & 3 & 15 & 2 & 7 & 4 & 2 & 0 & 0 & 0 & 9 & 6 \\
\hline & $32 \mathrm{CM} 1$ & 114 & 109 & 86 & 23 & 1 & 13 & 9 & 1 & 6 & 1 & 0 & 1 & 0 & 0 & 8 & 1 \\
\hline & 39AF1 & 68 & 61 & 51 & 10 & 0 & 4 & 6 & 1 & 3 & 0 & 1 & 1 & 0 & 0 & 5 & 1 \\
\hline & 39AM1 & 202 & 201 & 179 & 22 & 3 & 3 & 16 & 0 & 12 & 4 & 0 & 0 & 0 & 0 & 12 & 4 \\
\hline \multirow[t]{4}{*}{$\begin{array}{c}\text { Pre- } \\
\text { Meiotic }\end{array}$} & $32 \mathrm{EF} 1$ & 142 & 131 & 119 & 12 & 0 & 2 & 10 & 1 & 5 & 1 & 0 & 2 & 1 & 0 & 9 & 1 \\
\hline & 32EM1 & 73 & 70 & 56 & 14 & 0 & 3 & 11 & 1 & 6 & 0 & 1 & 1 & 2 & 0 & 10 & 1 \\
\hline & $39 \mathrm{FF} 1$ & 203 & 198 & 188 & 10 & 1 & 5 & 4 & 0 & 2 & 1 & 0 & 1 & 0 & 0 & 3 & 1 \\
\hline & 39FM1 & 83 & 81 & 48 & 33 & 2 & 3 & 28 & 0 & 8 & 9 & 8 & 3 & 0 & 0 & 11 & 17 \\
\hline
\end{tabular}

${ }^{1}$ Samples affected by the BaP exposure, in that they have more de novo mutations, are highlighted in yellow. The highlighted animals have higher number of mutations that match the mutational profile of BaP than the other animals (Fisher's Exact $\mathrm{P}=0.0001$ ).

${ }^{2}$ False-positive

${ }^{3}$ True-positive

${ }^{4}$ Embryonic 
Table C14 Summary of embryonic mutations detected in this study.

\begin{tabular}{|c|c|c|c|c|c|c|c|c|c|c|c|}
\hline Group & Sample $^{1}$ & Embryonic & $\begin{array}{l}\mathrm{A}: \mathrm{T} \rightarrow \\
\mathrm{G}: \mathrm{C}\end{array}$ & $\begin{array}{l}\text { G:C } \rightarrow \\
\text { A:T }\end{array}$ & $\begin{array}{c}\text { G:C } \rightarrow \\
\text { T:A }\end{array}$ & $\begin{array}{c}\text { G:C } \rightarrow \\
\text { C:G }\end{array}$ & $\begin{array}{l}\mathrm{A}: \mathrm{T} \rightarrow \\
\mathrm{T}: \mathrm{A}\end{array}$ & $\begin{array}{l}\text { A:T } \rightarrow \\
\text { C:G }\end{array}$ & Indel & $\begin{array}{l}\text { Non-BaP } \\
\text { Type }\end{array}$ & $\begin{array}{l}\text { BaP } \\
\text { Type }\end{array}$ \\
\hline \multirow[t]{4}{*}{ Control } & 3HF1 & 2 & 0 & 1 & 1 & 0 & 0 & 0 & 0 & 1 & 1 \\
\hline & 3HM1 & 0 & 0 & 0 & 0 & 0 & 0 & 0 & 0 & 0 & 0 \\
\hline & $4 \mathrm{HF} 1$ & 4 & 0 & 1 & 1 & 1 & 1 & 0 & 0 & 2 & 2 \\
\hline & 4HM1 & 3 & 0 & 1 & 0 & 1 & 0 & 1 & 0 & 2 & 1 \\
\hline \multirow{4}{*}{$\begin{array}{l}\text { Post- } \\
\text { Meiotic }\end{array}$} & $32 \mathrm{CF} 1$ & 3 & 0 & 2 & 1 & 0 & 0 & 0 & 0 & 2 & 1 \\
\hline & $32 \mathrm{CM} 1$ & 13 & 0 & 1 & 8 & 4 & 0 & 0 & 0 & 1 & 12 \\
\hline & 39AF1 & 4 & 0 & 1 & 1 & 1 & 0 & 1 & 0 & 2 & 2 \\
\hline & 39AM1 & 3 & 0 & 1 & 0 & 2 & 0 & 0 & 0 & 1 & 2 \\
\hline \multirow[t]{4}{*}{$\begin{array}{l}\text { Pre- } \\
\text { Meiotic }\end{array}$} & $32 \mathrm{EF} 1$ & 2 & 0 & 1 & 0 & 0 & 1 & 0 & 0 & 2 & 0 \\
\hline & 32EM1 & 3 & 0 & 0 & 1 & 1 & 0 & 0 & 1 & 1 & 2 \\
\hline & $39 \mathrm{FF} 1$ & 5 & 0 & 1 & 2 & 0 & 0 & 0 & 2 & 3 & 2 \\
\hline & 39FM1 & 3 & 0 & 1 & 1 & 1 & 0 & 0 & 0 & 1 & 2 \\
\hline
\end{tabular}

${ }^{1}$ The sample affected by the BaP exposure is highlighted in yellow. This animal has a higher number of mutations that match the mutational profile of $\mathrm{BaP}$ than the other animals (Fisher's Exact $\mathrm{P}=0.0046$ ). 
Table C15 Consequences of mutations detected in this study.

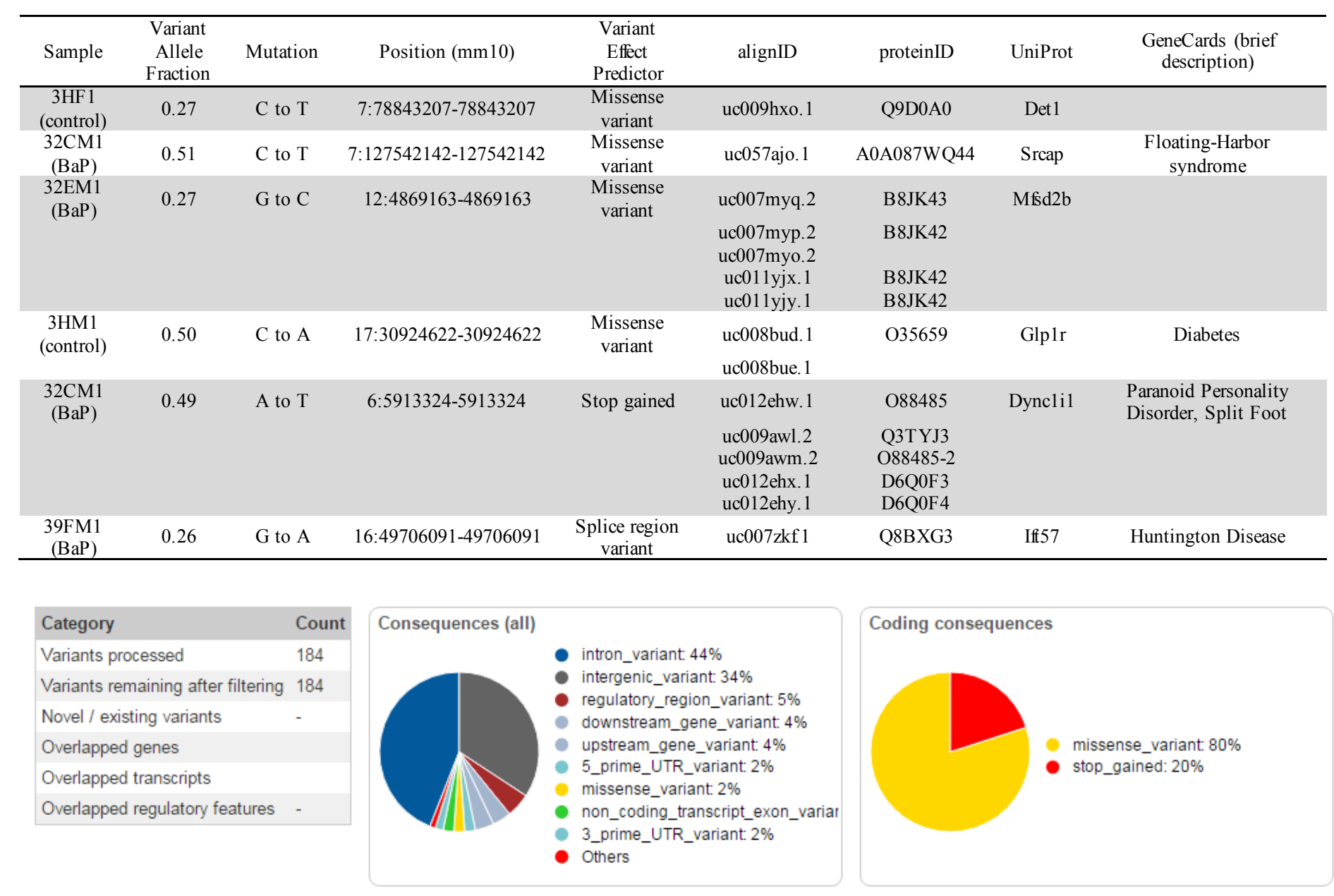


Table C16 Parental origin of SNVs with haplotype information.

\begin{tabular}{cccccccccc}
\hline Group & Sample & Chromosome & Position & Reference & Mutation & $\begin{array}{c}\text { Phasing } \\
\text { Position }\end{array}$ & $\begin{array}{c}\text { Distance } \\
\text { (bp) }\end{array}$ & $\begin{array}{c}\text { Variant } \\
\text { Allele } \\
\text { Fraction }\end{array}$ & $\begin{array}{c}\text { Parental } \\
\text { Origin }\end{array}$ \\
\hline Control & $4 \mathrm{HF} 1$ & 18 & 70636373 & $\mathrm{C}$ & $\mathrm{T}$ & 70636256 & -117 & 0.51 & $\mathrm{P}$ \\
$\mathrm{BaP}$ & $32 \mathrm{CF} 1$ & 2 & 63007102 & $\mathrm{C}$ & $\mathrm{G}$ & 63007071 & -31 & 0.50 & $\mathrm{M}$ \\
$\mathrm{BaP}$ & $32 \mathrm{CM} 1$ & 4 & 139695288 & $\mathrm{G}$ & $\mathrm{T}$ & 139695318 & 30 & 0.31 & $\mathrm{P}$ \\
$\mathrm{BaP}$ & $32 \mathrm{CM} 1$ & 10 & 125272711 & $\mathrm{G}$ & $\mathrm{A}$ & 125272693 & -18 & 0.43 & $\mathrm{P}$ \\
$\mathrm{BaP}$ & $32 \mathrm{CM} 1$ & 9 & 110894808 & $\mathrm{G}$ & $\mathrm{A}$ & 110894804 & -4 & 0.48 & $\mathrm{P}$ \\
$\mathrm{BaP}$ & $32 \mathrm{EF} 1$ & 14 & 59791120 & $\mathrm{~A}$ & $\mathrm{G}$ & 59791125 & 5 & 0.47 & $\mathrm{P}$ \\
$\mathrm{BaP}$ & $32 \mathrm{EF} 1$ & 11 & 28424214 & $\mathrm{C}$ & $\mathrm{T}$ & 28424224 & 10 & 0.34 & $\mathrm{P}$ \\
$\mathrm{BaP}$ & $32 \mathrm{EM} 1$ & 9 & 92281765 & $\mathrm{C}$ & $\mathrm{T}$ & 92281691 & -74 & 0.48 & $\mathrm{P}$ \\
$\mathrm{BaP}$ & $32 \mathrm{EM} 1$ & 10 & 92269288 & $\mathrm{~A}$ & $\mathrm{G}$ & 92269306 & 18 & 0.46 & $\mathrm{P}$ \\
$\mathrm{BaP}$ & $32 \mathrm{EM} 1$ & 19 & 57509070 & $\mathrm{C}$ & $\mathrm{T}$ & 57508970 & -100 & 0.49 & $\mathrm{P}$ \\
$\mathrm{BaP}$ & $39 \mathrm{AM} 1$ & 2 & 171738999 & $\mathrm{G}$ & $\mathrm{A}$ & 171739004 & 5 & 0.53 & $\mathrm{M}$ \\
$\mathrm{BaP}$ & $39 \mathrm{AM} 1$ & 10 & 105262209 & $\mathrm{G}$ & $\mathrm{C}$ & 105262158 & -51 & 0.28 & $\mathrm{P}$ \\
$\mathrm{BaP}$ & $39 \mathrm{AM} 1$ & 8 & 94983437 & $\mathrm{C}$ & $\mathrm{T}$ & 94983430 & -7 & 0.40 & $\mathrm{P}$ \\
$\mathrm{BaP}$ & $39 \mathrm{AM} 1$ & 3 & 48662684 & $\mathrm{~A}$ & $\mathrm{G}$ & 48662793 & 109 & 0.48 & $\mathrm{M}$ \\
$\mathrm{BaP}$ & $39 \mathrm{AM} 1$ & 11 & 4685927 & $\mathrm{G}$ & $\mathrm{A}$ & 4685949 & 22 & 0.40 & $\mathrm{P}$ \\
$\mathrm{BaP}$ & $39 \mathrm{FM} 1$ & 8 & 125400104 & $\mathrm{G}$ & $\mathrm{A}$ & 125400043 & -61 & 0.47 & $\mathrm{P}$ \\
$\mathrm{BaP}$ & $39 \mathrm{FM} 1$ & 6 & 108002478 & $\mathrm{C}$ & $\mathrm{T}$ & 108002501 & 23 & 0.49 & $\mathrm{P}$ \\
$\mathrm{BaP}$ & $39 \mathrm{FM} 1$ & 3 & 50269511 & $\mathrm{G}$ & $\mathrm{C}$ & 50269532 & 21 & 0.47 & $\mathrm{P}$ \\
\hline & & & & & & & & &
\end{tabular}

${ }^{1} \mathrm{P}$ is paternal and $\mathrm{M}$ is maternal. 


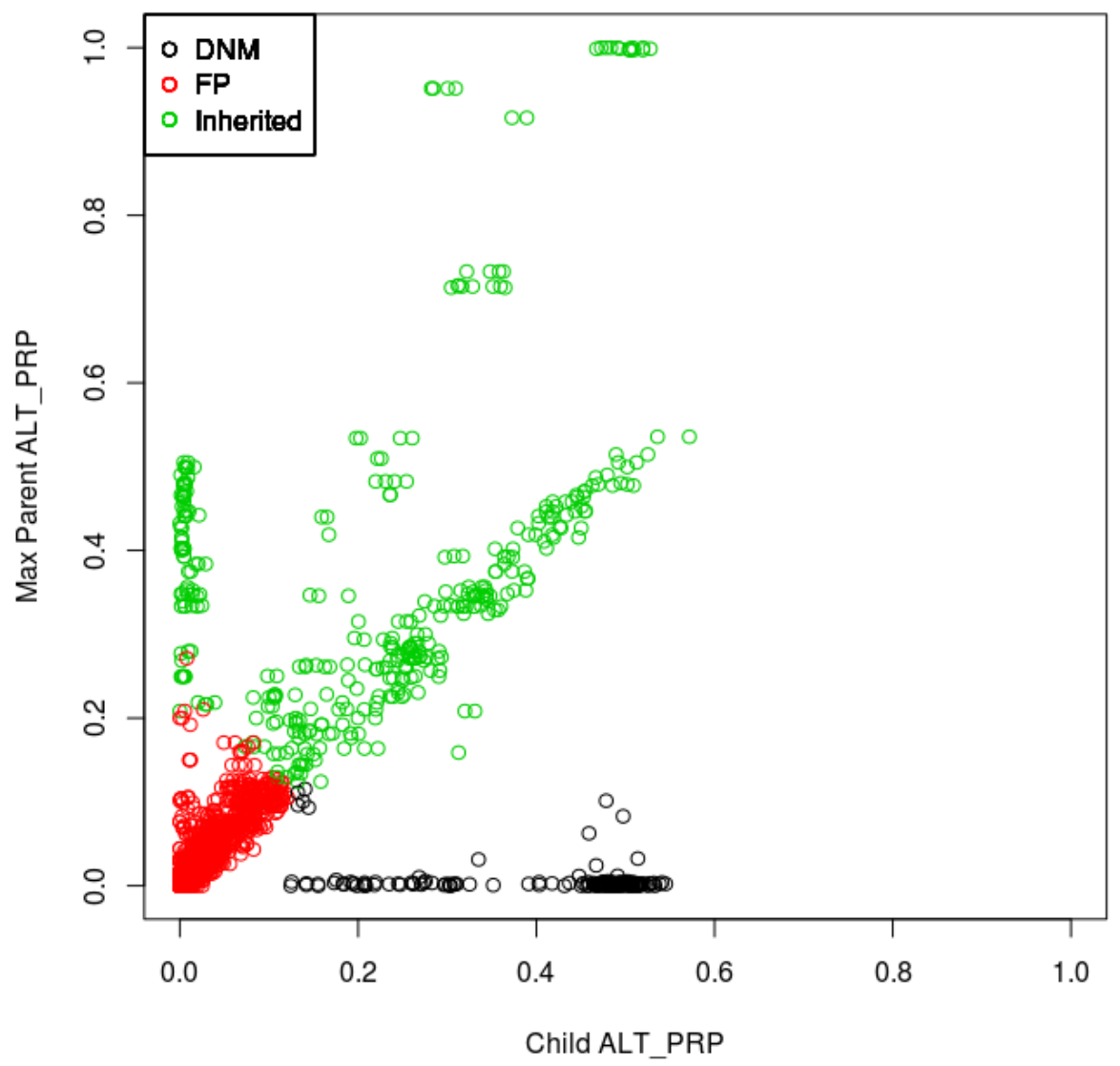

Figure C14. Validation of variants as de novo mutations, false-positives, or inherited. Each circle represents a variant call in an offspring. The $\mathrm{Y}$-axis shows the maximum parental VAF for the variant and the X-axis shows the VAF in the offspring. Variants were called de novo here if the variant allele fraction was below $15 \%$ in parents and above $15 \%$ in offspring. The mutations were further filtered to eliminate mosaic and embryonic events. 


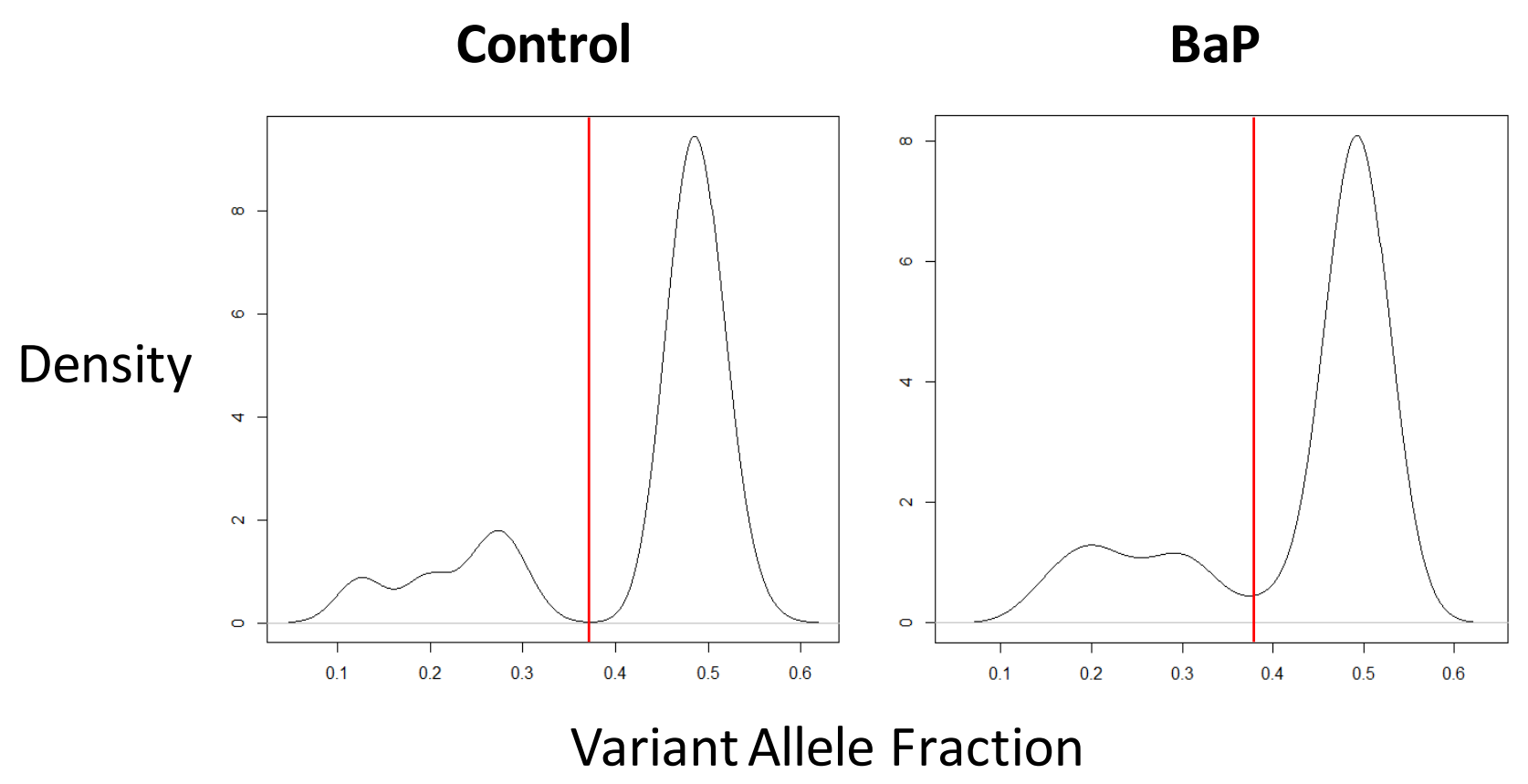

Figure C15. Density plots of variant allele fractions reveal 2 modes: embryonic mutations and de novo mutations. The red line (VAF

$=0.39)$ indicates the threshold separating embryonic mutations from de novo mutations. 


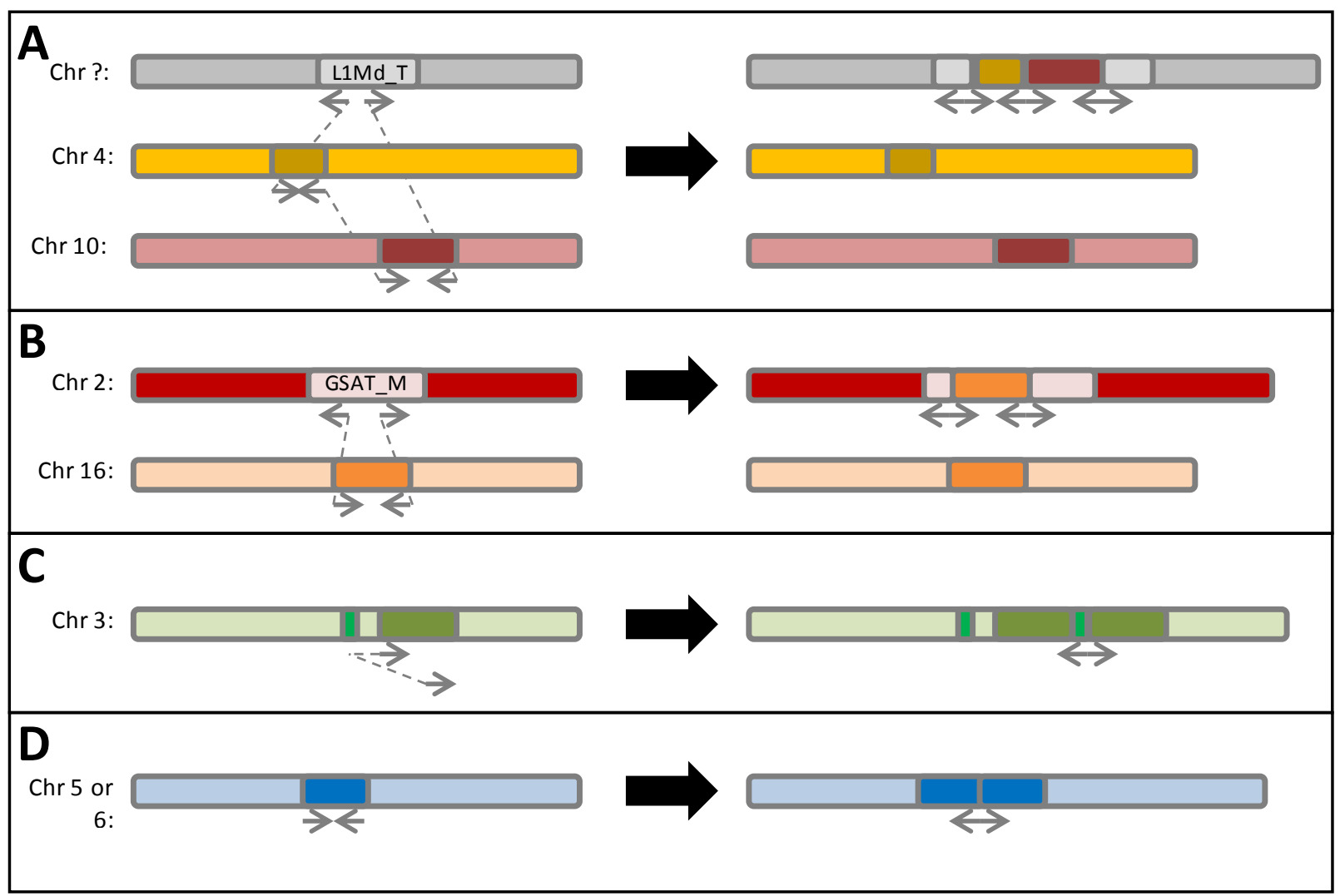

Figure C16 Overview of insertions and duplications detected in this study. The left side shows how the chromosomes appear in the reference genome (not to scale). The grey arrows indicate discordant mate pairs and the dashed lines indicate their partners. Using this information, the CNV structure could be identified (right side). A) Sections of chromosomes 4 and 10 were inserted into a large L1Md_T element at an unidentified location in the genome. B) A segment of chromosome was inserted into a GSAT_M element, likely on chromosome 2. C) A tandem duplication occurred on chromosome 3. The breakpoint contained DNA from a $28 \mathrm{bp}$ region $16 \mathrm{~kb}$ upstream of the duplication. D) Two simple tandem duplications occurred in this study, 1 animal had the duplication on chromosome 5 and the other had it on chromosome 6 . 


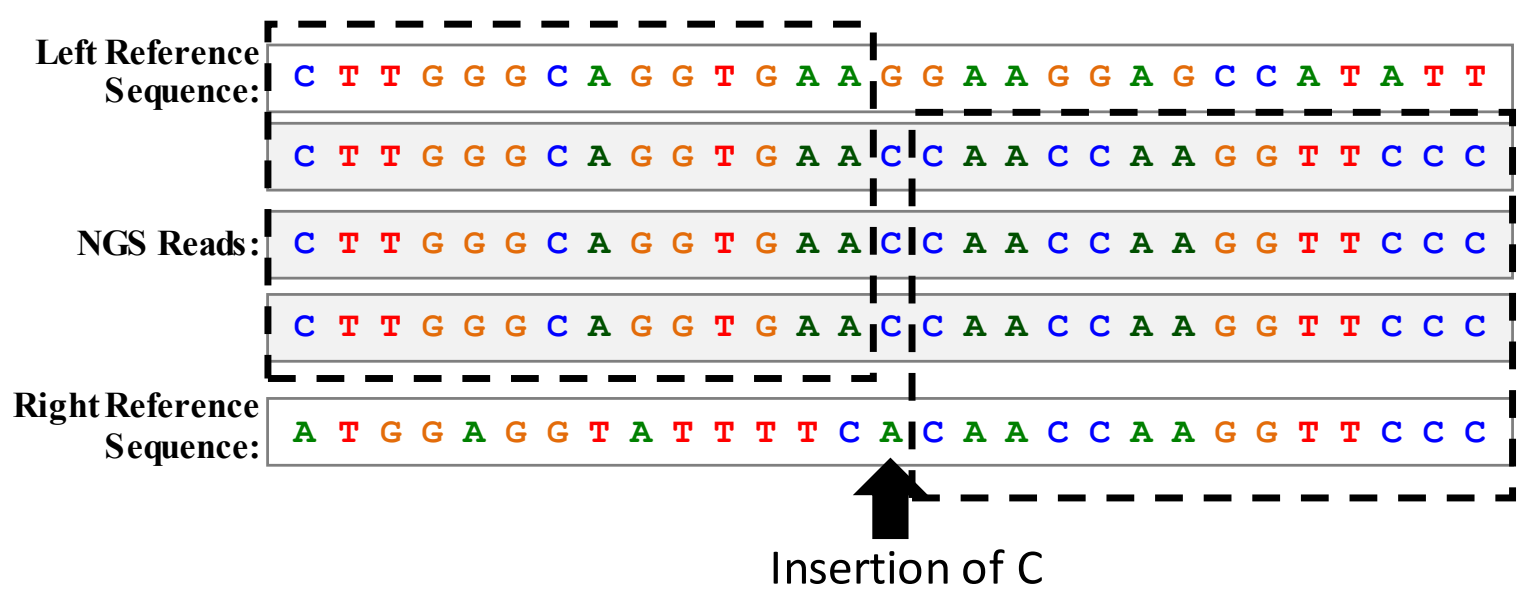

1)

$$
\begin{array}{r}
\mathrm{A}-\mathrm{A}-\mathrm{C}-\mathrm{C}-\mathrm{A}-\mathrm{A}-\mathrm{G}-\mathrm{G}- \\
\mathrm{G}-\mathrm{T}\} \mathrm{S}-\mathrm{T}-\mathrm{T}-\mathrm{G}-\mathrm{G}-\mathrm{T}-\mathrm{T}-\mathrm{C}-\mathrm{C}-
\end{array}
$$

2)

$$
\begin{array}{r}
C-A-A, \\
A-A-G-G- \\
\text { G-T } 2\}-T-T-G-G-T-T-C-C-
\end{array}
$$

3)

$$
\begin{gathered}
\mathrm{C}-\mathrm{A}-\mathrm{A} \\
\mathrm{C}-\mathrm{A}-\mathrm{C}-\mathrm{C}-\mathrm{A}-\mathrm{A}-\mathrm{G}-\mathrm{G}- \\
\mathrm{G}-\mathrm{T} \mathrm{S}-\mathrm{T}-\mathrm{T}-\mathrm{G}-\mathrm{G}-\mathrm{T}-\mathrm{T}-\mathrm{C}-\mathrm{C}-
\end{gathered}
$$

4)

$$
\begin{aligned}
& \begin{array}{r}
G-G-G-C-A-G-G \quad A-A-C-C-A-A-C-C-A-A-G-G- \\
T-T-G-G-T-T-C-C-
\end{array} \\
& C-C-C-G-T-C-C-A-C-T-T-C-C-T-T-C
\end{aligned}
$$

Figure C17 Different possible mechanisms that explain the germline mosaic CNV in animals 33FF1, 33FF3, and 33FF5. The top panel shows how the NGS reads align to the reference genome on the left and right sides of the CNV. The Cytosine nucleotide does not match either break sequence and may represent an insertion through NonHomologous End-Joining. The bottom panel demonstrates a scenario where replication 
slippage can explain the mutation formation. (1) Replicating daughter strand pauses at damaged nucleotide. (2) Replication strand slips and re-anneals at incorrect template. (3) Replication re-initiates and duplicates sequence. (4) Failing to proceed on the template, the strand slips again and invades another replication fork downstream, using the microhomology of AA, resulting in a $196 \mathrm{~kb}$ deletion. 


\section{C.6 Chapter 8}

\section{C.6.1 Tables and Figures}

Table C17 Human evidence for sperm parameters impaired by tobacco smoke that may be related to mutagenicity.

\begin{tabular}{|c|c|c|c|c|c|c|c|c|c|c|c|c|c|}
\hline Fertility Parameter & Cigarettes/Day & Pack-Years & $\begin{array}{l}\text { Cotinine } \\
(\mathrm{ng} / \mathrm{mL})\end{array}$ & $\mathrm{N}-\mathrm{S}$ & $\mathrm{S}$ & $\mathrm{L}$ & M & $\mathrm{H}$ & $\mathrm{F} ?$ & D & FD & P-Value & Ref. \\
\hline \multirow[t]{17}{*}{ Sperm Concentration } & - & - & - & 30 & 22 & - & - & - & I & $\downarrow$ & 2.9 & $<0.05$ & $\begin{array}{c}\text { (Omran et al., } \\
2013)\end{array}$ \\
\hline & $1-20 \mathrm{~L},>21 \mathrm{H}$ & - & - & 126 & 178 & 98 & - & 80 & I & $\downarrow$ & $\begin{array}{l}1.7- \\
2.6\end{array}$ & 0.001 & $\begin{array}{l}\text { (Mitra et al., } \\
\text { 2012) }\end{array}$ \\
\hline & - & $3.8 \mathrm{~L}, 15.1 \mathrm{H}$ & - & 56 & 54 & 29 & - & 25 & $\begin{array}{l}\text { I } \\
\text { (V) }\end{array}$ & $\downarrow$ & 2.2 & 0.038 & $\begin{array}{l}\text { (Fariello et al., } \\
\text { 2012) }\end{array}$ \\
\hline & 20 & - & - & 15 & 10 & - & - & - & F & $\downarrow$ & 2.2 & $<0.001$ & $\begin{array}{l}\text { (Rubes et al., } \\
\text { 1998) }\end{array}$ \\
\hline & $\begin{array}{c}1-10 \mathrm{~L}, 11-19 \mathrm{M} \\
\geq 20 \mathrm{H}\end{array}$ & - & - & 153 & 118 & 42 & 25 & 51 & I & $\downarrow$ & 2.1 & $<0.05$ & $\begin{array}{c}\text { (Collodel et al., } \\
2010)\end{array}$ \\
\hline & $\begin{array}{c}1-10 \mathrm{~L}, 11-19 \mathrm{M}, \\
\geq 20 \mathrm{H}\end{array}$ & - & - & 39 & 121 & 49 & 36 & 36 & $\begin{array}{l}\text { I } \\
\text { (V) }\end{array}$ & $\downarrow$ & 2.0 & $<0.05$ & $\begin{array}{l}\text { (Collodel et al., } \\
\text { 2009) }\end{array}$ \\
\hline & $13.3 \pm 9.5$ & - & - & 19 & 9 & - & - & - & F & $\downarrow$ & 1.87 & 0.02 & $\begin{array}{l}\text { (Härkönen et al., } \\
1999 \text { ) }\end{array}$ \\
\hline & - & $14.1 \pm 7.3$ & - & 27 & 30 & - & - & - & $\mathrm{F}$ & $\downarrow$ & 1.7 & - & (Yu et al., 2013) \\
\hline & $\begin{array}{c}5 \pm 2.44 \mathrm{~L}, 14.84 \pm \\
3.43 \mathrm{M}, 22.90 \pm 1.7 \\
\mathrm{H}\end{array}$ & - & - & 30 & 40 & 10 & 19 & 11 & I & $\downarrow$ & 1.7 & $<0.001$ & $\begin{array}{l}\text { (El-Melegy and } \\
\text { Ali, 2011) }\end{array}$ \\
\hline & $<20 \mathrm{~L},>20 \mathrm{H}$ & - & - & 69 & 62 & 43 & - & 19 & I & $\downarrow$ & $\begin{array}{l}1.6- \\
1.7\end{array}$ & $\leq 0.0053$ & $\begin{array}{l}\text { (Reina Bouvet et } \\
\text { al., 2007) }\end{array}$ \\
\hline & $>20$ & - & $\begin{array}{c}60.44 \pm 31.32 \\
\text { (seminal) }\end{array}$ & 19 & 35 & - & - & - & $\mathrm{F}$ & $\downarrow$ & 1.6 & 0.002 & $\begin{array}{l}\text { (Hamad et al., } \\
\text { 2014) }\end{array}$ \\
\hline & $<20 \mathrm{~L},>20 \mathrm{H}$ & - & - & 13 & 49 & 22 & - & 27 & $\mathrm{~F}$ & $\downarrow$ & 1.6 & $<0.05$ & (Joo et al., 2012) \\
\hline & $\begin{array}{c}<10 \mathrm{~L}, 10-19 \mathrm{M} \\
\geq 20 \mathrm{H}\end{array}$ & - & - & 298 & 920 & 256 & 365 & 299 & I & $\downarrow$ & 1.6 & $<0.05$ & (Cui et al., 2016) \\
\hline & $\begin{array}{c}7.89 \pm 0.9 \mathrm{~L}, 17.8 \pm \\
0.5 \mathrm{M}, 33.75 \pm 1.3 \\
\mathrm{H}\end{array}$ & - & - & $<40$ & 40 & 9 & 15 & 16 & I & $\downarrow$ & 1.6 & $<0.05$ & $\begin{array}{c}\text { (Tawadrous et al, } \\
\text { 2011) }\end{array}$ \\
\hline & $\begin{array}{c}1-10 \mathrm{~L}, 11-20 \mathrm{M} \\
21-40 \mathrm{H}\end{array}$ & & 173.9 (seminal) & 30 & 35 & 19 & 11 & 5 & $\mathrm{~F}$ & $\downarrow$ & 1.5 & $<0.05$ & $\begin{array}{c}\text { (Chen and Kuo, } \\
\text { 2007) }\end{array}$ \\
\hline & $\geq 21$ & - & - & 24 & 26 & - & - & - & I & $\downarrow$ & 1.5 & $<0.001$ & $\begin{array}{l}\text { (Kiziler et al., } \\
\text { 2007) }\end{array}$ \\
\hline & - & - & 63.30 (saliva) & 117 & 87 & - & - & - & I & $\downarrow$ & 1.5 & $<0.001$ & $\begin{array}{c}\text { (Jurewicz et al., } \\
2014 \text { ) }\end{array}$ \\
\hline
\end{tabular}




\begin{tabular}{|c|c|c|c|c|c|c|c|c|c|c|c|c|}
\hline $\begin{array}{c}7.5 \pm 0.43 \mathrm{~L}, 15.92 \\
\pm 0.7 \mathrm{M}, 26.44 \pm \\
1.4 \mathrm{H}\end{array}$ & - & - & $<40$ & 40 & 10 & 12 & 18 & $\mathrm{~F}$ & $\downarrow$ & 1.4 & $<0.05$ & $\begin{array}{c}\text { (Tawadrous et al, } \\
\text { 2011) }\end{array}$ \\
\hline $\begin{array}{c}<10 \mathrm{~L}, 10-30 \mathrm{M} \\
>30 \mathrm{H}\end{array}$ & - & - & 132 & 48 & 31 & 6 & 11 & I & $\downarrow$ & 1.4 & 0.03 & $\begin{array}{c}\text { (Aryanpur et al., } \\
\text { 2011) }\end{array}$ \\
\hline- & - & - & 337 & 194 & - & - & - & I & $\downarrow$ & 1.4 & $<0.05$ & $\begin{array}{c}\text { (Kunzle et al., } \\
\text { 2004) }\end{array}$ \\
\hline $19 \pm 7$ & - & - & 17 & 25 & - & - & - & I & $\downarrow$ & 1.4 & $<0.001$ & $\begin{array}{l}\text { (Laudat et al., } \\
\text { 2004) }\end{array}$ \\
\hline- & $<10 \mathrm{~L}, \geq 10 \mathrm{H}$ & - & 473 & 137 & 75 & - & 62 & I & $\downarrow$ & $\begin{array}{l}1.1- \\
1.4\end{array}$ & $<0.04$ & (Chia et al., 1994) \\
\hline $10-19 \mathrm{~L}, 20 \mathrm{H}$ & - & - & 382 & 293 & 57 & - & 236 & $\mathrm{~F}$ & $\downarrow$ & 1.3 & $<0.04$ & $\begin{array}{l}\text { (Lewin et al., } \\
\text { 1991) }\end{array}$ \\
\hline$>20$ & - & - & 20 & 20 & - & - & - & $\begin{array}{c}\mathrm{I} \\
\text { (A) }\end{array}$ & $\downarrow$ & 1.3 & $<0.05$ & $\begin{array}{l}\text { (Mostafa et al., } \\
2006)\end{array}$ \\
\hline $\begin{array}{c}1-10 \mathrm{~L}, 11-20 \mathrm{M} \\
>20 \mathrm{H}\end{array}$ & - & - & 161 & 197 & 57 & 115 & 25 & I & $\downarrow$ & 1.3 & $<0.005$ & $\begin{array}{l}\text { (Merino et al., } \\
\text { 1998) }\end{array}$ \\
\hline $\begin{array}{c}<5 \mathrm{~L}, 5-20 \mathrm{M}, \geq 20 \\
\mathrm{H}\end{array}$ & - & - & 79 & 68 & 14 & 21 & 33 & I & $\downarrow$ & 1.3 & $<0.05$ & (Liu et al., 2010) \\
\hline$<20 \mathrm{~L}, \geq 20 \mathrm{H}$ & - & - & - & 396 & 164 & - & 232 & I & $\downarrow$ & 1.3 & $<0.005$ & $\begin{array}{l}\text { (Meri et al., } \\
\text { 2013) }\end{array}$ \\
\hline $\begin{array}{c}\leq 10 \mathrm{~L}, \leq 20 \mathrm{M},>20 \\
\mathrm{H}\end{array}$ & - & - & 83 & 64 & 26 & 30 & 8 & $\mathrm{~F}$ & $\downarrow$ & 1.2 & 0.001 & $\begin{array}{c}\text { (Ghaffari and } \\
\text { Rostami, 2013) }\end{array}$ \\
\hline $16 \pm 7.5$ & - & - & 83 & 64 & - & - & - & $\mathrm{F}$ & $\downarrow$ & 1.2 & $\leq 0.01$ & $\begin{array}{c}\text { (Ghaffari and } \\
\text { Rostami, 2012) }\end{array}$ \\
\hline- & $13.2 \pm 9.2$ & - & 448 & 200 & - & - & - & I & $\downarrow$ & 1.2 & 0.004 & $\begin{array}{l}\text { (Caserta et al., } \\
\text { 2013) }\end{array}$ \\
\hline- & - & - & 21 & 15 & - & - & - & I & $\downarrow$ & 1.2 & - & $\begin{array}{c}\text { (Colagar et al., } \\
2009)\end{array}$ \\
\hline$\geq 10$ & - & - & 93 & 111 & - & - & - & $\mathrm{F}$ & $\downarrow$ & 1.2 & $<0.001$ & $\begin{array}{l}\text { (Al-Matubsi et } \\
\text { al., 2011) }\end{array}$ \\
\hline $\begin{array}{c}1-10 \mathrm{~L}, 11-20 \mathrm{M} \\
>20 \mathrm{H}\end{array}$ & - & - & 110 & 191 & 58 & 68 & 65 & I & $\downarrow$ & 1.2 & $<0.01$ & $\begin{array}{l}\text { (Zhang et al., } \\
2000 \text { ) }\end{array}$ \\
\hline$<20 \mathrm{~L},>20 \mathrm{H}$ & - & - & 7 & 10 & 4 & - & 6 & $\mathrm{~F}$ & $\downarrow$ & 1.2 & - & $\begin{array}{l}\text { (Pereira et al., } \\
\text { 2014) }\end{array}$ \\
\hline$\geq 1$ & - & - & 1131 & 655 & - & - & - & I & $\downarrow$ & 1.2 & 0.0004 & $\begin{array}{l}\text { (Künzle et al., } \\
\text { 2003) }\end{array}$ \\
\hline- & - & - & 168 & 90 & - & - & - & I & $\downarrow$ & 1.2 & 0.07 & (Al-Turki, 2015) \\
\hline$\leq 7 \mathrm{~L},>7 \mathrm{H}$ & - & - & 32 & 23 & 11 & - & 12 & I & $\downarrow$ & 1.2 & $<0.05$ & $\begin{array}{c}\text { (Colagar et al., } \\
\text { 2007) }\end{array}$ \\
\hline$<20 \mathrm{~L}, \geq 20 \mathrm{H}$ & - & - & 38 & 87 & 40 & - & 47 & I & $\downarrow$ & 1.2 & - & $\begin{array}{c}\text { (Al-Bader et al., } \\
\text { 1999) }\end{array}$ \\
\hline$<20 \mathrm{~L}, \geq 20 \mathrm{H}$ & - & - & 40 & 40 & 23 & - & 17 & I & $\downarrow$ & $\begin{array}{l}1.0- \\
1.2\end{array}$ & $<0.05$ & $\begin{array}{c}\text { (Omu et al., } \\
1998)\end{array}$ \\
\hline - & - & - & 19 & 17 & - & - & - & $\mathrm{F}$ & $\downarrow$ & 1.1 & $<0.05$ & $\begin{array}{c}\text { (Colagar et al., } \\
\text { 2009) }\end{array}$ \\
\hline$\leq 7 \mathrm{~L},>7 \mathrm{H}$ & - & - & 21 & 25 & 14 & - & 11 & $\mathrm{~F}$ & $\downarrow$ & 1.1 & $<0.05$ & $\begin{array}{c}\text { (Colagar et al., } \\
\text { 2007) }\end{array}$ \\
\hline $12.96 \pm 6.93$ & - & - & 30 & 30 & - & - & - & $\mathrm{F}$ & $\downarrow$ & 1.1 & - & $\begin{array}{c}\text { (El-Melegy and } \\
\text { Ali, 2011) }\end{array}$ \\
\hline - & - & - & 829 & 371 & - & - & - & F & $\downarrow$ & - & $<0.01$ & $\begin{array}{l}\text { (Tang et al., } \\
2015 \text { ) }\end{array}$ \\
\hline
\end{tabular}




\begin{tabular}{|c|c|c|c|c|c|c|c|c|c|c|c|c|}
\hline- & - & $\begin{array}{c}276.8 \pm 107.2 \\
\text { (seminal) }\end{array}$ & 4 & 4 & - & - & - & $\mathrm{F}$ & $\downarrow$ (NS) & 2.2 & NS & $\begin{array}{l}\text { (Linschooten et } \\
\text { al., 2009) }\end{array}$ \\
\hline $13.75(10-20)$ & - & - & 8 & 8 & - & - & - & I & $\downarrow$ (NS) & 2.1 & 0.065 & $\begin{array}{c}\text { (Metzler- } \\
\text { Guillemain et al., } \\
2015)\end{array}$ \\
\hline$>20$ & - & - & 36 & 34 & - & - & - & I & $\downarrow$ (NS) & 1.9 & NS & $\begin{array}{c}\text { (Elshal et al., } \\
\text { 2009) }\end{array}$ \\
\hline- & - & - & 71 & 23 & - & - & - & $\mathrm{F}$ & $\downarrow$ (NS) & 1.5 & NS & $\begin{array}{l}\text { (Handelsman et } \\
\text { al., 1984) }\end{array}$ \\
\hline $18(6-40)$ & - & - & 69 & 18 & - & - & - & I & $\downarrow(\mathrm{NS})$ & 1.5 & NS & (Mak et al., 2000) \\
\hline$\geq 10$ & - & - & 20 & 20 & - & - & - & I & ND & ND & NS & $\begin{array}{c}\text { (Antoniassi et al., } \\
2016)\end{array}$ \\
\hline- & - & - & 322 & 194 & - & - & - & I & ND & $\mathrm{ND}$ & NS & (Al-Turki, 2016) \\
\hline $2-40$ & - & - & 372 & 704 & - & - & - & I & ND & ND & NS & $\begin{array}{l}\text { (Zhang et al., } \\
\text { 2016) }\end{array}$ \\
\hline- & - & - & 245 & 46 & - & - & - & $\mathrm{F}$ & ND & $\mathrm{ND}$ & NS & (Hart et al., 2015) \\
\hline- & - & - & 593 & 171 & - & - & - & F & ND & ND & NS & $\begin{array}{l}\text { (Yang et al., } \\
\text { 2015) }\end{array}$ \\
\hline- & - & - & 32 & 18 & - & - & - & I & ND & ND & NS & $\begin{array}{l}\text { (Stramova and } \\
\text { Kandar, 2015) }\end{array}$ \\
\hline $10-20$ & - & - & 30 & 33 & - & - & - & I & ND & ND & NS & $\begin{array}{c}\text { (Yousefniapasha } \\
\text { et al., 2015) }\end{array}$ \\
\hline- & - & - & 229 & 109 & - & - & - & I & ND & $\mathrm{ND}$ & NS & $\begin{array}{l}\text { (Lotti et al., } \\
2015 \text { ) }\end{array}$ \\
\hline- & $\geq 10$ & 203 (seminal) & 175 & 147 & - & - & - & I & ND & $\mathrm{ND}$ & NS & (Yu et al., 2014) \\
\hline- & $0-1 \mathrm{~L},>1 \mathrm{H}$ & - & 998 & 109 & 76 & - & 33 & I & ND & ND & NS & $\begin{array}{c}\text { (Anifandis et al., } \\
2014 \text { ) }\end{array}$ \\
\hline $\begin{array}{c}<10 \operatorname{light~L,~} 10-19 \\
\mathrm{M}, \geq 20 \mathrm{H}\end{array}$ & - & - & 47 & 26 & 7 & 14 & 5 & I & ND & ND & NS & $\begin{array}{c}\text { (De Bantel et al., } \\
2014)\end{array}$ \\
\hline $18.4 \pm 5.8$ & - & - & 72 & 84 & - & - & - & I & ND & ND & NS & $\begin{array}{l}\text { (Taha et al., } \\
\text { 2014) }\end{array}$ \\
\hline$>10$ & - & - & 71 & 39 & - & - & - & $\mathrm{F} / \mathrm{I}$ & ND & $\mathrm{ND}$ & NS & $\begin{array}{l}\text { (Moretti et al., } \\
\text { 2014) }\end{array}$ \\
\hline - & - & - & 32 & 18 & - & - & - & I & ND & ND & NS & $\begin{array}{l}\text { (Kand'ár et al., } \\
\text { 2014) }\end{array}$ \\
\hline- & - & $>10$ (saliva) & 143 & 110 & - & - & - & I & ND & ND & NS & $\begin{array}{c}\text { (Jurewicz et al., } \\
2013 \text { ) }\end{array}$ \\
\hline- & $14.1 \pm 7.3$ & - & 314 & 314 & - & - & - & $\mathrm{F}$ & ND & ND & - & (Yu et al., 2013) \\
\hline- & - & - & 13 & 13 & - & - & - & I & ND & ND & NS & $\begin{array}{c}\text { (Salama and El- } \\
\text { Sawy, 2013) }\end{array}$ \\
\hline- & - & - & 93 & 30 & - & - & - & $\mathrm{F} / \mathrm{I}$ & ND & ND & NS & (Guz et al., 2013) \\
\hline$\leq 9 \mathrm{~L},>9 \mathrm{H}$ & - & - & 238 & 57 & 37 & - & 20 & F & ND & $\mathrm{ND}$ & NS & $\begin{array}{c}\text { (Axelsson et al., } \\
\text { 2013) }\end{array}$ \\
\hline- & $<8 \mathrm{~L},>30 \mathrm{H}$ & - & 30 & 40 & 20 & - & 20 & $\mathrm{~F}$ & ND & ND & NS & (Selit et al., 2013) \\
\hline- & $<8 \mathrm{~L},>30 \mathrm{H}$ & - & 30 & 40 & 20 & - & 20 & I & ND & $\mathrm{ND}$ & NS & (Selit et al., 2013) \\
\hline
\end{tabular}




\begin{tabular}{|c|c|c|c|c|c|c|c|c|c|c|c|c|}
\hline $\begin{array}{c}<5 \mathrm{~L}, 5-20 \mathrm{M},>20 \\
\mathrm{H}\end{array}$ & - & - & 775 & 737 & 180 & 327 & 230 & I & ND & ND & NS & $\begin{array}{l}\text { (Zhang et al., } \\
\text { 2013) }\end{array}$ \\
\hline - & - & - & 150 & 50 & - & - & - & I & ND & ND & NS & $\begin{array}{l}\text { (Habibi et al., } \\
\text { 2012) }\end{array}$ \\
\hline$>10$ & - & - & 98 & 53 & - & - & - & $\mathrm{F}$ & ND & ND & NS & $\begin{array}{c}\text { (Davar et al., } \\
\text { 2012) }\end{array}$ \\
\hline $11.1 \pm 5.8$ & - & - & 80 & 80 & - & - & - & $\mathrm{F}$ & ND & ND & NS & $\begin{array}{c}\text { (Taha et al., } \\
\text { 2012) }\end{array}$ \\
\hline $3.7 \pm 0.5$ & - & - & 9 & 12 & - & - & - & $\mathrm{F}$ & ND & ND & NS & $\begin{array}{l}\text { (Linschooten et } \\
\text { al., 2011) }\end{array}$ \\
\hline$>10$ & - & - & 73 & 39 & - & - & - & $\mathrm{F} / \mathrm{I}$ & ND & ND & NS & $\begin{array}{l}\text { (Moretti et al., } \\
\text { 2011) }\end{array}$ \\
\hline- & - & - & 1321 & 762 & - & - & - & $\mathrm{F}$ & ND & ND & NS & $\begin{array}{c}\text { (Ravnborg et al., } \\
2011 \text { ) }\end{array}$ \\
\hline$>20$ & - & $\begin{array}{c}99.55 \pm 64.58 \\
\text { (seminal) }\end{array}$ & 63 & 53 & - & - & - & I & ND & ND & NS & $\begin{array}{l}\text { (Hammadeh et } \\
\text { al., 2010) }\end{array}$ \\
\hline- & - & - & 57 & 35 & - & - & - & I & ND & ND & NS & $\begin{array}{l}\text { (Wegner et al., } \\
2010 \text { ) }\end{array}$ \\
\hline $11.6 \pm 6.1$ & - & - & 58 & 20 & - & - & - & I & ND & ND & NS & $\begin{array}{c}\text { (Chohan and } \\
\text { Badawy, 2010) }\end{array}$ \\
\hline$<10 \mathrm{~L}, \geq 10 \mathrm{H}$ & - & - & 509 & 799 & 347 & - & 452 & $\mathrm{~F}$ & ND & ND & NS & (Li et al., 2009b) \\
\hline$<10 \mathrm{~L}, \geq 10 \mathrm{H}$ & - & - & 217 & 85 & 47 & - & 38 & $\mathrm{~F}$ & ND & ND & NS & $\begin{array}{c}\text { (Richthoffet al., } \\
\text { 2008) }\end{array}$ \\
\hline$\geq 21$ & - & - & 22 & 23 & - & - & - & $\mathrm{F}$ & ND & ND & NS & $\begin{array}{l}\text { (Kiziler et al., } \\
\text { 2007) }\end{array}$ \\
\hline$\leq 10 \mathrm{~L},>10 \mathrm{H}$ & - & - & 731 & 241 & 120 & - & 121 & F & ND & ND & NS & $\begin{array}{l}\text { (Teijon et al., } \\
2007 \text { ) }\end{array}$ \\
\hline $\begin{array}{c}1-10 \mathrm{~L}, 11-20 \mathrm{M} \\
>20 \mathrm{H}\end{array}$ & - & - & 1490 & 1052 & 436 & 522 & 94 & $\mathrm{~F}$ & ND & ND & NS & $\begin{array}{l}\text { (Ramlau-Hansen } \\
\text { et al., 2007) }\end{array}$ \\
\hline$>20$ & - & - & 20 & 20 & - & - & - & F & ND & ND & $>0.05$ & $\begin{array}{c}\text { (Mostafa et al., } \\
2006)\end{array}$ \\
\hline$>20$ & - & - & 20 & 30 & - & - & - & $\begin{array}{l}\mathrm{I} \\
(\mathrm{O})\end{array}$ & ND & ND & $>0.05$ & $\begin{array}{l}\text { (Mostafa et al., } \\
\text { 2006) }\end{array}$ \\
\hline- & - & - & 19 & 29 & - & - & - & $\mathrm{F}$ & ND & ND & NS & (Hsu et al., 2006) \\
\hline- & - & - & 935 & 239 & - & - & - & $\mathrm{F}$ & ND & ND & NS & $\begin{array}{c}\text { (Hellstrom et al., } \\
2006)\end{array}$ \\
\hline $\begin{array}{c}<10 \mathrm{~L}, 11-20 \mathrm{M} \\
>20 \mathrm{H}\end{array}$ & - & - & 522 & 367 & 143 & 154 & 70 & F & ND & ND & NS & $\begin{array}{l}\text { (Pasqualotto et } \\
\text { al., 2006) }\end{array}$ \\
\hline $12.2 \pm 6.5$ & - & - & 137 & 48 & - & - & - & I & ND & ND & NS & $\begin{array}{c}\text { (Khademi et al., } \\
2005 \text { ) }\end{array}$ \\
\hline $1-19 \mathrm{~L}, \geq 20 \mathrm{H}$ & - & - & 30 & 25 & 10 & - & 15 & I & ND & ND & - & $\begin{array}{l}\text { (Viloria et al., } \\
\text { 2005) }\end{array}$ \\
\hline - & - & - & 60 & 73 & - & - & - & $\mathrm{F}$ & ND & ND & NS & $\begin{array}{l}\text { (Tsarev et al., } \\
2005 \text { ) }\end{array}$ \\
\hline $\begin{array}{c}1-10 \mathrm{~L}, 11-20 \mathrm{M} \\
>20 \mathrm{H}\end{array}$ & - & - & 324 & 176 & 80 & 66 & 30 & $\mathrm{~F}$ & ND & ND & NS & $\begin{array}{c}\text { (Sobreiro et al., } \\
2005 \text { ) }\end{array}$ \\
\hline $1-19 \mathrm{~L}, \geq 20 \mathrm{H}$ & - & - & 83 & 175 & 69 & - & 106 & I & $\mathrm{ND}$ & ND & NS & $\begin{array}{l}\text { (Ozgur et al., } \\
2005 \text { ) }\end{array}$ \\
\hline- & - & - & 227 & 22 & - & - & - & I & ND & ND & NS & $\begin{array}{l}\text { (Chen et al., } \\
\text { 2004) }\end{array}$ \\
\hline
\end{tabular}




\begin{tabular}{|c|c|c|c|c|c|c|c|c|c|c|c|c|c|}
\hline & $\leq 20 \mathrm{~L},>20 \mathrm{H}$ & - & - & 3194 & 472 & 388 & - & 84 & I & ND & ND & NS & $\begin{array}{c}\text { (Martini et al., } \\
\text { 2004) }\end{array}$ \\
\hline & $\begin{array}{c}9.0 \pm 6.1 \text { control, } \\
15.2 \pm 3.9 \text { exposed }\end{array}$ & - & - & 15 & 16 & - & - & - & $\mathrm{F}$ & ND & ND & NS & $\begin{array}{c}\text { (Naccarati et al., } \\
2003 \text { ) }\end{array}$ \\
\hline & $0-40$ & - & - & 151 & 74 & - & - & - & $\mathrm{F}$ & ND & ND & NS & (Loft et al., 2003) \\
\hline & $<10 \mathrm{~L},>10 \mathrm{H}$ & - & - & 783 & 203 & 135 & - & 68 & $\mathrm{~F}$ & ND & ND & NS & $\begin{array}{l}\text { (Swan et al., } \\
2003 \text { ) }\end{array}$ \\
\hline & $>20$ & - & - & 21 & 12 & - & - & - & I & ND & ND & - & $\begin{array}{l}\text { (Saleh et al., } \\
\text { 2002) }\end{array}$ \\
\hline & $\begin{array}{c}1-10 \mathrm{~L}, 11-20 \mathrm{M} \\
>20 \mathrm{H}\end{array}$ & - & - & 517 & 478 & 124 & 244 & 110 & I & ND & ND & NS & $\begin{array}{l}\text { (Trummer et al., } \\
2002 \text { ) }\end{array}$ \\
\hline & $19 \pm 7$ & - & - & 24 & 24 & - & - & - & $\mathrm{F}$ & ND & ND & NS & $\begin{array}{c}\text { (Wallock et al., } \\
2001 \text { ) }\end{array}$ \\
\hline & $7.70 \pm 5.32$ & - & - & 49 & 81 & - & - & - & $\mathrm{F}$ & ND & ND & NS & $\begin{array}{l}\text { (Wang et al., } \\
2001 \text { ) }\end{array}$ \\
\hline & $1-19 \mathrm{~L}, \geq 20 \mathrm{H}$ & - & - & 69 & 28 & 17 & - & 11 & $\mathrm{~F}$ & ND & ND & NS & $\begin{array}{l}\text { (Sergerie et al., } \\
2000)\end{array}$ \\
\hline & - & - & - & 152 & 91 & - & - & - & $\mathrm{F}$ & ND & ND & NS & (Chia et al., 1998) \\
\hline & $9.4 \pm 1.6$ & - & - & 32 & 28 & - & - & - & $\mathrm{F}$ & ND & ND & NS & $\begin{array}{l}\text { (Shen et al.., } \\
\text { 1997) }\end{array}$ \\
\hline & $>20$ & - & - & 28 & 49 & - & - & - & F & ND & ND & NS & $\begin{array}{c}\text { (Sofikitis et al., } \\
1995 \text { ) }\end{array}$ \\
\hline & $1-10 \mathrm{~L}, 11-50 \mathrm{H}$ & - & - & 16 & 26 & 5 & - & 21 & I & ND & ND & NS & $\begin{array}{c}\text { (Goverde et al., } \\
1995 \text { ) }\end{array}$ \\
\hline & $1-10 \mathrm{~L}, 11-50 \mathrm{H}$ & - & - & 35 & 30 & 9 & - & 21 & $\mathrm{~F}$ & ND & ND & NS & $\begin{array}{c}\text { (Goverde et al., } \\
1995 \text { ) }\end{array}$ \\
\hline & - & - & - & $<162$ & $<162$ & - & - & - & I & ND & ND & NS & $\begin{array}{c}\text { (Keck et al., } \\
\text { 1995) }\end{array}$ \\
\hline & - & - & - & 21 & 40 & - & - & - & I & ND & ND & NS & $\begin{array}{c}\text { (Banerjee et al., } \\
\text { 1993) }\end{array}$ \\
\hline & $\geq 10$ & - & - & 50 & 40 & - & - & - & I & ND & ND & NS & $\begin{array}{c}\text { (Holzki et al., } \\
\text { 1991) }\end{array}$ \\
\hline & $1-14 \mathrm{~L}, 15-40 \mathrm{H}$ & - & - & 203 & 147 & 68 & - & 79 & I & ND & ND & NS & $\begin{array}{c}\text { (Oldereid et al., } \\
\text { 1989) }\end{array}$ \\
\hline & $\leq 16 \mathrm{~L},>16 \mathrm{H}$ & - & - & 110 & 54 & 24 & - & 30 & $\mathrm{~F}$ & ND & ND & NS & $\begin{array}{c}\text { (Saaranen et al., } \\
\text { 1987) }\end{array}$ \\
\hline & $\begin{array}{c}1-10 \mathrm{~L}, 11-20 \mathrm{M} \\
>20 \mathrm{H}\end{array}$ & - & - & 50 & 60 & 14 & 32 & 14 & I & ND & ND & NS & $\begin{array}{c}(\text { Rantala and } \\
\text { Koskimies, 1987) }\end{array}$ \\
\hline & - & - & - & 34 & 20 & - & - & - & I & ND & ND & NS & (Lähdetie, 1986) \\
\hline & $<10 \mathrm{~L}, \geq 10 \mathrm{H}$ & - & - & 86 & 147 & 48 & - & 99 & I & ND & ND & NS & $\begin{array}{c}\text { (Andersen et al., } \\
1984 \text { ) }\end{array}$ \\
\hline Total Sperm Count & - & $3.8 \mathrm{~L}, 15.1 \mathrm{H}$ & - & 56 & 54 & 29 & - & 25 & $\begin{array}{l}\text { I } \\
\text { (V) }\end{array}$ & $\downarrow$ & 2.8 & 0.0001 & $\begin{array}{l}\text { (Fariello et al., } \\
\text { 2012) }\end{array}$ \\
\hline & $13.6 \pm 9.8$ & - & - & 50 & 50 & - & - & - & I & $\downarrow$ & 2.2 & $<0.01$ & $\begin{array}{l}\text { (Hussein et al., } \\
\text { 2011) }\end{array}$ \\
\hline & $<20 \mathrm{~L},>20 \mathrm{H}$ & - & - & 7 & 10 & 4 & - & 6 & F & $\downarrow$ & 2.0 & - & $\begin{array}{c}\text { (Pereira et al., } \\
\text { 2014) }\end{array}$ \\
\hline & - & - & - & 32 & 38 & - & - & - & I & $\downarrow$ & 1.8 & $<0.05$ & $\begin{array}{c}\text { (Kumosani et al., } \\
2008)\end{array}$ \\
\hline
\end{tabular}




\begin{tabular}{|c|c|c|c|c|c|c|c|c|c|c|c|c|}
\hline $\begin{array}{c}1-10 \mathrm{~L}, 11-20 \mathrm{M} \\
21-40 \mathrm{H}\end{array}$ & - & 173.9 (seminal) & 30 & 35 & 19 & 11 & 5 & $\mathrm{~F}$ & $\downarrow$ & 1.7 & $<0.05$ & $\begin{array}{c}\text { (Chen and Kuo, } \\
\text { 2007) }\end{array}$ \\
\hline- & - & - & 71 & 23 & - & - & - & $\mathrm{F}$ & $\downarrow$ & 1.7 & 0.01 & $\begin{array}{l}\text { (Handelsman et } \\
\text { al., 1984) }\end{array}$ \\
\hline $\begin{array}{c}1-10 \mathrm{~L}, 11-20 \mathrm{M} \\
>20 \mathrm{H}\end{array}$ & - & - & 50 & 60 & 14 & 32 & 14 & I & $\downarrow$ & 1.7 & $<0.05$ & $\begin{array}{c}\text { (Rantala and } \\
\text { Koskimies, 1987) }\end{array}$ \\
\hline- & - & - & 21 & 15 & - & - & - & I & $\downarrow$ & 1.6 & - & $\begin{array}{c}\text { (Colagar et al., } \\
2009)\end{array}$ \\
\hline- & $14.1 \pm 7.3$ & - & 27 & 30 & - & - & - & $\mathrm{F}$ & $\downarrow$ & 1.6 & - & (Yu et al., 2013) \\
\hline- & - & - & 337 & 194 & - & - & - & I & $\downarrow$ & 1.5 & $<0.05$ & $\begin{array}{c}\text { (Kunzle et al., } \\
\text { 2004) }\end{array}$ \\
\hline $19 \pm 7$ & - & - & 17 & 25 & - & - & - & I & $\downarrow$ & 1.5 & $<0.001$ & $\begin{array}{l}\text { (Laudat et al., } \\
\text { 2004) }\end{array}$ \\
\hline$<10 \mathrm{~L}, \geq 10 \mathrm{H}$ & - & - & 217 & 85 & 47 & - & 38 & $\mathrm{~F}$ & $\downarrow$ & 1.4 & 0.02 & $\begin{array}{c}\text { (Richthoffet al., } \\
2008)\end{array}$ \\
\hline $\begin{array}{c}<10 \mathrm{~L}, 10-19 \mathrm{M} \\
\geq 20 \mathrm{H}\end{array}$ & - & - & 298 & 920 & 256 & 365 & 299 & I & $\downarrow$ & 1.3 & $<0.05$ & (Cui et al., 2016) \\
\hline$>20$ & - & - & 15 & 29 & - & - & - & I & $\downarrow$ & 1.3 & $<0.05$ & $\begin{array}{c}\text { (Zavos et al., } \\
1998)\end{array}$ \\
\hline- & - & - & 593 & 171 & - & - & - & F & $\downarrow$ & 1.2 & $<0.05$ & $\begin{array}{l}\text { (Yang et al., } \\
\text { 2015) }\end{array}$ \\
\hline- & $\geq 10$ & 203 (seminal) & 175 & 147 & - & - & - & I & $\downarrow$ & 1.2 & $<0.05$ & (Yu et al., 2014) \\
\hline$\geq 1$ & - & - & 1131 & 655 & - & - & - & I & $\downarrow$ & 1.2 & 0.0001 & $\begin{array}{l}\text { (Künzle et al., } \\
2003 \text { ) }\end{array}$ \\
\hline $\begin{array}{c}1-10 \mathrm{~L}, 11-20 \mathrm{M}, \\
>20 \mathrm{H}\end{array}$ & - & - & 130 & 214 & 66 & 76 & 72 & I & $\downarrow$ & 1.2 & $<0.05$ & $\begin{array}{l}\text { (Mehrannia, } \\
\text { 2007) }\end{array}$ \\
\hline $\begin{array}{c}1-10 \mathrm{~L}, 11-20 \mathrm{M} \\
>20 \mathrm{H}\end{array}$ & - & - & 1490 & 1052 & 436 & 522 & 94 & $\mathrm{~F}$ & $\downarrow$ & 1.2 & 0.01 & $\begin{array}{l}\text { (Ramlau-Hansen } \\
\text { et al., 2007) }\end{array}$ \\
\hline- & - & - & 21 & 40 & - & - & - & I & $\downarrow$ & $\sim 1.2$ & $<0.05$ & $\begin{array}{c}\text { (Banerjee et al., } \\
1993 \text { ) }\end{array}$ \\
\hline- & - & - & 19 & 17 & - & - & - & $\mathrm{F}$ & $\downarrow$ & 1.1 & $<0.05$ & $\begin{array}{c}\text { (Colagar et al., } \\
\text { 2009) }\end{array}$ \\
\hline - & - & - & 829 & 371 & - & - & - & $\mathrm{F}$ & $\downarrow$ & - & $<0.05$ & $\begin{array}{l}\text { (Tang et al., } \\
2015 \text { ) }\end{array}$ \\
\hline 20 & - & - & 15 & 10 & - & - & - & $\mathrm{F}$ & $\downarrow(\mathrm{NS})$ & 1.7 & NS & $\begin{array}{c}\text { (Rubes et al., } \\
1998)\end{array}$ \\
\hline$\geq 10$ & - & - & 20 & 20 & - & - & - & I & $\downarrow(\mathrm{NS})$ & 1.7 & NS & $\begin{array}{c}\text { (Antoniassiet al., } \\
2016)\end{array}$ \\
\hline$\leq 16 \mathrm{~L},>16 \mathrm{H}$ & - & - & 110 & 54 & 24 & - & 30 & $\mathrm{~F}$ & $\downarrow$ (NS) & 1.5 & NS & $\begin{array}{c}\text { (Saaranen et al., } \\
\text { 1987) }\end{array}$ \\
\hline- & - & - & 322 & 194 & - & - & - & I & ND & ND & NS & (Al-Turki, 2016) \\
\hline- & - & - & 245 & 46 & - & - & - & F & ND & ND & NS & (Hart et al., 2015) \\
\hline- & - & - & 32 & 18 & - & - & - & I & ND & $\mathrm{ND}$ & NS & $\begin{array}{c}\text { (Stramova and } \\
\text { Kandar, 2015) }\end{array}$ \\
\hline $10-20$ & - & - & 30 & 33 & - & - & - & I & ND & ND & NS & $\begin{array}{l}\text { (Yousefniapasha } \\
\text { et al., 2015) }\end{array}$ \\
\hline - & - & - & 229 & 109 & - & - & - & I & ND & ND & NS & $\begin{array}{c}\text { (Lotti et al., } \\
2015)\end{array}$ \\
\hline $14.6 \pm 5.5$ & - & - & 21 & 38 & - & - & - & $\mathrm{F} / \mathrm{I}$ & ND & ND & NS & (Jong et al., 2014) \\
\hline
\end{tabular}




\begin{tabular}{|c|c|c|c|c|c|c|c|c|c|c|c|c|c|}
\hline & - & - & - & 32 & 18 & - & - & - & I & ND & ND & NS & $\begin{array}{c}\text { (Kand'ár et al., } \\
2014)\end{array}$ \\
\hline & - & $14.1 \pm 7.3$ & - & 314 & 314 & - & - & - & F & ND & $\mathrm{ND}$ & - & (Yu et al., 2013) \\
\hline & - & - & - & 93 & 30 & - & - & - & $\mathrm{F} / \mathrm{I}$ & ND & ND & NS & (Guz et al., 2013) \\
\hline & $\leq 9 \mathrm{~L},>9 \mathrm{H}$ & - & - & 238 & 57 & 37 & - & 20 & $\mathrm{~F}$ & ND & ND & NS & $\begin{array}{l}\text { (Axelsson et al., } \\
\text { 2013) }\end{array}$ \\
\hline & - & - & - & 1321 & 762 & - & - & - & F & ND & ND & NS & $\begin{array}{c}\text { (Ravnborg et al., } \\
2011 \text { ) }\end{array}$ \\
\hline & $16.0 \pm 6.2$ & - & - & 42 & 37 & - & - & - & I & ND & ND & NS & $\begin{array}{c}\text { (Perrin et al., } \\
\text { 2011) }\end{array}$ \\
\hline & $\geq 10$ & - & - & 93 & 111 & - & - & - & $\mathrm{F}$ & ND & ND & NS & $\begin{array}{c}\text { (Al-Matubsi et } \\
\text { al., 2011) }\end{array}$ \\
\hline & $<10 \mathrm{~L}, \geq 10 \mathrm{H}$ & - & - & 509 & 799 & 347 & - & 452 & $\mathrm{~F}$ & ND & ND & NS & (Li et al., 2009b) \\
\hline & - & - & - & 66 & 23 & - & - & - & $\mathrm{F}$ & ND & ND & NS & $\begin{array}{c}\text { (Kumosani et al., } \\
2008 \text { ) }\end{array}$ \\
\hline & $\leq 7 \mathrm{~L},>7 \mathrm{H}$ & - & - & 21 & 25 & 14 & - & 11 & $\mathrm{~F}$ & ND & ND & NS & $\begin{array}{c}\text { (Colagar et al., } \\
\text { 2007) }\end{array}$ \\
\hline & $\leq 7 \mathrm{~L},>7 \mathrm{H}$ & - & - & 32 & 23 & 11 & - & 12 & I & ND & ND & NS & $\begin{array}{c}\text { (Colagar et al., } \\
\text { 2007) }\end{array}$ \\
\hline & - & - & - & 935 & 239 & - & - & - & $\mathrm{F}$ & ND & ND & NS & $\begin{array}{c}\text { (Hellstromet al., } \\
\text { 2006) }\end{array}$ \\
\hline & $0-40$ & - & - & 151 & 74 & - & - & - & $\mathrm{F}$ & ND & $\mathrm{ND}$ & NS & (Loft et al., 2003) \\
\hline & $19 \pm 7$ & - & - & 24 & 24 & - & - & - & $\mathrm{F}$ & ND & ND & NS & $\begin{array}{l}\text { (Wallock et al., } \\
\text { 2001) }\end{array}$ \\
\hline & $7.70 \pm 5.32$ & - & - & 49 & 81 & - & - & - & F & ND & $\mathrm{ND}$ & NS & $\begin{array}{l}\text { (Wang et al., } \\
2001 \text { ) }\end{array}$ \\
\hline & $9.4 \pm 1.6$ & - & - & 32 & 28 & - & - & - & $\mathrm{F}$ & ND & ND & NS & $\begin{array}{l}\text { (Shen et al., } \\
\text { 1997) }\end{array}$ \\
\hline & $<20 \mathrm{~L}, \geq 20 \mathrm{H}$ & - & - & 21 & 37 & 18 & - & 19 & I & ND & ND & NS & $\begin{array}{c}\text { (Oldereid et al., } \\
1994)\end{array}$ \\
\hline & $\geq 10$ & - & - & 50 & 40 & - & - & - & I & ND & ND & NS & $\begin{array}{c}\text { (Holzki et al., } \\
\text { 1991) }\end{array}$ \\
\hline & $1-14 \mathrm{~L}, 15-40 \mathrm{H}$ & - & - & 203 & 147 & 68 & - & 79 & I & ND & ND & NS & $\begin{array}{c}\text { (Oldereid et al., } \\
1989)\end{array}$ \\
\hline & - & - & - & 34 & 20 & - & - & - & I & ND & ND & NS & (Lähdetie, 1986) \\
\hline \multirow[t]{7}{*}{$\begin{array}{c}\text { \% Normal } \\
\text { Morphology }\end{array}$} & $\begin{array}{l}1-10 \mathrm{~L}, 11-19 \mathrm{M} \\
\geq 20 \mathrm{H}\end{array}$ & - & - & 39 & 121 & 49 & 36 & 36 & $\begin{array}{c}\mathrm{I} \\
(\mathrm{V})\end{array}$ & $\downarrow$ & 14.0 & $<0.05$ & $\begin{array}{c}\text { (Collodel et al., } \\
2009)\end{array}$ \\
\hline & - & - & - & 30 & 22 & - & - & - & I & $\downarrow$ & 3.0 & $<0.05$ & $\begin{array}{c}\text { (Omran et al., } \\
2013)\end{array}$ \\
\hline & $1-19 \mathrm{~L}, \geq 20 \mathrm{H}$ & - & - & 31 & 25 & 10 & - & 15 & I & $\downarrow$ & 2.2 & $?$ & $\begin{array}{c}\text { (Viloria et al., } \\
2005 \text { ) }\end{array}$ \\
\hline & $<20 \mathrm{~L},>20 \mathrm{H}$ & - & - & 7 & 10 & 4 & - & 6 & $\mathrm{~F}$ & $\downarrow$ & 1.7 & $<0.036$ & $\begin{array}{l}\text { (Pereira et al., } \\
\text { 2014) }\end{array}$ \\
\hline & $>20$ & - & - & 28 & 49 & - & - & - & $\mathrm{F}$ & $\downarrow$ & $\begin{array}{l}1.4- \\
1.7\end{array}$ & $<0.05$ & $\begin{array}{c}\text { (Sofikitis et al., } \\
1995 \text { ) }\end{array}$ \\
\hline & $\leq 7 \mathrm{~L},>7 \mathrm{H}$ & - & - & 32 & 23 & 11 & - & 12 & I & $\downarrow$ & 1.6 & $<0.05$ & $\begin{array}{c}\text { (Colagar et al., } \\
\text { 2007) }\end{array}$ \\
\hline & $\begin{array}{l}7.89 \pm 0.9 \mathrm{~L}, 17.8 \pm \\
0.5 \mathrm{M}, 33.75 \pm 1.3\end{array}$ & - & - & $<40$ & 40 & 9 & 15 & 16 & I & $\downarrow$ & 1.6 & $<0.05$ & $\begin{array}{c}\text { (Tawadrous et al, } \\
2011 \text { ) }\end{array}$ \\
\hline
\end{tabular}




\begin{tabular}{|c|c|c|c|c|c|c|c|c|c|c|c|c|}
\hline$>20$ & - & $\begin{array}{l}99.55 \pm 64.58 \\
\text { (seminal) }\end{array}$ & 63 & 53 & - & - & - & I & $\downarrow$ & 1.5 & $<0.001$ & $\begin{array}{l}\text { (Hammadeh et } \\
\text { al., 2010) }\end{array}$ \\
\hline $\begin{array}{c}<5 \mathrm{~L}, 5-20 \mathrm{M}, \geq 20 \\
\mathrm{H}\end{array}$ & - & - & 79 & 68 & 14 & 21 & 33 & I & $\downarrow$ & 1.5 & $<0.05$ & (Liu et al., 2010) \\
\hline$<20 \mathrm{~L},>20 \mathrm{H}$ & - & - & 69 & 62 & 43 & - & 19 & I & $\downarrow$ & 1.5 & $\leq 0.0015$ & $\begin{array}{c}\text { (Reina Bouvet et } \\
\text { al., 2007) }\end{array}$ \\
\hline- & - & - & 21 & 15 & - & - & - & I & $\downarrow$ & 1.4 & - & $\begin{array}{c}\text { (Colagar et al., } \\
\text { 2009) }\end{array}$ \\
\hline $\begin{array}{c}5 \pm 2.44 \mathrm{~L}, 14.84 \pm \\
3.43 \mathrm{M}, 22.90 \pm 1.7 \\
\mathrm{H}\end{array}$ & - & - & 30 & 40 & 10 & 19 & 11 & I & $\downarrow$ & 1.4 & $<0.001$ & $\begin{array}{l}\text { (El-Melegy and } \\
\text { Ali, 2011) }\end{array}$ \\
\hline$>20$ & - & - & 15 & 29 & - & - & - & I & $\downarrow$ & 1.4 & $<0.05$ & $\begin{array}{c}\text { (Zavos et al., } \\
1998 \text { ) }\end{array}$ \\
\hline$>20$ & - & - & 36 & 34 & - & - & - & I & $\downarrow$ & 1.4 & $<0.05$ & $\begin{array}{l}\text { (Elshal et al., } \\
\text { 2009) }\end{array}$ \\
\hline $\begin{array}{c}7.5 \pm 0.43 \mathrm{~L}, 15.92 \\
\pm 0.7 \mathrm{M}, 26.44 \pm \\
1.4 \mathrm{H}\end{array}$ & - & - & $<40$ & 40 & 10 & 12 & 18 & $\mathrm{~F}$ & $\downarrow$ & 1.3 & $<0.05$ & $\begin{array}{c}\text { (Tawadrous et al, } \\
2011 \text { ) }\end{array}$ \\
\hline- & - & - & 19 & 17 & - & - & - & $\mathrm{F}$ & $\downarrow$ & 1.3 & $<0.05$ & $\begin{array}{c}\text { (Colagar et al., } \\
\text { 2009) }\end{array}$ \\
\hline $\begin{array}{c}\leq 10 \mathrm{~L}, \leq 20 \mathrm{M},>20 \\
\mathrm{H}\end{array}$ & - & - & 83 & 64 & 26 & 30 & 8 & $\mathrm{~F}$ & $\downarrow$ & 1.3 & 0.002 & $\begin{array}{c}\text { (Ghaffari and } \\
\text { Rostami, 2013) }\end{array}$ \\
\hline $16 \pm 7.5$ & - & - & 83 & 64 & - & - & - & $\mathrm{F}$ & $\downarrow$ & 1.3 & $\leq 0.01$ & $\begin{array}{l}\text { (Ghaffari and } \\
\text { Rostami, 2012) }\end{array}$ \\
\hline$<20 \mathrm{~L}, \geq 20 \mathrm{H}$ & - & - & 38 & 87 & 40 & - & 47 & I & $\downarrow$ & 1.3 & - & $\begin{array}{c}\text { (Al-Bader et al., } \\
\text { 1999) }\end{array}$ \\
\hline- & $<10 \mathrm{~L}, \geq 10 \mathrm{H}$ & - & 473 & 137 & 75 & - & 62 & I & $\downarrow$ & $\begin{array}{l}1.2- \\
1.3\end{array}$ & $<0.001$ & (Chia et al., 1994) \\
\hline $\begin{array}{c}1-10 \mathrm{~L}, 11-20 \mathrm{M} \\
>20 \mathrm{H}\end{array}$ & - & - & 161 & 197 & 57 & 115 & 25 & I & $\downarrow$ & $\begin{array}{l}1.1- \\
1.3\end{array}$ & $<0.007$ & $\begin{array}{l}\text { (Merino et al., } \\
1998 \text { ) }\end{array}$ \\
\hline $18.4 \pm 5.8$ & - & - & 72 & 84 & - & - & - & I & $\downarrow$ & 1.2 & $<0.05$ & $\begin{array}{l}\text { (Taha et al., } \\
\text { 2014) }\end{array}$ \\
\hline$\geq 21$ & - & - & 24 & 26 & - & - & - & I & $\downarrow$ & 1.2 & $<0.001$ & $\begin{array}{l}\text { (Kiziler et al., } \\
\text { 2007) }\end{array}$ \\
\hline- & - & - & 337 & 194 & - & - & - & I & $\downarrow$ & 1.2 & $<0.05$ & $\begin{array}{l}\text { (Kunzle et al., } \\
\text { 2004) }\end{array}$ \\
\hline $12.96 \pm 6.93$ & - & - & 30 & 30 & - & - & - & $\mathrm{F}$ & $\downarrow$ & 1.1 & - & $\begin{array}{l}\text { (El-Melegy and } \\
\text { Ali, 2011) }\end{array}$ \\
\hline$\geq 1$ & - & - & 1131 & 655 & - & - & - & I & $\downarrow$ & 1.1 & 0.0007 & $\begin{array}{c}\text { (Künzle et al., } \\
\text { 2003) }\end{array}$ \\
\hline $\begin{array}{c}<15 \mathrm{~L}, 20-25 \mathrm{M} \\
>30 \mathrm{H}\end{array}$ & - & - & 43 & 43 & 6 & 27 & 10 & I & $\downarrow$ & 1.1 & $<0.01$ & $\begin{array}{c}\text { (Evans et al., } \\
1981 \text { ) }\end{array}$ \\
\hline $\begin{array}{c}<5 \mathrm{~L}, 5-20 \mathrm{M},>20 \\
\mathrm{H}\end{array}$ & - & - & 615 & 508 & 127 & 242 & 139 & I & $\downarrow$ & 1.1 & $<0.01$ & $\begin{array}{l}\text { (Zhang et al., } \\
\text { 2013) }\end{array}$ \\
\hline$>5$ & - & - & 164 & 137 & - & - & - & I & $\downarrow$ & 1.1 & 0.04 & $\begin{array}{c}\text { (Zitzmann et al., } \\
2003 \text { ) }\end{array}$ \\
\hline- & $13.2 \pm 9.2$ & - & 448 & 200 & - & - & - & I & $\downarrow(\mathrm{NS})$ & 1.1 & 0.07 & $\begin{array}{c}\text { (Caserta et al., } \\
\text { 2013) }\end{array}$ \\
\hline$\geq 10$ & - & - & 20 & 20 & - & - & - & I & ND & ND & NS & $\begin{array}{c}\text { (Antoniassi et al., } \\
2016)\end{array}$ \\
\hline
\end{tabular}




\begin{tabular}{|c|c|c|c|c|c|c|c|c|c|c|c|c|}
\hline- & - & - & 593 & 171 & - & - & - & $\mathrm{F}$ & ND & ND & NS & $\begin{array}{c}\text { (Yang et al., } \\
2015 \text { ) }\end{array}$ \\
\hline- & - & - & 829 & 371 & - & - & - & $\mathrm{F}$ & ND & ND & NS & $\begin{array}{c}\text { (Tang et al., } \\
2015 \text { ) }\end{array}$ \\
\hline- & - & - & 32 & 18 & - & - & - & I & ND & ND & NS & $\begin{array}{l}\text { (Stramova and } \\
\text { Kandar, 2015) }\end{array}$ \\
\hline $10-20$ & - & - & 30 & 33 & - & - & - & I & ND & ND & NS & $\begin{array}{l}\text { (Yousefiniapasha } \\
\text { et al., 2015) }\end{array}$ \\
\hline $14.6 \pm 5.5$ & - & - & 21 & 38 & - & - & - & $\mathrm{F} / \mathrm{I}$ & ND & ND & NS & (Jong et al., 2014) \\
\hline $\begin{array}{c}<10 \text { light } \mathrm{L}, 10-19 \\
\mathrm{M}, \geq 20 \mathrm{H}\end{array}$ & - & - & 47 & 26 & 7 & 14 & 5 & I & ND & ND & NS & $\begin{array}{c}\text { (De Bantel et al., } \\
2014)\end{array}$ \\
\hline$>10$ & - & - & 71 & 39 & - & - & - & $\mathrm{F} / \mathrm{I}$ & ND & ND & NS & $\begin{array}{l}\text { (Moretti et al., } \\
\text { 2014) }\end{array}$ \\
\hline- & - & - & 32 & 18 & - & - & - & I & ND & ND & NS & $\begin{array}{l}\text { (Kand'ár et al., } \\
\text { 2014) }\end{array}$ \\
\hline- & - & - & 13 & 13 & - & - & - & I & ND & ND & NS & $\begin{array}{c}\text { (Salama and El- } \\
\text { Sawy, 2013) }\end{array}$ \\
\hline- & - & - & 93 & 30 & - & - & - & $\mathrm{F} / \mathrm{I}$ & ND & ND & NS & (Guz et al., 2013) \\
\hline - & - & - & 150 & 50 & - & - & - & I & ND & ND & NS & $\begin{array}{l}\text { (Habibi et al., } \\
\text { 2012) }\end{array}$ \\
\hline$<20 \mathrm{~L},>20 \mathrm{H}$ & - & - & 13 & 49 & 22 & - & 27 & $\mathrm{~F}$ & ND & ND & NS & (Joo et al., 2012) \\
\hline- & $3.8 \mathrm{~L}, 15.1 \mathrm{H}$ & - & 56 & 54 & 29 & - & 25 & $\begin{array}{l}\text { I } \\
\text { (V) }\end{array}$ & ND & ND & NS & $\begin{array}{l}\text { (Fariello et al., } \\
\text { 2012) }\end{array}$ \\
\hline $11.1 \pm 5.8$ & - & - & 80 & 80 & - & - & - & F & ND & ND & NS & $\begin{array}{c}\text { (Taha et al., } \\
2012 \text { ) }\end{array}$ \\
\hline $\begin{array}{c}<10 \mathrm{~L}, 10-30 \mathrm{M} \\
>30 \mathrm{H}\end{array}$ & - & - & 132 & 48 & 31 & 6 & 11 & I & ND & ND & NS & $\begin{array}{c}\text { (Aryanpur et al., } \\
2011 \text { ) }\end{array}$ \\
\hline $3.7 \pm 0.5$ & - & - & 9 & 12 & - & - & - & $\mathrm{F}$ & ND & ND & NS & $\begin{array}{l}\text { (Linschooten et } \\
\text { al., 2011) }\end{array}$ \\
\hline$>10$ & - & - & 73 & 39 & - & - & - & $\mathrm{F} / \mathrm{I}$ & ND & ND & NS & $\begin{array}{l}\text { (Moretti et al., } \\
\text { 2011) }\end{array}$ \\
\hline- & - & - & 729 & 400 & - & - & - & $\mathrm{F}$ & ND & ND & NS & $\begin{array}{c}\text { (Ravnborg et al., } \\
2011 \text { ) }\end{array}$ \\
\hline $16.0 \pm 6.2$ & - & - & 42 & 37 & - & - & - & I & ND & ND & NS & $\begin{array}{l}\text { (Perrin et al., } \\
\text { 2011) }\end{array}$ \\
\hline- & - & - & 57 & 35 & - & - & - & I & ND & ND & NS & $\begin{array}{c}\text { (Wegner et al., } \\
2010 \text { ) }\end{array}$ \\
\hline $11.6 \pm 6.1$ & - & - & 58 & 20 & - & - & - & I & ND & ND & NS & $\begin{array}{c}\text { (Chohan and } \\
\text { Badawy, 2010) }\end{array}$ \\
\hline - & - & $\begin{array}{c}276.8 \pm 107.2 \\
\text { (seminal) }\end{array}$ & 4 & 4 & - & - & - & $\mathrm{F}$ & ND & ND & NS & $\begin{array}{l}\text { (Linschooten et } \\
\text { al., 2009) }\end{array}$ \\
\hline$\geq 21$ & - & - & 22 & 23 & - & - & - & $\mathrm{F}$ & ND & ND & NS & $\begin{array}{l}\text { (Kiziler et al., } \\
\text { 2007) }\end{array}$ \\
\hline$\leq 7 \mathrm{~L},>7 \mathrm{H}$ & - & - & 21 & 25 & 14 & - & 11 & $\mathrm{~F}$ & ND & ND & NS & $\begin{array}{c}\text { (Colagar et al., } \\
\text { 2007) }\end{array}$ \\
\hline- & - & - & 935 & 239 & - & - & - & $\mathrm{F}$ & ND & ND & NS & $\begin{array}{c}\text { (Hellstromet al., } \\
2006)\end{array}$ \\
\hline $\begin{array}{c}<10 \mathrm{~L}, 11-20 \mathrm{M}, \\
>20 \mathrm{H}\end{array}$ & - & - & 522 & 367 & 143 & 154 & 70 & $\mathrm{~F}$ & ND & ND & NS & $\begin{array}{l}\text { (Pasqualotto et } \\
\text { al., 2006) }\end{array}$ \\
\hline
\end{tabular}




\begin{tabular}{|c|c|c|c|c|c|c|c|c|c|c|c|c|c|}
\hline & $12.2 \pm 6.5$ & - & - & 137 & 48 & - & - & - & I & ND & ND & NS & $\begin{array}{l}\text { (Khademi et al., } \\
\text { 2005) }\end{array}$ \\
\hline & $\begin{array}{c}1-10 \mathrm{~L}, 11-20 \mathrm{M} \\
>20 \mathrm{H}\end{array}$ & - & - & 324 & 176 & 80 & 66 & 30 & $\mathrm{~F}$ & ND & ND & NS & $\begin{array}{c}\text { (Sobreiro et al., } \\
2005 \text { ) }\end{array}$ \\
\hline & $1-19 \mathrm{~L}, \geq 20 \mathrm{H}$ & - & - & 83 & 175 & 69 & - & 106 & I & ND & ND & NS & $\begin{array}{l}\text { (Ozgur et al., } \\
2005 \text { ) }\end{array}$ \\
\hline & $\leq 20 \mathrm{~L},>20 \mathrm{H}$ & - & - & 3194 & 472 & 388 & - & 84 & I & ND & $\mathrm{ND}$ & NS & $\begin{array}{l}\text { (Martini et al., } \\
\text { 2004) }\end{array}$ \\
\hline & $0-40$ & - & - & 151 & 74 & - & - & - & $\mathrm{F}$ & ND & $\mathrm{ND}$ & NS & (Loft et al., 2003) \\
\hline & $<10 \mathrm{~L},>10 \mathrm{H}$ & - & - & 783 & 203 & 135 & - & 68 & $\mathrm{~F}$ & ND & $\mathrm{ND}$ & NS & $\begin{array}{l}\text { (Swan et al., } \\
\text { 2003) }\end{array}$ \\
\hline & $18(6-40)$ & - & - & 69 & 18 & - & - & - & I & ND & $\mathrm{ND}$ & NS & (Mak et al., 2000) \\
\hline & $1-19 \mathrm{~L}, \geq 20 \mathrm{H}$ & - & - & 69 & 28 & 17 & - & 11 & $\mathrm{~F}$ & ND & $\mathrm{ND}$ & NS & $\begin{array}{l}\text { (Sergerie et al., } \\
2000 \text { ) }\end{array}$ \\
\hline & 20 & - & - & 15 & 10 & - & - & - & $\mathrm{F}$ & ND & ND & NS & $\begin{array}{c}\text { (Rubes et al., } \\
1998)\end{array}$ \\
\hline & - & - & - & 152 & 91 & - & - & - & $\mathrm{F}$ & ND & $\mathrm{ND}$ & NS & (Chia et al., 1998) \\
\hline & $9.4 \pm 1.6$ & - & - & 32 & 28 & - & - & - & $\mathrm{F}$ & ND & ND & NS & $\begin{array}{l}\text { (Shen et al., } \\
\text { 1997) }\end{array}$ \\
\hline & - & - & - & $<100$ & $<100$ & - & - & - & I & $\mathrm{ND}$ & ND & NS & $\begin{array}{c}\text { (Hossain et al., } \\
\text { 1997) }\end{array}$ \\
\hline & $1-10 \mathrm{~L}, 11-50 \mathrm{H}$ & - & - & 16 & 26 & 5 & - & 21 & I & ND & ND & NS & $\begin{array}{c}\text { (Goverde et al., } \\
1995 \text { ) }\end{array}$ \\
\hline & $1-10 \mathrm{~L}, 11-50 \mathrm{H}$ & - & - & 35 & 30 & 9 & - & 21 & $\mathrm{~F}$ & ND & $\mathrm{ND}$ & NS & $\begin{array}{c}\text { (Goverde et al., } \\
\text { 1995) }\end{array}$ \\
\hline & $\geq 10$ & - & - & 50 & 40 & - & - & - & I & ND & ND & NS & $\begin{array}{c}\text { (Holzki et al., } \\
1991)\end{array}$ \\
\hline & $\leq 16 \mathrm{~L},>16 \mathrm{H}$ & - & - & 110 & 54 & 24 & - & 30 & $\mathrm{~F}$ & ND & $\mathrm{ND}$ & NS & $\begin{array}{l}\text { (Saaranen et al., } \\
\text { 1987) }\end{array}$ \\
\hline & - & - & - & $<162$ & $<162$ & - & - & - & I & ND & ND & NS & $\begin{array}{l}\text { (Keck et al., } \\
1995 \text { ) }\end{array}$ \\
\hline & - & - & - & 229 & 109 & - & - & - & I & $\uparrow$ & 1.7 & $<0.05$ & $\begin{array}{l}\text { (Lotti et al., } \\
2015)\end{array}$ \\
\hline \multirow[t]{9}{*}{$\begin{array}{l}\text { \% Abnormal } \\
\text { Morphology }\end{array}$} & $19 \pm 7$ & - & - & 17 & 25 & - & - & - & I & $\uparrow$ & $\begin{array}{l}1.6- \\
3.0\end{array}$ & $<0.001$ & $\begin{array}{l}\text { (Laudat et al., } \\
\text { 2004) }\end{array}$ \\
\hline & - & - & - & 21 & 40 & - & - & - & I & $\uparrow$ & $\sim 2.7$ & $<0.001$ & $\begin{array}{c}\text { (Banerjee et al., } \\
1993 \text { ) }\end{array}$ \\
\hline & - & - & - & 5 & 11 & - & - & - & F & $\uparrow$ & 1.3 & $?$ & (Hsu et al., 2006) \\
\hline & $>20$ & - & - & 20 & 20 & - & - & - & $\begin{array}{l}\mathrm{I} \\
\text { (A) }\end{array}$ & $\uparrow$ & 1.2 & $<0.05$ & $\begin{array}{l}\text { (Mostafa et al., } \\
2006)\end{array}$ \\
\hline & $\begin{array}{c}<10 \mathrm{~L}, 10-19 \mathrm{M} \\
\geq 20 \mathrm{H}\end{array}$ & - & - & 298 & 920 & 256 & 365 & 299 & I & $\uparrow$ & 1.2 & $<0.05$ & (Cui et al., 2016) \\
\hline & $<20 \mathrm{~L}, \geq 20 \mathrm{H}$ & - & - & - & 396 & 164 & - & 232 & I & $\uparrow$ & $\begin{array}{l}1.1- \\
1.2\end{array}$ & $<0.005$ & $\begin{array}{l}\text { (Meri et al., } \\
\text { 2013) }\end{array}$ \\
\hline & $<20 \mathrm{~L}, \geq 20 \mathrm{H}$ & - & - & 40 & 40 & 23 & - & 17 & I & $\uparrow$ & $\begin{array}{l}1.0- \\
1.2\end{array}$ & $<0.01$ & $\begin{array}{c}\text { (Omu et al., } \\
1998 \text { ) }\end{array}$ \\
\hline & $\begin{array}{c}1-10 \mathrm{~L}, 11-19 \mathrm{M}, \\
\geq 20 \mathrm{H}\end{array}$ & - & - & 39 & 121 & 49 & 36 & 36 & $\begin{array}{l}\text { I } \\
\text { (V) }\end{array}$ & $\uparrow$ & 1.1 & $<0.05$ & $\begin{array}{c}\text { (Collodel et al., } \\
\text { 2009) }\end{array}$ \\
\hline & - & $<10 \mathrm{~L}, \geq 10 \mathrm{H}$ & - & 473 & 137 & 75 & - & 62 & I & $\uparrow$ & 1.1 & $<0.01$ & (Chia et al., 1994) \\
\hline
\end{tabular}




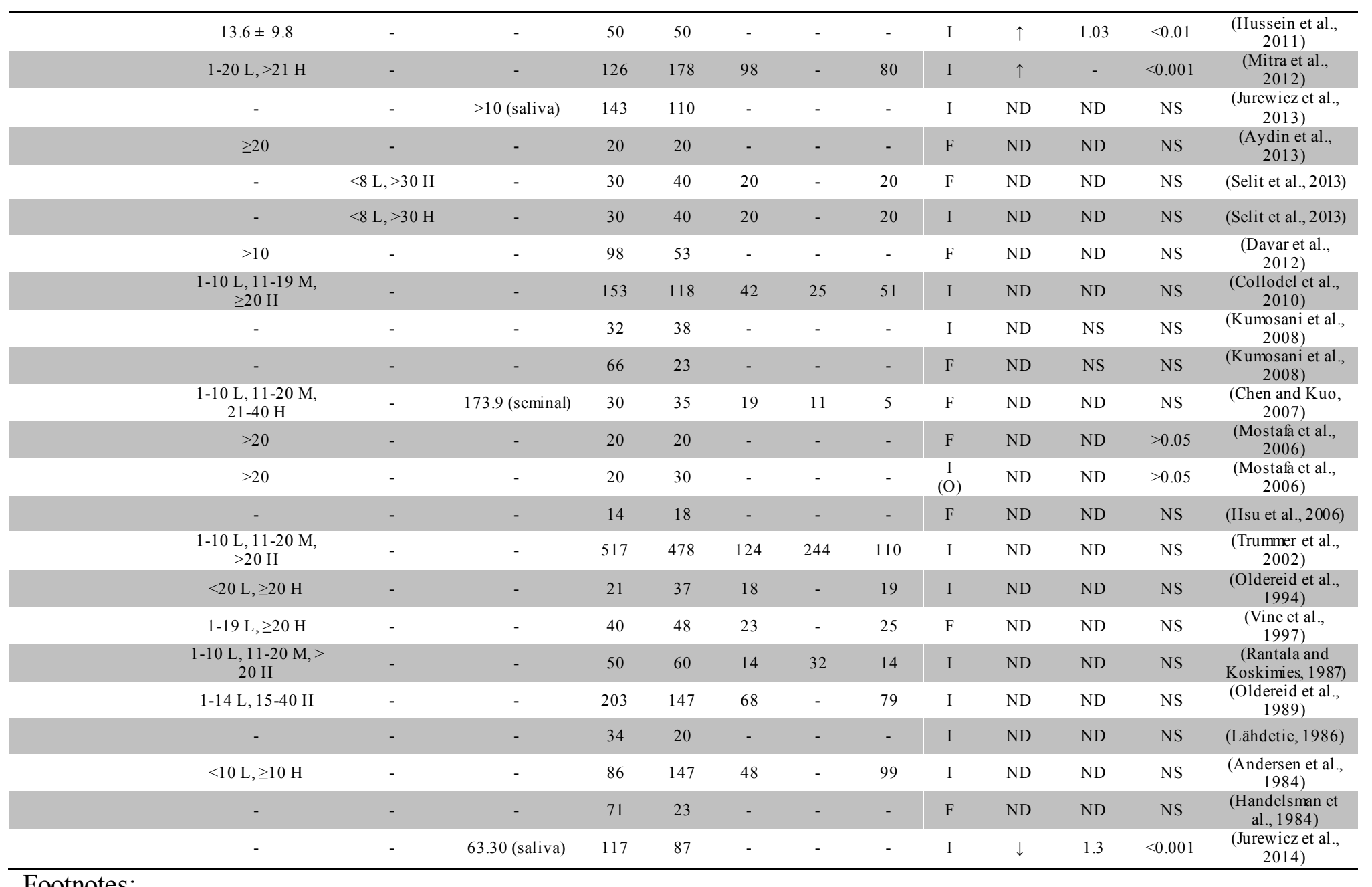

Footnotes:

N-S: Non-smokers, S: Smokers 
L: Light, M: Medium, H: Heavy

F?: Fertile?

D: Direction

FD: Fold-difference

F: Fertile or assumed fertile (can include couples attending fertility clinic if normospermatic subjects were selected)

I: Declared infertile or attending fertility clinic with no selection for normospermatic subjects

O: Oligospermia; A: Asthenospermia; V: Varicocele

ND: No difference; NS: Not significant (note fold difference presented if FD $\geq 1.5$ or $\mathrm{P}<0.1$ ) 
Table C18 Animal studies with evidence related to the germ cell mutagenicity of tobacco smoke.

\begin{tabular}{|c|c|c|c|c|c|c|c|c|c|c|c|c|}
\hline $\begin{array}{l}\text { Smoking-Associated } \\
\text { Outcome }\end{array}$ & Animal (strain) & $\begin{array}{l}\text { Exposure } \\
\text { Duration }\end{array}$ & $\begin{array}{l}\text { Cigarettes } \\
\text { Per Day }\end{array}$ & $\begin{array}{c}\text { Cotinine } \\
(\mathrm{ng} / \mathrm{mL})\end{array}$ & $\mathrm{TPM}\left(\mathrm{mg} / \mathrm{m}^{3}\right)$ & Age & C & E & $\mathrm{D}$ & FD & P-Value & Ref. \\
\hline \multirow[t]{2}{*}{$\begin{array}{c}\text { Tandem Repeat } \\
\text { Mutations in Sperm }\end{array}$} & $\begin{array}{c}\text { Mouse (C57BL/6 x } \\
\text { CBA F1) }\end{array}$ & 12 weeks & 2,6 & - & - & $\begin{array}{c}8-10 \\
\text { weeks }\end{array}$ & 10 & 10 & $\uparrow$ & $1.4-1.7$ & 0.02 & (Yauk et al., 2007) \\
\hline & Mouse (B6C3F1) & 2 weeks & 3,16 & $\begin{array}{l}15.7 \pm 2.9 ; 95.4 \\
\pm 23.5 \text { (plasma) }\end{array}$ & $\begin{array}{c}30.2 \pm 5.5,74.5 \\
\quad \pm 18.9\end{array}$ & $\begin{array}{c}6-8 \\
\text { weeks }\end{array}$ & 6 & $6 \mathrm{~L}, 6 \mathrm{H}$ & $\uparrow$ & $2.6-3.1$ & 0.02 & $\begin{array}{l}\text { (Marchetti et al., } \\
\text { 2011) }\end{array}$ \\
\hline $\begin{array}{l}\text { Dominant Lethal } \\
\text { Mutations }\end{array}$ & $\begin{array}{l}\text { Mosue (BALB/c, } \\
\text { BDF1 and } \mathrm{H})\end{array}$ & 8 Weeks & 8,16 & - & - & $\begin{array}{l}12-13 \\
\text { weeks }\end{array}$ & 46 & $46 \mathrm{~L}, 46 \mathrm{H}$ & $\uparrow$ & $1.0-2.0$ & $<0.001$ & $\begin{array}{l}\text { (Stoichev et al., } \\
\text { 1993) }\end{array}$ \\
\hline \multirow[t]{4}{*}{$\begin{array}{c}\text { SpermDNA } \\
\text { Fragmentation }\end{array}$} & Rat (Wistar) & 9 weeks & 20 & - & - & $\begin{array}{c}60 \\
\text { days }\end{array}$ & 10 & 10 & $\uparrow$ & $4.0-9.2$ & $<0.05$ & $\begin{array}{l}\text { (Garcia et al., } \\
2012 \text { ) }\end{array}$ \\
\hline & Mouse (A/J) & 10 weeks & 22.5 & $\sim 175$ (plasma) & $96.5 \pm 1.38$ & $\begin{array}{c}12 \\
\text { hours }\end{array}$ & 25 & 23 & $\uparrow$ & 1.3 & $<0.05$ & $\begin{array}{c}\text { (La Maestra et al., } \\
2015 \text { ) }\end{array}$ \\
\hline & $\begin{array}{c}\text { Mouse } \\
(\mathrm{B} 6 \mathrm{C} 3 \mathrm{~F} 1 / \mathrm{Crl})\end{array}$ & 2 weeks & 3,16 & $\begin{array}{l}15.7 \pm 2.9 ; 95.4 \\
\pm 23.5 \text { (plasma) }\end{array}$ & $\begin{array}{c}30.2 \pm 5.5,74.5 \\
\pm 18.9\end{array}$ & $\begin{array}{c}8-9 \\
\text { weeks }\end{array}$ & 11 & $6 \mathrm{~L}, 6 \mathrm{H}$ & $\uparrow$ & $1.1-1.7$ & $<0.05$ & $\begin{array}{c}\text { (Polyzos et al., } \\
\text { 2009) }\end{array}$ \\
\hline & $\begin{array}{l}\text { Rhesus Macaques } \\
\text { (Macaca mulatta) }\end{array}$ & 6 months & - & $>100$ (serum) & $3.69 \pm 1.57$ & - & - & 4 & $\mathrm{ND}$ & ND & NS & (Hung et al., 2009) \\
\hline \multirow[t]{4}{*}{ Oxidative stress } & $\begin{array}{l}\text { Rat (Sprague } \\
\text { Dawley) }\end{array}$ & 13 weeks & 30 & - & - & $\begin{array}{c}10 \\
\text { weeks }\end{array}$ & 8 & 8 & $\uparrow$ & 2.3 & $<0.05$ & $\begin{array}{c}\text { (Mohamed et al., } \\
\text { 2011) }\end{array}$ \\
\hline & Rat (Wistar-Albino) & 60 days & 8 & - & - & - & 7 & 7 & $\uparrow$ & $1.3-2.2$ & $<0.05$ & $\begin{array}{l}\text { (Ozyurt et al., } \\
\text { 2006) }\end{array}$ \\
\hline & Rat (Wistar) & 63 days & 20 & - & - & $\begin{array}{c}8-9 \\
\text { weeks }\end{array}$ & 10 & 10 & $\uparrow$ & 2.0 & $<0.05$ & $\begin{array}{l}\text { (Sankako et al., } \\
\text { 2012) }\end{array}$ \\
\hline & $\begin{array}{l}\text { Rat (Sprague } \\
\text { Dawley) }\end{array}$ & $\begin{array}{l}2,14,28 \\
\text { days }\end{array}$ & 20 & - & - & $\begin{array}{c}4 \\
\text { weeks }\end{array}$ & - & - & $\uparrow$ & 1.7 & $\leq 0.05$ & $\begin{array}{l}\text { (Abdul-Ghani et } \\
\text { al., 2014) }\end{array}$ \\
\hline $\begin{array}{l}\text { Reactive Oxygen } \\
\text { Species }\end{array}$ & Mouse (A/J) & 10 weeks & 22.5 & $\sim 175$ (plasma) & $96.5 \pm 1.38$ & $\begin{array}{c}12 \\
\text { hours }\end{array}$ & 25 & 23 & $\uparrow$ & 1.3 & $<0.05$ & $\begin{array}{c}\text { (La Maestra et al., } \\
2015 \text { ) }\end{array}$ \\
\hline \multirow[t]{2}{*}{$\%$ of Animals Fertile } & Rat (Wistar) & $\begin{array}{c}7-10 \\
\text { weeks }\end{array}$ & 20 & $\begin{array}{c}180 \pm 86 \\
\text { (serum) }\end{array}$ & - & $\begin{array}{c}6 \\
\text { weeks }\end{array}$ & 20 & 40 & $\downarrow$ & $2.9-3.3$ & $<0.05$ & $\begin{array}{l}\text { (Kapawa et al., } \\
\text { 2004) }\end{array}$ \\
\hline & Rat (Wistar) & 10 weeks & 20 & $\begin{array}{c}274 \pm 19 \\
\text { (serum) }\end{array}$ & - & $\begin{array}{c}4 \\
\text { weeks }\end{array}$ & 10 & 20 & $\downarrow$ & 2.5 & $<0.05$ & $\begin{array}{c}\text { (Yamamoto et al., } \\
\text { 1998) }\end{array}$ \\
\hline \multirow[t]{2}{*}{$\begin{array}{c}\text { Fertlization and Early } \\
\text { Embryonic } \\
\text { Development Rate }\end{array}$} & $\begin{array}{c}\text { Mouse } \\
(\mathrm{B} 6 \mathrm{C} 3 \mathrm{~F} 1 / \mathrm{Crl})\end{array}$ & 2 weeks & 3,16 & $\begin{array}{l}15.7 \pm 2.9 ; 95.4 \\
\pm 23.5 \text { (plasma) }\end{array}$ & $\begin{array}{c}30.2 \pm 5.5,745 \\
\quad \pm 18.9\end{array}$ & $\begin{array}{c}8-9 \\
\text { weeks }\end{array}$ & 11 & $6 \mathrm{~L}, 6 \mathrm{H}$ & $\downarrow$ & $1.1-1.3$ & $<0.05$ & $\begin{array}{l}\text { (Polyzos et al., } \\
\text { 2009) }\end{array}$ \\
\hline & Rat (Wistar) & $\begin{array}{c}7-10 \\
\text { weeks }\end{array}$ & 20 & $\begin{array}{c}180 \pm 86 \\
(\text { serum })\end{array}$ & - & $\begin{array}{c}6 \\
\text { weeks }\end{array}$ & 20 & 40 & $\downarrow$ & $2.6-4.5$ & $<0.05$ & $\begin{array}{l}\text { (Kapawa et al., } \\
\text { 2004) }\end{array}$ \\
\hline Testis Weight & Mouse (A/J) & 10 weeks & 22.5 & $\sim 175$ (plasma) & $96.5 \pm 1.38$ & $\begin{array}{c}12 \\
\text { hours }\end{array}$ & 25 & 23 & $\downarrow$ & 1.1 & $<0.05$ & $\begin{array}{c}\text { (La Maestra et al., } \\
2015)\end{array}$ \\
\hline $\begin{array}{c}\text { \% Seminiferous } \\
\text { Tubule with Germ Cell } \\
\text { Loss }\end{array}$ & $\begin{array}{l}\text { Rat (Sprague } \\
\text { Dawley) }\end{array}$ & 13 weeks & 30 & - & - & $\begin{array}{c}10 \\
\text { weeks }\end{array}$ & 8 & 8 & $\uparrow$ & $\infty$ & $<0.05$ & $\begin{array}{c}\text { (Mohamed et al., } \\
\text { 2011) }\end{array}$ \\
\hline $\begin{array}{c}\text { Seminiferous } \\
\text { Epithelial Height }\end{array}$ & $\begin{array}{l}\text { Rat (Sprague } \\
\text { Dawley) }\end{array}$ & 13 weeks & 30 & - & - & $\begin{array}{c}10 \\
\text { weeks }\end{array}$ & 8 & 8 & $\downarrow$ & 1.2 & $<0.05$ & $\begin{array}{c}\text { (Mohamed et al., } \\
\text { 2011) }\end{array}$ \\
\hline \multirow[t]{2}{*}{$\begin{array}{l}\text { Seminiferous Tubule } \\
\text { Diameter }\end{array}$} & Rat (Sprague) & 60 days & 10 & - & - & $\begin{array}{c}10 \\
\text { weeks }\end{array}$ & 15 & 15 & $\downarrow$ & 1.2 & 0.04 & $\begin{array}{c}\text { (Ahmadnia et al., } \\
\text { 2007) }\end{array}$ \\
\hline & $\begin{array}{l}\text { Rat (Sprague } \\
\text { Dawley) }\end{array}$ & 13 weeks & 30 & - & - & $\begin{array}{c}10 \\
\text { weeks }\end{array}$ & 8 & 8 & $\downarrow$ & 1.1 & $<0.05$ & $\begin{array}{l}\text { (Mohamed et al., } \\
\text { 2011) }\end{array}$ \\
\hline $\begin{array}{c}\text { Normal } \\
\text { Spermatogenesis }\end{array}$ & Rat (Sprague) & 60 days & 10 & - & - & $\begin{array}{c}10 \\
\text { weeks }\end{array}$ & 15 & 15 & $\downarrow$ & 11.0 & $<0.001$ & $\begin{array}{c}\text { (Ahmadnia et al., } \\
\text { 2007) }\end{array}$ \\
\hline
\end{tabular}




\begin{tabular}{|c|c|c|c|c|c|c|c|c|c|c|c|c|}
\hline \multirow[t]{4}{*}{$\begin{array}{c}\text { Sperm } \\
\text { Viability/Vitality (\%) }\end{array}$} & Rat (Wistar) & 9 weeks & 20 & - & - & $\begin{array}{c}60 \\
\text { days }\end{array}$ & 10 & 10 & $\downarrow$ & 1.1 & $<0.05$ & $\begin{array}{l}\text { (Garcia et al., } \\
2012)\end{array}$ \\
\hline & Rat (Wistar) & 63 days & 20 & - & - & $\begin{array}{c}8-9 \\
\text { weeks }\end{array}$ & 10 & 10 & $\downarrow$ & 1.0 & $<0.05$ & $\begin{array}{l}\text { (Sankako et al., } \\
2012 \text { ) }\end{array}$ \\
\hline & Rat (Wistar) & 60 days & 20 & - & - & $\begin{array}{c}8-9 \\
\text { weeks }\end{array}$ & 4 & 4 & ND & ND & NS & $\begin{array}{l}\text { (Sankako et al., } \\
\text { 2013) }\end{array}$ \\
\hline & $\begin{array}{l}\text { Rhesus Macaques } \\
\text { (Macaca mulatta) }\end{array}$ & 6 months & - & >100 (serum) & $3.69 \pm 1.57$ & - & - & 4 & ND & ND & NS & (Hung et al., 2009) \\
\hline \multirow[t]{2}{*}{ Sperm Concentration } & Rat (Wistar) & 63 days & 20 & - & - & $\begin{array}{c}8-9 \\
\text { weeks }\end{array}$ & 10 & 10 & $\downarrow$ & 1.2 & $<0.05$ & $\begin{array}{c}\text { (Sankako et al., } \\
2012 \text { ) }\end{array}$ \\
\hline & Rat (Wistar) & 9 weeks & 20 & - & - & $\begin{array}{l}60 \\
\text { days }\end{array}$ & 10 & 10 & ND & ND & NS & $\begin{array}{l}\text { (Garcia et al., } \\
2012 \text { ) }\end{array}$ \\
\hline \multirow[t]{7}{*}{ Total Sperm Count } & Rat (Wistar) & 60 days & 20 & - & - & $\begin{array}{c}8-9 \\
\text { weeks }\end{array}$ & 4 & 4 & $\downarrow$ & 1.7 & $<0.05$ & $\begin{array}{l}\text { (Sankako et al., } \\
\text { 2013) }\end{array}$ \\
\hline & $\begin{array}{l}\text { Rat (Sprague } \\
\text { Dawley) }\end{array}$ & $\begin{array}{l}2,14,28 \\
\text { days }\end{array}$ & 20 & - & - & $\begin{array}{c}4 \\
\text { weeks }\end{array}$ & - & - & $\downarrow$ & $1.3-1.7$ & $\leq 0.05$ & $\begin{array}{l}\text { (Abdul-Ghani et } \\
\text { al., 2014) }\end{array}$ \\
\hline & Rat (Wistar) & 8 weeks & - & - & - & $\begin{array}{c}6-8 \\
\text { weeks }\end{array}$ & 10 & 10 & $\downarrow$ & 1.6 & $<0.01$ & $\begin{array}{c}\text { (Chen and Jiang, } \\
2015)\end{array}$ \\
\hline & Rat (Wistar) & 10 weeks & 20 & $\begin{array}{c}274 \pm 19 \\
\text { (serum) }\end{array}$ & - & $\begin{array}{c}4 \\
\text { weeks }\end{array}$ & 10 & 20 & $\downarrow$ & 1.5 & $<0.05$ & $\begin{array}{c}\text { (Yamamoto et al., } \\
\text { 1998) }\end{array}$ \\
\hline & Mouse (A/J) & 10 weeks & 22.5 & $\sim 175$ (plasma) & $96.5 \pm 1.38$ & $\begin{array}{c}12 \\
\text { hours }\end{array}$ & 25 & 23 & $\downarrow$ & 1.3 & $<0.05$ & $\begin{array}{c}\text { (La Maestra et al., } \\
2015 \text { ) }\end{array}$ \\
\hline & $\begin{array}{l}\text { Rhesus Macaques } \\
\text { (Macaca mulatta) }\end{array}$ & 6 months & - & $>100$ (serum) & $3.69 \pm 1.57$ & - & - & 4 & ND & ND & NS & (Hung et al., 2009) \\
\hline & Rat (Wistar) & $\begin{array}{c}\text { 7-10 } \\
\text { weeks }\end{array}$ & 20 & $\begin{array}{c}180 \pm 86 \\
(\text { serum) }\end{array}$ & - & $\begin{array}{c}6 \\
\text { weeks }\end{array}$ & 20 & 40 & ND & ND & NS & $\begin{array}{c}\text { (Kapawa et al., } \\
2004)\end{array}$ \\
\hline \multirow[t]{3}{*}{$\begin{array}{c}\text { \% Normal } \\
\text { Morphology }\end{array}$} & Rat (Wistar) & 60 days & 20 & - & - & $\begin{array}{c}8-9 \\
\text { weeks }\end{array}$ & 4 & 4 & $\downarrow$ & 1.0 & $<0.05$ & $\begin{array}{l}\text { (Sankako et al., } \\
\text { 2013) }\end{array}$ \\
\hline & Rat (Wistar) & 63 days & 20 & - & - & $\begin{array}{c}8-9 \\
\text { weeks }\end{array}$ & 10 & 10 & $\downarrow$ & 1.0 & $<0.05$ & $\begin{array}{l}\text { (Sankako et al., } \\
\text { 2012) }\end{array}$ \\
\hline & Rat (Wistar) & 9 weeks & 20 & - & - & $\begin{array}{c}60 \\
\text { days }\end{array}$ & 10 & 10 & $\downarrow$ & 1.0 & $<0.05$ & $\begin{array}{l}\text { (Garcia et al., } \\
2012 \text { ) }\end{array}$ \\
\hline \multirow[t]{2}{*}{$\begin{array}{l}\text { \% Abnormal } \\
\text { Morphology }\end{array}$} & Rat (Wistar) & 8 weeks & - & - & - & $\begin{array}{c}6-8 \\
\text { weeks }\end{array}$ & 10 & 10 & $\uparrow$ & 3.3 & $<0.01$ & $\begin{array}{c}\text { (Chen and Jiang, } \\
2015)\end{array}$ \\
\hline & Mouse (A/J) & 10 weeks & 22.5 & $\sim 175$ (plasma) & $96.5 \pm 1.38$ & $\begin{array}{c}12 \\
\text { hours }\end{array}$ & 25 & 23 & $\uparrow$ & 1.4 & $<0.05$ & $\begin{array}{c}\text { (La Maestra et al., } \\
2015 \text { ) }\end{array}$ \\
\hline $\begin{array}{l}\text { Altered Protein/mRNA } \\
\text { Expression }\end{array}$ & Mouse (C57BL/6J) & 6 weeks & - & - & - & $\begin{array}{c}7 \\
\text { weeks }\end{array}$ & 20 & 20 & $\uparrow \downarrow$ & 1.5 & $<0.05$ & (Chen et al., 2015) \\
\hline
\end{tabular}

\section{Footnotes:}

\section{TPM: Total Particulate Matter}

\section{C: Control}

E: Exposed 


\section{D: Direction}

FD: Fold-difference

L: Low dose; H: High dose

ND: No difference; NS: Not significant 
Table C19 Evidence that tobacco smoke is a germ cell mutagen in humans.

\begin{tabular}{|c|c|c|c|c|c|c|c|c|c|c|c|c|c|}
\hline Smoking-Associated Outcome & Cigarettes/Day & $\begin{array}{l}\text { Pack- } \\
\text { Years } \\
\end{array}$ & $\begin{array}{l}\text { Cotinine } \\
(\mathrm{ng} / \mathrm{mL})\end{array}$ & $\mathrm{N}-\mathrm{S}$ & $\mathrm{S}$ & $\mathrm{L}$ & M & $\mathrm{H}$ & $\mathrm{F} ?$ & $\mathrm{D}$ & FD & P-Value & Ref. \\
\hline \multirow[t]{19}{*}{ Childhood Cancer Risk } & - & - & - & 117 & 63 & - & - & - & $\mathrm{F}$ & $\uparrow$ & 3.9 & 0.003 & (Grufferman et al., 1982) \\
\hline & $\begin{array}{c}\text { 1-9 L, } 10-14 \mathrm{M} \\
\geq 15 \mathrm{H}\end{array}$ & - & - & 60 & 102 & 35 & 38 & 29 & $\mathrm{~F}$ & $\uparrow$ & $1.5-3.8$ & - & (Ji et al., 1997) \\
\hline & 10 (median) & - & - & 446 & 168 & - & - & - & $\mathrm{F}$ & $\uparrow$ & $1.3-3.8$ & - & (Chang et al., 2006) \\
\hline & $<20 \mathrm{~L}, \geq 20 \mathrm{H}$ & - & - & 63 & 93 & 50 & - & 43 & $\mathrm{~F}$ & $\uparrow$ & $2.0-3.2$ & $<0.05$ & (Rudant et al., 2008) \\
\hline & $<20 \mathrm{~L}, \geq 20 \mathrm{H}$ & - & - & 998 & 839 & 478 & - & 361 & $\mathrm{~F}$ & $\uparrow$ & 3.1 & 0.04 & (Plichart et al., 2008) \\
\hline & - & - & - & 112 & 51 & - & - & - & $\mathrm{F}$ & $\uparrow$ & 2.0 & - & (McCredie et al., 1994) \\
\hline & - & - & - & 132 & 65 & - & - & - & $\mathrm{F}$ & $\uparrow$ & 1.8 & $<0.05$ & (Edraki and Rambod, 2011) \\
\hline & $\begin{array}{c}<10 \mathrm{~L}, 10-19 \mathrm{M}, \\
\geq 20 \mathrm{H}\end{array}$ & - & - & 46 & 568 & 60 & - & 508 & $\mathrm{~F}$ & $\uparrow$ & $1.5-1.6$ & - & (Sorahan et al., 2001) \\
\hline & $\begin{array}{c}1-10 \mathrm{~L}, 11-20 \mathrm{M}, \\
>20 \mathrm{H}\end{array}$ & - & - & - & - & - & - & - & $\mathrm{F}$ & $\uparrow$ & $1.3-1.6$ & - & (Shu et al., 1996) \\
\hline & $\begin{array}{c}1-10 \mathrm{~L}, 11-20 \mathrm{M}, \\
>20 \mathrm{H}\end{array}$ & - & - & 82 & 106 & 23 & 48 & 35 & $\mathrm{~F}$ & $\uparrow$ & $1.2-1.6$ & - & (John et al., 1991) \\
\hline & $1-14 \mathrm{~L}, \geq 15 \mathrm{H}$ & - & - & 709 & 368 & 134 & - & 234 & $\mathrm{~F}$ & $\uparrow$ & 1.4 & - & (Milne et al., 2012) \\
\hline & $1-19 \mathrm{~L}, \geq 20 \mathrm{H}$ & - & - & 4625 & 3783 & 1586 & - & 2197 & $\mathrm{~F}$ & $\uparrow$ & 1.2 & $<0.05$ & (Pang et al., 2003) \\
\hline & $\begin{array}{c}<10 \mathrm{~L}, 10-19 \mathrm{M} \\
\geq 20 \mathrm{H}\end{array}$ & - & - & 623 & 515 & 65 & 139 & 311 & $\mathrm{~F}$ & ND & ND & NS & (MacArthur et al., 2008) \\
\hline & - & - & - & 273 & 296 & - & - & - & $\mathrm{F}$ & ND & ND & NS & (Ruckart et al., 2013) \\
\hline & $\leq 20,>20$ & - & - & 690 & 662 & 573 & - & 89 & $\mathrm{~F}$ & ND & ND & NS & (Menegaux et al., 2007) \\
\hline & $\begin{array}{c}<10 \mathrm{~L}, 10-19 \mathrm{M} \\
\geq 20 \mathrm{H}\end{array}$ & - & - & - & - & - & - & - & $\mathrm{F}$ & ND & ND & NS & (Brondumet al., 1999) \\
\hline & $\begin{array}{c}1-10 \mathrm{~L}, 11-20 \mathrm{M}, \\
>20 \mathrm{H}\end{array}$ & - & - & $\begin{array}{c}\sim 241 \\
0\end{array}$ & $\sim 2125$ & $\sim 521$ & $\begin{array}{c}\sim 121 \\
1\end{array}$ & $\sim 393$ & $\mathrm{~F}$ & ND & ND & NS & (Schüz et al., 1999) \\
\hline & $<20 \mathrm{~L}, \geq 20 \mathrm{H}$ & - & - & 591 & 663 & 135 & - & 528 & $\mathrm{~F}$ & ND & ND & NS & (Gold et al., 1993) \\
\hline & - & - & - & - & - & - & - & - & $\mathrm{F}$ & ND & ND & NS & (Magnani et al., 1990) \\
\hline \multirow[t]{5}{*}{ Congenital Malformations } & $\begin{array}{c}1-9 \mathrm{~L}, 10-19 \mathrm{M} \\
\geq 20 \mathrm{H}\end{array}$ & - & - & 248 & 405 & 276 & 110 & 19 & $\mathrm{~F}$ & $\uparrow$ & $2.0-13.1$ & - & (Deng et al., 2013) \\
\hline & - & - & - & 20 & 5 & - & - & - & $\mathrm{F}$ & $\uparrow$ & 6.4 & $<0.05$ & (Hearey et al., 1984) \\
\hline & - & - & - & 177 & 149 & - & - & - & $\mathrm{F}$ & $\uparrow$ & 3.8 & $<0.05$ & (Pierik et al., 2004) \\
\hline & $\begin{array}{c}<10 \mathrm{~L}, 10-20 \mathrm{M} \\
>20 \mathrm{H}\end{array}$ & - & - & 564 & 793 & 415 & 339 & 39 & $\mathrm{~F}$ & $\uparrow$ & $1.5-3.0$ & $<0.05$ & $\begin{array}{c}\text { (Kučienè and Dulskienè, } \\
2010)\end{array}$ \\
\hline & $1-10 \mathrm{~L},>10 \mathrm{H}$ & - & - & 2563 & 2598 & 1089 & - & 1509 & $\mathrm{~F}$ & $\uparrow$ & $1.8-2.6$ & $<0.01$ & (Mau and Netter, 1974) \\
\hline
\end{tabular}




\begin{tabular}{|c|c|c|c|c|c|c|c|c|c|c|c|c|c|}
\hline & $1-19 \mathrm{~L}, \geq 20 \mathrm{H}$ & - & - & 21 & 62 & 25 & - & 37 & $\mathrm{~F}$ & $\uparrow$ & $1.7-2.4$ & - & (Savitz et al., 1991) \\
\hline & $\sim 20$ & - & - & 3306 & 362 & - & - & - & $\mathrm{F}$ & $\uparrow$ & 2.4 & - & (Steinberger et al., 2002) \\
\hline & - & - & - & 140 & 35 & - & - & - & $\mathrm{F}$ & $\uparrow$ & 2.4 & 0.003 & (Palma et al., 2013) \\
\hline & $1-19 \mathrm{~L}, \geq 20 \mathrm{H}$ & - & - & 1083 & 591 & 365 & - & 226 & $\mathrm{~F}$ & $\uparrow$ & 2.1 & - & (Wasserman et al., 1996) \\
\hline & $<15 \mathrm{~L}, \geq 15 \mathrm{H}$ & - & - & 214 & 146 & - & - & - & $\mathrm{F}$ & $\uparrow$ & $1.7-2.1$ & $<0.02$ & (Cresci et al., 2011) \\
\hline & - & - & - & 71 & 137 & - & - & - & $\mathrm{F}$ & $\uparrow$ & 1.9 & - & (Kurahashi et al., 2005) \\
\hline & - & - & - & 432 & 251 & - & - & - & $\mathrm{F}$ & $\uparrow$ & 1.8 & - & (van Rooij et al., 2010) \\
\hline & $1-10 \mathrm{~L},>10 \mathrm{H}$ & - & - & 377 & 191 & 76 & - & 115 & $\mathrm{~F}$ & $\uparrow$ & 1.5 & - & (Krapels et al., 2006) \\
\hline & - & - & - & 131 & 81 & - & - & - & $\mathrm{F}$ & $\uparrow$ & 1.4 & 0.03 & (Cardy et al., 2007) \\
\hline & - & - & - & 1685 & 495 & - & - & - & $\mathrm{F}$ & $\uparrow$ & 1.3 & $<0.01$ & (Sun et al., 2013) \\
\hline & $\begin{array}{c}1-9 \mathrm{~L}, 10-19 \mathrm{M} \\
\geq 20 \mathrm{H}\end{array}$ & - & - & 792 & 132 & 420 & 488 & 324 & $\mathrm{~F}$ & $\uparrow$ & 1.2 & - & (Zhang et al., 1992) \\
\hline & - & - & - & 273 & 296 & - & - & - & $\mathrm{F}$ & ND & ND & NS & (Ruckart et al., 2013) \\
\hline & - & - & - & 167 & 114 & - & - & - & $\mathrm{F}$ & ND & ND & NS & (Krapels et al., 2008) \\
\hline & - & - & - & 164 & 101 & - & - & - & $\mathrm{F}$ & ND & ND & NS & (Carmichael et al., 2004) \\
\hline & $<20 \mathrm{~L}, \geq 20 \mathrm{H}$ & - & - & 447 & 269 & 149 & - & 120 & $\mathrm{~F}$ & ND & ND & NS & (Shaw et al., 1996) \\
\hline & - & - & - & 125 & 135 & - & - & - & $\mathrm{F}$ & ND & ND & NS & (Yuan et al., 1995) \\
\hline & $\leq \sim 30 \mathrm{~L},>\sim 30 \mathrm{H}$ & - & - & 9838 & 7314 & 6140 & - & 1174 & $\mathrm{~F}$ & ND & ND & NS & (Seidman et al., 1990) \\
\hline & - & - & - & 60 & 60 & - & - & - & $\mathrm{F}$ & ND & ND & NS & $\begin{array}{c}\text { (Holmberg and Nurminen, } \\
1980)\end{array}$ \\
\hline Perinatal Mortality & $1-10 \mathrm{~L},>10 \mathrm{H}$ & - & - & 2575 & 2608 & 1085 & - & 1523 & $\mathrm{~F}$ & $\uparrow$ & 1.5 & $<0.01$ & (Mau and Netter, 1974) \\
\hline Mutations in Children & $12.3 \pm 7.0$ & - & - & 59 & 19 & - & - & - & $\mathrm{F}$ & $\uparrow$ & 4.0 & 0.061 & (Linschooten et al., 2013) \\
\hline $\begin{array}{l}\text { DNA Damage in Newborn } \\
\text { Cord Blood }\end{array}$ & - & - & - & 10 & 4 & - & - & - & $\mathrm{F}$ & $\uparrow$ & $1.2-1.5$ & - & (Laubenthal et al., 2012) \\
\hline \multirow[t]{6}{*}{ SpermDNA Adducts } & $16.0 \pm 6.2$ & - & - & 42 & 37 & - & - & - & I & $\uparrow$ & $1.2-7.6$ & $<0.002$ & (Perrin et al., 2011) \\
\hline & $20.6 \pm 0.7$ & - & - & 12 & 11 & - & - & - & $\mathrm{F}$ & $\uparrow$ & 1.9 & $<0.0001$ & (Zenzes et al., 1999a) \\
\hline & - & - & - & 48 & 22 & - & - & - & $\mathrm{F}$ & $\uparrow$ & 1.7 & 0.026 & (Horak et al., 2003) \\
\hline & - & - & - & 42 & 30 & - & - & - & I & ND & ND & NS & (Horak et al., 2003) \\
\hline & - & - & - & 88 & 69 & - & - & - & I & ND & ND & NS & (Gaspari et al., 2003) \\
\hline & $1-19 \mathrm{~L}, \geq 20 \mathrm{H}$ & - & - & 12 & 24 & 12 & - & 12 & $\mathrm{~F}$ & ND & ND & NS & (Gallagher et al., 1993) \\
\hline $\begin{array}{c}\text { Aneuploidy: } \mathrm{X}-\mathrm{X} / \mathrm{Y}-\mathrm{Y} / 3-3 \\
\text { Diploidies }\end{array}$ & $<20 \mathrm{~L},>20 \mathrm{H}$ & - & - & 7 & 10 & 4 & - & 6 & $\mathrm{~F}$ & $\uparrow$ & $1.9-4.0$ & $\begin{array}{l}<0.02- \\
0.0002 \\
\end{array}$ & (Pereira et al., 2014) \\
\hline
\end{tabular}




\begin{tabular}{|c|c|c|c|c|c|c|c|c|c|c|c|c|c|}
\hline 13-13 Disomies & $\sim 7 \mathrm{~L}, \geq 20 \mathrm{H}$ & - & - & 10 & 21 & 11 & - & 10 & $\mathrm{~F}$ & $\uparrow$ & $2.7-3.0$ & $<0.0001$ & (Shi et al., 2001) \\
\hline X-Y, 3-3 Disomies & $<20 \mathrm{~L},>20 \mathrm{H}$ & - & - & 7 & 10 & 4 & - & 6 & $\mathrm{~F}$ & $\uparrow$ & $1.7-2.3$ & $\begin{array}{l}<0.01- \\
0.0001\end{array}$ & (Pereira et al., 2014) \\
\hline 21-21 Disomies & $\geq 10$ & - & - & 14 & 14 & - & - & - & I & $\uparrow$ & 2.2 & 0.01 & (Faure et al., 2007) \\
\hline $\mathrm{X}$-X and $\mathrm{Y}$-Y Disomies & $\begin{array}{l}9.0 \pm 6.1 \text { control, } \\
15.2 \pm 3.9 \text { exposed }\end{array}$ & - & - & 15 & 16 & - & - & - & $\mathrm{F}$ & $\uparrow$ & 1.9 & 0.0189 & (Naccarati et al., 2003) \\
\hline $\mathrm{X}$-X Disomies & $1-19 \mathrm{~L}, \geq 20 \mathrm{H}$ & - & - & 28 & 17 & 7 & - & 10 & $\mathrm{~F}$ & $\uparrow$ & 1.9 & $<0.05$ & (Robbins et al., 1997) \\
\hline X-X-18-18 Diploidies & $1-19 \mathrm{~L}, \geq 20 \mathrm{H}$ & - & - & 28 & 17 & 7 & - & 10 & $\mathrm{~F}$ & $\uparrow$ & 1.8 & $<0.05$ & (Robbins et al., 1997) \\
\hline Y-Y-18-18 Diploidies & $1-19 \mathrm{~L}, \geq 20 \mathrm{H}$ & - & - & 28 & 17 & 7 & - & 10 & $\mathrm{~F}$ & $\uparrow$ & 1.7 & $<0.05$ & (Robbins et al., 1997) \\
\hline 18-18 Disomies & $1-19 \mathrm{~L}, \geq 20 \mathrm{H}$ & - & - & 28 & 17 & 7 & - & 10 & $\mathrm{~F}$ & $\uparrow$ & 1.6 & $<0.05$ & (Robbins et al., 1997) \\
\hline 1-1,7-7 Disomies/Diploidies & $13.3 \pm 9.5$ & - & - & 19 & 9 & - & - & - & $\mathrm{F}$ & $\uparrow$ & 1.3 & 0.03 & (Härkönen et al., 1999) \\
\hline $\mathrm{X}-\mathrm{X}, \mathrm{Y}-\mathrm{Y}$, and 8-8 Disomies & 20 & - & - & 15 & 11 & - & - & - & $\mathrm{F}$ & $\uparrow$ & 1.3 & $<0.01$ & (Rubes et al., 1998) \\
\hline X-Y Disomies & $1-19 \mathrm{~L}, \geq 20 \mathrm{H}$ & - & - & 28 & 17 & 7 & - & 10 & $\mathrm{~F}$ & $\uparrow$ & 1.2 & $<0.05$ & (Robbins et al., 1997) \\
\hline X-Y-18-18 Diploidies & $1-19 \mathrm{~L}, \geq 20 \mathrm{H}$ & - & - & 28 & 17 & 7 & - & 10 & $\mathrm{~F}$ & $\begin{array}{l}\uparrow \\
(\mathrm{N} \\
\mathrm{S})\end{array}$ & 1.5 & NS & (Robbins et al., 1997) \\
\hline Y-Y Disomies & $1-19 \mathrm{~L}, \geq 20 \mathrm{H}$ & - & - & 28 & 17 & 7 & - & 10 & $\mathrm{~F}$ & $\begin{array}{l}\uparrow \\
(\mathrm{N} \\
\mathrm{S})\end{array}$ & 1.5 & NS & (Robbins et al., 1997) \\
\hline X-Y Disomies & 20 & - & - & 15 & 11 & - & - & - & $\mathrm{F}$ & $\begin{array}{l}\uparrow \\
(\mathrm{N} \\
\mathrm{S})\end{array}$ & 1.5 & NS & (Rubes et al., 1998) \\
\hline X-X, Y-Y Disomies & $<20 \mathrm{~L},>20 \mathrm{H}$ & - & - & 7 & 10 & 4 & - & 6 & $\mathrm{~F}$ & ND & ND & NS & (Pereira et al., 2014) \\
\hline $\begin{array}{c}\mathrm{X}-\mathrm{X}, \mathrm{Y}-\mathrm{Y}, 13-13,18-18,21- \\
21 \text { Disomies }\end{array}$ & - & - & $\begin{array}{l}63.30 \\
\text { (saliva) }\end{array}$ & 117 & 87 & - & - & - & I & ND & ND & NS & (Jurewicz et al., 2014) \\
\hline 13-13, 18-18, 21-21 Disomies & $1-19 \mathrm{~L}, \geq 20 \mathrm{H}$ & - & - & 31 & 25 & 10 & - & 15 & I & ND & ND & - & (Viloria et al., 2005) \\
\hline $\begin{array}{c}\text { 21-21, X-X, Y-Y, X-Y } \\
\text { Disomies }\end{array}$ & $\sim 7 \mathrm{~L}, \geq 20 \mathrm{H}$ & - & - & 10 & 21 & 11 & - & 10 & F & ND & ND & NS & (Shi et al., 2001) \\
\hline $\begin{array}{c}\mathrm{X}-\mathrm{X}-8-8, \mathrm{Y}-\mathrm{Y}-8-8, \mathrm{X}-\mathrm{Y}-8-8 \\
\text { Diploidies }\end{array}$ & 20 & - & - & 15 & 11 & - & - & - & $\mathrm{F}$ & ND & ND & NS & (Rubes et al., 1998) \\
\hline \multirow[t]{6}{*}{$\begin{array}{l}\text { SpermDNA Fragmentation } \\
\text { (TUNEL/TdTa) }\end{array}$} & - & - & - & 78 & 35 & - & - & - & I & $\uparrow$ & 4.3 & 0.01 & (Sun et al., 1997) \\
\hline & $>10$ & - & - & 35 & 35 & - & - & - & $\mathrm{F}$ & $\uparrow$ & 1.6 & $<0.05$ & (Potts et al., 1999) \\
\hline & $>20$ & - & $\begin{array}{c}99.55 \pm \\
64.58 \\
\text { (seminal) }\end{array}$ & 63 & 53 & - & - & - & I & $\uparrow$ & 1.5 & $<0.001$ & (Hammadeh et al., 2010) \\
\hline & $\geq 20$ & - & - & 20 & 20 & - & - & - & $\mathrm{F}$ & $\uparrow$ & 1.3 & $<0.0001$ & (Aydin et al., 2013) \\
\hline & $10.3 \pm 7.74$ & - & - & 57 & 51 & - & - & - & I & $\uparrow$ & 1.2 & $<0.01$ & (Sepaniak et al., 2006) \\
\hline & $\begin{array}{c}<10 \text { light } \mathrm{L}, 10-19 \\
\mathrm{M}, \geq 20 \mathrm{H}\end{array}$ & - & - & 47 & 26 & 7 & 14 & 5 & I & ND & $\mathrm{ND}$ & NS & (De Bantel et al., 2014) \\
\hline
\end{tabular}




\begin{tabular}{|c|c|c|c|c|c|c|c|c|c|c|c|c|c|}
\hline & - & - & - & 459 & 45 & - & - & - & I & ND & ND & NS & (Varshini et al., 2012) \\
\hline & $1-19 \mathrm{~L}, \geq 20 \mathrm{H}$ & - & - & 69 & 28 & 17 & - & 11 & $\mathrm{~F}$ & ND & ND & NS & (Sergerie et al., 2000) \\
\hline \multirow[t]{5}{*}{$\begin{array}{l}\text { Sperm DNA Fragmentation } \\
\text { (Comet) }\end{array}$} & $\geq 10$ & - & - & 20 & 20 & - & - & - & I & $\uparrow$ & $4.2-5.0$ & $<0.05$ & (Antoniassi et al., 2016) \\
\hline & - & $\begin{array}{c}3.8 \mathrm{~L}, 15.1 \\
\mathrm{H}\end{array}$ & - & 56 & 54 & 29 & - & 25 & $\mathrm{I}(\mathrm{V})$ & $\uparrow$ & 3.2 & $<0.0001$ & (Fariello et al., 2012) \\
\hline & $3.7 \pm 0.5$ & - & - & 9 & 12 & - & - & - & $\mathrm{F}$ & $\uparrow$ & 1.3 & $<0.01$ & (Linschooten et al., 2011) \\
\hline & $7.67 \pm 7.56$ & - & - & 62 & 53 & - & - & - & $\mathrm{F}$ & ND & ND & NS & (Oh et al., 2005) \\
\hline & $>20$ & - & - & 15 & 25 & - & - & - & $\mathrm{F}$ & ND & ND & NS & (Belcheva et al., 2004) \\
\hline \multirow[t]{10}{*}{$\begin{array}{l}\text { Sperm DNA Fragmentation } \\
\text { (SCSA) }\end{array}$} & $>20$ & - & - & 36 & 34 & - & - & - & $\mathrm{I}$ & $\uparrow$ & 2.0 & $<0.001$ & (Elshal et al., 2009) \\
\hline & - & - & - & 33 & 74 & - & - & - & $\mathrm{F}$ & $\uparrow$ & $1.3-1.6$ & $<0.05$ & (Kumar et al., 2015) \\
\hline & - & - & - & 4 & 22 & - & - & - & $\mathrm{F}$ & $\uparrow$ & 1.2 & 0.02 & (Gautamet al., 2015) \\
\hline & - & - & $\begin{array}{l}>10 \\
\text { (saliva) }\end{array}$ & 143 & 110 & - & - & - & I & ND & ND & NS & (Jurewicz et al., 2013) \\
\hline & - & - & - & 135 & 51 & - & - & - & I & ND & ND & NS & (Bojar et al., 2013) \\
\hline & 15 & - & - & 187 & 92 & - & - & - & I & ND & ND & NS & (Smit et al., 2010) \\
\hline & $>5$ & - & - & 131 & 46 & - & - & - & $\mathrm{F} / \mathrm{I}$ & ND & ND & NS & (Rybar et al., 2009) \\
\hline & - & - & - & 19 & 29 & - & - & - & $\mathrm{F}$ & ND & ND & NS & (Hsu et al., 2006) \\
\hline & - & - & - & 147 & 68 & - & - & - & $\mathrm{F}$ & ND & ND & NS & (Spano et al., 1998) \\
\hline & 20 & - & - & 15 & 11 & - & - & - & $\mathrm{F}$ & ND & ND & NS & (Rubes et al., 1998) \\
\hline \multirow[t]{10}{*}{$\begin{array}{c}\text { SpermDNA Fragmentation } \\
\text { (Other) }\end{array}$} & - & - & - & 30 & 22 & - & - & - & I & $\uparrow$ & 2.1 & $<0.02$ & (Omran et al., 2013) \\
\hline & $\begin{array}{c}<10 \mathrm{~L}, 10-19 \mathrm{M} \\
\geq 20 \mathrm{H}\end{array}$ & - & - & 298 & 920 & 256 & 365 & 299 & I & $\uparrow$ & $\sim 2.1$ & $\mathrm{P}<0.05$ & (Cui et al., 2016) \\
\hline & $11.1 \pm 5.8$ & - & - & 80 & 80 & - & - & - & $\mathrm{F}$ & $\uparrow$ & 1.9 & $<0.05$ & (Taha et al., 2012) \\
\hline & - & - & - & 51 & 48 & - & - & - & $\mathrm{F}$ & $\uparrow$ & $\sim 1.5$ & 0.028 & (Viloria et al., 2007) \\
\hline & $\begin{array}{c}5 \pm 2.44 \mathrm{~L}, 14.84 \\
\pm 3.43 \mathrm{M}, 22.90 \pm \\
1.7 \mathrm{H}\end{array}$ & - & - & 30 & 40 & 10 & 19 & 11 & I & $\uparrow$ & 1.4 & $<0.001$ & (El-Melegy and Ali, 2011) \\
\hline & $12.96 \pm 6.93$ & - & - & 30 & 30 & - & - & - & $\mathrm{F}$ & $\uparrow$ & 1.3 & - & (El-Melegy and Ali, 2011) \\
\hline & $18.4 \pm 5.8$ & - & - & 72 & 84 & - & - & - & I & $\uparrow$ & 1.3 & $<0.05$ & (Taha et al., 2014) \\
\hline & - & $\begin{array}{c}0-1 \mathrm{~L},>1 \\
\mathrm{H}\end{array}$ & - & 998 & 109 & 76 & - & 33 & I & $\uparrow$ & 1.1 & $<0.05$ & (Anifandis et al., 2014) \\
\hline & $1-20 \mathrm{~L},>21 \mathrm{H}$ & - & - & 126 & 178 & 98 & - & 80 & I & $\uparrow$ & - & 0.008 & (Mitra et al., 2012) \\
\hline & $\begin{array}{r}7.5 \pm 0.43 \mathrm{~L} \\
15.92 \pm 0.7 \mathrm{M}\end{array}$ & - & - & 40 & 80 & 19 & 27 & 34 & $\mathrm{~F} / \mathrm{I}$ & $\uparrow$ & - & $<0.05$ & (Tawadrous et al., 2011) \\
\hline
\end{tabular}




\begin{tabular}{|c|c|c|c|c|c|c|c|c|c|c|c|c|c|}
\hline & $26.44 \pm 1.4 \mathrm{H}$ & & & & & & & & & & & & \\
\hline & - & - & - & 51 & 48 & - & - & - & $\mathrm{F}$ & ND & ND & NS & (Viloria et al., 2007) \\
\hline Sperm Micronuclei (\%) & - & - & - & 34 & 20 & - & - & - & I & ND & ND & NS & (Lähdetie, 1986) \\
\hline \multirow[t]{2}{*}{ SpermDNA Intact } & - & $\begin{array}{c}<8 \mathrm{~L},>30 \\
\mathrm{H}\end{array}$ & - & 30 & 40 & 20 & - & 20 & I & $\downarrow$ & 12.0 & $<0.05$ & (Selit et al., 2013) \\
\hline & - & $\begin{array}{c}<8 \mathrm{~L},>30 \\
\mathrm{H}\end{array}$ & - & 30 & 40 & 20 & - & 20 & $\mathrm{~F}$ & $\downarrow$ & 5.0 & $<0.05$ & (Selit et al., 2013) \\
\hline \multirow[t]{2}{*}{$\begin{array}{l}\text { Abnormal DNA Condensation } \\
\text { Pattern }\end{array}$} & $>20$ & - & $\begin{array}{c}99.55 \pm \\
64.58 \\
\text { (seminal) }\end{array}$ & 63 & 53 & - & - & - & I & $\uparrow$ & 1.2 & $<0.001$ & (Hammadeh et al., 2010) \\
\hline & $1-14 \mathrm{~L}, 15-40 \mathrm{H}$ & - & - & 203 & 147 & 68 & - & 79 & I & ND & ND & NS & (Oldereid et al., 1989) \\
\hline \multirow[t]{16}{*}{ Oxidative Stress } & - & - & - & 33 & 74 & - & - & - & $\mathrm{F}$ & $\uparrow$ & $1.9-5.2$ & $<0.05$ & (Kumar et al., 2015) \\
\hline & $>20$ & - & $\begin{array}{c}99.55 \pm \\
64.58 \\
\text { (seminal) }\end{array}$ & 63 & 53 & - & - & - & I & $\uparrow$ & $1.4-3.7$ & $<0.001$ & (Hammadeh et al., 2010) \\
\hline & $>20$ & - & - & 36 & 34 & - & - & - & I & $\uparrow$ & 2.0 & $<0.001$ & (Elshal et al., 2009) \\
\hline & $16 \pm 7.5$ & - & - & 83 & 64 & - & - & - & $\mathrm{F}$ & $\uparrow$ & 1.9 & $\leq 0.01$ & $\begin{array}{l}\text { (Ghaffari and Rostami, } \\
\text { 2012) }\end{array}$ \\
\hline & - & - & - & 4 & 22 & - & - & - & $\mathrm{F}$ & $\uparrow$ & 1.7 & 0.05 & (Gautamet al., 2015) \\
\hline & $\geq 21$ & - & - & 24 & 26 & - & - & - & I & $\uparrow$ & $1.6-1.7$ & $<0.001$ & (Kiziler et al., 2007) \\
\hline & $>20$ & - & $\begin{array}{c}60.44 \pm \\
31.32 \\
\text { (seminal) }\end{array}$ & 19 & 35 & - & - & - & $\mathrm{F}$ & $\uparrow$ & 1.6 & 0.0001 & (Hamad et al., 2014) \\
\hline & $9.4 \pm 1.6$ & - & - & 32 & 28 & - & - & - & $\mathrm{F}$ & $\uparrow$ & 1.6 & $<0.001$ & (Shen et al., 1997) \\
\hline & - & - & - & 29 & 25 & - & - & - & $\mathrm{F}$ & $\uparrow$ & 1.6 & $<0.01$ & (Shen et al., 1999) \\
\hline & $22 \pm 1.8$ & - & - & 22 & 19 & - & - & - & $\mathrm{F}$ & $\uparrow$ & 1.5 & 0.005 & (Fraga et al., 1996) \\
\hline & - & $\begin{array}{c}3.8 \mathrm{~L}, 15.1 \\
\mathrm{H}\end{array}$ & - & 56 & 54 & 29 & - & 25 & $\mathrm{I}(\mathrm{V})$ & $\uparrow$ & 1.5 & $<0.0001$ & (Fariello et al., 2012) \\
\hline & - & $<5 \mathrm{~L}, \geq 5 \mathrm{H}$ & - & 149 & 271 & 115 & - & 156 & I & $\uparrow$ & 1.2 & $<0.001$ & (Ji et al., 2013) \\
\hline & $\geq 21$ & - & - & 22 & 23 & - & - & - & $\mathrm{F}$ & $\uparrow$ & 1.2 & $<0.001$ & (Kiziler et al., 2007) \\
\hline & - & - & - & 30 & 25 & - & - & - & I & ND & ND & NS & (Viloria et al., 2010) \\
\hline & $0-40$ & - & - & 151 & 74 & - & - & - & $\mathrm{F}$ & ND & ND & NS & (Loft et al., 2003) \\
\hline & - & - & - & 46 & 14 & - & - & - & I & ND & ND & NS & (Shen et al., 1999) \\
\hline \multirow[t]{3}{*}{ Reactive Oxygen Species } & $>20$ & - & - & 21 & 12 & - & - & - & I & $\uparrow$ & 19.7 & 0.04 & (Saleh et al., 2002) \\
\hline & $\geq 21$ & - & - & 22 & 23 & - & - & - & $\mathrm{F}$ & $\uparrow$ & 2.8 & $<0.001$ & (Kiziler et al., 2007) \\
\hline & $11.1 \pm 5.8$ & - & - & 80 & 80 & - & - & - & $\mathrm{F}$ & $\uparrow$ & 2.7 & $<0.05$ & (Taha et al., 2012) \\
\hline
\end{tabular}




\begin{tabular}{|c|c|c|c|c|c|c|c|c|c|c|c|c|c|}
\hline & $>20$ & - & $\begin{array}{c}99.55 \pm \\
64.58 \\
\text { (seminal) }\end{array}$ & 63 & 53 & - & - & - & I & $\uparrow$ & 2.1 & $<0.001$ & (Hammadeh et al., 2010) \\
\hline & $\geq 21$ & - & - & 24 & 26 & - & - & - & I & $\uparrow$ & 2.0 & $<0.001$ & (Kiziler et al., 2007) \\
\hline & - & - & - & 33 & 74 & - & - & - & $\mathrm{F}$ & $\uparrow$ & $1.6-1.9$ & $<0.05$ & (Kumar et al., 2015) \\
\hline & - & - & - & 4 & 22 & - & - & - & $\mathrm{F}$ & $\uparrow$ & 1.2 & 0.046 & (Gautamet al., 2015) \\
\hline & $18.4 \pm 5.8$ & - & - & 72 & 84 & - & - & - & I & $\uparrow$ & 1.2 & $<0.05$ & (Taha et al., 2014) \\
\hline & - & - & - & 27 & 15 & - & - & - & $\mathrm{I}(\mathrm{V})$ & $\begin{array}{l}\uparrow \\
(\mathrm{N} \\
\mathrm{S})\end{array}$ & 1.5 & NS & (Moein et al., 2009) \\
\hline \multirow[t]{2}{*}{ Apoptotic Markers } & $\begin{array}{c}5 \pm 2.44 \mathrm{~L}, 14.84 \\
\pm 3.43 \mathrm{M}, 22.90 \pm \\
1.7 \mathrm{H}\end{array}$ & - & - & 30 & 40 & 10 & 19 & 11 & I & $\uparrow$ & $1.1-1.2$ & $<0.01$ & (El-Melegy and Ali, 2011) \\
\hline & $12.96 \pm 6.93$ & - & - & 30 & 30 & - & - & - & $\mathrm{F}$ & $\uparrow$ & $1.1-1.2$ & - & (El-Melegy and Ali, 2011) \\
\hline \multirow[t]{4}{*}{ Apoptotic Sperm } & $>20$ & - & - & 15 & 25 & - & - & - & $\mathrm{F}$ & $\uparrow$ & 2.5 & $<0.01$ & (Belcheva et al., 2004) \\
\hline & $\begin{array}{c}1-10 \mathrm{~L}, 11-19 \mathrm{M} \\
\geq 20 \mathrm{H}\end{array}$ & - & - & 39 & 121 & 49 & 36 & 36 & $\mathrm{I}(\mathrm{V})$ & $\uparrow$ & 1.2 & $<0.05$ & (Collodel et al., 2009) \\
\hline & $>10$ & - & - & 71 & 39 & - & - & - & $\mathrm{F} / \mathrm{I}$ & ND & ND & NS & (Moretti et al., 2014) \\
\hline & $\begin{array}{c}1-10 \mathrm{~L}, 11-19 \mathrm{M}, \\
\geq 20 \mathrm{H}\end{array}$ & - & - & 153 & 118 & 42 & 25 & 51 & I & ND & ND & NS & (Collodel et al., 2010) \\
\hline \multirow[t]{3}{*}{ Necrotic Sperm } & $\begin{array}{c}1-10 \mathrm{~L}, 11-19 \mathrm{M} \\
\geq 20 \mathrm{H}\end{array}$ & - & - & 39 & 121 & 49 & 36 & 36 & $\mathrm{I}(\mathrm{V})$ & $\uparrow$ & 1.2 & $<0.05$ & (Collodel et al., 2009) \\
\hline & $>10$ & - & - & 71 & 39 & - & - & - & $\mathrm{F} / \mathrm{I}$ & ND & ND & NS & (Moretti et al., 2014) \\
\hline & $\begin{array}{c}1-10 \mathrm{~L}, 11-19 \mathrm{M} \\
\geq 20 \mathrm{H}\end{array}$ & - & - & 153 & 118 & 42 & 25 & 51 & I & ND & ND & NS & (Collodel et al., 2010) \\
\hline Dead Sperm & $>20$ & - & - & 15 & 25 & - & - & - & $\mathrm{F}$ & ND & ND & NS & (Belcheva et al., 2004) \\
\hline \multirow[t]{9}{*}{ Sperm Viability/Vitality (\%) } & $\begin{array}{c}1-10 \mathrm{~L}, 11-19 \mathrm{M} \\
\geq 20 \mathrm{H}\end{array}$ & - & - & 153 & 118 & 42 & 25 & 51 & I & $\downarrow$ & 4 & $<0.05$ & (Collodel et al., 2010) \\
\hline & - & - & - & 30 & 22 & - & - & - & I & $\downarrow$ & 2.7 & $<0.05$ & (Omran et al., 2013) \\
\hline & $\begin{array}{c}<10 \mathrm{~L}, 10-19 \mathrm{M} \\
\geq 20 \mathrm{H}\end{array}$ & - & - & 298 & 920 & 256 & 365 & 299 & I & $\uparrow$ & $\sim 1.5$ & $<0.05$ & (Cui et al., 2016) \\
\hline & $>20$ & - & $\begin{array}{c}60.44 \pm \\
31.32 \\
\text { (seminal) }\end{array}$ & 19 & 35 & - & - & - & $\mathrm{F}$ & $\downarrow$ & 1.3 & 0.009 & (Hamad et al., 2014) \\
\hline & $>20$ & - & $\begin{array}{c}99.55 \pm \\
64.58 \\
\text { (seminal) }\end{array}$ & 63 & 53 & - & - & - & I & $\downarrow$ & 1.2 & 0.030 & (Hammadeh et al., 2010) \\
\hline & $\begin{array}{c}1-10 \mathrm{~L}, 11-20 \mathrm{M} \\
>20 \mathrm{H}\end{array}$ & - & - & 110 & 191 & 58 & 68 & 65 & I & $\downarrow$ & $1.2-1.3$ & $<0.01$ & (Zhang et al., 2000) \\
\hline & $>20$ & - & - & 15 & 25 & - & - & - & $\mathrm{F}$ & $\downarrow$ & 1.2 & $<0.05$ & (Belcheva et al., 2004) \\
\hline & $\begin{array}{c}1-10 \mathrm{~L}, 11-20 \mathrm{M} \\
>20 \mathrm{H}\end{array}$ & - & - & 130 & 214 & 66 & 76 & 72 & I & $\downarrow$ & $1.1-1.2$ & $<0.05$ & (Mehrannia, 2007) \\
\hline & $2-40$ & - & - & 372 & 704 & - & - & - & I & $\downarrow$ & 1.1 & 0.005 & (Zhang et al., 2016) \\
\hline
\end{tabular}




\begin{tabular}{|c|c|c|c|c|c|c|c|c|c|c|c|c|c|}
\hline & - & - & - & 57 & 35 & - & - & - & I & $\downarrow$ & 1.1 & $<0.005$ & (Wegner et al., 2010) \\
\hline & $\begin{array}{c}1-10 \mathrm{~L}, 11-20 \mathrm{M} \\
>20 \mathrm{H}\end{array}$ & - & - & 161 & 197 & 57 & 115 & 25 & I & $\downarrow$ & $1.0-1.1$ & $<0.007$ & (Merino et al., 1998) \\
\hline & - & - & - & 829 & 371 & - & - & - & $\mathrm{F}$ & ND & ND & NS & (Tang et al.., 2015) \\
\hline & - & $\geq 10$ & $\begin{array}{c}203 \\
\text { (seminal) }\end{array}$ & 175 & 147 & - & - & - & I & ND & ND & NS & (Yu et al., 2014) \\
\hline & $\begin{array}{c}<5 \mathrm{~L}, 5-20 \mathrm{M},>20 \\
\mathrm{H}\end{array}$ & - & - & 775 & 737 & 180 & 327 & 230 & I & ND & ND & NS & (Zhang et al., 2013) \\
\hline & - & $14.1 \pm 7.3$ & - & 314 & 314 & - & - & - & $\mathrm{F}$ & ND & ND & - & (Yu et al., 2013) \\
\hline & - & $14.1 \pm 7.3$ & - & 27 & 30 & - & - & - & $\mathrm{F}$ & ND & ND & - & (Yu et al., 2013) \\
\hline & $\begin{array}{c}1-10 \mathrm{~L}, 11-20 \mathrm{M} \\
21-40 \mathrm{H}\end{array}$ & - & $\begin{array}{c}173.9 \\
\text { (seminal) }\end{array}$ & 30 & 35 & 19 & 11 & 5 & $\mathrm{~F}$ & ND & ND & NS & (Chen and Kuo, 2007) \\
\hline & - & - & - & 337 & 194 & - & - & - & I & ND & ND & NS & (Kunzle et al., 2004) \\
\hline & $\geq 1$ & - & - & 1131 & 655 & - & - & - & I & ND & ND & NS & (Künzle et al., 2003) \\
\hline & $7.70 \pm 5.32$ & - & - & 49 & 81 & - & - & - & $\mathrm{F}$ & ND & ND & NS & (Wang et al., 2001) \\
\hline & - & - & - & 152 & 91 & - & - & - & $\mathrm{F}$ & ND & ND & NS & (Chia et al., 1998) \\
\hline & $9.4 \pm 1.6$ & - & - & 32 & 28 & - & - & - & $\mathrm{F}$ & ND & ND & NS & (Shen et al., 1997) \\
\hline & $1-14 \mathrm{~L}, 15-40 \mathrm{H}$ & - & - & 203 & 147 & 68 & - & 79 & I & ND & ND & NS & (Oldereid et al., 1989) \\
\hline ART Pregnancy Success & $>5$ & - & - & 164 & 137 & - & - & - & I & $\downarrow$ & $1.7-1.8$ & $\begin{array}{c}<0.05- \\
0.01\end{array}$ & (Zitzmann et al., 2003) \\
\hline \multirow[t]{2}{*}{ Pregnancy Rate } & $14.6 \pm 5.5$ & - & - & 21 & 38 & - & - & - & $\mathrm{F} / \mathrm{I}$ & ND & ND & NS & (Jong et al., 2014) \\
\hline & - & - & $\begin{array}{c}>20 \\
\text { (seminal) }\end{array}$ & 118 & 96 & - & - & - & I & ND & ND & NS & (Cinar et al., 2014) \\
\hline Fertilization Rate & - & - & $\begin{array}{c}>20 \\
\text { (seminal) }\end{array}$ & 118 & 96 & - & - & - & I & ND & ND & NS & (Cinar et al., 2014) \\
\hline \multirow[t]{9}{*}{ Spontaneous Abortion } & $<20 \mathrm{~L}, \geq 20 \mathrm{H}$ & - & - & 216 & 310 & 239 & - & 71 & $\mathrm{~F}$ & $\uparrow$ & 1.8 & 0.049 & (Venners et al., 2004) \\
\hline & $\begin{array}{c}1-9 \mathrm{~L}, 10-19 \mathrm{M} \\
>20 \mathrm{H}\end{array}$ & - & - & 358 & 507 & 176 & 170 & 161 & $\mathrm{~F}$ & $\uparrow$ & 1.4 & - & (Zhang and Cai, 1992) \\
\hline & - & - & - & 124 & 62 & - & - & - & $\mathrm{F}$ & $\uparrow$ & 2.2 & 0.029 & (Firns et al., 2015) \\
\hline & - & - & - & 58 & 49 & - & - & - & $\mathrm{F}$ & $\begin{array}{l}\uparrow \\
(\mathrm{N} \\
\mathrm{S})\end{array}$ & 2.9 & NS & (Blanco-Muñozet al., 2009) \\
\hline & - & - & - & 192 & 409 & - & - & - & $\mathrm{F}$ & ND & ND & NS & (Jaleel and Khan, 2013) \\
\hline & $\begin{array}{c}<5-10 \mathrm{~L}, 11-20 \mathrm{M} \\
>20 \mathrm{H}\end{array}$ & - & - & 4419 & 2143 & 669 & 950 & 524 & $\mathrm{~F}$ & ND & ND & NS & (Maconochie et al., 2007) \\
\hline & - & - & - & 7422 & 15898 & - & - & - & $\mathrm{F}$ & ND & ND & NS & $\begin{array}{l}\text { (Nybo Andersen et al., } \\
2004)\end{array}$ \\
\hline & - & - & - & 1318 & 1856 & - & - & - & $\mathrm{F}$ & ND & ND & NS & $\begin{array}{c}\text { (de la Rochebrochard and } \\
\text { Thonneau, 2002) }\end{array}$ \\
\hline & $\leq 10 \mathrm{~L},>10 \mathrm{H}$ & - & - & 975 & 1044 & 385 & - & 659 & $\mathrm{~F}$ & ND & ND & NS & (Chatenoud et al., 1998) \\
\hline
\end{tabular}




\begin{tabular}{|c|c|c|c|c|c|c|c|c|c|c|c|c|c|}
\hline & $\begin{array}{c}1-10 \mathrm{~L}, 11-20 \mathrm{M}, \\
>20 \mathrm{H}\end{array}$ & - & - & 1361 & 526 & 220 & 197 & 109 & $\mathrm{~F}$ & ND & ND & NS & (Windhamet al., 1992) \\
\hline & - & - & - & 172 & 199 & - & - & - & $\mathrm{F}$ & ND & ND & NS & (Taskinen et al., 1989) \\
\hline & $1-10 \mathrm{~L},>10 \mathrm{H}$ & - & - & - & - & - & - & - & $\mathrm{F}$ & ND & ND & NS & (Mau and Netter, 1974) \\
\hline $\begin{array}{l}\text { Anti-apoptotic Gene } \\
\text { Expression }\end{array}$ & - & - & $\begin{array}{c}276.8 \pm \\
107.2 \\
\text { (seminal) }\end{array}$ & 4 & 4 & - & - & - & $\mathrm{F}$ & $\uparrow \downarrow$ & $1.6-3.2$ & $<0.05$ & (Linschooten et al., 2009) \\
\hline \multirow[t]{2}{*}{$\begin{array}{l}\text { Differential miRNA } \\
\text { Expression }\end{array}$} & $13.75(10-20)$ & - & - & 4 & 4 & - & - & - & I & $\uparrow \downarrow$ & $1.3-1.8$ & $<0.01$ & $\begin{array}{l}\text { (Metzler-Guillemain et al., } \\
\text { 2015) }\end{array}$ \\
\hline & - & - & - & 7 & 6 & - & - & - & $\mathrm{F}$ & $\uparrow \downarrow$ & $1.3-1.6$ & $<0.05$ & (Marczylo et al., 2012) \\
\hline \multirow[t]{2}{*}{$\begin{array}{l}\text { Differential Protein } \\
\text { Expression }\end{array}$} & 20 & - & - & 30 & 30 & - & - & - & $\mathrm{F}$ & $\downarrow$ & 1.5 & $<0.05$ & (Chen et al., 2015) \\
\hline & $\geq 10$ & - & - & 20 & 20 & - & - & - & I & $\uparrow \downarrow$ & 1.3 & $<0.05$ & (Antoniassi et al., 2016) \\
\hline
\end{tabular}

Footnotes:

N-S: Non-smokers, S: Smokers

L: Light, M: Medium, H: Heavy

F?: Fertile?

D: Direction

FD: Fold-difference

F: Fertile or assumed fertile (can include couples attending fertility clinic if normospermatic subjects were selected)

I: Declared infertile or attending fertility clinic with no selection for normospermatic subjects

V: Varicocele

ND: No difference; NS: Not significant (note fold difference presented if $\mathrm{FD} \geq 1.5$ or $\mathrm{P}<0.1$ ) 


\section{C.6.2 Supple me ntary Information}

Genetic Disease Burden of Tobacco Smoke:

Intellectual disability disease genes (IDDG; Disability Disease Gene Panel 2.4x

(Vissers et al., 2015)) were examined as a model to understand the disease burden

resulting from paternal tobacco smoke exposure. For this purpose, exon sequences from the disease genes were acquired from BioMart (Kinsella et al., 2011). Using the exon sequences, a Variant Call Format file was generated that represented all potential mutations matching the mutational fingerprint of tobacco smoke (G:C $\rightarrow$ T:A) (IARC, 2012a). Variant Effect Predictor (McLaren et al., 2010) was used to predict the most severe consequence of the $\mathrm{G}: \mathrm{C} \rightarrow \mathrm{T}: \mathrm{A}$ variants representing tobacco smoke mutations (only 1 prediction per nucleotide per transcript):

\begin{tabular}{cc}
\hline Consequence Type & Count \\
splice_donor_variant & 6,088 \\
splice_acceptor_variant & 5,906 \\
stop_gained & 150,325 \\
stop_lost & 22,842 \\
start_lost & 4,778 \\
missense_variant & $1,569,797$ \\
splice_region_variant & 44,266 \\
incomplete_terminal_codon_variant & 121 \\
TOTA_ & $\mathbf{1 , 8 0 4 , 1 2 3}$ \\
synonymous_variant & 386,249 \\
coding_sequence_variant & 46 \\
mature_miRNA_variant & 228 \\
5_prime_UTR_variant & 475,524 \\
3_prime_UTR_variant & $1,328,784$ \\
non_coding_transcript_exon_variant & 45,1593 \\
\hline
\end{tabular}

Based on the Variant Effect Predictor results, there is a maximum of $\underline{1.8 \times 10^{6}}$

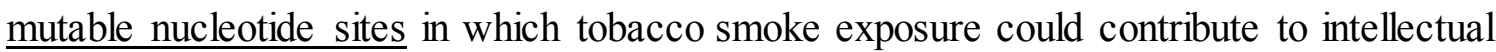


disability. The spontaneous rate of mutation in human gametes is $\sim 1 \times 10^{-8}$ per

nucleotide, and G:C $\rightarrow \mathrm{T}: \mathrm{A}$ mutations represent $10 \%$ of this $=1 \times 10^{-9}$ mutations /

nucleotide. A $25 \%$ increase of the gamete mutation rate would lead to an additional $\underline{2.5 \times}$ $\underline{10^{-10} \text { mutations / nucleotide. }}$

$$
\begin{gathered}
\left(1.8 \times 10^{6} \text { nucleotides }\right) \times\left(2.5 \times 10^{-10} \text { mutations / nucleotide }\right) \\
=0 . \underline{5 \times 10^{-3} \text { mutations per gamete }}
\end{gathered}
$$

It has been demonstrated that mainstream cigarette smoke can induce frameshift mutations (Thorne et al., 2015). There are approximately $4 \times 10^{6}$ nucleotides where an indel can cause a frameshift mutation. Similar to G:C $\rightarrow$ T:A mutations the spontaneous indel rate is $1 \times 10^{-9}$ (Kloosterman et al., 2015) so a $25 \%$ increase in rate would lead to an additional $2.5 \times 10^{-10}$ indels / nucleotides.

$$
\begin{gathered}
\left(4 \times 10^{6} \text { nucleotides }\right) \times\left(2.5 \times 10^{-10} \text { mutations / nucleotide }\right) \\
=\underline{1 \times 10^{-3} \text { mutations per gamete }}
\end{gathered}
$$

Therefore, the increase in the number of IDDG mutations at an individual level would be small. However, at the population level the disease burden is much more apparent. More than 800 million men smoke worldwide (Eriksen et al., 2012), and if on average those men have 2 children each, there would be $1.6 \times 10^{9}$ children born to smoking males.

$$
\begin{gathered}
\left(1.6 \times 10^{9} \text { children }\right) \times\left((1+0.5) \times 10^{-3} \text { mutations } / \text { child }\right) \\
=\mathbf{2 . 4} \times \mathbf{1 0}^{\mathbf{6}} \mathbf{e x t r a} \text { IDDG mutations worldwide }
\end{gathered}
$$

It can be argued that not all missense mutations will lead to loss-of-function (LOF). In this model, $90 \%$ of the base substitutions are missense and base substitutions make up $33 \%$ of the IDDG mutations: $90 \% \times 33 \% \times 2.4$ million $=\underline{0.7 \text { million mutations. }}$. 
As stated in refs. (Boyko et al., 2008; Hurles, 2012), approximately 30\% of missense mutations in human genes will lead to LOF and will be highly deleterious: $30 \% \times 0.7$ million $=\underline{0.2 \text { million mutations }}$.

$$
(0.2+(2.4-0.7)) \times 10^{-3} \text { mutations }
$$

\section{$=1.9 \times 10^{6}$ extra LOF IDDG mutations worldwide}

Approximately $13 \%$ of those mutations will be on the X-chromosome. Hence, $\sim 0.2$ million mutations will be carried by female progeny and will be hemizygous if inherited by males in the F2 generation.

Based on the inheritance pattern of the genes in the IDDG panel (Vissers et al., 2015), $30 \%$ of the 1.9 million mutations will be dominant. Thus, there will be 510,000 autosomal dominant mutations ( $30 \%$ of 1.7 million), 1,190,000 autosomal recessive mutations (70\%), 60,000 X-linked dominant mutations (30\% of 0.2 million), and 140,000 $\mathrm{X}$-linked recessive mutations $(70 \%)$. In total, there will be 570,000 mutations $(30 \%$ of 1.9 million) that will be dominant that can lead to ID. To estimate the healthcare cost of these mutations, we used the lifelong cost of providing care for individuals with Autism Spectrum Disorder or mental retardation. It has been estimated that these ID disorders cost the healthcare system approximately 1 million dollars to treat 1 individual throughout their life (Buescher et al., 2014; Honeycutt et al., 2003), and many of the 570,000 extra mutations can contribute to the development of varying forms of ID.

$$
\begin{gathered}
\left(5.7 \times 10^{5} \text { ID mutations }\right) \times\left(1 \times 10^{6} \text { dollars } / \mathrm{ID} / \text { generation }\right) \\
=\mathbf{5 . 7} \times 10^{11} \text { dollars per generation }
\end{gathered}
$$

